\title{
Enantioselective Assembly of Cycloenones with a Nitrile-Containing All-Carbon Quaternary Center from Malononitriles Enabled by Ni Catalysis
}

Zhiwu Lu, $\uparrow$ Xu-Dong Hu, $\uparrow$ Hui Zhang, Xiao-Wen Zhang, Jinhui Cai, Muhammad Usman, Hengjiang Cong, and Wen-Bo Liu*

Sauvage Center for Molecular Sciences; Engineering Research Center of Organosilicon Compounds \& Materials (Ministry of Education); College of Chemistry and Molecular Sciences, Wuhan University, 299 Bayi Rd, Wuhan 430072, Hubei, China

E-mail: wenboliu@whu.edu.cn

${ }^{\dagger}$ These authors contributed equally to this work

\section{Table of content}

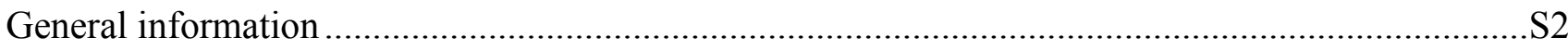

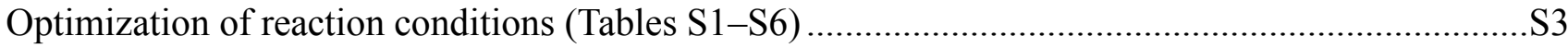

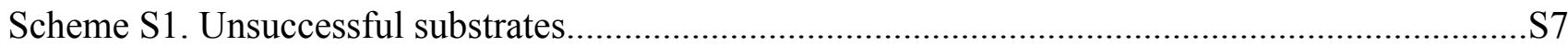

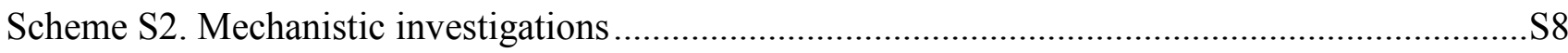

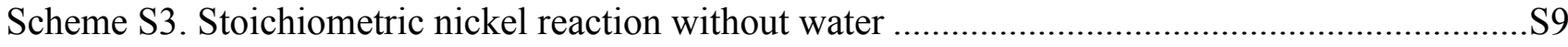

Figure S1. ${ }^{31} \mathrm{P}$ NMR spectra of the ligand, nickel complex, control reactions, and reaction mixture

General procedures for the preparation of substrates 1

General procedure for synthesis of $\mathbf{3}$ by Ni-catalyzed desymmetrization of malononitrile and characterization data

General procedure for synthesis of $\mathbf{4}$ by Ni-catalyzed desymmetrization of malononitrile and characterization data

Natural abundant ${ }^{13} \mathrm{C}$ kinetic isotope effect studies S5 1

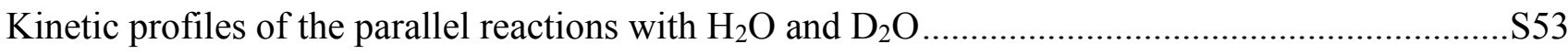

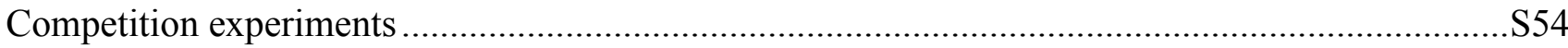

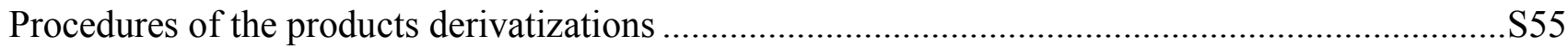

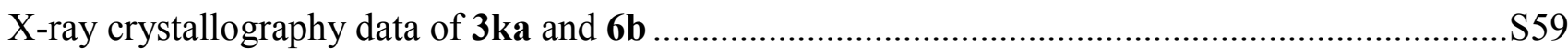

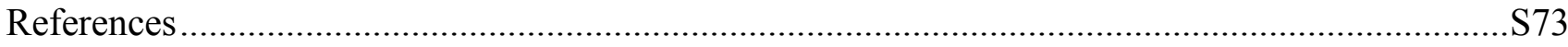

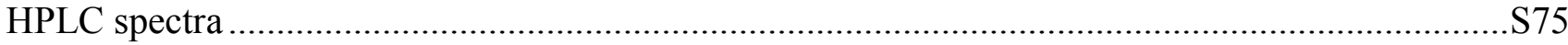

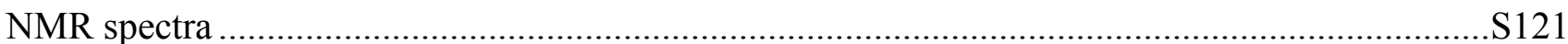




\section{General information}

Unless otherwise stated, all experiments were carried out in oven-dried glassware using argon manifolds or in a glovebox. Reactions were monitored by thin-layer chromatography (TLC). TLC was performed using Huanghai $8 \pm 0.2 \mu \mathrm{m}$ precoated glass plates $(0.25 \mathrm{~mm})$ and visualized by UV fluorescence quenching, $\mathrm{KMnO}_{4}$, p-anisaldehyde, or phosphomolybdic acid staining. Huanghai silica gel (particle size 300 - 400 or 200 - 300 mesh) was used for chromatography. ${ }^{1} \mathrm{H}$ NMR spectra were recorded at room temperature on a Bruker ADVANCE III $400 \mathrm{MHz}$ spectrometer and were reported relative to residual $\mathrm{CDCl}_{3}(\delta 7.26 \mathrm{ppm}) .{ }^{13} \mathrm{C}$ NMR spectra were recorded on a Bruker ADVANCE III $400 \mathrm{MHz}$ spectrometer $(100 \mathrm{MHz})$ and were reported relative to $\mathrm{CDCl}_{3}(\delta 77.16 \mathrm{ppm})$. Data for ${ }^{1} \mathrm{H}$ NMR were reported as follows: chemical shift $(\delta \mathrm{ppm})$ (multiplicity, coupling constant $(\mathrm{Hz})$, integration). Multiplicities were reported as follows: $\mathrm{s}=$ singlet, $\mathrm{d}=$ doublet, $\mathrm{t}=$ triplet, $\mathrm{q}=$ quartet, $\mathrm{m}$ $=$ multiplet. Data for ${ }^{13} \mathrm{C}$ NMR and ${ }^{19}$ F NMR were reported in terms of chemical shifts $(\delta$ ppm $)$. High resolution mass spectra (HRMS) were obtained by use of a Bruker Compact TOF mass spectrometer or a Thermo Scientific Quadrupole-Orbitrap Mass Spectrometer in electrospray ionization mode $\left(\mathrm{ESI}^{+}\right)$. Single crystal diffraction data were collected using a Bruker SMART APEXII diffractometer. Enantiomeric ratio (er) was determined by an Agilent 1260 Series HPLC utilizing DAICEL Chiralpak (AD-H, IB, AS-H or IC) or Chiralcel (OD-H, OJ-H) columns (4.6 mm x $250 \mathrm{~mm})$. Optical rotations

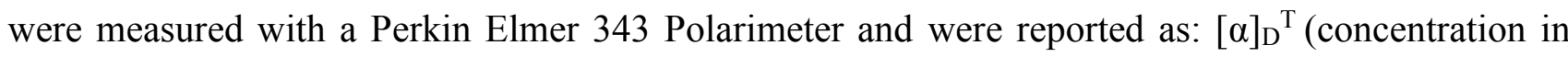
$\mathrm{g} / 100 \mathrm{~mL}$, solvent).

Unless otherwise noted, all chemicals were purchased from Strem, Acros, Alfa Aesar, Adamas-beta, TCI, J\&K, and Energy, and used as received. Petroleum ether (PE, $60 \sim 90{ }^{\circ} \mathrm{C}$ ) was used as eluent for silica gel chromatography. Dry solvents were purchased commercially or were dried by passage through an activated alumina column under argon.

\section{List of abbreviations:}

er - enantiomeric ratio, dr - diastereomeric ratio, PE - petroleum ether, EA - ethyl acetate, HPLC high-performance liquid chromatography, TLC - thin-layer chromatography, THF - tetrahydrofuran, IPA - isopropanol. 


\section{Optimization of reaction conditions (Tables S1-S6)}

A typical procedure for condition optimization: To a resealable Schlenk tube equipped with a magnetic stirring bar were added [Ni] $(0.01 \mathrm{mmol}, 10 \mathrm{~mol} \%)$, ligand $(0.012 \mathrm{mmol}, 12 \mathrm{~mol} \%)$, and 1.0 $\mathrm{mL}$ of solvent in a glovebox. After the resultant mixture was stirred at room temperature for $30 \mathrm{~min}$, substrate 1a (28.5 mg, $0.1 \mathrm{mmol}, 1.0$ equiv), boronic acid $2 \mathbf{a}(24.4 \mathrm{mg}, 0.2 \mathrm{mmol}, 2.0$ equiv), another $1.0 \mathrm{~mL}$ of solvent were added. The tube was sealed with a teflon valve, removed from the glovebox, and then connected to a Schlenk line. Under a positive pressure of argon, the Teflon valve was removed and $\mathrm{H}_{2} \mathrm{O}$ (8 ul, $0.4 \mathrm{mmol}, 4.0$ equiv) was added rapidly. The tube was resealed and stirred at the indicated temperature for the indicated time. Then the resultant mixture was cooled to room temperature, and $1.0 \mathrm{~mL}$ of $\mathrm{HCl}(1 \mathrm{M})$ and $1.0 \mathrm{~mL}$ of EA were added to the tube. After stirred for additional $30 \mathrm{~min}$, the mixture was extracted with EA $(8 \mathrm{~mL}$ x 3). The combined organic phase was washed with brine, dried over $\mathrm{Na}_{2} \mathrm{SO}_{4}$, and filtered. The solvents were removed under reduced pressure to give a crude mixture. The yield was determined by ${ }^{1} \mathrm{H}$ NMR analysis of the crude mixture using 1,3,5-trimethoxybenzene as an internal standard. The er was determined by HPLC analysis (Chiralpak AD-H) after quick separation of the product using prep TLC.

Table S1. Screening of nickel precursors ${ }^{a}$

\begin{tabular}{|c|c|c|c|}
\hline & $\begin{array}{c}\mathrm{PhB}(\mathrm{OH})_{2} \\
2 \mathrm{a}\end{array}$ & لل & $\begin{array}{l}\mathrm{N} \\
\mathrm{Ph} \mathrm{h}_{2} \\
\mathrm{Bu} \text {-phox }\end{array}$ \\
\hline entry & {$[\mathrm{Ni}]$} & yield $(\%)^{b}$ & $\mathrm{er}^{c}$ \\
\hline 1 & $\mathrm{Ni}(\mathrm{OAc})_{2} \bullet 4 \mathrm{H}_{2} \mathrm{O}$ & trace & $74: 26$ \\
\hline 2 & $\mathrm{Ni}(\mathrm{acac})_{2}$ & 78 & $71.5: 28.5$ \\
\hline 3 & $\mathrm{NiCl}_{2} \bullet \mathrm{DME}$ & trace & - \\
\hline 4 & $\mathrm{NiBr}_{2} \bullet \mathrm{DME}$ & 39 & $66.5: 33.5$ \\
\hline 5 & $\mathrm{NiI}_{2}$ & 5 & $66.5: 33.5$ \\
\hline 6 & $\mathrm{Ni}\left(\mathrm{NO}_{3}\right)_{2} \bullet 6 \mathrm{H}_{2} \mathrm{O}$ & 50 & $74.5: 25.5$ \\
\hline 7 & $\mathrm{Ni}\left(\mathrm{ClO}_{4}\right)_{2} \cdot 6 \mathrm{H}_{2} \mathrm{O}$ & 58 & $92.5: 7.5$ \\
\hline 8 & $\mathrm{Ni}(\mathrm{OTf})_{2}$ & $95(93)$ & $94: 6$ \\
\hline
\end{tabular}




\begin{tabular}{cccc}
\hline 9 & $\mathrm{Ni}(\mathrm{OTs})_{2} \bullet 6 \mathrm{H}_{2} \mathrm{O}$ & 84 & $85: 15$ \\
10 & $\mathrm{Ni}\left(\mathrm{BF}_{4}\right)_{2} \cdot 6 \mathrm{H}_{2} \mathrm{O}$ & 73 & $90: 10$ \\
\hline
\end{tabular}

${ }^{a}$ The reactions conducted with [Ni] (10 mol\%), L1 (12 mol\%), 1a $(0.1 \mathrm{mmol}), \mathbf{2 a}\left(0.2 \mathrm{mmol}, 2.0\right.$ equiv) and $\mathrm{H}_{2} \mathrm{O}$ (4.0 equiv) in toluene $\left(1.0 \mathrm{~mL}\right.$ ) at $100^{\circ} \mathrm{C}$ for $14 \mathrm{~h} .{ }^{b}$ Determined by ${ }^{1} \mathrm{H}$ NMR analysis of the crude mixture using $1,3,5-$ trimethoxybenzene as an internal standard with isolated yield in the parentheses. ${ }^{c}$ Determined by HPLC analysis (Chiralpak AD-H).

\section{Table S2. Investigation of ligands ${ }^{a}$}

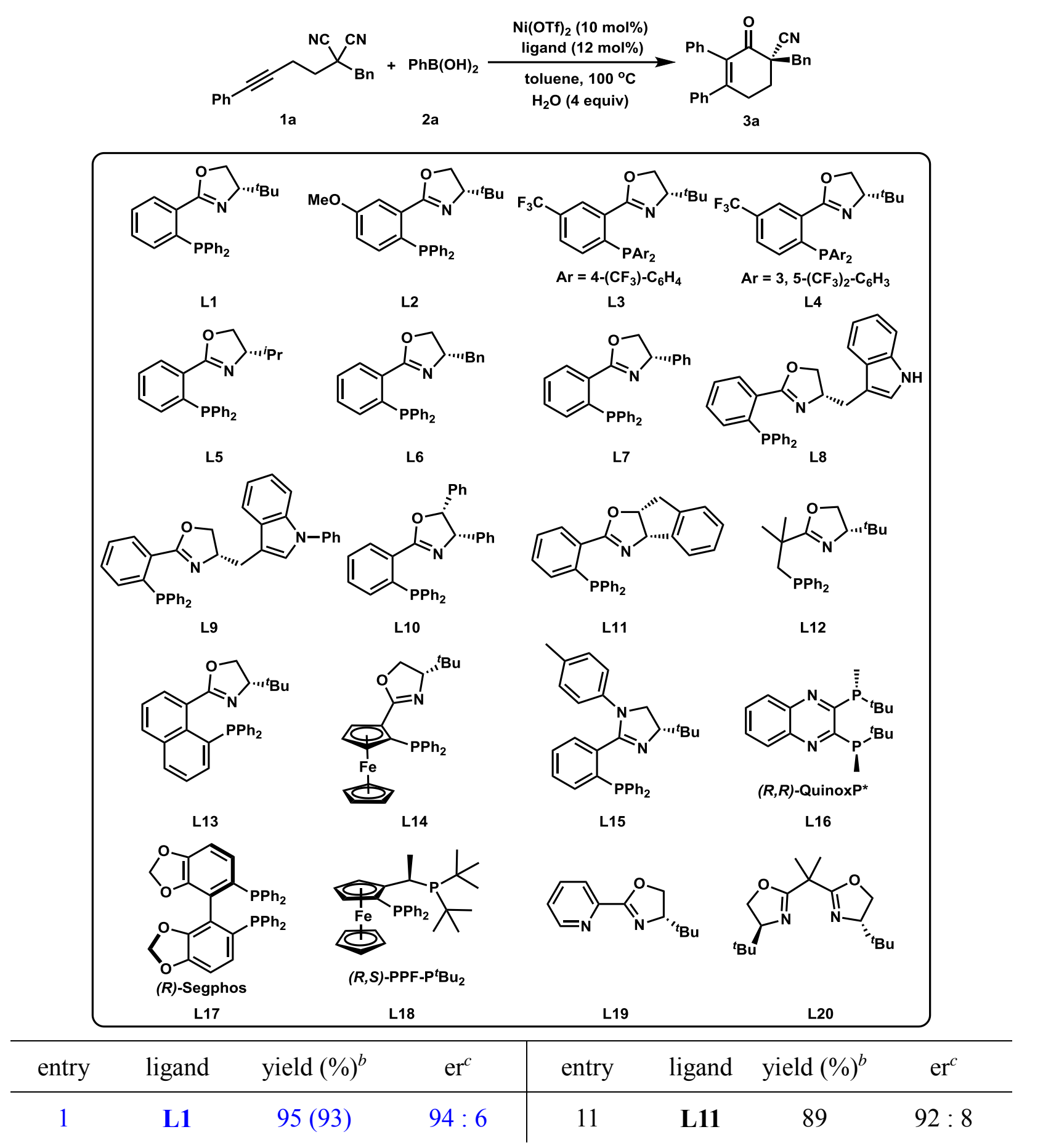




\begin{tabular}{rrrr|cccc}
2 & $\mathbf{L 2}$ & 91 & $94: 6$ & 12 & $\mathbf{L 1 2}$ & 75 & $92.5: 7.5$ \\
3 & $\mathbf{L 3}$ & 69 & $91: 9$ & 13 & $\mathbf{L 1 3}$ & 19 & $24: 76$ \\
4 & $\mathbf{L 4}$ & 43 & $77: 23$ & 14 & $\mathbf{L 1 4}$ & 56 & $85.5: 14.5$ \\
5 & $\mathbf{L 5}$ & 86 & $89: 11$ & 15 & $\mathbf{L 1 5}$ & 73 & $86.5: 13.5$ \\
6 & $\mathbf{L 6}$ & 96 & $90: 10$ & 16 & $\mathbf{L 1 6}$ & 49 & $68: 32$ \\
7 & $\mathbf{L 7}$ & 91 & $94: 6$ & 17 & $\mathbf{L 1 7}$ & 6 & $47.5: 51.5$ \\
8 & $\mathbf{L 8}$ & 94 & $86: 14$ & 18 & $\mathbf{L 1 8}$ & 59 & $34.5: 65.5$ \\
9 & $\mathbf{L 9}$ & 95 & $89.5: 10.5$ & 19 & $\mathbf{L 1 9}$ & 28 & $53: 47$ \\
10 & $\mathbf{L 1 0}$ & 92 & $93.5: 6.5$ & 20 & $\mathbf{L 2 0}$ & $\mathrm{NR}$ & - \\
\hline
\end{tabular}

${ }^{a}$ The reactions conducted with $\mathrm{Ni}(\mathrm{OTf})_{2}(10 \mathrm{~mol} \%)$, ligand $(12 \mathrm{~mol} \%), \mathbf{1 a}(0.1 \mathrm{mmol}), \mathbf{2 a}(0.2 \mathrm{mmol}, 2.0$ equiv) and $\mathrm{H}_{2} \mathrm{O}$ (4.0 equiv) in toluene $(1.0 \mathrm{~mL})$ at $100{ }^{\circ} \mathrm{C}$ for $14 \mathrm{~h} .{ }^{b}$ Determined by ${ }^{1} \mathrm{H}$ NMR analysis of the crude mixture using 1,3,5-trimethoxybenzene as an internal standard standard with isolated yield in the parentheses. ${ }^{c}$ Determined by HPLC analysis (Chiralpak AD-H).

Table S3. Screening of solvents ${ }^{a}$

\begin{tabular}{|c|c|c|c|}
\hline & $\begin{array}{c}\mathrm{N} \\
2 \mathrm{Ph}\end{array}$ & ${ }^{P h}{ }_{11}$ & 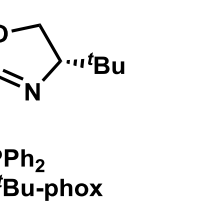 \\
\hline entry & solvent & yield $(\%)^{b}$ & $\mathrm{er}^{c}$ \\
\hline 1 & toluene & $95(93)$ & $94: 6$ \\
\hline 2 & $\mathrm{PhCl}$ & 91 & $83.5: 16.5$ \\
\hline 3 & benzene & 92 & $94: 6$ \\
\hline 4 & $\mathrm{MeCN}$ & 89 & $57: 43$ \\
\hline 5 & dioxane & 95 & $89.5: 10.5$ \\
\hline 6 & THF & 93 & $79: 21$ \\
\hline 7 & DME & 96 & $79.5: 20.5$ \\
\hline 8 & MTBE & 88 & $87: 13$ \\
\hline 9 & DCE & 83 & $62.5: 37.5$ \\
\hline 10 & $\mathrm{CHCl}_{3}$ & 80 & $83: 17$ \\
\hline 11 & cyclohexane & 10 & $93.5: 6.5$ \\
\hline 12 & 2-propanol & $<5$ & - \\
\hline
\end{tabular}




13 DMF trace

${ }^{a}$ The reaction conducted with $\mathrm{Ni}(\mathrm{OTf})_{2}(10 \mathrm{~mol} \%), \mathrm{L} 1(12 \mathrm{~mol} \%), \mathbf{1 a}(0.1 \mathrm{mmol}), \mathbf{2 a}(0.2 \mathrm{mmol}, 2.0$ equiv $)$ and $\mathrm{H}_{2} \mathrm{O}$ (4.0 equiv) in toluene $\left(1.0 \mathrm{~mL}\right.$ ) at $100^{\circ} \mathrm{C}$ for $14 \mathrm{~h} .{ }^{b}$ Determined by ${ }^{1} \mathrm{H}$ NMR analysis of the crude mixture using $1,3,5$ trimethoxybenzene as an internal standard standard with isolated yield in the parentheses. ${ }^{c}$ Determined by HPLC analysis (Chiralpak AD-H).

Table S4. Investigation of the effects of concentration, temperature, and additives ${ }^{a}$

\begin{tabular}{|c|c|c|c|c|c|c|}
\hline & 1a & $\begin{array}{r}B n+P h B(C \\
2 a\end{array}$ & $\begin{array}{c}\mathrm{Ni}(\mathrm{OTf})_{2}(10 \mathrm{~mol} \% \\
\mathrm{L} 1 \text { (12 mol\%) } \\
\text { toluene, temp } \\
\text { additive (20 mol\% } \\
\mathrm{H}_{2} \mathrm{O} \text { (4 equiv) }\end{array}$ & YI & 17 & \\
\hline entry & conc. (M) & additive & temp $\left({ }^{\circ} \mathrm{C}\right)$ & time (h) & yield $(\%)^{b}$ & $\mathrm{er}^{c}$ \\
\hline 1 & 0.1 & - & 100 & 14 & $95(93)$ & $94: 6$ \\
\hline 2 & 0.1 & - & 80 & 15 & $86(89)$ & $94.5: 5.5$ \\
\hline 3 & 0.1 & - & 60 & 15 & $37(41)$ & $95.5: 4.5$ \\
\hline 4 & 0.1 & $\mathrm{Zn}(\mathrm{OTf})_{2}$ & 60 & 14.5 & 72 & $95.5: 4.5$ \\
\hline 5 & 0.1 & $\mathrm{Cu}(\mathrm{OTf})_{2}$ & 60 & 14.5 & N.R & - \\
\hline 6 & 0.1 & $\mathrm{Fe}(\mathrm{OTf})_{2}$ & 60 & 23 & 65 & $95: 5$ \\
\hline 7 & 0.05 & - & 80 & 23 & $92(89)$ & $95: 5$ \\
\hline 8 & 0.033 & - & 80 & 23 & 91 & $95.5: 4.5$ \\
\hline
\end{tabular}

${ }^{a}$ The reaction conducted with $\mathrm{Ni}(\mathrm{OTf})_{2}(10 \mathrm{~mol} \%), \mathbf{L 1}(12 \mathrm{~mol} \%), \mathbf{1 a}(0.1 \mathrm{mmol}), \mathbf{2 a}(0.2 \mathrm{mmol}, 2.0$ equiv), additive (20 mmol\%, if added), and $\mathrm{H}_{2} \mathrm{O}$ (4.0 equiv) in toluene (indicated volume) at the indicated temperature for the indicated time. ${ }^{b}$ Determined by ${ }^{1} \mathrm{H}$ NMR analysis of the crude mixture using 1,3,5-trimethoxybenzene as an internal standard with isolated yields in the parentheses. ${ }^{c}$ Determined by HPLC analysis (Chiralpak AD-H).

Table S5. Examination of the catalyst loading and Ni/L ratio ${ }^{a}$

\begin{tabular}{|c|c|c|c|c|c|}
\hline & $\mathcal{N}^{N C}{ }_{B n}^{C N}+$ & $\begin{array}{c}\mathrm{Ni}(\mathrm{OTf})_{2}(\mathrm{x} \mathrm{mo} \\
\mathrm{L} 1(\mathrm{y} \mathrm{mol} \% \\
\text { toluene, tem } \\
\mathrm{H}_{2} \mathrm{O}(4 \text { equi } \\
20-23 \mathrm{~h}\end{array}$ & I & $\mathrm{L} 1=(S$ & \\
\hline entry & $\mathrm{Ni}(\mathrm{OTf})_{2}(\mathrm{x} \mathrm{mol} \%)$ & L1 (y mol\%) & temp $\left({ }^{\circ} \mathrm{C}\right)$ & yield $(\%)^{b}$ & $\mathrm{er}^{c}$ \\
\hline 1 & $10 \mathrm{~mol} \%$ & $12 \mathrm{~mol} \%$ & 80 & $92(89)$ & $95: 5$ \\
\hline 2 & $10 \mathrm{~mol} \%$ & $22 \mathrm{~mol} \%$ & 80 & 96 & $95.5: 4.5$ \\
\hline 3 & $10 \mathrm{~mol} \%$ & $5 \mathrm{~mol} \%$ & 80 & 84 & $94.5: 5.5$ \\
\hline
\end{tabular}




$\begin{array}{cccccc}4 & 5 \mathrm{~mol} \% & 6 \mathrm{~mol} \% & 80 & 78(82) & 94.5: 5.5 \\ 5 & 2.5 \mathrm{~mol} \% & 3 \mathrm{~mol} \% & 80 & 50 & 94.5: 5.5 \\ 6 & 5 \mathrm{~mol} \% & 6 \mathrm{~mol} \% & 100 & 81(86) & 94: 6 \\ 7^{d} & 10 \mathrm{~mol} \% & 12 \mathrm{~mol} \% & 80 & 50 & 94: 6\end{array}$

${ }^{a}$ The reaction conducted with $\mathrm{Ni}(\mathrm{OTf})_{2}(\mathrm{x} \mathrm{mol} \%), \mathbf{L 1}(\mathrm{y} \mathrm{mol} \%), \mathbf{1 a}(0.1 \mathrm{mmol}), \mathbf{2 a}\left(0.2 \mathrm{mmol}, 2.0\right.$ equiv), and $\mathrm{H}_{2} \mathrm{O}$ (4.0 equiv) in toluene $(2.0 \mathrm{~mL})$ at the indicated temperature for $23 \mathrm{~h} .{ }^{b}$ Determined by ${ }^{1} \mathrm{H}$ NMR analysis of the crude mixture using 1,3,5-trimethoxybenzene as an internal standard with isolated yields in the parentheses. ${ }^{c}$ Determined by HPLC analysis (Chiralpak AD-H). ${ }^{d}$ Under air.

Table S6. Investigation of the effect of water ${ }^{a}$

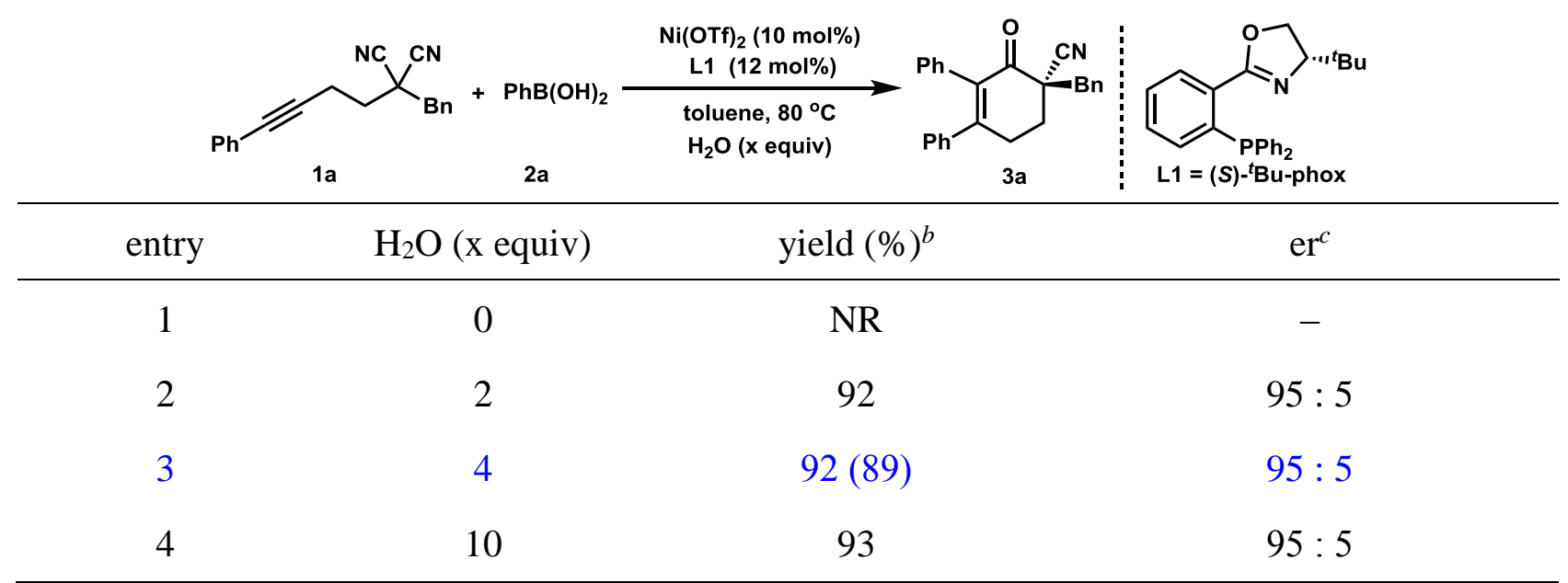

${ }^{a}$ The reaction conducted with $\mathrm{Ni}(\mathrm{OTf})_{2}(10 \mathrm{~mol} \%), \mathbf{L 1}(12 \mathrm{~mol} \%), \mathbf{1 a}(0.1 \mathrm{mmol}), \mathbf{2 a}\left(0.2 \mathrm{mmol}, 2.0\right.$ equiv) and $\mathrm{H}_{2} \mathrm{O}$ (x equiv) in toluene $(2.0 \mathrm{~mL})$ at $80{ }^{\circ} \mathrm{C}$ for $24 \mathrm{~h} .{ }^{b}$ Determined by ${ }^{1} \mathrm{H}$ NMR analysis of the crude mixture using $1,3,5-$ trimethoxybenzene as an internal standard with isolated yield in parentheses. ${ }^{c}$ Determined by HPLC analysis (Chiralpak AD-H).

\section{Scheme S1. Unsuccessful substrates}<smiles>COc1cccnc1</smiles> 


\section{Scheme S2. Mechanistic investigations}

a. Competition experiments of arylboronic acids

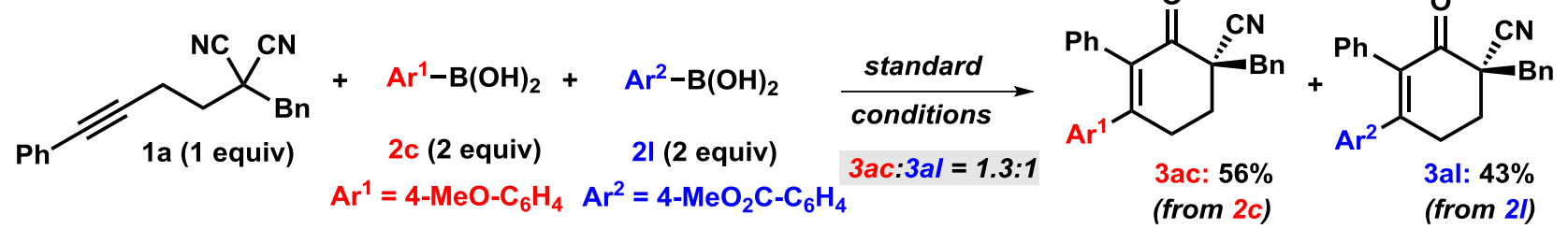

b. Competition experiments of alkynes

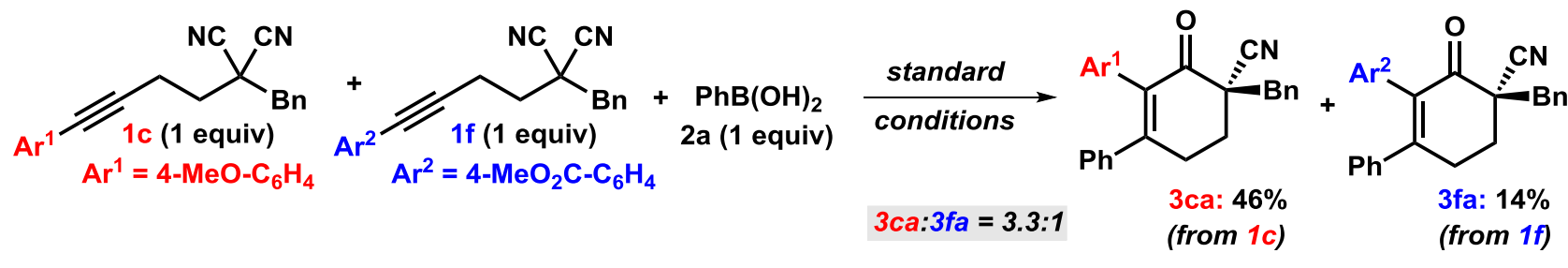

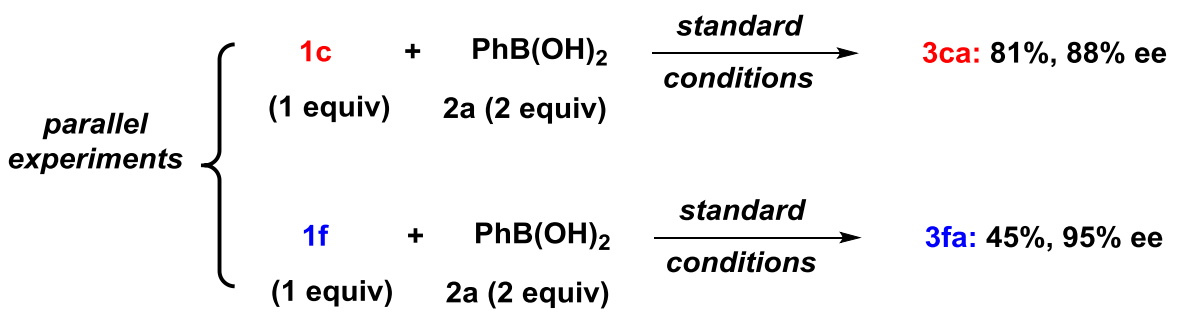

C. Parallel reactions with $\mathrm{H}_{2} \mathrm{O}$ and $\mathrm{D}_{2} \mathrm{O}$

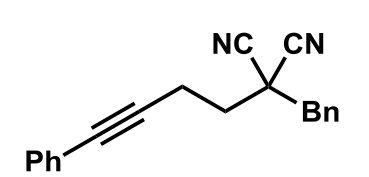

$1 \mathrm{a}$

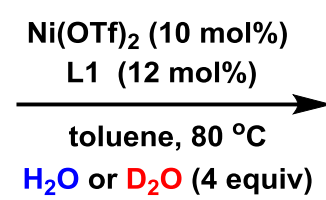

$5 a$

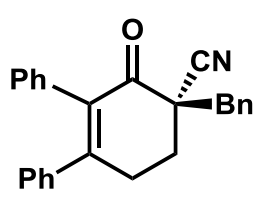

3aa

(I): with $\mathrm{H}_{2} \mathrm{O}$ (4 equiv): $\frac{\mathrm{42 \%}(1 \mathrm{~h}) ; 66 \%(2 \mathrm{~h})}{43 \%(1 \mathrm{~h}) ; 69 \%(2 \mathrm{~h})}$

(II): with $\mathrm{D}_{2} \mathrm{O}$ (4 equiv): $43 \%(1 \mathrm{~h}) ; 69 \%(2 \mathrm{~h})$

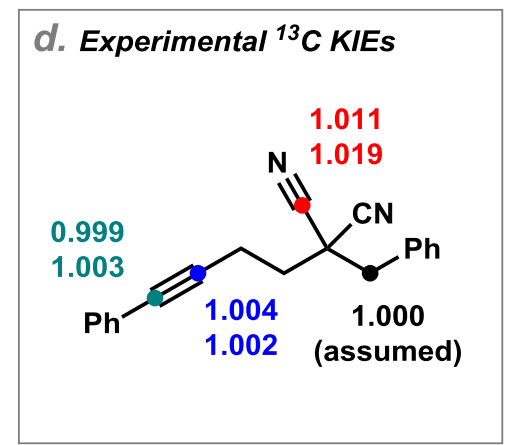

To get insights into the possible reaction pathways, a number of experiments were conducted. Recently, Sanford et al. observed that under base-free conditions, transmetalation of an aryl nickel fluoride complex with electron-rich aryl boronic acids is slower than with the electron-deficient ones. ${ }^{1}$ In our chemistry, adding equal amounts of methoxy (2c) and ester (2l) substituted aryl boronic acids resulted in major product 3ac, formed from the more electron-rich boronic acid (Scheme S2a). Second, the alkyne insertion into the aryl-nickel species was investigated by subjecting equal amounts of methoxy (1c) and ester (1f) substituted aryl alkynes to the reaction conditions (Scheme S2b). The ratio of the corresponding products $\mathbf{3 c a}$ and $\mathbf{3 f a}$ was determined as 3.3:1, showing that the electron-rich alkyne reacts faster. The same trend was observed in the corresponding parellel competition reactions. Third, 
parallel experiments with $\mathrm{H}_{2} \mathrm{O}$ and $\mathrm{D}_{2} \mathrm{O}$ were performed using $(\mathrm{PhBO})_{3}$ without the observation of kinetic isotopic effect (Scheme S2c). Finally, ${ }^{13} \mathrm{C}$ KIE studies of the substrate 1a at natural abundance were carried out. A significant nitrile carbon ${ }^{13} \mathrm{C}$ KIE was observed by comparing the changes in ${ }^{13} \mathrm{C}$ isotopic composition of the recovered 1a to the original starting material 1a (Scheme S2d). This result implies that the addition to the nitrile group is most likely involved in the rate-determing step (RDS). However, transmetalation as the RDS can not be ruled out at this stage. Attempts to study the kinetic behavior of aryl boronic acids by in situ NMR or IR failed due to the poor solubility of the catalyst in situ formed (see Figure S1).

\section{Scheme S3. Stoichiometric nickel reaction without water}
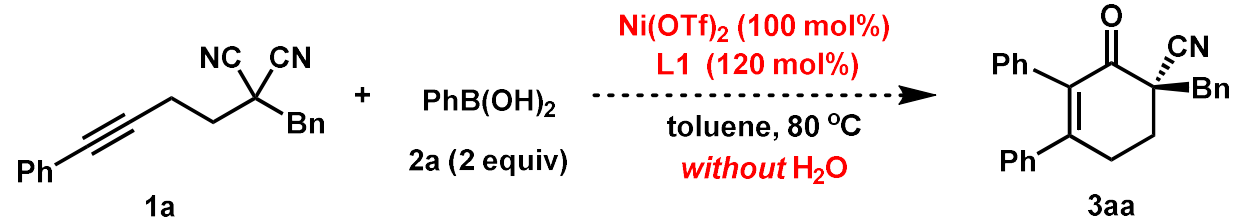

no reaction

\section{Scheme S4. An alternative proposed mechanism}

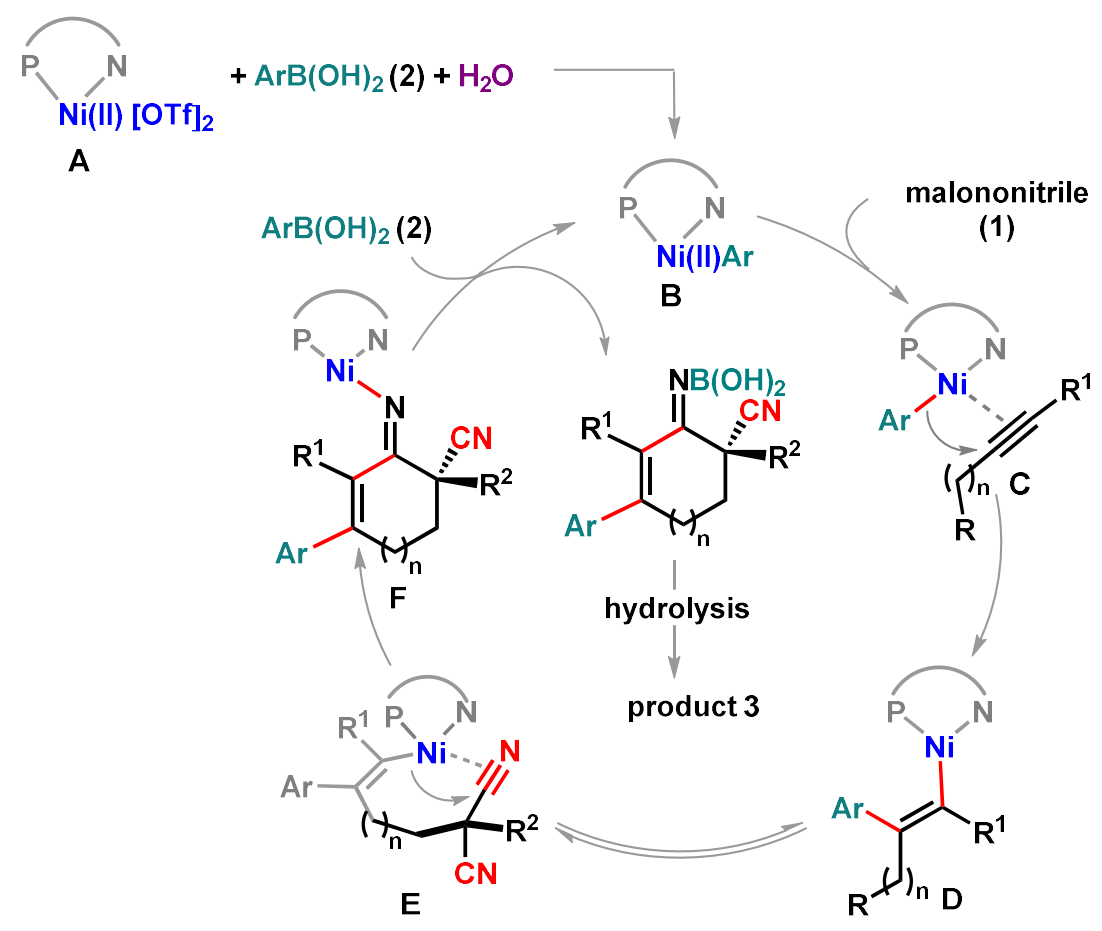


Figure S1. ${ }^{31} \mathrm{P}$ NMR spectra of the ligand, nickel complex, control reactions, and

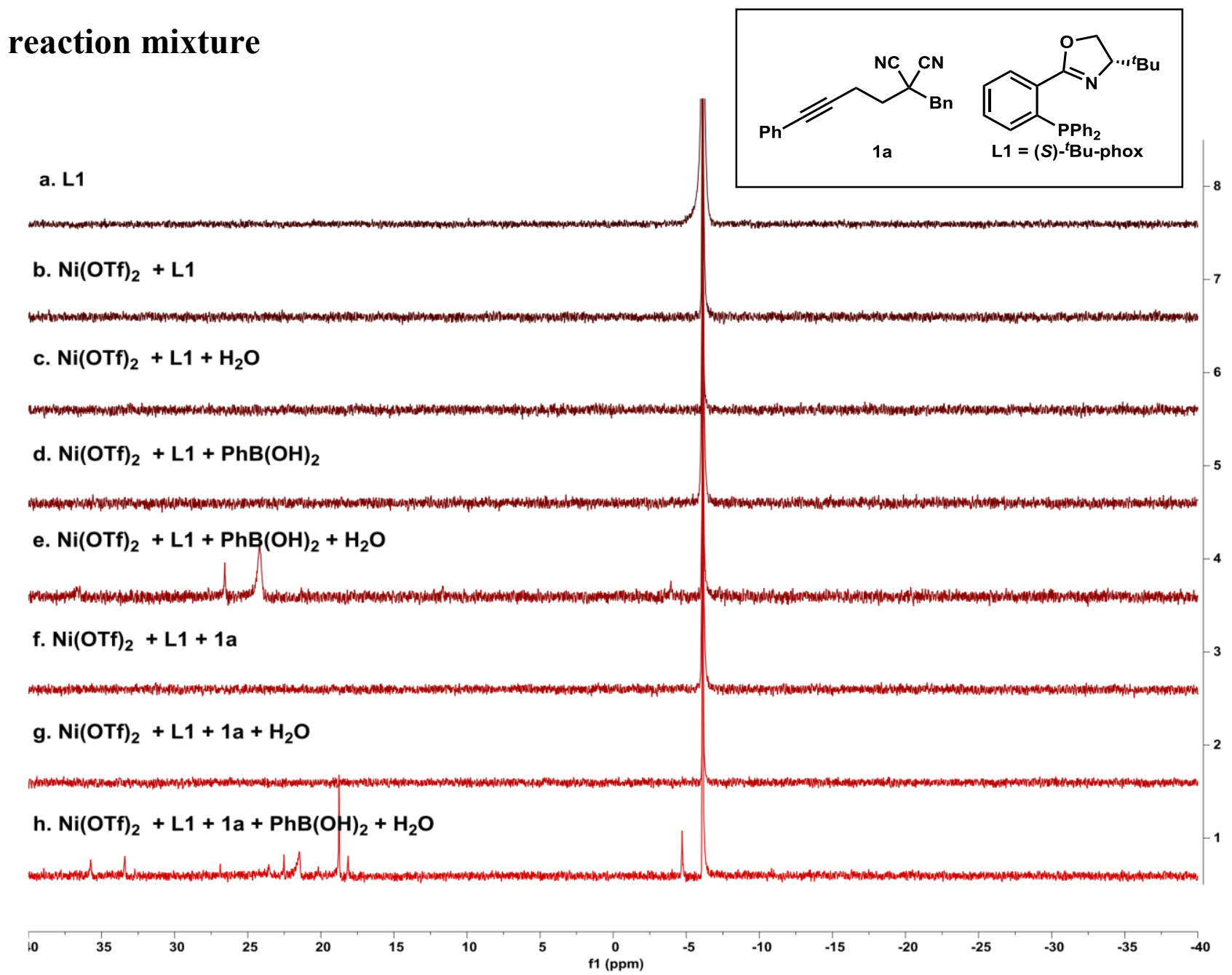

a) ${ }^{31}$ P NMR spectrum of ligand L1: L1 (ca $5.0 \mathrm{mg}$ ) was dissolved in $0.6 \mathrm{~mL}$ of toluene in a NMR tube and the spectrum was measured at room temperature $(-6.12 \mathrm{ppm})$.

b) ${ }^{31} \mathrm{P}$ NMR spectrum of in situ formed nickel complex: $\mathrm{Ni}(\mathrm{OTf})_{2}(3.6 \mathrm{mg}, 0.01 \mathrm{mmol}), \mathbf{L 1}$ (4.7 $\mathrm{mg}, 0.012 \mathrm{mmol})$ and $1.0 \mathrm{~mL}$ of toluene were added to a resealable Schlenk tube under argon. The tube was sealed, and stirred at room temperature for $20 \mathrm{~min}$. A suspension solution was obtained. The mixture was stirred at $80{ }^{\circ} \mathrm{C}$ for 45 minutes, the suspension solution remained. After cooled to room temperature, the mixture was transferred to a $J$-Young NMR tube and only the trace of the ligand was observed by ${ }^{13} \mathrm{P}$ NMR, indicating that the in situ formed nickel complex is likely insoluble in toluene. Indeed, this solid in the suspension solution was isolated by centrifugation in a scale-up reaction $(0.2 \mathrm{mmol})$, dissolved in $\mathrm{CD}_{3} \mathrm{CN}$, and characterized by ${ }^{1} H$ NMR (Figure S2), ${ }^{31}$ P NMR (Figure S3), and ${ }^{19}$ F NMR (Figure S4). 
c) ${ }^{31}$ P NMR spectrum of in situ formed nickel complex with the addition of water: To a in situ formed nickel complex suspension solution prepared according b) was added $\mathrm{H}_{2} \mathrm{O}(8 \mathrm{uL}$, $0.4 \mathrm{mmol}$ ) and the mixture stirred at $80{ }^{\circ} \mathrm{C}$ for $45 \mathrm{~min}$. After cooled to room temperature, the mixture was added to a $J$-Young NMR tube. A spectrum identical to b) was obtained.

d) ${ }^{31} \mathrm{P}$ NMR spectrum of in situ formed nickel complex with the addition $\mathrm{PhB}(\mathrm{OH})_{2}$ : To a in situ formed nickel complex suspension solution prepared according b) was added $\mathrm{PhB}(\mathrm{OH})_{2}$ (24.4 mg, $0.2 \mathrm{mmol}$ ) and the mixture was stirred at $80{ }^{\circ} \mathrm{C}$ for $45 \mathrm{~min}$. After cooled to room temperature, the mixture was added to a $J$-Young NMR tube. No new peak was detected which indicates that transmetalation does not occur under those conditions.

e) ${ }^{31} \mathrm{P}$ NMR spectrum of in situ formed nickel complex with the addition $\mathrm{PhB}(\mathrm{OH})_{2}$ and water: To a in situ formed nickel complex suspension solution prepared according $b$ ) were added $\mathrm{PhB}(\mathrm{OH})_{2}(24.4 \mathrm{mg}, 0.2 \mathrm{mmol})$ and $\mathrm{H}_{2} \mathrm{O}(8 \mathrm{uL}, 0.4 \mathrm{mmol})$. The mixture was stirred at $80{ }^{\circ} \mathrm{C}$ for $45 \mathrm{~min}$. After cooled to room temperature, the solution was added to a $J$-Young NMR tube. A new broad peak at $24.20 \mathrm{ppm}$ was observed, which indicates that the transmetalation may take place in the presence of water.

f) and g) ${ }^{31}$ P NMR spectra of the control experiments with the addition of substrate 1a and with or without water: To a in situ formed nickel complex suspension solution prepared according b) was added $1 \mathbf{a}(28.4 \mathrm{mg}, 0.1 \mathrm{mmol})$ and the mixture was stirred at $80{ }^{\circ} \mathrm{C}$ for 45 min. After cooled to room temperature, the mixture was added to a $J$-Young NMR tube to measure the ${ }^{31} \mathrm{P}$ NMR. For $\mathrm{g}$ ), the same procedure was followed except the addition of water ( $8 \mathrm{uL}, 0.4 \mathrm{mmol})$. In both cases, no new peak was observed.

h) ${ }^{31}$ P NMR spectrum of the reaction mixture: To a in situ formed nickel complex suspension solution prepared according b) were added $1 \mathrm{a}(28.4 \mathrm{mg}, 0.1 \mathrm{mmol}), \mathrm{PhB}(\mathrm{OH})_{2}(24.4 \mathrm{mg}, 0.2$ mmol) and $\mathrm{H}_{2} \mathrm{O}(8 \mathrm{uL}, 0.4 \mathrm{mmol})$. The mixture was stirred at $80{ }^{\circ} \mathrm{C}$ for $45 \mathrm{~min}$. After cooled to room temperature, the mixture was added to a $J$-Young NMR tube and a complex ${ }^{31} \mathrm{P}$ NMR spectrum of the mixture was obtained with major new peaks at 21.51 (br), 18.75 and -6.12 ppm. 
Figure S2. ${ }^{1} \mathrm{H}$ NMR spectra of $\mathrm{L} 1$ and in situ formed nickel complex in $\mathrm{CD}_{3} \mathrm{CN}$.<smiles>CC(C)(C)[C@H]1COC(c2ccccc2-c2ccccc2)=N1</smiles>
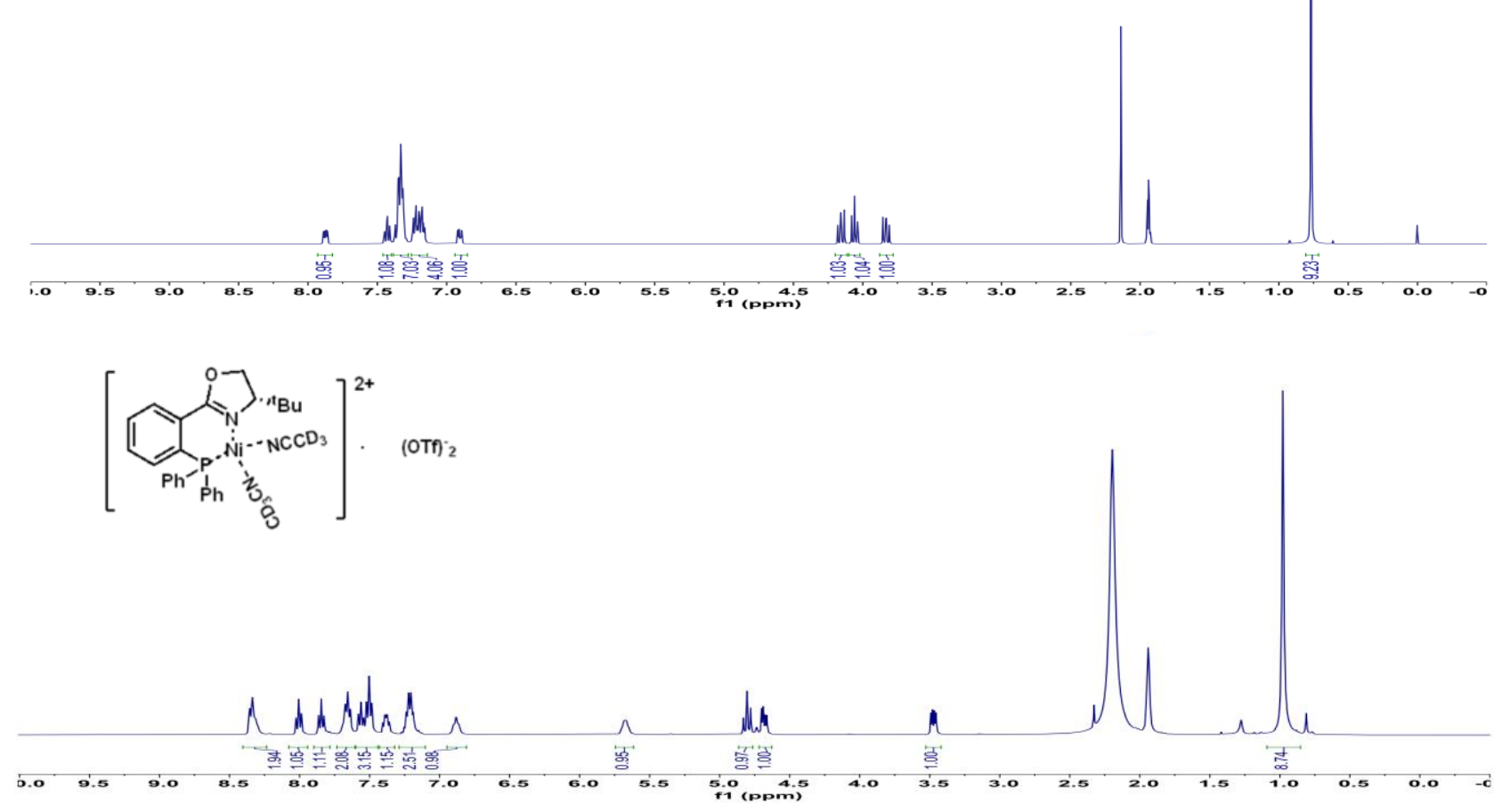

Figure S3. ${ }^{31} \mathrm{P}$ NMR spectra of $\mathrm{L} 1$ and in situ formed nickel complex in $\mathrm{CD}_{3} \mathrm{CN}$.

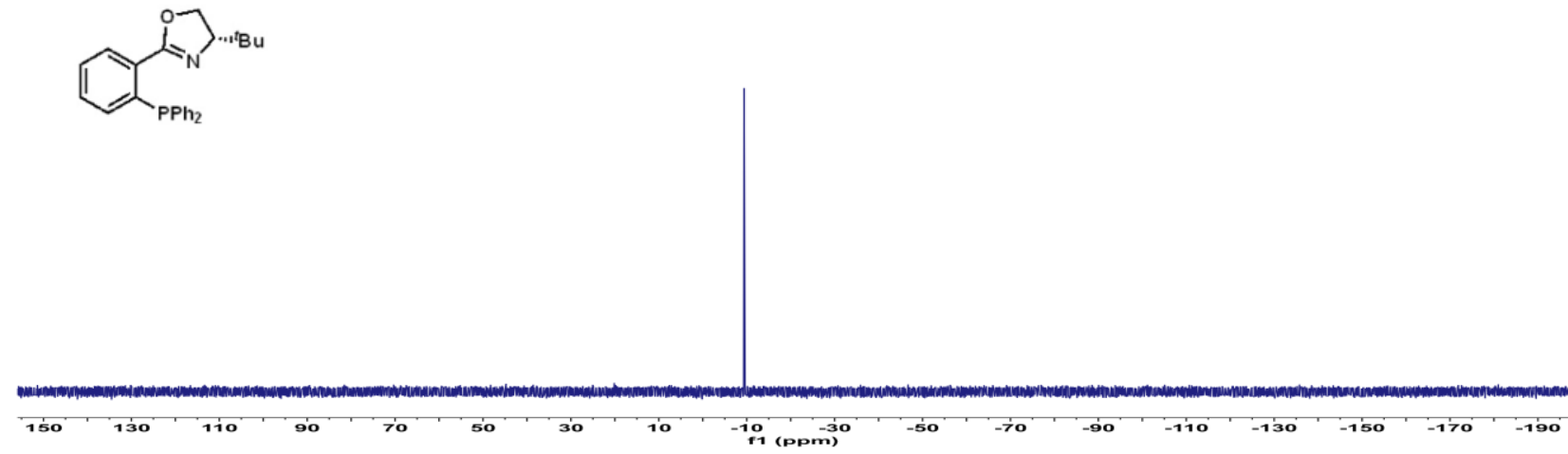

$$
[\underbrace{}_{(\mathrm{OTf})_{2}}
$$

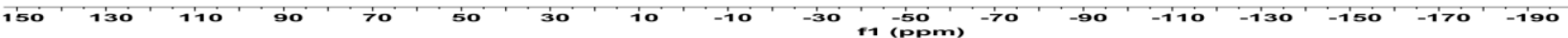


Figure S4. ${ }^{19} \mathrm{~F}$ NMR spectra of in situ formed nickel complex in $\mathrm{CD}_{3} \mathrm{CN}$.

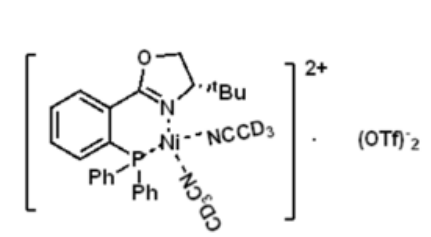

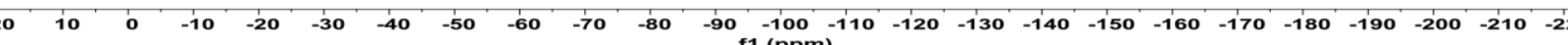

\section{General procedures for the preparation of substrates 1}

Synthesis of 2-benzylmalononitrile (S1)

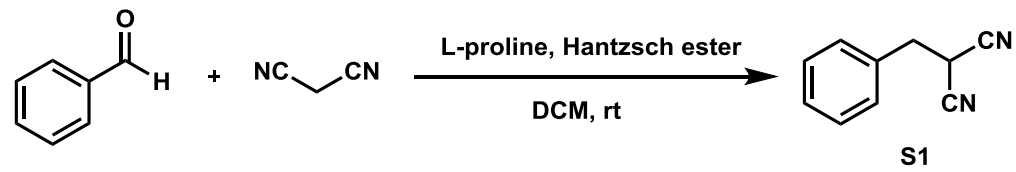

To a solution of benzaldehyde $(31.84 \mathrm{~g}, 300.0 \mathrm{mmol}, 3.0$ equiv) in DCM (150 mL) were added malononitrile (6.61 g, $100.0 \mathrm{mmol}, 1.0$ equiv), $L$-proline ( $2.31 \mathrm{~g}, 20.0 \mathrm{mmol}, 0.2$ equiv), and Hantzsch ester (25.33 g, $100.0 \mathrm{mmol}, 1.0$ equiv) sequentially at room temperature. The mixture was stirred at the room temperature for $12 \mathrm{~h}$. The solvent was removed under reduced pressure to give a crude mixture. The crude mixture was purified by flash column chromatography to give the desired product S1 (15.58 g, 99\% yield) as a white solid. $R_{f}=0.4(\mathrm{PE}: \mathrm{EA}=5: 1) .{ }^{1} \mathbf{H}$ NMR $\left(400 \mathrm{MHz}, \mathrm{CDCl}_{3}\right) \delta$ $7.44-7.37(\mathrm{~m}, 3 \mathrm{H}), 7.35-7.32(\mathrm{~m}, 2 \mathrm{H}), 3.91(\mathrm{t}, J=6.9 \mathrm{~Hz}, 1 \mathrm{H}), 3.29(\mathrm{~d}, J=6.9 \mathrm{~Hz}, 2 \mathrm{H})$. The NMR spectra are in agreement with those reported in the literature. ${ }^{2}$

\section{Synthesis of (4-bromobut-1-yn-1-yl)benzene (S3)}

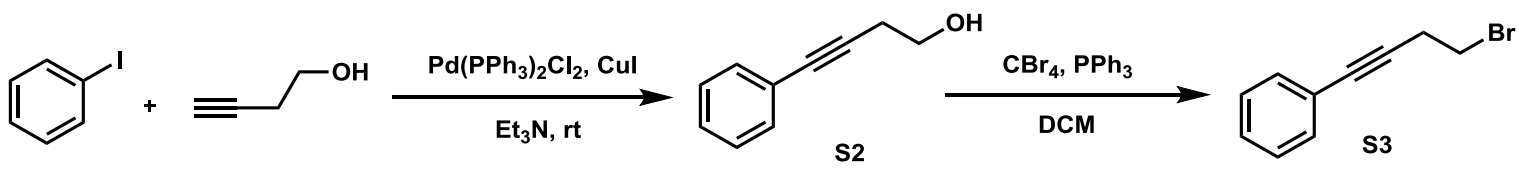

To a Schlenk flask with a magnetic stirring bar were added CuI $(95.3 \mathrm{mg}, 0.5 \mathrm{mmol}, 1 \mathrm{~mol} \%)$, $\mathrm{Pd}\left(\mathrm{PPh}_{3}\right)_{2} \mathrm{Cl}_{2}(350.9 \mathrm{mg}, 0.5 \mathrm{mmol}, 1 \mathrm{~mol} \%)$ and $15 \mathrm{~mL} \mathrm{Et}_{3} \mathrm{~N}$ at room temperature. After the resulting solution was stirred at room temperature for $15 \mathrm{~min}$, iodobenzene $(11.22 \mathrm{~g}, 55.0 \mathrm{mmol}$, $1.1 \mathrm{equiv})$, but-3-yn-1-ol (3.51 g, $50.0 \mathrm{mmol}, 1.0$ equiv), and another $15 \mathrm{~mL}^{\text {of }} \mathrm{Et}_{3} \mathrm{~N}$ were added. The mixture 
was stirred at the room temperature for $10 \mathrm{~h}$. Then the resultant mixture was concentrated, filtered through a celite pad, and washed with EA. The solvents were removed under reduced pressure to give a crude mixture. The crude mixture was purified by flash column chromatography to give the desired product S2 (7.22 g, 99\% yield) as a colorless oil. $R_{f}=0.3(\mathrm{PE}: \mathrm{EA}=2: 1) .{ }^{1} \mathbf{H}$ NMR $(400 \mathrm{MHz}$, $\left.\mathrm{CDCl}_{3}\right) \delta 7.43-7.39(\mathrm{~m}, 2 \mathrm{H}), 7.31-7.27(\mathrm{~m}, 3 \mathrm{H}), 3.82(\mathrm{t}, J=6.3 \mathrm{~Hz}, 2 \mathrm{H}), 2.69(\mathrm{t}, J=6.3 \mathrm{~Hz}, 2 \mathrm{H})$, $1.89(\mathrm{~s}, 1 \mathrm{H})$. The NMR spectra are in agreement with those reported in the literature. ${ }^{3}$

To a solution of above synthesized 4-phenylbut-3-yn-1-ol S2 (7.16 g, 49 mmol, 1.0 equiv) in DCM (150 mL) were added $\mathrm{PPh}_{3}$ (16.06 g, 61.25 mmol, 1.25 equiv) and $\mathrm{CBr}_{4}$ (19.31g, 58.8 mmol, 1.2 equiv) sequentially at $0{ }^{\circ} \mathrm{C}$. The mixture was stirred at the room temperature for $3 \mathrm{~h}$. The solvents were removed under reduced pressure to give a crude mixture. The crude mixture was purified by flash column chromatography to give the desired product $\mathbf{S 3}(15.58 \mathrm{~g}, 99 \%$ yield $)$ as a colorless oil. $R_{f}=0.8$ $(\mathrm{PE}: \mathrm{EA}=10: 1) .{ }^{1} \mathbf{H}$ NMR $\left(400 \mathrm{MHz}, \mathrm{CDCl}_{3}\right) \delta 7.44-7.37(\mathrm{~m}, 2 \mathrm{H}), 7.31-7.28(\mathrm{~m}, 3 \mathrm{H}), 3.53(\mathrm{t}, J$ $=7.4 \mathrm{~Hz}, 2 \mathrm{H}), 2.98(\mathrm{t}, J=7.4 \mathrm{~Hz}, 2 \mathrm{H})$. The NMR spectra are in agreement with those reported in the literature. $^{4}$

\section{Synthesis of 2-benzyl-2-(but-3-yn-1-yl)malononitrile (S5)}

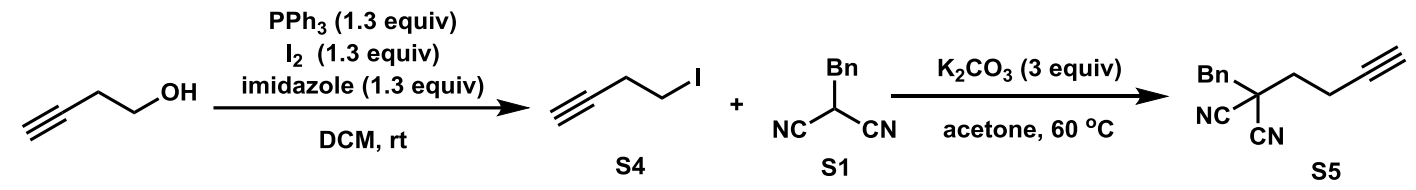

A mixture of $\mathrm{PPh}_{3}(33.81 \mathrm{~g}, 129.0 \mathrm{mmol}, 1.29$ equiv), imidazole (8.87 g, $130.0 \mathrm{mmol}, 1.3$ equiv) in DCM $(150 \mathrm{~mL})$ were added to a $500 \mathrm{~mL}$ round bottom flask. The mixture was stirred at room temperature until the solid was dissolved completely. Subsequently, iodine (33.05 g, $130.0 \mathrm{mmol}, 1.3$ equiv) was added slowly to the flask at $0{ }^{\circ} \mathrm{C}$. After the solution was stirred for about $10 \mathrm{~min}$ at $0{ }^{\circ} \mathrm{C}$, 3-butyn-1-ol (7.15 g, $100.0 \mathrm{mmol}, 1.0$ equiv) was added dropwise over $5 \mathrm{~min}$, then, the resultant mixture was warmed up to room temperature and stirred for another $4 \mathrm{~h}$. The mixture was filtered and washed with DCM (100 mL). The filtrate was concentrated carefully in an ice bath (caution: volatile) to give a residue which was purified by flash column chromatography to provide the desired product S4 (12.15 g, 68\% yield) as a colorless oil. $R_{f}=0.4(\mathrm{PE}: \mathrm{EA}=50: 1) .{ }^{1} \mathrm{H} \mathrm{NMR}\left(400 \mathrm{MHz}, \mathrm{CDCl}_{3}\right) \delta$ $3.22(\mathrm{t}, J=7.3 \mathrm{~Hz}, 2 \mathrm{H}), 2.77(\mathrm{td}, J=7.3,2.6 \mathrm{~Hz}, 2 \mathrm{H}), 2.15(\mathrm{t}, J=2.6 \mathrm{~Hz}, 1 \mathrm{H})$. The NMR data was compared to the reported literature. ${ }^{5}$ 
A $100 \mathrm{~mL}$ Schlenk tube were added with 2-benzylmalononitrile (1.22 g, $7.7 \mathrm{mmol}, 1$ equiv), 4-iodobut1-yne S4 (2.81 g, $15.4 \mathrm{mmol}, 2$ equiv), $\mathrm{K}_{2} \mathrm{CO}_{3}$ (3.23 g, $23.1 \mathrm{mmol}, 3.0$ equiv) and acetone (30 mL). The tube was sealed and the mixture was stirred at $60{ }^{\circ} \mathrm{C}$ for $18 \mathrm{~h}$. After cooled to room temperature, the mixture was filtered and washed with EA $(50 \mathrm{~mL})$. The filtrates were concentrated and the residue was purified by silica gel chromatography to give the desired product S5 (1.54 g, 96\% yield) as a white solid. $R_{f}=0.4(\mathrm{PE}: \mathrm{EA}=10: 1)$. ${ }^{1} \mathbf{H}$ NMR $\left(400 \mathrm{MHz}, \mathrm{CDCl}_{3}\right) \delta 7.43-7.36(\mathrm{~m}, 5 \mathrm{H}), 3.26(\mathrm{~s}, 2 \mathrm{H})$, $2.65-2.60(\mathrm{~m}, 2 \mathrm{H}), 2.25-2.20(\mathrm{~m}, 2 \mathrm{H}), 2.09(\mathrm{t}, J=2.6 \mathrm{~Hz}, 1 \mathrm{H}) .{ }^{13} \mathbf{C}$ NMR $\left(100 \mathrm{MHz}, \mathrm{CDCl}_{3}\right) \delta$ 131.6, 130.4, 129.2, 129.1, 114.7, 80.2, 71.1, 43.5, 38.7, 36.1, 15.7. HRMS (ESI $\left.{ }^{+}\right) m / z$ calc'd for $\mathrm{C}_{14} \mathrm{H}_{12} \mathrm{~N}_{2} \mathrm{Na}[\mathrm{M}+\mathrm{Na}]^{+}: 231.0893$, found 231.0891 .

General procedure for the synthesis of substrates $1 \mathrm{a}$ and $1 \mathbf{j}-1 \mathbf{u}$ (general procedure $A$ )

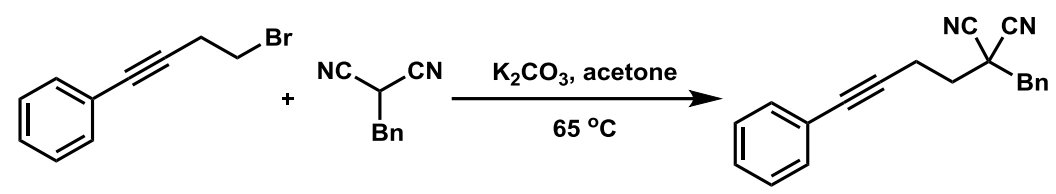

Procedure A: To a Schlenk flask equipped with a magnetic stirring bar were added (4-bromobut-1yn-1-yl) benzene (3.14 g, 15.0 mmol, 1.2 equiv), 2-benzyl malononitrile (1.95 g, 12.5 mmol, 1.0 equiv), $\mathrm{K}_{2} \mathrm{CO}_{3}(6.22 \mathrm{~g}, 45.0 \mathrm{mmol}, 3.0$ equiv), and $30 \mathrm{~mL}$ of acetone at room temperature. The flask was sealed and the mixture was stirred at the $65^{\circ} \mathrm{C}$ for $12 \mathrm{~h}$. Then the mixture was cooled to room temperature, filtered through a celite pad, and washed with EA $(10 \mathrm{~mL} \times 3)$. The solvents were removed under reduced pressure to give a crude mixture. The crude mixture was purified by flash column chromatography to give the desired product 2-benzyl-2-(4-phenylbut-3-yn-1-yl)malononitrile (1a) (3.29 g, 91\% yield) as a yellow solid. $R_{f}=0.5(\mathrm{PE}: \mathrm{EA}=5: 1) .{ }^{1} \mathbf{H}$ NMR $\left(400 \mathrm{MHz}, \mathrm{CDCl}_{3}\right) \delta 7.45$ - $7.38(\mathrm{~m}, 7 \mathrm{H}), 7.32-7.28(\mathrm{~m}, 3 \mathrm{H}), 3.29$ (s, 2H), $2.88-2.84(\mathrm{~m}, 2 \mathrm{H}), 2.32-2.28(\mathrm{~m}, 2 \mathrm{H}) .{ }^{13} \mathbf{C}$ NMR $\left(100 \mathrm{MHz} \mathrm{CDCl}_{3}\right) \delta 131.8,131.7,130.4,129.2,129.1,128.5,128.4,123.0,114.8,85.5,83.0,43.6$, 38.7, 36.2, 16.7. HRMS $\left(\mathrm{ESI}^{+}\right) \mathrm{m} / z$ calc'd for $\mathrm{C}_{20} \mathrm{H}_{16} \mathrm{~N}_{2} \mathrm{Na}[\mathrm{M}+\mathrm{Na}]^{+}: 307.1206$, found 307.1205.

General procedure for the synthesis of substrates $1 \mathrm{~b}-1 \mathrm{i}$ and $1 \mathrm{v}$ (general procedure B)

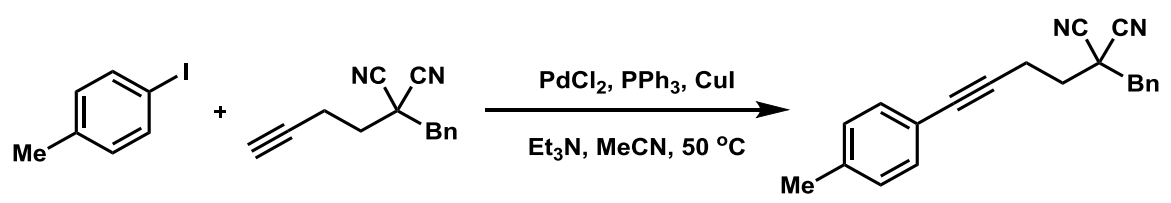

Procedure B: To a Schlenk tube equipped with a magnetic stirring bar were added CuI (29.8 mg, 0.11 mmol, $3.6 \mathrm{~mol} \%$ ), $\mathrm{PdCl}_{2}$ (19.6 mg, $\left.0.1 \mathrm{mmol}, 2.5 \mathrm{~mol} \%\right), \mathrm{PPh}_{3}(40.5 \mathrm{mg}, 0.15 \mathrm{mmol}, 5 \mathrm{~mol} \%)$, and 10 
$\mathrm{mL}$ of acetonitrile at room temperature. After the mixture was stirred at room temperature for $15 \mathrm{~min}$, 1-iodo-4 methylbenzene (722.0 mg, 3.3 mmol, 1.1 equiv), 2-benzyl-2-(but-3-yn-1-yl) malononitrile (626.5 mg, $3.0 \mathrm{mmol}, 1.0$ equiv), $\mathrm{Et}_{3} \mathrm{~N}(1.5 \mathrm{~mL}, 11.9 \mathrm{mmol}, 3$ equiv), and another $10 \mathrm{~mL}$ of acetonitrile were added. The mixture was stirred at $50{ }^{\circ} \mathrm{C}$ for $10 \mathrm{~h}$. Then the mixture was filtered through a celite pad and washed with EA ( $5 \mathrm{ml} \times 3)$. The solvents were removed under reduced pressure to give a crude mixture. The crude mixture was purified by flash column chromatography to give the desired product 2-benzyl-2-(4-(p-tolyl)but-3-yn-1-yl)malononitrile (1b) $\left(773.9 \mathrm{mg}, 91 \%\right.$ yield) as a white solid. $R_{f}$ $=0.5(\mathrm{PE}: \mathrm{EA}=10: 1) .{ }^{1} \mathbf{H}$ NMR $\left(400 \mathrm{MHz}, \mathrm{CDCl}_{3}\right) \delta 7.44-7.38(\mathrm{~m}, 5 \mathrm{H}), 7.30(\mathrm{~d}, J=8.0 \mathrm{~Hz}, 2 \mathrm{H})$, $7.10(\mathrm{~d}, J=7.9 \mathrm{~Hz}, 2 \mathrm{H}), 3.29(\mathrm{~s}, 2 \mathrm{H}), 2.84(\mathrm{t}, J=7.6 \mathrm{~Hz}, 2 \mathrm{H}), 2.34(\mathrm{~s}, 3 \mathrm{H}), 2.29$ (t, $J=8.2 \mathrm{~Hz}, 2 \mathrm{H})$. ${ }^{13} \mathrm{C}$ NMR $\left(100 \mathrm{MHz}, \mathrm{CDCl}_{3}\right) \delta 138.5,131.8,131.6,130.4,129.21,129.19,129.1,119.9,114.8,84.8$, 83.1, 43.6, 38.7, 36.3, 21.6, 16.7. HRMS $\left(\mathrm{ESI}^{+}\right) \mathrm{m} / z$ calc'd for $\mathrm{C}_{21} \mathrm{H}_{18} \mathrm{~N}_{2} \mathrm{Na}[\mathrm{M}+\mathrm{Na}]^{+}: 321.1362$, found 321.1351.

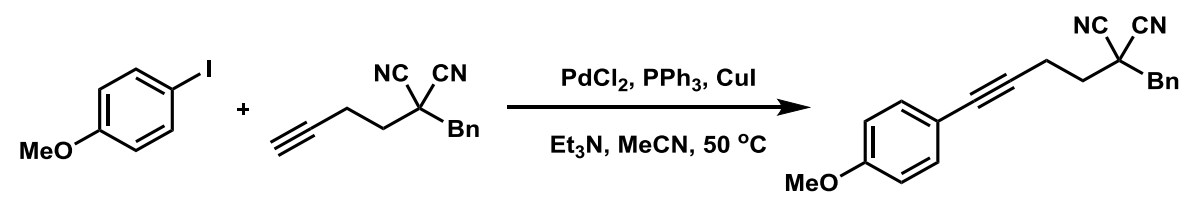

\section{2-Benzyl-2-(4-(4-methoxyphenyl)but-3-yn-1-yl)malononitrile (1c)}

Following the general procedure $\mathrm{B}$, the desired product $1 \mathrm{c}$ was obtained in $83 \%$ yield $(487.6 \mathrm{mg})$ as a white solid from $\mathrm{CuI}(18.9 \mathrm{mg}, 0.07 \mathrm{mmol}, 3.6 \mathrm{~mol} \%), \mathrm{PdCl}_{2}$ (8.0 mg, $\left.0.05 \mathrm{mmol}, 2.5 \mathrm{~mol} \%\right), \mathrm{PPh}_{3}$ (24.1 mg, $0.1 \mathrm{mmol}, 5 \mathrm{~mol} \%$ ), 1-iodo-4- methoxybenzene (492.2 mg, $2.2 \mathrm{mmol}, 1.1$ equiv), 2-benzyl2-(but-3-yn-1-yl) malononitrile (408.2 mg, 2.0 mmol, 1.0 equiv), Et $3 \mathrm{~N}$ (1.0 mL, 7.9 mmol, 3.0 equiv), and $8 \mathrm{~mL}$ of acetonitrile. $R_{f}=0.2(\mathrm{PE}: \mathrm{EA}=20: 1) .{ }^{1} \mathbf{H} \mathbf{N M R}\left(400 \mathrm{MHz}, \mathrm{CDCl}_{3}\right) \delta 7.44-7.39(\mathrm{~m}$, 5H), $7.38-7.33(\mathrm{~m}, 2 \mathrm{H}), 6.82(\mathrm{~d}, J=8.7 \mathrm{~Hz}, 2 \mathrm{H}), 3.80(\mathrm{~s}, 3 \mathrm{H}), 3.29(\mathrm{~s}, 2 \mathrm{H}), 2.87-2.82(\mathrm{t}, J=7.8$ $\mathrm{Hz}, 2 \mathrm{H}), 2.30-2.27(\mathrm{t}, J=8.4 \mathrm{~Hz}, 2 \mathrm{H}) .{ }^{13} \mathbf{C} \mathbf{N M R}\left(100 \mathrm{MHz}, \mathrm{CDCl}_{3}\right) \delta 159.6,133.1,131.8,130.4$, 129.2, 129.0, 115.1, 114.9, 114.1, 84.0, 82.8, 55.4, 43.6, 38.7, 36.3, 16.6. HRMS $\left(\mathrm{ESI}^{+}\right) \mathrm{m} / z$ calc'd for $\mathrm{C}_{21} \mathrm{H}_{18} \mathrm{~N}_{2} \mathrm{ONa}[\mathrm{M}+\mathrm{Na}]^{+}: 337.1311$, found 337.1314.

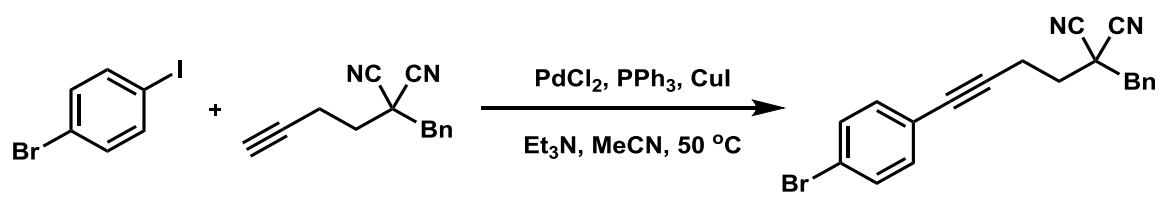

2-Benzyl-2-(4-(4-bromophenyl)but-3-yn-1-yl)malononitrile (1d) 
Following the general procedure B, the desired product 1d was obtained in 97\% yield (1.06 $\mathrm{g})$ as a yellow solid from CuI (25.6 mg, $0.11 \mathrm{mmol}, 3.6 \mathrm{~mol} \%$ ), $\mathrm{PdCl}_{2}$ (15.2 mg, $\left.0.08 \mathrm{mmol}, 2.5 \mathrm{~mol} \%\right), \mathrm{PPh}_{3}$ (39.9 mg, $0.15 \mathrm{mmol}, 5 \mathrm{~mol} \%$ ), 1-bromo-4-iodobenzene (1.02 g, $3.6 \mathrm{mmol}, 1.2$ equiv), 2-benzyl-2(but-3-yn-1-yl) malononitrile (622.9 mg, $3.0 \mathrm{mmol}, 1.0$ equiv), Et $3 \mathrm{~N}$ (1.5 mL, 11.9 mmol, 3.0 equiv), and $10 \mathrm{~mL}$ of acetonitrile. $R_{f}=0.4(\mathrm{PE}: \mathrm{EA}=20: 1) .{ }^{1} \mathbf{H} \mathbf{N M R}\left(400 \mathrm{MHz}, \mathrm{CDCl}_{3}\right) \delta 7.44-7.38(\mathrm{~m}$, $7 \mathrm{H}), 7.27(\mathrm{~d}, J=7.6 \mathrm{~Hz}, 2 \mathrm{H}), 3.29(\mathrm{~s}, 2 \mathrm{H}), 2.84(\mathrm{t}, J=7.7 \mathrm{~Hz}, 2 \mathrm{H}), 2.28(\mathrm{t}, J=8.0 \mathrm{~Hz}, 2 \mathrm{H}) .{ }^{13} \mathbf{C} \mathbf{N M R}$ $\left(100 \mathrm{MHz}, \mathrm{CDCl}_{3}\right) \delta 133.2,131.71,131.65,130.4,129.2,129.1,122.6,121.9,114.8,86.7,82.1,43.7$, 38.7, 36.0, 16.7. HRMS $\left(\mathrm{ESI}^{+}\right) \mathrm{m} / z$ calc'd for $\mathrm{C}_{20} \mathrm{H}_{15} \mathrm{BrN}_{2} \mathrm{Na}[\mathrm{M}+\mathrm{Na}]^{+}: 385.0311$, found 385.0298.

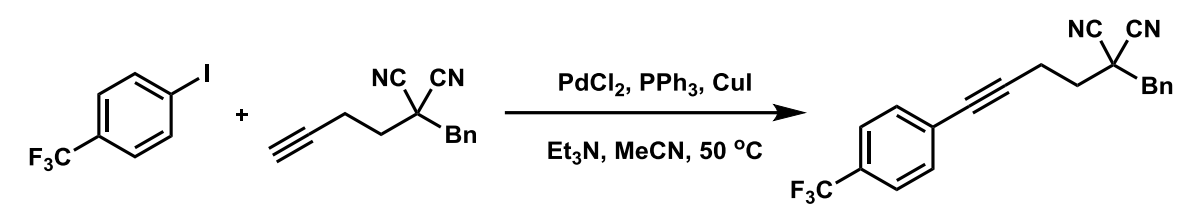

\section{2-Benzyl-2-(4-(4-(trifluoromethyl)phenyl)but-3-yn-1-yl)malononitrile (1e)}

Following the general procedure $\mathrm{B}$, the desired product $1 \mathrm{e}(0.86 \mathrm{~g})$ was obtained in $66 \%$ yield as a white solid from CuI (25.4 mg, $0.13 \mathrm{mmol}, 3.6 \mathrm{~mol} \%$ ), $\mathrm{PdCl}_{2}$ (16.3 mg, $\left.0.10 \mathrm{mmol}, 2.5 \mathrm{~mol} \%\right), \mathrm{PPh}_{3}$ (49.0 mg, $0.20 \mathrm{mmol}, 5 \mathrm{~mol} \%$ ), 2-benzyl -2-(but-3-yn-1-yl) malononitrile (771.0 mg, $3.7 \mathrm{mmol}$ ) and 1-iodo-4-(trifluoromethyl) benzene (2.07 g, $7.4 \mathrm{mmol}), \mathrm{Et}_{3} \mathrm{~N}(1.5 \mathrm{~mL}, 11.1 \mathrm{mmol}, 3$ equiv), and 10 mL of acetonitrile. $R_{f}=0.3(\mathrm{PE}: \mathrm{EA}=10: 1) .{ }^{1} \mathbf{H} \mathbf{~ N M R}\left(400 \mathrm{MHz}, \mathrm{CDCl}_{3}\right) \delta 7.56(\mathrm{~d}, J=8.4 \mathrm{~Hz}, 2 \mathrm{H})$, $7.51(\mathrm{~d}, J=8.4 \mathrm{~Hz}, 2 \mathrm{H}), 7.45-7.38(\mathrm{~m}, 5 \mathrm{H}), 3.30(\mathrm{~s}, 2 \mathrm{H}), 2.88(\mathrm{t}, J=8.0 \mathrm{~Hz}, 2 \mathrm{H}), 2.30(\mathrm{t}, J=7.6 \mathrm{~Hz}$, 2H). ${ }^{13}$ C NMR $\left(100 \mathrm{MHz}, \mathrm{CDCl}_{3}\right) \delta 132.0,131.6,130.4,130.1$ (q, $\left.J=33.0 \mathrm{~Hz}\right), 129.22,129.14,126.8$, $125.7(\mathrm{q}, J=275.6 \mathrm{~Hz}), 125.4(\mathrm{q}, J=3.7 \mathrm{~Hz}), 114.8,88.1,81.9,43.7,38.6,35.9,16.7 .{ }^{19} \mathrm{~F}$ NMR $(376$ $\left.\mathrm{MHz}, \mathrm{CDCl}_{3}\right) \delta-62.83$. HRMS $\left(\mathrm{ESI}^{+}\right) \mathrm{m} / z$ calc'd for $\mathrm{C}_{21} \mathrm{H}_{15} \mathrm{~F}_{3} \mathrm{~N}_{2} \mathrm{Na}[\mathrm{M}+\mathrm{Na}]^{+}:$375.1080, found 375.1080 .

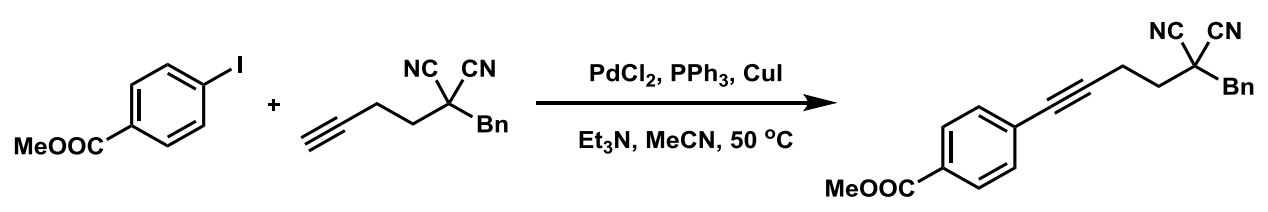

\section{Methyl 4-(5,5-dicyano-6-phenylhex-1-yn-1-yl)benzoate (1f)}

Following the general procedure B, the desired product 1 f was obtained in 56\% yield $(574.7 \mathrm{mg})$ as a yellow solid from CuI (24.3 mg, $0.11 \mathrm{mmol}, 3.6 \mathrm{~mol} \%), \mathrm{PdCl}_{2}(14.2 \mathrm{mg}, 0.08 \mathrm{mmol}, 2.5 \mathrm{~mol} \%), \mathrm{PPh}_{3}$ (44.6 mg, $0.17 \mathrm{mmol}, 6 \mathrm{~mol} \%$ ), methyl 4-iodobenzoate (863.5 mg, $3.3 \mathrm{mmol}, 1.1$ equiv), 2-benzyl-2(but-3-yn-1-yl) malononitrile (627.9 mg, $3.0 \mathrm{mmol}, 1.0$ equiv), $\mathrm{Et}_{3} \mathrm{~N}$ (1.5 mL, 11.9 mmol, 3.0 equiv), 
and $20 \mathrm{~mL}$ of acetonitrile. $R_{f}=0.2(\mathrm{PE}: \mathrm{EA}=10: 1) .{ }^{1} \mathbf{H} \mathbf{~ N M R}\left(400 \mathrm{MHz}, \mathrm{CDCl}_{3}\right) \delta 7.96-7.87(\mathrm{~m}$, 2H), $7.48-7.45(\mathrm{~m}, 2 \mathrm{H}), 7.43-7.39(\mathrm{~m}, 5 \mathrm{H}), 3.91(\mathrm{~s}, 3 \mathrm{H}), 3.29(\mathrm{~s}, 2 \mathrm{H}), 2.90-2.86(\mathrm{~m}, 2 \mathrm{H}), 2.32-$ 2.28 (m, 2H). ${ }^{13} \mathbf{C}$ NMR (100 MHz, $\left.\mathrm{CDCl}_{3}\right) \delta 166.7,131.7,131.6,130.4,129.7,129.6,129.2,129.1$, 127.7, 114.8, 88.7, 82.4, 52.4, 43.7, 38.8, 36.0, 16.7. HRMS $\left(\mathrm{ESI}^{+}\right) \mathrm{m} / z$ calc'd for $\mathrm{C}_{22} \mathrm{H}_{18} \mathrm{~N}_{2} \mathrm{O}_{2} \mathrm{Na}$ $[\mathrm{M}+\mathrm{Na}]^{+}: 365.1260$, found 365.1252 .

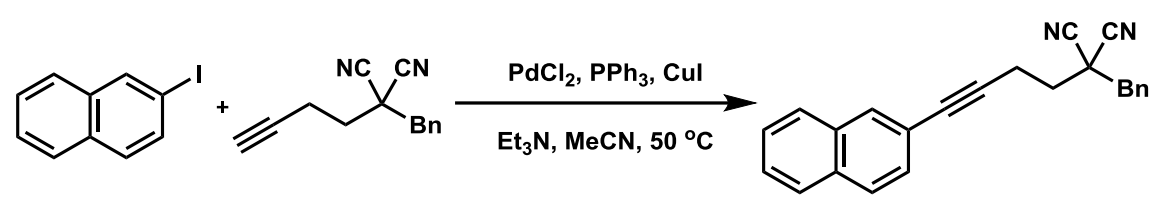

\section{2-Benzyl-2-(4-(naphthalen-2-yl)but-3-yn-1-yl)malononitrile (1g)}

Following the general procedure B, the desired product $1 \mathrm{~g}$ was obtained in 39\% yield (390.6 mg) as a yellow solid from CuI (25.9 mg, $0.11 \mathrm{mmol}, 3.6 \mathrm{~mol} \%$ ), $\mathrm{PdCl}_{2}$ (15.3 mg, $0.08 \mathrm{mmol}, 2.5 \mathrm{~mol} \%$ ), $\mathrm{PPh}_{3}$ (49.5 mg, $0.19 \mathrm{mmol}, 6 \mathrm{~mol} \%$ ), 2-iodonaphthalene (690.9 mg, $3.3 \mathrm{mmol}, 1.1$ equiv), 2-benzyl-2-(but3-yn-1-yl)malononitrile S5 (625.7 mg, $3.0 \mathrm{mmol}, 1.0$ equiv), Et $3 \mathrm{~N}$ (1.5 mL, $11.9 \mathrm{mmol}, 3.0$ equiv), and $20 \mathrm{~mL}$ of acetonitrile. $R_{f}=0.6(\mathrm{PE}: \mathrm{EA}=5: 1) .{ }^{1} \mathbf{H} \mathbf{N M R}\left(400 \mathrm{MHz}, \mathrm{CDCl}_{3}\right) \delta 7.93(\mathrm{~s}, 1 \mathrm{H}), 7.86$ - $7.76(\mathrm{~m}, 3 \mathrm{H}), 7.51-7.40(\mathrm{~m}, 8 \mathrm{H}), 3.31(\mathrm{~s}, 2 \mathrm{H}), 2.93-2.89(\mathrm{~m}, 2 \mathrm{H}), 2.36-2.32(\mathrm{~m}, 2 \mathrm{H}) .{ }^{13} \mathbf{C}$ NMR $\left(100 \mathrm{MHz}, \mathrm{CDCl}_{3}\right) \delta 133.0,132.9,131.7,131.6,130.5,129.2,129.1,128.5,128.1,127.9,127.8,126.8$, 126.7, 120.3, 114.8, 85.8, 83.4, 43.7, 38.8, 36.3, 16.8. HRMS $\left(\mathrm{ESI}^{+}\right) \mathrm{m} / z$ calc'd for $\mathrm{C}_{24} \mathrm{H}_{18} \mathrm{~N}_{2} \mathrm{Na}$ $[\mathrm{M}+\mathrm{Na}]^{+}:$357.1362, found 357.1355 .

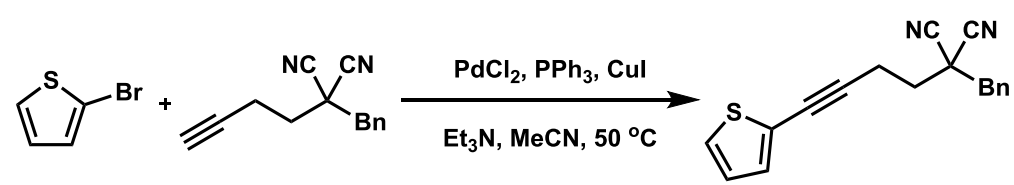

\section{2-Benzyl-2-(4-(thiophen-2-yl)but-3-yn-1-yl)malononitrile (1h)}

Following the general procedure B, the desired product $\mathbf{1 h}$ was obtained in $79 \%$ yield $(0.69 \mathrm{~g})$ as a white solid from CuI (20.5 mg, $0.11 \mathrm{mmol}, 3.6 \mathrm{~mol} \%), \mathrm{PdCl}_{2}(13.7 \mathrm{mg}, 0.08 \mathrm{mmol}, 2.5 \mathrm{~mol} \%), \mathrm{PPh}_{3}$ (39.3 mg, $0.15 \mathrm{mmol}, 5 \mathrm{~mol} \%$ ), 2-benzyl -2-(but-3-yn-1-yl) malononitrile (624.7 mg, $3.0 \mathrm{mmol}$ ), 2bromothiophene $(0.98 \mathrm{~g}, 6.0 \mathrm{mmol}), \mathrm{Et}_{3} \mathrm{~N}$ (1.2 mL, 9.0 mmol, 3.0 equiv), and $10 \mathrm{~mL}$ of acetonitrile. $R_{f}=0.3(\mathrm{PE}: \mathrm{EA}=10: 1) .{ }^{1} \mathbf{H} \mathbf{N M R}\left(400 \mathrm{MHz}, \mathrm{CDCl}_{3}\right) \delta 7.48-7.35(\mathrm{~m}, 5 \mathrm{H}), 7.23(\mathrm{dd}, J=5.2,0.8$ $\mathrm{Hz}, 1 \mathrm{H}), 7.18(\mathrm{dd}, J=3.6,0.8 \mathrm{~Hz}, 1 \mathrm{H}), 6.96(\mathrm{dd}, J=5.2,3.6 \mathrm{~Hz}, 1 \mathrm{H}), 3.28(\mathrm{~s}, 2 \mathrm{H}), 2.87$ (t, $J=8.0 \mathrm{~Hz}$, 2H), $2.29(\mathrm{t}, J=7.6 \mathrm{~Hz}, 2 \mathrm{H}) .{ }^{13} \mathbf{C} \mathbf{N M R}\left(100 \mathrm{MHz}, \mathrm{CDCl}_{3}\right) \delta 132.0,131.7,130.4,129.2,129.1,127.04$, 
126.98, 122.9, 114.7, 89.5, 76.4, 43.5, 38.7, 36.0, 16.9. HRMS $\left(\mathrm{ESI}^{+}\right) \mathrm{m} / z$ calc'd for $\mathrm{C}_{18} \mathrm{H}_{14} \mathrm{~N}_{2} \mathrm{SNa}$ $[\mathrm{M}+\mathrm{Na}]^{+}:$313.0770, found 313.0770.

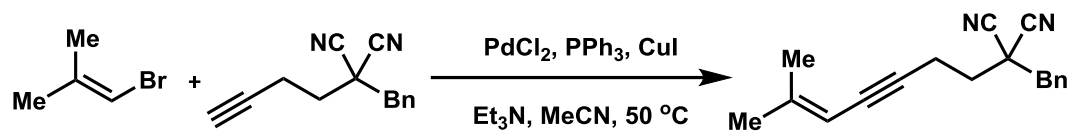

\section{2-Benzyl-2-(6-methylhept-5-en-3-yn-1-yl)malononitrile (1i)}

Following the general procedure B, the desired product $1 \mathbf{i}$ was obtained in $16 \%$ yield $(129.9 \mathrm{mg})$ as a yellow solid from $\mathrm{CuI}(27.0 \mathrm{mg}, 0.11 \mathrm{mmol}, 3.6 \mathrm{~mol} \%), \mathrm{PdCl}_{2}(13.8 \mathrm{mg}, 0.08 \mathrm{mmol}, 2.5 \mathrm{~mol} \%), \mathrm{PPh}_{3}$ (41.2 mg, 0.15 mmol, 5 mol\%), 1-bromo-2-methylprop-1-ene (340 ul, $3.3 \mathrm{mmol}, 1.1$ equiv), 2-benzyl2-(but-3-yn-1-yl)malononitrile S5 (626.3 mg, 3.0 mmol, 1.0 equiv), Et $3 \mathrm{~N}$ (1.5 mL, 11.9 mmol, 3.0 equiv), and $20 \mathrm{~mL}$ of acetonitrile. $R_{f}=0.6(\mathrm{PE}: \mathrm{EA}=10: 1) .{ }^{1} \mathbf{H} \mathbf{N M R}\left(400 \mathrm{MHz}, \mathrm{CDCl}_{3}\right) \delta 7.43-$ $7.38(\mathrm{~m}, 5 \mathrm{H}), 5.22-5.21(\mathrm{~m}, 1 \mathrm{H}), 3.26(\mathrm{~s}, 2 \mathrm{H}), 2.79-2.75(\mathrm{~m}, 2 \mathrm{H}), 2.26-2.19(\mathrm{~m}, 2 \mathrm{H}), 1.87(\mathrm{~s}, 3 \mathrm{H})$, 1.79 (s, 3H). ${ }^{13} \mathbf{C}$ NMR (100 MHz, $\left.\mathrm{CDCl}_{3}\right) \delta 148.9,131.8,130.4,129.2,129.0,114.8,104.7,87.2$, 81.0, 43.5, 38.7, 36.6, 24.9, 21.1, 16.7. HRMS $\left(\mathrm{ESI}^{+}\right) \mathrm{m} / z$ calc'd for $\mathrm{C}_{18} \mathrm{H}_{18} \mathrm{~N}_{2} \mathrm{Na}[\mathrm{M}+\mathrm{Na}]^{+}: 285.1362$, found 285.1362 .

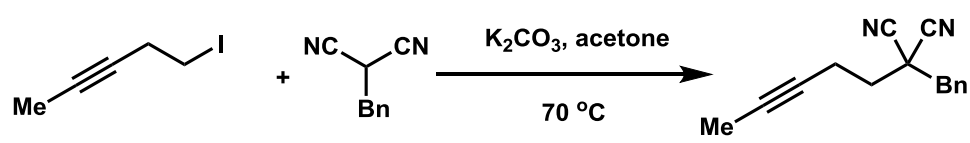

\section{2-Benzyl-2-(pent-3-yn-1-yl)malononitrile (1j)}

Following the general procedure A, the desired product $\mathbf{1} \mathbf{j}$ was obtained in $90 \%$ yield (1.21g) as a white solid from 2-benzylmalononitrile ( $1.04 \mathrm{~g}, 6.0 \mathrm{mmol}, 1.0$ equiv), 5-iodopent-2-yne ${ }^{6}$ (3.49 g, 18.0 mmol, 3.0 equiv), $\mathrm{K}_{2} \mathrm{CO}_{3}\left(2.48 \mathrm{~g}, 18 \mathrm{mmol}, 3.0\right.$ equiv), and $30 \mathrm{~mL}$ of acetone. $R_{f}=0.3$ (PE : EA = 20 : 1). ${ }^{1}$ HNMR (400 MHz, CDCl3) $\delta 7.45-7.35(\mathrm{~m}, 5 \mathrm{H}), 3.24(\mathrm{~s}, 2 \mathrm{H}), 2.61-2.49(\mathrm{~m}, 2 \mathrm{H}), 2.16(\mathrm{t}, J=$ $8.4 \mathrm{~Hz}, 2 \mathrm{H}), 1.78$ (t, $J=2.4 \mathrm{~Hz}, 3 \mathrm{H}) .{ }^{13} \mathrm{CNMR}(100 \mathrm{MHz}, \mathrm{CDCl} 3) \delta 131.8,130.4,129.1,129.0,114.9$, 78.8, 75.1, 43.5, 38.6, 36.5, 16.0, 3.6. HRMS $\left(\mathrm{ESI}^{+}\right) \mathrm{m} / \mathrm{z}$ calc'd for $\mathrm{C}_{15} \mathrm{H}_{14} \mathrm{~N}_{2} \mathrm{Na}[\mathrm{M}+\mathrm{Na}]^{+}:$245.1049, found 245.1049 .

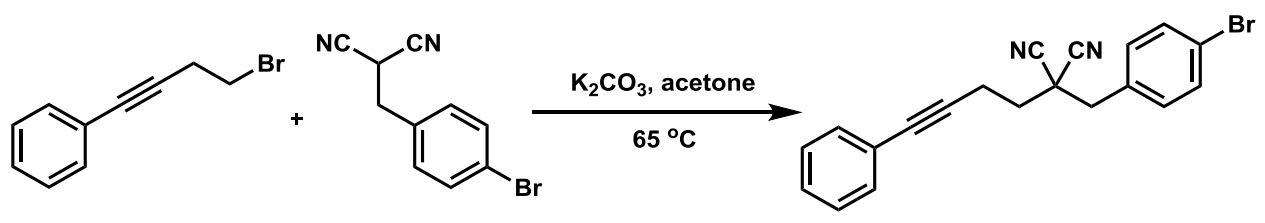

\section{2-(4-Bromobenzyl)-2-(4-phenylbut-3-yn-1-yl)malononitrile (1k)}

Following the general procedure A, the desired product $1 \mathbf{k}$ was obtained in $61 \%$ yield $(533.6 \mathrm{mg})$ as a yellow solid from (4-bromobut-1-yn-1-yl)benzene (602.2 mg, $2.88 \mathrm{mmol}, 1.2$ equiv), 2-(4- 
bromobenzyl)malononitrile ${ }^{7}$ (564.3 mg, $2.4 \mathrm{mmol}, 1.0$ equiv), $\mathrm{K}_{2} \mathrm{CO}_{3}$ (995.1 mg, $7.2 \mathrm{mmol}, 3.0$ equiv), and $10 \mathrm{~mL}$ of acetone. $R_{f}=0.5(\mathrm{PE}: \mathrm{EA}=5: 1) .{ }^{1} \mathbf{H}$ NMR $\left(400 \mathrm{MHz}, \mathrm{CDCl}_{3}\right) \delta 7.57-7.55(\mathrm{~m}, 2 \mathrm{H})$, $7.43-7.40(\mathrm{~m}, 2 \mathrm{H}), 7.32-7.26(\mathrm{~m}, 5 \mathrm{H}), 3.25(\mathrm{~s}, 2 \mathrm{H}), 2.86(\mathrm{t}, J=7.8 \mathrm{~Hz}, 2 \mathrm{H}), 2.30(\mathrm{t}, J=7.8 \mathrm{~Hz}$, 2H). ${ }^{13} \mathbf{C}$ NMR $\left(100 \mathrm{MHz}, \mathrm{CDCl}_{3}\right) \delta 132.4,132.0,131.7,130.7,128.5,128.4,123.6,122.9,114.6$, 85.3, 83.2, 43.0, 38.6, 36.3, 16.7. HRMS $\left(\mathrm{ESI}^{+}\right) \mathrm{m} / \mathrm{z}$ calc'd for $\mathrm{C}_{20} \mathrm{H}_{15} \mathrm{BrN}_{2} \mathrm{Na}[\mathrm{M}+\mathrm{Na}]^{+}: 385.0311$, found 385.0312 .

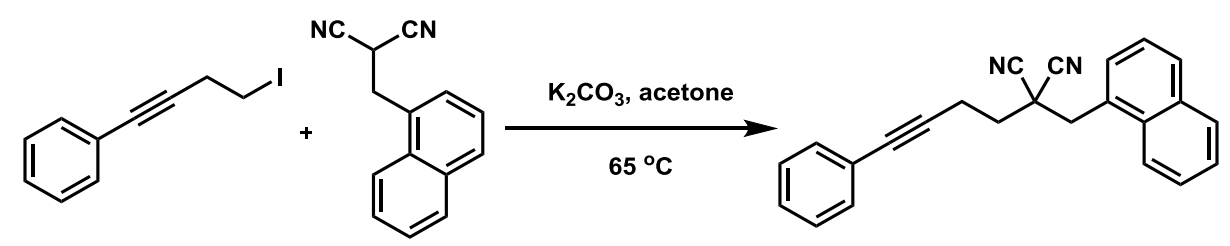

\section{2-(Naphthalen-1-ylmethyl)-2-(4-phenylbut-3-yn-1-yl)malononitrile (11)}

Following the general procedure A, the desired product 11 was obtained in $79 \%$ yield (3.27 g) as a yellow solid from 2-(naphthalen-1-ylmethyl)malononitrile ${ }^{8}$ (2.55 g, $12.4 \mathrm{mmol}, 1.0$ equiv), (4-iodobut1-yn-1-yl)benzene (9.30 g, $36.0 \mathrm{mmol}, 2.9$ equiv), $\mathrm{K}_{2} \mathrm{CO}_{3}(5.01 \mathrm{~g}, 36 \mathrm{mmol}, 2.9$ equiv) and $15 \mathrm{~mL}$ of acetone. $R_{f}=0.4(\mathrm{PE}: \mathrm{EA}=5: 1) .{ }^{1} \mathbf{H} \mathbf{N M R}\left(400 \mathrm{MHz}, \mathrm{CDCl}_{3}\right) \delta 8.10(\mathrm{~d}, J=8.4 \mathrm{~Hz}, 1 \mathrm{H}), 7.92(\mathrm{~d}, J$ $=8.0 \mathrm{~Hz}, 2 \mathrm{H}), 7.66(\mathrm{~d}, J=6.8 \mathrm{~Hz}, 1 \mathrm{H}), 7.62-7.50(\mathrm{~m}, 3 \mathrm{H}), 7.46-7.38(\mathrm{~m}, 2 \mathrm{H}), 7.34-7.27(\mathrm{~m}, 3 \mathrm{H})$, $3.84(\mathrm{~s}, 2 \mathrm{H}), 2.88(\mathrm{t}, J=8.0 \mathrm{~Hz}, 2 \mathrm{H}), 2.39(\mathrm{t}, J=7.6 \mathrm{~Hz}, 2 \mathrm{H}) .{ }^{13} \mathbf{C} \mathbf{N M R}\left(100 \mathrm{MHz}, \mathrm{CDCl}_{3}\right) \delta 134.2$, $132.2,131.7,129.9,129.6,129.3,128.44,128.37,128.0,126.9,126.4,125.5,123.4,123.0,115.1,85.5$, 83.1, 39.1, 38.6, 36.7, 16.8. HRMS $\left(\mathrm{ESI}^{+}\right) \mathrm{m} / \mathrm{z}$ calc'd for $\mathrm{C}_{24} \mathrm{H}_{18} \mathrm{~N}_{2} \mathrm{Na}[\mathrm{M}+\mathrm{Na}]^{+}:$357.1362, found 357.1363.

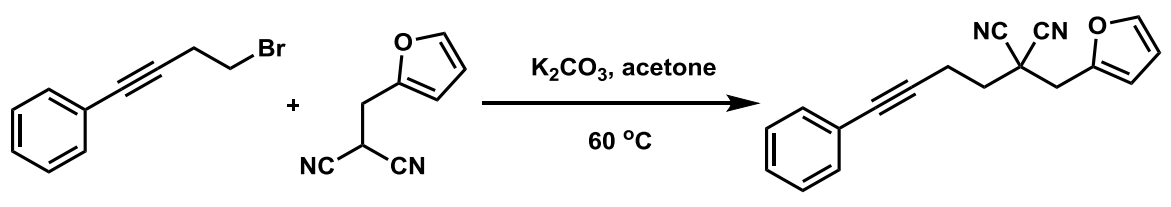

\section{2-(Furan-2-ylmethyl)-2-(4-phenylbut-3-yn-1-yl)malononitrile (1m)}

Following the general procedure A, the desired product $1 \mathrm{~m}$ was obtained in $63 \%$ yield (344.0 $\mathrm{mg})$ as a white solid from (4-bromobut-1-yn-1-yl)benzene (534.9 mg, $2.6 \mathrm{mmol}, 1.3$ equiv), 2-(furan-2ylmethyl)malononitrile ${ }^{9}$ (300.4 mg, 2 mmol, 1.0 equiv), $\mathrm{K}_{2} \mathrm{CO}_{3}$ (855.7 mg, $6.0 \mathrm{mmol}, 3.0$ equiv), and $4.5 \mathrm{~mL}$ of acetone. $R_{f}=0.3(\mathrm{PE}: \mathrm{EA}=20: 1) .{ }^{1} \mathbf{H} \mathbf{N M R}\left(400 \mathrm{MHz}, \mathrm{CDCl}_{3}\right) \delta 7.470-7.468(\mathrm{~m}, 1 \mathrm{H})$, $7.44-7.41(\mathrm{~m}, 2 \mathrm{H}), 7.32-7.29(\mathrm{~m}, 3 \mathrm{H}), 6.48-6.41(\mathrm{~m}, 2 \mathrm{H}), 3.41(\mathrm{~s}, 2 \mathrm{H}), 2.89-2.82(\mathrm{~m}, 2 \mathrm{H}), 2.30$ - $2.26(\mathrm{~m}, 2 \mathrm{H}) .{ }^{13} \mathrm{C}$ NMR $\left(100 \mathrm{MHz}, \mathrm{CDCl}_{3}\right) \delta 145.9,143.8,131.7,128.44,128.38,123.0,114.6$, 
111.2, 111.1, 85.4, 83.1, 37.1, 36.3, 35.6, 16.6. HRMS $\left(\mathrm{ESI}^{+}\right) \mathrm{m} / \mathrm{z}$ calc'd for $\mathrm{C}_{18} \mathrm{H}_{14} \mathrm{~N}_{2} \mathrm{ONa}[\mathrm{M}+\mathrm{Na}]^{+}$: 297.0998, found 297.1002.

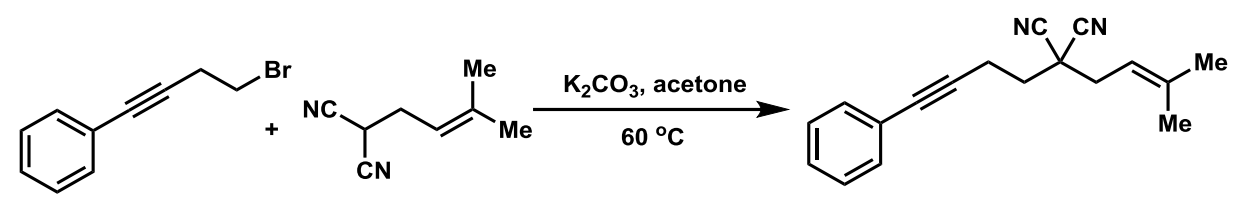

\section{2-(3-Methylbut-2-en-1-yl)-2-(4-phenylbut-3-yn-1-yl)malononitrile (1n)}

Following the general procedure A, the desired product 1n was obtained in $40 \%$ yield $(142.9 \mathrm{mg})$ as a colorless oil from (4-bromobut-1-yn-1-yl)benzene (378.4 mg, $1.8 \mathrm{mmol}, 1.3$ equiv), 2-(3-methylbut2-en-1-yl)malononitrile ${ }^{10}$ (184.6 mg, $1.4 \mathrm{mmol}, 1.0$ equiv), $\mathrm{K}_{2} \mathrm{CO}_{3}(572.5 \mathrm{mg}, 4.1 \mathrm{mmol}, 3.0$ equiv), and $2.0 \mathrm{~mL}$ of acetone. $R_{f}=0.4(\mathrm{PE}: \mathrm{EA}=20: 1) .{ }^{1} \mathbf{H} \mathbf{~ N M R}\left(400 \mathrm{MHz}, \mathrm{CDCl}_{3}\right) \delta 7.44-7.39(\mathrm{~m}$, 2H), $7.32-7.29(\mathrm{~m}, 3 \mathrm{H}), 5.31(\mathrm{t}, J=7.6 \mathrm{~Hz}, 1 \mathrm{H}), 2.84-2.80(\mathrm{~m}, 2 \mathrm{H}), 2.75(\mathrm{~d}, J=7.6 \mathrm{~Hz}, 2 \mathrm{H}), 2.28$ - 2.24 (m, 2H), 1.83 (s, 3H), 1.74 (s, 3H). $\left.{ }^{13} \mathbf{C ~ N M R ~ ( 1 0 0 ~ M H z , ~} \mathrm{CDCl}_{3}\right) \delta 141.3,131.7,128.5,128.4$, 123.0, 115.2, 114.4, 85.7, 82.9, 37.3, 36.6, 35.8, 26.2, 18.6, 16.6. HRMS $\left(\mathrm{ESI}^{+}\right) \mathrm{m} / \mathrm{z}$ calc'd for $\mathrm{C}_{18} \mathrm{H}_{18} \mathrm{~N}_{2} \mathrm{Na}[\mathrm{M}+\mathrm{Na}]^{+}:$285.1362, found 285.1359.

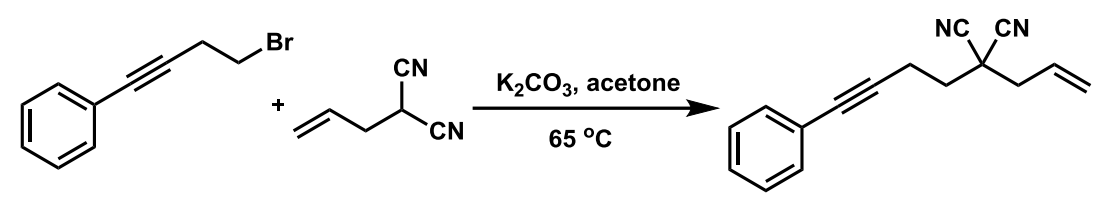

\section{2-Allyl-2-(4-phenylbut-3-yn-1-yl)malononitrile (10)}

Following the general procedure A, the desired product 10 was obtained in $84 \%$ yield $(587.2 \mathrm{mg})$ as a colorless oil from (4-bromobut-1-yn-1-yl)benzene (752.7 mg, $3.6 \mathrm{mmol}, 1.2$ equiv), 2allylmalononitrile ${ }^{11}$ (318.4 mg, $3 \mathrm{mmol}, 1.0$ equiv), $\mathrm{K}_{2} \mathrm{CO}_{3}$ (1243.9 mg, 9 mmol, 3.0 equiv), and 10 mL of acetone. $R_{f}=0.4(\mathrm{PE}: \mathrm{EA}=10: 1) .{ }^{1} \mathbf{H}$ NMR $\left(400 \mathrm{MHz}, \mathrm{CDCl}_{3}\right) \delta 7.44-7.39(\mathrm{~m}, 2 \mathrm{H}), 7.32-$ $7.29(\mathrm{~m}, 3 \mathrm{H}), 5.98-5.87(\mathrm{~m}, 1 \mathrm{H}), 5.48-5.41(\mathrm{~m}, 2 \mathrm{H}), 2.85-2.81(\mathrm{~m}, 2 \mathrm{H}), 2.77(\mathrm{~d}, J=7.3 \mathrm{~Hz}, 2 \mathrm{H})$, 2.29 - $2.25(\mathrm{~m}, 2 \mathrm{H}) .{ }^{13} \mathbf{C} \mathbf{N M R}\left(100 \mathrm{MHz}, \mathrm{CDCl}_{3}\right) \delta 131.7,128.5,128.4,128.3,123.8,123.0,114.8$, 85.5, 83.0, 41.8, 36.9, 35.9, 16.5. HRMS $\left(\mathrm{ESI}^{+}\right) \mathrm{m} / z$ calc'd for $\mathrm{C}_{16} \mathrm{H}_{14} \mathrm{~N}_{2} \mathrm{Na}[\mathrm{M}+\mathrm{Na}]^{+}: 257.1049$, found 257.1050 .

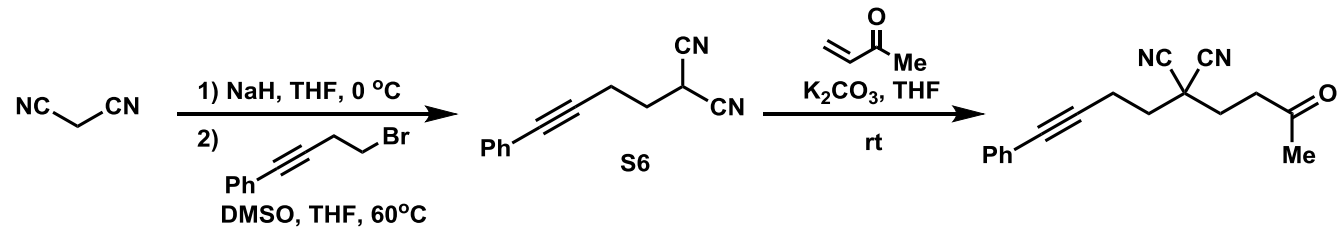

2-(3-Oxobutyl)-2-(4-phenylbut-3-yn-1-yl)malononitrile (1p) 
To a stirred solution of malononitrile (2.64 g, $40.0 \mathrm{mmol}, 4.0$ equiv) in THF (150 mL) was added $\mathrm{NaH}$ ( $1.60 \mathrm{~g}, 40.0 \mathrm{mmol}, 4.0$ equiv) at $0^{\circ} \mathrm{C}$. After stirred for $30 \mathrm{~min}$, (4-bromobut-1-yn-1-yl)benzene (2.10 g, $10 \mathrm{mmol}, 1.0$ equiv) and DMSO $(10 \mathrm{ml})$ were added slowly. The resulting mixture was stirred at room temperature for $4 \mathrm{~h}$, and reaction was quenched with saturated $\mathrm{NH}_{4} \mathrm{Cl}$ aqueous solution. The mixture was extracted with EA (30 mL x 3). The combined organic phase was washed with brine, dried over $\mathrm{Na}_{2} \mathrm{SO}_{4}$, and filtered. After the solvents were removed under reduced pressure, the residue was purified by flash chromatography to give $\mathbf{S 6}$ in $58 \%$ yield $\left(1.13 \mathrm{~g}\right.$ ) as a yellow solid. $R_{f}=0.3$ (PE : EA $=10$ : 1). ${ }^{1} \mathbf{H}$ NMR $\left(400 \mathrm{MHz}, \mathrm{CDCl}_{3}\right) \delta 7.44-7.41(\mathrm{~m}, 2 \mathrm{H}), 7.36-7.30$ (m, 3H), 4.07 (t, J= 7.5 Hz, $1 \mathrm{H}), 2.78(\mathrm{t}, J=6.6 \mathrm{~Hz}, 2 \mathrm{H}), 2.32(\mathrm{dt}, J=7.6,6.7 \mathrm{~Hz}, 2 \mathrm{H}) .{ }^{13} \mathbf{C ~ N M R}\left(100 \mathrm{MHz}, \mathrm{CDCl}_{3}\right) \delta 131.8$, 128.6, 128.5, 122.5, 112.3, 84.4, 84.2, 30.1, 21.5, 17.1. HRMS $\left(\mathrm{ESI}^{+}\right) \mathrm{m} / z$ calc'd for $\mathrm{C}_{13} \mathrm{H}_{10} \mathrm{~N}_{2} \mathrm{Na}$ $[\mathrm{M}+\mathrm{Na}]^{+}: 217.0736$, found 217.0728 .

To a Schlenk flask equipped with a magnetic stirring bar were added 2-(4-phenylbut-3-yn-1yl)malononitrile S6 (485.8 mg, $2.5 \mathrm{mmol}, 1.0$ equiv), but-3-en-2-one (262.5 mg, $3.75 \mathrm{mmol}, 1.5$ equiv), $\mathrm{K}_{2} \mathrm{CO}_{3}(1.04 \mathrm{~g}, 7.5 \mathrm{mmol}, 3.0$ equiv), and $15 \mathrm{~mL}$ of THF at room temperature. The mixture was stirred at the room temperature for $23 \mathrm{~h}$, and filtered through a celite pad. The solvents were removed under reduced pressure to give a crude mixture. The crude mixture was purified by flash column chromatography to give the desired product 1 p $(398.5 \mathrm{mg}, 60 \%)$ as a white solid. $R_{f}=0.3(\mathrm{PE}: \mathrm{EA}=$ 5 : 1). ${ }^{1} \mathbf{H}$ NMR (400 MHz, $\left.\mathrm{CDCl}_{3}\right) \delta 7.44-7.38$ (m, 2H), 7.33 - 7.27 (m, 3H), $2.91-2.87$ (m, 2H), 2.85-2.81 (m, 2H), $2.34-2.28(\mathrm{~m}, 4 \mathrm{H}), 2.25(\mathrm{~s}, 3 \mathrm{H}) .{ }^{13} \mathbf{C ~ N M R}\left(100 \mathrm{MHz}, \mathrm{CDCl}_{3}\right) \delta$ 204.6, 131.7, 128.5, 128.4, 122.9, 114.8, 85.2, 83.2, 39.3, 36.7, 36.4, 31.8, 30.2, 16.6. HRMS $\left(\mathrm{ESI}^{+}\right) \mathrm{m} / \mathrm{z}$ calc'd for $\mathrm{C}_{17} \mathrm{H}_{16} \mathrm{~N}_{2} \mathrm{ONa}[\mathrm{M}+\mathrm{Na}]^{+}:$287.1155, found 287.1156.

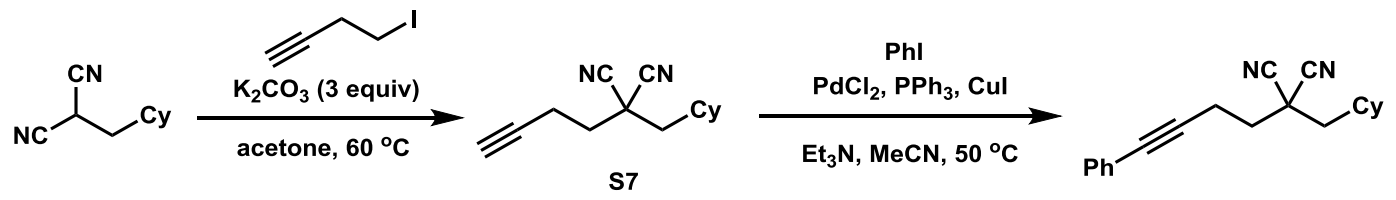

\section{2-(Cyclohexylmethyl)-2-(4-phenylbut-3-yn-1-yl)malononitrile (1q)}

Synthesis of S7: Following the general procedure A, 2-(but-3-yn-1-yl)-2-(cyclohexylmethyl)malononitrile (S7) was obtained in 59\% yield (2.52 g) as a white solid from 4-iodobut-1-yne (4.68 g, 26.0 mmol, 1.3 equiv), 2-(cyclohexylmethyl)malononitrile ${ }^{8}$ (3.24 g, 20.0 mmol, 1.0 equiv), $\mathrm{K}_{2} \mathrm{CO}_{3}$ (8.31 g, $60.0 \mathrm{mmol}, 3.0$ equiv), and $30 \mathrm{~mL}$ of acetone. $R_{f}=0.4(\mathrm{PE}: \mathrm{EA}=30: 1) .{ }^{1} \mathbf{H} \mathbf{~ N M R}(400 \mathrm{MHz}$, $\left.\mathrm{CDCl}_{3}\right) \delta 2.58(\mathrm{ddd}, J=11.0,6.3,2.7 \mathrm{~Hz}, 2 \mathrm{H}), 2.21-2.17(\mathrm{~m}, 2 \mathrm{H}), 2.08(\mathrm{t}, J=2.7 \mathrm{~Hz}, 1 \mathrm{H}), 1.94-$ 
$1.89(\mathrm{~m}, 2 \mathrm{H}), 1.84(\mathrm{~d}, J=6.5 \mathrm{~Hz}, 2 \mathrm{H}), 1.80-1.63(\mathrm{~m}, 4 \mathrm{H}), 1.31(\mathrm{qt}, J=12.6,3.3 \mathrm{~Hz}, 2 \mathrm{H}), 1.18(\mathrm{tt}, J$ $=12.5,3.2 \mathrm{~Hz}, 1 \mathrm{H}), 1.13-1.03(\mathrm{~m}, 2 \mathrm{H}) .{ }^{13} \mathbf{C} \mathbf{N M R}\left(100 \mathrm{MHz}, \mathrm{CDCl}_{3}\right) \delta 115.4,80.3,70.9,44.6,37.7$, 35.6, 35.3, 33.4, 25.9, 25.8, 15.4. HRMS $\left(\mathrm{ESI}^{+}\right) \mathrm{m} / z$ calc'd for $\mathrm{C}_{14} \mathrm{H}_{19} \mathrm{~N}_{2}[\mathrm{M}+\mathrm{H}]^{+}: 215.1543$, found 215.1539.

Synthesis of 1q: Following the general procedure B, the desired product 1q was obtained in $93 \%$ yield $(811.7 \mathrm{mg})$ as a white solid from $\mathrm{CuI}(20.6 \mathrm{mg}, 0.11 \mathrm{mmol}, 3.6 \mathrm{~mol} \%), \mathrm{PdCl}_{2}(13.3 \mathrm{mg}, 0.08 \mathrm{mmol}$, $2.5 \mathrm{~mol} \%$ ), $\mathrm{PPh}_{3}$ (39.4 mg, $0.15 \mathrm{mmol}, 5 \mathrm{~mol} \%$ ), iodobenzene (673.3 mg, $\left.3.3 \mathrm{mmol}, 1.1 \mathrm{equiv}\right), \mathbf{S} 7$ (642.9 mg, $3.0 \mathrm{mmol}, 1.0$ equiv), $\mathrm{Et}_{3} \mathrm{~N}$ (1.5 mL, $9.0 \mathrm{mmol}, 3$ equiv), and $20 \mathrm{~mL}$ of acetonitrile. $R_{f}=$ $0.3(\mathrm{PE}: \mathrm{EA}=50: 1) .{ }^{1} \mathbf{H}$ NMR $\left(400 \mathrm{MHz}, \mathrm{CDCl}_{3}\right) \delta 7.44-7.38(\mathrm{~m}, 2 \mathrm{H}), 7.33-7.28(\mathrm{~m}, 3 \mathrm{H}), 2.84$ - $2.80(\mathrm{~m}, 2 \mathrm{H}), 2.32-2.26(\mathrm{~m}, 2 \mathrm{H}), 1.98-1.92(\mathrm{~m}, 2 \mathrm{H}), 1.89(\mathrm{~d}, J=6.4 \mathrm{~Hz}, 2 \mathrm{H}), 1.83-1.66(\mathrm{~m}$, 4H), 1.33 (qt, $J=12.5,3.3 \mathrm{~Hz}, 2 \mathrm{H}), 1.24-1.04(\mathrm{~m}, 3 \mathrm{H}) .{ }^{13} \mathbf{C}$ NMR $\left(100 \mathrm{MHz}, \mathrm{CDCl}_{3}\right) \delta 131.7,128.4$, 128.3, 123.1, 115.6, 85.7, 82.8, 44.8, 38.0, 35.7, 35.3, 33.5, 25.9, 16.4. HRMS $\left(\mathrm{ESI}^{+}\right) \mathrm{m} / z$ calc'd for $\mathrm{C}_{20} \mathrm{H}_{22} \mathrm{~N}_{2} \mathrm{Na}[\mathrm{M}+\mathrm{Na}]^{+}: 313.1675$, found 313.1675 .

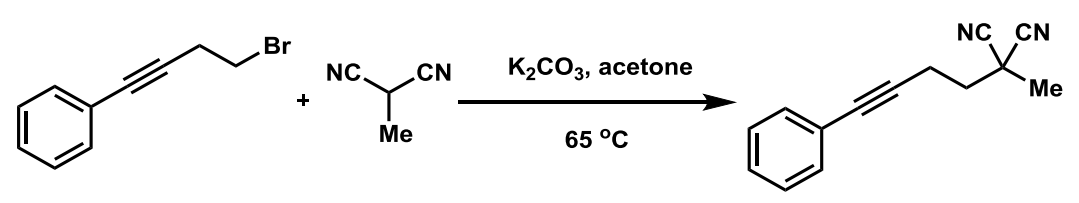

\section{2-Methyl-2-(4-phenylbut-3-yn-1-yl)malononitrile (1r)}

Following the general procedure A, the desired product $1 \mathbf{r}$ was obtained in $41 \%$ yield $(110.9 \mathrm{mg})$ as a colorless oil from (4-bromobut-1-yn-1-yl)benzene (328.3 mg, $1.57 \mathrm{mmol}, 1.2$ equiv), 2methylmalononitrile ${ }^{11}$ (104.9 mg, $1.31 \mathrm{mmol}, 1.0$ equiv) and $\mathrm{K}_{2} \mathrm{CO}_{3}(539.1 \mathrm{mg}, 3.9 \mathrm{mmol}, 3.0$ equiv), $R_{f}=0.3(\mathrm{PE}: \mathrm{EA}=10: 1) .{ }^{1} \mathbf{H}$ NMR $\left(400 \mathrm{MHz} \mathrm{CDCl}_{3}\right) \delta 7.44-7.40(\mathrm{~m}, 2 \mathrm{H}), 7.34-7.27(\mathrm{~m}, 3 \mathrm{H})$, $2.82(\mathrm{t}, J=7.7 \mathrm{~Hz}, 2 \mathrm{H}), 2.28(\mathrm{t}, J=7.7 \mathrm{~Hz}, 2 \mathrm{H}), 1.88(\mathrm{~s}, 3 \mathrm{H}) .{ }^{13} \mathbf{C} \mathbf{N M R}\left(100 \mathrm{MHz}, \mathrm{CDCl}_{3}\right) \delta 131.7$, 128.4, 128.3, 122.9, 115.7, 85.4, 83.0, 37.8, 31.2, 25.0, 16.5. HRMS $\left(\mathrm{ESI}^{+}\right) \mathrm{m} / z$ calc'd for $\mathrm{C}_{14} \mathrm{H}_{12} \mathrm{~N}_{2} \mathrm{Na}$ $[\mathrm{M}+\mathrm{Na}]^{+}: 231.0893$, found 231.0890 .

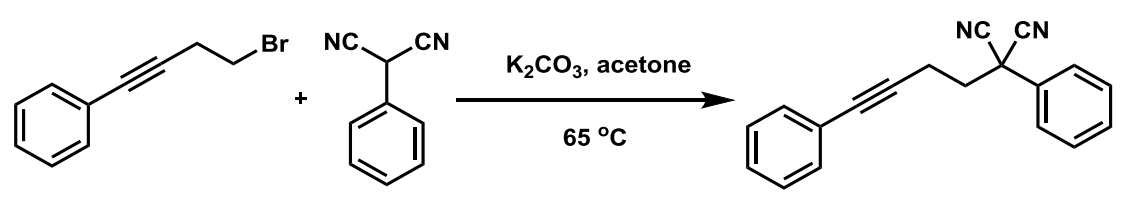

\section{2-Phenyl-2-(4-phenylbut-3-yn-1-yl)malononitrile (1s)}

Following the general procedure A, the desired product $1 \mathrm{~s}$ was obtained in $56 \%$ yield $(393.5 \mathrm{mg})$ as a white solid from (4-bromobut-1-yn-1-yl)benzene (652.3 mg, 3.12 mmol, 1.2 equiv), 2- 
phenylmalononitrile ${ }^{12}$ (364.5 mg, $2.6 \mathrm{mmol}, 1.0$ equiv), $\mathrm{K}_{2} \mathrm{CO}_{3}$ (1.08 g, $7.8 \mathrm{mmol}, 3.0$ equiv), and 40 mL of acetone. $R_{f}=0.5(\mathrm{PE}: \mathrm{EA}=10: 1) .{ }^{1} \mathbf{H} \mathbf{N M R}\left(400 \mathrm{MHz}, \mathrm{CDCl}_{3}\right) \delta 7.62-7.59(\mathrm{~m}, 2 \mathrm{H}), 7.55-$ $7.47(\mathrm{~m}, 3 \mathrm{H}), 7.39-7.37(\mathrm{~m}, 2 \mathrm{H}), 7.31-7.27(\mathrm{~m}, 3 \mathrm{H}), 2.75-2.71(\mathrm{~m}, 2 \mathrm{H}), 2.60-2.56(\mathrm{~m}, 2 \mathrm{H}) .{ }^{13} \mathbf{C}$ NMR $\left(100 \mathrm{MHz}_{2} \mathrm{CDCl}_{3}\right) \delta 131.7,131.5,130.3,130.0,128.4,128.3,125.9,123.0,114.6,85.2,83.0$, 41.6, 41.2, 16.5. HRMS $\left(\mathrm{ESI}^{+}\right) \mathrm{m} / z$ calc'd for $\mathrm{C}_{19} \mathrm{H}_{14} \mathrm{~N}_{2} \mathrm{Na}[\mathrm{M}+\mathrm{Na}]^{+}: 293.1049$, found 293.1050.

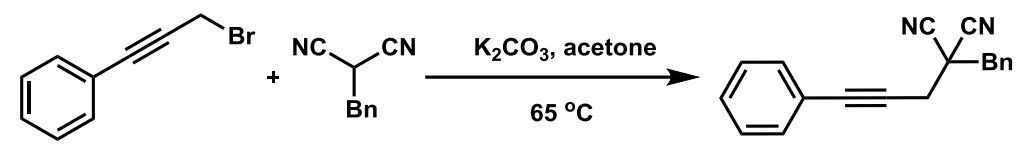

\section{2-Benzyl-2-(3-phenylprop-2-yn-1-yl)malononitrile (1t)}

Following the general procedure A, the desired product $1 \mathbf{k}$ was obtained in $98 \%$ yield $(801.8 \mathrm{mg})$ as a white solid from (3-bromoprop-1-yn-1-yl)benzene ${ }^{13}$ (702.2 mg, 3.6 mmol, 1.2 equiv), 2benzylmalononitrile (468.2 mg, $3 \mathrm{mmol}, 1.0$ equiv), $\mathrm{K}_{2} \mathrm{CO}_{3}(1.24 \mathrm{~g}, 9 \mathrm{mmol}, 3.0$ equiv), and $30 \mathrm{~mL}$ of acetone. $R_{f}=0.5(\mathrm{PE}: \mathrm{EA}=5: 1) .{ }^{1} \mathbf{H}$ NMR $\left(400 \mathrm{MHz}, \mathrm{CDCl}_{3}\right) \delta 7.53-7.50(\mathrm{~m}, 2 \mathrm{H}), 7.45-7.40$ (m, 5H), $7.39-7.33$ (m, 3H), 3.42 (s, 2H), 3.11 (s, 2H). ${ }^{13}$ C NMR (100 MHz, $\left.\mathrm{CDCl}_{3}\right) \delta$ 132.1, 131.5, 130.5, 129.3, 129.2, 128.6, 121.8, 114.7, 87.6, 79.8, 41.9, 38.4, 28.7. HRMS (ESI $\left.{ }^{+}\right) \mathrm{m} / z$ calc'd for $\mathrm{C}_{19} \mathrm{H}_{14} \mathrm{~N}_{2} \mathrm{Na}[\mathrm{M}+\mathrm{Na}]^{+}: 293.1049$, found 293.1050.

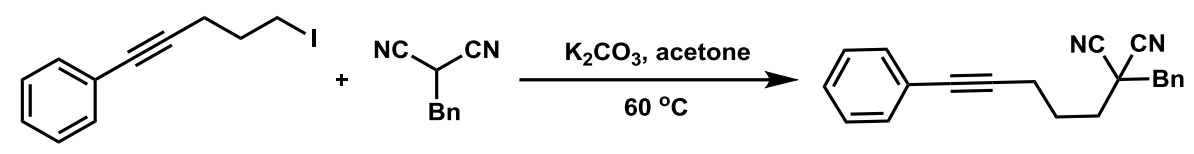

\section{2-Benzyl-2-(5-phenylpent-4-yn-1-yl)malononitrile (1u)}

Following the general procedure A, the desired product $1 \mathbf{u}$ was obtained in $97 \%$ yield (1.84 $\mathrm{g})$ as a white solid from (5-iodopent-1-yn-1-yl)benzene ${ }^{14}(2.25 \mathrm{~g}, 8.33 \mathrm{mmol}, \quad 1.3$ equiv), 2benzylmalononitrile (997.0 mg, $6.41 \mathrm{mmol}, 1.0$ equiv), $\mathrm{K}_{2} \mathrm{CO}_{3}$ (2.68 g, $19.2 \mathrm{mmol}, 3.0$ equiv), and 15 mL of acetone. $R_{f}=0.2(\mathrm{PE}: \mathrm{EA}=30: 1) .{ }^{1} \mathbf{H} \mathbf{N M R}\left(400 \mathrm{MHz}, \mathrm{CDCl}_{3}\right) \delta 7.39-7.36(\mathrm{~m}, 7 \mathrm{H}), 7.31$ - $7.30(\mathrm{~m}, 3 \mathrm{H}), 3.25(\mathrm{~s}, 2 \mathrm{H}), 2.59(\mathrm{t}, J=6.5 \mathrm{~Hz}, 2 \mathrm{H}), 2.19-2.15(\mathrm{~m}, 2 \mathrm{H}), 2.10-1.99(\mathrm{~m}, 2 \mathrm{H}) .{ }^{13} \mathbf{C}$ NMR $\left(100 \mathrm{MHz}, \mathrm{CDCl}_{3}\right) \delta 132.0,131.7,130.4,129.2,129.0,128.4,128.1,123.4,115.3,87.6,82.4$, 43.5, 39.3, 36.7, 24.9, 18.9. HRMS $\left(\mathrm{ESI}^{+}\right) \mathrm{m} / z$ calc'd for $\mathrm{C}_{21} \mathrm{H}_{18} \mathrm{~N}_{2} \mathrm{Na}[\mathrm{M}+\mathrm{Na}]^{+}:$321.1362, found 321.1351 .
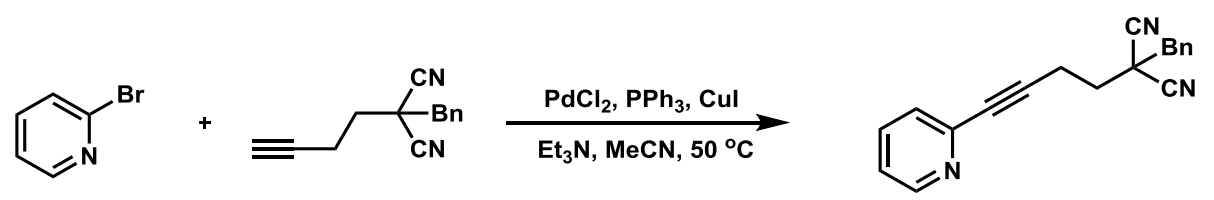
Following the general procedure $\mathrm{B}$, the desired product $\mathbf{1 v}$ was obtained in $70 \%$ yield $(811.7 \mathrm{mg})$ as a yellow solid from $\mathrm{CuI}$ (20.9 mg, $0.108 \mathrm{mmol}, 3.6 \mathrm{~mol} \%$ ), $\mathrm{PdCl}_{2}$ (15.6 mg, $0.075 \mathrm{mmol}, 2.5 \mathrm{~mol} \%$ ), $\mathrm{PPh}_{3}$ (41.2 mg, $0.15 \mathrm{mmol}, 5 \mathrm{~mol} \%$ ), 2-bromopyridine (320 uL, $3.6 \mathrm{mmol}, 1.1$ equiv), 2-benzyl-2-(but3-yn-1-yl)malononitrile S5 (625.9 mg, 3.0 mmol, 1.0 equiv), $\mathrm{Et}_{3} \mathrm{~N}$ (1.5 mL, $11.9 \mathrm{mmol}, 3$ equiv) and $20 \mathrm{~mL}$ of acetonitrile. $R_{f}=0.2(\mathrm{PE}: \mathrm{EA}=5: 1) .{ }^{1} \mathbf{H} \mathbf{N M R}\left(400 \mathrm{MHz}, \mathrm{CDCl}_{3}\right) \delta 8.56-8.55(\mathrm{~m}, 1 \mathrm{H})$, $7.65(\mathrm{td}, J=7.7,1.8 \mathrm{~Hz}, 1 \mathrm{H}), 7.43-7.37(\mathrm{~m}, 6 \mathrm{H}), 7.25-7.22(\mathrm{~m}, 1 \mathrm{H}), 3.28(\mathrm{~s}, 2 \mathrm{H}), 2.89-2.86(\mathrm{~m}$, 2H), $2.34-2.30(\mathrm{~m}, 2 \mathrm{H}) .{ }^{13} \mathbf{C}$ NMR (100 MHz, $\left.\mathrm{CDCl}_{3}\right) \delta 150.0,143.0,136.4,131.6,130.4,129.2$, 129.1, 127.1, 123.1, 114.7, 86.0, 82.4, 43.6, 38.7, 35.9, 16.6. HRMS $\left(\mathrm{ESI}^{+}\right) \mathrm{m} / z$ calc'd for $\mathrm{C}_{19} \mathrm{H}_{16} \mathrm{~N}_{3}$ $[\mathrm{M}+\mathrm{H}]^{+}:$286.1339, found 286.1327 .

\section{General procedure for synthesis of 3 by Ni-catalyzed desymmetrization of}

\section{malononitrile and characterization data}

Please note that the absolute configuration was determined only for compound $3 \mathbf{k a}$ via X-ray analysis (vide infra). The absolute configuration for all other products 3 and $\mathbf{4}$ has been inferred by analogy.

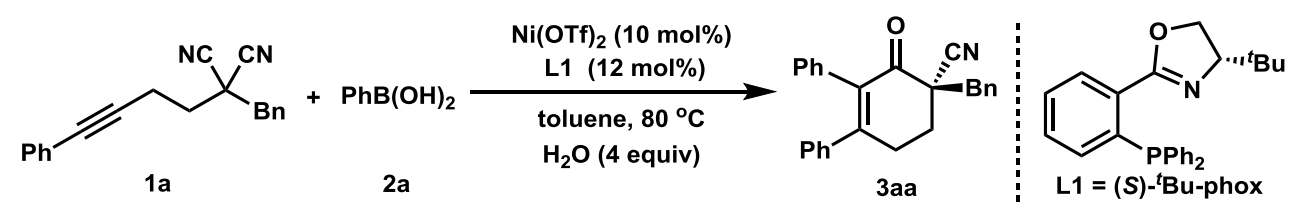

General Procedure: Ni(OTf) 2 (7.2 mg, $0.02 \mathrm{mmol}, 10 \mathrm{~mol} \%$ ), (S)- ${ }^{t} \mathrm{Bu}-\mathrm{phox}(9.3 \mathrm{mg}, 0.024 \mathrm{mmol}, 12$ $\mathrm{mol} \%$ ) and $2.0 \mathrm{~mL}$ of toluene were added to resealable Schlenk tube under argon. The resultant solution was stirred at room temperature for $30 \mathrm{~min}$, then $1 \mathrm{a}(56.9 \mathrm{mg}, 0.2 \mathrm{mmol}, 1.0$ equiv) and phenylboronic acid $2 \mathrm{a}$ (48.8 mg, $0.4 \mathrm{mmol}, 2.0$ equiv), toluene (2.0 mL), and $\mathrm{H}_{2} \mathrm{O}(15 \mathrm{uL}, 0.8 \mathrm{mmol}, 4.0$ equiv) were added. The tube was sealed, and stirred at $80{ }^{\circ} \mathrm{C}$ for $24 \mathrm{~h}$. After the mixture was cooled to room temperature, $1 \mathrm{M} \mathrm{HCl}(1.0 \mathrm{~mL})$ and $\mathrm{EA}(1.0 \mathrm{~mL})$ were added to the tube and the mixture was stirred for additional $30 \mathrm{~min}$. The mixture was then extracted with EA $(10 \mathrm{~mL}$ x 3). The combined organic phase was washed with brine, dried over $\mathrm{Na}_{2} \mathrm{SO}_{4}$, and filtered. After the solvents were removed under reduced pressure, the residue was purified by silica gel column chromatography to provide (R)-4'benzyl-3'-oxo-3',4',5',6'-tetrahydro-[1,1':2',1'-terphenyl]-4'-carbonitrile (3aa) in 89\% yield (64.8 $\mathrm{mg})$ as a white solid. er $=95: 5 .[\alpha]_{\mathrm{D}}^{25}=-116.88\left(c 1.73, \mathrm{CHCl}_{3}\right) \cdot R_{f}=0.3(\mathrm{PE}: \mathrm{EA}=10: 1) .{ }^{1} \mathbf{H}$ NMR $\left(400 \mathrm{MHz}, \mathrm{CDCl}_{3}\right) \delta 7.40$ - $7.32(\mathrm{~m}, 5 \mathrm{H}), 7.19$ - $7.17(\mathrm{~m}, 6 \mathrm{H}), 7.05-7.02(\mathrm{~m}, 2 \mathrm{H}), 6.96-6.94$ $(\mathrm{m}, 2 \mathrm{H}), 3.54(\mathrm{~d}, J=14.0 \mathrm{~Hz}, 1 \mathrm{H}), 3.14-3.06(\mathrm{~m}, 2 \mathrm{H}), 2.93(\mathrm{dt}, J=19.6,4.6 \mathrm{~Hz}, 1 \mathrm{H}), 2.39(\mathrm{dt}, J=$ 
13.7, 4.6 Hz, 1H), $2.24-2.17(\mathrm{~m}, 1 \mathrm{H}) .{ }^{13} \mathrm{C} \mathrm{NMR}\left(100 \mathrm{MHz}, \mathrm{CDCl}_{3}\right) \delta 190.2,158.5,139.5,135.8$, $134.8,134.5,130.9,130.7,128.8,128.7,128.24,128.20,127.9,127.8,127.5,118.7,47.7,39.7,30.2$, 29.8. HRMS $\left(\mathrm{ESI}^{+}\right) \mathrm{m} / \mathrm{z}$ calc'd for $\mathrm{C}_{26} \mathrm{H}_{21} \mathrm{NONa}[\mathrm{M}+\mathrm{Na}]^{+}: 386.1515$, found 386.1518. HPLC conditions: hexane/2-propanol $95: 5,0.8 \mathrm{~mL} / \mathrm{min}, \lambda=280 \mathrm{~nm}$, Chiralpak AD-H column (4.6 mm x $250 \mathrm{~mm}), \mathrm{t}_{\mathrm{r}}($ minor $)=16.2 \mathrm{~min}, \mathrm{t}_{\mathrm{r}}($ major $)=18.3 \mathrm{~min}$.<smiles>Cc1ccc(C2=C(c3ccccc3)C(=O)[C@@](C)(C#N)CC2)cc1</smiles>

(R)-4'-Benzyl-4-methyl-3'-oxo-3',4',5',6'-tetrahydro-[1,1':2',1'-terphenyl]-4' -carbonitrile (3ab)

Following the general procedure, the reaction was performed with 1a (56.9 $\mathrm{mg}, 0.2 \mathrm{mmol}, 1.0$ equiv), p-tolylboronic acid $2 \mathbf{b}$ (54.4 mg, $0.4 \mathrm{mmol}, 2.0$ equiv), $\mathrm{H}_{2} \mathrm{O}$ (15 uL, $0.8 \mathrm{mmol}, 4.0$ equiv), Ni(OTf) (7.2 mg, $0.02 \mathrm{mmol}, 10 \mathrm{~mol} \%$ ), and (S)- ${ }^{t} \mathrm{Bu}-$ phox (9.3 mg, $0.024 \mathrm{mmol}, 12 \mathrm{~mol} \%$ ) in $4.0 \mathrm{~mL}$ of toluene at $80{ }^{\circ} \mathrm{C}$ for $24 \mathrm{~h}$. The desired product 3ab was obtained in $71 \%$ yield $(53.3 \mathrm{mg})$ as a white solid. er $=$ $95: 5 .[\alpha]_{\mathrm{D}}{ }^{25}=-133.14\left(c 2.1, \mathrm{CHCl}_{3}\right) . R_{f}=0.3(\mathrm{PE}: \mathrm{EA}=10: 1) .{ }^{1} \mathbf{H} \mathbf{N M R}\left(400 \mathrm{MHz}, \mathrm{CDCl}_{3}\right) \delta$ $7.38-7.35(\mathrm{~m}, 5 \mathrm{H}), 7.22-7.18(\mathrm{~m}, 3 \mathrm{H}), 6.99-6.92(\mathrm{~m}, 6 \mathrm{H}), 3.53(\mathrm{AB}, J=14.0 \mathrm{~Hz}, 1 \mathrm{H}), 3.12-3.04$ (m, 2H), $2.92(\mathrm{dt}, J=19.5,4.6 \mathrm{~Hz}, 1 \mathrm{H}), 2.38(\mathrm{dt}, J=13.7,4.6 \mathrm{~Hz}, 1 \mathrm{H}), 2.26(\mathrm{~s}, 3 \mathrm{H}), 2.23-2.16(\mathrm{~m}$, 1H). ${ }^{13} \mathbf{C}$ NMR $\left(100 \mathrm{MHz}, \mathrm{CDCl}_{3}\right) \delta 190.2,158.5,138.9,136.5,135.4,135.1,134.6,130.9,130.6$, 128.9, 128.7, 128.3 128.0, 127.8, 127.4, 118.8, 47.6, 39.7, 30.1, 29.7, 21.4. HRMS (ESI $\left.{ }^{+}\right) \mathrm{m} / z$ calc'd for $\mathrm{C}_{27} \mathrm{H}_{23} \mathrm{NONa}[\mathrm{M}+\mathrm{Na}]^{+}:$400.1672, found 400.1674. HPLC conditions: hexane/2-propanol $95: 5$, $0.8 \mathrm{~mL} / \mathrm{min}, \lambda=300 \mathrm{~nm}$, Chiralpak AD-H column $(4.6 \mathrm{~mm} \times 250 \mathrm{~mm}), \mathrm{t}_{\mathrm{r}}($ minor $)=15.9 \mathrm{~min}, \mathrm{t}_{\mathrm{r}}($ major $)$ $=18.5 \mathrm{~min}$.<smiles>CCC1([N+]#N)CCC(c2ccc(OC)cc2)=C(c2ccccc2)C1=O</smiles>

(R)-4'-Benzyl-4-methoxy-3'-oxo-3',4',5',6'-tetrahydro-[1,1':2',1' '-terphenyl]-4'-carbonitrile (3ac) Following the general procedure, the reaction was performed with 1a (56.9 mg, $0.2 \mathrm{mmol}, 1.0$ equiv), (4-methoxyphenyl)boronic acid $2 c$ (60.8 mg, $0.4 \mathrm{mmol}, 2.0$ equiv), $\mathrm{H}_{2} \mathrm{O}$ (15 uL, $0.8 \mathrm{mmol}, 4.0$ equiv), $\mathrm{Ni}(\mathrm{OTf})_{2}(7.2 \mathrm{mg}, 0.02 \mathrm{mmol}, 10 \mathrm{~mol} \%)$, and $(S)-{ }^{t} \mathrm{Bu}-$ phox $(9.3 \mathrm{mg}, 0.024 \mathrm{mmol}, 12 \mathrm{~mol} \%)$ in $4.0 \mathrm{~mL}$ of toluene at $80{ }^{\circ} \mathrm{C}$ for $24 \mathrm{~h}$. The desired product 3ac was obtained in $86 \%$ yield $(67.3 \mathrm{mg})$ as a white 
solid. er $=94: 6 .[\alpha]_{\mathrm{D}}{ }^{25}=-118.89\left(c 1.2, \mathrm{CHCl}_{3}\right) . R_{f}=0.2(\mathrm{PE}: \mathrm{EA}=10: 1) .{ }^{\mathbf{1}} \mathbf{H} \mathbf{~ N M R}(400 \mathrm{MHz}$, $\left.\mathrm{CDCl}_{3}\right) \delta 7.40-7.31(\mathrm{~m}, 5 \mathrm{H}), 7.24-7.17(\mathrm{~m}, 3 \mathrm{H}), 7.00-6.96(\mathrm{~m}, 4 \mathrm{H}), 6.68(\mathrm{~d}, J=8.8 \mathrm{~Hz}, 2 \mathrm{H}), 3.74$ (s, 3H), $3.53(\mathrm{AB}, J=14.0 \mathrm{~Hz}, 1 \mathrm{H}), 3.10(\mathrm{BA}, J=13.9 \mathrm{~Hz}, 1 \mathrm{H}), 3.05(\mathrm{dd}, J=9.9,4.9 \mathrm{~Hz}, 1 \mathrm{H}), 2.93$ $(\mathrm{dt}, J=19.5,4.6 \mathrm{~Hz}, 1 \mathrm{H}), 2.37(\mathrm{dt}, J=13.7,4.6 \mathrm{~Hz}, 1 \mathrm{H}), 2.23-2.14(\mathrm{~m}, 1 \mathrm{H}) .{ }^{13} \mathrm{C}$ NMR $(100 \mathrm{MHz}$, $\left.\mathrm{CDCl}_{3}\right) \delta 190.1,159.9,158.0,135.4,135.0,134.6,131.5,131.0,130.6,130.1,128.7,128.1,127.7$, 127.4, 118.9, 113.6, 55.3, 47.6, 39.7, 30.0, 29.5. HRMS $\left(\mathrm{ESI}^{+}\right) \mathrm{m} / z$ calc'd for $\mathrm{C}_{27} \mathrm{H}_{23} \mathrm{O}_{2} \mathrm{Na}[\mathrm{M}+\mathrm{Na}]^{+}$: 416.1621, found 416.1627. HPLC conditions: hexane/2-propanol $95: 5,0.8 \mathrm{~mL} / \mathrm{min}, \lambda=300 \mathrm{~nm}$, Chiralpak AD-H column $(4.6 \mathrm{~mm} \times 250 \mathrm{~mm}), \mathrm{t}_{\mathrm{r}}($ minor $)=27.7 \mathrm{~min}, \mathrm{t}_{\mathrm{r}}($ major $)=34.7 \mathrm{~min}$.<smiles>C[C@@]1(C#N)CCC(c2ccc(F)cc2)=C(c2ccccc2)C1=O</smiles>

\section{(R)-4'-Benzyl-4-fluoro-3'-oxo-3',4',5',6'-tetrahydro-[1,1':2',1''-terphenyl]-4'-carbonitrile (3ad)}

Following the general procedure, the reaction was performed with 1a (56.9 $\mathrm{mg}, 0.2 \mathrm{mmol}, 1.0$ equiv), (4-fluorophenyl)boronic acid $\mathbf{2 d}\left(56.0 \mathrm{mg}, 0.4 \mathrm{mmol}, 2.0\right.$ equiv), $\mathrm{H}_{2} \mathrm{O}$ (15 uL, $0.8 \mathrm{mmol}, 4.0$ equiv), $\mathrm{Ni}(\mathrm{OTf})_{2}(7.2 \mathrm{mg}, 0.02 \mathrm{mmol}, 10 \mathrm{~mol} \%)$, and $(S)-{ }^{t} \mathrm{Bu}-$ phox $(9.3 \mathrm{mg}, 0.024 \mathrm{mmol}, 12 \mathrm{~mol} \%)$ in $4.0 \mathrm{~mL}$ of toluene at $80{ }^{\circ} \mathrm{C}$ for $24 \mathrm{~h}$. The desired product 3ad was obtained in $80 \%$ yield $(60.9 \mathrm{mg})$ as a white solid. er $=95: 5 .[\alpha]_{\mathrm{D}}{ }^{25}=-156.60\left(c 1.5, \mathrm{CHCl}_{3}\right) . R_{f}=0.3(\mathrm{PE}: \mathrm{EA}=10: 1) .{ }^{1} \mathbf{H}$ NMR $(400 \mathrm{MHz}$, $\left.\mathrm{CDCl}_{3}\right) \delta 7.40-7.30(\mathrm{~m}, 5 \mathrm{H}), 7.22-7.19(\mathrm{~m}, 3 \mathrm{H}), 7.05-6.99(\mathrm{~m}, 2 \mathrm{H}), 6.96-6.92(\mathrm{~m}, 2 \mathrm{H}), 6.89-$ $6.83(\mathrm{~m}, 2 \mathrm{H}), 3.53(\mathrm{~d}, J=14.0 \mathrm{~Hz}, 1 \mathrm{H}), 3.12-3.04(\mathrm{~m}, 2 \mathrm{H}), 2.89(\mathrm{dt}, J=19.6,4.5 \mathrm{~Hz}, 1 \mathrm{H}), 2.38(\mathrm{dt}$, $J=13.7,4.5 \mathrm{~Hz}, 1 \mathrm{H}), 2.23-2.16(\mathrm{~m}, 1 \mathrm{H}) .{ }^{13} \mathbf{C} \mathbf{N M R}\left(100 \mathrm{MHz}, \mathrm{CDCl}_{3}\right) \delta 190.1,162.6(\mathrm{~d}, J=249.9$ Hz), 157.1, 136.0, 135.5 (d, $J=3.1 \mathrm{~Hz}), 134.7,134.5,130.9,130.7,130.3$ (d, $J=8.1 \mathrm{~Hz}), 128.8,128.1$, $127.7(\mathrm{~d}, J=18.4 \mathrm{~Hz}), 118.6,115.5,115.3,47.6,39.6,30.1,29.7 .{ }^{19} \mathbf{F} \mathbf{N M R}\left(376 \mathrm{MHz}, \mathrm{CDCl}_{3}\right) \delta-$ 111.69. HRMS $\left(\mathrm{ESI}^{+}\right) \mathrm{m} / \mathrm{z}$ calc'd for $\mathrm{C}_{26} \mathrm{H}_{20} \mathrm{NOFNa}[\mathrm{M}+\mathrm{Na}]^{+}:$404.1421, found 404.1423. HPLC conditions: hexane/2-propanol 95:5, $0.8 \mathrm{~mL} / \mathrm{min}, \lambda=300 \mathrm{~nm}$, Chiralpak AD-H column (4.6 mm x $250 \mathrm{~mm}), \mathrm{t}_{\mathrm{r}}($ minor $)=20.0 \mathrm{~min}, \mathrm{t}_{\mathrm{r}}($ major $)=24.5 \mathrm{~min}$.<smiles>C[C@@]1(C#N)CCC(c2ccc(Cl)cc2)=C(c2ccccc2)C1=O</smiles>

(R)-4'-Benzyl-4-chloro-3'-oxo-3',4',5',6'-tetrahydro-[1,1':2',1''-terphenyl]-4' -carbonitrile (3ae) 
Following the general procedure, the reaction was performed with $1 \mathbf{a}(56.9 \mathrm{mg}, 0.2 \mathrm{mmol}, 1.0 \mathrm{equiv})$, (4-chlorophenyl)boronic acid $2 \mathrm{e}\left(62.5 \mathrm{mg}, 0.4 \mathrm{mmol}, 2.0\right.$ equiv), $\mathrm{H}_{2} \mathrm{O}$ (15 uL, $0.8 \mathrm{mmol}, 4.0$ equiv), $\mathrm{Ni}(\mathrm{OTf})_{2}(7.2 \mathrm{mg}, 0.02 \mathrm{mmol}, 10 \mathrm{~mol} \%)$, and $(S)-{ }^{t} \mathrm{Bu}-\mathrm{phox}(9.3 \mathrm{mg}, 0.024 \mathrm{mmol}, 12 \mathrm{~mol} \%)$ in $4.0 \mathrm{~mL}$ of toluene at $80{ }^{\circ} \mathrm{C}$ for $24 \mathrm{~h}$. The desired product 3ae was obtained in $82 \%$ yield $(65.0 \mathrm{mg})$ as a white solid. er $=91: 9 .[\alpha]_{\mathrm{D}}{ }^{25}=-114.45\left(c 1.5, \mathrm{CHCl}_{3}\right) . R_{f}=0.3(\mathrm{PE}: \mathrm{EA}=10: 1) .{ }^{1} \mathbf{H} \mathrm{NMR}(400 \mathrm{MHz}$, $\left.\mathrm{CDCl}_{3}\right) \delta 7.40-7.31(\mathrm{~m}, 5 \mathrm{H}), 7.23-7.20(\mathrm{~m}, 3 \mathrm{H}), 7.15(\mathrm{~d}, J=8.3 \mathrm{~Hz}, 2 \mathrm{H}), 7.00-6.92(\mathrm{~m}, 4 \mathrm{H}), 3.53$ $(\mathrm{d}, J=13.9 \mathrm{~Hz}, 1 \mathrm{H}), 3.12-3.03(\mathrm{~m}, 2 \mathrm{H}), 2.88(\mathrm{dt}, J=19.5,4.4 \mathrm{~Hz}, 1 \mathrm{H}), 2.39(\mathrm{dt}, J=13.8,4.4 \mathrm{~Hz}$, 1H), 2.23 - $2.16(\mathrm{~m}, 1 \mathrm{H}) .{ }^{13} \mathrm{C}$ NMR $\left(100 \mathrm{MHz}, \mathrm{CDCl}_{3}\right) \delta 190.1,156.8,137.9,136.1,134.7,134.5$, $134.4,130.8,130.7,129.7,128.8,128.6,128.2,127.8,127.7,118.6,47.6,39.6,30.1,29.6$. HRMS $\left(\mathrm{ESI}^{+}\right) \mathrm{m} / z$ calc'd for $\mathrm{C}_{26} \mathrm{H}_{20} \mathrm{NOClNa}[\mathrm{M}+\mathrm{Na}]^{+}:$420.1126, found 420.1126. HPLC conditions: hexane/2-propanol $95: 5,0.8 \mathrm{~mL} / \mathrm{min}, \lambda=300 \mathrm{~nm}$, Chiralpak AD-H column (4.6 mm x $250 \mathrm{~mm})$, $\mathrm{t}_{\mathrm{r}}$ $($ minor $)=21.7 \min , \mathrm{t}_{\mathrm{r}}($ major $)=25.6 \mathrm{~min}$.<smiles>C[C@@H]1CCC(c2ccc(Br)cc2)=C(c2ccccc2)C1=O</smiles>

(R)-4'-Benzyl-4-bromo-3'-oxo-3',4',5',6'-tetrahydro-[1,1':2',1''-terphenyl]-4' -carbonitrile (3af)

Following the general procedure, the reaction was performed with $1 \mathrm{a}(56.9 \mathrm{mg}, 0.2 \mathrm{mmol}, 1.0 \mathrm{equiv})$, (4-bromophenyl)boronic acid $2 \mathbf{f}$ ( $80.3 \mathrm{mg}, 0.4 \mathrm{mmol}, 2.0$ equiv), $\mathrm{H}_{2} \mathrm{O}$ (15 uL, $0.8 \mathrm{mmol}, 4.0$ equiv), $\mathrm{Ni}(\mathrm{OTf})_{2}(7.2 \mathrm{mg}, 0.02 \mathrm{mmol}, 10 \mathrm{~mol} \%)$, and $(S)-{ }^{t} \mathrm{Bu}-\mathrm{phox}(9.3 \mathrm{mg}, 0.024 \mathrm{mmol}, 12 \mathrm{~mol} \%)$ in $4.0 \mathrm{~mL}$ of toluene at $80{ }^{\circ} \mathrm{C}$ for $24 \mathrm{~h}$. The desired product 3af was obtained in $67 \%$ yield $(58.8 \mathrm{mg})$ as a white solid. er $=92: 8 .[\alpha]_{\mathrm{D}}{ }^{25}=-109.30\left(c 0.9, \mathrm{CHCl}_{3}\right) . R_{f}=0.3(\mathrm{PE}: \mathrm{EA}=10: 1) .{ }^{1} \mathbf{H}$ NMR $(400 \mathrm{MHz}$, $\left.\mathrm{CDCl}_{3}\right) \delta 7.39-7.29(\mathrm{~m}, 7 \mathrm{H}), 7.23-7.19(\mathrm{~m}, 3 \mathrm{H}), 6.96-6.89(\mathrm{~m}, 4 \mathrm{H}), 3.52(\mathrm{~d}, J=14.0 \mathrm{~Hz}, 1 \mathrm{H}), 3.11$ - $3.03(\mathrm{~m}, 2 \mathrm{H}), 2.87$ (dt, $J=19.6,4.5 \mathrm{~Hz}, 1 \mathrm{H}), 2.38$ (dt, $J=13.8,4.5 \mathrm{~Hz}, 1 \mathrm{H}), 2.23-2.15(\mathrm{~m}, 1 \mathrm{H})$. ${ }^{13} \mathrm{C}$ NMR $\left(100 \mathrm{MHz}, \mathrm{CDCl}_{3}\right) \delta 190.1,156.8,138.4,136.1,134.5,134.4,131.5,130.8,130.7,129.9$, 128.8, 128.2, 127.9, 127.8, 123.0, 118.6, 47.6, 39.6, 30.1, 29.6. HRMS $\left(\mathrm{ESI}^{+}\right) \mathrm{m} / \mathrm{z}$ calc'd for $\mathrm{C}_{26} \mathrm{H}_{20} \mathrm{NOBrNa}[\mathrm{M}+\mathrm{Na}]^{+}:$464.0620, found 464.0624. HPLC conditions: hexane/2-propanol $95: 5$, $0.8 \mathrm{~mL} / \mathrm{min}, \lambda=300 \mathrm{~nm}$, Chiralpak AD-H column $(4.6 \mathrm{~mm} \times 250 \mathrm{~mm}), \mathrm{t}_{\mathrm{r}}($ minor $)=24.4 \mathrm{~min}, \mathrm{t}_{\mathrm{r}}$ (major) $=28.9 \mathrm{~min}$. 
(N)

(R)-4'-Benzyl-3'-oxo-4-(trifluoromethyl)-3',4',5',6'-tetrahydro-[1,1':2',1''-terphenyl]-4'carbonitrile (3ag)

Following the general procedure, the reaction was performed with $1 \mathbf{a}(56.9 \mathrm{mg}, 0.2 \mathrm{mmol}, 1.0$ equiv), (4-(trifluoromethyl)phenyl)boronic acid $2 \mathrm{~g}$ (76.0 mg, $0.4 \mathrm{mmol}, 2.0$ equiv), $\mathrm{H}_{2} \mathrm{O}$ (15 uL, $0.8 \mathrm{mmol}$, 4.0 equiv), $\mathrm{Ni}(\mathrm{OTf})_{2}(7.2 \mathrm{mg}, 0.02 \mathrm{mmol}, 10 \mathrm{~mol} \%)$, and $(S)-{ }^{t} \mathrm{Bu}-\mathrm{phox}(9.3 \mathrm{mg}, 0.024 \mathrm{mmol}, 12 \mathrm{~mol} \%)$ in $4.0 \mathrm{~mL}$ of toluene at $80{ }^{\circ} \mathrm{C}$ for $24 \mathrm{~h}$. The desired product $3 \mathrm{ag}$ was obtained in $80 \%$ yield $(68.9 \mathrm{mg})$ as a white solid. er $=89: 11 .[\alpha]_{\mathrm{D}}{ }^{25}=-95.75\left(c 2.1, \mathrm{CHCl}_{3}\right) . R_{f}=0.2(\mathrm{PE}: \mathrm{EA}=15: 1) .{ }^{1} \mathbf{H} \mathbf{N M R}(400$ $\left.\mathrm{MHz}, \mathrm{CDCl}_{3}\right) 7.44(\mathrm{~d}, J=8.1 \mathrm{~Hz}, 2 \mathrm{H}), 7.40-7.32(\mathrm{~m}, 5 \mathrm{H}), 7.22-7.18(\mathrm{~m}, 3 \mathrm{H}), 7.15(\mathrm{~d}, J=8.1 \mathrm{~Hz}$, 2H), $6.95-6.90(\mathrm{~m}, 2 \mathrm{H}), 3.54(\mathrm{~d}, J=14.0 \mathrm{~Hz}, 1 \mathrm{H}), 3.15-3.06(\mathrm{~m}, 2 \mathrm{H}), 2.88(\mathrm{dt}, J=19.6,4.4 \mathrm{~Hz}$, 1H), $2.41(\mathrm{dt}, J=13.8,4.5 \mathrm{~Hz}, 1 \mathrm{H}), 2.26-2.19(\mathrm{~m}, 1 \mathrm{H}) .{ }^{13} \mathbf{C ~ N M R}\left(100 \mathrm{MHz}, \mathrm{CDCl}_{3}\right) \delta 190.1,156.4$, 143.2, 136.7, 134.3, 134.1, 130.7, 130.6, 130.4 (q, $J=32.7 \mathrm{~Hz}), 128.8,128.5,128.2,127.91,127.87$, $125.3(\mathrm{q}, J=3.8 \mathrm{~Hz}), 123.8(\mathrm{q}, J=272.3 \mathrm{~Hz}), 118.5,47.6,39.6,30.2,29.6 .{ }^{19} \mathbf{F}$ NMR $(376 \mathrm{MHz}$, $\left.\mathrm{CDCl}_{3}\right) \delta-62.82$. HRMS $\left(\mathrm{ESI}^{+}\right) \mathrm{m} / z$ calc'd for $\mathrm{C}_{27} \mathrm{H}_{20} \mathrm{~F}_{3} \mathrm{NONa}[\mathrm{M}+\mathrm{Na}]^{+}:$454.1389, found 454.1390. HPLC conditions: hexane/2-propanol $95: 5,0.8 \mathrm{~mL} / \mathrm{min}, \lambda=300 \mathrm{~nm}$, Chiralpak AD-H column (4.6 $\mathrm{mm} \times 250 \mathrm{~mm}), \mathrm{t}_{\mathrm{r}}($ minor $)=18.0 \mathrm{~min}, \mathrm{t}_{\mathrm{r}}($ major $)=21.9 \mathrm{~min}$.<smiles>CC1(C)CCC(c2ccc(OC(F)(F)F)cc2)=C(c2ccccc2)C1=O</smiles>

\section{(R)-4'-Benzyl-3' -oxo-4-(trifluoromethoxy)-3',4',5',6'-tetrahydro-[1,1':2',1'--terphenyl]-4'-} carbonitrile (3ah)

Following the general procedure, the reaction was performed with $1 \mathrm{a}(56.9 \mathrm{mg}, 0.2 \mathrm{mmol}, 1.0 \mathrm{equiv})$, (4-(trifluoromethoxy)phenyl)boronic acid $\mathbf{2 h}\left(82.4 \mathrm{mg}, 0.4 \mathrm{mmol}, 2.0\right.$ equiv), $\mathrm{H}_{2} \mathrm{O}$ (15 uL, $0.8 \mathrm{mmol}$, 4.0 equiv), $\mathrm{Ni}(\mathrm{OTf})_{2}\left(7.2 \mathrm{mg}, 0.02 \mathrm{mmol}, 10 \mathrm{~mol} \%\right.$ ), and $(S)-{ }^{t} \mathrm{Bu}-\mathrm{phox}(9.3 \mathrm{mg}, 0.024 \mathrm{mmol}, 12 \mathrm{~mol} \%)$ in $4.0 \mathrm{~mL}$ of toluene at $80{ }^{\circ} \mathrm{C}$ for $24 \mathrm{~h}$. The desired product $\mathbf{3 a h}$ was obtained in $56 \%$ yield $(50.2 \mathrm{mg})$ as a white solid. er $=90.5: 9.5 .[\alpha]_{\mathrm{D}}{ }^{25}=-99.18\left(c 2.1, \mathrm{CHCl}_{3}\right) \cdot R_{f}=0.3(\mathrm{PE}: \mathrm{EA}=10: 1) .{ }^{1} \mathbf{H} \mathbf{~ N M R}$ $\left(400 \mathrm{MHz} \mathrm{CDCl}_{3}\right) \delta 7.40-7.32(\mathrm{~m}, 5 \mathrm{H}), 7.22-7.20(\mathrm{~m}, 3 \mathrm{H}), 7.07(\mathrm{~d}, J=8.6 \mathrm{~Hz}, 2 \mathrm{H}), 7.02(\mathrm{~d}, J=$ 
$8.5 \mathrm{~Hz}, 2 \mathrm{H}), 6.95-6.93(\mathrm{~m}, 2 \mathrm{H}), 3.54(\mathrm{~d}, J=14.0 \mathrm{~Hz}, 1 \mathrm{H}), 3.13-3.05(\mathrm{~m}, 2 \mathrm{H}), 2.90$ (dt, $J=19.6$, $4.5 \mathrm{~Hz}, 1 \mathrm{H}), 2.40(\mathrm{dt}, J=13.8,4.5 \mathrm{~Hz}, 1 \mathrm{H}), 2.25-2.18(\mathrm{~m}, 1 \mathrm{H}) .{ }^{13} \mathbf{C} \mathbf{N M R}\left(100 \mathrm{MHz}, \mathrm{CDCl}_{3}\right) \delta 190.1$, $156.6,149.1,138.0,136.3,134.4,130.8,130.6,129.9,128.8,128.1,127.8,127.7,120.5,120.4(\mathrm{q}, J=$ 256.3 Hz), 118.6, 47.6, 39.6, 30.1, 29.6. ${ }^{19} \mathbf{F ~ N M R ~}\left(376 \mathrm{MHz}, \mathrm{CDCl}_{3}\right) \delta-57.78 . \mathbf{H R M S}\left(\mathrm{ESI}^{+}\right) \mathrm{m} / \mathrm{z}$ calc'd for $\mathrm{C}_{27} \mathrm{H}_{20} \mathrm{NO}_{2} \mathrm{~F}_{3} \mathrm{Na}[\mathrm{M}+\mathrm{Na}]^{+}$: 470.1338, found 470.1329. HPLC conditions: hexane/2propanol $95: 5,0.8 \mathrm{~mL} / \mathrm{min}, \lambda=300 \mathrm{~nm}$, Chiralpak AD-H column $(4.6 \mathrm{~mm} \times 250 \mathrm{~mm}), \mathrm{t}_{\mathrm{r}}(\operatorname{minor})=$ $16.8 \mathrm{~min}, \mathrm{t}_{\mathrm{r}}($ major $)=18.9 \mathrm{~min}$.<smiles>N#Cc1ccc(C2=C(c3ccccc3)C(=O)[C@@](Br)(C#N)CC2)cc1</smiles>

\section{(R)-4'-Benzyl-3'-oxo-3',4',5',6'-tetrahydro-[1,1':2',1''-terphenyl]-4,4'-dicarbonitrile (3ai)}

Following the general procedure, the reaction was performed with 1a (56.9 $\mathrm{mg}, 0.2 \mathrm{mmol}, 1.0$ equiv), (4-cyanophenyl)boronic acid $2 \mathbf{i}\left(58.8 \mathrm{mg}, 0.4 \mathrm{mmol}, 2.0\right.$ equiv), $\mathrm{H}_{2} \mathrm{O}$ (15 uL, $0.8 \mathrm{mmol}, 4.0$ equiv), $\mathrm{Ni}(\mathrm{OTf})_{2}(7.2 \mathrm{mg}, 0.02 \mathrm{mmol}, 10 \mathrm{~mol} \%)$, and $(S)-{ }^{t} \mathrm{Bu}-$ phox $(9.3 \mathrm{mg}, 0.024 \mathrm{mmol}, 12 \mathrm{~mol} \%)$ in $4.0 \mathrm{~mL}$ of toluene at $80{ }^{\circ} \mathrm{C}$ for $24 \mathrm{~h}$. The desired product 3ai was obtained in $80 \%$ yield $(62.0 \mathrm{mg})$ as a white solid. er $=92.5: 7.5 .[\alpha]_{\mathrm{D}}{ }^{25}=-103.27\left(c 1.0, \mathrm{CHCl}_{3}\right) . R_{f}=0.2(\mathrm{PE}: \mathrm{EA}=5: 1) .{ }^{1} \mathbf{H} \mathbf{N M R}(400 \mathrm{MHz}$, $\left.\mathrm{CDCl}_{3}\right) \delta 7.48-7.45(\mathrm{~m}, 2 \mathrm{H}), 7.40-7.31(\mathrm{~m}, 5 \mathrm{H}), 7.24-7.17(\mathrm{~m}, 3 \mathrm{H}), 7.16-7.12(\mathrm{~m}, 2 \mathrm{H}), 6.93-$ $6.89(\mathrm{~m}, 2 \mathrm{H}), 3.53(\mathrm{AB}, J=14.0 \mathrm{~Hz}, 1 \mathrm{H}), 3.14-3.05(\mathrm{~m}, 2 \mathrm{H}), 2.86(\mathrm{dt}, J=19.6,4.4 \mathrm{~Hz}, 1 \mathrm{H}), 2.44-$ $2.38(\mathrm{~m}, 1 \mathrm{H}), 2.25-2.18(\mathrm{~m}, 1 \mathrm{H}) .{ }^{13} \mathbf{C}$ NMR $\left(100 \mathrm{MHz}, \mathrm{CDCl}_{3}\right) \delta 189.9,155.7,144.2,137.0,134.2$, $133.8,132.1,130.7,130.6,128.9,128.8,128.3,128.1,127.9,118.3,112.3,47.6,39.5,30.2,29.4$. HRMS $\left(\mathrm{ESI}^{+}\right) \mathrm{m} / \mathrm{z}$ calc'd for $\mathrm{C}_{27} \mathrm{H}_{20} \mathrm{~N}_{2} \mathrm{ONa}[\mathrm{M}+\mathrm{Na}]^{+}:$411.1468, found 411.1457. HPLC conditions: hexane/2-propanol $70: 30,0.8 \mathrm{~mL} / \mathrm{min}, \lambda=280 \mathrm{~nm}$, Chiralcel OD-H column (4.6 mm x $250 \mathrm{~mm}), \mathrm{t}_{\mathrm{r}}$ $($ minor $)=21.2 \min , \mathrm{t}_{\mathrm{r}}($ major $)=24.7 \mathrm{~min}$.<smiles>CC1(C)CCC(c2ccc(C=O)cc2)=C(c2ccccc2)C1=O</smiles>

\section{(R)-4'-Benzyl-4-formyl-3'-oxo-3',4',5',6'-tetrahydro-[1,1':2',1'-terphenyl]-4' -carbonitrile (3aj)}

Following the general procedure, the reaction was performed with $1 \mathbf{a}(56.9 \mathrm{mg}, 0.2 \mathrm{mmol}, 1.0$ equiv), (4-formylphenyl)boronic acid $\mathbf{2 j}$ (60.0 mg, $0.4 \mathrm{mmol}, 2.0$ equiv), $\mathrm{H}_{2} \mathrm{O}$ (15 uL, $0.8 \mathrm{mmol}, 4.0$ equiv), 
$\mathrm{Ni}(\mathrm{OTf})_{2}(7.2 \mathrm{mg}, 0.02 \mathrm{mmol}, 10 \mathrm{~mol} \%)$, and $(S)-{ }^{t} \mathrm{Bu}-$ phox $(9.3 \mathrm{mg}, 0.024 \mathrm{mmol}, 12 \mathrm{~mol} \%)$ in $4.0 \mathrm{~mL}$ of toluene at $80{ }^{\circ} \mathrm{C}$ for $24 \mathrm{~h}$. The desired product 3aj was obtained in $72 \%$ yield $(56.3 \mathrm{mg})$ as a white solid. er $=96.5: 3.5 .[\alpha]_{\mathrm{D}}{ }^{25}=-122.02\left(c 1.6, \mathrm{CHCl}_{3}\right) . R_{f}=0.2(\mathrm{PE}: \mathrm{EA}=5: 1) .{ }^{1} \mathbf{H} \mathbf{~ N M R}(400 \mathrm{MHz}$, $\left.\mathrm{CDCl}_{3}\right) \delta 9.91(\mathrm{~s}, 1 \mathrm{H}), 7.69(\mathrm{~d}, J=8.3 \mathrm{~Hz}, 2 \mathrm{H}), 7.41-7.32(\mathrm{~m}, 5 \mathrm{H}), 7.21-7.15(\mathrm{~m}, 5 \mathrm{H}), 6.96-6.91$ (m, 2H), $3.54(\mathrm{AB}, J=14.0 \mathrm{~Hz}, 1 \mathrm{H}), 3.16-3.07(\mathrm{~m}, 2 \mathrm{H}), 2.90(\mathrm{dt}, J=19.6,4.5 \mathrm{~Hz}, 1 \mathrm{H}), 2.42(\mathrm{dt}, J$ $=13.8,4.5 \mathrm{~Hz}, 1 \mathrm{H}), 2.27-2.20(\mathrm{~m}, 1 \mathrm{H}) .{ }^{13} \mathbf{C ~ N M R}\left(100 \mathrm{MHz}, \mathrm{CDCl}_{3}\right) \delta 191.6,190.0,156.6,145.7$, $136.8,135.9,134.3,134.1,130.8,130.7,129.6,128.8,128.2,128.0,127.9,118.5,47.6,39.6,30.3$, 29.6. HRMS $\left(\mathrm{ESI}^{+}\right) \mathrm{m} / z$ calc'd for $\mathrm{C}_{27} \mathrm{H}_{21} \mathrm{NO}_{2} \mathrm{Na}[\mathrm{M}+\mathrm{Na}]^{+}:$414.1465, found 414.1468. HPLC conditions: hexane/2-propanol $70: 30,1.0 \mathrm{~mL} / \mathrm{min}, \lambda=300 \mathrm{~nm}$, Chiralcel OD-H column $(4.6 \mathrm{~mm} \mathrm{x}$ $250 \mathrm{~mm}), \mathrm{t}_{\mathrm{r}}($ minor $)=18.3 \mathrm{~min}, \mathrm{t}_{\mathrm{r}}($ major $)=22.8 \mathrm{~min}$.<smiles>CC(=O)c1ccc(C2=C(c3ccccc3)C(=O)C(C)([N+]#N)CC2)cc1</smiles>

\section{(R)-4-Acetyl-4'-benzyl-3'-oxo-3',4',5',6'-tetrahydro-[1,1':2',1''-terphenyl]-4' -carbonitrile (3ak)}

Following the general procedure, the reaction was performed with $1 \mathrm{a}(56.9 \mathrm{mg}, 0.2 \mathrm{mmol}, 1.0$ equiv), (4-acetylphenyl)boronic acid 2k (65.6 mg, 0.4 mmol, 2.0 equiv), $\mathrm{H}_{2} \mathrm{O}$ (15 uL, $0.8 \mathrm{mmol}, 4.0$ equiv), $\mathrm{Ni}(\mathrm{OTf})_{2}(7.2 \mathrm{mg}, 0.02 \mathrm{mmol}, 10 \mathrm{~mol} \%)$, and $(S)-{ }^{t} \mathrm{Bu}-\mathrm{phox}(9.3 \mathrm{mg}, 0.024 \mathrm{mmol}, 12 \mathrm{~mol} \%)$ in $4.0 \mathrm{~mL}$ of toluene at $80{ }^{\circ} \mathrm{C}$ for $24 \mathrm{~h}$. The desired product 3ak was obtained in $84 \%$ yield $(68.0 \mathrm{mg})$ as a white solid. er $=93.5: 6.5 .[\alpha]_{\mathrm{D}}{ }^{25}=-145.63\left(c 1.9, \mathrm{CHCl}_{3}\right) . R_{f}=0.2(\mathrm{PE}: \mathrm{EA}=5: 1) .{ }^{1} \mathbf{H} \mathrm{NMR}(400 \mathrm{MHz}$, $\left.\mathrm{CDCl}_{3}\right) \delta 7.76(\mathrm{~d}, J=8.4 \mathrm{~Hz}, 2 \mathrm{H}), 7.42-7.30(\mathrm{~m}, 5 \mathrm{H}), 7.20-7.17(\mathrm{~m}, 3 \mathrm{H}), 7.13(\mathrm{~d}, J=8.4 \mathrm{~Hz}, 2 \mathrm{H})$, $6.96-6.91(\mathrm{~m}, 2 \mathrm{H}), 3.53(\mathrm{~d}, J=14.0 \mathrm{~Hz}, 1 \mathrm{H}), 3.16-3.04(\mathrm{~m}, 2 \mathrm{H}), 2.90(\mathrm{dt}, J=19.6,4.5 \mathrm{~Hz}, 1 \mathrm{H})$, $2.52(\mathrm{~s}, 3 \mathrm{H}), 2.41(\mathrm{dt}, J=13.7,4.5 \mathrm{~Hz}, 1 \mathrm{H}), 2.26-2.19(\mathrm{~m}, 1 \mathrm{H}) .{ }^{13} \mathbf{C}$ NMR $\left(100 \mathrm{MHz}, \mathrm{CDCl}_{3}\right) \delta$ 197.5, 190.0, 156.9, 144.2, 136.6, 136.5, 134.3, 134.2, 131.0, 130.8, 130.6, 128.8, 128.5, 128.3, 128.1, 127.9, 118.5, 47.6, 39.6, 30.2, 29.5, 26.7. HRMS $\left(\mathrm{ESI}^{+}\right) \mathrm{m} / \mathrm{z}$ calc'd for $\mathrm{C}_{28} \mathrm{H}_{23} \mathrm{NO}_{2} \mathrm{Na}[\mathrm{M}+\mathrm{Na}]^{+}$: 428.1621, found 428.1613. HPLC conditions: hexane/2-propanol $75: 25,1.0 \mathrm{~mL} / \mathrm{min}, \lambda=254 \mathrm{~nm}$, Chiralpak AD-H column $(4.6 \mathrm{~mm}$ x $250 \mathrm{~mm}), \mathrm{t}_{\mathrm{r}}($ minor $)=10.3 \mathrm{~min}, \mathrm{t}_{\mathrm{r}}($ major $)=11.6 \mathrm{~min}$.<smiles>CC(=O)c1ccc(C2=C(c3ccccc3)C(=O)C(C#N)(C#N)CC2)cc1</smiles> 


\section{(R)-Methyl-4'-benzyl-4'-cyano-3'-oxo-3',4',5',6'-tetrahydro-[1, 1':2',1'-terphenyl]-4-carboxylate}

(3al)

Following the general procedure, the reaction was performed with $1 \mathbf{a}(56.9 \mathrm{mg}, 0.2 \mathrm{mmol}, 1.0$ equiv), (4-(methoxycarbonyl)phenyl)boronic acid 21 (71.9 mg, 0.4 mmol, 2.0 equiv), $\mathrm{H}_{2} \mathrm{O}$ (15 uL, $0.8 \mathrm{mmol}$, 4.0 equiv), $\mathrm{Ni}(\mathrm{OTf})_{2}(7.2 \mathrm{mg}, 0.02 \mathrm{mmol}, 10 \mathrm{~mol} \%)$, and $(S){ }^{t} \mathrm{Bu}-\mathrm{phox}(9.3 \mathrm{mg}, 0.024 \mathrm{mmol}, 12 \mathrm{~mol} \%)$ in $4.0 \mathrm{~mL}$ of toluene at $80{ }^{\circ} \mathrm{C}$ for $24 \mathrm{~h}$. The desired product 3al was obtained in $92 \%$ yield $(76.9 \mathrm{mg})$ as a white solid. $\mathrm{er}=96.5: 3.5 .[\alpha]_{\mathrm{D}}{ }^{25}=-118.40\left(c 1.0, \mathrm{CHCl}_{3}\right) . R_{f}=0.2(\mathrm{PE}: \mathrm{EA}=5: 1) .{ }^{1} \mathbf{H}$ NMR $\left(400 \mathrm{MHz}, \mathrm{CDCl}_{3}\right) \delta 7.84(\mathrm{~d}, J=8.1 \mathrm{~Hz}, 2 \mathrm{H}), 7.40-7.31(\mathrm{~m}, 5 \mathrm{H}), 7.20-7.17(\mathrm{~m}, 3 \mathrm{H}), 7.11(\mathrm{~d}, J=$ $8.1 \mathrm{~Hz}, 2 \mathrm{H}), 6.95-6.91(\mathrm{~m}, 2 \mathrm{H}), 3.87(\mathrm{~s}, 3 \mathrm{H}), 3.53(\mathrm{~d}, J=14.0 \mathrm{~Hz}, 1 \mathrm{H}), 3.14-3.06(\mathrm{~m}, 2 \mathrm{H}), 2.90$ $(\mathrm{dt}, J=19.6,4.5 \mathrm{~Hz}, 1 \mathrm{H}), 2.41(\mathrm{dt}, J=13.8,4.5 \mathrm{~Hz}, 1 \mathrm{H}), 2.26-2.19(\mathrm{~m}, 1 \mathrm{H}) .{ }^{13} \mathbf{C}$ NMR $(100 \mathrm{MHz}$, $\left.\mathrm{CDCl}_{3}\right) \delta 190.0,166.5,157.1,144.1,136.4,134.3,134.2,130.7,130.6,130.0,129.5,128.8,128.2$, 128.1, 127.83, 127.79, 118.5, 52.3, 47.6, 39.5, 30.2, 29.5. HRMS $\left(\mathrm{ESI}^{+}\right) \mathrm{m} / z$ calc'd for $\mathrm{C}_{28} \mathrm{H}_{23} \mathrm{NO}_{3} \mathrm{Na}$ $[\mathrm{M}+\mathrm{Na}]^{+}:$444.1570, found 444.1558. HPLC conditions: hexane/2-propanol $80: 20,1.0 \mathrm{~mL} / \mathrm{min}, \lambda=$ $254 \mathrm{~nm}$, Chiralpak AS-H column $(4.6 \mathrm{~mm}$ x $250 \mathrm{~mm}), \mathrm{t}_{\mathrm{r}}($ major $)=19.6 \mathrm{~min}, \mathrm{t}_{\mathrm{r}}(\operatorname{minor})=23.8 \mathrm{~min}$.<smiles>CN(C)C(=O)c1ccc(C2=C(c3ccccc3)C(=O)C(C)(Br)CC2)cc1</smiles>

\section{(R)-4'-Benzyl-4'-cyano-N,N-dimethyl-3'-oxo-3',4',5',6'-tetrahydro-[1,1':2',1''-terphenyl]-4-} carboxamide (3am)

Following the general procedure, the reaction was performed with 1a (56.9 $\mathrm{mg}, 0.2 \mathrm{mmol}, 1.0$ equiv), (4-(dimethylcarbamoyl)phenyl)boronic acid $2 \mathrm{~m}$ (77.2 mg, $0.4 \mathrm{mmol}, 2.0$ equiv), $\mathrm{H}_{2} \mathrm{O}$ (15 uL, 0.8 mmol, 4.0 equiv), Ni(OTf) 2 (7.2 mg, $0.02 \mathrm{mmol}, 10 \mathrm{~mol} \%$ ), and $(S){ }^{t}{ }^{\mathrm{Bu}} \mathrm{-phox}(9.3 \mathrm{mg}, 0.024 \mathrm{mmol}$, $12 \mathrm{~mol} \%$ ) in $4.0 \mathrm{~mL}$ of toluene at $80^{\circ} \mathrm{C}$ for $24 \mathrm{~h}$. The desired product $3 \mathrm{am}$ was obtained in $63 \%$ yield $(45.2 \mathrm{mg})$ as a white solid. er $=97: 3 .[\alpha]_{\mathrm{D}}{ }^{25}=-95.02\left(c 1.7, \mathrm{CHCl}_{3}\right) . R_{f}=0.2(\mathrm{PE}: \mathrm{EA}=1: 1) .{ }^{1} \mathbf{H}$ NMR $\left(400 \mathrm{MHz}, \mathrm{CDCl}_{3}\right) \delta 7.39-7.29(\mathrm{~m}, 5 \mathrm{H}), 7.22(\mathrm{~d}, J=8.0 \mathrm{~Hz}, 2 \mathrm{H}), 7.18-7.14(\mathrm{~m}, 3 \mathrm{H}), 7.06$ $(\mathrm{d}, J=8.0 \mathrm{~Hz}, 2 \mathrm{H}), 6.95-6.91(\mathrm{~m}, 2 \mathrm{H}), 3.52(\mathrm{AB}, J=13.9 \mathrm{~Hz}, 1 \mathrm{H}), 3.12-2.87(\mathrm{~m}, 9 \mathrm{H}), 2.39(\mathrm{dt}, J$ $=13.7,4.5 \mathrm{~Hz}, 1 \mathrm{H}), 2.24-2.17(\mathrm{~m}, 1 \mathrm{H}) .{ }^{13} \mathbf{C}$ NMR $\left(100 \mathrm{MHz}, \mathrm{CDCl}_{3}\right) \delta 190.1,170.8,157.3,140.8$, 136.2 , 136.1 134.5, 134.4, 130.8, 130.6, 128.7, 128.2 , 128.0, 127.8, 127.7, 127.1, 118.6, 47.6, 39.6, 
30.1, 35.4 (br), 29.6. HRMS $\left(\mathrm{ESI}^{+}\right) \mathrm{m} / z$ calc'd for $\mathrm{C}_{29} \mathrm{H}_{26} \mathrm{~N}_{2} \mathrm{O}_{2} \mathrm{Na}[\mathrm{M}+\mathrm{Na}]^{+}$: 457.1886, found 457.1892. HPLC conditions: hexane/2-propanol $75: 25,0.8 \mathrm{~mL} / \mathrm{min}, \lambda=300 \mathrm{~nm}$, Chiralpak IB column (4.6 $\mathrm{mm} \times 250 \mathrm{~mm}), \mathrm{t}_{\mathrm{r}}($ minor $)=26.9 \mathrm{~min}, \mathrm{t}_{\mathrm{r}}($ major $)=29.8 \mathrm{~min}$.<smiles>CC(=O)c1cccc(C2=C(c3ccccc3)C(=O)C([18O])([18O])CC2)c1</smiles>

(R)- Methyl-4'- benzyl-4'- cyano-3'- oxo $-3^{\prime}, 4^{\prime}, 5^{\prime}, 6^{\prime}$-tetrahydro- $\left[1,1^{\prime}: 2^{\prime}, 1^{\prime \prime}-\right.$ terphenyl $\quad-3$ carboxylate (3an)

Following the general procedure, the reaction was performed with $\mathbf{1 a}(56.9 \mathrm{mg}, 0.2 \mathrm{mmol}, 1.0 \mathrm{equiv})$, (3-(methoxycarbonyl)phenyl)boronic acid 2n (71.9 mg, $0.4 \mathrm{mmol}, 2.0$ equiv), $\mathrm{H}_{2} \mathrm{O}$ (15 uL, $0.8 \mathrm{mmol}$, 4.0 equiv), $\mathrm{Ni}(\mathrm{OTf})_{2}(7.2 \mathrm{mg}, 0.02 \mathrm{mmol}, 10 \mathrm{~mol} \%)$, and $(S)-{ }^{t} \mathrm{Bu}-\mathrm{phox}(9.3 \mathrm{mg}, 0.024 \mathrm{mmol}, 12 \mathrm{~mol} \%)$ in $4.0 \mathrm{~mL}$ of toluene at $80{ }^{\circ} \mathrm{C}$ for $24 \mathrm{~h}$. The desired product $3 \mathrm{an}$ was obtained in $91 \%$ yield $(76.3 \mathrm{mg})$ as a white solid. er $=97: 3 .[\alpha]_{\mathrm{D}}{ }^{25}=-127.94\left(c 1.6, \mathrm{CHCl}_{3}\right) . R_{f}=0.2(\mathrm{PE}: \mathrm{EA}=10: 1) .{ }^{1} \mathbf{H}$ NMR $\left(400 \mathrm{MHz}, \mathrm{CDCl}_{3}\right) \delta 7.87-7.83(\mathrm{~m}, 2 \mathrm{H}), 7.41-7.31(\mathrm{~m}, 5 \mathrm{H}), 7.21-7.16(\mathrm{~m}, 4 \mathrm{H}), 7.12(\mathrm{dt}, J=7.8$, $1.6 \mathrm{~Hz}, 1 \mathrm{H}), 6.97-6.92(\mathrm{~m}, 2 \mathrm{H}), 3.88(\mathrm{~s}, 3 \mathrm{H}), 3.53(\mathrm{AB}, J=14.0 \mathrm{~Hz}, 1 \mathrm{H}), 3.15-3.06(\mathrm{~m}, 2 \mathrm{H}), 2.95$ $(\mathrm{dt}, J=19.7,4.6 \mathrm{~Hz}, 1 \mathrm{H}), 2.41(\mathrm{dt}, J=13.7,4.6 \mathrm{~Hz}, 1 \mathrm{H}), 2.26-2.19(\mathrm{~m}, 1 \mathrm{H}) .{ }^{13} \mathrm{C}$ NMR $(100 \mathrm{MHz}$, $\left.\mathrm{CDCl}_{3}\right) \delta 190.1,166.5,157.1,139.8,136.4,134.4,134.3,132.9,130.8,130.6,130.3,129.7,129.0$, 128.8, 128.4, 128.1, 127.8, 127.7, 118.6, 52.4, 47.6, 39.6, 30.1, 29.6. HRMS (ESI $\left.{ }^{+}\right) \mathrm{m} / z$ calc'd for $\mathrm{C}_{28} \mathrm{H}_{23} \mathrm{NO}_{3} \mathrm{Na}[\mathrm{M}+\mathrm{Na}]^{+}: 444.1570$, found 444.1578. HPLC conditions: hexane/2-propanol $95: 5,0.8$ $\mathrm{mL} / \mathrm{min}, \lambda=300 \mathrm{~nm}$, Chiralpak AD-H column $(4.6 \mathrm{~mm} \times 250 \mathrm{~mm}) ; \mathrm{t}_{\mathrm{r}}($ minor $)=28.8 \mathrm{~min}, \mathrm{t}_{\mathrm{r}}$ (major) $=34.6 \mathrm{~min}$.<smiles>C=Cc1ccc(C2=C(c3ccccc3)C(=O)C3([18O])CCC2C3=O)cc1</smiles>

(R)-4'-Benzyl-3'-oxo-4-vinyl-3',4',5',6'-tetrahydro-[1,1':2',1'-terphenyl]-4'-carbonitrile (3ao)

Following the general procedure, the reaction was performed with 1a (56.9 mg, $0.2 \mathrm{mmol}, 1.0$ equiv), (4-vinylphenyl)boronic acid 20 (59.2 mg, $0.4 \mathrm{mmol}, 2.0$ equiv), $\mathrm{H}_{2} \mathrm{O}$ (15 uL, $0.8 \mathrm{mmol}, 4.0$ equiv), $\mathrm{Ni}(\mathrm{OTf})_{2}(7.2 \mathrm{mg}, 0.02 \mathrm{mmol}, 10 \mathrm{~mol} \%)$, and $(S)-{ }^{t} \mathrm{Bu}-\mathrm{phox}(9.3 \mathrm{mg}, 0.024 \mathrm{mmol}, 12 \mathrm{~mol} \%)$ in $4.0 \mathrm{~mL}$ of toluene at $80{ }^{\circ} \mathrm{C}$ for $24 \mathrm{~h}$. The desired product 3ao was obtained in $39 \%$ yield $(30.2 \mathrm{mg})$ as a white 
solid. er $=96: 4 .[\alpha]_{\mathrm{D}}{ }^{25}=-108.60\left(c 0.6, \mathrm{CHCl}_{3}\right) . R_{f}=0.3(\mathrm{PE}: \mathrm{EA}=10: 1) .{ }^{1} \mathbf{H}$ NMR $(400 \mathrm{MHz}$, $\left.\mathrm{CDCl}_{3}\right) \delta 7.42-7.30(\mathrm{~m}, 5 \mathrm{H}), 7.22-7.19(\mathrm{~m}, 5 \mathrm{H}), 7.02-6.94(\mathrm{~m}, 4 \mathrm{H}), 6.61(\mathrm{dd}, J=17.6,10.9 \mathrm{~Hz}$, 1H), $5.71(\mathrm{~d}, J=17.6 \mathrm{~Hz}, 1 \mathrm{H}), 5.25(\mathrm{~d}, J=10.9 \mathrm{~Hz}, 1 \mathrm{H}), 3.54(\mathrm{AB}, J=14.0 \mathrm{~Hz}, 1 \mathrm{H}), 3.13-3.05(\mathrm{~m}$, 2H), $2.92(\mathrm{dt}, J=19.5,4.4 \mathrm{~Hz}, 1 \mathrm{H}), 2.39(\mathrm{dt}, J=13.7,4.5 \mathrm{~Hz}, 1 \mathrm{H}), 2.24-2.17(\mathrm{~m}, 1 \mathrm{H}) .{ }^{13} \mathbf{C} \mathbf{~ N M R}$ $\left(100 \mathrm{MHz}, \mathrm{CDCl}_{3}\right) \delta 190.2,157.9,138.8,137.9,136.1,135.6,134.9,134.5,130.9,130.6,128.8,128.6$, 128.0, 127.8, 127.5, 126.0, 118.7, 115.2, 47.7, 39.7, 30.2, 29.5. HRMS $\left(\mathrm{ESI}^{+}\right) \mathrm{m} / z$ calc'd for $\mathrm{C}_{28} \mathrm{H}_{24} \mathrm{NO}$ $[\mathrm{M}+\mathrm{H}]^{+}:$390.1852, found 390.1846. HPLC conditions: hexane/2-propanol $95: 5,0.8 \mathrm{~mL} / \mathrm{min}, \lambda=$ $254 \mathrm{~nm}$, Chiralpak AD-H column $(4.6 \mathrm{~mm} \times 250 \mathrm{~mm}), \mathrm{t}_{\mathrm{r}}($ minor $)=18.6 \mathrm{~min}, \mathrm{t}_{\mathrm{r}}($ major $)=22.4 \mathrm{~min}$.

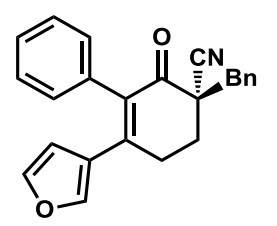

\section{(R)-3-Benzyl-6-(furan-3-yl)-2-oxo-2,3,4,5-tetrahydro-[1,1'-biphenyl]-3-carbonitrile (3ap)}

Following the general procedure, the reaction was performed with $1 \mathrm{a}(56.9 \mathrm{mg}, 0.2 \mathrm{mmol}, 1.0 \mathrm{equiv})$, furan-3-yl boronic acid $\mathbf{2 p}$ (44.8 mg, $0.4 \mathrm{mmol}, 2.0$ equiv), $\mathrm{H}_{2} \mathrm{O}$ (15 uL, $0.8 \mathrm{mmol}, 4.0$ equiv), $\mathrm{Ni}(\mathrm{OTf})_{2}$ (7.2 mg, $0.02 \mathrm{mmol}, 10 \mathrm{~mol} \%$ ), and (S)- ${ }^{t} \mathrm{Bu}-$ phox (9.3 mg, $0.024 \mathrm{mmol}, 12 \mathrm{~mol} \%$ ) in $4.0 \mathrm{~mL}$ of toluene at $80{ }^{\circ} \mathrm{C}$ for $24 \mathrm{~h}$. The desired product 3ap was obtained in $70 \%$ yield $(49.7 \mathrm{mg})$ as a white solid. er $=$ $85: 15 .[\alpha]_{\mathrm{D}}^{25}=-62.63\left(c 1.0, \mathrm{CHCl}_{3}\right) \cdot R_{f}=0.3(\mathrm{PE}: \mathrm{EA}=10: 1) .{ }^{1} \mathbf{H} \mathbf{N M R}\left(400 \mathrm{MHz}, \mathrm{CDCl}_{3}\right) \delta$ $7.44-7.29(\mathrm{~m}, 8 \mathrm{H}), 7.19-7.16(\mathrm{~m}, 2 \mathrm{H}), 7.12-7.08(\mathrm{~m}, 2 \mathrm{H}), 5.66-5.65(\mathrm{~m}, 1 \mathrm{H}), 3.51(\mathrm{AB}, J=14.0$ $\mathrm{Hz}, 1 \mathrm{H}), 3.10-3.02(\mathrm{~m}, 2 \mathrm{H}), 2.92(\mathrm{dt}, J=18.8,4.7 \mathrm{~Hz}, 1 \mathrm{H}), 2.36(\mathrm{dt}, J=13.7,4.7 \mathrm{~Hz}, 1 \mathrm{H}), 2.19-$ $2.12(\mathrm{~m}, 1 \mathrm{H}) .{ }^{13} \mathbf{C} \mathbf{N M R}\left(100 \mathrm{MHz}, \mathrm{CDCl}_{3}\right) \delta 189.8,147.7,144.5,143.2,136.1,134.6,134.0,130.6$, 129.9, 128.9, 128.7, 128.3, 127.7, 124.7, 118.8, 110.0, 47.5, 39.6, 29.8, 27.0. HRMS (ESI $\left.{ }^{+}\right) \mathrm{m} / z$ calc'd for $\mathrm{C}_{24} \mathrm{H}_{19} \mathrm{NO}_{2} \mathrm{Na}[\mathrm{M}+\mathrm{Na}]^{+}: 376.1308$, found 376.1309. HPLC conditions: hexane/2-propanol $95: 5$, $0.8 \mathrm{~mL} / \mathrm{min}, \lambda=280 \mathrm{~nm}$, Chiralpak AD-H column $(4.6 \mathrm{~mm} \times 250 \mathrm{~mm}), \mathrm{t}_{\mathrm{r}}($ major $)=24.7 \mathrm{~min}, \mathrm{t}_{\mathrm{r}}($ minor $)$ $=34.3 \mathrm{~min}$.<smiles>N#[13C]C1CCC(c2ccsc2)=C(c2ccccc2)C1=O</smiles>

\section{(R)-3-Benzyl-2-oxo-6-(thiophen-3-yl)-2,3,4,5-tetrahydro-[1,1'-biphenyl]-3-carbonitrile (3aq)}

Following the general procedure, the reaction was performed with $1 \mathbf{a}(56.9 \mathrm{mg}, 0.2 \mathrm{mmol}, 1.0 \mathrm{equiv})$, thiophen-3-ylboronic acid $2 q\left(51.2 \mathrm{mg}, 0.4 \mathrm{mmol}, 2.0\right.$ equiv), $\mathrm{H}_{2} \mathrm{O}$ (15 uL, $0.8 \mathrm{mmol}, 4.0$ equiv), 
$\mathrm{Ni}(\mathrm{OTf})_{2}(7.2 \mathrm{mg}, 0.02 \mathrm{mmol}, 10 \mathrm{~mol} \%)$, and $(S)-{ }^{t} \mathrm{Bu}-\mathrm{phox}(9.3 \mathrm{mg}, 0.024 \mathrm{mmol}, 12 \mathrm{~mol} \%)$ in $4.0 \mathrm{~mL}$ of toluene at $80{ }^{\circ} \mathrm{C}$ for $24 \mathrm{~h}$. The desired product $3 \mathbf{a q}$ was obtained in $86 \%$ yield $(63.3 \mathrm{mg})$ as a white solid. er $=91: 9 .[\alpha]_{\mathrm{D}}{ }^{25}=-89.42\left(c 1.5, \mathrm{CHCl}_{3}\right) . R_{f}=0.3(\mathrm{PE}: \mathrm{EA}=10: 1) .{ }^{1} \mathbf{H}$ NMR $(400 \mathrm{MHz}$, $\left.\mathrm{CDCl}_{3}\right) \delta 7.39-7.31(\mathrm{~m}, 8 \mathrm{H}), 7.13-7.03(\mathrm{~m}, 4 \mathrm{H}), 6.56(\mathrm{dd}, J=5.2,1.3 \mathrm{~Hz}, 1 \mathrm{H}), 3.52(\mathrm{AB}, J=14.0$ $\mathrm{Hz}, 1 \mathrm{H}), 3.18-2.98(\mathrm{~m}, 3 \mathrm{H}), 2.38(\mathrm{dt}, J=13.7,4.6 \mathrm{~Hz}, 1 \mathrm{H}), 2.21-2.14(\mathrm{~m}, 1 \mathrm{H}) .{ }^{13} \mathbf{C}$ NMR $(100$ $\left.\mathrm{MHz}, \mathrm{CDCl}_{3}\right) \delta 190.3,150.9,139.7,135.9,134.6,130.6,130.4,128.8,128.6,128.1,128.0,127.8$, 127.7, 125.2, 118.8, 47.5, 39.7, 29.9, 28.7. HRMS $\left(\mathrm{ESI}^{+}\right) \mathrm{m} / z$ calc'd for $\mathrm{C}_{24} \mathrm{H}_{19} \mathrm{NOSNa}[\mathrm{M}+\mathrm{Na}]^{+}$: 392.1080, found 392.1083. HPLC conditions: hexane/2-propanol $95: 5,0.8 \mathrm{~mL} / \mathrm{min}, \lambda=280 \mathrm{~nm}$, Chiralpak AD-H column $(4.6 \mathrm{~mm}$ x $250 \mathrm{~mm}), \mathrm{t}_{\mathrm{r}}($ major $)=28.1 \mathrm{~min}, \mathrm{t}_{\mathrm{r}}($ minor $)=30.5 \mathrm{~min}$.<smiles>Cc1ccc(C2=C(c3ccccc3)CC[C@@](C)([N+]#N)C2=O)cc1</smiles>

$(R)-4$ '-Benzyl-4' '-methyl-3'-oxo-3',4',5',6'-tetrahydro-[1,1':2',1'-terphenyl]-4'-carbonitrile (3ba) Following the general procedure, the reaction was performed with $\mathbf{1 b}(59.7 \mathrm{mg}, 0.2 \mathrm{mmol}, 1.0 \mathrm{equiv})$, phenylboronic acid $\mathbf{2 a}$ (48.8 mg, $0.4 \mathrm{mmol}, 2.0$ equiv), $\mathrm{H}_{2} \mathrm{O}$ (15 uL, $0.8 \mathrm{mmol}, 4.0$ equiv), Ni(OTf) (7.2 mg, $0.02 \mathrm{mmol}, 10 \mathrm{~mol} \%$ ), and (S)- ${ }^{t} \mathrm{Bu}-$ phox (9.3 mg, $0.024 \mathrm{mmol}, 12 \mathrm{~mol} \%$ ) in $4.0 \mathrm{~mL}$ of toluene at $80{ }^{\circ} \mathrm{C}$ for $24 \mathrm{~h}$. The desired product $3 \mathrm{ba}$ was obtained in $80 \%$ yield $(60.1 \mathrm{mg})$ as a white solid. er $=$ 94.5 : 5.5. $[\alpha]_{\mathrm{D}}{ }^{25}=-145.67\left(c 1.0, \mathrm{CHCl}_{3}\right) . R_{f}=0.3(\mathrm{PE}: \mathrm{EA}=10: 1) .{ }^{1} \mathbf{H} \mathbf{~ N M R}\left(400 \mathrm{MHz}, \mathrm{CDCl}_{3}\right) \delta$ $7.40-7.31(\mathrm{~m}, 5 \mathrm{H}), 7.21-7.16(\mathrm{~m}, 3 \mathrm{H}), 7.07-7.04(\mathrm{~m}, 2 \mathrm{H}), 6.99(\mathrm{AB}, J=7.8 \mathrm{~Hz}, 2 \mathrm{H}), 6.84(\mathrm{BA}$, $J=7.7 \mathrm{~Hz}, 2 \mathrm{H}), 3.53(\mathrm{AB}, J=13.9 \mathrm{~Hz}, 1 \mathrm{H}), 3.13-3.04(\mathrm{~m}, 2 \mathrm{H}), 2.92(\mathrm{dt}, J=19.6,4.6 \mathrm{~Hz}, 1 \mathrm{H}), 2.38$ $(\mathrm{dt}, J=13.7,4.6 \mathrm{~Hz}, 1 \mathrm{H}), 2.27$ (s, 3H), $2.23-2.16(\mathrm{~m}, 1 \mathrm{H}) .{ }^{13} \mathbf{C} \mathbf{N M R}\left(100 \mathrm{MHz}, \mathrm{CDCl}_{3}\right) \delta 190.4$, 158.0, 139.7, 137.2, 135.6, 134.6, 131.7, 130.7, 130.6, 128.74, 128.71, 128.6, 128.23, 128.22, 127.8, 118.8, 47.6, 39.6, 30.1, 29.8, 21.4. HRMS $\left(\mathrm{ESI}^{+}\right) \mathrm{m} / \mathrm{z}$ calc'd for $\mathrm{C}_{27} \mathrm{H}_{23} \mathrm{NONa}[\mathrm{M}+\mathrm{Na}]^{+}:$400.1672, found 400.1677. HPLC conditions: hexane/2-propanol $95: 5,0.8 \mathrm{~mL} / \mathrm{min}, \lambda=300 \mathrm{~nm}$, Chiralpak AD-H column $(4.6 \mathrm{~mm} \times 250 \mathrm{~mm}), \mathrm{t}_{\mathrm{r}}($ minor $)=15.7 \mathrm{~min}, \mathrm{t}_{\mathrm{r}}($ major $)=17.3 \mathrm{~min}$.<smiles>COc1ccc(C2=C(c3ccccc3)CC[C@@H](Br)C2=O)cc1</smiles> 


\section{(R)-4'-Benzyl-4''-methoxy-3'-oxo-3',4',5',6'-tetrahydro-[1,1':2',1'-terphenyl]-4'-carbonitrile}

(3ca)

Following the general procedure, the reaction was performed with $1 \mathrm{c}(62.9 \mathrm{mg}, 0.2 \mathrm{mmol}, 1.0 \mathrm{equiv})$, phenylboronic acid 2a (48.8 mg, $0.4 \mathrm{mmol}, 2.0$ equiv), $\mathrm{H}_{2} \mathrm{O}$ (15 uL, $0.8 \mathrm{mmol}, 4.0$ equiv), Ni(OTf) (7.2 mg, $0.02 \mathrm{mmol}, 10 \mathrm{~mol} \%$ ), and $(S)-{ }^{t} \mathrm{Bu}-\mathrm{phox}(9.3 \mathrm{mg}, 0.024 \mathrm{mmol}, 12 \mathrm{~mol} \%)$ in $4.0 \mathrm{~mL}$ of toluene at $80{ }^{\circ} \mathrm{C}$ for $24 \mathrm{~h}$. The desired product 3ca was obtained in $81 \%$ yield $(63.4 \mathrm{mg})$ as a white solid. er $=$ $94: 6 .[\alpha]_{\mathrm{D}}{ }^{25}=-157.10\left(c\right.$ 1.3, $\left.\mathrm{CHCl}_{3}\right) . R_{f}=0.3(\mathrm{PE}: \mathrm{EA}=10: 1) .{ }^{1} \mathbf{H} \mathbf{N M R}\left(400 \mathrm{MHz}, \mathrm{CDCl}_{3}\right) \delta$ $7.40-7.31(\mathrm{~m}, 5 \mathrm{H}), 7.20-7.18(\mathrm{~m}, 3 \mathrm{H}), 7.07-7.03(\mathrm{~m}, 2 \mathrm{H}), 6.86(\mathrm{~d}, J=8.7 \mathrm{~Hz}, 2 \mathrm{H}), 6.72(\mathrm{~d}, J=$ $8.6 \mathrm{~Hz}, 2 \mathrm{H}), 3.75(\mathrm{~s}, 3 \mathrm{H}), 3.53(\mathrm{~d}, J=14.0 \mathrm{~Hz}, 1 \mathrm{H}), 3.13-3.03(\mathrm{~m}, 2 \mathrm{H}), 2.91(\mathrm{dt}, J=19.6,4.6 \mathrm{~Hz}$, 1H), $2.38(\mathrm{dt}, J=13.7,4.6 \mathrm{~Hz}, 1 \mathrm{H}), 2.19(\mathrm{ddd}, J=14.1,9.6,5.0 \mathrm{~Hz}, 1 \mathrm{H}) .{ }^{13} \mathbf{C ~ N M R}\left(100 \mathrm{MHz}, \mathrm{CDCl}_{3}\right)$ $\delta 190.6,158.8,157.9,139.8,135.3,134.6,132.2,130.7,128.8,128.6,128.3,128.2,127.8,127.0$, 118.8, 113.5, 55.3, 47.7, 39.7, 30.2, 29.8. HRMS $\left(\mathrm{ESI}^{+}\right) \mathrm{m} / \mathrm{z}$ calc'd for $\mathrm{C}_{27} \mathrm{H}_{23} \mathrm{NO}_{2} \mathrm{Na}[\mathrm{M}+\mathrm{Na}]^{+}$: 416.1621, found 416.1618. HPLC conditions: hexane/2-propanol $85: 15,1.0 \mathrm{~mL} / \mathrm{min}, \lambda=254 \mathrm{~nm}$, Chiralpak AS-H column $(4.6 \mathrm{~mm} \times 250 \mathrm{~mm}), \mathrm{t}_{\mathrm{r}}($ major $)=18.1 \mathrm{~min}, \mathrm{t}_{\mathrm{r}}($ minor $)=23.2 \mathrm{~min}$.<smiles>C[C@@]1(C#N)CCC(c2ccccc2)=C(c2ccc(Br)cc2)C1=O</smiles>

(R)-4'-Benzyl-4"'-bromo-3'-oxo-3',4',5',6'-tetrahydro-[1,1':2',1''-terphenyl]-4' -carbonitrile (3da) Following the general procedure, the reaction was performed with $1 \mathbf{d}$ (72.7 $\mathrm{mg}, 0.2 \mathrm{mmol}, 1.0$ equiv), phenylboronic acid 2a (48.8 mg, $0.4 \mathrm{mmol}, 2.0$ equiv), $\mathrm{H}_{2} \mathrm{O}$ (15 uL, $0.8 \mathrm{mmol}, 4.0$ equiv), Ni(OTf) (7.2 mg, $0.02 \mathrm{mmol}, 10 \mathrm{~mol} \%$ ), and (S)- ${ }^{t} \mathrm{Bu}-$ phox (9.3 mg, $0.024 \mathrm{mmol}, 12 \mathrm{~mol} \%$ ) in $4.0 \mathrm{~mL}$ of toluene at $80{ }^{\circ} \mathrm{C}$ for $24 \mathrm{~h}$. The desired product 3da was obtained in $63 \%$ yield $(55.9 \mathrm{mg})$ as a white solid. er $=$ $97: 3 \cdot[\alpha]_{\mathrm{D}}{ }^{25}=-120.87\left(c 1.0, \mathrm{CHCl}_{3}\right) \cdot R_{f}=0.3(\mathrm{PE}: \mathrm{EA}=10: 1) .{ }^{1} \mathbf{H} \mathbf{N M R}\left(400 \mathrm{MHz}, \mathrm{CDCl}_{3}\right) \delta$ $7.40-7.30(\mathrm{~m}, 7 \mathrm{H}), 7.24-7.18(\mathrm{~m}, 3 \mathrm{H}), 7.03-7.01(\mathrm{~m}, 2 \mathrm{H}), 6.82(\mathrm{~d}, J=8.5 \mathrm{~Hz}, 2 \mathrm{H}), 3.52(\mathrm{~d}, J=$ $14.0 \mathrm{~Hz}, 1 \mathrm{H}), 3.13-3.05(\mathrm{~m}, 2 \mathrm{H}), 2.92(\mathrm{dt}, J=19.7,4.5 \mathrm{~Hz}, 1 \mathrm{H}), 2.39$ (dt, $J=13.8,4.6 \mathrm{~Hz}, 1 \mathrm{H}), 2.20$ $(\mathrm{ddd}, J=14.2,9.7,4.8 \mathrm{~Hz}, 1 \mathrm{H}) .{ }^{13} \mathbf{C}$ NMR $\left(100 \mathrm{MHz}, \mathrm{CDCl}_{3}\right) \delta 190.0,159.2,139.2,134.6,134.4$, 133.7, 132.6, 131.2, 130.6, 129.0, 128.8, 128.5, 128.1, 127.9, 121.8, 118.6, 47.6, 39.6, 30.1, 29.8. HRMS $\left(\mathrm{ESI}^{+}\right) \mathrm{m} / z$ calc'd for $\mathrm{C}_{26} \mathrm{H}_{20} \mathrm{BrNONa}[\mathrm{M}+\mathrm{Na}]^{+}: 464.0620$, found 464.0623. HPLC conditions: 
hexane/2-propanol $85: 15,1.0 \mathrm{~mL} / \mathrm{min}, \lambda=300 \mathrm{~nm}$, Chiralpak AS-H column (4.6 mm x $250 \mathrm{~mm}), \mathrm{t}_{\mathrm{r}}$ $($ major $)=13.4 \mathrm{~min}, \mathrm{t}_{\mathrm{r}}($ minor $)=19.5 \mathrm{~min}$.<smiles>N#CC1(C2CCCCC2)CCC(c2ccccc2)=C(c2ccc(C(F)(F)F)cc2)C1=O</smiles>

\section{(R)-4'-Benzyl-3'-oxo-4' '-(trifluoromethyl)-3',4',5',6'-tetrahydro-[1,1':2',1'"-terphenyl]-4'-} carbonitrile (3ea)

Following the general procedure, the reaction was performed with $1 \mathrm{e}(70.5 \mathrm{mg}, 0.2 \mathrm{mmol}, 1.0$ equiv), phenylboronic acid 2a (48.8 mg, $0.4 \mathrm{mmol}, 2.0$ equiv), $\mathrm{H}_{2} \mathrm{O}$ (15 uL, $0.8 \mathrm{mmol}, 4.0$ equiv), Ni(OTf) (7.2 mg, $0.02 \mathrm{mmol}, 10 \mathrm{~mol} \%$ ), and (S)- ${ }^{t} \mathrm{Bu}-$ phox (9.3 mg, $0.024 \mathrm{mmol}, 12 \mathrm{~mol} \%$ ) in $4.0 \mathrm{~mL}$ of toluene at $100{ }^{\circ} \mathrm{C}$ for $24 \mathrm{~h}$. The desired product 3ea was obtained in $78 \%$ yield $(67.3 \mathrm{mg})$ as a white solid. er $=95.5: 4.5 .[\alpha]_{\mathrm{D}}{ }^{25}=-127.63\left(c 1.0, \mathrm{CHCl}_{3}\right) . R_{f}=0.3(\mathrm{PE}: \mathrm{EA}=10: 1) .{ }^{\mathbf{1}} \mathbf{H} \mathbf{N M R}\left(400 \mathrm{MHz}, \mathrm{CDCl}_{3}\right)$ $\delta 7.45(\mathrm{~d}, J=8.1 \mathrm{~Hz}, 2 \mathrm{H}), 7.41-7.33(\mathrm{~m}, 5 \mathrm{H}), 7.25-7.18(\mathrm{~m}, 3 \mathrm{H}), 7.08(\mathrm{~d}, J=8.0 \mathrm{~Hz}, 2 \mathrm{H}), 7.04-$ $7.00(\mathrm{~m}, 2 \mathrm{H}), 3.54(\mathrm{AB}, J=14.0 \mathrm{~Hz}, 1 \mathrm{H}), 3.17-3.09(\mathrm{~m}, 2 \mathrm{H}), 2.95(\mathrm{dt}, J=19.7,4.5 \mathrm{~Hz}, 1 \mathrm{H}), 2.42$ $(\mathrm{dt}, J=13.8,4.6 \mathrm{~Hz}, 1 \mathrm{H}), 2.27-2.19(\mathrm{~m}, 1 \mathrm{H}) .{ }^{13} \mathbf{C}$ NMR $\left(100 \mathrm{MHz}, \mathrm{CDCl}_{3}\right) \delta 189.9,159.9,138.9$, $138.7,134.6,134.3,131.4,130.6,129.5$ (q, $J=32.7 \mathrm{~Hz}), 129.2,128.8,128.5,128.1,127.9,124.9$ (q, $J=3.7 \mathrm{~Hz}), 124.2(\mathrm{q}, J=272.5 \mathrm{~Hz}), 118.5,47.6,39.6,30.1,29.9 .{ }^{19} \mathbf{F} \mathbf{N M R}\left(376 \mathrm{MHz}, \mathrm{CDCl}_{3}\right) \delta-$ 62.59. HRMS $\left(\mathrm{ESI}^{+}\right) \mathrm{m} / z$ calc'd for $\mathrm{C}_{27} \mathrm{H}_{20} \mathrm{NOF}_{3} \mathrm{Na}[\mathrm{M}+\mathrm{Na}]^{+}:$454.1389, found 454.1391. HPLC conditions: hexane/2-propanol $90: 10,0.8 \mathrm{~mL} / \mathrm{min}, \lambda=280 \mathrm{~nm}$, Chiralpak AS-H column (4.6 mm x $250 \mathrm{~mm}), \mathrm{t}_{\mathrm{r}}($ major $)=13.5 \mathrm{~min}, \mathrm{t}_{\mathrm{r}}($ minor $)=23.6 \mathrm{~min}$.<smiles>CC(=O)c1ccc(C2=C(c3ccccc3)CC[C@@](C(N)=O)(C(=O)c3ccccc3)C2=O)cc1</smiles>

(R)-Methyl-5'-benzyl-5'-cyano-6'-oxo-3',4',5',6'-tetrahydro-[1,1':2',1'--terphenyl]-4-carboxylate (3fa)

Following the general procedure, the reaction was performed with $\mathbf{1 f}(68.5 \mathrm{mg}, 0.2 \mathrm{mmol}$, 1.0 equiv), phenylboronic acid $2 \mathrm{a}$ (48.8 mg, $0.4 \mathrm{mmol}, 2.0$ equiv), $\mathrm{H}_{2} \mathrm{O}$ (15 uL, $0.8 \mathrm{mmol}, 4.0$ equiv), Ni(OTf) 2 (7.2 mg, $0.02 \mathrm{mmol}, 10 \mathrm{~mol} \%$ ), and $(S)-{ }^{t} \mathrm{Bu}-$ phox (9.3 mg, $0.024 \mathrm{mmol}, 12 \mathrm{~mol} \%$ ) in $4.0 \mathrm{~mL}$ of toluene at $100{ }^{\circ} \mathrm{C}$ for $24 \mathrm{~h}$. The desired product 3 fa was obtained in $80 \%$ yield $(67.4 \mathrm{mg})$ as a white solid. er $=$ 
$96: 4 \cdot[\alpha]_{\mathrm{D}}{ }^{25}=-138.37\left(c 1.0, \mathrm{CHCl}_{3}\right) \cdot R_{f}=0.3(\mathrm{PE}: \mathrm{EA}=10: 1) .{ }^{\mathbf{1}} \mathbf{H} \mathbf{~ N M R}\left(400 \mathrm{MHz}, \mathrm{CDCl}_{3}\right) \delta$ $7.86(\mathrm{~d}, J=8.3 \mathrm{~Hz}, 2 \mathrm{H}), 7.40-7.31(\mathrm{~m}, 5 \mathrm{H}), 7.23-7.13(\mathrm{~m}, 3 \mathrm{H}), 7.04-6.99(\mathrm{~m}, 4 \mathrm{H}), 3.87(\mathrm{~s}, 3 \mathrm{H})$, $3.53(\mathrm{~d}, J=13.9 \mathrm{~Hz}, 1 \mathrm{H}), 3.16-3.07(\mathrm{~m}, 2 \mathrm{H}), 2.94(\mathrm{dt}, J=19.7,4.5 \mathrm{~Hz}, 1 \mathrm{H}), 2.40$ (dt, $J=13.8,4.6$ $\mathrm{Hz}, 1 \mathrm{H}), 2.25-2.18(\mathrm{~m}, 1 \mathrm{H}) .{ }^{13} \mathbf{C}$ NMR $\left(100 \mathrm{MHz}, \mathrm{CDCl}_{3}\right) \delta 189.8,166.9,159.6,139.9,139.0,135.0$, 134.4, 131.1, 130.6, 129.2, 129.1, 129.0, 128.8, 128.4, 128.1, 127.9, 118.5, 52.2, 47.6, 39.6, 30.1, 29.9. HRMS $\left(\mathrm{ESI}^{+}\right) m / z$ calc'd for $\mathrm{C}_{28} \mathrm{H}_{23} \mathrm{NO}_{3} \mathrm{Na}[\mathrm{M}+\mathrm{Na}]^{+}:$444.1570, found 444.1571. HPLC conditions: hexane/2-propanol $70: 30,1.0 \mathrm{~mL} / \mathrm{min}, \lambda=254 \mathrm{~nm}$, Chiralpak AS-H column (4.6 mm x $250 \mathrm{~mm}), \mathrm{t}_{\mathrm{r}}$ $($ major $)=13.1 \mathrm{~min}, \mathrm{t}_{\mathrm{r}}($ minor $)=18.3 \mathrm{~min}$.<smiles>C[C@@]1(C#N)CCC(c2ccccc2)=C(c2ccc3ccccc3c2)C1=O</smiles>

(R)-4-Benzyl-6-(naphthalen-2-yl)-5-oxo-2,3,4,5-tetrahydro-[1,1'-biphenyl]-4-carbonitrile (3ga) Following the general procedure, the reaction was performed with $1 \mathrm{~g}$ ( $66.9 \mathrm{mg}, 0.2 \mathrm{mmol}, 1.0$ equiv), phenylboronic acid 2a (48.8 mg, $0.4 \mathrm{mmol}, 2.0$ equiv), $\mathrm{H}_{2} \mathrm{O}$ (15 uL, $0.8 \mathrm{mmol}, 4.0$ equiv), $\mathrm{Ni}(\mathrm{OTf})_{2}$ (7.2 mg, $0.02 \mathrm{mmol}, 10 \mathrm{~mol} \%$ ), and (S)- ${ }^{t} \mathrm{Bu}-$ phox (9.3 mg, $0.024 \mathrm{mmol}, 12 \mathrm{~mol} \%$ ) in $4.0 \mathrm{~mL}$ of toluene at $80{ }^{\circ} \mathrm{C}$ for $24 \mathrm{~h}$. The desired product $3 \mathrm{ga}$ was obtained in $80 \%$ yield $(58.0 \mathrm{mg})$ as a white solid. er $=$ $94: 6 .[\alpha]_{\mathrm{D}}{ }^{25}=-133.45\left(c 0.85, \mathrm{CHCl}_{3}\right) \cdot R_{f}=0.3(\mathrm{PE}: \mathrm{EA}=10: 1) .{ }^{1} \mathbf{H} \mathbf{N M R}\left(400 \mathrm{MHz}, \mathrm{CDCl}_{3}\right) \delta$ $7.76-7.73(\mathrm{~m}, 1 \mathrm{H}), 7.68-7.64(\mathrm{~m}, 2 \mathrm{H}), 7.47-7.46(\mathrm{~m}, 1 \mathrm{H}), 7.45-7.32(\mathrm{~m}, 7 \mathrm{H}), 7.15-7.03(\mathrm{~m}$, $6 \mathrm{H}), 3.57(\mathrm{AB}, J=14.0 \mathrm{~Hz}, 1 \mathrm{H}), 3.19-3.11(\mathrm{~m}, 2 \mathrm{H}), 2.98(\mathrm{dt}, J=19.7,4.6 \mathrm{~Hz}, 1 \mathrm{H}), 2.43(\mathrm{dt}, J=$ 13.7, $4.6 \mathrm{~Hz}, 1 \mathrm{H}), 2.28-2.13(\mathrm{~m}, 1 \mathrm{H}) .{ }^{13} \mathrm{C} \mathrm{NMR}\left(100 \mathrm{MHz}, \mathrm{CDCl}_{3}\right) \delta 190.4,158.8,139.5,135.6$, 134.6, 133.1, 132.6, 132.4, 130.7, 130.4, 128.8, 128.7, 128.6, 128.4, 128.3, 128.2, 127.8, 127.7, 127.4, 126.2, 126.0, 118.8, 47.8, 39.7, 30.2, 29.9. HRMS $\left(\mathrm{ESI}^{+}\right) \mathrm{m} / z$ calc'd for $\mathrm{C}_{30} \mathrm{H}_{23} \mathrm{NONa}[\mathrm{M}+\mathrm{Na}]^{+}$: 436.1672, found 436.1675. HPLC conditions: hexane/2-propanol $80: 20,1.0 \mathrm{~mL} / \mathrm{min}, \lambda=280 \mathrm{~nm}$, Chiralcel OD-H column $(4.6 \mathrm{~mm}$ x $250 \mathrm{~mm}), \mathrm{t}_{\mathrm{r}}($ minor $)=12.3 \mathrm{~min}, \mathrm{t}_{\mathrm{r}}($ major $)=23.3 \mathrm{~min}$.

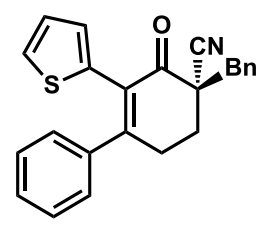

(R)-4-Benzyl-5-oxo-6-(thiophen-2-yl)-2,3,4,5-tetrahydro-[1,1'-biphenyl]-4-carbonitrile (3ha) 
Following the general procedure, the reaction was performed with $\mathbf{1 h}(58.1 \mathrm{mg}, 0.2 \mathrm{mmol}, 1.0$ equiv), phenylboronic acid 2a (48.8 mg, $0.4 \mathrm{mmol}, 2.0$ equiv), $\mathrm{H}_{2} \mathrm{O}$ (15 uL, $0.8 \mathrm{mmol}, 4.0$ equiv), Ni(OTf) (7.2 mg, $0.02 \mathrm{mmol}, 10 \mathrm{~mol} \%$ ), and $(S)-{ }^{t} \mathrm{Bu}-$ phox (9.3 mg, $0.024 \mathrm{mmol}, 12 \mathrm{~mol} \%$ ) in $4.0 \mathrm{~mL}$ of toluene at $80{ }^{\circ} \mathrm{C}$ for $24 \mathrm{~h}$. The desired product 3 ha was obtained in $81 \%$ yield $(59.7 \mathrm{mg})$ as a white solid. er $=$ $88: 12 .[\alpha]_{\mathrm{D}}{ }^{25}=-85.12\left(c 1.4, \mathrm{CHCl}_{3}\right) . R_{f}=0.4(\mathrm{PE}: \mathrm{EA}=10: 1) .{ }^{1} \mathbf{H} \mathbf{~ N M R}\left(400 \mathrm{MHz}, \mathrm{CDCl}_{3}\right) \delta$ $7.41-7.32(\mathrm{~m}, 5 \mathrm{H}), 7.30-7.26(\mathrm{~m}, 3 \mathrm{H}), 7.24(\mathrm{dd}, J=5.1,1.2 \mathrm{~Hz}, 1 \mathrm{H}), 7.18-7.13(\mathrm{~m}, 2 \mathrm{H}), 6.84(\mathrm{dd}$, $J=5.1,3.6 \mathrm{~Hz}, 1 \mathrm{H}), 6.68(\mathrm{dd}, J=3.6,1.2 \mathrm{~Hz}, 1 \mathrm{H}), 3.56(\mathrm{AB}, J=14.0 \mathrm{~Hz}, 1 \mathrm{H}), 3.13-3.05(\mathrm{~m}, 2 \mathrm{H})$, $2.94(\mathrm{dt}, J=20.0,4.7 \mathrm{~Hz}, 1 \mathrm{H}), 2.39(\mathrm{dt}, J=13.8,4.7 \mathrm{~Hz}, 1 \mathrm{H}), 2.24-2.17(\mathrm{~m}, 1 \mathrm{H}) .{ }^{13} \mathbf{C}$ NMR $(100$ $\left.\mathrm{MHz}, \mathrm{CDCl}_{3}\right) \delta 189.6,159.6,139.9,134.8,134.4,130.6,130.1,129.1,128.9,128.8,128.6,127.9$, 127.8, 127.1, 126.3, 118.6, 47.9, 39.8, 30.4, 30.0. HRMS $\left(\mathrm{ESI}^{+}\right) \mathrm{m} / z$ calc'd for $\mathrm{C}_{24} \mathrm{H}_{19} \mathrm{NOSNa}$ $[\mathrm{M}+\mathrm{Na}]^{+}:$392.1080, found 392.1081. HPLC conditions: hexane/2-propanol $95: 5,0.8 \mathrm{~mL} / \mathrm{min}, \lambda=$ $254 \mathrm{~nm}$, Chiralpak AD-H column $(4.6 \mathrm{~mm} \times 250 \mathrm{~mm}), \mathrm{t}_{\mathrm{r}}($ minor $)=18.6 \mathrm{~min}, \mathrm{t}_{\mathrm{r}}($ major $)=22.3 \mathrm{~min}$.

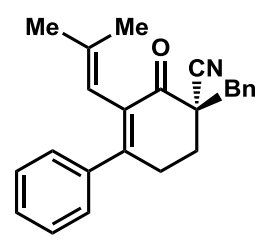

\section{(R)-4-Benzyl-6-(2-methylprop-1-en-1-yl)-5-oxo-2,3,4,5-tetrahydro-[1,1'-biphenyl]-4- carbonitrile (3ia)}

Following the general procedure, the reaction was performed with $1 \mathbf{i}(52.5 \mathrm{mg}, 0.2 \mathrm{mmol}, 1.0$ equiv), phenylboronic acid 2a (48.8 mg, $0.4 \mathrm{mmol}, 2.0$ equiv), $\mathrm{H}_{2} \mathrm{O}$ (15 uL, $0.8 \mathrm{mmol}, 4.0$ equiv), Ni(OTf) (7.2 mg, $0.02 \mathrm{mmol}, 10 \mathrm{~mol} \%$ ), and $(S)-{ }^{t}$ Bu-phox (9.3 mg, $0.024 \mathrm{mmol}, 12 \mathrm{~mol} \%$ ) in $4.0 \mathrm{~mL}$ of toluene at $80{ }^{\circ} \mathrm{C}$ for $24 \mathrm{~h}$. The desired product 3ia was obtained in $63 \%$ yield $(42.9 \mathrm{mg})$ as a white solid. er $=$ $91: 9 .[\alpha]_{\mathrm{D}}{ }^{25}=-113.06\left(c 1.0, \mathrm{CHCl}_{3}\right) \cdot R_{f}=0.4(\mathrm{PE}: \mathrm{EA}=10: 1) .{ }^{\mathbf{1}} \mathbf{H} \mathbf{N M R}\left(400 \mathrm{MHz}, \mathrm{CDCl}_{3}\right) \delta 7.41$ - $7.30(\mathrm{~m}, 8 \mathrm{H}), 7.28-7.26(\mathrm{~m}, 2 \mathrm{H}), 5.64(\mathrm{~s}, 1 \mathrm{H}), 3.49(\mathrm{~d}, J=14.0 \mathrm{~Hz}, 1 \mathrm{H}), 3.05-2.94(\mathrm{~m}, 2 \mathrm{H}), 2.82$ $(\mathrm{dtd}, J=19.6,4.6,1.6 \mathrm{~Hz}, 1 \mathrm{H}), 2.30(\mathrm{dt}, J=13.7,4.6 \mathrm{~Hz}, 1 \mathrm{H}), 2.11-2.04(\mathrm{~m}, 1 \mathrm{H}), 1.66(\mathrm{~d}, J=1.5$ $\mathrm{Hz}, 3 \mathrm{H}), 1.21(\mathrm{~d}, J=1.3 \mathrm{~Hz}, 3 \mathrm{H}) .{ }^{13} \mathbf{C} \mathbf{N M R}\left(100 \mathrm{MHz}, \mathrm{CDCl}_{3}\right) \delta 190.9,157.6,140.2,138.8,134.6$, 132.3, 130.6, 128.8, 128.7, 128.2, 127.9, 127.7, 118.8, 118.2, 47.6, 39.6, 30.3, 29.2, 25.5, 20.1. HRMS $\left(\mathrm{ESI}^{+}\right) \mathrm{m} / z$ calc'd for $\mathrm{C}_{24} \mathrm{H}_{23} \mathrm{NONa}[\mathrm{M}+\mathrm{Na}]^{+}: 364.1672$, found 364.1679. HPLC conditions: hexane/2propanol $95: 5,0.5 \mathrm{~mL} / \mathrm{min}, \lambda=280 \mathrm{~nm}$, Chiralpak AD-H column $(4.6 \mathrm{~mm} \times 250 \mathrm{~mm}), \mathrm{t}_{\mathrm{r}}($ minor $)=$ $18.9 \min , \mathrm{t}_{\mathrm{r}}$ (major) $=21.0 \mathrm{~min}$. 


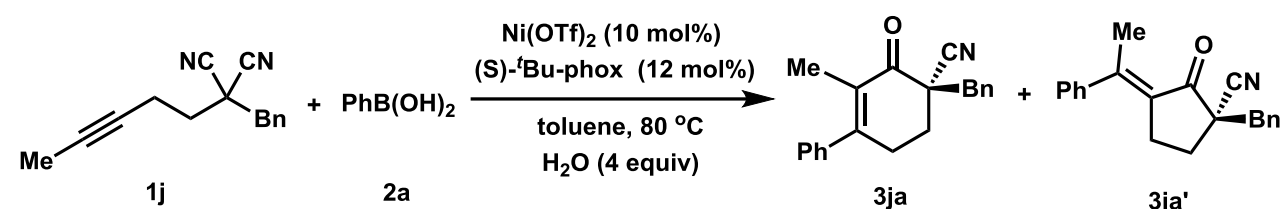

Following the general procedure, the reaction was performed with $\mathbf{1 j}$ ( $22.3 \mathrm{mg}, 0.1 \mathrm{mmol}, 1.0$ equiv), phenylboronic acid $\mathbf{2 a}\left(24.4 \mathrm{mg}, 0.2 \mathrm{mmol}, 2.0\right.$ equiv), $\mathrm{H}_{2} \mathrm{O}$ (8 ul, $0.4 \mathrm{mmol}, 4.0$ equiv), $\mathrm{Ni}(\mathrm{OTf})_{2}(3.6$ $\mathrm{mg}, 0.01 \mathrm{mmol}, 10 \mathrm{~mol} \%)$, and $(S)-{ }^{t} \mathrm{Bu}-\mathrm{phox}(4.7 \mathrm{mg}, 0.012 \mathrm{mmol}, 12 \mathrm{~mol} \%)$ in $2.0 \mathrm{~mL}$ of toluene at $80{ }^{\circ} \mathrm{C}$ for $24 \mathrm{~h}$. A mixture of regioseletive isomers $\mathbf{3 j a}(6.8 \mathrm{mg}, 23 \%$ yield) and $\mathbf{3 j a}$ ' (16.3 mg, 54\% yield) were obtained. The stereochemistry of the these proudcts were assigned by 2D NMR experiments.

For (R)-4-benzyl-6-methyl-5-oxo-2,3,4,5-tetrahydro-[1,1'-biphenyl]-4-carbonitrile (3ja): White solid. er $=91.5: 8.5 .[\alpha]_{\mathrm{D}}{ }^{25}=-64.45\left(c 0.4, \mathrm{CHCl}_{3}\right) . R_{f}=0.3(\mathrm{PE}: \mathrm{EA}=15: 1) .{ }^{1} \mathbf{H} \mathbf{~ N M R}(400 \mathrm{MHz}$, $\left.\mathrm{CDCl}_{3}\right) \delta 7.44-7.28(\mathrm{~m}, 8 \mathrm{H}), 7.23-7.19(\mathrm{~m}, 2 \mathrm{H}), 3.48(\mathrm{AB}, J=13.9 \mathrm{~Hz}, 1 \mathrm{H}), 3.03(\mathrm{BA}, J=13.9$ $\mathrm{Hz}, 1 \mathrm{H}), 2.94-2.85(\mathrm{~m}, 1 \mathrm{H}), 2.74-2.65(\mathrm{~m}, 1 \mathrm{H}), 2.28(\mathrm{dt}, J=13.6,4.8 \mathrm{~Hz}, 1 \mathrm{H}), 2.07$ (ddd, $J=13.9$, 9.3, $4.8 \mathrm{~Hz}, 1 \mathrm{H}), 1.80(\mathrm{t}, J=1.9 \mathrm{~Hz}, 3 \mathrm{H}) .{ }^{13} \mathbf{C} \mathbf{N M R}\left(100 \mathrm{MHz}, \mathrm{CDCl}_{3}\right) \delta 191.4,157.3,140.1,134.6$, 130.6, 130.3, 128.8, 128.7, 128.6, 127.8, 127.1, 119.0, 47.4, 39.8, 30.4, 29.7, 13.9. HRMS (ESI $\left.{ }^{+}\right) \mathrm{m} / z$ calc'd for $\mathrm{C}_{21} \mathrm{H}_{19} \mathrm{NONa}[\mathrm{M}+\mathrm{Na}]^{+}: 324.1359$, found 324.1359. HPLC conditions: hexane/2-propanol $95: 5,0.5 \mathrm{~mL} / \mathrm{min}, \lambda=280 \mathrm{~nm}$, Chiralpak IC column $(4.6 \mathrm{~mm}$ x $250 \mathrm{~mm}), \mathrm{t}_{\mathrm{r}}($ minor $)=18.8 \mathrm{~min}, \mathrm{t}_{\mathrm{r}}$ $($ major $)=22.5 \mathrm{~min}$.

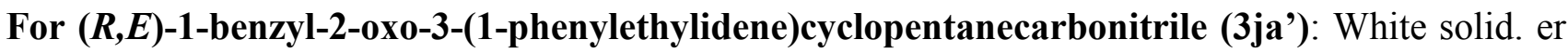
$=51.5: 48.5 \cdot[\alpha]_{\mathrm{D}}{ }^{25}=-14.30\left(c 0.3, \mathrm{CHCl}_{3}\right) \cdot R_{f}=0.3(\mathrm{PE}: \mathrm{EA}=15: 1) .{ }^{1} \mathbf{H} \mathbf{N M R}\left(400 \mathrm{MHz}, \mathrm{CDCl}_{3}\right)$ $\delta 7.42-7.28(\mathrm{~m}, 7 \mathrm{H}), 7.27-7.24(\mathrm{~m}, 1 \mathrm{H}), 7.21-7.16(\mathrm{~m}, 2 \mathrm{H}), 3.18(\mathrm{AB}, J=13.9 \mathrm{~Hz}, 1 \mathrm{H}), 2.88-$ $2.79(\mathrm{~m}, 2 \mathrm{H}), 2.74-2.65(\mathrm{~m}, 1 \mathrm{H}), 2.27(\mathrm{ddd}, J=13.2,8.1,5.1 \mathrm{~Hz}, 1 \mathrm{H}), 2.18(\mathrm{~s}, 3 \mathrm{H}), 2.04(\mathrm{dt}, J=$ 13.3, 8.0 Hz, 1H). ${ }^{13} \mathbf{C}$ NMR $\left(100 \mathrm{MHz}, \mathrm{CDCl}_{3}\right) \delta$ 194.4, 154.0, 140.6, 134.9, 130.2, 128.9, 128.8, 128.4, 128.2, 127.7, 127.2, 119.6, 51.6, 39.6, 29.7, 26.1, 25.4. HRMS $\left(\mathrm{ESI}^{+}\right) \mathrm{m} / \mathrm{z}$ calc'd for $\mathrm{C}_{21} \mathrm{H}_{19} \mathrm{NONa}[\mathrm{M}+\mathrm{Na}]^{+}:$324.1359, found 324.1360. HPLC conditions: hexane/2-propanol $95: 5,0.8$ $\mathrm{mL} / \mathrm{min}, \lambda=280 \mathrm{~nm}$, Chiralpak AD-H column $(4.6 \mathrm{~mm} \times 250 \mathrm{~mm}), \mathrm{t}_{\mathrm{r}}($ major $)=13.7 \mathrm{~min}, \mathrm{t}_{\mathrm{r}}($ minor $)$ $=16.2 \mathrm{~min}$.

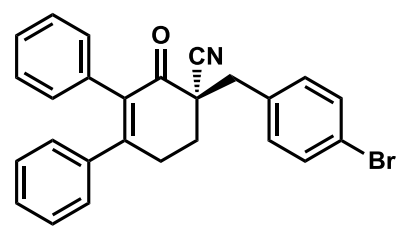




\section{(R)-4' -(4-Bromobenzyl)-3'-oxo-3',4',5',6'-tetrahydro-[1,1':2',1''-terphenyl]-4' -carbonitrile (3ka)}

Following the general procedure, the reaction was performed with $1 \mathbf{k}(72.7 \mathrm{mg}, 0.2 \mathrm{mmol}, 1.0$ equiv), phenylboronic acid $\mathbf{2 a}$ (48.8 mg, $0.4 \mathrm{mmol}, 2.0$ equiv), $\mathrm{H}_{2} \mathrm{O}$ (15 uL, $0.8 \mathrm{mmol}, 4.0$ equiv), Ni(OTf) (7.2 mg, $0.02 \mathrm{mmol}, 10 \mathrm{~mol} \%)$, and (S)- ${ }^{t} \mathrm{Bu}-$ phox $(9.3 \mathrm{mg}, 0.024 \mathrm{mmol}, 12 \mathrm{~mol} \%)$ in $4.0 \mathrm{~mL}$ of toluene at $80{ }^{\circ} \mathrm{C}$ for $24 \mathrm{~h}$. The desired product 3ka was obtained in $77 \%$ yield $(68.3 \mathrm{mg})$ as a white solid. er $=$ $97: 3 .[\alpha]_{\mathrm{D}}{ }^{25}=-59.91\left(c 0.5, \mathrm{CHCl}_{3}\right) . R_{f}=0.3(\mathrm{PE}: \mathrm{EA}=10: 1)$. Enantiopure product $(>99.9 \%$ ee $)$ was obtained after recrystallization in $n$-hexane and DCM. Crystals were obtained by recrystallization again and the absolute configuration was determined by X-ray analyses as $(R) .{ }^{1} \mathbf{H}$ NMR $(400 \mathrm{MHz}$, $\left.\mathrm{CDCl}_{3}\right) \delta 7.50(\mathrm{~d}, J=8.3 \mathrm{~Hz}, 2 \mathrm{H}), 7.23(\mathrm{~d}, J=8.4 \mathrm{~Hz}, 2 \mathrm{H}), 7.20-7.15(\mathrm{~m}, 6 \mathrm{H}), 7.06-7.02(\mathrm{~m}, 2 \mathrm{H})$, $6.97-6.92(\mathrm{~m}, 2 \mathrm{H}), 3.48(\mathrm{~d}, J=14.0 \mathrm{~Hz}, 1 \mathrm{H}), 3.16-3.06(\mathrm{~m}, 2 \mathrm{H}), 2.93(\mathrm{dt}, J=19.7,4.4 \mathrm{~Hz}, 1 \mathrm{H})$, $2.37(\mathrm{dt}, J=13.6,4.4 \mathrm{~Hz}, 1 \mathrm{H}), 2.19(\mathrm{ddd}, J=14.1,10.1,4.8 \mathrm{~Hz}, 1 \mathrm{H}) .{ }^{13} \mathbf{C} \mathbf{N M R}\left(100 \mathrm{MHz}, \mathrm{CDCl}_{3}\right) \delta$ $189.9,158.6,139.4,135.7,134.7,133.5,132.3,131.9,130.9,128.7,128.3,128.2,128.0,127.5,122.0$, 118.4, 47.4, 39.1, 30.2, 29.8. HRMS $\left(\mathrm{ESI}^{+}\right) \mathrm{m} / \mathrm{z}$ calc'd for $\mathrm{C}_{26} \mathrm{H}_{20} \mathrm{BrNONa}[\mathrm{M}+\mathrm{Na}]^{+}:$464.0620, found 464.0612. HPLC conditions: hexane/2-propanol $95: 5,0.8 \mathrm{~mL} / \mathrm{min}, \lambda=280 \mathrm{~nm}$, Chiralpak AD-H column $(4.6 \mathrm{~mm} \times 250 \mathrm{~mm}), \mathrm{t}_{\mathrm{r}}($ minor $)=18.9 \mathrm{~min}, \mathrm{t}_{\mathrm{r}}($ major $)=21.9 \mathrm{~min}$.<smiles>N#C[C@]1(Cc2cccc3ccccc23)CCC(c2ccccc2)=C(c2ccccc2)C1=O</smiles>

\section{(R)-4'-(Naphthalen-1-ylmethyl)-3'-oxo-3',4',5',6'-tetrahydro-[1,1':2',1''-terphenyl]-4'- carbonitrile (3la)}

Following the general procedure, the reaction was performed with 11 (33.5 $\mathrm{mg}, 0.1 \mathrm{mmol}, 1.0$ equiv), phenylboronic acid $\mathbf{2 a}\left(24.4 \mathrm{mg}, 0.2 \mathrm{mmol}, 2.0\right.$ equiv), $\mathrm{H}_{2} \mathrm{O}$ (8 ul, $0.4 \mathrm{mmol}, 4.0$ equiv), $\mathrm{Ni}(\mathrm{OTf})_{2}$ (3.6 $\mathrm{mg}, 0.01 \mathrm{mmol}, 10 \mathrm{~mol} \%)$, and $(S)-{ }^{t} \mathrm{Bu}-$ phox $(4.7 \mathrm{mg}, 0.012 \mathrm{mmol}, 12 \mathrm{~mol} \%)$ in $2.0 \mathrm{~mL}$ of toluene at $80{ }^{\circ} \mathrm{C}$ for $24 \mathrm{~h}$. The desired product 3la was obtained in $98 \%$ yield $(40.5 \mathrm{mg})$ as a white solid. er $=97$ : 3. $[\mathrm{a}]_{\mathrm{D}}{ }^{25}=-136.29\left(c 1.2, \mathrm{CHCl}_{3}\right) \cdot R_{f}=0.3(\mathrm{PE}: \mathrm{EA}=10: 1) .{ }^{1} \mathbf{H} \mathbf{N M R}\left(400 \mathrm{MHz}, \mathrm{CDCl}_{3}\right) \delta 8.15(\mathrm{~d}$, $J=8.2 \mathrm{~Hz}, 1 \mathrm{H}), 7.91(\mathrm{dd}, J=8.0,1.6 \mathrm{~Hz}, 1 \mathrm{H}), 7.86(\mathrm{~d}, J=8.2 \mathrm{~Hz}, 1 \mathrm{H}), 7.62(\mathrm{~d}, J=7.0 \mathrm{~Hz}, 1 \mathrm{H}), 7.60$ $-7.48(\mathrm{~m}, 3 \mathrm{H}), 7.23-7.14(\mathrm{~m}, 6 \mathrm{H}), 7.05-6.95(\mathrm{~m}, 4 \mathrm{H}), 4.09(\mathrm{AB}, J=14.8 \mathrm{~Hz}, 1 \mathrm{H}), 3.64(\mathrm{BA}, J=$ 14.7 Hz, 1H), 3.06 (dt, $J=19.7,7.7 \mathrm{~Hz}, 1 \mathrm{H}), 2.85$ (dt, $J=19.7,4.1 \mathrm{~Hz}, 1 \mathrm{H}), 2.29$ (dd, $J=7.7,4.1 \mathrm{~Hz}$, 2H). ${ }^{13} \mathrm{C}$ NMR $\left(100 \mathrm{MHz}, \mathrm{CDCl}_{3}\right) \delta 190.4,158.7,139.5,135.8,134.9,134.1,132.9,131.1,130.9$, 
129.3, 129.1, 128.7, 128.5, 128.21, 128.19, 127.9, 127.5, 126.4, 125.9, 125.6, 124.0, 118.7, 48.1, 34.8, 30.9, 30.0. HRMS $\left(\mathrm{ESI}^{+}\right) \mathrm{m} / \mathrm{z}$ calc'd for $\mathrm{C}_{30} \mathrm{H}_{23} \mathrm{NONa}[\mathrm{M}+\mathrm{Na}]^{+}:$436.1672, found 436.1674. HPLC conditions: hexane/2-propanol $90: 10,0.8 \mathrm{~mL} / \mathrm{min}, \lambda=280 \mathrm{~nm}$, Chiralpak IB column (4.6 mm x 250 $\mathrm{mm}), \mathrm{t}_{\mathrm{r}}($ minor $)=17.9 \mathrm{~min}, \mathrm{t}_{\mathrm{r}}($ major $)=22.7 \mathrm{~min}$.<smiles>C[C@@]1(C#N)CCC(c2ccccc2)=C(c2ccccc2)C1=O</smiles>

\section{(R)-4' -(Furan-2-ylmethyl)-3'-oxo-3',4',5',6'-tetrahydro-[1,1':2',1''-terphenyl]-4' -carbonitrile} (3ma)

Following the general procedure, the reaction was performed with $1 \mathbf{m}(54.9 \mathrm{mg}, 0.2 \mathrm{mmol}, 1.0 \mathrm{equiv})$, phenylboronic acid 2a (48.8 mg, $0.4 \mathrm{mmol}, 2.0$ equiv), $\mathrm{H}_{2} \mathrm{O}$ (15 uL, $0.8 \mathrm{mmol}, 4.0$ equiv), $\mathrm{Ni}(\mathrm{OTf})_{2}$ (7.2 mg, $0.02 \mathrm{mmol}, 10 \mathrm{~mol} \%$ ), and (S)- ${ }^{t} \mathrm{Bu}-$ phox (9.3 mg, $0.024 \mathrm{mmol}, 12 \mathrm{~mol} \%$ ) in $4.0 \mathrm{~mL}$ of toluene at $80{ }^{\circ} \mathrm{C}$ for $24 \mathrm{~h}$. The desired product $3 \mathrm{ma}$ was obtained in $66 \%$ yield $(44.6 \mathrm{mg})$ as a white solid. er $=$ $93.5: 6.5 .[\alpha]_{\mathrm{D}}{ }^{25}=-115.35\left(c 1.0, \mathrm{CHCl}_{3}\right) . R_{f}=0.3(\mathrm{PE}: \mathrm{EA}=10: 1) .{ }^{1} \mathbf{H} \mathbf{~ N M R}\left(400 \mathrm{MHz}, \mathrm{CDCl}_{3}\right)$ $\delta 7.42(\mathrm{~s}, 1 \mathrm{H}), 7.19-7.15(\mathrm{~m}, 6 \mathrm{H}), 7.06-7.04(\mathrm{~m}, 2 \mathrm{H}), 6.96-6.94(\mathrm{~m}, 2 \mathrm{H}), 6.39-6.35(\mathrm{~m}, 2 \mathrm{H})$, $3.53(\mathrm{AB}, J=15.2 \mathrm{~Hz}, 1 \mathrm{H}), 3.27(\mathrm{BA}, J=15.2 \mathrm{~Hz}, 1 \mathrm{H}), 3.15(\mathrm{ddd}, J=19.7,9.7,4.8 \mathrm{~Hz}, 1 \mathrm{H}), 2.97$ $(\mathrm{dt}, J=19.7,4.7 \mathrm{~Hz}, 1 \mathrm{H}), 2.53(\mathrm{dt}, J=13.9,4.6 \mathrm{~Hz}, 1 \mathrm{H}), 2.26(\mathrm{ddd}, J=14.2,9.7,4.8 \mathrm{~Hz}, 1 \mathrm{H}) .{ }^{13} \mathrm{C}$ NMR $\left(100 \mathrm{MHz}, \mathrm{CDCl}_{3}\right) \delta 189.7,158.7,149.1,142.6,139.5,135.6,134.7,130.9,128.7,128.2,128.1$, $127.9,127.5,118.5,110.9,109.8,47.2,32.5,30.5,29.7$. HRMS $\left(\mathrm{ESI}^{+}\right) \mathrm{m} / z$ calc'd for $\mathrm{C}_{24} \mathrm{H}_{20} \mathrm{NO}_{2}$ $[\mathrm{M}+\mathrm{H}]^{+}:$354.1489, found 354.1482. HPLC conditions: hexane/2-propanol $95: 5,0.8 \mathrm{~mL} / \mathrm{min}, \lambda=$ $300 \mathrm{~nm}$, Chiralpak AD-H column $(4.6 \mathrm{~mm} \times 250 \mathrm{~mm}), \mathrm{t}_{\mathrm{r}}($ minor $)=14.8 \mathrm{~min}, \mathrm{t}_{\mathrm{r}}($ major $)=16.5 \mathrm{~min}$.<smiles>CC(C)=CC[C@]1(C#N)CCC(c2ccccc2)=C(c2ccccc2)C1=O</smiles>

\section{(R)-4'-(3-Methylbut-2-en-1-yl)-3'-oxo-3',4',5',6'-tetrahydro-[1,1':2',1''-terphenyl]-4'-} carbonitrile (3na)

Following the general procedure, the reaction was performed with $1 \mathrm{n}(26.3 \mathrm{mg}, 0.1 \mathrm{mmol}, 1.0$ equiv) and phenylboronic acid $\mathbf{2 a}\left(24.4 \mathrm{mg}, 0.2 \mathrm{mmol}, 2.0\right.$ equiv), $\mathrm{H}_{2} \mathrm{O}$ (8 ul, $0.4 \mathrm{mmol}, 4.0$ equiv), Ni(OTf) (3.6 mg, $0.01 \mathrm{mmol}, 10 \mathrm{~mol} \%$ ), and $(S)-{ }^{t} \mathrm{Bu}-$ phox (4.7 mg, $0.012 \mathrm{mmol}, 12 \mathrm{~mol} \%$ ) in $2.0 \mathrm{~mL}$ of toluene 
at $80{ }^{\circ} \mathrm{C}$ for $24 \mathrm{~h}$. The desired product 3na was obtained in $87 \%$ yield $(29.7 \mathrm{mg})$ as a yellow oil. er $=$ $93: 7$ er. $[\alpha]_{\mathrm{D}}{ }^{25}=-110.47\left(c 0.6, \mathrm{CHCl}_{3}\right) . R_{f}=0.3(\mathrm{PE}: \mathrm{EA}=10: 1) .{ }^{1} \mathbf{H} \mathbf{~ N M R}\left(400 \mathrm{MHz}, \mathrm{CDCl}_{3}\right) \delta$ $7.19-7.15(\mathrm{~m}, 6 \mathrm{H}), 7.05-7.03(\mathrm{~m}, 2 \mathrm{H}), 6.94-6.92(\mathrm{~m}, 2 \mathrm{H}), 5.30(\mathrm{t}, J=7.5 \mathrm{~Hz}, 1 \mathrm{H}), 3.13(\mathrm{ddd}, J=$ $19.6,8.9,4.9 \mathrm{~Hz}, 1 \mathrm{H}), 2.96-2.88(\mathrm{~m}, 2 \mathrm{H}), 2.59-2.53(\mathrm{~m}, 2 \mathrm{H}), 2.29$ (ddd, $J=13.8,9.0,4.9 \mathrm{~Hz}, 1 \mathrm{H})$, 1.80 (s, 3H), 1.72 (s, 3H). ${ }^{13} \mathbf{C}$ NMR (100 MHz, $\left.\mathbf{C D C l}_{3}\right) \delta 190.7,158.0,139.6,137.9,135.7,134.8$, $130.9,128.6,128.23,128.19,127.9,127.4,119.2,116.9,47.2,32.5,30.4,29.7,26.2,18.4$. HRMS $\left(\mathrm{ESI}^{+}\right) \mathrm{m} / \mathrm{z}$ calc'd for $\mathrm{C}_{24} \mathrm{H}_{23} \mathrm{NONa}[\mathrm{M}+\mathrm{Na}]^{+}:$364.1672, found 364.1678; HPLC conditions: hexane/2-propanol $95: 5,0.8 \mathrm{~mL} / \mathrm{min}, \lambda=280 \mathrm{~nm}$, Chiralcel AD-H column (4.6 mm x $250 \mathrm{~mm}), \mathrm{t}_{\mathrm{r}}$ $($ minor $)=9.0 \mathrm{~min}, \mathrm{t}_{\mathrm{r}}($ major $)=10.1 \mathrm{~min}$.<smiles>C=CC[C@]1(C#N)CCC(c2ccccc2)=C(c2ccccc2)C1=O</smiles>

\section{(R)-4' -Allyl-3'-oxo-3',4',5',6'-tetrahydro-[1,1':2',1'-terphenyl]-4'-carbonitrile (3oa)}

Following the general procedure, the reaction was performed with 10 ( $26.3 \mathrm{mg}, 0.1 \mathrm{mmol}, 1.0$ equiv) and phenylboronic acid $2 \mathrm{a}\left(24.4 \mathrm{mg}, 0.2 \mathrm{mmol}, 2.0\right.$ equiv), $\mathrm{H}_{2} \mathrm{O}$ ( 8 ul, $0.4 \mathrm{mmol}, 4.0$ equiv), $\mathrm{Ni}(\mathrm{OTf})_{2}$ (3.6 mg, $0.01 \mathrm{mmol}, 10 \mathrm{~mol} \%$ ), and $(S)-{ }^{t} \mathrm{Bu}-$ phox (4.7 mg, $0.012 \mathrm{mmol}, 12 \mathrm{~mol} \%$ ) in $2.0 \mathrm{~mL}$ of toluene at $80{ }^{\circ} \mathrm{C}$ for $24 \mathrm{~h}$. The desired product $30 a$ was obtained in $87 \%$ yield $(29.7 \mathrm{mg})$ as a yellow oil. er $=$ $93: 7 .[\alpha]_{\mathrm{D}}{ }^{25}=-123.89\left(c 1.0, \mathrm{CHCl}_{3}\right) \cdot R_{f}=0.3(\mathrm{PE}: \mathrm{EA}=10: 1) .{ }^{1} \mathbf{H} \mathbf{N M R}\left(400 \mathrm{MHz}, \mathrm{CDCl}_{3}\right) \delta 7.20$ $-7.15(\mathrm{~m}, 6 \mathrm{H}), 7.07-7.03(\mathrm{~m}, 2 \mathrm{H}), 6.96-6.91(\mathrm{~m}, 2 \mathrm{H}), 5.99-5.89(\mathrm{~m}, 1 \mathrm{H}), 5.33-5.28(\mathrm{~m}, 2 \mathrm{H})$, $3.15(\mathrm{ddd}, J=19.7,9.1,5.0 \mathrm{~Hz}, 1 \mathrm{H}), 2.98-2.91(\mathrm{~m}, 2 \mathrm{H}), 2.62-2.53(\mathrm{~m}, 2 \mathrm{H}), 2.29(\mathrm{ddd}, J=13.9$, 9.1, $4.9 \mathrm{~Hz}, 1 \mathrm{H}) .{ }^{13} \mathbf{C} \mathbf{N M R}\left(100 \mathrm{MHz}, \mathrm{CDCl}_{3}\right) \delta 190.3,158.2,139.6,135.7,134.8,131.4,130.9$, 128.7, 128.3, 128.2, 127.9, 127.5, 121.1, 118.7, 46.6, 38.3, 30.5, 29.7. HRMS $\left(\mathrm{ESI}^{+}\right) \mathrm{m} / \mathrm{z}$ calc'd for $\mathrm{C}_{22} \mathrm{H}_{19} \mathrm{NONa}[\mathrm{M}+\mathrm{Na}]^{+}:$336.1359, found 336.1363. HPLC conditions: hexane/2-propanol $95: 5,0.5$ $\mathrm{mL} / \mathrm{min}, \lambda=300 \mathrm{~nm}$, Chiralpak AD-H column $(4.6 \mathrm{~mm} \times 250 \mathrm{~mm}), \mathrm{t}_{\mathrm{r}}($ minor $)=15.8 \mathrm{~min}, \mathrm{t}_{\mathrm{r}}$ (major) $=17.6 \mathrm{~min}$.<smiles>CC(=O)CC[C@]1(C)CCC(c2ccccc2)=C(c2ccccc2)C1=O</smiles>

(R)-3'-Oxo-4' -(3-oxobutyl)-3',4',5',6'-tetrahydro-[1,1':2',1' '-terphenyl]-4' -carbonitrile (3pa) 
Following the general procedure, the reaction was performed with $1 \mathbf{p}(53.2 \mathrm{mg}, 0.2 \mathrm{mmol}, 1.0$ equiv), phenylboronic acid $2 \mathbf{a}$ (48.8 mg, $0.4 \mathrm{mmol}, 2.0$ equiv), $\mathrm{H}_{2} \mathrm{O}$ (15 uL, $0.8 \mathrm{mmol}, 4.0$ equiv), Ni(OTf) (7.2 mg, $0.02 \mathrm{mmol}, 10 \mathrm{~mol} \%$ ), and $(S)-{ }^{t} \mathrm{Bu}-$ phox $(9.3 \mathrm{mg}, 0.024 \mathrm{mmol}, 12 \mathrm{~mol} \%$ ) in $4.0 \mathrm{~mL}$ of toluene at $80{ }^{\circ} \mathrm{C}$ for $24 \mathrm{~h}$. The desired product 3pa was obtained in $41 \%$ yield $(28.0 \mathrm{mg})$ as a colorless liquid. er $=91.5: 8.5 .[\alpha]_{\mathrm{D}}{ }^{25}=-60.65\left(c 0.4, \mathrm{CHCl}_{3}\right) . R_{f}=0.2(\mathrm{PE}: \mathrm{EA}=5: 1) .{ }^{1} \mathbf{H} \mathbf{N M R}\left(400 \mathrm{MHz}, \mathrm{CDCl}_{3}\right)$ $\delta 7.20-7.14(\mathrm{~m}, 6 \mathrm{H}), 7.06-7.02(\mathrm{~m}, 2 \mathrm{H}), 6.94-6.90(\mathrm{~m}, 2 \mathrm{H}), 3.11(\mathrm{ddd}, J=19.7,7.5,5.2 \mathrm{~Hz}, 1 \mathrm{H})$, $3.03-2.96(\mathrm{~m}, 1 \mathrm{H}), 2.96-2.88(\mathrm{~m}, 1 \mathrm{H}), 2.79(\mathrm{ddd}, J=18.3,9.5,5.4 \mathrm{~Hz}, 1 \mathrm{H}), 2.61(\mathrm{ddd}, J=13.8$, 6.4, 5.1 Hz, 1H), 2.45 (ddd, $J=14.4,9.6,5.7 \mathrm{~Hz}, 1 \mathrm{H}), 2.35$ (ddd, $J=13.7,7.5,5.0 \mathrm{~Hz}, 1 \mathrm{H}), 2.21$ (s, 3H), $2.12(\mathrm{ddd}, J=14.4,9.7,5.5 \mathrm{~Hz}, 1 \mathrm{H}) .{ }^{13} \mathbf{C} \mathbf{N M R}\left(100 \mathrm{MHz}, \mathrm{CDCl}_{3}\right) \delta 206.9,190.9,157.9,139.4$, 135.5, 134.6, 130.9, 128.7, 128.3, 128.2, 127.9, 127.5, 118.9, 46.7, 39.4, 32.0, 30.2, 29.4, 27.8. HRMS $\left(\mathrm{ESI}^{+}\right) \mathrm{m} / \mathrm{z}$ calc'd for $\mathrm{C}_{23} \mathrm{H}_{21} \mathrm{NO}_{2} \mathrm{Na}[\mathrm{M}+\mathrm{Na}]^{+}:$366.1465, found 366.1469. HPLC conditions: hexane/2-propanol $95: 5,0.8 \mathrm{~mL} / \mathrm{min}, \lambda=254 \mathrm{~nm}$, Chiralpak AD-H column (4.6 mm x $250 \mathrm{~mm}), \mathrm{t}_{\mathrm{r}}$ $($ major $)=27.4 \mathrm{~min}, \mathrm{t}_{\mathrm{r}}($ minor $)=30.4 \mathrm{~min}$.

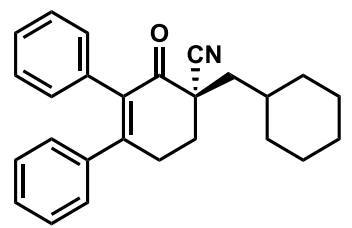

(R)-4'-(Cyclohexylmethyl)-3'-oxo-3',4',5',6'-tetrahydro-[1,1':2',1'-terphenyl]-4'-carbonitrile (3qa)

Following the general procedure, the reaction was performed with $1 \mathbf{q}(58.1 \mathrm{mg}, 0.2 \mathrm{mmol}, 1.0$ equiv), phenylboronic acid 2a (48.8 mg, $0.4 \mathrm{mmol}, 2.0$ equiv), $\mathrm{H}_{2} \mathrm{O}$ (15 uL, $0.8 \mathrm{mmol}, 4.0$ equiv), Ni(OTf) (7.2 mg, $0.02 \mathrm{mmol}, 10 \mathrm{~mol} \%$ ), and $(S)-{ }^{t} \mathrm{Bu}-$ phox (9.3 mg, $0.024 \mathrm{mmol}, 12 \mathrm{~mol} \%$ ) in $4.0 \mathrm{~mL}$ of toluene at $80{ }^{\circ} \mathrm{C}$ for $24 \mathrm{~h}$. The desired product 3qa was obtained in $61 \%$ yield $(44.7 \mathrm{mg}$ ) as a colorless liquid. er $=94: 6 \cdot[\alpha]_{\mathrm{D}}{ }^{25}=-46.82\left(c 1.6, \mathrm{CHCl}_{3}\right) \cdot R_{f}=0.4(\mathrm{PE}: \mathrm{EA}=10: 1) .{ }^{1} \mathbf{H} \mathbf{~ N M R}\left(400 \mathrm{MHz}, \mathrm{CDCl}_{3}\right) \delta$ $7.20-7.13(\mathrm{~m}, 6 \mathrm{H}), 7.06-7.01(\mathrm{~m}, 2 \mathrm{H}), 6.94-6.91(\mathrm{~m}, 2 \mathrm{H}), 3.11(\mathrm{ddd}, J=19.6,7.6,5.0 \mathrm{~Hz}, 1 \mathrm{H})$, $2.93(\mathrm{ddd}, J=19.6,6.4,4.9 \mathrm{~Hz}, 1 \mathrm{H}), 2.63(\mathrm{ddd}, J=13.7,6.4,5.0 \mathrm{~Hz}, 1 \mathrm{H}), 2.36$ (ddd, $J=13.7,7.6$, $4.9 \mathrm{~Hz}, 1 \mathrm{H}), 2.08(\mathrm{dd}, J=14.4,6.5 \mathrm{~Hz}, 1 \mathrm{H}), 1.99-1.90(\mathrm{~m}, 1 \mathrm{H}), 1.87-1.81(\mathrm{~m}, 1 \mathrm{H}), 1.80-1.58(\mathrm{~m}$, $5 \mathrm{H}), 1.37-1.25(\mathrm{~m}, 2 \mathrm{H}), 1.19(\mathrm{tt}, J=12.5,3.1 \mathrm{~Hz}, 1 \mathrm{H}), 1.15-1.04(\mathrm{~m}, 2 \mathrm{H}) .{ }^{13} \mathrm{C}$ NMR $(100 \mathrm{MHz}$, $\left.\mathrm{CDCl}_{3}\right) \delta 191.4,157.3,139.6,135.4,134.9,130.9,128.6,128.23,128.20,127.9,127.4,119.8,46.5$, 40.6, 34.8, 34.1, 31.4, 29.5, 26.4, 26.3, 26.2. HRMS $\left(\mathrm{ESI}^{+}\right) \mathrm{m} / z$ calc'd for $\mathrm{C}_{26} \mathrm{H}_{27} \mathrm{NONa}[\mathrm{M}+\mathrm{Na}]^{+}$: 
392.1985, found 392.1992. HPLC conditions: hexane/2-propanol $95: 5,0.8 \mathrm{~mL} / \mathrm{min}, \lambda=300 \mathrm{~nm}$, Chiralpak AD-H column $(4.6 \mathrm{~mm}$ x $250 \mathrm{~mm}), \mathrm{t}_{\mathrm{r}}($ minor $)=10.3 \mathrm{~min}, \mathrm{t}_{\mathrm{r}}($ major $)=13.0 \mathrm{~min}$.<smiles>C[C@H]1CCC(c2ccccc2)=C(c2ccccc2)C1=O</smiles>

\section{(S)-4'-Methyl-3'-oxo-3',4',5',6'-tetrahydro-[1,1':2',1''-terphenyl]-4'-carbonitrile (3ra)}

Following the general procedure, the reaction was performed with $\mathbf{1 r}(20.8 \mathrm{mg}, 0.1 \mathrm{mmol}, 1.0$ equiv) and phenylboronic acid $2 \mathrm{a}\left(24.4 \mathrm{mg}, 0.2 \mathrm{mmol}, 2.0\right.$ equiv), $\mathrm{H}_{2} \mathrm{O}$ (8 ul, $0.4 \mathrm{mmol}, 4.0$ equiv), $\mathrm{Ni}(\mathrm{OTf})_{2}$ (3.6 mg, $0.01 \mathrm{mmol}, 10 \mathrm{~mol} \%$ ), and (S)- ${ }^{t} \mathrm{Bu}-$ phox (4.7 mg, $0.012 \mathrm{mmol}, 12 \mathrm{~mol} \%$ ) in $2.0 \mathrm{~mL}$ of toluene at $80{ }^{\circ} \mathrm{C}$ for $24 \mathrm{~h}$. The desired product 3ra was obtained in $73 \%$ yield $(21.0 \mathrm{mg})$ as a colorless liquid. er $=91.5: 8.5 .[\alpha]_{\mathrm{D}}{ }^{25}=-168.90\left(c 0.9, \mathrm{CHCl}_{3}\right) . R_{f}=0.3(\mathrm{PE}: \mathrm{EA}=10: 1) .{ }^{1} \mathbf{H} \mathbf{~ N M R}(400 \mathrm{MHz}$, $\left.\mathrm{CDCl}_{3}\right) \delta 7.20-7.15(\mathrm{~m}, 6 \mathrm{H}), 7.07-7.04(\mathrm{~m}, 2 \mathrm{H}), 6.95-6.92(\mathrm{~m}, 2 \mathrm{H}), 3.21(\mathrm{ddd}, J=19.6,9.8,4.9$ Hz, 1H), $2.95(\mathrm{dt}, J=19.6,4.5 \mathrm{~Hz}, 1 \mathrm{H}), 2.57(\mathrm{dt}, J=13.7,4.6 \mathrm{~Hz}, 1 \mathrm{H}), 2.30(\mathrm{ddd}, J=14.2,9.8,4.8$ $\mathrm{Hz}, 1 \mathrm{H}), 1.67$ (s, 3H). ${ }^{13} \mathbf{C} \mathbf{N M R}\left(100 \mathrm{MHz}, \mathrm{CDCl}_{3}\right) \delta 190.9,158.2,139.6,135.4,134.8,130.9,128.6$, 128.24, 128.21, 127.9, 127.4, 119.8, 42.3, 33.6, 29.9, 21.3. HRMS $\left(\mathrm{ESI}^{+}\right) \mathrm{m} / z$ calc'd for $\mathrm{C}_{20} \mathrm{H}_{17} \mathrm{NONa}$ $[\mathrm{M}+\mathrm{Na}]^{+}:$310.1202, found 310.1205. HPLC conditions: hexane/2-propanol $95: 5,0.8 \mathrm{~mL} / \mathrm{min}, \lambda=$ $280 \mathrm{~nm}$, Chiralpak AD-H column $(4.6 \mathrm{~mm} \times 250 \mathrm{~mm}), \mathrm{t}_{\mathrm{r}}($ minor $)=10.4 \mathrm{~min}, \mathrm{t}_{\mathrm{r}}($ major $)=11.2 \mathrm{~min}$.

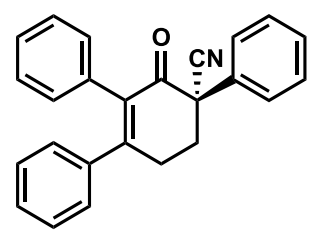

\section{(R)-3'-Oxo-4'-phenyl-3',4',5',6'-tetrahydro-[1,1':2',1"'-terphenyl]-4'-carbonitrile (3sa)}

Following the general procedure, the reaction was performed with $1 \mathrm{~s}$ ( $27.1 \mathrm{mg}, 0.1 \mathrm{mmol}, 1.0$ equiv) and phenylboronic acid $\mathbf{2 a}\left(24.4 \mathrm{mg}, 0.2 \mathrm{mmol}, 2.0\right.$ equiv), $\mathrm{H}_{2} \mathrm{O}$ (8 ul, $0.4 \mathrm{mmol}, 4.0$ equiv), $\mathrm{Ni}(\mathrm{OTf})_{2}$ (3.6 mg, $0.01 \mathrm{mmol}, 10 \mathrm{~mol} \%$ ), and $(S)$ - ${ }^{t} \mathrm{Bu}-\mathrm{phox}(4.7 \mathrm{mg}, 0.012 \mathrm{mmol}, 12 \mathrm{~mol} \%$ ) in $2.0 \mathrm{~mL}$ of toluene at $80{ }^{\circ} \mathrm{C}$ for $24 \mathrm{~h}$. The desired product 3sa was obtained in $62 \%$ yield $(21.6 \mathrm{mg})$ as a white solid. er $=$ $86: 14 .[\alpha]_{\mathrm{D}}{ }^{25}=49.83\left(c 1.4, \mathrm{CHCl}_{3}\right) . R_{f}=0.3(\mathrm{PE}: \mathrm{EA}=5: 1) .{ }^{1} \mathbf{H} \mathbf{N M R}\left(400 \mathrm{MHz}, \mathrm{CDCl}_{3}\right) \delta 7.51$ - $7.36(\mathrm{~m}, 5 \mathrm{H}), 7.22-7.13(\mathrm{~m}, 6 \mathrm{H}), 7.04-6.96(\mathrm{~m}, 4 \mathrm{H}), 3.14-3.02(\mathrm{~m}, 1 \mathrm{H}), 2.98-2.81(\mathrm{~m}, 3 \mathrm{H})$. ${ }^{13}$ C NMR $\left(100 \mathrm{MHz} \mathrm{CDCl}_{3}\right) \delta 189.8,158.3,139.5,136.3,134.6,134.4,131.0,129.3,129.0,128.7$, 128.2, 128.1, 127.9, 127.5, 127.1, 119.1, 52.5, 33.9, 29.6. HRMS $\left(\mathrm{ESI}^{+}\right) \mathrm{m} / z$ calc'd for $\mathrm{C}_{25} \mathrm{H}_{19} \mathrm{NONa}$ 
$[\mathrm{M}+\mathrm{Na}]^{+}:$372.1359, found 372.1363. HPLC conditions: hexane/2-propanol $75: 25,0.5 \mathrm{~mL} / \mathrm{min}, \lambda=$ $300 \mathrm{~nm}$, Chiralcel OD-H column $(4.6 \mathrm{~mm} \times 250 \mathrm{~mm}), \mathrm{t}_{\mathrm{r}}($ minor $)=20.2 \mathrm{~min}, \mathrm{t}_{\mathrm{r}}($ major $)=21.7 \mathrm{~min}$.

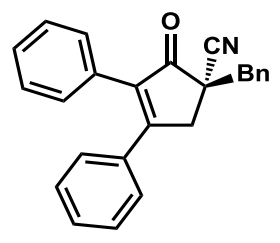

\section{(S)-1-Benzyl-2-oxo-3,4-diphenylcyclopent-3-enecarbonitrile (3ta)}

Following the general procedure, the reaction was performed with $1 \mathrm{t}(27.1 \mathrm{mg}, 0.1 \mathrm{mmol}, 1.0$ equiv) and phenylboronic acid $\mathbf{2 a}\left(24.4 \mathrm{mg}, 0.2 \mathrm{mmol}, 2.0\right.$ equiv), $\mathrm{H}_{2} \mathrm{O}$ (8 ul, $0.4 \mathrm{mmol}, 4.0$ equiv), Ni(OTf) (3.6 mg, $0.01 \mathrm{mmol}, 10 \mathrm{~mol} \%$ ), and $(S)-{ }^{t} \mathrm{Bu}-$ phox (4.7 mg, $0.012 \mathrm{mmol}, 12 \mathrm{~mol} \%$ ) in $2.0 \mathrm{~mL}$ of toluene at $80{ }^{\circ} \mathrm{C}$ for $24 \mathrm{~h}$. The desired product 3ta was obtained in $52 \%$ yield $(21.6 \mathrm{mg})$ as a white solid. er $=$ $86.5: 13.5 \cdot[\alpha]_{\mathrm{D}}{ }^{25}=56.33\left(c 1.2, \mathrm{CHCl}_{3}\right) . R_{f}=0.3(\mathrm{PE}: \mathrm{EA}=10: 1) .{ }^{1} \mathbf{H} \mathbf{~ N M R}\left(400 \mathrm{MHz}, \mathrm{CDCl}_{3}\right) \delta$ $7.39-7.26(\mathrm{~m}, 11 \mathrm{H}), 7.17-7.14(\mathrm{~m}, 2 \mathrm{H}), 7.13-7.10(\mathrm{~m}, 2 \mathrm{H}), 3.46-3.40(\mathrm{~m}, 2 \mathrm{H}), 3.32-3.27(\mathrm{~m}$, 2H). ${ }^{13} \mathrm{C}$ NMR $\left(100 \mathrm{MHz}, \mathrm{CDCl}_{3}\right) \delta 198.7,166.2,137.4,134.0,133.8,130.9,130.8,130.2,129.4$, 128.9, 128.8, 128.7, 128.2, 128.1, 119.9, 46.7, 42.1, 39.8. HRMS $\left(\mathrm{ESI}^{+}\right) \mathrm{m} / z$ calc'd for $\mathrm{C}_{25} \mathrm{H}_{19} \mathrm{NONa}$ $[\mathrm{M}+\mathrm{Na}]^{+}: 372.1359$, found 372.1361. HPLC conditions: hexane/2-propanol $80: 20,0.3 \mathrm{~mL} / \mathrm{min}, \lambda=$ $300 \mathrm{~nm}$, Chiralpak AD-H column $(4.6 \mathrm{~mm} \times 250 \mathrm{~mm}), \mathrm{t}_{\mathrm{r}}($ minor $)=24.7 \mathrm{~min}, \mathrm{t}_{\mathrm{r}}($ major $)=26.4 \mathrm{~min}$.

General procedure for synthesis of 4 by Ni-catalyzed desymmetrization of malononitrile and characterization data

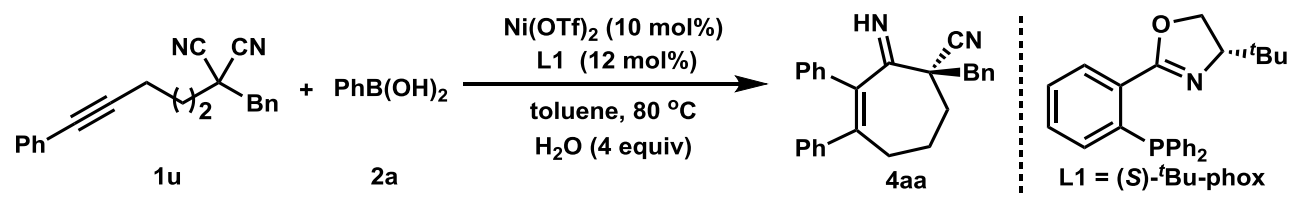

General Procedure: $\mathrm{Ni}(\mathrm{OTf})_{2}(7.2 \mathrm{mg}, 0.02 \mathrm{mmol}, 10 \mathrm{~mol} \%),(S)-{ }^{t} \mathrm{Bu}-\mathrm{phox}(9.3 \mathrm{mg}, 0.024 \mathrm{mmol}, 12$ mol\%) and $2.0 \mathrm{~mL}$ of toluene were added to a Schlenk tube under argon. The resultant solution was stirred at room temperature for $30 \mathrm{~min}$, then $1 \mathbf{u}(60.1 \mathrm{mg}, 0.2 \mathrm{mmol}, 1.0$ equiv), phenylboronic acid 2a (48.8 mg, $0.4 \mathrm{mmol}, 2.0$ equiv), and toluene $(2.0 \mathrm{~mL})$ were added, followed by the addition of $\mathrm{H}_{2} \mathrm{O}$ $(15 \mathrm{uL}, 0.8 \mathrm{mmol})$. The tube was sealed and the mixture was stirred at $80{ }^{\circ} \mathrm{C}$ for $24 \mathrm{~h}$. The reaction was cooled to room temperature and the solvent was directly removed under reduced pressure. The residue was purified by silica gel column chromatography to provide $(\boldsymbol{S})$-1-benzyl-2-imino-3,4diphenylcyclohept-3-ene-1-carbonitrile (4aa) (54.1 mg, 72\% yield) as a white solid. er = $99: 1$. 
$[\alpha]_{\mathrm{D}}^{25}=-128.00\left(c 1.0, \mathrm{CH}_{3} \mathrm{CN}\right) . R_{f}=0.3(\mathrm{PE}: \mathrm{EA}=10: 1) .{ }^{1} \mathbf{H} \mathbf{N M R}\left(400 \mathrm{MHz}, \mathrm{C}_{6} \mathrm{D}_{6}\right) \delta 9.73(\mathrm{~s}$, 1H), $7.62-7.40(\mathrm{~m}, 2 \mathrm{H}), 7.23-7.09(\mathrm{~m}, 3 \mathrm{H}), 7.08-7.02(\mathrm{~m}, 2 \mathrm{H}), 6.94-6.73(\mathrm{~m}, 8 \mathrm{H}), 3.64(\mathrm{AB}, J$ $=13.5 \mathrm{~Hz}, 1 \mathrm{H}), 3.09(\mathrm{BA}, J=13.5 \mathrm{~Hz}, 1 \mathrm{H}), 2.65-2.44(\mathrm{~m}, 1 \mathrm{H}), 2.44-2.28(\mathrm{~m}, 1 \mathrm{H}), 1.98-1.80(\mathrm{~m}$, 1H), $1.78-1.64(\mathrm{~m}, 2 \mathrm{H}), 1.54-1.34(\mathrm{~m}, 1 \mathrm{H}) .{ }^{13} \mathbf{C} \mathbf{N M R}\left(100 \mathrm{MHz}, \mathrm{C}_{6} \mathrm{D}_{6}\right) \delta 180.5,142.6,141.9$, $139.3,138.4,136.4,131.7,130.7,129.2,128.50,128.46,128.1,127.9,127.5,127.4,121.9,51.3,43.2$, 39.1, 36.2, 22.8. HRMS $\left(\mathrm{ESI}^{+}\right) \mathrm{m} / z$ calc'd for $\mathrm{C}_{27} \mathrm{H}_{24} \mathrm{~N}_{2} \mathrm{Na}[\mathrm{M}+\mathrm{Na}]^{+}$: 399.1832, found 399.1824. HPLC conditions: hexane/2-propanol $95: 5,1.0 \mathrm{~mL} / \mathrm{min}, \lambda=254 \mathrm{~nm}$, Chiralpak AD-H column (4.6 $\mathrm{mm} \times 250 \mathrm{~mm}), \mathrm{t}_{\mathrm{r}}($ major $)=10.2 \mathrm{~min}, \mathrm{t}_{\mathrm{r}}($ minor $)=13.8 \mathrm{~min}$.

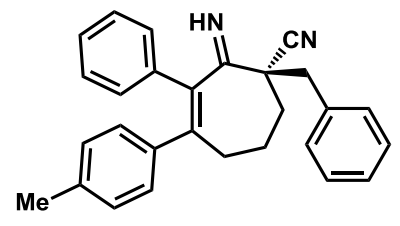

\section{(S)-1-Benzyl-2-imino-3-phenyl-4-(p-tolyl)cyclohept-3-ene-1-carbonitrile (4ab):}

Following the general procedure, the reaction was performed with $\mathbf{1 u}(59.6 \mathrm{mg}, 0.2 \mathrm{mmol}, 1.0 \mathrm{equiv})$, p-tolylboronic acid $2 \mathbf{b}$ (54.4 mg, 0.4 mmol, 2.0 equiv), $\mathrm{H}_{2} \mathrm{O}$ (16 ul, $0.8 \mathrm{mmol}, 4.0$ equiv), $\mathrm{Ni}(\mathrm{OTf})_{2}$ (7.4 mg, $0.02 \mathrm{mmol}, 10 \mathrm{~mol} \%$ ), and (S)- ${ }^{t} \mathrm{Bu}-$ phox (9.6 mg, $0.024 \mathrm{mmol}, 12 \mathrm{~mol} \%$ ) in $4.0 \mathrm{~mL}$ of toluene at $80{ }^{\circ} \mathrm{C}$ for $24 \mathrm{~h}$. The desired product 4 ab was obtained in $68 \%$ yield $(53.1 \mathrm{mg})$ as a yellow soild. er $=99: 1 .[\alpha]_{\mathrm{D}}^{25}=-77.20\left(c 1.0, \mathrm{CH}_{3} \mathrm{CN}\right) \cdot R_{f}=0.2(\mathrm{PE}: \mathrm{EA}=10: 1) .{ }^{1} \mathbf{H} \mathbf{N M R}\left(400 \mathrm{MHz}, \mathrm{CD}_{3} \mathrm{COCD}_{3}\right)$ $\delta 10.21(\mathrm{~s}, 1 \mathrm{H}), 7.51-7.37(\mathrm{~m}, 2 \mathrm{H}), 7.36-7.28(\mathrm{~m}, 3 \mathrm{H}), 7.11-7.00(\mathrm{~m}, 5 \mathrm{H}), 7.01-6.96(\mathrm{~m}, 2 \mathrm{H})$, $6.95-6.82(\mathrm{~m}, 2 \mathrm{H}), 3.55(\mathrm{AB}, J=13.5 \mathrm{~Hz}, 1 \mathrm{H}), 3.22(\mathrm{BA}, J=13.4 \mathrm{~Hz}, 1 \mathrm{H}), 2.78-2.65(\mathrm{~m}, 2 \mathrm{H})$, $2.29-2.16(\mathrm{~m}, 5 \mathrm{H}), 2.03-1.87(\mathrm{~m}, 2 \mathrm{H}) .{ }^{13} \mathbf{C}$ NMR $\left(100 \mathrm{MHz}, \mathrm{CD}_{3} \mathrm{COCD}_{3}\right) \delta 181.0,142.0,140.0$, $139.9,138.2$, 137.5, 137.0, 131.9, 131.1, 129.6, 129.5, 128.9, 128.7, 128.0, 127.7, 122.2, 51.6, 43.7, 38.3, 36.3, 22.9, 21.1. HRMS $\left(\mathrm{ESI}^{+}\right) \mathrm{m} / z$ calc'd for $\mathrm{C}_{28} \mathrm{H}_{27} \mathrm{~N}_{2}[\mathrm{M}+\mathrm{H}]^{+}$: 391.2169, found 391.2174. HPLC conditions: hexane/2-propanol $95: 5,1.0 \mathrm{~mL} / \mathrm{min}, \lambda=254 \mathrm{~nm}$, Chiralpak AD-H column (4.6 $\mathrm{mm} \times 250 \mathrm{~mm}), \mathrm{t}_{\mathrm{r}}($ major $)=11.5 \mathrm{~min}, \mathrm{t}_{\mathrm{r}}($ minor $)=15.5 \mathrm{~min}$.

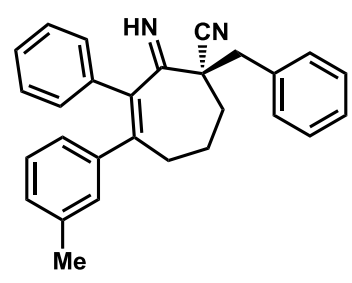

(S)-1-Benzyl-2-imino-3-phenyl-4-(m-tolyl)cyclohept-3-ene-1-carbonitrile (4ac): 
Following the general procedure, the reaction was performed with $1 \mathbf{u}(59.7 \mathrm{mg}, 0.2 \mathrm{mmol}, 1.0$ equiv), $m$-tolylboronic acid 2 c (54.4 mg, 0.4 mmol, 2.0 equiv), $\mathrm{H}_{2} \mathrm{O}$ (16 ul, $0.8 \mathrm{mmol}, 4.0$ equiv), Ni(OTf) 2 (7.2 mg, $0.02 \mathrm{mmol}, 10 \mathrm{~mol} \%$ ), and $(S)-{ }^{t} \mathrm{Bu}-$ phox (9.6 mg, $0.024 \mathrm{mmol}, 12 \mathrm{~mol} \%$ ) in $4.0 \mathrm{~mL}$ of toluene at $80{ }^{\circ} \mathrm{C}$ for $24 \mathrm{~h}$. The desired product 4 ac was obtained in $66 \%$ yield $(51.4 \mathrm{mg})$ as a yellow soild. er $=99: 1 .[\alpha]_{\mathrm{D}}{ }^{25}=-103.33\left(c 1.0, \mathrm{CH}_{3} \mathrm{CN}\right) \cdot R_{f}=0.2(\mathrm{PE}: \mathrm{EA}=10: 1) .{ }^{1} \mathbf{H} \mathbf{~ N M R}\left(400 \mathrm{MHz}, \mathrm{CD}_{3} \mathrm{CN}\right)$ $\delta 10.01(\mathrm{~s}, 1 \mathrm{H}), 7.46-7.24(\mathrm{~m}, 5 \mathrm{H}), 7.14-7.04(\mathrm{~m}, 3 \mathrm{H}), 7.04-7.00(\mathrm{~m}, 1 \mathrm{H}), 6.99-6.94(\mathrm{~m}, 2 \mathrm{H})$, $6.94-6.82(\mathrm{~m}, 3 \mathrm{H}), 3.50(\mathrm{AB}, J=13.5 \mathrm{~Hz}, 1 \mathrm{H}), 3.17(\mathrm{BA}, J=13.6 \mathrm{~Hz}, 1 \mathrm{H}), 2.73-2.60(\mathrm{~m}, 2 \mathrm{H})$, $2.21-2.16(\mathrm{~m}, 5 \mathrm{H}), 1.99-1.88(\mathrm{~m}, 2 \mathrm{H}) .{ }^{13} \mathrm{C} \mathbf{N M R}\left(100 \mathrm{MHz}, \mathrm{CD}_{3} \mathrm{CN}\right) \delta 181.0,143.1,142.6,139.8$, 138.6, 137.9, 137.1, 131.9, 131.1, 130.1, 129.0, 128.83, 128.8, 128.79, 128.2, 128.0, 126.7, 122.6, 51.8, 43.6, 38.1, 36.3, 22.9, 21.3. HRMS $\left(\mathrm{ESI}^{+}\right) \mathrm{m} / z$ calc'd for $\mathrm{C}_{28} \mathrm{H}_{27} \mathrm{~N}_{2}[\mathrm{M}+\mathrm{H}]^{+}:$391.2169, found 391.2160. HPLC conditions: hexane/2-propanol $95: 5,1.0 \mathrm{~mL} / \mathrm{min}, \lambda=254 \mathrm{~nm}$, Chiralpak AD-H column (4.6 $\mathrm{mm} \times 250 \mathrm{~mm}), \mathrm{t}_{\mathrm{r}}($ major $)=8.3 \mathrm{~min}, \mathrm{t}_{\mathrm{r}}($ minor $)=10.1 \mathrm{~min}$.

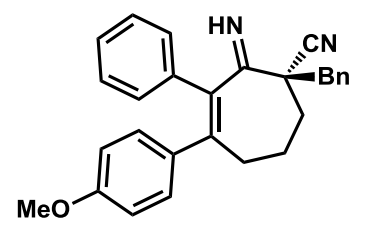

(S)-1-Benzyl-2-imino-4-(4-methoxyphenyl)-3-phenylcyclohept-3-ene-1-carbonitrile (4ad):

Following the general procedure, the reaction was performed with $1 \mathbf{u}(59.3 \mathrm{mg}, 0.2 \mathrm{mmol}, 1.0$ equiv), (4-methoxyphenyl)boronic acid $2 d$ (60.8 mg, 0.4 mmol, 2.0 equiv), $\mathrm{H}_{2} \mathrm{O}$ (16 ul, $0.8 \mathrm{mmol}, 4.0$ equiv), $\mathrm{Ni}(\mathrm{OTf})_{2}(7.2 \mathrm{mg}, 0.02 \mathrm{mmol}, 10 \mathrm{~mol} \%)$, and $(S)-{ }^{t} \mathrm{Bu}-$ phox $(9.3 \mathrm{mg}, 0.024 \mathrm{mmol}, 12 \mathrm{~mol} \%)$ in $4.0 \mathrm{~mL}$ of toluene at $80{ }^{\circ} \mathrm{C}$ for $24 \mathrm{~h}$. The desired product $4 \mathbf{a d}$ was obtained in $70 \%$ yield $(56.2 \mathrm{mg})$ as a yellow soild. er $=99: 1 .[\alpha]_{\mathrm{D}}{ }^{25}=-81.27\left(c 1.0, \mathrm{CH}_{3} \mathrm{CN}\right) . R_{f}=0.2(\mathrm{PE}: \mathrm{EA}=10: 1) .{ }^{1} \mathbf{H}$ NMR $(400 \mathrm{MHz}$, $\left.\mathrm{CD}_{3} \mathrm{CN}\right) \delta 9.97(\mathrm{br}, 1 \mathrm{H}), 7.45-7.26(\mathrm{~m}, 5 \mathrm{H}), 7.12-7.07(\mathrm{~m}, 3 \mathrm{H}), 7.04(\mathrm{~d}, J=8.8 \mathrm{~Hz}, 2 \mathrm{H}), 6.94-$ $6.82(\mathrm{~m}, 2 \mathrm{H}), 6.75-6.66(\mathrm{~m}, 2 \mathrm{H}), 3.70(\mathrm{~s}, 3 \mathrm{H}), 3.49(\mathrm{AB}, J=13.7 \mathrm{~Hz}, 1 \mathrm{H}), 3.16(\mathrm{BA}, J=13.6 \mathrm{~Hz}$, 1H), $2.75-2.60(\mathrm{~m}, 2 \mathrm{H}), 2.29-2.12(\mathrm{~m}, 2 \mathrm{H}), 1.96-1.86(\mathrm{~m}, 2 \mathrm{H}) .{ }^{13} \mathbf{C} \mathbf{N M R}\left(100 \mathrm{MHz}, \mathrm{CD}_{3} \mathrm{CN}\right) \delta$ 181.4, 159.7, 142.2, 140.1, 137.5, 137.1, 135.0, 131.9, 131.2, 131.1, 129.0, 128.9, 128.2, 127.9, 122.6, 114.3, 55.8, 51.7, 43.7, 37.9, 36.2, 22.8. HRMS $\left(\mathrm{ESI}^{+}\right) \mathrm{m} / z$ calc'd for $\mathrm{C}_{28} \mathrm{H}_{27} \mathrm{~N}_{2} \mathrm{O}[\mathrm{M}+\mathrm{H}]^{+}:$: 407.2118, found 407.2116. HPLC conditions: hexane/2-propanol $95: 5,1.0 \mathrm{~mL} / \mathrm{min}, \lambda=254 \mathrm{~nm}$, Chiralpak AD-H column $(4.6 \mathrm{~mm} \times 250 \mathrm{~mm}), \mathrm{t}_{\mathrm{r}}($ major $)=16.9 \mathrm{~min}, \mathrm{t}_{\mathrm{r}}($ minor $)=24.7 \mathrm{~min}$. 
<smiles>C[C@@]1(C#N)CCCC(c2ccc(F)cc2)=C(c2ccccc2)C1=N</smiles>

(S)-1-Benzyl-4-(4-fluorophenyl)-2-imino-3-phenylcyclohept-3-ene-1-carbonitrile (4ae):

Following the general procedure, the reaction was performed with $1 \mathbf{u}(59.4 \mathrm{mg}, 0.2 \mathrm{mmol}, 1.0$ equiv), (4-fluorophenyl)boronic acid 2 (56.2 mg, $0.4 \mathrm{mmol}, 2.0$ equiv), $\mathrm{H}_{2} \mathrm{O}$ (16 ul, $0.8 \mathrm{mmol}, 4.0$ equiv), $\mathrm{Ni}(\mathrm{OTf})_{2}(7.3 \mathrm{mg}, 0.02 \mathrm{mmol}, 10 \mathrm{~mol} \%)$, and $(S)-{ }^{t} \mathrm{Bu}-\mathrm{phox}(9.7 \mathrm{mg}, 0.024 \mathrm{mmol}, 12 \mathrm{~mol} \%)$ in $4.0 \mathrm{~mL}$ of toluene at $80{ }^{\circ} \mathrm{C}$ for $24 \mathrm{~h}$. The desired product 4ae was obtained in $58 \%$ yield $(45.5 \mathrm{mg})$ as a white soild. er $=99: 1 .[\alpha]_{\mathrm{D}}{ }^{25}=-130.50\left(c 1.0, \mathrm{CH}_{3} \mathrm{CN}\right) \cdot R_{f}=0.2(\mathrm{PE}: \mathrm{EA}=10: 1) .{ }^{1} \mathbf{H} \mathbf{N M R}(400 \mathrm{MHz}$, $\left.\mathrm{C}_{6} \mathrm{D}_{6}\right) \delta 9.71(\mathrm{~s}, 1 \mathrm{H}), 7.81-7.34(\mathrm{~m}, 2 \mathrm{H}), 7.22$ - 7.08(m, 4H), $6.90-6.68(\mathrm{~m}, 6 \mathrm{H}), 6.61-6.48(\mathrm{~m}$, 2H), $3.78-3.46(\mathrm{~m}, 1 \mathrm{H}), 3.16-2.95(\mathrm{~m}, 1 \mathrm{H}), 2.52-2.36(\mathrm{~m}, 1 \mathrm{H}), 2.33-2.19(\mathrm{~m}, 1 \mathrm{H}), 1.97-1.77$ $(\mathrm{m}, 1 \mathrm{H}), 1.78-1.60(\mathrm{~m}, 2 \mathrm{H}), 1.48-1.32(\mathrm{~m}, 1 \mathrm{H}) .{ }^{13} \mathrm{C} \mathbf{N M R}\left(100 \mathrm{MHz}, \mathrm{C}_{6} \mathrm{D}_{6}\right) \delta 180.1,162.2(\mathrm{~d}, J=$ $246.8 \mathrm{~Hz}$ ), 140.8, 139.1, 138.4, 136.3, 131.7, 131.0 (d, $J=8.0 \mathrm{~Hz}), 130.6,128.5,128.4$ 127.6, 127.5, 122.0, $115.4(\mathrm{~d}, J=21.4 \mathrm{~Hz}), 51.5,43.1,39.2,36.1,22.8 .{ }^{19} \mathbf{F}$ NMR $\left(376 \mathrm{MHz}, \mathrm{C}_{6} \mathrm{D}_{6}\right) \delta-114.25$. HRMS $\left(\mathrm{ESI}^{+}\right) m / z$ calc'd for $\mathrm{C}_{27} \mathrm{H}_{24} \mathrm{~N}_{2} \mathrm{~F}[\mathrm{M}+\mathrm{H}]^{+}:$395.1918, found 395.1913. HPLC conditions: hexane/2-propanol 95:5, $1.0 \mathrm{~mL} / \mathrm{min}, \lambda=254 \mathrm{~nm}$, Chiralpak AD-H column $(4.6 \mathrm{~mm} \times 250 \mathrm{~mm}), \mathrm{t}_{\mathrm{r}}$ $($ major $)=12.17 \mathrm{~min}, \mathrm{t}_{\mathrm{r}}(\operatorname{minor})=15.32 \mathrm{~min}$.<smiles>N#C[C@]1([18O])CCCC(c2ccc(Cl)cc2)=C1c1ccccc1</smiles>

\section{(S)-1-Benzyl-4-(4-chlorophenyl)-2-imino-3-phenylcyclohept-3-ene-1-carbonitrile (4af)}

Following the general procedure, the reaction was performed with $1 \mathbf{u}(59.7 \mathrm{mg}, 0.2 \mathrm{mmol}, 1.0$ equiv), (4-chlorophenyl)boronic acid 2f (62.6 mg, $0.4 \mathrm{mmol}, 2.0$ equiv), $\mathrm{H}_{2} \mathrm{O}$ (16 ul, $0.8 \mathrm{mmol}, 4.0$ equiv), $\mathrm{Ni}(\mathrm{OTf})_{2}(7.2 \mathrm{mg}, 0.02 \mathrm{mmol}, 10 \mathrm{~mol} \%)$ and $(S)-{ }^{t} \mathrm{Bu}-\mathrm{phox}(9.5 \mathrm{mg}, 0.024 \mathrm{mmol}, 12 \mathrm{~mol} \%)$ in $4.0 \mathrm{~mL}$ of toluene at $80{ }^{\circ} \mathrm{C}$ for $24 \mathrm{~h}$. The desired product 4 af was obtained in $62 \%$ yield $(50.8 \mathrm{mg})$ as a yellow oil. er $=99: 1 .[\alpha]_{\mathrm{D}}{ }^{25}=-117.27\left(c 1.0, \mathrm{CH}_{3} \mathrm{CN}\right) . R_{f}=0.4(\mathrm{PE}: \mathrm{EA}=5: 1) .{ }^{1} \mathbf{H} \mathbf{~ N M R}(400 \mathrm{MHz}$, $\left.\mathrm{CD}_{3} \mathrm{CN}\right) \delta 10.04(\mathrm{~s}, 1 \mathrm{H}), 7.44-7.26(\mathrm{~m}, 5 \mathrm{H}), 7.19-7.14(\mathrm{~m}, 2 \mathrm{H}), 7.14-7.05(\mathrm{~m}, 5 \mathrm{H}), 6.96-6.79$ $(\mathrm{m}, 2 \mathrm{H}), 3.50(\mathrm{AB}, J=13.6 \mathrm{~Hz}, 1 \mathrm{H}), 3.17(\mathrm{BA}, J=13.6 \mathrm{~Hz}, 1 \mathrm{H}), 2.73-2.60(\mathrm{~m}, 2 \mathrm{H}), 2.22-2.13(\mathrm{~m}$, 2H), $2.00-1.87(\mathrm{~m}, 2 \mathrm{H}) .{ }^{13} \mathrm{C}$ NMR $\left(100 \mathrm{MHz}, \mathrm{CD}_{3} \mathrm{CN}\right) \delta 180.6,141.9,141.5,139.5,138.8,137.0$, 
$133.2,131.9,131.4,131.1,129.02,129.00,128.95,128.2,122.6,52.0,43.4,38.4,36.2$, 22.9. HRMS $\left(\mathrm{ESI}^{+}\right) \mathrm{m} / z$ calc'd for $\mathrm{C}_{27} \mathrm{H}_{24} \mathrm{~N}_{2} \mathrm{Cl}[\mathrm{M}+\mathrm{H}]^{+}: 411.1623$, found 411.1633; HPLC conditions: hexane/2propanol 95:5, $1.0 \mathrm{~mL} / \mathrm{min}, \lambda=230 \mathrm{~nm}$, Chiralpak AD-H column $(4.6 \mathrm{~mm} \times 250 \mathrm{~mm})$; $\mathrm{t}_{\mathrm{r}}($ minor $)=$ $17.5 \min , \mathrm{t}_{\mathrm{r}}($ major $)=13.8 \min$.<smiles>C[C@]1(C#N)CCCC(c2ccc(Br)cc2)=C(c2ccccc2)C1=N</smiles>

(S)-1-Benzyl-4-(4-bromophenyl)-2-imino-3-phenylcyclohept-3-ene-1-carbonitrile (4ag):

Following the general procedure, the reaction was performed with $1 \mathbf{u}(59.5 \mathrm{mg}, 0.2 \mathrm{mmol}, 1.0$ equiv), (4-bromophenyl)boronic acid $2 \mathrm{~g}$ ( $80.0 \mathrm{mg}, 0.4 \mathrm{mmol}, 2.0$ equiv), $\mathrm{H}_{2} \mathrm{O}$ (16 ul, $0.8 \mathrm{mmol}, 4.0$ equiv), $\mathrm{Ni}(\mathrm{OTf})_{2}(7.3 \mathrm{mg}, 0.02 \mathrm{mmol}, 10 \mathrm{~mol} \%)$, and $(S)-{ }^{t} \mathrm{Bu}-$ phox $(9.7 \mathrm{mg}, 0.024 \mathrm{mmol}, 12 \mathrm{~mol} \%)$ in $4.0 \mathrm{~mL}$ of toluene at $80{ }^{\circ} \mathrm{C}$ for $24 \mathrm{~h}$. The desired product $4 \mathrm{ag}$ was obtained in $61 \%$ yield $(56.2 \mathrm{mg})$ as a white soild. er $=99: 1 .[\alpha]_{\mathrm{D}}{ }^{25}=-118.27\left(c 1.0, \mathrm{CH}_{3} \mathrm{CN}\right) . R_{f}=0.3(\mathrm{PE}: \mathrm{EA}=10: 1) .{ }^{\mathbf{1}} \mathbf{H} \mathbf{N M R}(400 \mathrm{MHz}$, $\left.\mathrm{CD}_{3} \mathrm{CN}\right) \delta 10.04(\mathrm{~s}, 1 \mathrm{H}), 7.45-7.24(\mathrm{~m}, 7 \mathrm{H}), 7.17-7.06(\mathrm{~m}, 3 \mathrm{H}), 7.02(\mathrm{~d}, J=8.5 \mathrm{~Hz}, 2 \mathrm{H}), 6.96-$ $6.80(\mathrm{~m}, 2 \mathrm{H}), 3.50(\mathrm{AB}, J=13.5 \mathrm{~Hz}, 1 \mathrm{H}), 3.16(\mathrm{BA}, J=13.6 \mathrm{~Hz}, 1 \mathrm{H}), 2.73-2.61(\mathrm{~m}, 2 \mathrm{H}), 2.27-$ 2.12 (m, 2H), $2.01-1.88$ (m, 2H). ${ }^{13} \mathbf{C}$ NMR (100 MHz, $\left.\mathrm{CD}_{3} \mathrm{CN}\right) \delta 180.6,142.4,141.5,139.4,138.8$, 137.0, 132.0, 131.9, 131.7, 131.1, 129.03, 128.97, 128.2, 128.2, 122.6, 121.4, 52.0, 43.5, 38.4, 36.2, 23.0. HRMS $\left(\mathrm{ESI}^{+}\right) \mathrm{m} / z$ calc' $\mathrm{d}$ for $\mathrm{C}_{27} \mathrm{H}_{24} \mathrm{~N}_{2} \mathrm{Br}[\mathrm{M}+\mathrm{H}]^{+}: 455.1118$, found 455.1106. HPLC conditions: hexane/2-propanol $95: 5,1.0 \mathrm{~mL} / \mathrm{min}, \lambda=254 \mathrm{~nm}$, Chiralpak AD-H column (4.6 mm x $250 \mathrm{~mm}), \mathrm{t}_{\mathrm{r}}$ $($ major $)=15.3 \min , \mathrm{t}_{\mathrm{r}}($ minor $)=20.3 \mathrm{~min}$.

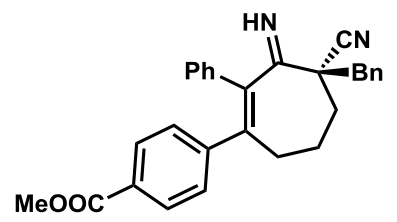

(S)-Methyl-4-(4-Benzyl-4-cyano-3-imino-2-phenylcyclohept-1-en-1-yl)benzoate (4ah):

Following the general procedure, the reaction was performed with $1 \mathbf{u}(59.8 \mathrm{mg}, 0.2 \mathrm{mmol}, 1.0$ equiv), (4-(methoxycarbonyl)phenyl)boronic acid $2 \mathrm{~h}$ (74.2 mg, $0.4 \mathrm{mmol}, 2.0$ equiv), $\mathrm{H}_{2} \mathrm{O}$ (16 ul, $0.8 \mathrm{mmol}$, 4.0 equiv), $\mathrm{Ni}(\mathrm{OTf})_{2}(7.4 \mathrm{mg}, 0.02 \mathrm{mmol}, 10 \mathrm{~mol} \%)$, and $(S){ }^{t} \mathrm{Bu}-\mathrm{phox}$ (10.0 mg, $0.024 \mathrm{mmol}, 12$ $\mathrm{mol} \%$ ) in $4.0 \mathrm{~mL}$ of toluene at $80{ }^{\circ} \mathrm{C}$ for $24 \mathrm{~h}$. The desired product 4 ah was obtained in $48 \%$ yield (46.6 mg) as a yellow oil. er $=99: 1 .[\alpha]_{\mathrm{D}}{ }^{25}=-133.05\left(c 1.0, \mathrm{CH}_{3} \mathrm{CN}\right) . R_{f}=0.2(\mathrm{PE}: \mathrm{EA}=5: 1) .{ }^{1} \mathbf{H}$ NMR (400 MHz, $\left.\mathrm{C}_{6} \mathrm{D}_{6}\right) \delta 9.74$ (br s, 1H), 7.87 (d, $\left.J=8.3 \mathrm{~Hz}, 2 \mathrm{H}\right), 7.54-7.36$ (m, 2H), $7.22-7.08$ 
(m, 3H), 6.99 (d, J = 8.4 Hz, 2H), $6.88-6.63(\mathrm{~m}, 5 \mathrm{H}), 3.76-3.48(\mathrm{~m}, 1 \mathrm{H}), 3.40(\mathrm{~s}, 3 \mathrm{H}), 3.17-2.88$ $(\mathrm{m}, 1 \mathrm{H}), 2.55-2.35(\mathrm{~m}, 1 \mathrm{H}), 2.33-2.16(\mathrm{~m}, 1 \mathrm{H}), 1.97-1.79(\mathrm{~m}, 1 \mathrm{H}), 1.78-1.62(\mathrm{~m}, 2 \mathrm{H}), 1.46-$ $1.31(\mathrm{~m}, 1 \mathrm{H}) .{ }^{13} \mathrm{C}$ NMR (100 MHz, $\left.\mathrm{C}_{6} \mathrm{D}_{6}\right) \delta 179.8,166.3,147.2,141.0,139.2,138.7,136.2,131.7$, $130.5,129.9,129.5,129.2,128.5,128.4,127.7,127.6,121.9,51.6,51.4,43.1,39.0,35.7,22.9$. HRMS $\left(\mathrm{ESI}^{+}\right) \mathrm{m} / z$ calc'd for $\mathrm{C}_{29} \mathrm{H}_{27} \mathrm{~N}_{2} \mathrm{O}_{2}[\mathrm{M}+\mathrm{H}]^{+}:$435.2067, found 435.2054. HPLC conditions: hexane/2propanol $85: 15,1.0 \mathrm{~mL} / \mathrm{min}, \lambda=230 \mathrm{~nm}$, Chiralpak AD-H column $(4.6 \mathrm{~mm} \times 250 \mathrm{~mm}), \mathrm{t}_{\mathrm{r}}($ major $)=$ $10.6 \min , \mathrm{t}_{\mathrm{r}}($ minor $)=19.5 \mathrm{~min}$.

\section{Natural abundant ${ }^{13} \mathrm{C}$ kinetic isotope effect studies}

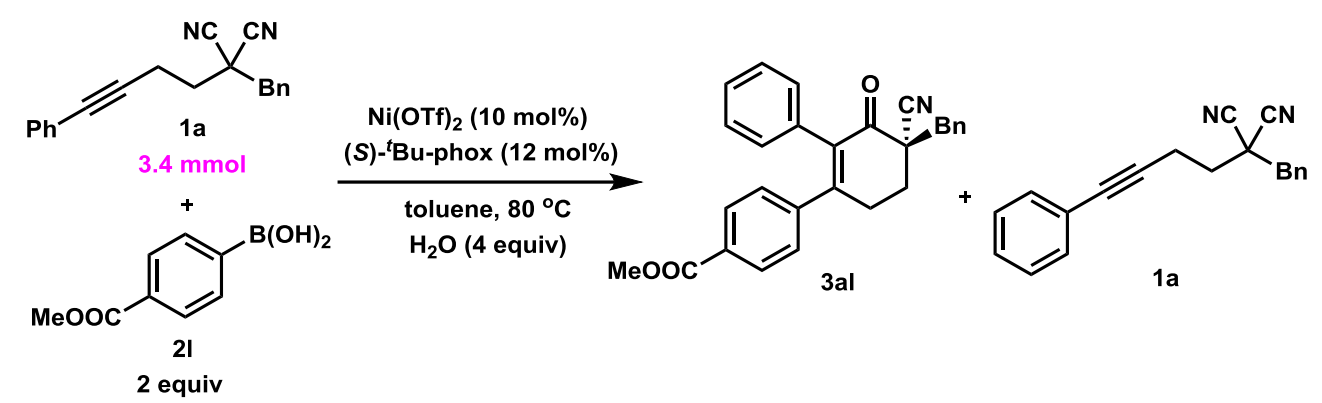

To a resealable Schlenk flask equipped with a magnetic stirring bar were added $\mathrm{Ni}(\mathrm{OTf})_{2}(121.3 \mathrm{mg}$, $0.34 \mathrm{mmol}, 10 \mathrm{~mol} \%),(S)-{ }^{t}$ Bu-phox $(158.1 \mathrm{mg}, 0.41 \mathrm{mmol}, 12 \mathrm{~mol} \%)$ and $34 \mathrm{~mL}$ of toluene in a glovebox. After the solution was stirred at room temperature for $30 \mathrm{~min}$, substrate 1a $(966.8 \mathrm{mg}, 3.4$ mmol, 1.0 equiv), (4-(methoxycarbonyl)phenyl)boronic acid 21 (1223.8 mg, $6.8 \mathrm{mmol}, 2.0$ equiv), another $34.0 \mathrm{~mL}$ of toluene were added. The flask was sealed with a Teflon valve, removed from the glovebox, and connected to a Schlenk line. Under a positive pressure of argon, the Teflon valve was removed and $\mathrm{H}_{2} \mathrm{O}$ (245 ul, 13.6 mmol, 4.0 equiv) was added rapidly. The flask was resealed and the mixture was stirred at $80{ }^{\circ} \mathrm{C}$ for the $22 \mathrm{~h}$. Then the mixture was cooled to room temperature, and 12 $\mathrm{mL}$ of $\mathrm{HCl}(1 \mathrm{M})$ and $10 \mathrm{~mL}$ of EA were added to the flask. After stirred for additional $30 \mathrm{~min}$, the mixture was extracted with EA $(20 \mathrm{~mL} x 3)$. The combined organic phase was washed with brine, dried over $\mathrm{Na}_{2} \mathrm{SO}_{4}$, and filtered. After vacuum evaporation of the solvents, the residue was purified by silica gel column chromatography to provide the recovered 1 a $(127.9 \mathrm{mg}, 13 \%$ yield, named as recovered A) and the desired product 3al (1.23 g, 86\% yield). The second reaction using starting material 1a from the same bath as above was carried out followed the same procedure with the recovered 1a (147.2 mg, $15 \%$ yield, named as recovered B) and the desired product $\mathbf{3 a l}$ (1.22 g, 85\% yield). 
The ${ }^{13} \mathrm{C}$ NMR analyses of the recovered (A and $\left.\mathbf{B}\right)$ and virgin samples of $\mathbf{1 a}$ were performed by following Singleton's method. ${ }^{15}$ All the samples (recovered A and $\mathbf{B}$, and virgin 1a) used for the the ${ }^{13} \mathrm{C}$ NMR experiments were came from the same batch, and the recovered material integrations were compared with starting material from the same lot. The NMR samples of virgin and recovered 1a were prepared equivalently as follows: a $J$-Young NMR tube was charged with 1a (102.6 mg, $0.36 \mathrm{mmol})$, then $\mathrm{CDCl}_{3}(320 \mathrm{ul})$ was added to a total height of $3.0 \mathrm{~cm}$.

The ${ }^{13} \mathrm{C}$ NMR spectra were recorded with inverse gated decoupling and calibrated $45{ }^{\circ} \mathrm{C}$ pulses on 100 MHz NMR spectrometer. $70 \mathrm{~s}$ delays between calibrated $\pi / 2$ pulses and $5 \mathrm{~s}$ acquisition time were used $(\mathrm{d} 1=70 \mathrm{~s}$, at $=5 \mathrm{~s}, \mathrm{nt}=1024)$. Back to back experiments in the order of recovered $\mathbf{A}$, virgin 1a, and recovered $\mathbf{B}$, were carried out and each sample was run three times successively. The simulated spectra were determined by Gaussian fitting of Bruker Topspin 4.0.7 deconvolution module. A zero order baseline correction was applied to process the date. Integrations were determined automatically using a constant integration region for each peak. The NMR data are summarized in Table S7.

Table S7. ${ }^{13} \mathrm{C}$ NMR integration of recovered and virgin samples of 1a

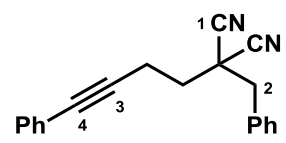

Recovered A vs Virgin 1a

\begin{tabular}{cccc}
\hline Carbon atom & Virgin $\left(\mathbf{R}_{\mathbf{0}}\right)$ & Recovered A $(\mathbf{R})$ & $\mathbf{R} / \mathbf{R}_{\mathbf{0}}$ \\
\hline 1 & 2.094 & 2.116 & $1.021^{a}$ \\
2 & 1.000 & 1.000 & 1.000 \\
3 & 1.011 & 1.019 & 1.008 \\
4 & 1.036 & 1.033 & 0.998 \\
\hline
\end{tabular}

Recovered B vs Virgin 1a

\begin{tabular}{cccc}
\hline Carbon atom & Virgin $\left(\mathbf{R}_{\mathbf{0}}\right)$ & Recovered B (R) & $\mathbf{R} / \mathbf{R}_{\mathbf{0}}$ \\
\hline 1 & 2.094 & 2.134 & $1.039^{a}$ \\
2 & 1.000 & 1.000 & 1.000 \\
3 & 1.011 & 1.016 & 1.005 \\
4 & 1.036 & 1.042 & 1.006 \\
\hline
\end{tabular}

${ }^{a}$ Because only one of the two nitriles was reacted, ${ }^{13} \mathrm{C}$ KIE ratio $\left(\mathrm{R} / \mathrm{R}_{0}\right)$ of $\mathrm{C} 1$ was revised according the equation of $\left(\mathrm{R}-\mathrm{R}_{0} / 2\right) / \mathrm{R}_{0} * 2$. 


\section{Kinetic profiles of the parallel reactions with $\mathrm{H}_{2} \mathrm{O}$ and $\mathrm{D}_{2} \mathrm{O}$}

$\mathrm{Ni}(\mathrm{OTf})_{2}(7.2 \mathrm{mg}, 0.02 \mathrm{mmol}, 10 \mathrm{~mol} \%),(S)-{ }^{t} \mathrm{Bu}-\mathrm{phox}(9.3 \mathrm{mg}, 0.024 \mathrm{mmol}, 12 \mathrm{~mol} \%)$ and $2.0 \mathrm{~mL}$ toluene were added to resealable Schlenk tube under argon. The resultant solution was stirred at room temperature for $30 \mathrm{~min}$, then $1 \mathrm{a}\left(56.9 \mathrm{mg}, 0.2 \mathrm{mmol}, 1.0\right.$ equiv), $(\mathrm{PhBO})_{3}(49.8 \mathrm{mg}, 0.16 \mathrm{mmol}, 0.8$ equiv), 1,3,5-trimethoxybenzene (16.8 $\mathrm{mg}, 0.1 \mathrm{mmol}$, as an internal standard), toluene $(2.0 \mathrm{~mL})$, and $\mathrm{H}_{2} \mathrm{O}$ (15 uL, $0.8 \mathrm{mmol}, 4.0$ equiv) were added. The tube was sealed and stirred at $80{ }^{\circ} \mathrm{C}$ under argon on a preheated stirring plate. At the indicated time points as shown in Table S8, an aliquot $(0.2 \mathrm{~mL})$ was removed with a syringe. The aliquot was diluted with DCM $(1 \mathrm{~mL})$, filtered through a celit pad, and concentrated under reduced pressure. The the yield of the product $\mathbf{3 a a}$ was determined by ${ }^{1} \mathrm{H}$ NMR analysis of the crude mixture using 1,3,5-trimethoxybenzene as an internal standard (in blue, Table S8). A parallel reaction was set up following the same procedure except use $\mathrm{D}_{2} \mathrm{O}$ instead of $\mathrm{H}_{2} \mathrm{O}$ (in red, Table S8). All the experiments were repeated for twice.

Table S8. Kinetic profiles of the parallel reactions with $\mathrm{H}_{2} \mathrm{O}$ and $\mathrm{D}_{2} \mathrm{O}^{a}$

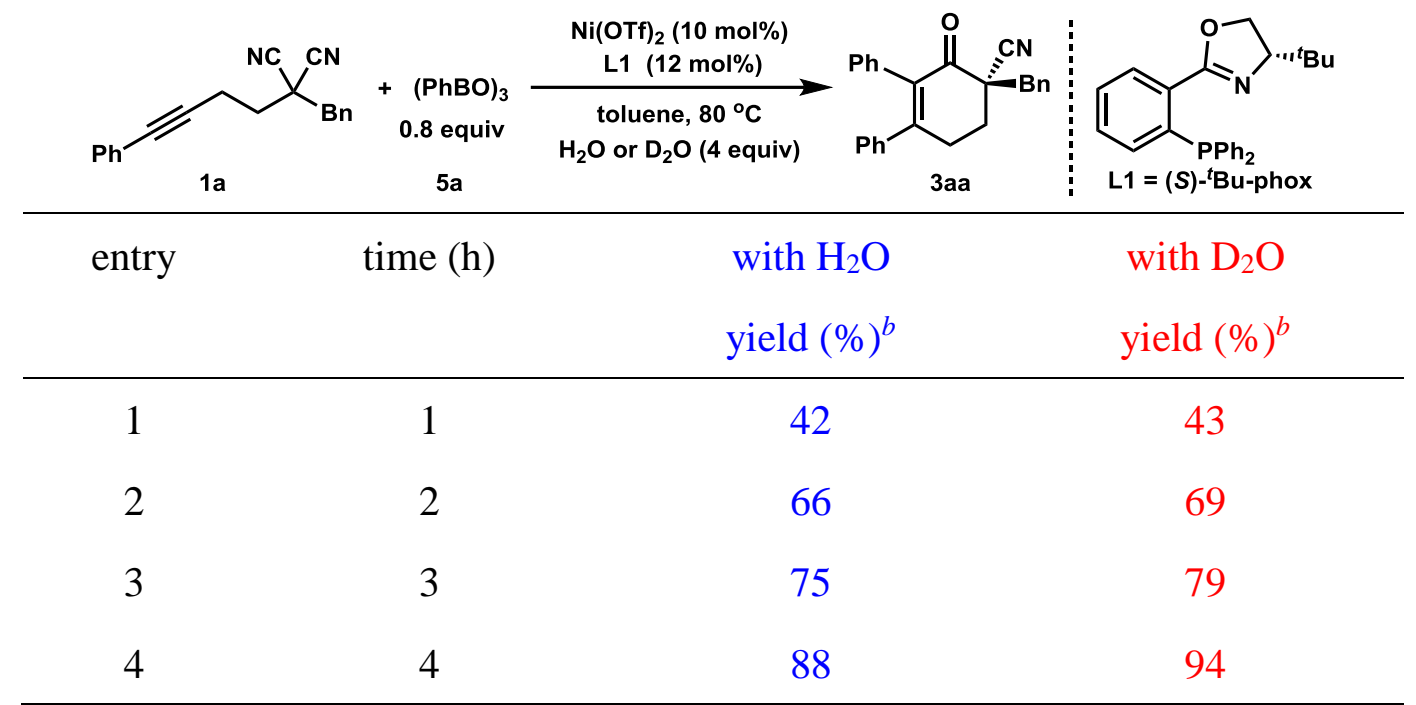

${ }^{a}$ The reaction conducted with $\mathrm{Ni}(\mathrm{OTf})_{2}(10 \mathrm{~mol} \%), \mathbf{L 1}(12 \mathrm{~mol} \%), \mathbf{1 a}(0.1 \mathrm{mmol}), \mathbf{5 a}(0.16 \mathrm{mmol}, 0.8 \mathrm{equiv})$, and $\mathrm{H}_{2} \mathrm{O}$ or $\mathrm{D}_{2} \mathrm{O}$ (4.0 equiv) in toluene $(4.0 \mathrm{~mL})$ at $80{ }^{\circ} \mathrm{C}$ for the indicated time. ${ }^{b}$ Determined by ${ }^{1} \mathrm{H}$ NMR analysis of the crude mixture using 1,3,5-trimethoxybenzene as an internal standard. 


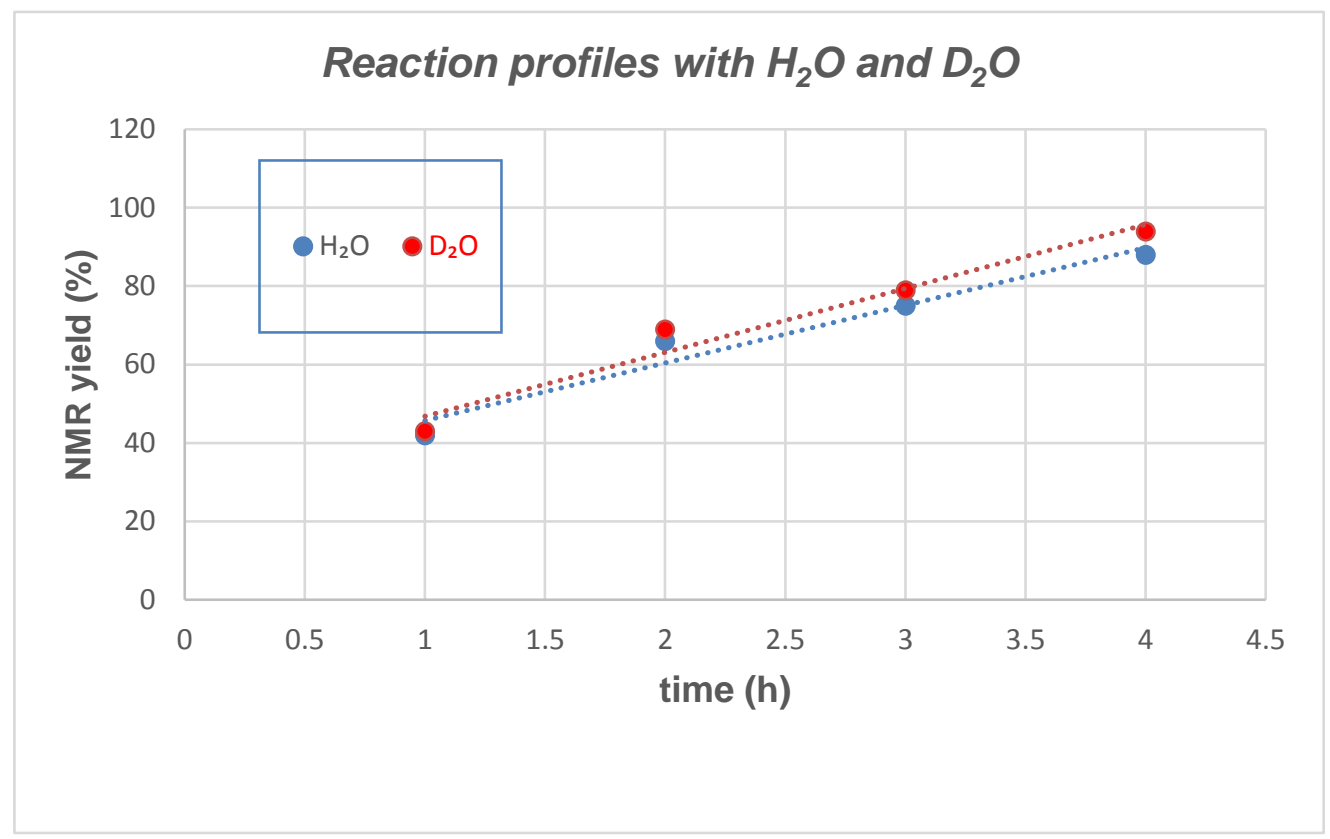

\section{Competition experiments}

\section{Competition experiments of arylboronic acids}

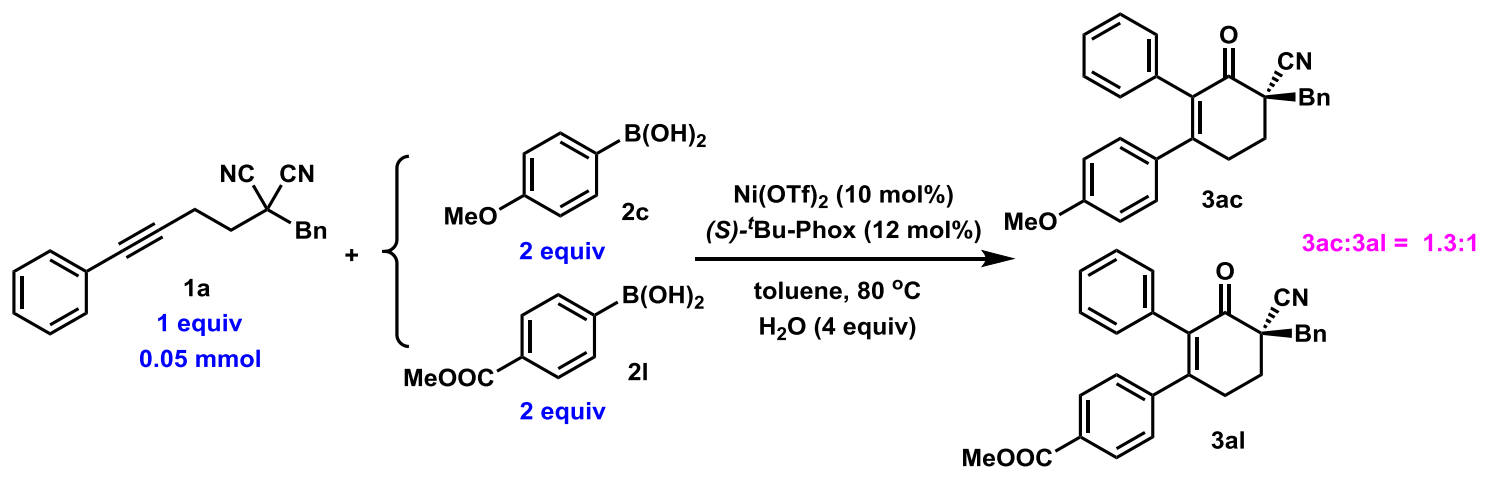

To a resealable Schlenk tube equipped with a magnetic stirring bar were added $\mathrm{Ni}(\mathrm{OTf})_{2}(0.005 \mathrm{mmol}$, $10 \mathrm{~mol} \%),(S)-{ }^{t} \mathrm{Bu}-$ phox $(0.006 \mathrm{mmol}, 12 \mathrm{~mol} \%)$, and $0.5 \mathrm{~mL}$ of toluene in a glovebox. After the resultant mixture was stirred at room temperature for $30 \mathrm{~min}$, substrate $1 \mathrm{a}(14.2 \mathrm{mg}, 0.05 \mathrm{mmol}, 1.0$ equiv), (4-methoxyphenyl)boronic acid $\quad \mathbf{2 c} \quad(15.2 \mathrm{mg}, \quad 0.1 \quad \mathrm{mmol}, \quad 2.0 \quad$ equiv), (4(methoxycarbonyl)phenyl)boronic acid 21 (18.0 mg, $0.1 \mathrm{mmol}, 2.0$ equiv), another $0.5 \mathrm{~mL}$ of toluene were added. The tube was sealed with a Teflon valve, removed from the glovebox, and then connected to a Schlenk line. Under a positive pressure of argon, the Teflon valve was removed and $\mathrm{H}_{2} \mathrm{O}(4 \mathrm{ul}, 0.2$ mmol, 4.0 equiv) was added rapidly. The tube was resealed and stirred at the indicated temperature for the $16 \mathrm{~h}$. Then the resultant mixture was cooled to room temperature, and $1.0 \mathrm{~mL}$ of $\mathrm{HCl}(1 \mathrm{M})$ and $1.0 \mathrm{~mL}$ of EA were added to the tube. After stirred for additional $30 \mathrm{~min}$, the mixture was extracted 
with EA ( $8 \mathrm{~mL} \times 3)$. The combined organic phase was washed with brine, dried over $\mathrm{Na}_{2} \mathrm{SO}_{4}$, and filtered. The solvents were removed under reduced pressure to give a crude mixture. The yield was determined by ${ }^{1} \mathrm{H}$ NMR analysis of the crude mixture using 1,3,5-trimethoxybenzene as an internal standard.

\section{Competition experiments of arylalkynes}

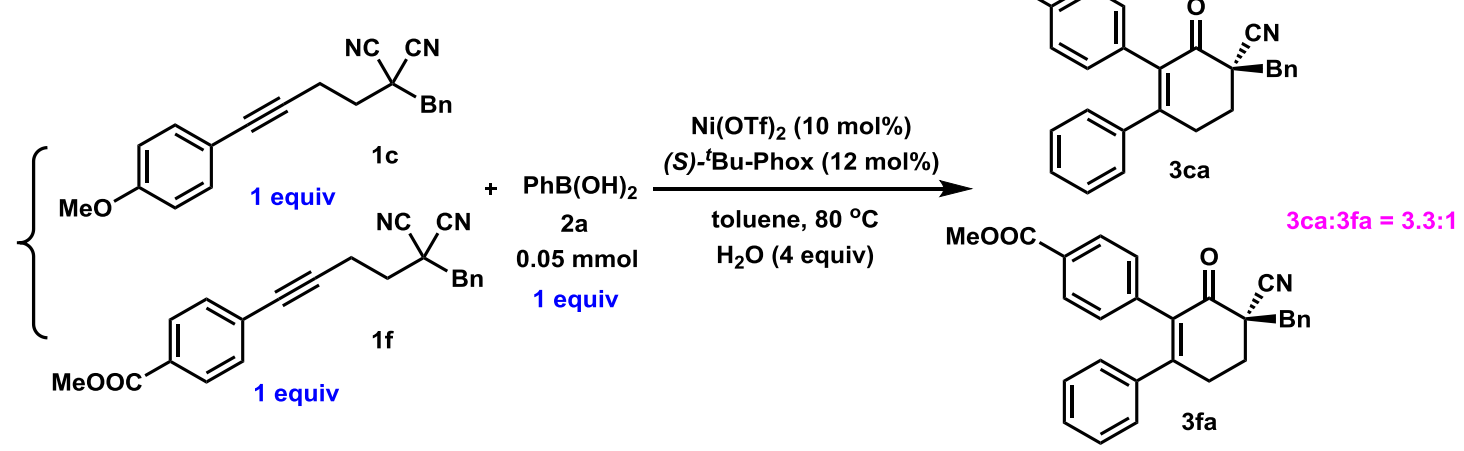

To a resealable Schlenk tube equipped with a magnetic stirring bar were added $\mathrm{Ni}(\mathrm{OTf})_{2}(0.005 \mathrm{mmol}$, $10 \mathrm{~mol} \%),(S)-{ }^{t} \mathrm{Bu}-$ phox $(0.006 \mathrm{mmol}, 12 \mathrm{~mol} \%)$, and $0.5 \mathrm{~mL}$ of toluene in a glovebox. After the resultant mixture was stirred at room temperature for $30 \mathrm{~min}$, substrate $1 \mathrm{c}(15.8 \mathrm{mg}, 0.05 \mathrm{mmol}, 1.0$ equiv), 1 f (17.2 mg, $0.05 \mathrm{mmol}, 1.0$ equiv), boronic acid $\mathbf{2 a}$ (6.1 $\mathrm{mg}, 0.05 \mathrm{mmol}, 1.0$ equiv), another $0.5 \mathrm{~mL}$ of toluene were added. The tube was sealed with a Teflon valve, removed from the glovebox, and then connected to a Schlenk line. Under a positive pressure of argon, the Teflon valve was removed and $\mathrm{H}_{2} \mathrm{O}$ (4 ul, $0.2 \mathrm{mmol}, 4.0$ equiv) was added rapidly. The tube was resealed and stirred at the indicated temperature for the indicated time. Then the resultant mixture was cooled to room temperature, and $1.0 \mathrm{~mL}$ of $\mathrm{HCl}(1 \mathrm{M})$ and $1.0 \mathrm{~mL}$ of EA were added to the tube. After stirred for additional $30 \mathrm{~min}$, the mixture was extracted with $\mathrm{EA}(8 \mathrm{~mL} \times 3)$. The combined organic phase was washed with brine, dried over $\mathrm{Na}_{2} \mathrm{SO}_{4}$, and filtered. The solvents were removed under reduced pressure to give a crude mixture. The yield was determined by ${ }^{1} \mathrm{H}$ NMR analysis of the crude mixture using 1,3,5-trimethoxybenzene as an internal standard.

\section{Procedures of the products derivatizations}

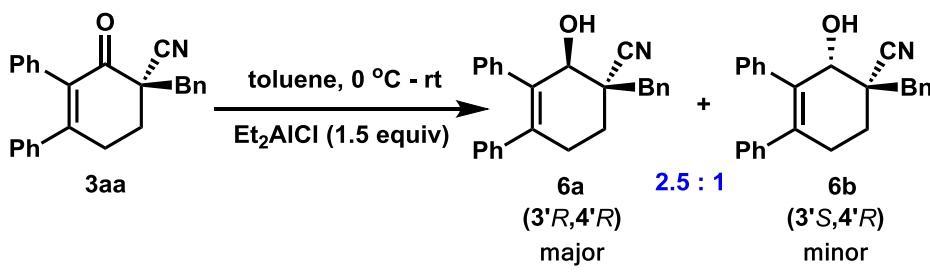


To a solution of compound 3aa $(12.1 \mathrm{mg}, 0.033 \mathrm{mmol})$ in toluene $(1.0 \mathrm{~mL})$ was added $\mathrm{Et}_{2} \mathrm{AlCl}(50 \mathrm{ul}$, $0.0495 \mathrm{mmol}, 1.5$ equiv, $1.0 \mathrm{M}$ solution in hexanes) at $0{ }^{\circ} \mathrm{C}$. The mixture was stirred at room temperature for $14 \mathrm{~h}$, after which the reaction was quenched with potassium sodium tartrate aqueous solution. The mixture was extracted with EA $(5 \mathrm{~mL}$ x 3). The combined organic phase was washed with brine, dried over $\mathrm{Na}_{2} \mathrm{SO}_{4}$, and filtered. After vacuum evaporation of the solvents, the residue was purified by flash chromatography to give product $\mathbf{6 a}\left(3^{\prime} R, 4^{\prime} R\right)$ in $66 \%$ yield $(8.0 \mathrm{mg})$ and $\mathbf{6 b}\left(3^{\prime} S, 4^{\prime} R\right)$ in $26 \%$ yield $(3.2 \mathrm{mg})$.

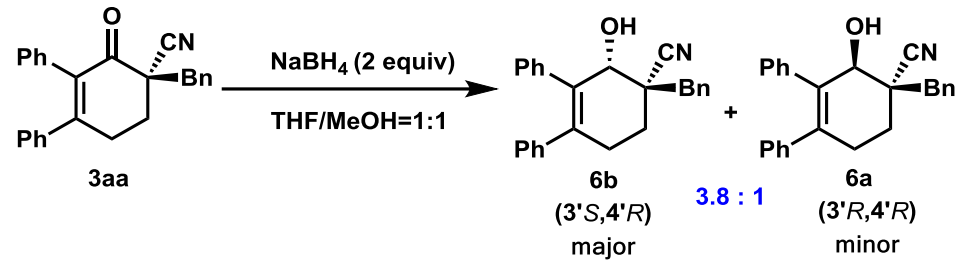

To a solution of compound 3aa $(13.5 \mathrm{mg}, 0.04 \mathrm{mmol})$ in THF $(1.0 \mathrm{~mL})$ and $\mathrm{MeOH}(1.0 \mathrm{~mL})$ was added $\mathrm{NaBH}_{4}(2.8 \mathrm{mg}, 0.07 \mathrm{mmol}, 2.0$ equiv) at room temperature. The mixture was stirred at room temperature for $6 \mathrm{~h}$. The mixture was diluted with EA $(1.0 \mathrm{~mL})$, and filtered through a celite pad, washed with EA ( $5 \mathrm{ml} \times 3)$, and concentrated under reduced pressure. The residue was purified by flash chromatography to give product $\mathbf{6 b}\left(3^{\prime} S, 4^{\prime} R\right)$ in $70 \%$ yield $(9.4 \mathrm{mg})$ and $\mathbf{6 a}\left(3^{\prime} R, 4^{\prime} R\right)$ in $19 \%$ yield (2.5 mg).

For $\left(3^{\prime} R, 4^{\prime} R\right)-4$ '- benzyl-3'- hydroxy-3',4',5',6'- tetrahydro- $\left[1,1^{\prime}: 2^{\prime}, 1^{\prime \prime}\right.$-terphenyl]-4'-carbonitrile (6a) : white soild. $[\alpha]_{\mathrm{D}}{ }^{25}=-212.53\left(c 1.0, \mathrm{CHCl}_{3}\right) . R_{f}=0.3(\mathrm{PE}: \mathrm{EA}=10: 1) .{ }^{1} \mathbf{H}$ NMR $(400 \mathrm{MHz}$, $\left.\mathrm{CDCl}_{3}\right) \delta 7.48-7.45(\mathrm{~m}, 2 \mathrm{H}), 7.39-7.29(\mathrm{~m}, 3 \mathrm{H}), 7.15-7.08(\mathrm{~m}, 6 \mathrm{H}), 7.05-7.00(\mathrm{~m}, 4 \mathrm{H}), 4.36(\mathrm{~s}$, 1H), $3.20(\mathrm{AB}, J=13.5 \mathrm{~Hz}, 1 \mathrm{H}), 3.04(\mathrm{BA}, J=13.6 \mathrm{~Hz}, 1 \mathrm{H}), 2.76-2.72$ (m, 2H), 2.19 (ddd, $J=13.4$, 10.5, $7.3 \mathrm{~Hz}, 1 \mathrm{H}), 2.02-1.97(\mathrm{~m}, 1 \mathrm{H}), 1.81($ br s, $1 \mathrm{H}) .{ }^{13} \mathbf{C} \mathbf{N M R}\left(100 \mathrm{MHz}, \mathrm{CDCl}_{3}\right) \delta 141.2,140.3$, $139.9,135.3,134.8,130.6,129.7,128.8,128.7,128.2,128.0,127.5,127.0,121.4,71.0,43.5,40.8$, 30.3, 25.6. HRMS $\left(\mathrm{ESI}^{+}\right) \mathrm{m} / z$ calc'd for $\mathrm{C}_{26} \mathrm{H}_{23} \mathrm{NONa}[\mathrm{M}+\mathrm{Na}]^{+}: 388.1672$, found 388.1672.

For (3'S,4'R)-4'- benzyl-3'- hydroxy-3',4',5',6'- tetrahydro-[1,1':2',1'- terphenyl]-4'-carbonitrile (6b) : white soild. $[\alpha]_{\mathrm{D}}{ }^{25}=53.56\left(c 0.6, \mathrm{CHCl}_{3}\right) . R_{f}=0.2(\mathrm{PE}: \mathrm{EA}=10: 1) .{ }^{1} \mathbf{H} \mathbf{~ N M R}(400 \mathrm{MHz}$, $\left.\mathrm{CDCl}_{3}\right) \delta 7.41-7.32(\mathrm{~m}, 5 \mathrm{H}), 7.19-7.09(\mathrm{~m}, 6 \mathrm{H}), 7.06-7.02(\mathrm{~m}, 2 \mathrm{H}), 7.01-6.97(\mathrm{~m}, 2 \mathrm{H}), 4.51(\mathrm{~s}$, 1H), $3.32(\mathrm{AB}, J=13.6 \mathrm{~Hz}, 1 \mathrm{H}), 3.07(\mathrm{BA}, J=13.6 \mathrm{~Hz}, 1 \mathrm{H}), 2.94-2.85$ (m, 1H), 2.45 (dt, $J=19.0$, $5.8 \mathrm{~Hz}, 1 \mathrm{H}), 2.28(\mathrm{dt}, J=13.6,5.7 \mathrm{~Hz}, 1 \mathrm{H}), 1.93-1.86(\mathrm{~m}, 2 \mathrm{H}) .{ }^{13} \mathbf{C} \mathbf{N M R}\left(100 \mathrm{MHz}, \mathrm{CDCl}_{3}\right) \delta 141.5$, 
$138.1,137.9,134.9,134.4,130.7,130.0,128.7,128.5,128.4,128.0,127.7,127.2,126.9,121.7,72.2$, 44.9, 40.8, 29.1, 26.9. HRMS $\left(\mathrm{ESI}^{+}\right) \mathrm{m} / z$ calc'd for $\mathrm{C}_{26} \mathrm{H}_{23} \mathrm{NONa}[\mathrm{M}+\mathrm{Na}]^{+}: 388.1672$, found 388.1673 .

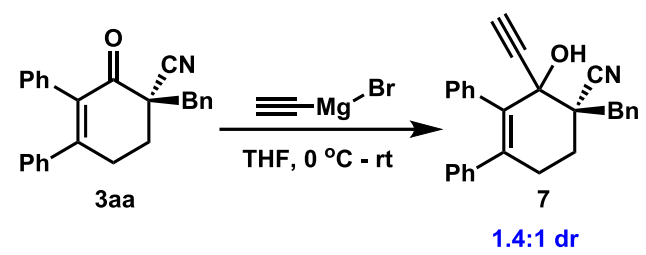

To a solution of compound 3aa $(25.6 \mathrm{mg}, 0.07 \mathrm{mmol})$ in THF $(1.5 \mathrm{~mL})$ was added ethynylmagnesium bromide (356 ul, $0.178 \mathrm{mmol}, 2.6$ equiv, $0.5 \mathrm{M}$ solution in THF) at $0{ }^{\circ} \mathrm{C}$. The mixture was stirred at room temperature for $8 \mathrm{~h}$, after which the reaction was quenched with saturated aqueous $\mathrm{NH}_{4} \mathrm{Cl}$ solution. The mixture was extracted with EA ( $5 \mathrm{ml}$ x 3$)$. The combined organic phase was washed with brine, dried over $\mathrm{Na}_{2} \mathrm{SO}_{4}$, and filtered. After vacuum evaporation of the solvent, the residue was purified by flash chromatography to give product 7 (major: $13.2 \mathrm{mg}, 51 \%$ yield; minor: $9.5 \mathrm{mg}, 37 \%$ yield ).

For the major isomer of 7: white solid. $[\alpha]_{\mathrm{D}}{ }^{25}=17.57\left(c 1.1, \mathrm{CHCl}_{3}\right) . R_{f}=0.3(\mathrm{PE}: \mathrm{EA}=10: 1)$.

${ }^{1} \mathbf{H}$ NMR $\left(400 \mathrm{MHz}, \mathrm{CDCl}_{3}\right) \delta 7.45$ - $7.42(\mathrm{~m}, 2 \mathrm{H}), 7.41$ - $7.37(\mathrm{~m}, 2 \mathrm{H}), 7.35$ - $7.32(\mathrm{~m}, 1 \mathrm{H}), 7.31$ $7.27(\mathrm{~m}, 2 \mathrm{H}), 7.23-7.14(\mathrm{~m}, 3 \mathrm{H}), 7.11-7.04(\mathrm{~m}, 3 \mathrm{H}), 7.03-6.99$ (m, 2H), $3.62(\mathrm{AB}, J=13.6 \mathrm{~Hz}$, 1H), $3.16(\mathrm{BA}, J=13.6 \mathrm{~Hz}, 1 \mathrm{H}), 2.95$ (s, 1H), 2.80 (ddd, $J=19.0,11.0,6.7 \mathrm{~Hz}, 1 \mathrm{H}), 2.53-2.46$ (m, 2H), $2.25(\mathrm{ddd}, J=14.0,11.0,6.3 \mathrm{~Hz}, 1 \mathrm{H}), 2.09(\mathrm{ddd}, J=14.0,6.7,1.7 \mathrm{~Hz}, 1 \mathrm{H}) .{ }^{13} \mathbf{C}$ NMR $(100 \mathrm{MHz}$, $\left.\mathrm{CDCl}_{3}\right) \delta 141.2,138.6,136.4,135.6,135.3,131.4,131.0,128.6,128.3,128.2,127.9,127.8,127.5$, 126.8, 120.0, 83.1, 78.2, 72.6, 48.8, 39.6, 29.6, 27.0. HRMS (ESI $\left.{ }^{+}\right) \mathrm{m} / z$ calc'd for $\mathrm{C}_{28} \mathrm{H}_{23} \mathrm{NONa}$ $[\mathrm{M}+\mathrm{Na}]^{+}:$412.1672, found 412.1675 .

For the minor isomer of 7: white solid. $[\alpha]_{\mathrm{D}}{ }^{25}=-22.18\left(c 0.7, \mathrm{CHCl}_{3}\right), R_{f}=0.2(\mathrm{PE}: \mathrm{EA}=10: 1)$. ${ }^{1} \mathbf{H}$ NMR $\left(400 \mathrm{MHz}, \mathrm{CDCl}_{3}\right) \delta 7.46-7.44(\mathrm{~m}, 2 \mathrm{H}), 7.42-7.37(\mathrm{~m}, 2 \mathrm{H}), 7.36-7.31(\mathrm{~m}, 1 \mathrm{H}), 7.22-$ $7.19(\mathrm{~m}, 2 \mathrm{H}), 7.18-7.14(\mathrm{~m}, 3 \mathrm{H}), 7.12-7.03(\mathrm{~m}, 3 \mathrm{H}), 7.01-6.98(\mathrm{~m}, 2 \mathrm{H}), 3.56(\mathrm{AB}, J=13.6 \mathrm{~Hz}$, 1H), $3.09(\mathrm{BA}, J=13.7 \mathrm{~Hz}, 1 \mathrm{H}), 2.74(\mathrm{~s}, 1 \mathrm{H}), 2.72-2.53(\mathrm{~m}, 3 \mathrm{H}), 2.21-2.06(\mathrm{~m}, 2 \mathrm{H}) .{ }^{13} \mathbf{C} \mathbf{N M R}$ $\left(100 \mathrm{MHz}, \mathrm{CDCl}_{3}\right) \delta 141.3,139.2,137.6,135.5,135.1,131.3,130.9,128.7,128.3,127.9,127.7,127.6$, $127.3,126.9,121.1,83.1,78.1,72.3,48.4,37.5,29.1,24.9 .[\alpha]_{\mathrm{D}}{ }^{25}=-22.17\left(c 0.7, \mathrm{CHCl}_{3}\right) . \mathbf{H R M S}$ $\left(\mathrm{ESI}^{+}\right) \mathrm{m} / z$ calc'd for $\mathrm{C}_{28} \mathrm{H}_{23} \mathrm{NONa}[\mathrm{M}+\mathrm{Na}]^{+}:$412.1672, found 412.1674 . 


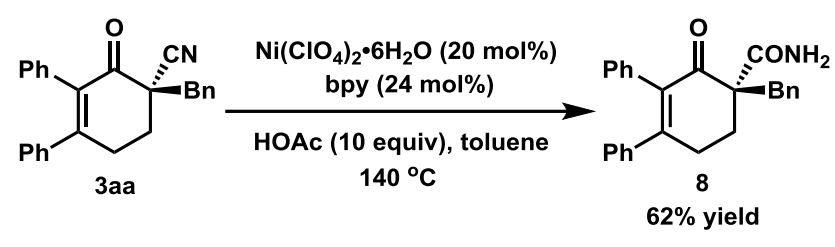

\section{(S)-4'-Benzyl-3'-oxo-3',4',5',6'-tetrahydro-[1,1':2',1'-terphenyl]-4'-carboxamide (8)}

To a resealable Schlenk tube were added $\mathrm{Ni}\left(\mathrm{ClO}_{4}\right)_{2} \bullet 6 \mathrm{H}_{2} \mathrm{O}(6.2 \mathrm{mg}, 0.017 \mathrm{mmol}, 20 \mathrm{~mol} \%)$, bpy (3.2 $\mathrm{mg}, 0.02 \mathrm{mmol}, 24 \mathrm{~mol} \%$ ), and $2.0 \mathrm{~mL}$ of toluene under argon. The resultant solution was stirred at room temperature for $30 \mathrm{~min}$, then $3 \mathbf{a a}(30.1 \mathrm{mg}, 0.083 \mathrm{mmol})$ and $\mathrm{AcOH}(47 \mathrm{ul}, 0.83 \mathrm{mmol}, 10$ equiv) were added. The mixture was stirred at $140^{\circ} \mathrm{C}$ for $24 \mathrm{~h}$, then cooled to room temperature. After vacuum evaporation of the solvents, the residue was purified by silica gel column chromatography to provide the desired product $8(19.6 \mathrm{mg}, 62 \%$ yield $)$ as a colorless oil. $[\alpha]_{\mathrm{D}}{ }^{25}=-35.56\left(c 1.3, \mathrm{CHCl}_{3}\right) . R_{f}=0.3$ $(\mathrm{PE}: \mathrm{EA}=2: 1) .{ }^{1} \mathbf{H}$ NMR $\left(400 \mathrm{MHz}, \mathrm{CDCl}_{3}\right) \delta 7.33-7.26(\mathrm{~m}, 3 \mathrm{H}), 7.23-7.16(\mathrm{~m}, 5 \mathrm{H}), 7.14-7.09$ (m, 3H), $6.96-6.93(\mathrm{~m}, 2 \mathrm{H}), 6.89-6.86(\mathrm{~m}, 2 \mathrm{H}), 6.75$ (br s, 1H), 5.61 (br s, 1H), 3.47 (AB, J= 13.5 $\mathrm{Hz}, 1 \mathrm{H}), 3.20(\mathrm{BA}, J=13.5 \mathrm{~Hz}, 1 \mathrm{H}), 3.11(\mathrm{ddd}, J=19.1,10.1,4.6 \mathrm{~Hz}, 1 \mathrm{H}), 2.68(\mathrm{dt}, J=19.0,4.6 \mathrm{~Hz}$, 1H), $2.58(\mathrm{dt}, J=13.6,4.5 \mathrm{~Hz}, 1 \mathrm{H}), 2.12(\mathrm{ddd}, J=14.2,10.0,4.8 \mathrm{~Hz}, 1 \mathrm{H}) .{ }^{13} \mathbf{C} \mathbf{N M R}(100 \mathrm{MHz}$, $\left.\mathrm{CDCl}_{3}\right) \delta 199.4,171.8,160.1,140.2,137.2,136.4,135.7,130.9,130.5,128.4,128.3,128.1,128.0$, 127.9, 127.2, 57.5, 43.6, 29.8, 28.3. HRMS $\left(\mathrm{ESI}^{+}\right) \mathrm{m} / z$ calc'd for $\mathrm{C}_{26} \mathrm{H}_{23} \mathrm{NO}_{2} \mathrm{Na}[\mathrm{M}+\mathrm{Na}]^{+}: 404.1621$, found 404.1625 .

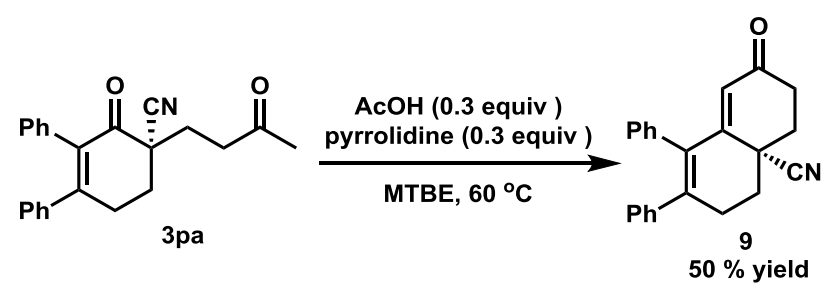

\section{(S)-2-Oxo-7,8- diphenyl -2,3,4,4a,5,6-hexahydro naphthalene-4a-carbonitrile (9)}

To a solution of compound 3pa $(18.6 \mathrm{mg}, 0.05 \mathrm{mmol})$ in MTBE $(1.0 \mathrm{~mL})$ were added pyrrolidine $(1.2$ $\mathrm{mg}, 0.02 \mathrm{mmol}, 0.3$ equiv) and $\mathrm{AcOH}(1.0 \mathrm{mg}, 0.02 \mathrm{mmol}, 0.3$ equiv) at room temperature. The mixture was stirred at $60{ }^{\circ} \mathrm{C}$ for $8 \mathrm{~h}$. After vacuum evaporation of the solvent, the residue was purified by flash chromatography to give product 9 in $50 \%$ yield $(8.7 \mathrm{mg})$ as a white solid. $[\alpha]_{\mathrm{D}}{ }^{25}=-357.15(c$ $\left.0.7, \mathrm{CHCl}_{3}\right) . R_{f}=0.3(\mathrm{PE}: \mathrm{EA}=5: 1) .{ }^{1} \mathbf{H} \mathbf{N M R}\left(400 \mathrm{MHz}, \mathrm{CDCl}_{3}\right) \delta 7.18-7.08(\mathrm{~m}, 6 \mathrm{H}), 7.01-$ $6.94(\mathrm{~m}, 4 \mathrm{H}), 5.71(\mathrm{~s}, 1 \mathrm{H}), 3.15(\mathrm{ddd}, J=18.1,12.3,4.9 \mathrm{~Hz}, 1 \mathrm{H}), 2.94-2.85(\mathrm{~m}, 2 \mathrm{H}), 2.65-2.60(\mathrm{~m}$, 1H), $2.56-2.51(\mathrm{~m}, 1 \mathrm{H}), 2.43(\mathrm{dd}, J=13.4,4.8 \mathrm{~Hz}, 1 \mathrm{H}), 2.19-2.03(\mathrm{~m}, 2 \mathrm{H}) .{ }^{13} \mathrm{C}$ NMR $(100 \mathrm{MHz}$, 
$\left.\mathrm{CDCl}_{3}\right) \delta 197.7,152.3,148.2,141.1,137.0,134.6,130.8,128.4,128.3,128.0,127.6,127.4,126.8$, 119.8, 36.5, 34.8, 34.4, 33.5, 30.5. HRMS $\left(\mathrm{ESI}^{+}\right) \mathrm{m} / z$ calc'd for $\mathrm{C}_{23} \mathrm{H}_{19} \mathrm{NONa}[\mathrm{M}+\mathrm{Na}]^{+}: 348.1359$, found 348.1363 .

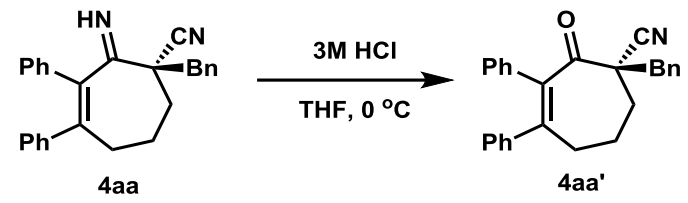

(R)-1-Benzyl-2-oxo-3,4-diphenylcyclohept-3-ene-1-carbonitrile (4aa')

To a solution of 4aa $(20.7 \mathrm{mg}, 0.55 \mathrm{mmol})$ in $1 \mathrm{~mL}$ THF was added $1 \mathrm{~mL}$ aqueous solution of $\mathrm{HCl}$ $(3 \mathrm{M})$ at $0{ }^{\circ} \mathrm{C}$. The reaction was stirred at $0{ }^{\circ} \mathrm{C}$ until $4 \mathbf{a a}$ was converted completely as monitored with TLC. Saturated sodium bicarbonate was added to quench the reaction, and extracted with EA. The combine organic phase was dried over $\mathrm{Na}_{2} \mathrm{SO}_{4}$, concentrated. Then the residue purified by column chromatography $(\mathrm{PE}: \mathrm{EA}=10: 1)$ to give product 4aa' as a white solid in $78 \%$ yield $(16.1 \mathrm{mg})$. er $=$ $99.5: 0.5 .[\alpha]_{\mathrm{D}}{ }^{25}=-221.77\left(c 1.0, \mathrm{CHCl}_{3}\right) . R_{f}=0.4(\mathrm{PE}: \mathrm{EA}=10: 1) .{ }^{1} \mathbf{H} \mathbf{~ N M R}\left(400 \mathrm{MHz}, \mathrm{CDCl}_{3}\right)$ $\delta 7.30-7.27(\mathrm{~m}, 5 \mathrm{H}), 7.19-7.12(\mathrm{~m}, 3 \mathrm{H}), 7.11-7.01(\mathrm{~m}, 5 \mathrm{H}), 6.88-6.80(\mathrm{~m}, 2 \mathrm{H}), 3.37(\mathrm{AB}, J=$ $13.6 \mathrm{~Hz}, 1 \mathrm{H}), 3.02(\mathrm{BA}, J=13.6 \mathrm{~Hz}, 1 \mathrm{H}), 2.97-2.75(\mathrm{~m}, 2 \mathrm{H}), 2.30-2.12(\mathrm{~m}, 3 \mathrm{H}), 2.12-2.00(\mathrm{~m}$, 1H). ${ }^{13} \mathbf{C ~ N M R ~}\left(100 \mathrm{MHz}, \mathrm{CDCl}_{3}\right) \delta 201.2,145.7,141.2,138.6,136.5,134.6,130.8,129.8,128.6$, 128.5, 128.3, 128.2, 127.7, 127.7, 127.4, 120.4, 55.5, 41.9, 36.6, 35.7, 23.4. HRMS (ESI $\left.{ }^{+}\right) m / z$ calc'd for $\mathrm{C}_{27} \mathrm{H}_{23} \mathrm{NNaO}^{+}[\mathrm{M}+\mathrm{Na}]^{+}:$400.1672, found 400.1664; HPLC conditions: hexane/2-propanol 95:5, $1.0 \mathrm{~mL} / \mathrm{min}, \lambda=230 \mathrm{~nm}$, Chiralpak AD-H column $(4.6 \mathrm{~mm} \times 250 \mathrm{~mm}) ; \mathrm{t}_{\mathrm{r}}($ minor $)=8.4 \mathrm{~min}, \mathrm{t}_{\mathrm{r}}$ (major) $=6.9 \mathrm{~min}$.

\section{X-ray crystallography data of $3 \mathrm{ka}$ and $6 \mathrm{~b}$}

General methods: all the crystallographic data were collected on a Bruker KAPPA APEX DUO diffractometer equipped with a CCD area detector. The X-ray source used was amolybdenum fine focus sealed tube $(50 \mathrm{kV} 30 \mathrm{~mA})$ combined with a TRIUMPH monochromator $(\lambda=0.71073 \AA)$. All ellipsoids in ORTEP diagrams are displayed at the 50\% probability level unless noted otherwise.

\section{X-ray crystallographic data of $3 \mathrm{ka}$}



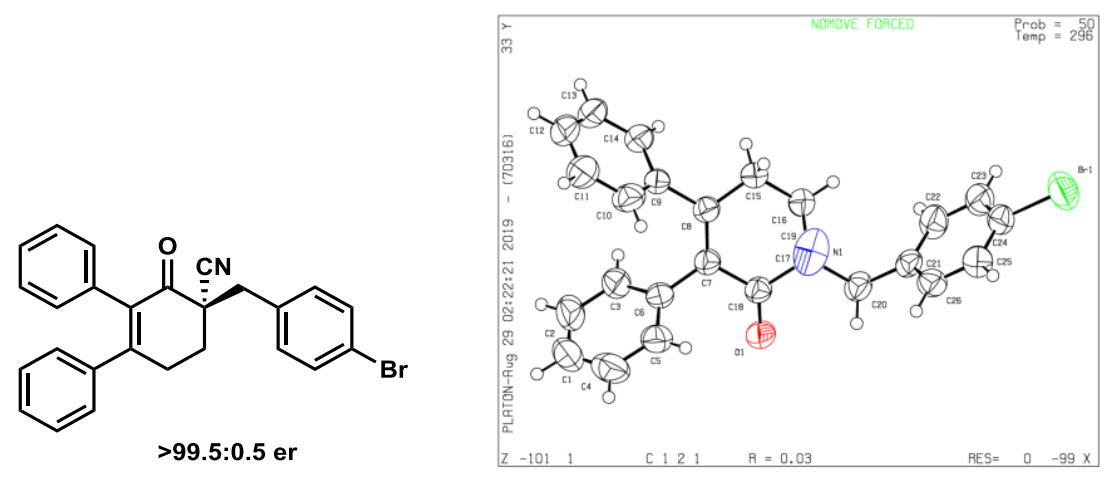

Table S9. Crystal data and structure refinement for $3 \mathrm{ka}$

\begin{tabular}{|c|c|}
\hline [Identification code & CCDC 1971345 \\
\hline Empirical formula & $\mathrm{C}_{26} \mathrm{H}_{20} \mathrm{BrNO}$ \\
\hline Formula weight & 442.34 \\
\hline Temperature/K & 296.15 \\
\hline Crystal system & monoclinic \\
\hline Space group & $\mathrm{C} 2$ \\
\hline $\mathrm{a} / \AA$ & $23.866(2)$ \\
\hline $\mathrm{b} / \AA$ & $6.6629(6)$ \\
\hline $\mathrm{c} / \AA$ & $13.5743(11)$ \\
\hline$\alpha /^{\circ}$ & 90 \\
\hline$\beta /{ }^{\circ}$ & $94.136(3)$ \\
\hline$\gamma /{ }^{\circ}$ & 90 \\
\hline Volume $/ \AA^{3}$ & $2152.9(3)$ \\
\hline $\mathrm{Z}$ & 4 \\
\hline$\rho_{\text {calc }} \mathrm{g} / \mathrm{cm}^{3}$ & 1.365 \\
\hline$\mu / \mathrm{mm}^{-1}$ & 2.718 \\
\hline $\mathrm{F}(000)$ & 904.0 \\
\hline Crystal size $/ \mathrm{mm}^{3}$ & $0.12 \times 0.08 \times 0.08 \mathrm{~mm}^{3}$ \\
\hline Radiation & $\mathrm{CuK} \alpha(\lambda=1.54178)$ \\
\hline \multicolumn{2}{|c|}{$2 \Theta$ range for data collection $/{ }^{\circ} 6.528$ to 130.67} \\
\hline Index ranges & $-28 \leq \mathrm{h} \leq 28,-7 \leq \mathrm{k} \leq 7,-16 \leq 1 \leq 15$ \\
\hline Reflections collected & 12461 \\
\hline Independent reflections & $3628\left[\mathrm{R}_{\text {int }}=0.0393, \mathrm{R}_{\text {sigma }}=0.0432\right]$ \\
\hline Data/restraints/parameters & $3628 / 1 / 262$ \\
\hline Goodness-of-fit on $\mathrm{F}^{2}$ & 1.045 \\
\hline Final $R$ indexes $[\mathrm{I}>=2 \sigma(\mathrm{I})]$ & $\mathrm{R}_{1}=0.0324, \mathrm{wR}_{2}=0.0863$ \\
\hline Final $\mathrm{R}$ indexes [all data] & $\mathrm{R}_{1}=0.0331, \mathrm{wR}_{2}=0.0874$ \\
\hline \multicolumn{2}{|c|}{ Largest diff. peak/hole / e $\AA^{-3} 0.28 /-0.30$} \\
\hline Flack parameter & $0.059(8)$ \\
\hline
\end{tabular}


Table S10. Fractional atomic coordinates $\left(\times 10^{4}\right)$ and equivalent isotropic displacement parameters $\left(\AA^{2} \times 10^{3}\right)$ for $\mathrm{CCDC}$ 1971345. $U_{\text {eq }}$ is defined as $1 / 3$ of of the trace of the orthogonalised $U_{I J}$ tensor.

\begin{tabular}{|c|c|c|c|c|}
\hline Atom & $x$ & $y$ & $z$ & $\mathbf{U}(\mathbf{e q})$ \\
\hline $\operatorname{Br}(1)$ & $4145.9(2)$ & $8942.8(11)$ & $10382.6(3)$ & $92.6(2)$ \\
\hline $\mathrm{O}(1)$ & 2624.0(9) & 5591(5) & $4471.2(19)$ & 70.6(7) \\
\hline $\mathrm{C}(17)$ & $3417.5(12)$ & $5525(5)$ & $5660(2)$ & $46.9(6)$ \\
\hline $\mathrm{C}(9)$ & $4382.5(12)$ & $3245(5)$ & $3302(2)$ & $51.4(7)$ \\
\hline$C(6)$ & $3224.0(12)$ & $4669(5)$ & 2812(3) & $52.4(7)$ \\
\hline $\mathrm{C}(8)$ & $3991.4(11)$ & $3768(5)$ & 4074(2) & $48.4(6)$ \\
\hline$C(7)$ & $3469.8(11)$ & 4478(5) & $3842(2)$ & $48.0(6)$ \\
\hline $\mathrm{C}(21)$ & $3259.2(13)$ & $6560(5)$ & $7423(2)$ & $50.5(7)$ \\
\hline$C(26)$ & $3314.9(14)$ & $8589(5)$ & $7621(2)$ & $57.2(7)$ \\
\hline $\mathrm{C}(14)$ & $4625.4(15)$ & 1361(6) & $3276(3)$ & $62.4(8)$ \\
\hline $\mathrm{C}(24)$ & $3784.2(14)$ & 7918(6) & 9191(2) & $60.2(8)$ \\
\hline $\mathrm{C}(15)$ & 4231.1(13) & $3505(6)$ & $5113(2)$ & $56.9(8)$ \\
\hline $\mathrm{C}(18)$ & $3114.9(12)$ & $5220(5)$ & $4625(2)$ & $48.0(6)$ \\
\hline $\mathrm{C}(25)$ & $3572.6(14)$ & $9279(6)$ & 8497(3) & $61.0(8)$ \\
\hline $\mathrm{C}(19)$ & $3767.9(15)$ & $7319(6)$ & $5573(3)$ & $58.6(8)$ \\
\hline $\mathrm{C}(16)$ & $3796.9(13)$ & $3696(6)$ & $5875(2)$ & $56.0(7)$ \\
\hline $\mathrm{N}(1)$ & $4048.0(18)$ & $8671(7)$ & $5482(3)$ & $95.7(12)$ \\
\hline$C(5)$ & $3030.1(15)$ & $6523(7)$ & 2447(3) & $67.2(9)$ \\
\hline $\mathrm{C}(22)$ & $3463.7(18)$ & $5232(6)$ & $8145(3)$ & $67.2(9)$ \\
\hline $\mathrm{C}(3)$ & $3203.8(15)$ & $3026(7)$ & $2177(3)$ & $67.8(10)$ \\
\hline $\mathrm{C}(23)$ & $3729.8(18)$ & $5905(7)$ & $9028(3)$ & $72.0(10)$ \\
\hline $\mathrm{C}(13)$ & $4983.0(16)$ & $890(8)$ & $2542(3)$ & $76.2(11)$ \\
\hline $\mathrm{C}(20)$ & 2991.1(13) & $5851(6)$ & $6447(2)$ & $56.2(7)$ \\
\hline $\mathrm{C}(12)$ & $5102.3(17)$ & 2314(9) & $1852(3)$ & $82.0(13)$ \\
\hline $\mathrm{C}(10)$ & $4511.2(14)$ & $4661(6)$ & $2606(3)$ & $67.8(10)$ \\
\hline $\mathrm{C}(2)$ & $3008.6(19)$ & $3260(10)$ & 1194(4) & $93.2(18)$ \\
\hline $\mathrm{C}(11)$ & $4872.3(16)$ & $4177(10)$ & 1884(3) & $83.8(12)$ \\
\hline $\mathrm{C}(4)$ & $2829.9(19)$ & $6707(10)$ & $1466(4)$ & $95.1(17)$ \\
\hline $\mathrm{C}(1)$ & $2828(2)$ & $5092(12)$ & $849(3)$ & $101.9(19)$ \\
\hline
\end{tabular}

Table S11. Anisotropic displacement parameters $\left(\AA^{2} \times 10^{3}\right)$ for CCDC 1971345.

The anisotropic displacement factor exponent takes the form: $-2 \pi^{2}\left[h^{2} a^{* 2} U_{11}+2 h k a * b * U_{12}+\ldots\right]$.

\begin{tabular}{ccccccc}
\hline Atom & $\mathbf{U}_{\mathbf{1 1}}$ & $\mathbf{U}_{\mathbf{2 2}}$ & $\mathbf{U}_{\mathbf{3 3}}$ & $\mathbf{U}_{\mathbf{2 3}}$ & $\mathbf{U}_{\mathbf{1 3}}$ & $\mathbf{U}_{\mathbf{1 2}}$ \\
\hline $\mathrm{Br}(1)$ & $85.6(3)$ & $132.9(5)$ & $58.0(2)$ & $-5.7(3)$ & $-4.63(17)$ & $-6.9(3)$ \\
$\mathrm{O}(1)$ & $43.0(12)$ & $105(2)$ & $63.6(14)$ & $-14.4(14)$ & $1.5(10)$ & $12.0(12)$ \\
$\mathrm{C}(17)$ & $41.8(13)$ & $52.0(16)$ & $47.1(15)$ & $-6.3(12)$ & $4.8(11)$ & $-1.7(11)$
\end{tabular}




\begin{tabular}{|c|c|c|c|c|c|c|}
\hline $\mathrm{C}(9)$ & $39.4(13)$ & $68.2(19)$ & $46.1(15)$ & $-7.7(12)$ & $-0.2(11)$ & $6.5(12)$ \\
\hline $\mathrm{C}(6)$ & $35.9(13)$ & $69(2)$ & $52.2(17)$ & $-2.0(13)$ & $4.3(11)$ & 1.1(12) \\
\hline $\mathrm{C}(8)$ & $44.6(13)$ & $50.9(15)$ & 49.6(14) & $-3.5(14)$ & $2.9(10)$ & $2.4(13)$ \\
\hline $\mathrm{C}(7)$ & $41.8(14)$ & $53.6(16)$ & $48.5(15)$ & $-4.6(12)$ & $2.9(11)$ & $0.6(11)$ \\
\hline$C(21)$ & $48.1(15)$ & $54.8(17)$ & $50.4(16)$ & $2.4(13)$ & $16.4(12)$ & $2.5(12)$ \\
\hline$C(26)$ & $60.7(17)$ & $54.7(19)$ & $56.4(17)$ & $8.2(15)$ & $4.8(13)$ & $6.8(14)$ \\
\hline$C(14)$ & $59.3(18)$ & $66(2)$ & $60.8(19)$ & $-11.0(16)$ & $-1.1(15)$ & $12.7(15)$ \\
\hline $\mathrm{C}(24)$ & $53.6(17)$ & $82(2)$ & $46.6(17)$ & 4.1(15) & 11.1(14) & $1.7(15)$ \\
\hline$C(15)$ & $48.7(15)$ & $72(2)$ & $50.1(15)$ & $-1.1(15)$ & $1.7(12)$ & $13.3(14)$ \\
\hline $\mathrm{C}(18)$ & $40.6(15)$ & $52.2(15)$ & $51.2(16)$ & $-4.8(13)$ & $3.1(11)$ & $-0.9(12)$ \\
\hline$C(25)$ & $69.1(19)$ & $53.6(18)$ & $60.7(18)$ & $1.8(15)$ & $6.8(15)$ & $-2.5(15)$ \\
\hline$C(19)$ & $58.9(18)$ & $64(2)$ & $54.6(18)$ & $-10.5(15)$ & $13.5(14)$ & $-8.2(16)$ \\
\hline$C(16)$ & $59.4(16)$ & $62.4(18)$ & $46.6(14)$ & $1.8(15)$ & $6.3(12)$ & $10.6(15)$ \\
\hline $\mathrm{N}(1)$ & $109(3)$ & $80(3)$ & $102(3)$ & $-20(2)$ & $38(2)$ & $-39(2)$ \\
\hline $\mathrm{C}(5)$ & $54.5(18)$ & $83(2)$ & $65(2)$ & $7.5(18)$ & $5.1(16)$ & $10.8(17)$ \\
\hline$C(22)$ & $96(3)$ & $52.3(17)$ & $55(2)$ & $7.9(15)$ & $18.8(18)$ & 7.7(17) \\
\hline $\mathrm{C}(3)$ & $52.7(18)$ & $90(3)$ & $60(2)$ & $-16.5(17)$ & $-3.5(15)$ & $1.0(16)$ \\
\hline $\mathrm{C}(23)$ & $93(3)$ & $73(2)$ & $51.3(19)$ & $15.2(17)$ & 16.1(18) & $21(2)$ \\
\hline $\mathrm{C}(13)$ & $64(2)$ & $92(3)$ & $72(2)$ & $-26(2)$ & $0.2(18)$ & $21.5(19)$ \\
\hline $\mathrm{C}(20)$ & $49.4(16)$ & $64.7(19)$ & $55.8(17)$ & $-4.9(15)$ & $12.9(13)$ & $-0.5(14)$ \\
\hline $\mathrm{C}(12)$ & $54(2)$ & $128(4)$ & $64(2)$ & $-15(3)$ & $10.0(17)$ & $16(2)$ \\
\hline $\mathrm{C}(10)$ & $51.8(18)$ & $78(2)$ & $75(2)$ & $7.2(17)$ & $13.2(16)$ & $8.7(15)$ \\
\hline $\mathrm{C}(2)$ & $64(2)$ & 153(6) & $61(2)$ & $-33(3)$ & $-6.9(19)$ & $2(3)$ \\
\hline $\mathrm{C}(11)$ & $61(2)$ & 123(4) & $70(2)$ & $9(3)$ & $20.4(16)$ & $9(3)$ \\
\hline $\mathrm{C}(4)$ & $68(2)$ & $139(5)$ & $79(3)$ & $44(3)$ & $8(2)$ & $21(3)$ \\
\hline $\mathrm{C}(1)$ & $69(3)$ & $184(6)$ & $52(2)$ & $6(3)$ & $-2.6(19)$ & $19(3)$ \\
\hline
\end{tabular}

Table S12. Bond lengths for CCDC 1971345.

\begin{tabular}{lcll}
\hline Atom Atom & Length/A & Atom Atom & Length/A \\
\hline $\mathrm{Br}(1) \mathrm{C}(24)$ & $1.904(4)$ & $\mathrm{C}(21) \mathrm{C}(22)$ & $1.384(5)$ \\
$\mathrm{O}(1) \mathrm{C}(18)$ & $1.201(4)$ & $\mathrm{C}(21) \mathrm{C}(20)$ & $1.504(5)$ \\
$\mathrm{C}(17) \mathrm{C}(18)$ & $1.547(4)$ & $\mathrm{C}(26) \mathrm{C}(25)$ & $1.378(5)$ \\
$\mathrm{C}(17) \mathrm{C}(19)$ & $1.469(5)$ & $\mathrm{C}(14) \mathrm{C}(13)$ & $1.394(5)$ \\
$\mathrm{C}(17) \mathrm{C}(16)$ & $1.533(4)$ & $\mathrm{C}(24) \mathrm{C}(25)$ & $1.377(5)$ \\
$\mathrm{C}(17) \mathrm{C}(20)$ & $1.544(4)$ & $\mathrm{C}(24) \mathrm{C}(23)$ & $1.364(6)$ \\
$\mathrm{C}(9) \mathrm{C}(8)$ & $1.494(4)$ & $\mathrm{C}(15) \mathrm{C}(16)$ & $1.521(4)$ \\
$\mathrm{C}(9) \mathrm{C}(14)$ & $1.384(5)$ & $\mathrm{C}(19) \mathrm{N}(1)$ & $1.134(5)$ \\
$\mathrm{C}(9) \mathrm{C}(10)$ & $1.385(5)$ & $\mathrm{C}(5) \mathrm{C}(4)$ & $1.388(6)$ \\
$\mathrm{C}(6) \mathrm{C}(7)$ & $1.483(5)$ & $\mathrm{C}(22) \mathrm{C}(23)$ & $1.390(6)$
\end{tabular}




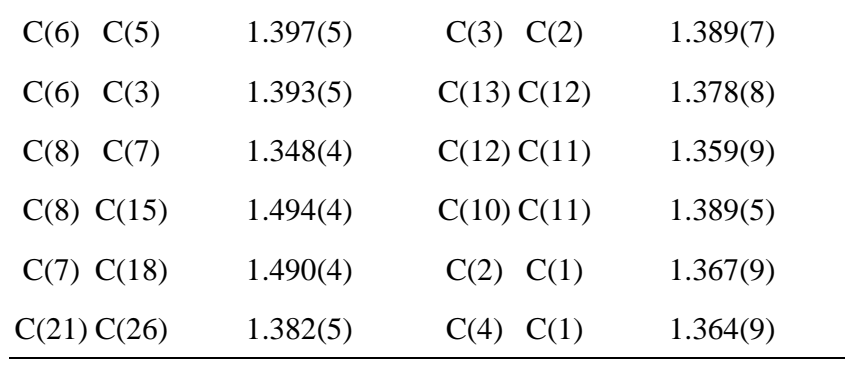

Table S13. Bond angles for CCDC 1971345.

\begin{tabular}{|c|c|c|c|}
\hline Atom Atom Atom & Angle $/^{\circ}$ & Atom Atom Atom & Angle ${ }^{\circ}$ \\
\hline $\mathrm{C}(19) \mathrm{C}(17) \mathrm{C}(18)$ & $105.4(3)$ & $\mathrm{C}(25) \mathrm{C}(24) \mathrm{Br}(1)$ & $117.8(3)$ \\
\hline$C(19) C(17) C(16)$ & 109.3(3) & $\mathrm{C}(23) \mathrm{C}(24) \mathrm{Br}(1)$ & $121.5(3)$ \\
\hline$C(19) C(17) C(20)$ & $110.4(3)$ & $C(23) C(24) C(25)$ & $120.7(4)$ \\
\hline$C(16) C(17) C(18)$ & $107.4(2)$ & $C(8) C(15) C(16)$ & $113.5(2)$ \\
\hline$C(16) C(17) C(20)$ & $112.9(3)$ & $\mathrm{O}(1) \mathrm{C}(18) \mathrm{C}(17)$ & $121.1(3)$ \\
\hline $\mathrm{C}(20) \mathrm{C}(17) \mathrm{C}(18)$ & 111.1(2) & $\mathrm{O}(1) \mathrm{C}(18) \mathrm{C}(7)$ & $122.9(3)$ \\
\hline $\mathrm{C}(14) \mathrm{C}(9) \quad \mathrm{C}(8)$ & $120.9(3)$ & $C(7) C(18) C(17)$ & $116.0(2)$ \\
\hline$C(14) C(9) C(10)$ & 119.1(3) & $C(24) C(25) C(26)$ & $119.3(4)$ \\
\hline $\mathrm{C}(10) \mathrm{C}(9) \quad \mathrm{C}(8)$ & $120.0(3)$ & $\mathrm{N}(1) \mathrm{C}(19) \mathrm{C}(17)$ & $177.6(4)$ \\
\hline $\mathrm{C}(5) \quad \mathrm{C}(6) \quad \mathrm{C}(7)$ & $120.6(3)$ & $C(15) C(16) C(17)$ & $111.0(3)$ \\
\hline $\mathrm{C}(3) \quad \mathrm{C}(6) \quad \mathrm{C}(7)$ & $120.7(3)$ & $C(4) \quad C(5) \quad C(6)$ & $120.1(4)$ \\
\hline$C(3) \quad C(6) \quad C(5)$ & $118.7(3)$ & $C(21) C(22) C(23)$ & $121.4(4)$ \\
\hline $\mathrm{C}(9) \mathrm{C}(8) \mathrm{C}(15)$ & $114.8(2)$ & $C(2) \quad C(3) \quad C(6)$ & $120.0(4)$ \\
\hline $\mathrm{C}(7) \quad \mathrm{C}(8) \quad \mathrm{C}(9)$ & $122.1(3)$ & $C(24) C(23) C(22)$ & $119.3(3)$ \\
\hline$C(7) \quad C(8) C(15)$ & 123.1(3) & $C(12) C(13) C(14)$ & $119.9(4)$ \\
\hline$C(6) \quad C(7) C(18)$ & $115.9(2)$ & $C(21) C(20) C(17)$ & $113.2(2)$ \\
\hline$C(8) \quad C(7) \quad C(6)$ & $123.1(3)$ & $C(11) C(12) C(13)$ & $120.2(4)$ \\
\hline $\mathrm{C}(8) \quad \mathrm{C}(7) \mathrm{C}(18)$ & $120.9(3)$ & $C(9) C(10) C(11)$ & $120.2(4)$ \\
\hline$C(26) C(21) C(22)$ & $117.6(3)$ & $\mathrm{C}(1) \quad \mathrm{C}(2) \quad \mathrm{C}(3)$ & $120.4(5)$ \\
\hline$C(26) C(21) C(20)$ & $120.5(3)$ & $C(12) C(11) C(10)$ & $120.5(5)$ \\
\hline $\mathrm{C}(22) \mathrm{C}(21) \mathrm{C}(20)$ & $121.9(3)$ & $\mathrm{C}(1) \quad \mathrm{C}(4) \quad \mathrm{C}(5)$ & $120.4(5)$ \\
\hline$C(25) C(26) C(21)$ & $121.6(3)$ & $\mathrm{C}(4) \quad \mathrm{C}(1) \quad \mathrm{C}(2)$ & $120.5(4)$ \\
\hline$C(9) C(14) C(13)$ & $120.1(4)$ & & \\
\hline
\end{tabular}

Table S14. Hydrogen atom coordinates $\left(\AA \times 10^{4}\right)$ and isotropic displacement parameters $\left(\AA^{2} \times 10^{3}\right)$ for CCDC 1971345.

\begin{tabular}{ccccc}
\hline Atom & $\boldsymbol{x}$ & $\boldsymbol{y}$ & $\boldsymbol{z}$ & $\mathrm{U}(\mathbf{e q})$ \\
\hline $\mathrm{H}(26)$ & 3175.03 & 9510.44 & 7150.67 & 69 \\
$\mathrm{H}(14)$ & 4549.79 & 408.45 & 3749.63 & 75
\end{tabular}




\begin{tabular}{ccccc}
$\mathrm{H}(15 \mathrm{~A})$ & 4521.88 & 4503.67 & 5252.41 & 68 \\
$\mathrm{H}(15 \mathrm{~B})$ & 4405.62 & 2192.65 & 5177.78 & 68 \\
$\mathrm{H}(25)$ & 3603.24 & 10649.32 & 8618.47 & 73 \\
$\mathrm{H}(16 \mathrm{~A})$ & 3986.69 & 3828.06 & 6528.37 & 67 \\
$\mathrm{H}(16 \mathrm{~B})$ & 3568.77 & 2490.64 & 5867.63 & 67 \\
$\mathrm{H}(5)$ & 3035.15 & 7634.39 & 2862.2 & 81 \\
$\mathrm{H}(22)$ & 3422.16 & 3859.78 & 8037.69 & 81 \\
$\mathrm{H}(3)$ & 3321.13 & 1771 & 2410.28 & 81 \\
$\mathrm{H}(23)$ & 3869.37 & 4992.84 & 9502.93 & 86 \\
$\mathrm{H}(13)$ & 5140.93 & -383.19 & 2518.86 & 91 \\
$\mathrm{H}(20 \mathrm{~A})$ & 2714.65 & 6831.97 & 6203.31 & 67 \\
$\mathrm{H}(20 \mathrm{~B})$ & 2795.52 & 4599.69 & 6548.15 & 67 \\
$\mathrm{H}(12)$ & 5340.92 & 1999.89 & 1361.82 & 98 \\
$\mathrm{H}(10)$ & 4355.45 & 5939.27 & 2622.09 & 81 \\
$\mathrm{H}(2)$ & 3000.89 & 2163.5 & 769.51 & 112 \\
$\mathrm{H}(11)$ & 4957.39 & 5134.96 & 1419.42 & 101 \\
$\mathrm{H}(4)$ & 2696.44 & 7939.43 & 1227.71 & 114 \\
$\mathrm{H}(1)$ & 2702.49 & 5238.45 & 187.99 & 122 \\
\hline
\end{tabular}

\section{X-ray crystallographic data of $6 b$}
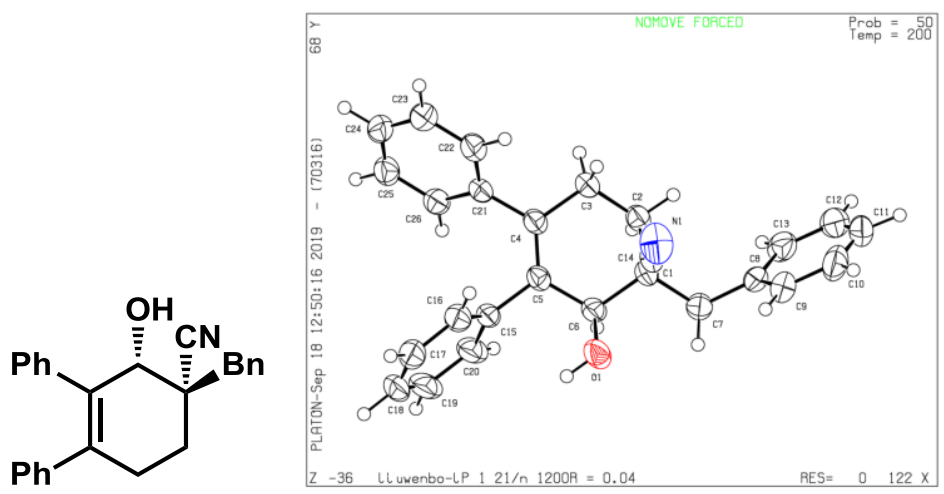

Table S15. Crystal data and structure refinement for $6 \mathbf{b}$.

Identification code

Empirical formula

Formula weight

Temperature

Wavelength

Crystal system

Space group

Unit cell dimensions
CCDC 1971344

$\mathrm{C}_{26} \mathrm{H}_{23} \mathrm{NO}$

365.45

200.00(10) K

$1.54184 \AA$

Monoclinic

P $121 /$ n 1

$a=6.43000(10) \AA$

$\alpha=90^{\circ}$.

$\mathrm{b}=12.18180(10) \AA$

$\beta=96.4970(10)^{\circ}$.

$c=25.8958(2) \AA$ 
Volume

Z

Density (calculated)

Absorption coefficient

$\mathrm{F}(000)$

Crystal size

Theta range for data collection

Index ranges

Reflections collected

Independent reflections

Completeness to theta $=67.684^{\circ}$

Absorption correction

Max. and min. transmission

Refinement method

Data / restraints / parameters

Goodness-of-fit on $\mathrm{F}^{2}$

Final $\mathrm{R}$ indices [I>2sigma(I)]

$\mathrm{R}$ indices (all data)

Extinction coefficient

Largest diff. peak and hole
2015.36(4) $\AA^{3}$

4

$1.204 \mathrm{Mg} / \mathrm{m}^{3}$

$0.562 \mathrm{~mm}^{-1}$

776

$0.08 \times 0.02 \times 0.02 \mathrm{~mm}^{3}$

3.436 to $74.082^{\circ}$.

$-7<=\mathrm{h}<=7,-15<=\mathrm{k}<=15,-31<=1<=31$

21974

$4006[\mathrm{R}(\mathrm{int})=0.0237]$

$99.3 \%$

Semi-empirical from equivalents

1.00000 and 0.67484

Full-matrix least-squares on $\mathrm{F}^{2}$

$4006 / 0 / 345$

1.026

$\mathrm{R} 1=0.0394, \mathrm{wR} 2=0.1009$

$\mathrm{R} 1=0.0409, \mathrm{wR} 2=0.1020$

$\mathrm{n} / \mathrm{a}$

0.189 and -0.166 e. $\AA^{-3}$

Table S16. Atomic coordinates $\left(\times 10^{4}\right)$ and equivalent isotropic displacement parameters $\left(\AA^{2} \times 10^{3}\right)$ for CCDC 1971344.

$U(e q)$ is defined as one third of the trace of the orthogonalized $U^{i j}$ tensor.

\begin{tabular}{lcccc}
\hline & $\mathrm{x}$ & $\mathrm{y}$ & $\mathrm{z}$ & $\mathrm{U}(\mathrm{eq})$ \\
\hline $\mathrm{O}(1)$ & $6850(2)$ & $5092(1)$ & $2866(1)$ & $64(1)$ \\
$\mathrm{C}(5)$ & $5915(2)$ & $4279(1)$ & $3666(1)$ & $35(1)$ \\
$\mathrm{C}(4)$ & $6487(2)$ & $4261(1)$ & $4181(1)$ & $37(1)$ \\
$\mathrm{N}(1)$ & $11242(2)$ & $5797(1)$ & $3676(1)$ & $68(1)$ \\
$\mathrm{C}(21)$ & $6381(2)$ & $3254(1)$ & $4503(1)$ & $38(1)$ \\
$\mathrm{C}(14)$ & $9507(2)$ & $5982(1)$ & $3636(1)$ & $41(1)$ \\
$\mathrm{C}(15)$ & $5112(2)$ & $3292(1)$ & $3364(1)$ & $38(1)$ \\
$\mathrm{C}(8)$ & $8084(2)$ & $8303(1)$ & $3474(1)$ & $38(1)$ \\
$\mathrm{C}(2)$ & $6694(2)$ & $6330(1)$ & $4171(1)$ & $40(1)$ \\
$\mathrm{C}(1)$ & $7260(2)$ & $6246(1)$ & $3613(1)$ & $35(1)$ \\
$\mathrm{C}(7)$ & $6789(2)$ & $7314(1)$ & $3297(1)$ & $42(1)$ \\
$\mathrm{C}(26)$ & $4530(2)$ & $2663(1)$ & $4509(1)$ & $43(1)$
\end{tabular}




\begin{tabular}{lllll}
$\mathrm{C}(3)$ & $7253(2)$ & $5284(1)$ & $4476(1)$ & $44(1)$ \\
$\mathrm{C}(6)$ & $5978(2)$ & $5304(1)$ & $3340(1)$ & $39(1)$ \\
$\mathrm{C}(9)$ & $9983(2)$ & $8489(1)$ & $3286(1)$ & $49(1)$ \\
$\mathrm{C}(22)$ & $8143(2)$ & $2902(1)$ & $4824(1)$ & $50(1)$ \\
$\mathrm{C}(16)$ & $6428(2)$ & $2426(1)$ & $3278(1)$ & $49(1)$ \\
$\mathrm{C}(24)$ & $6220(2)$ & $1404(1)$ & $5135(1)$ & $51(1)$ \\
$\mathrm{C}(25)$ & $4460(2)$ & $1743(1)$ & $4823(1)$ & $50(1)$ \\
$\mathrm{C}(20)$ & $3033(2)$ & $3252(1)$ & $3147(1)$ & $52(1)$ \\
$\mathrm{C}(13)$ & $7391(2)$ & $9058(1)$ & $3817(1)$ & $54(1)$ \\
$\mathrm{C}(23)$ & $8062(2)$ & $1982(1)$ & $5137(1)$ & $55(1)$ \\
$\mathrm{C}(10)$ & $11177(3)$ & $9404(1)$ & $3438(1)$ & $66(1)$ \\
$\mathrm{C}(12)$ & $8598(4)$ & $9975(1)$ & $3967(1)$ & $74(1)$ \\
$\mathrm{C}(17)$ & $5682(3)$ & $1530(1)$ & $2981(1)$ & $67(1)$ \\
$\mathrm{C}(18)$ & $3633(3)$ & $1493(1)$ & $2771(1)$ & $74(1)$ \\
$\mathrm{C}(11)$ & $10478(3)$ & $10148(1)$ & $3779(1)$ & $74(1)$ \\
$\mathrm{C}(19)$ & $2307(3)$ & $2336(2)$ & $2857(1)$ & $72(1)$ \\
\hline
\end{tabular}

Table S17. Bond lengths $[\AA ̊ \AA]$ and angles $\left[{ }^{\circ}\right]$ for CCDC 1971344.

\begin{tabular}{ll}
\hline $\mathrm{O}(1)-\mathrm{C}(6)$ & $1.4289(14)$ \\
$\mathrm{O}(1)-\mathrm{H}(1)$ & $0.90(2)$ \\
$\mathrm{C}(5)-\mathrm{C}(4)$ & $1.3413(15)$ \\
$\mathrm{C}(5)-\mathrm{C}(15)$ & $1.4945(14)$ \\
$\mathrm{C}(5)-\mathrm{C}(6)$ & $1.5109(15)$ \\
$\mathrm{C}(4)-\mathrm{C}(21)$ & $1.4893(15)$ \\
$\mathrm{C}(4)-\mathrm{C}(3)$ & $1.5146(14)$ \\
$\mathrm{N}(1)-\mathrm{C}(14)$ & $1.1318(16)$ \\
$\mathrm{C}(21)-\mathrm{C}(26)$ & $1.3923(16)$ \\
$\mathrm{C}(21)-\mathrm{C}(22)$ & $1.3949(17)$ \\
$\mathrm{C}(14)-\mathrm{C}(1)$ & $1.4749(14)$ \\
$\mathrm{C}(15)-\mathrm{C}(16)$ & $1.3865(17)$ \\
$\mathrm{C}(15)-\mathrm{C}(20)$ & $1.3911(17)$ \\
$\mathrm{C}(8)-\mathrm{C}(7)$ & $1.5060(15)$ \\
$\mathrm{C}(8)-\mathrm{C}(9)$ & $1.3846(17)$ \\
$\mathrm{C}(8)-\mathrm{C}(13)$ & $1.3870(17)$ \\
$\mathrm{C}(2)-\mathrm{C}(1)$ & $1.5344(15)$ \\
$\mathrm{C}(2)-\mathrm{C}(3)$ & $1.5194(16)$ \\
$\mathrm{C}(2)-\mathrm{H}(2 \mathrm{~A})$ & $1.009(13)$ \\
$\mathrm{C}(2)-\mathrm{H}(2 B)$ & $0.999(14)$ \\
&
\end{tabular}




\begin{tabular}{|c|c|}
\hline$C(1)-C(7)$ & $1.5472(15)$ \\
\hline $\mathrm{C}(1)-\mathrm{C}(6)$ & $1.5374(14)$ \\
\hline $\mathrm{C}(7)-\mathrm{H}(7 \mathrm{~A})$ & $0.982(15)$ \\
\hline $\mathrm{C}(7)-\mathrm{H}(7 \mathrm{~B})$ & $1.018(16)$ \\
\hline$C(26)-C(25)$ & $1.3894(17)$ \\
\hline $\mathrm{C}(26)-\mathrm{H}(26)$ & $0.978(13)$ \\
\hline $\mathrm{C}(3)-\mathrm{H}(3 \mathrm{~A})$ & $1.024(15)$ \\
\hline $\mathrm{C}(3)-\mathrm{H}(3 \mathrm{~B})$ & $1.017(16)$ \\
\hline $\mathrm{C}(6)-\mathrm{H}(6)$ & $0.999(13)$ \\
\hline $\mathrm{C}(9)-\mathrm{C}(10)$ & $1.3851(19)$ \\
\hline $\mathrm{C}(9)-\mathrm{H}(9)$ & $0.958(16)$ \\
\hline$C(22)-C(23)$ & $1.3883(18)$ \\
\hline $\mathrm{C}(22)-\mathrm{H}(22)$ & $1.005(15)$ \\
\hline $\mathrm{C}(16)-\mathrm{C}(17)$ & $1.3898(18)$ \\
\hline $\mathrm{C}(16)-\mathrm{H}(16)$ & $0.993(17)$ \\
\hline $\mathrm{C}(24)-\mathrm{C}(25)$ & $1.376(2)$ \\
\hline $\mathrm{C}(24)-\mathrm{C}(23)$ & $1.378(2)$ \\
\hline $\mathrm{C}(24)-\mathrm{H}(24)$ & $0.987(17)$ \\
\hline $\mathrm{C}(25)-\mathrm{H}(25)$ & $1.013(16)$ \\
\hline $\mathrm{C}(20)-\mathrm{C}(19)$ & $1.395(2)$ \\
\hline $\mathrm{C}(20)-\mathrm{H}(20)$ & $0.987(16)$ \\
\hline $\mathrm{C}(13)-\mathrm{C}(12)$ & $1.390(2)$ \\
\hline $\mathrm{C}(13)-\mathrm{H}(13)$ & $0.997(17)$ \\
\hline $\mathrm{C}(23)-\mathrm{H}(23)$ & $1.024(17)$ \\
\hline $\mathrm{C}(10)-\mathrm{C}(11)$ & $1.376(3)$ \\
\hline $\mathrm{C}(10)-\mathrm{H}(10)$ & $0.989(19)$ \\
\hline $\mathrm{C}(12)-\mathrm{C}(11)$ & $1.370(3)$ \\
\hline $\mathrm{C}(12)-\mathrm{H}(12)$ & $0.95(2)$ \\
\hline $\mathrm{C}(17)-\mathrm{C}(18)$ & $1.368(3)$ \\
\hline $\mathrm{C}(17)-\mathrm{H}(17)$ & $0.986(18)$ \\
\hline $\mathrm{C}(18)-\mathrm{C}(19)$ & $1.369(3)$ \\
\hline $\mathrm{C}(18)-\mathrm{H}(18)$ & $0.990(19)$ \\
\hline $\mathrm{C}(11)-\mathrm{H}(11)$ & $1.01(2)$ \\
\hline C(19)-H(19) & $0.97(2)$ \\
\hline $\mathrm{C}(6)-\mathrm{O}(1)-\mathrm{H}(1)$ & $108.5(13)$ \\
\hline $\mathrm{C}(4)-\mathrm{C}(5)-\mathrm{C}(15)$ & $123.17(9)$ \\
\hline$C(4)-C(5)-C(6)$ & $123.18(9)$ \\
\hline
\end{tabular}




\begin{tabular}{|c|c|}
\hline$C(15)-C(5)-C(6)$ & 113.64(9) \\
\hline $\mathrm{C}(5)-\mathrm{C}(4)-\mathrm{C}(21)$ & 122.97(9) \\
\hline $\mathrm{C}(5)-\mathrm{C}(4)-\mathrm{C}(3)$ & $121.64(10)$ \\
\hline$C(21)-C(4)-C(3)$ & $115.37(9)$ \\
\hline $\mathrm{C}(26)-\mathrm{C}(21)-\mathrm{C}(4)$ & $121.72(10)$ \\
\hline$C(26)-C(21)-C(22)$ & $118.15(11)$ \\
\hline$C(22)-C(21)-C(4)$ & $120.08(10)$ \\
\hline $\mathrm{N}(1)-\mathrm{C}(14)-\mathrm{C}(1)$ & $176.99(13)$ \\
\hline$C(16)-C(15)-C(5)$ & $121.03(10)$ \\
\hline$C(16)-C(15)-C(20)$ & $118.98(11)$ \\
\hline$C(20)-C(15)-C(5)$ & $119.95(11)$ \\
\hline $\mathrm{C}(9)-\mathrm{C}(8)-\mathrm{C}(7)$ & $120.22(11)$ \\
\hline $\mathrm{C}(9)-\mathrm{C}(8)-\mathrm{C}(13)$ & $118.49(12)$ \\
\hline $\mathrm{C}(13)-\mathrm{C}(8)-\mathrm{C}(7)$ & $121.27(11)$ \\
\hline $\mathrm{C}(1)-\mathrm{C}(2)-\mathrm{H}(2 \mathrm{~A})$ & $107.6(7)$ \\
\hline $\mathrm{C}(1)-\mathrm{C}(2)-\mathrm{H}(2 \mathrm{~B})$ & $109.6(8)$ \\
\hline $\mathrm{C}(3)-\mathrm{C}(2)-\mathrm{C}(1)$ & $111.49(9)$ \\
\hline $\mathrm{C}(3)-\mathrm{C}(2)-\mathrm{H}(2 \mathrm{~A})$ & $109.6(7)$ \\
\hline $\mathrm{C}(3)-\mathrm{C}(2)-\mathrm{H}(2 \mathrm{~B})$ & $110.7(8)$ \\
\hline $\mathrm{H}(2 \mathrm{~A})-\mathrm{C}(2)-\mathrm{H}(2 \mathrm{~B})$ & 107.9(10) \\
\hline $\mathrm{C}(14)-\mathrm{C}(1)-\mathrm{C}(2)$ & 108.10(9) \\
\hline$C(14)-C(1)-C(7)$ & 109.73(9) \\
\hline$C(14)-C(1)-C(6)$ & 109.11(9) \\
\hline$C(2)-C(1)-C(7)$ & 112.92(9) \\
\hline$C(2)-C(1)-C(6)$ & $108.12(8)$ \\
\hline$C(6)-C(1)-C(7)$ & $108.79(8)$ \\
\hline $\mathrm{C}(8)-\mathrm{C}(7)-\mathrm{C}(1)$ & $116.30(9)$ \\
\hline $\mathrm{C}(8)-\mathrm{C}(7)-\mathrm{H}(7 \mathrm{~A})$ & 109.2(8) \\
\hline $\mathrm{C}(8)-\mathrm{C}(7)-\mathrm{H}(7 \mathrm{~B})$ & 109.3(9) \\
\hline $\mathrm{C}(1)-\mathrm{C}(7)-\mathrm{H}(7 \mathrm{~A})$ & $104.9(8)$ \\
\hline $\mathrm{C}(1)-\mathrm{C}(7)-\mathrm{H}(7 \mathrm{~B})$ & $107.1(8)$ \\
\hline $\mathrm{H}(7 \mathrm{~A})-\mathrm{C}(7)-\mathrm{H}(7 \mathrm{~B})$ & $109.8(12)$ \\
\hline $\mathrm{C}(21)-\mathrm{C}(26)-\mathrm{H}(26)$ & $118.9(8)$ \\
\hline $\mathrm{C}(25)-\mathrm{C}(26)-\mathrm{C}(21)$ & $120.56(12)$ \\
\hline $\mathrm{C}(25)-\mathrm{C}(26)-\mathrm{H}(26)$ & $120.5(8)$ \\
\hline $\mathrm{C}(4)-\mathrm{C}(3)-\mathrm{C}(2)$ & $112.56(9)$ \\
\hline $\mathrm{C}(4)-\mathrm{C}(3)-\mathrm{H}(3 \mathrm{~A})$ & $108.8(8)$ \\
\hline $\mathrm{C}(4)-\mathrm{C}(3)-\mathrm{H}(3 \mathrm{~B})$ & $110.3(9)$ \\
\hline
\end{tabular}




\begin{tabular}{|c|c|}
\hline $\mathrm{C}(2)-\mathrm{C}(3)-\mathrm{H}(3 \mathrm{~A})$ & $109.8(8)$ \\
\hline $\mathrm{C}(2)-\mathrm{C}(3)-\mathrm{H}(3 \mathrm{~B})$ & $109.6(9)$ \\
\hline $\mathrm{H}(3 \mathrm{~A})-\mathrm{C}(3)-\mathrm{H}(3 \mathrm{~B})$ & $105.5(12)$ \\
\hline $\mathrm{O}(1)-\mathrm{C}(6)-\mathrm{C}(5)$ & 111.67(9) \\
\hline $\mathrm{O}(1)-\mathrm{C}(6)-\mathrm{C}(1)$ & $106.70(9)$ \\
\hline $\mathrm{O}(1)-\mathrm{C}(6)-\mathrm{H}(6)$ & $108.0(7)$ \\
\hline$C(5)-C(6)-C(1)$ & 114.01(9) \\
\hline $\mathrm{C}(5)-\mathrm{C}(6)-\mathrm{H}(6)$ & $109.5(7)$ \\
\hline $\mathrm{C}(1)-\mathrm{C}(6)-\mathrm{H}(6)$ & $106.7(7)$ \\
\hline $\mathrm{C}(8)-\mathrm{C}(9)-\mathrm{C}(10)$ & $120.96(14)$ \\
\hline $\mathrm{C}(8)-\mathrm{C}(9)-\mathrm{H}(9)$ & $119.8(9)$ \\
\hline $\mathrm{C}(10)-\mathrm{C}(9)-\mathrm{H}(9)$ & 119.2(9) \\
\hline $\mathrm{C}(21)-\mathrm{C}(22)-\mathrm{H}(22)$ & 119.3(9) \\
\hline $\mathrm{C}(23)-\mathrm{C}(22)-\mathrm{C}(21)$ & $121.00(12)$ \\
\hline $\mathrm{C}(23)-\mathrm{C}(22)-\mathrm{H}(22)$ & 119.7(9) \\
\hline$C(15)-C(16)-C(17)$ & $120.51(14)$ \\
\hline $\mathrm{C}(15)-\mathrm{C}(16)-\mathrm{H}(16)$ & $118.8(9)$ \\
\hline $\mathrm{C}(17)-\mathrm{C}(16)-\mathrm{H}(16)$ & $120.6(9)$ \\
\hline $\mathrm{C}(25)-\mathrm{C}(24)-\mathrm{C}(23)$ & $119.90(12)$ \\
\hline $\mathrm{C}(25)-\mathrm{C}(24)-\mathrm{H}(24)$ & 119.1(10) \\
\hline $\mathrm{C}(23)-\mathrm{C}(24)-\mathrm{H}(24)$ & $121.0(10)$ \\
\hline $\mathrm{C}(26)-\mathrm{C}(25)-\mathrm{H}(25)$ & $118.8(9)$ \\
\hline$C(24)-C(25)-C(26)$ & $120.43(12)$ \\
\hline $\mathrm{C}(24)-\mathrm{C}(25)-\mathrm{H}(25)$ & $120.7(9)$ \\
\hline$C(15)-C(20)-C(19)$ & $119.58(15)$ \\
\hline $\mathrm{C}(15)-\mathrm{C}(20)-\mathrm{H}(20)$ & 119.2(9) \\
\hline $\mathrm{C}(19)-\mathrm{C}(20)-\mathrm{H}(20)$ & 121.2(9) \\
\hline $\mathrm{C}(8)-\mathrm{C}(13)-\mathrm{C}(12)$ & $120.15(15)$ \\
\hline $\mathrm{C}(8)-\mathrm{C}(13)-\mathrm{H}(13)$ & $117.2(10)$ \\
\hline $\mathrm{C}(12)-\mathrm{C}(13)-\mathrm{H}(13)$ & $122.6(10)$ \\
\hline $\mathrm{C}(22)-\mathrm{C}(23)-\mathrm{H}(23)$ & $119.6(9)$ \\
\hline $\mathrm{C}(24)-\mathrm{C}(23)-\mathrm{C}(22)$ & $119.95(12)$ \\
\hline $\mathrm{C}(24)-\mathrm{C}(23)-\mathrm{H}(23)$ & $120.4(9)$ \\
\hline $\mathrm{C}(9)-\mathrm{C}(10)-\mathrm{H}(10)$ & 118.1(11) \\
\hline $\mathrm{C}(11)-\mathrm{C}(10)-\mathrm{C}(9)$ & $120.11(16)$ \\
\hline $\mathrm{C}(11)-\mathrm{C}(10)-\mathrm{H}(10)$ & $121.7(11)$ \\
\hline $\mathrm{C}(13)-\mathrm{C}(12)-\mathrm{H}(12)$ & $117.2(12)$ \\
\hline$C(11)-C(12)-C(13)$ & $120.75(15)$ \\
\hline
\end{tabular}




$\begin{array}{ll}\mathrm{C}(11)-\mathrm{C}(12)-\mathrm{H}(12) & 122.1(12) \\ \mathrm{C}(16)-\mathrm{C}(17)-\mathrm{H}(17) & 120.6(11) \\ \mathrm{C}(18)-\mathrm{C}(17)-\mathrm{C}(16) & 120.18(16) \\ \mathrm{C}(18)-\mathrm{C}(17)-\mathrm{H}(17) & 119.2(11) \\ \mathrm{C}(17)-\mathrm{C}(18)-\mathrm{C}(19) & 120.05(13) \\ \mathrm{C}(17)-\mathrm{C}(18)-\mathrm{H}(18) & 120.5(11) \\ \mathrm{C}(19)-\mathrm{C}(18)-\mathrm{H}(18) & 119.4(11) \\ \mathrm{C}(10)-\mathrm{C}(11)-\mathrm{H}(11) & 117.0(12) \\ \mathrm{C}(12)-\mathrm{C}(11)-\mathrm{C}(10) & 119.54(14) \\ \mathrm{C}(12)-\mathrm{C}(11)-\mathrm{H}(11) & 123.4(12) \\ \mathrm{C}(20)-\mathrm{C}(19)-\mathrm{H}(19) & 116.4(12) \\ \mathrm{C}(18)-\mathrm{C}(19)-\mathrm{C}(20) & 120.68(15) \\ \mathrm{C}(18)-\mathrm{C}(19)-\mathrm{H}(19) & 123.0(11)\end{array}$

Symmetry transformations used to generate equivalent atoms:

Table S18. Anisotropic displacement parameters $\quad\left(\AA^{2} \times 10^{3}\right)$ for CCDC 1971344.

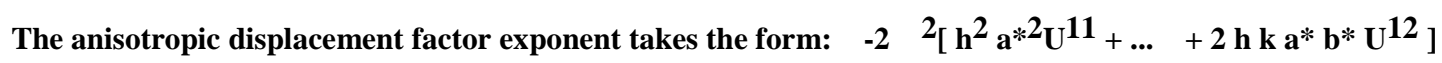

\begin{tabular}{|c|c|c|c|c|c|c|}
\hline & $\mathrm{U}^{11}$ & $\mathrm{U}^{22}$ & $\mathrm{U}^{33}$ & $\mathrm{U}^{23}$ & $\mathrm{U}^{13}$ & $\mathrm{U}^{12}$ \\
\hline $\mathrm{O}(1)$ & $96(1)$ & $59(1)$ & $41(1)$ & $-14(1)$ & $24(1)$ & $-36(1)$ \\
\hline $\mathrm{C}(5)$ & $32(1)$ & $36(1)$ & $38(1)$ & $-5(1)$ & $4(1)$ & $-6(1)$ \\
\hline$C(4)$ & $36(1)$ & $36(1)$ & $38(1)$ & $-5(1)$ & $3(1)$ & $-6(1)$ \\
\hline $\mathrm{N}(1)$ & $40(1)$ & $62(1)$ & $103(1)$ & $0(1)$ & $10(1)$ & $6(1)$ \\
\hline$C(21)$ & $47(1)$ & $35(1)$ & $33(1)$ & $-6(1)$ & $3(1)$ & $-6(1)$ \\
\hline $\mathrm{C}(14)$ & $39(1)$ & $33(1)$ & $52(1)$ & $-2(1)$ & $6(1)$ & $-2(1)$ \\
\hline $\mathrm{C}(15)$ & $44(1)$ & $39(1)$ & $31(1)$ & $-3(1)$ & $6(1)$ & $-12(1)$ \\
\hline$C(8)$ & $44(1)$ & $33(1)$ & $37(1)$ & $5(1)$ & $0(1)$ & $3(1)$ \\
\hline$C(2)$ & $43(1)$ & $36(1)$ & $41(1)$ & $-7(1)$ & $6(1)$ & $-4(1)$ \\
\hline $\mathrm{C}(1)$ & $32(1)$ & $34(1)$ & $40(1)$ & $-3(1)$ & $3(1)$ & $-4(1)$ \\
\hline$C(7)$ & $40(1)$ & $41(1)$ & $44(1)$ & $3(1)$ & $-2(1)$ & $-1(1)$ \\
\hline$C(26)$ & $47(1)$ & $45(1)$ & $38(1)$ & $-4(1)$ & $6(1)$ & $-8(1)$ \\
\hline$C(3)$ & $56(1)$ & $38(1)$ & $36(1)$ & $-5(1)$ & $2(1)$ & $-10(1)$ \\
\hline$C(6)$ & $41(1)$ & $43(1)$ & $34(1)$ & $-3(1)$ & $3(1)$ & $-11(1)$ \\
\hline $\mathrm{C}(9)$ & $53(1)$ & $40(1)$ & $55(1)$ & $7(1)$ & $9(1)$ & $-2(1)$ \\
\hline $\mathrm{C}(22)$ & $52(1)$ & $43(1)$ & $51(1)$ & $-1(1)$ & $-6(1)$ & $-9(1)$ \\
\hline$C(16)$ & $66(1)$ & $42(1)$ & $41(1)$ & $-4(1)$ & $12(1)$ & $-4(1)$ \\
\hline
\end{tabular}




$\begin{array}{lllllll}\mathrm{C}(24) & 77(1) & 37(1) & 41(1) & -1(1) & 10(1) & -4(1) \\ \mathrm{C}(25) & 62(1) & 44(1) & 45(1) & -5(1) & 15(1) & -14(1) \\ \mathrm{C}(20) & 48(1) & 67(1) & 40(1) & -7(1) & 0(1) & -18(1) \\ \mathrm{C}(13) & 72(1) & 42(1) & 48(1) & 4(1) & 11(1) & 11(1) \\ \mathrm{C}(23) & 68(1) & 44(1) & 50(1) & 3(1) & -6(1) & -2(1) \\ \mathrm{C}(10) & 63(1) & 50(1) & 81(1) & 22(1) & -5(1) & -16(1) \\ \mathrm{C}(12) & 132(2) & 36(1) & 51(1) & -4(1) & -2(1) & 7(1) \\ \mathrm{C}(17) & 122(1) & 40(1) & 41(1) & -7(1) & 22(1) & -11(1) \\ \mathrm{C}(18) & 131(2) & 58(1) & 35(1) & -11(1) & 12(1) & -46(1) \\ \mathrm{C}(11) & 107(1) & 40(1) & 68(1) & 12(1) & -25(1) & -22(1) \\ \mathrm{C}(19) & 77(1) & 94(1) & 41(1) & -6(1) & -4(1) & -49(1)\end{array}$

Table S19. Hydrogen coordinates $\left(\times 10^{4}\right)$ and isotropic displacement parameters $\left(\AA^{2} \times 10^{3}\right)$ for CCDC 1971344.

\begin{tabular}{|c|c|c|c|c|}
\hline & $\mathrm{x}$ & $\mathrm{y}$ & $\mathrm{z}$ & $\mathrm{U}(\mathrm{eq})$ \\
\hline $\mathrm{H}(6)$ & $4520(20)$ & $5588(10)$ & $3250(5)$ & $43(3)$ \\
\hline $\mathrm{H}(26)$ & $3260(20)$ & 2927(11) & $4301(5)$ & $45(3)$ \\
\hline $\mathrm{H}(7 \mathrm{~A})$ & $5310(20)$ & $7471(12)$ & $3325(5)$ & $55(4)$ \\
\hline $\mathrm{H}(2 \mathrm{~A})$ & $5140(20)$ & $6462(11)$ & $4152(5)$ & $44(3)$ \\
\hline $\mathrm{H}(3 \mathrm{~A})$ & $8840(20)$ & $5232(11)$ & $4565(6)$ & $53(4)$ \\
\hline $\mathrm{H}(2 \mathrm{~B})$ & $7420(20)$ & $6976(11)$ & $4348(5)$ & $49(3)$ \\
\hline $\mathrm{H}(3 \mathrm{~B})$ & $6660(20)$ & $5318(12)$ & $4824(6)$ & $61(4)$ \\
\hline $\mathrm{H}(25)$ & $3080(20)$ & $1340(13)$ & $4827(6)$ & $62(4)$ \\
\hline $\mathrm{H}(24)$ & $6140(30)$ & $746(14)$ & $5354(7)$ & $72(5)$ \\
\hline $\mathrm{H}(7 \mathrm{~B})$ & $6990(20)$ & $7142(12)$ & 2921(6) & $62(4)$ \\
\hline $\mathrm{H}(16)$ & $7910(30)$ & $2456(12)$ & $3434(6)$ & $65(4)$ \\
\hline $\mathrm{H}(22)$ & $9490(20)$ & $3315(12)$ & $4823(6)$ & $60(4)$ \\
\hline $\mathrm{H}(13)$ & $6010(30)$ & 8916(14) & $3943(6)$ & $69(5)$ \\
\hline $\mathrm{H}(9)$ & $10500(20)$ & 7974(13) & $3051(6)$ & $63(4)$ \\
\hline $\mathrm{H}(20)$ & $2090(20)$ & $3863(13)$ & $3210(6)$ & $60(4)$ \\
\hline $\mathrm{H}(10)$ & $12520(30)$ & $9503(15)$ & $3293(7)$ & $82(5)$ \\
\hline $\mathrm{H}(23)$ & $9380(30)$ & 1738(13) & $5369(7)$ & $71(4)$ \\
\hline $\mathrm{H}(12)$ & $8060(30)$ & 10471(16) & $4200(8)$ & $90(6)$ \\
\hline $\mathrm{H}(19)$ & $840(30)$ & $2342(15)$ & 2721(7) & $87(6)$ \\
\hline $\mathrm{H}(17)$ & $6610(30)$ & $910(15)$ & 2922(7) & $80(5)$ \\
\hline $\mathrm{H}(18)$ & $3090(30)$ & $854(15)$ & $2561(7)$ & $87(5)$ \\
\hline
\end{tabular}


$\mathrm{H}(11)$

11390(30)

10809(17)

3871(8)

102(6)

$\mathrm{H}(1)$

6140(30)

4534(18)

2701(8)

100(6) 


\section{References}

(1) Malapit, C. A.; Bour, J. R.; Laursen, S. R.; Sanford, M. S. Mechanism and Scope of NickelCatalyzed Decarbonylative Borylation of Carboxylic Acid Fluorides. J. Am. Chem. Soc. 2019, 141, $17322-17330$.

(2) Ramachary, D. B.; Kishor, M.; Reddy, Y. V. Development of Pharmaceutical Drugs, Drug Intermediates and Ingredients by Using Direct Organo-Click Reactions. Eur. J. Org. Chem. 2008, 6, 975-973.

(3) (a) Wu, H.; He, Y.-P.; Gong, L.-Z. The Combination of Relay and Cooperative Catalysis with a Gold/Palladium/Brønsted Acid Ternary System for the Cascade Hydroamination/Allylic Alkylation Reaction. Adv. Synth. Catal. 2012, 354, 975-980. (b) Tummatorn, J.; Ruchirawat, S.; Ploypradith, P. A Convergent General Strategy for the Functionalized 2-Aryl Cycloalkyl-Fused Chromans: Intramolecular Hetero-Diels--Alder Reactions of ortho-Quinone Methides. Chem. Eur. J. 2010, 16, $1445-1148$.

(4) Shintani, R.; Isobe, S.; Takeda, M.; Hayashi. T. Rhodium-Catalyzed Asymmetric Synthesis of Spirocarbocycles: Arylboron Reagents as Surrogates of 1,2-Dimetalloarenes. Angew. Chem. Int. Ed. 2010, 49, 3795-3798.

(5) Darses, B.; Michaelides, I. N.; Sladojevich, F.; Ward, J. W.; Rzepa, P. R.; Dixon, D. J. Expedient Construction of the [7-5-5] All-Carbon Tricyclic Core of the Daphniphyllum Alkaloids Daphnilongeranin B and Daphniyunnine D. Org. Lett. 2012, 14, 1684-1687.

(6) Hickmann,V.; Alcarazo, M.; Furstner, A. Protecting-Group-Free and Catalysis-Based Total Synthesis of the Ecklonialactones. J. Am. Chem. Soc. 2010, 132, 11042-11044.

(7) Zhang, Z.; Gao, J.; Xia, J.-J.; Wang, G.-W. Solvent-Free Mechanochemical and One-Pot Reductive Benzylizations of Malononitrile and 4-Methylaniline using Hantzsch 1,4-Dihydropyridine as the Reductant. Org. Biomol. Chem. 2005, 3, 1617-1619.

(8) Zhang, D.; Lian, M.; Liu, J.; Tang, S.; Liu, G.; Ma, C.; Meng, Q.; Peng, H.; Zhu, D. Preparation of O-Protected Cyanohydrins by Aerobic Oxidation of $\alpha$-Substituted Malononitriles in the Presence of Diarylphosphine Oxides. Org. Lett. 2019, 21, 2597-2601. 
(9) Chea, J.; Lam, Y. Polymer-Supported Hantzsch 1,4-Dihydropyridine Ester: An Efficient Biomimetic Hydrogen Source for the Reduction of Ketimines and Electron-Withdrawing Group Conjugated Olefins Adv. Synth. Catal. 2010, 352, 1752-1758.

(10) Maeda, H.; Wada, H.; Mukae, H.; Mizuno, K. Preparation of polycyclic compounds by intramolecular photospirocyclization and photocycloaddition reactions of 4-alkenyl-1cyanonaphthalene derivatives. J. Photochem. Photobiol. A: Chem. 2016, 331, 29-41.

(11) Ghorai, M. K.; Talukdar, R.; Tiwari, D. P. A Route to Highly Functionalized $\beta$-Enaminoesters via a Domino Ring-Opening Cyclization/Decarboxylative Tautomerization Sequence of Donor-Acceptor Cyclopropanes with Substituted Malononitriles. Org. Lett. 2014, 16, 2204-2207.

(12) Li, J.; Lear, M. J.; Hayashi, Y. Sterically Demanding Oxidative Amidation of $\alpha$-Substituted Malononitriles with Amines Using O 2 . Angew. Chem. Int. Ed. 2016, 55, 9060-9064.

(13) Kleinbeck, F.; Toste, F. D. Gold(I)-Catalyzed Enantioselective Ring Expansion of Allenylcyclopropanols. J. Am. Chem. Soc. 2009, 131, 9178-9179.

(14) Barber, D. M.; Sanganee, H. J.; Dixon, D. J. One-Pot Catalytic Enantioselective Synthesis of Tetrahydropyridines via a Nitro-Mannich/Hydroamination Cascade. Org. Lett. 2012, 14, 5290-5293. (15) (a) Singleton, D. A.; Thomas, A. A. High-Precision Simultaneous Determination of Multiple Small Kinetic Isotope Effects at Natural Abundance. J. Am. Chem. Soc. 1995, 117, 9357-9358; (b) Singleton, D. A.; Merrigan, S. R.; Liu, J.; Houk, K. N. Experimental Geometry of the Epoxidation Transition State. J. Am. Chem. Soc. 1997, 119, 3385-3386; (c) Singleton, D. A.; Schulmeier, B. E. Evidence for a Concerted Mechanism in a Palladium Trimethylenemethane Cycloaddition. J. Am. Chem. Soc. 1999, 121, 9313-9317; (d) Singleton, D. A.; Merrigan, S. R.; Kim, B. J.; Beak, P.; Phillips, L. M.; Lee, J. K. ${ }^{13} \mathrm{C}$ Kinetic Isotope Effects and the Mechanism of the Uncatalyzed Decarboxylation of Orotic Acid. J. Am. Chem. Soc. 2000, 122, 3296-3300. (e) Ashley, M. A.; Hirschi, J. S.; Izzo, J. A.; Vetticatt, M. J. Isotope Effects Reveal the Mechanism of Enamine Formation in 1-Proline-Catalyzed a-Amination of Aldehydes. J. Am. Chem. Soc. 2016, 138, 1756-1759. 


\section{HPLC Spectra}<smiles>CC1(C#N)CCC(c2ccccc2)=C(c2ccccc2)C1=O</smiles>

\section{3aa}

$\mathrm{AD}-\mathrm{H}, 95 \% \mathrm{HEX}, 30 \mathrm{~min}, 0.8 \mathrm{~mL} / \mathrm{min}$

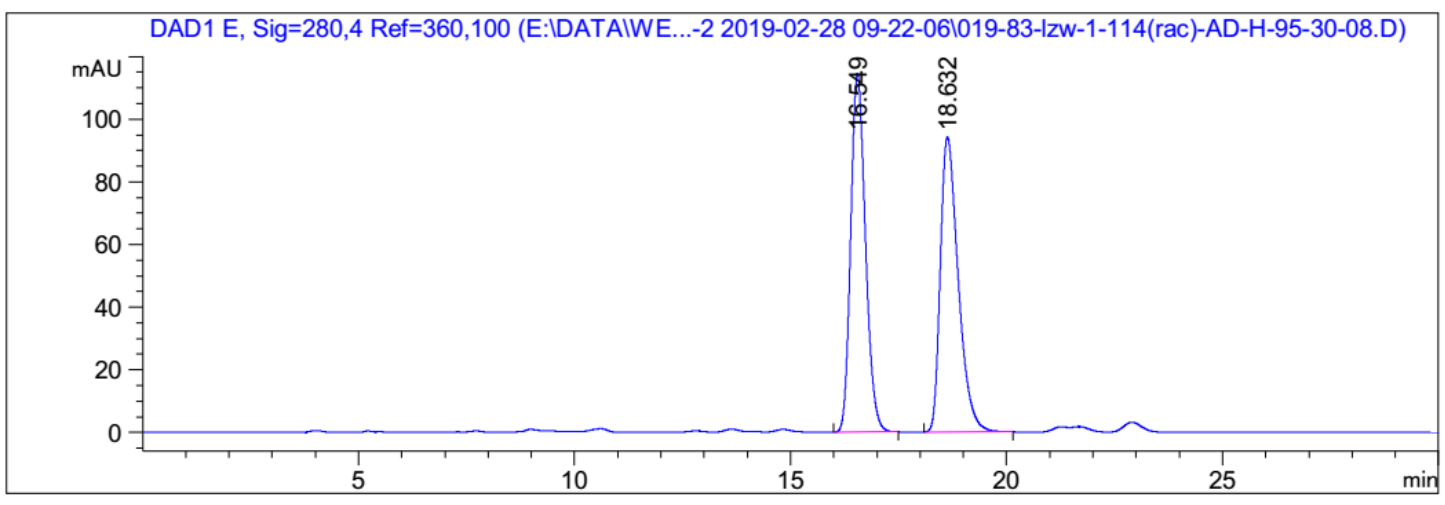

Signal 1: DAD1 E, Sig=280,4 Ref $=360,100$
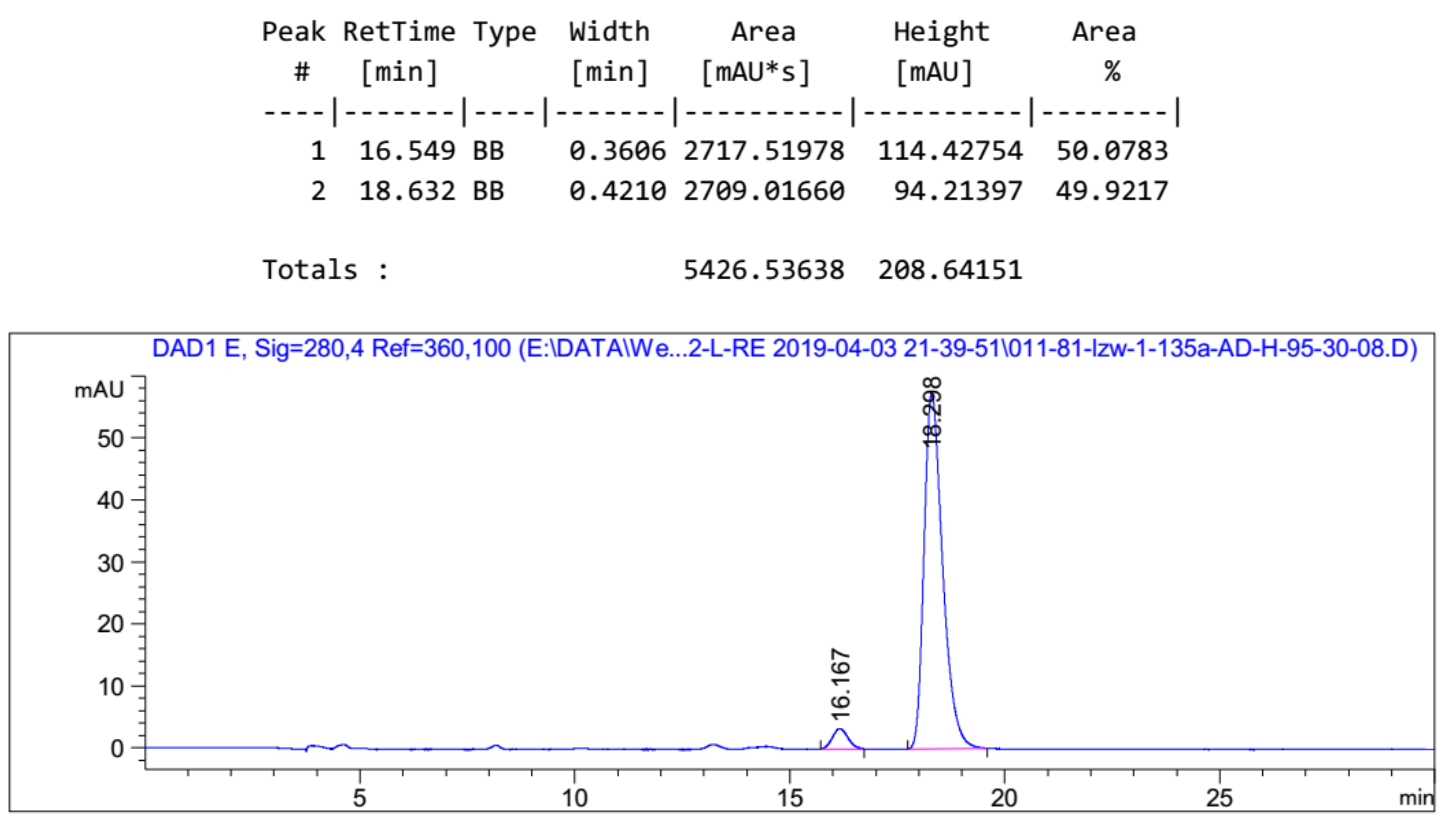

Signal 1: DAD1 E, Sig=280,4 $\operatorname{Ref}=360,100$

\begin{tabular}{|c|c|c|c|c|c|c|}
\hline $\begin{array}{c}\text { Peak } \\
\#\end{array}$ & $\begin{array}{c}\text { RetTime } \\
\text { [min] }\end{array}$ & Type & $\begin{array}{l}\text { Width } \\
\text { [min] }\end{array}$ & $\begin{array}{c}\text { Area } \\
{\left[\mathrm{mAU}^{*} \mathrm{~s}\right]}\end{array}$ & $\begin{array}{l}\text { Height } \\
\text { [mAU] }\end{array}$ & $\begin{array}{c}\text { Area } \\
\%\end{array}$ \\
\hline 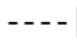 & & & & | - - & & \\
\hline 1 & 16.167 & BB & 0.2936 & 81.63767 & 3.27695 & 4.5136 \\
\hline 2 & 18.298 & BB & 0.4057 & 1727.08484 & 57.36832 & 95.4864 \\
\hline Tota. & ls: & & & 1808.72251 & 60.64527 & \\
\hline
\end{tabular}




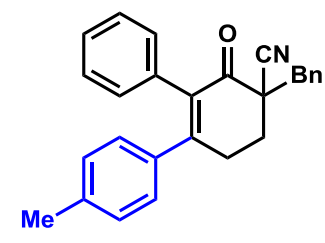

3ab

AD-H, 95\% HEX, $40 \mathrm{~min}, 0.8 \mathrm{~mL} / \mathrm{min}$

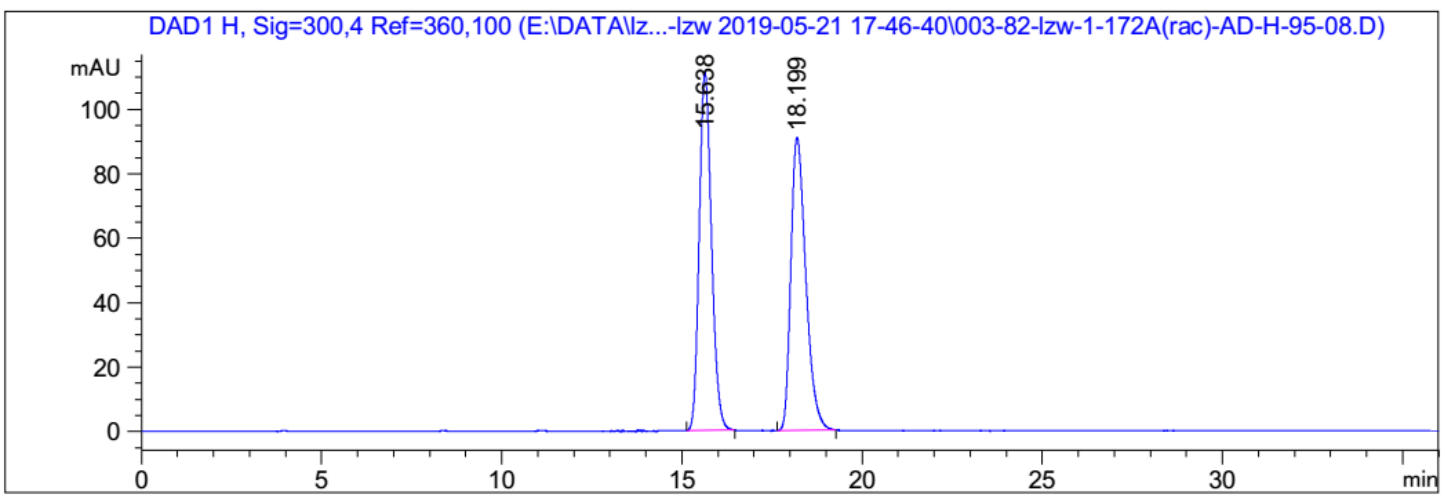

Signal 1: DAD1 H, Sig=300,4 Ref $=360,100$

\begin{tabular}{|c|c|c|c|c|c|c|}
\hline $\begin{array}{c}\text { Peak } \\
\#\end{array}$ & $\begin{array}{c}\text { RetTime } \\
\text { [min] }\end{array}$ & Type & $\begin{array}{l}\text { Width } \\
\text { [min] }\end{array}$ & $\begin{array}{c}\text { Area } \\
{\left[\mathrm{mAU}^{*} \mathrm{~s}\right]}\end{array}$ & $\begin{array}{l}\text { Height } \\
{[\mathrm{mAU}]}\end{array}$ & $\begin{array}{c}\text { Area } \\
\%\end{array}$ \\
\hline-1 & 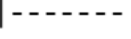 & & & 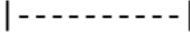 & - & - \\
\hline 1 & 15.638 & BB & 0.3520 & 2628.08594 & 111.27212 & 49.9998 \\
\hline 2 & 18.199 & BB & 0.4119 & 2628.10474 & 91.00531 & 50.0002 \\
\hline
\end{tabular}

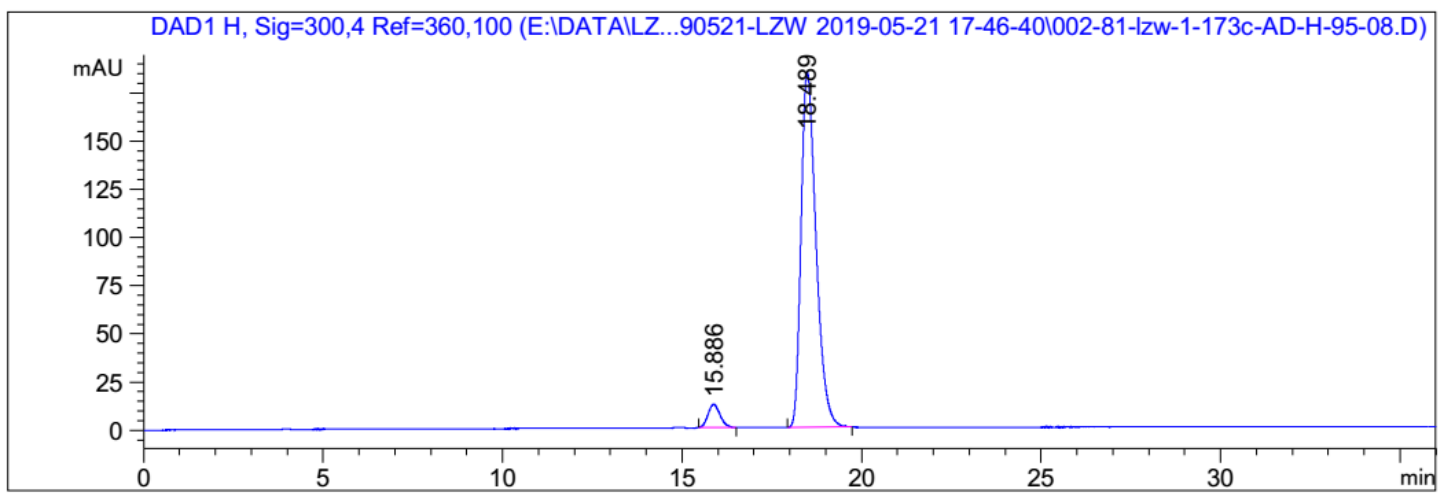

Signal 1: DAD1 H, Sig=300,4 Ref $=360,100$

\begin{tabular}{|c|c|c|c|c|c|c|}
\hline $\begin{array}{c}\text { Peak } \\
\quad \#\end{array}$ & $\begin{array}{c}\text { RetTime } \\
\text { [min] }\end{array}$ & Type & $\begin{array}{l}\text { Width } \\
\text { [min] }\end{array}$ & $\begin{array}{c}\text { Area } \\
{\left[\mathrm{mAU}^{*} \mathrm{~s}\right]}\end{array}$ & $\begin{array}{l}\text { Height } \\
{[\mathrm{mAU}]}\end{array}$ & $\begin{array}{c}\text { Area } \\
\%\end{array}$ \\
\hline--- & -- & & - & -- & $-\cdots$ & -----1 \\
\hline 1 & 15.886 & BB & 0.2781 & 283.73703 & 12.07753 & 5.0470 \\
\hline 2 & 18.489 & BB & 0.4342 & 5338.17090 & 184.14211 & 94.9530 \\
\hline Tota & . & & & 5621.90793 & 196.21963 & \\
\hline
\end{tabular}


<smiles>COc1ccc(C2=C(c3ccccc3)C(=O)C(C)(C(C)(C)C)CC2)cc1</smiles>

AD-H, 95\% HEX, $40 \mathrm{~min}, 0.8 \mathrm{~mL} / \mathrm{min}$

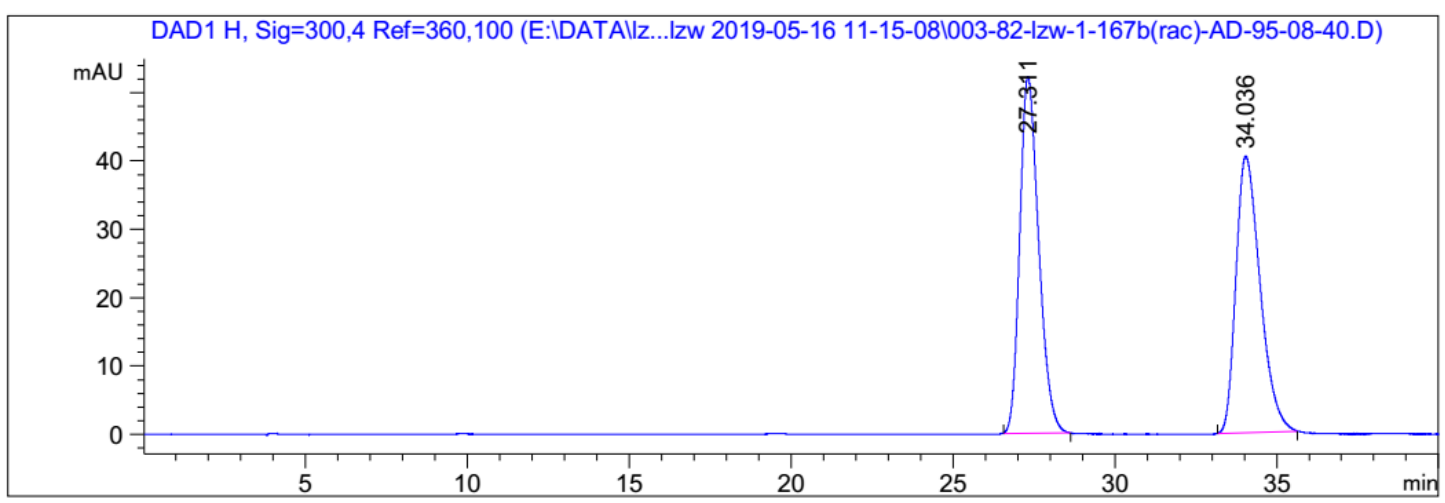

Signal 1: DAD1 H, Sig=300,4 Ref=360,100

\begin{tabular}{|c|c|c|c|c|c|c|}
\hline $\begin{array}{c}\text { Peak } \\
\quad \#\end{array}$ & $\begin{array}{c}\text { RetTime } \\
\text { [min] }\end{array}$ & Type & $\begin{array}{l}\text { Width } \\
\text { [min] }\end{array}$ & $\begin{array}{c}\text { Area } \\
{\left[\mathrm{mAU}^{*} \mathrm{~s}\right]}\end{array}$ & $\begin{array}{l}\text { Height } \\
{[\mathrm{mAU}]}\end{array}$ & $\begin{array}{c}\text { Area } \\
\%\end{array}$ \\
\hline--1 & & & & & & ------- \\
\hline 1 & 27.311 & BB & 0.4778 & 2121.85181 & 52.18815 & 50.2436 \\
\hline 2 & 34.036 & BB & 0.6103 & 2101.27856 & 40.46540 & 49.7564 \\
\hline Tota] & & & & 4223.13037 & 92.65355 & \\
\hline
\end{tabular}

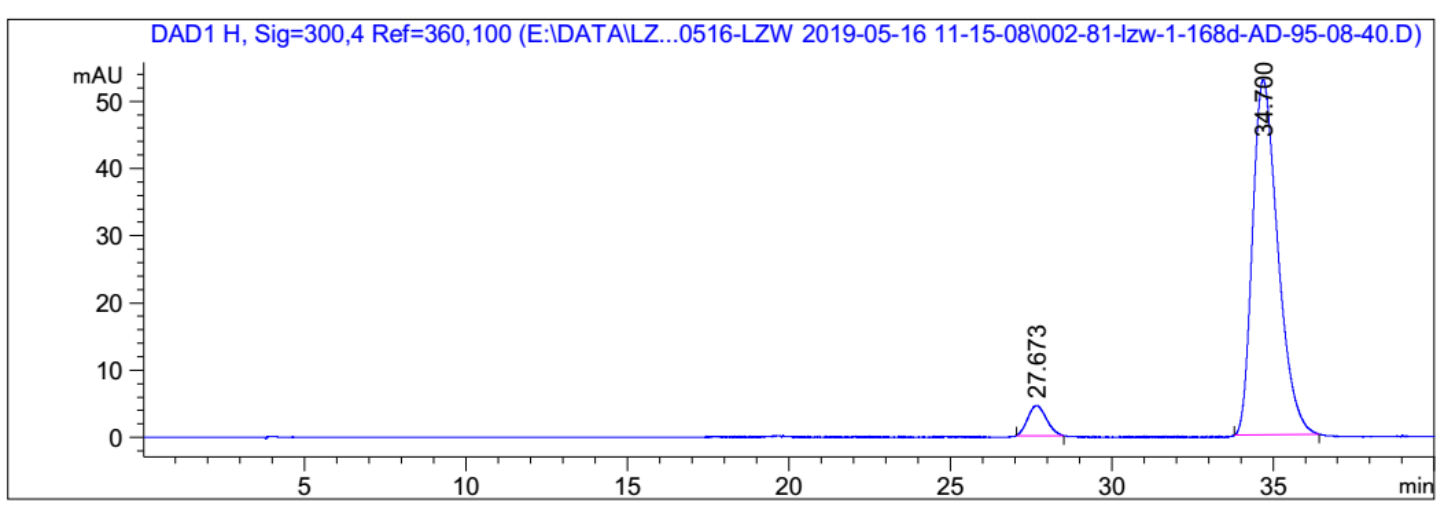

Signal 1: DAD1 H, Sig=300,4 Ref=360,100

\begin{tabular}{|c|c|c|c|c|c|c|}
\hline $\begin{array}{c}\text { Peak } \\
\#\end{array}$ & $\begin{array}{c}\text { RetTime } \\
\text { [min] }\end{array}$ & Type & $\begin{array}{l}\text { Width } \\
\text { [min] }\end{array}$ & $\begin{array}{c}\text { Area } \\
{\left[\mathrm{mAU}^{*} \mathrm{~s}\right]}\end{array}$ & $\begin{array}{l}\text { Height } \\
{[\mathrm{mAU}]}\end{array}$ & $\begin{array}{c}\text { Area } \\
\%\end{array}$ \\
\hline & & & & - & -- & ------- \\
\hline 1 & 27.673 & BB & 0.4704 & 178.89684 & 4.46509 & 5.8377 \\
\hline 2 & 34.700 & BB & 0.6408 & 2885.63330 & 52.82893 & 94.1623 \\
\hline Tota. & ls: & & & 3064.53014 & 57.29402 & \\
\hline
\end{tabular}




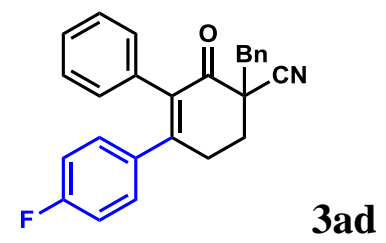

AD-H, 95\% HEX, $40 \mathrm{~min}, 0.8 \mathrm{~mL} / \mathrm{min}$

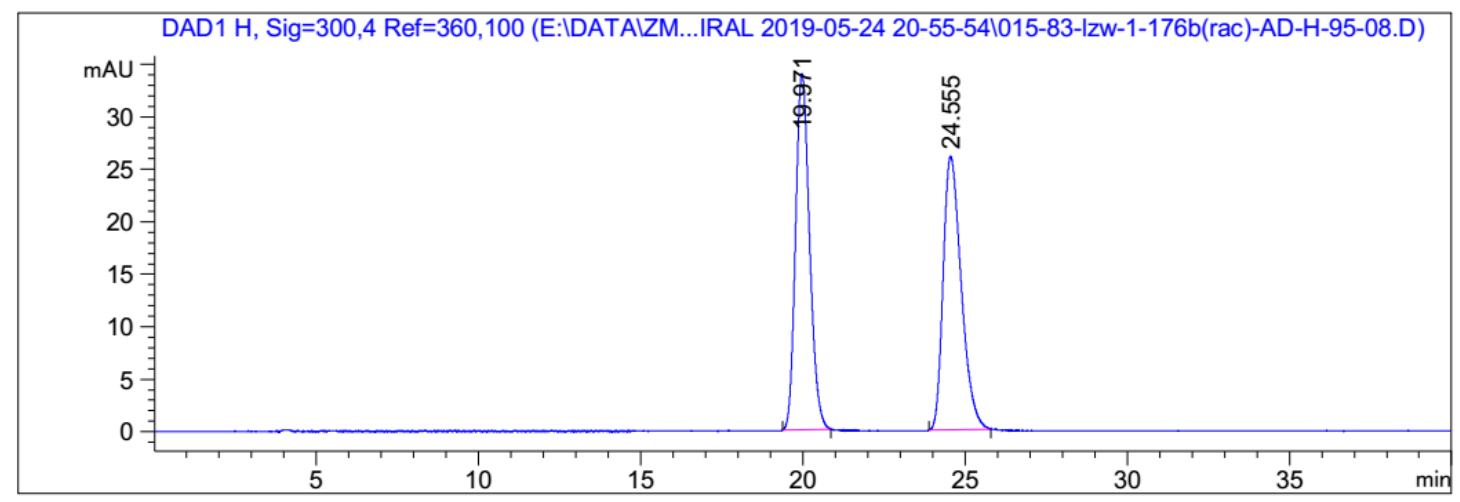

Signal 1: DAD1 H, Sig=300,4 Ref $=360,100$

\begin{tabular}{|c|c|c|c|c|c|}
\hline $\begin{array}{c}\text { Peak } \\
\#\end{array}$ & $\begin{array}{l}\text { RetTime Type } \\
\text { [min] }\end{array}$ & $\begin{array}{l}\text { Width } \\
\text { [min] }\end{array}$ & $\begin{array}{c}\text { Area } \\
{\left[\mathrm{mAU}^{*} \mathrm{~s}\right]}\end{array}$ & $\begin{array}{l}\text { Height } \\
\text { [mAU] }\end{array}$ & $\begin{array}{c}\text { Area } \\
\%\end{array}$ \\
\hline---1 & $\mid--$ & - & 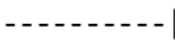 & | & - - - - \\
\hline 1 & 19.971 BB & 0.3568 & 1016.42285 & 33.94595 & 50.1668 \\
\hline 2 & 24.555 BB & 0.4555 & 1009.66260 & 26.06024 & 49.8332 \\
\hline \multicolumn{3}{|c|}{ Totals : } & 2026.08545 & 60.00619 & \\
\hline
\end{tabular}

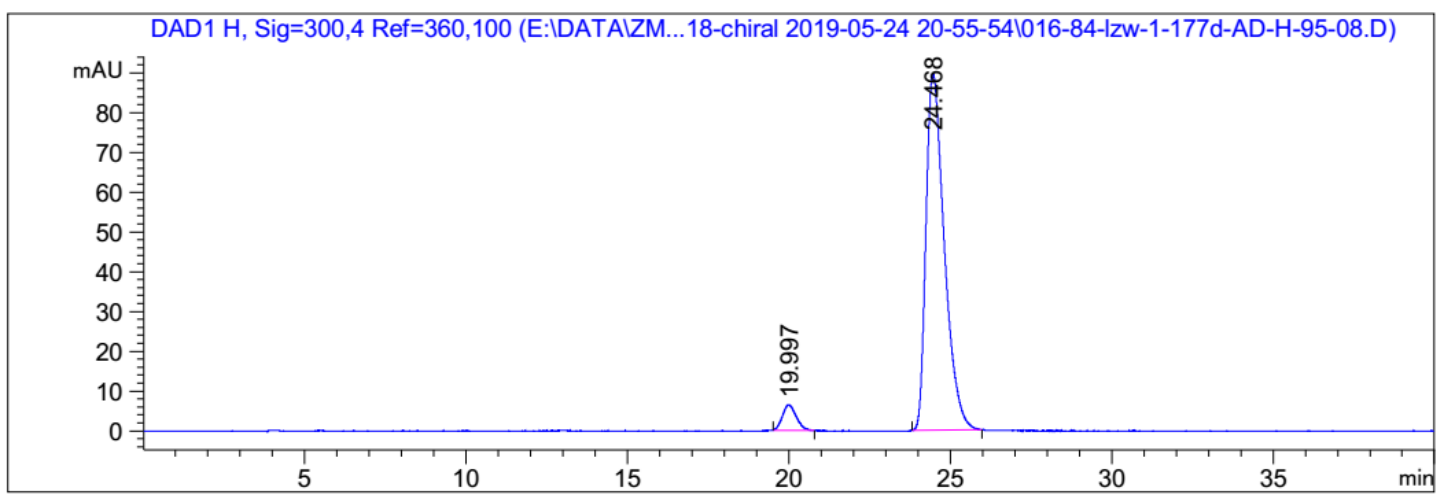

Signal 1: DAD1 H, Sig=300,4 Ref $=360,100$

\begin{tabular}{|c|c|c|c|c|c|}
\hline $\begin{array}{c}\text { Peak } \\
\#\end{array}$ & $\begin{array}{l}\text { RetTime Type } \\
\text { [min] }\end{array}$ & $\begin{array}{l}\text { Width } \\
\text { [min] }\end{array}$ & $\begin{array}{c}\text { Area } \\
{[\mathrm{mAU} * \mathrm{~s}]}\end{array}$ & $\begin{array}{l}\text { Height } \\
{[\mathrm{mAU}]}\end{array}$ & $\begin{array}{c}\text { Area } \\
\%\end{array}$ \\
\hline- & $|-----|--$ & 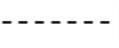 & | & | & $-\cdots-1$ \\
\hline 1 & 19.997 BB & 0.3474 & 189.65924 & 6.41500 & 5.0840 \\
\hline 2 & 24.468 BB & 0.5417 & 3540.87622 & 89.57404 & 94.9160 \\
\hline \multicolumn{3}{|c|}{ Totals : } & 3730.53546 & 95.98905 & \\
\hline
\end{tabular}


<smiles></smiles>

$\mathrm{AD}-\mathrm{H}, 95 \% \mathrm{HEX}, 40 \mathrm{~min}, 0.8 \mathrm{~mL} / \mathrm{min}$

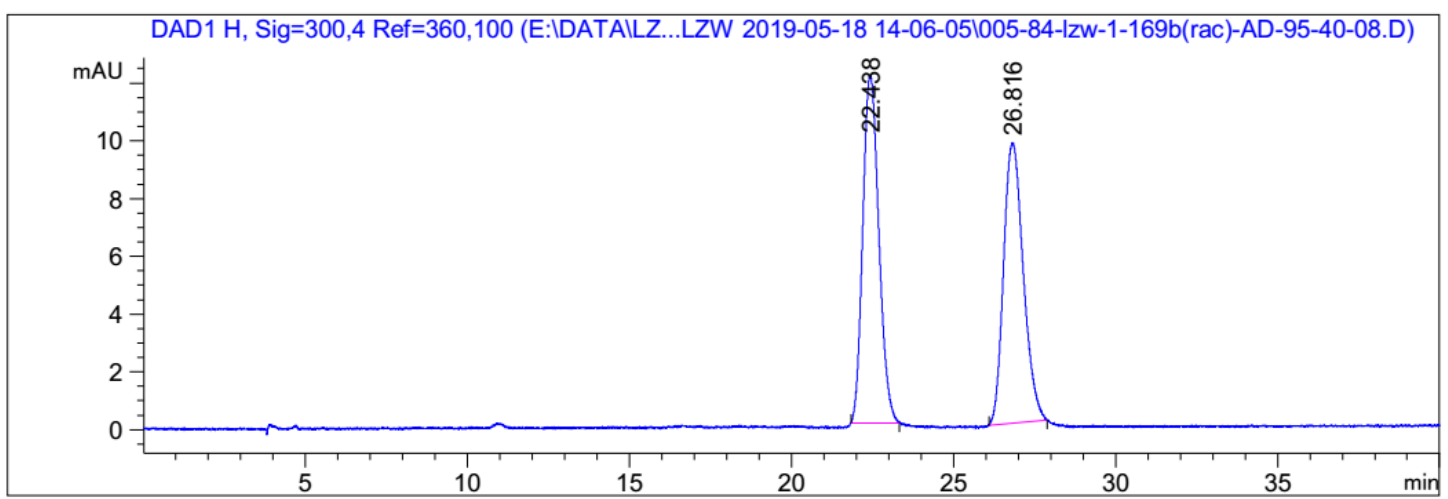

Signal 1: DAD1 H, Sig=300,4 Ref $=360,100$
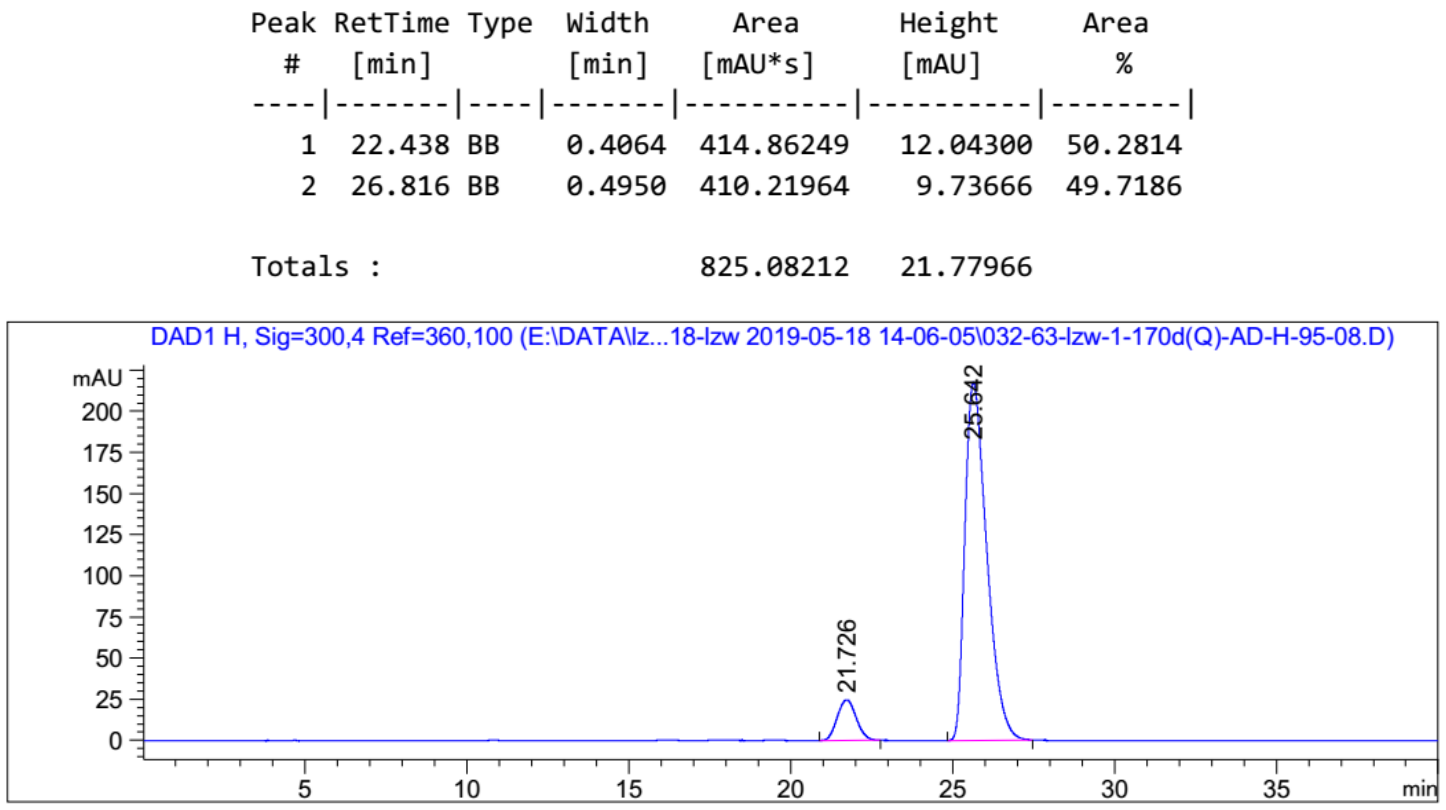

Signal 1: DAD1 H, Sig=300,4 Ref $=360,100$

\begin{tabular}{|c|c|c|c|c|c|}
\hline $\begin{array}{c}\text { Peak } \\
\#\end{array}$ & $\begin{array}{l}\text { RetTime Type } \\
\text { [min] }\end{array}$ & $\begin{array}{l}\text { Width } \\
\text { [min] }\end{array}$ & $\begin{array}{c}\text { Area } \\
{\left[\mathrm{mAU}^{*} \mathrm{~s}\right]}\end{array}$ & $\begin{array}{l}\text { Height } \\
{[\mathrm{mAU}]}\end{array}$ & $\begin{array}{c}\text { Area } \\
\%\end{array}$ \\
\hline 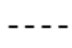 & & & & & 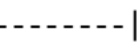 \\
\hline 1 & 21.726 вВ & 0.4 & 1008.43506 & 24.74788 & 8.9919 \\
\hline 2 & $25.642 \mathrm{BB}$ & 0.5924 & $1.02065 \mathrm{e} 4$ & 217.52884 & 91.0081 \\
\hline
\end{tabular}
Totals :
$1.12149 \mathrm{e} 4 \quad 242.27672$ 
$\underbrace{c}_{3 a f}$

$\mathrm{AD}-\mathrm{H}, 95 \% \mathrm{HEX}, 40 \mathrm{~min}, 0.8 \mathrm{~mL} / \mathrm{min}$

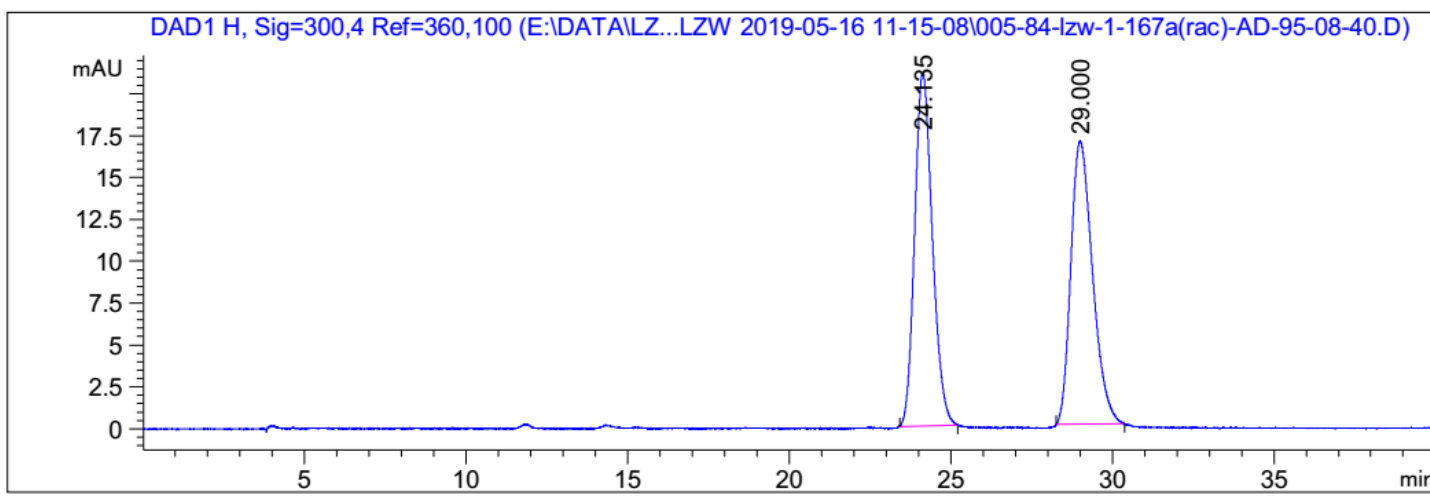

Signal 1: DAD1 H, Sig=300,4 Ref $=360,100$

\begin{tabular}{|c|c|c|c|c|c|c|}
\hline $\begin{array}{c}\text { Peak } \\
\#\end{array}$ & $\begin{array}{c}\text { RetTime } \\
\text { [min] }\end{array}$ & Type & $\begin{array}{l}\text { Width } \\
\text { [min] }\end{array}$ & $\begin{array}{c}\text { Area } \\
{\left[\mathrm{mAU}^{*} \mathrm{~s}\right]}\end{array}$ & $\begin{array}{l}\text { Height } \\
{[\mathrm{mAU}]}\end{array}$ & $\begin{array}{c}\text { Area } \\
\%\end{array}$ \\
\hline- & $\mid--$ & & -- & - - - & | & - - \\
\hline 1 & 24.135 & BB & 0.4471 & 802.67780 & 21.08880 & 50.4570 \\
\hline 2 & 29.000 & BB & 0.5460 & 788.13788 & 16.91813 & 49.5430 \\
\hline
\end{tabular}

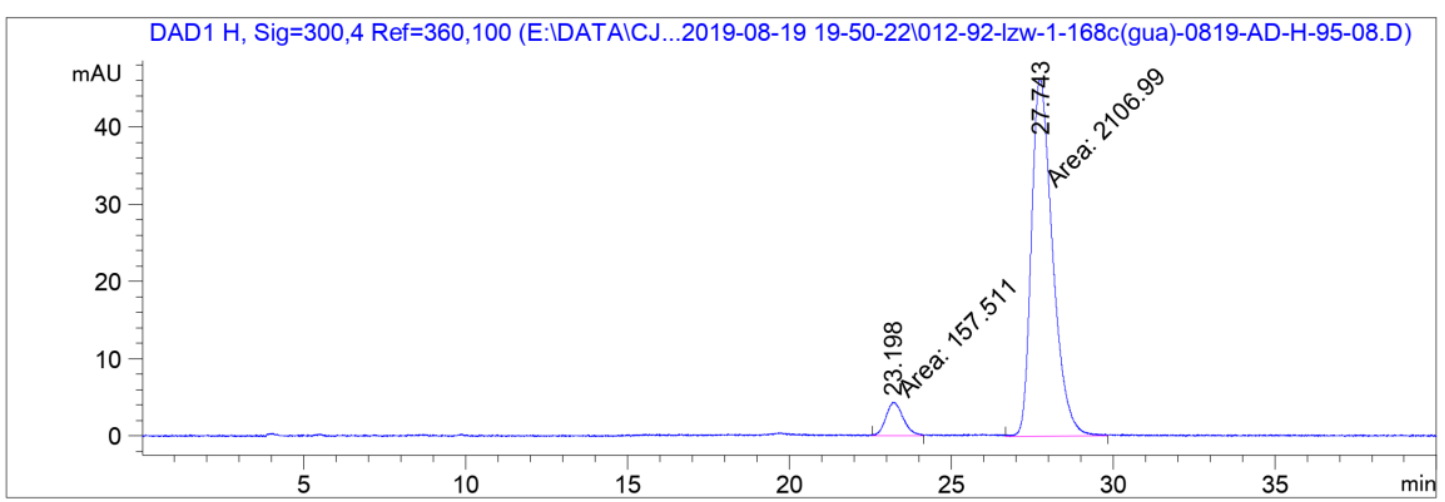

Signal 1: DAD1 H, Sig=300,4 Ref=360,100

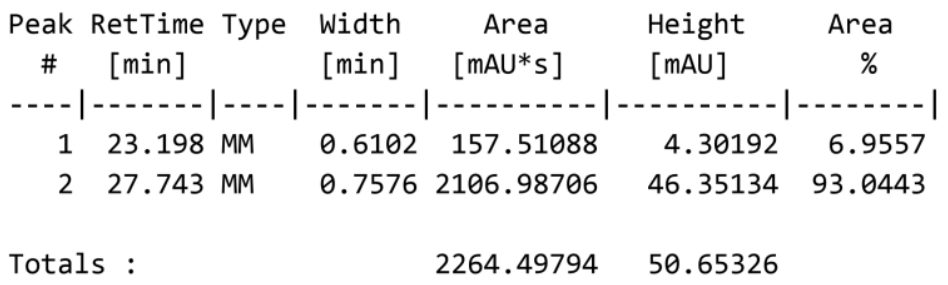


<smiles>N#CC1(Br)CCC(c2ccc(C(F)(F)F)cc2)=C(c2ccccc2)C1=O</smiles>

$\mathrm{AD}-\mathrm{H}, 95 \% \mathrm{HEX}, 40 \mathrm{~min}, 0.8 \mathrm{~mL} / \mathrm{min}$

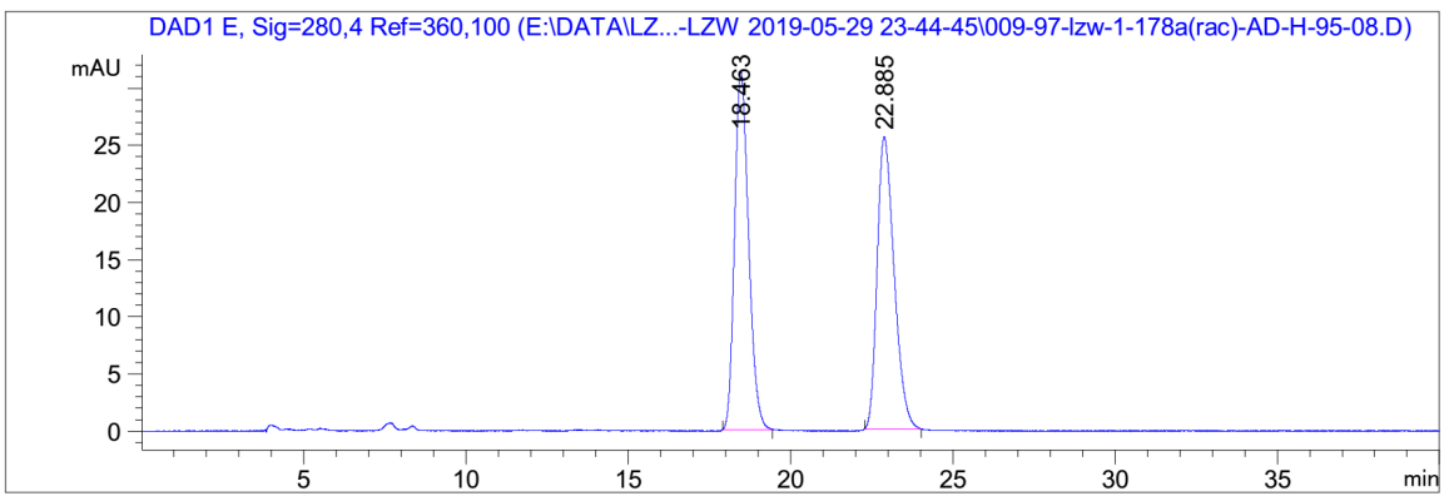

Signal 1: DAD1 E, Sig=280,4 Ref $=360,100$

\begin{tabular}{|c|c|c|c|c|c|c|}
\hline $\begin{array}{c}\text { Peak } \\
\quad \#\end{array}$ & $\begin{array}{c}\text { RetTime } \\
\text { [min] }\end{array}$ & Type & $\begin{array}{l}\text { Width } \\
\text { [min] }\end{array}$ & $\begin{array}{c}\text { Area } \\
{\left[\mathrm{mAU}^{*} \mathrm{~s}\right]}\end{array}$ & $\begin{array}{l}\text { Height } \\
{[\mathrm{mAU}]}\end{array}$ & $\begin{array}{c}\text { Area } \\
\%\end{array}$ \\
\hline & & & & --- & -1 & -- \\
\hline 1 & 18.463 & BB & 0.3585 & 941.03619 & 31.27589 & 50.2485 \\
\hline 2 & 22.885 & BB & 0.4313 & 931.72833 & 25.58786 & 49.7515 \\
\hline Tota & s: & & & 1872.76453 & 6.86375 & \\
\hline
\end{tabular}

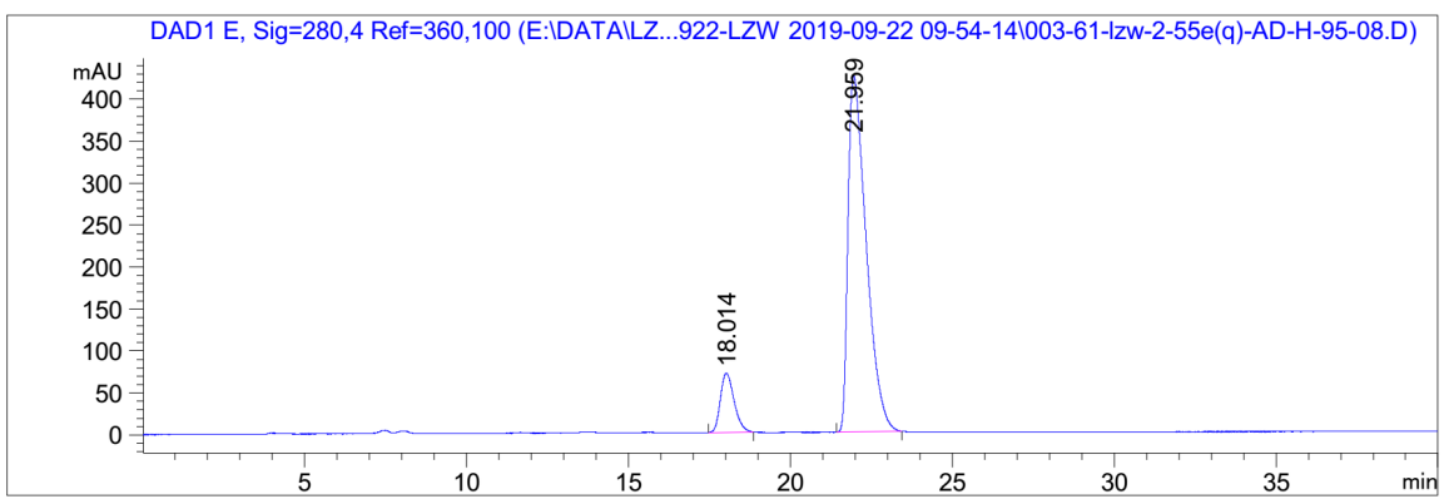

Signal 1: DAD1 E, Sig=280,4 Ref=360,100

\begin{tabular}{|c|c|c|c|c|c|c|}
\hline $\begin{array}{c}\text { Peak } \\
\#\end{array}$ & $\begin{array}{c}\text { RetTime } \\
\text { [min] }\end{array}$ & Type & $\begin{array}{l}\text { Width } \\
\text { [min] }\end{array}$ & $\begin{array}{c}\text { Area } \\
{\left[\mathrm{mAU}^{*} \mathrm{~s}\right]}\end{array}$ & $\begin{array}{l}\text { Height } \\
{[\mathrm{mAU}]}\end{array}$ & $\begin{array}{c}\text { Area } \\
\%\end{array}$ \\
\hline & & & & & 5 & ------ \\
\hline 1 & 18.014 & W R & 0.3480 & 2081.23975 & 70.37261 & 11.1684 \\
\hline 2 & 21.959 & $\mathrm{~W} R$ & 0.4587 & $1.65538 \mathrm{e} 4$ & 424.29568 & 88.8316 \\
\hline Tota & Is: & & & $1.86351 \mathrm{e} 4$ & 494.66830 & \\
\hline
\end{tabular}


<smiles>N#CC1(Br)CCC(c2ccc(OC(F)(F)F)cc2)=C(c2ccccc2)C1=O</smiles>

$\mathrm{AD}-\mathrm{H}, 95 \% \mathrm{HEX}, 40 \mathrm{~min}, 0.8 \mathrm{~mL} / \mathrm{min}$

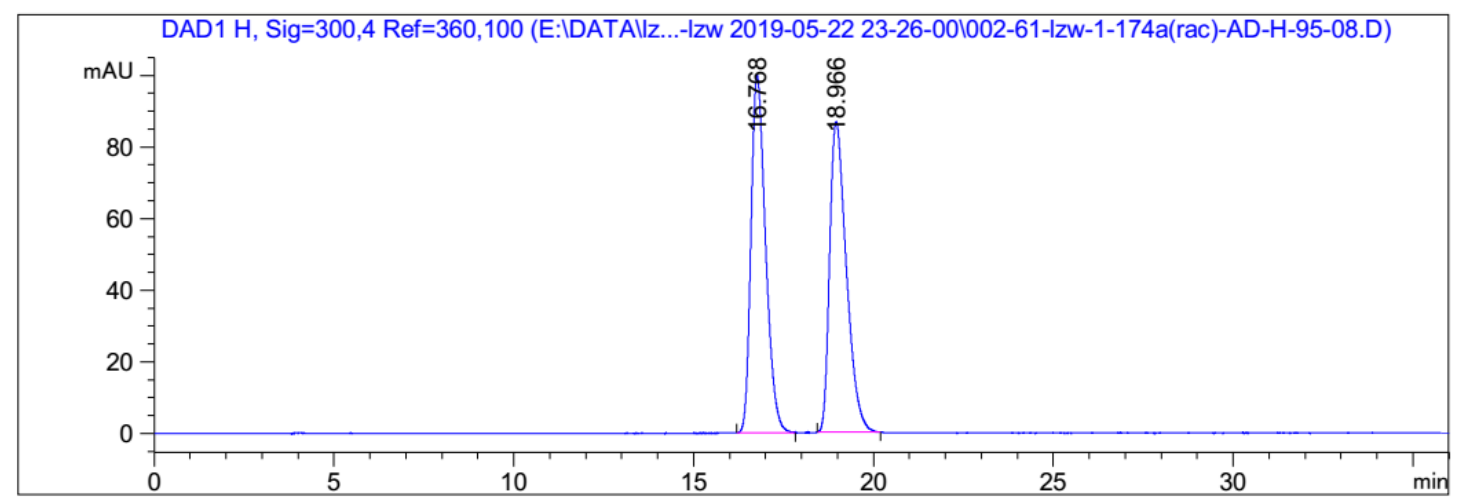

Signal 1: DAD1 H, Sig=300,4 Ref=360,100

\begin{tabular}{cccccc}
$\begin{array}{c}\text { Peak RetTime Type } \\
\#\end{array}$ & $\begin{array}{c}\text { Width } \\
\text { [min] }\end{array}$ & $\begin{array}{c}\text { Area } \\
{[\mathrm{min}]}\end{array}$ & $\begin{array}{c}\text { Height } \\
{[\mathrm{mAU} * \mathrm{~S}]}\end{array}$ & $\begin{array}{c}\text { Area } \\
{[\mathrm{mAU}]}\end{array}$ & $\%$ \\
\hline 1 & 16.768 BB & 0.3947 & 2808.68433 & 99.82922 & 50.0907 \\
2 & 18.966 BB & 0.4306 & 2798.50806 & 86.80452 & 49.9093
\end{tabular}

Totals :

$5607.19238 \quad 186.63374$

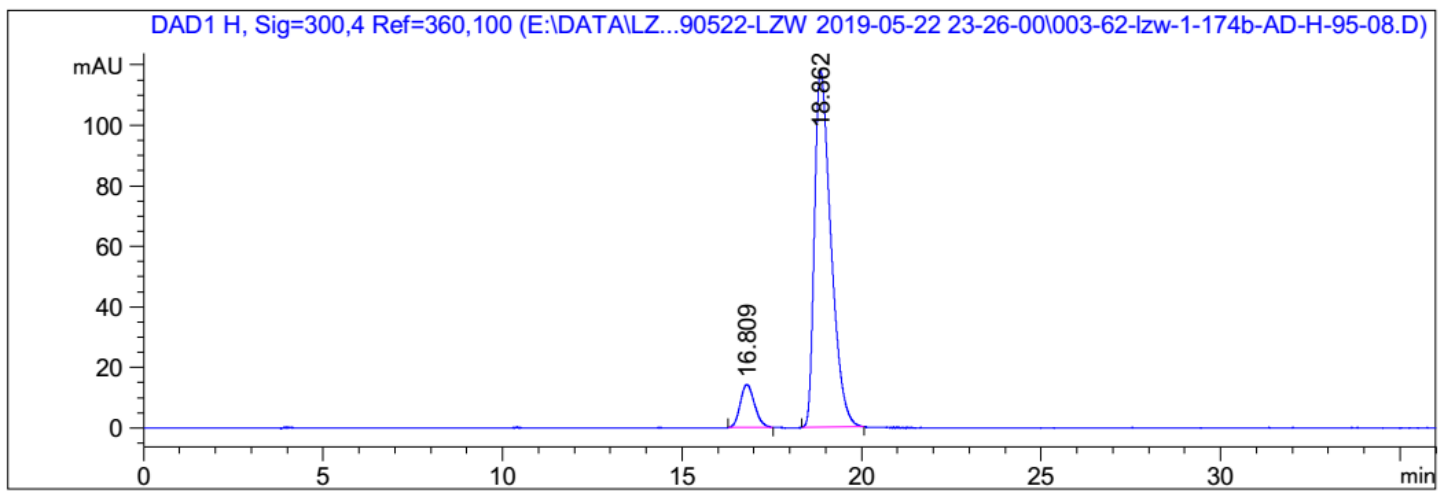

Signal 1: DAD1 H, Sig=300,4 Ref=360,100

\begin{tabular}{|c|c|c|c|c|c|c|}
\hline $\begin{array}{c}\text { Peak } \\
\#\end{array}$ & $\begin{array}{c}\text { RetTime } \\
\text { [min] }\end{array}$ & Type & $\begin{array}{l}\text { Width } \\
\text { [min] }\end{array}$ & $\begin{array}{c}\text { Area } \\
{\left[\mathrm{mAU}^{*} \mathrm{~s}\right]}\end{array}$ & $\begin{array}{l}\text { Height } \\
\text { [mAU] }\end{array}$ & $\begin{array}{c}\text { Area } \\
\%\end{array}$ \\
\hline 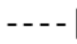 & & & & -- & -- & ---.-- \\
\hline 1 & 16.809 & BB & 0.3237 & 392.89270 & 14.22603 & 9.4604 \\
\hline 2 & 18.862 & BB & 0.4568 & 3760.14478 & 117.81679 & 90.5396 \\
\hline Tota] & Is: & & & 4153.03748 & 132.04282 & \\
\hline
\end{tabular}




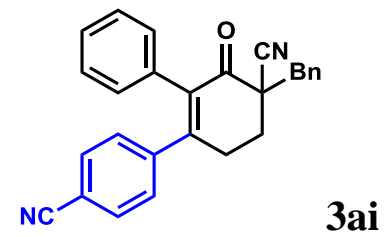

OD-H, 70\% HEX, 40min, $0.8 \mathrm{~mL} / \mathrm{min}$

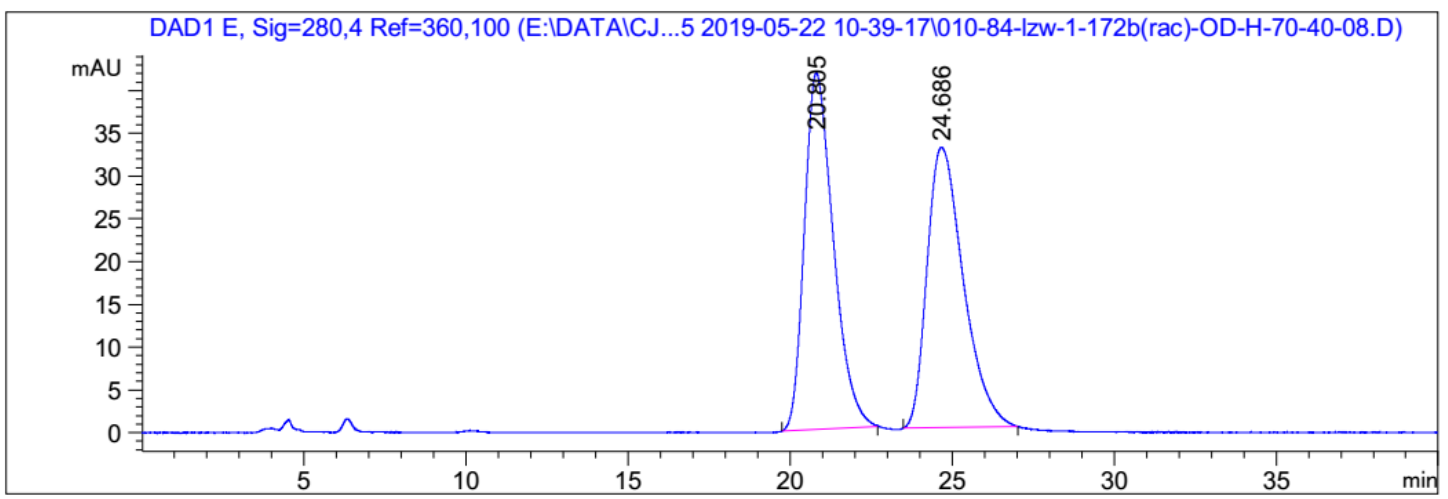

Signal 1: DAD1 E, Sig $=280,4$ Ref $=360,100$
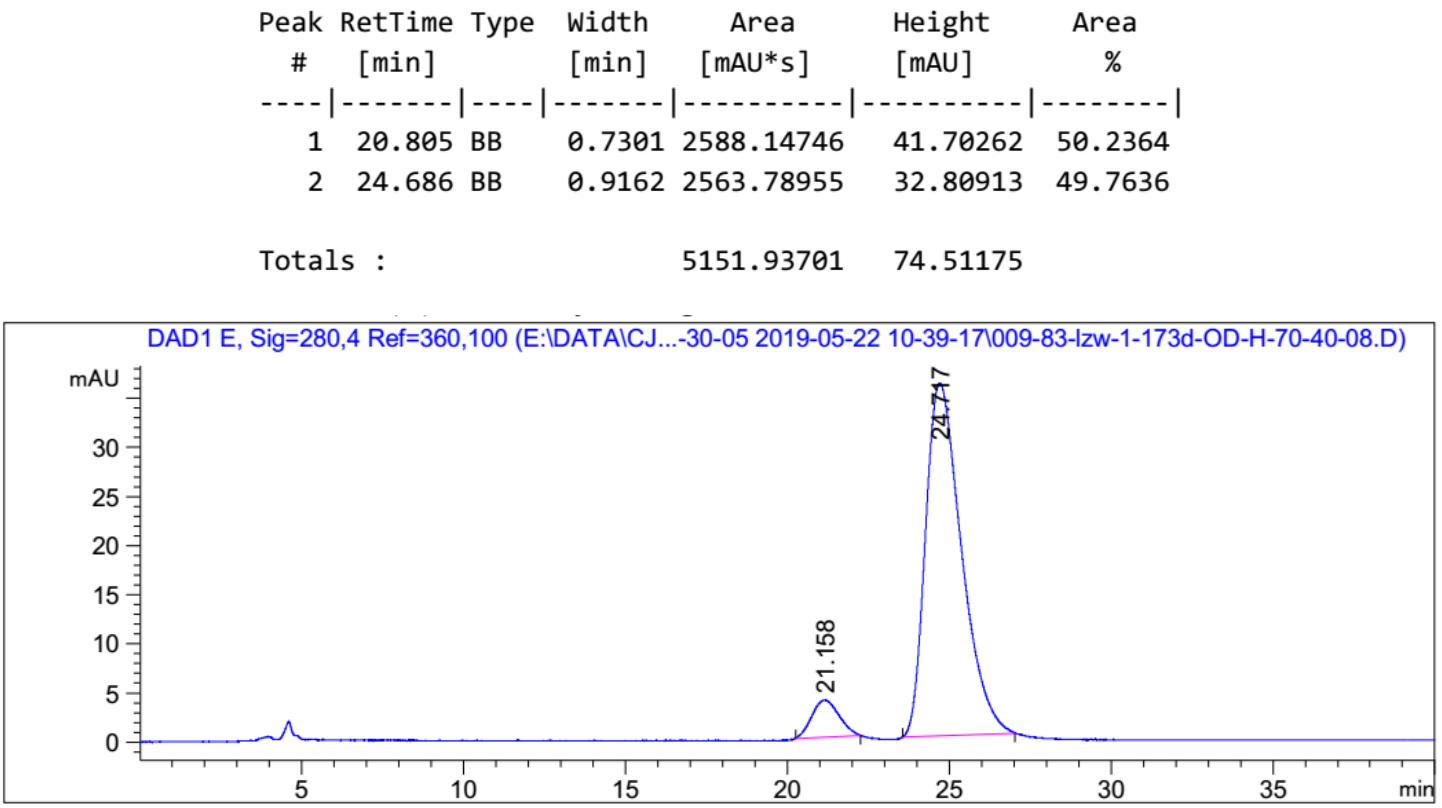

Signal 1: DAD1 E, Sig=280,4 Ref $=360,100$

\begin{tabular}{|c|c|c|c|c|c|}
\hline $\begin{array}{c}\text { Peak } \\
\#\end{array}$ & $\begin{array}{l}\text { RetTime Type } \\
\text { [min] }\end{array}$ & $\begin{array}{l}\text { Width } \\
\text { [min] }\end{array}$ & $\begin{array}{c}\text { Area } \\
{\left[\mathrm{mAU}^{*} \mathrm{~s}\right]}\end{array}$ & $\begin{array}{l}\text { Height } \\
{[\mathrm{mAU}]}\end{array}$ & $\begin{array}{c}\text { Area } \\
\%\end{array}$ \\
\hline-- & $-1-$ & $\cdots-1$ & -------- & $-\ldots$ & 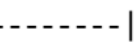 \\
\hline 1 & 21.158 BВ & 0.6815 & 220.42685 & 3.79005 & 7.3667 \\
\hline 2 & 24.717 BB & 0.9046 & 2771.77905 & 35.86761 & 92.6333 \\
\hline Tota & : & & 2992.20590 & 39.65766 & \\
\hline
\end{tabular}


<smiles>CC1(C)CCC2=C(c3ccc(C=O)cc3)C(=O)C1(C)CC2</smiles>

OD-H, 70\% HEX, 40min, $1 \mathrm{~mL} / \mathrm{min}$

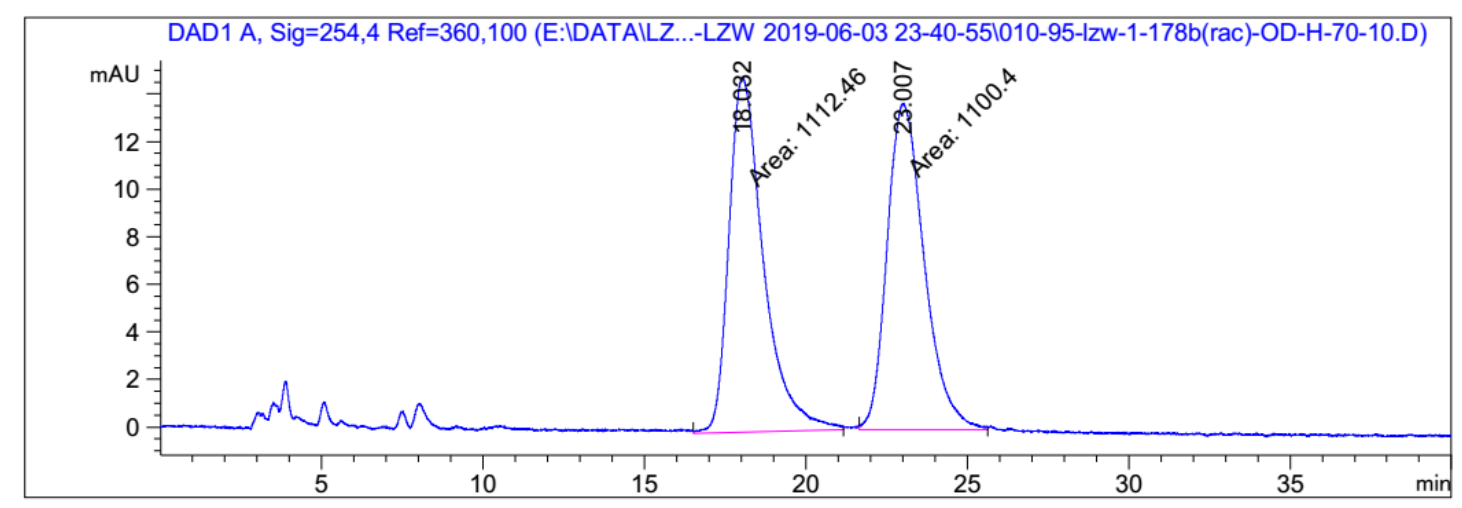

Signal 1: DAD1 A, Sig=254,4 $\operatorname{Ref}=360,100$
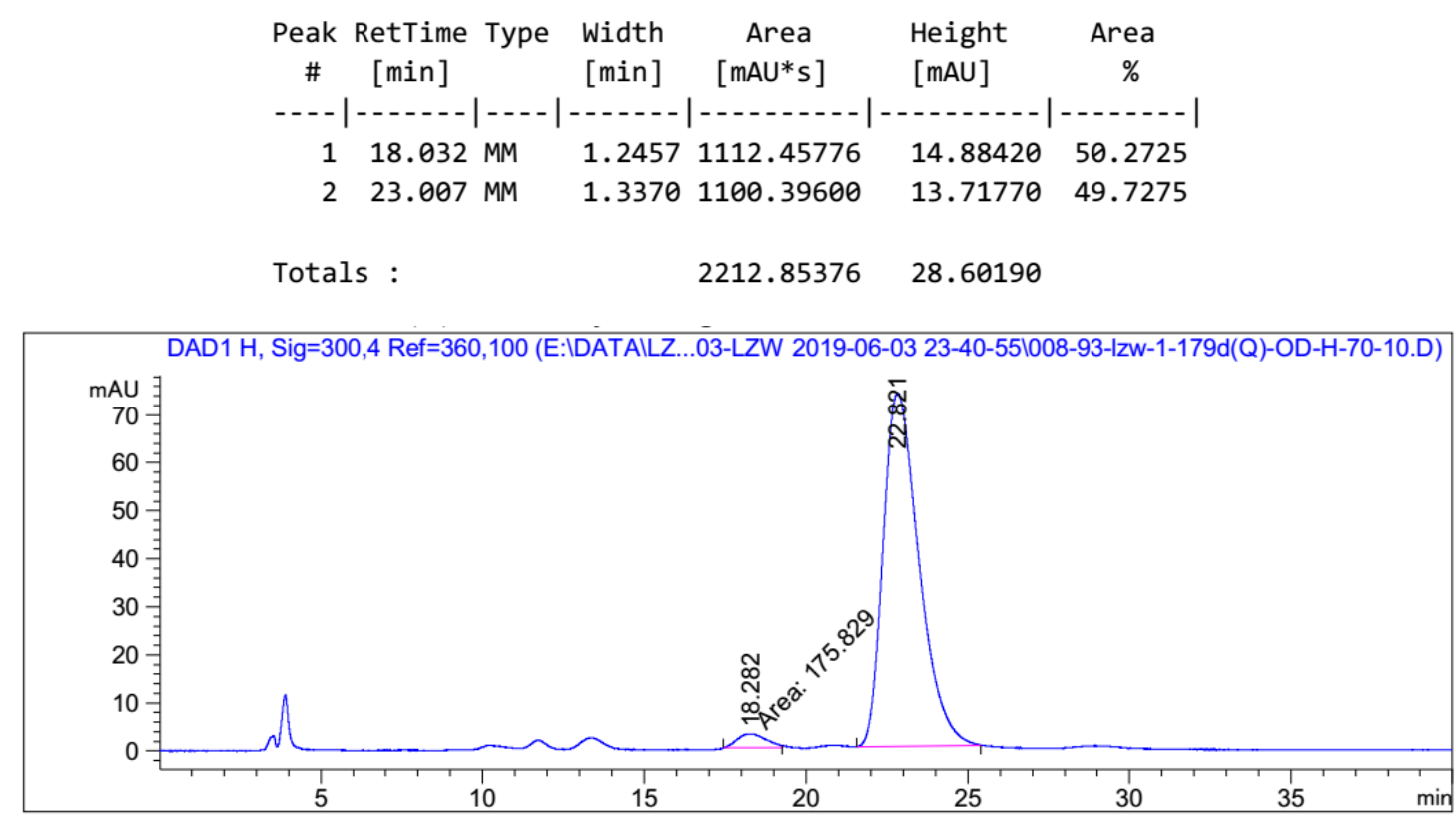

Signal 1: DAD1 H, Sig=300,4 Ref $=360,100$

\begin{tabular}{|c|c|c|c|c|c|c|}
\hline $\begin{array}{c}\text { Peak } \\
\#\end{array}$ & $\begin{array}{c}\text { RetTime } \\
\text { [min] }\end{array}$ & Type & $\begin{array}{l}\text { Width } \\
\text { [min] }\end{array}$ & $\begin{array}{c}\text { Area } \\
{\left[\mathrm{mAU}^{*} \mathrm{~s}\right]}\end{array}$ & $\begin{array}{l}\text { Height } \\
{[\mathrm{mAU}]}\end{array}$ & $\begin{array}{c}\text { Area } \\
\%\end{array}$ \\
\hline & & & & & 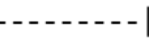 & ------1 \\
\hline 1 & 18.282 & MM & 1.0252 & 175.82883 & 2.85847 & 3.0112 \\
\hline 2 & 22.821 & BB & 0.9007 & 5663.29785 & 73.60427 & 96.9888 \\
\hline Tota. & Is: & & & 5839.12668 & 76.46274 & \\
\hline
\end{tabular}




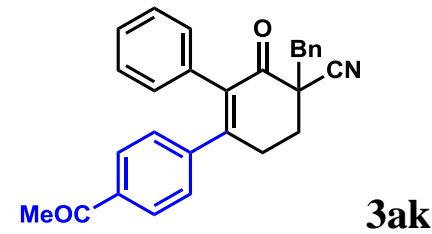

AD-H, AD-H, 75\% HEX, 45min, $1.0 \mathrm{~mL} / \mathrm{min}$

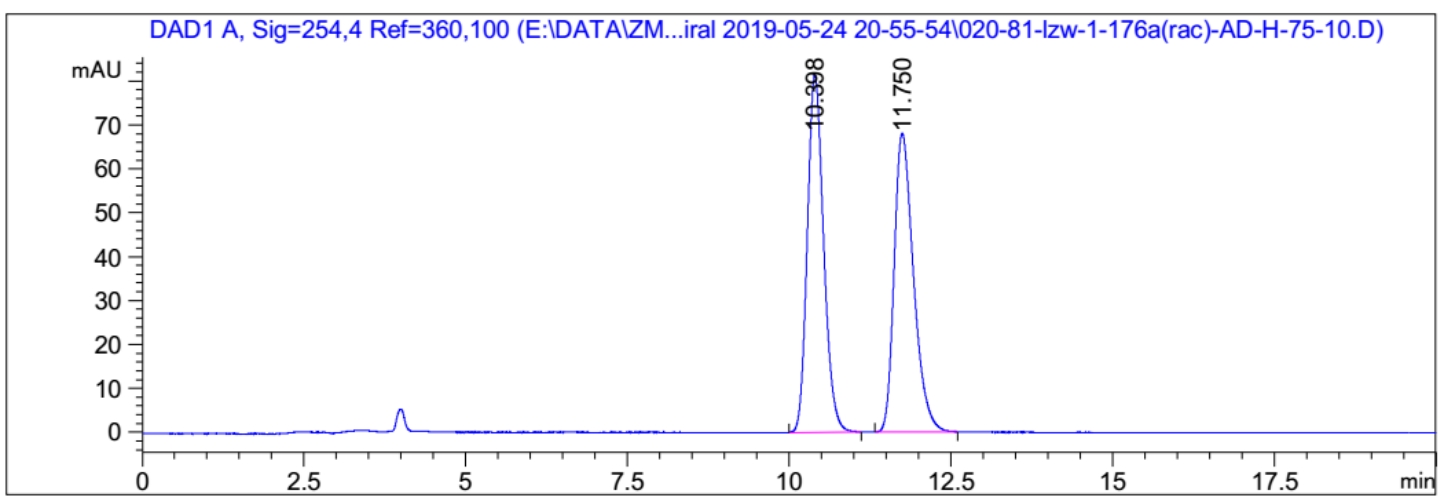

Signal 1: DAD1 A, Sig=254,4 Ref=360,100

\begin{tabular}{cccccc}
$\begin{array}{c}\text { Peak RetTime Type } \\
\#\end{array} \begin{array}{c}\text { Width } \\
{[\mathrm{min}]}\end{array}$ & $\begin{array}{c}\text { Area } \\
{[\mathrm{min}]}\end{array}$ & $\begin{array}{c}\text { Height } \\
{[\mathrm{mAU} \text { s }]}\end{array}$ & $\begin{array}{c}\text { Area } \\
{[\mathrm{mAU}]}\end{array}$ & $\%$ \\
\hline 1 & 10.398 BB & 0.2630 & 1427.86230 & 81.47675 & 50.1367 \\
2 & 11.750 BB & 0.3144 & 1420.07446 & 68.06273 & 49.8633
\end{tabular}

Totals :

$2847.93677 \quad 149.53947$

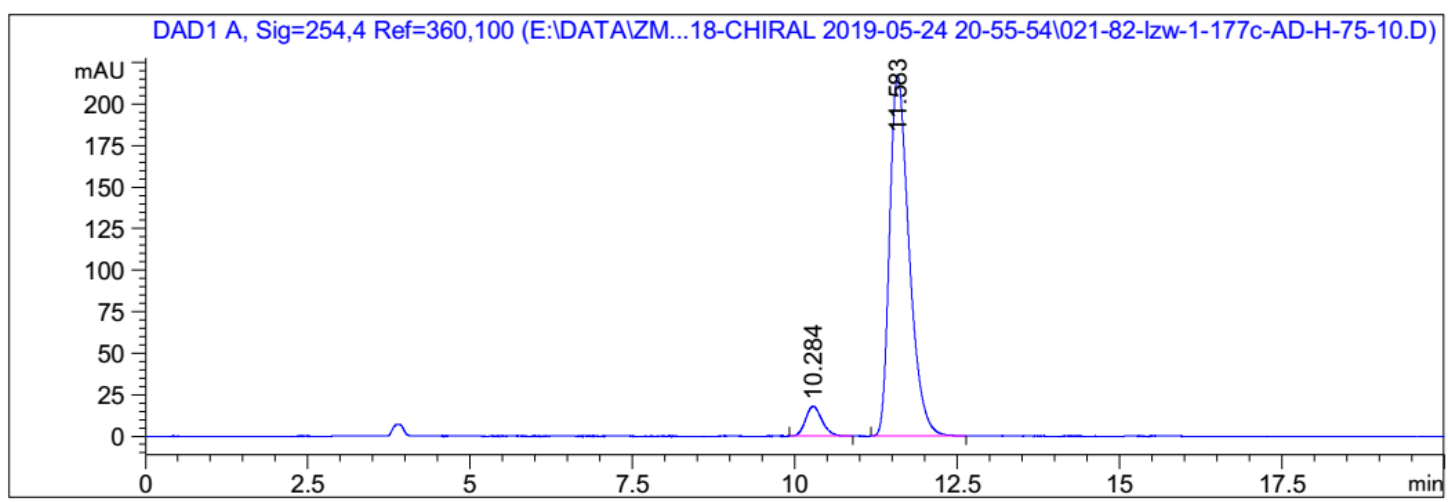

Signal 1: DAD1 A, Sig=254,4 Ref $=360,100$

\begin{tabular}{|c|c|c|c|c|c|c|}
\hline $\begin{array}{c}\text { Peak } \\
\quad \#\end{array}$ & $\begin{array}{c}\text { RetTime } \\
\text { [min] }\end{array}$ & Type & $\begin{array}{l}\text { Width } \\
\text { [min] }\end{array}$ & $\begin{array}{c}\text { Area } \\
{\left[\mathrm{mAU} \mathbf{U}^{*} \mathrm{~s}\right]}\end{array}$ & $\begin{array}{l}\text { Height } \\
\text { [mAU] }\end{array}$ & $\begin{array}{c}\text { Area } \\
\%\end{array}$ \\
\hline & & & & | - - - & --- & | \\
\hline 1 & 10.284 & BB & 0.2467 & 315.78299 & 18.00103 & 6.5096 \\
\hline 2 & 11.583 & BB & 0.3212 & 4535.25293 & 217.09393 & 93.4904 \\
\hline Tota & : & & & 4851.03592 & 235.09496 & \\
\hline
\end{tabular}


<smiles>CC(=O)c1ccc(C2=C(c3ccccc3)C(=O)C3(CC2)C[Se]3)cc1</smiles>

AS-H, 80\% HEX, 40min, $1 \mathrm{~mL} / \mathrm{min}$

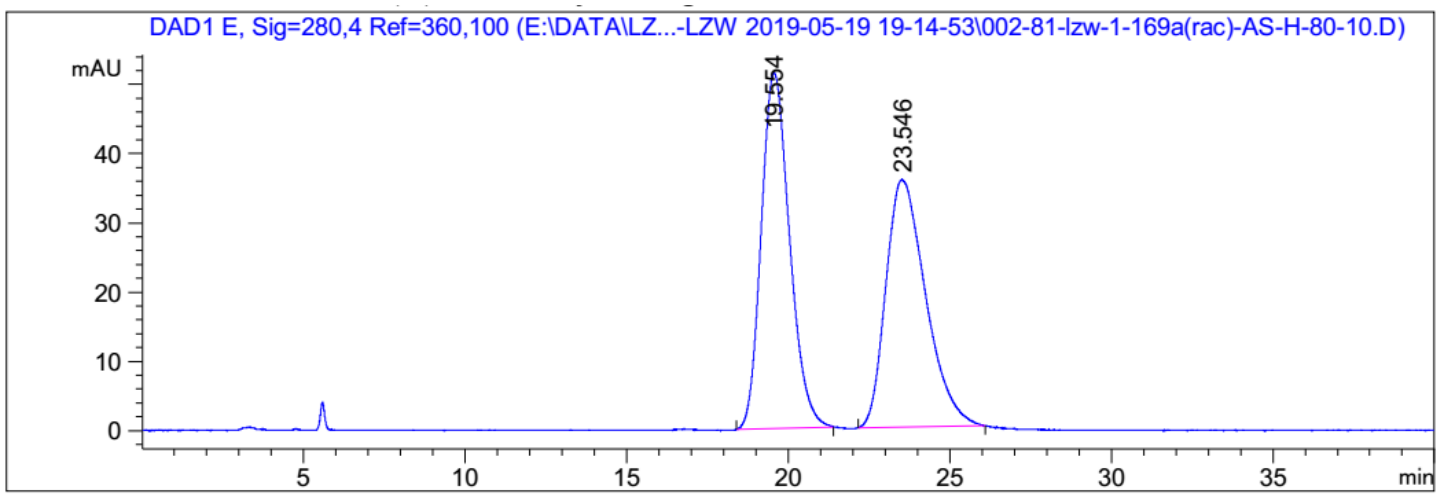

Signal 1: DAD1 E, Sig $=280,4$ Ref $=360,100$
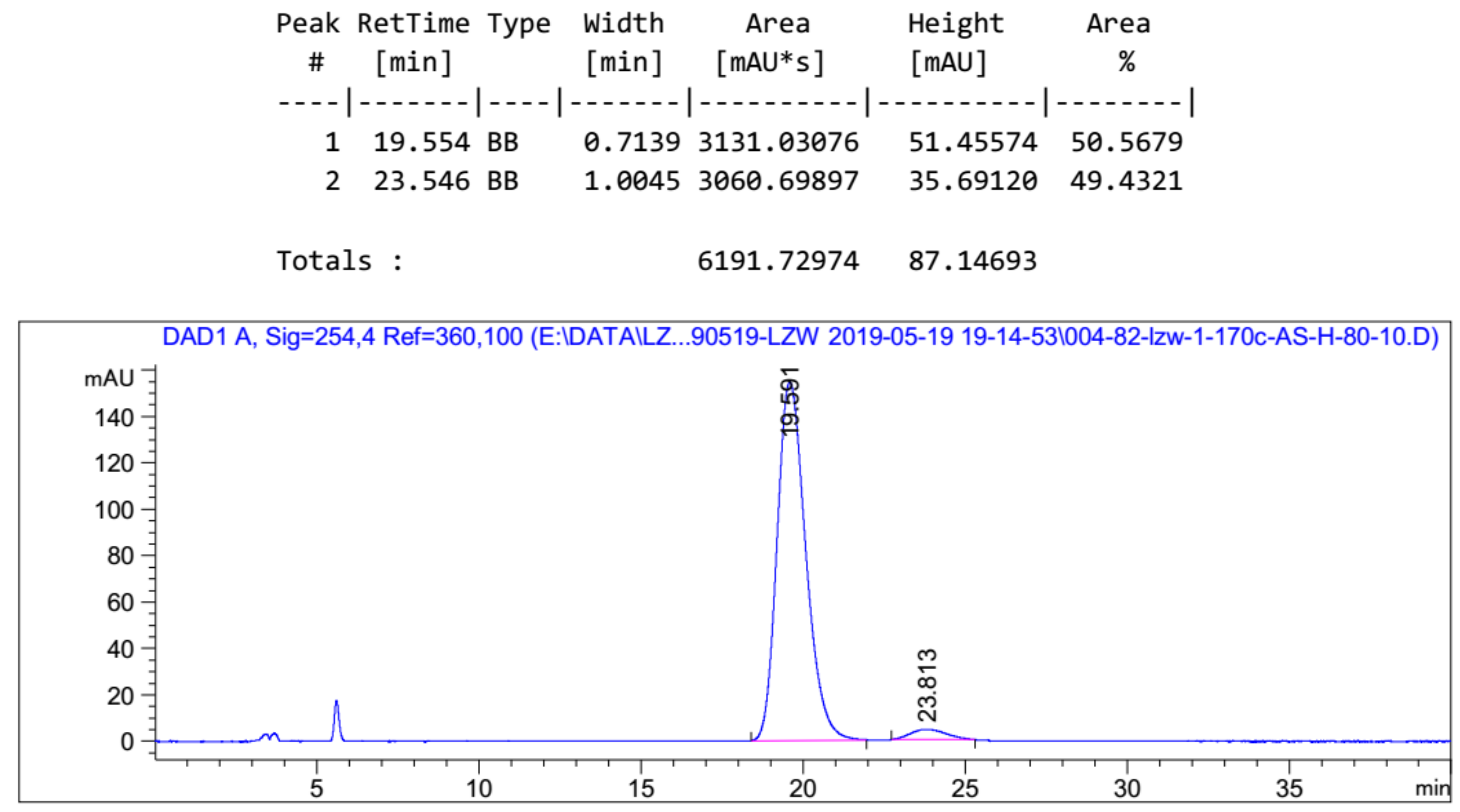

Signal 1: DAD1 A, Sig=254,4 Ref $=360,100$

\begin{tabular}{|c|c|c|c|c|c|}
\hline $\begin{array}{c}\text { Peak } \\
\#\end{array}$ & $\begin{array}{l}\text { RetTime Type } \\
\text { [min] }\end{array}$ & $\begin{array}{l}\text { Width } \\
\text { [min] }\end{array}$ & $\begin{array}{c}\text { Area } \\
{\left[\mathrm{mAU}^{*} \mathrm{~s}\right]}\end{array}$ & $\begin{array}{l}\text { Height } \\
{[\mathrm{mAU}]}\end{array}$ & $\begin{array}{c}\text { Area } \\
\%\end{array}$ \\
\hline--- & $-1-$ & 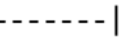 & |------ & $-\ldots$ & --- \\
\hline 1 & $19.591 \mathrm{BB}$ & 0.7251 & 9504.79395 & 154.41028 & 96.5223 \\
\hline 2 & 23.813 BB & 0.8999 & 342.46011 & 4.46948 & 3.4777 \\
\hline Tota & : & & 9847.25406 & 158.87976 & \\
\hline
\end{tabular}


<smiles>CN(C)C(=O)c1ccc(C2=C(c3ccccc3)C(=O)C3(CCCCC3)CC2)cc1</smiles>

3am

IB, 75\% HEX, $45 \mathrm{~min}, 0.8 \mathrm{~mL} / \mathrm{min}$

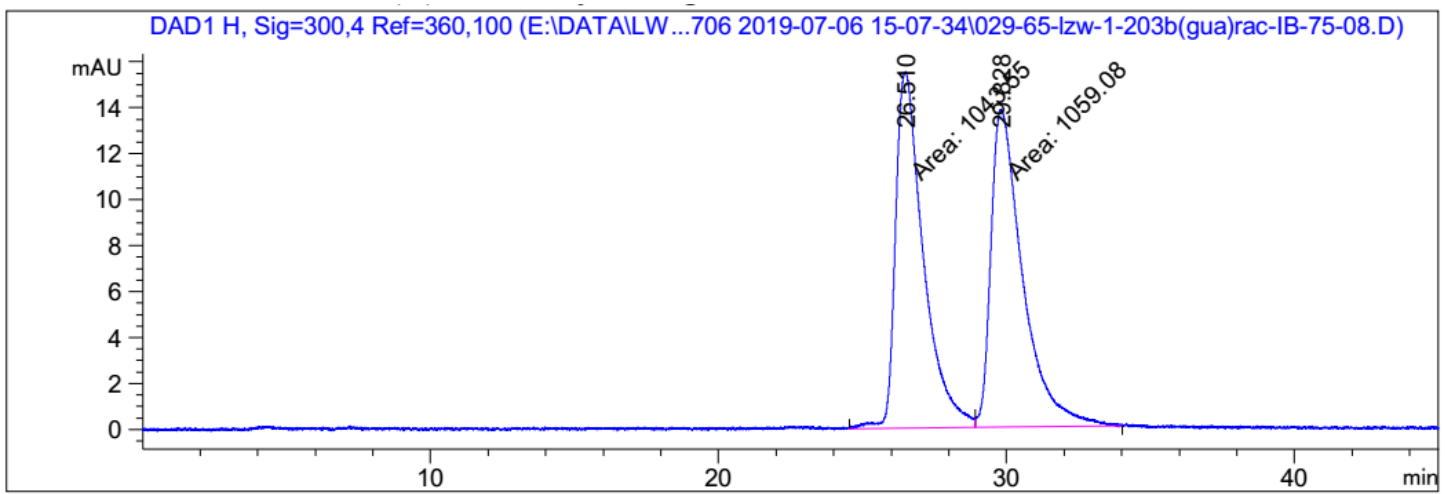

Signal 1: DAD1 H, Sig=300,4 Ref $=360,100$
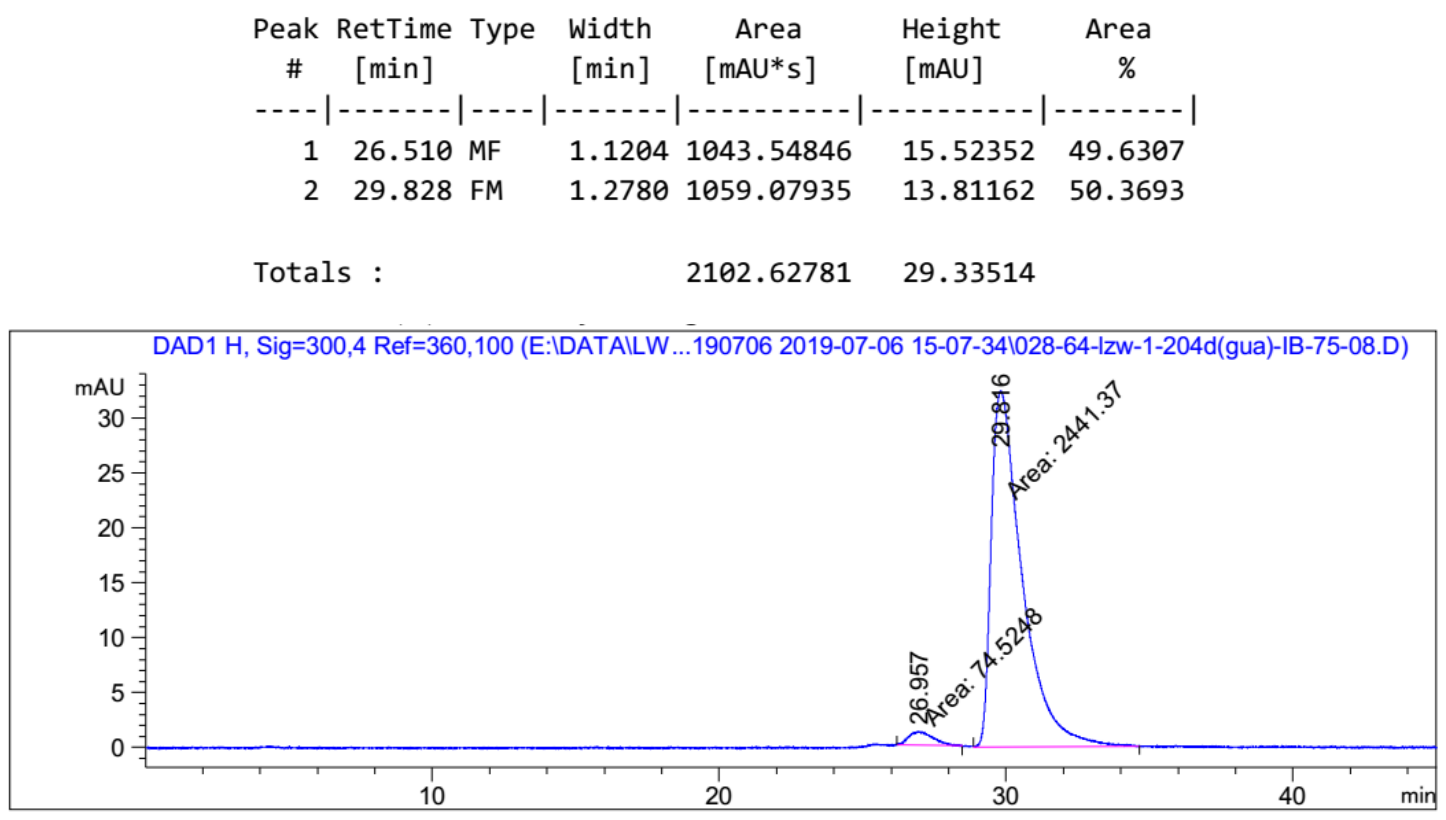

Signal 1: DAD1 H, Sig=300,4 Ref=360,100

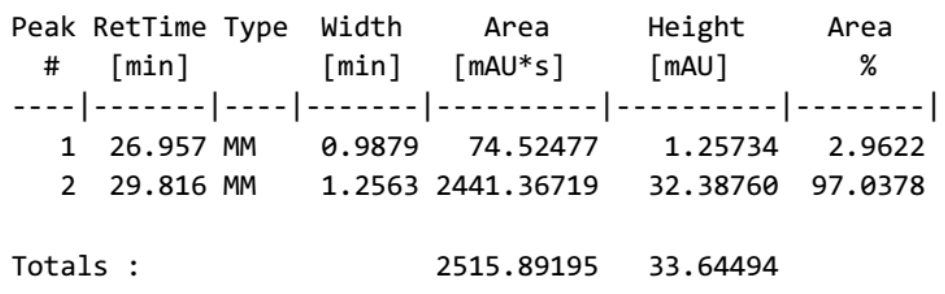


<smiles>CC(=O)c1cccc(C2=C(c3ccccc3)C(=O)C(Br)(Br)CC2)c1</smiles>

3an

AD-H, 95\% HEX, $40 \mathrm{~min}, 0.8 \mathrm{~mL} / \mathrm{min}$

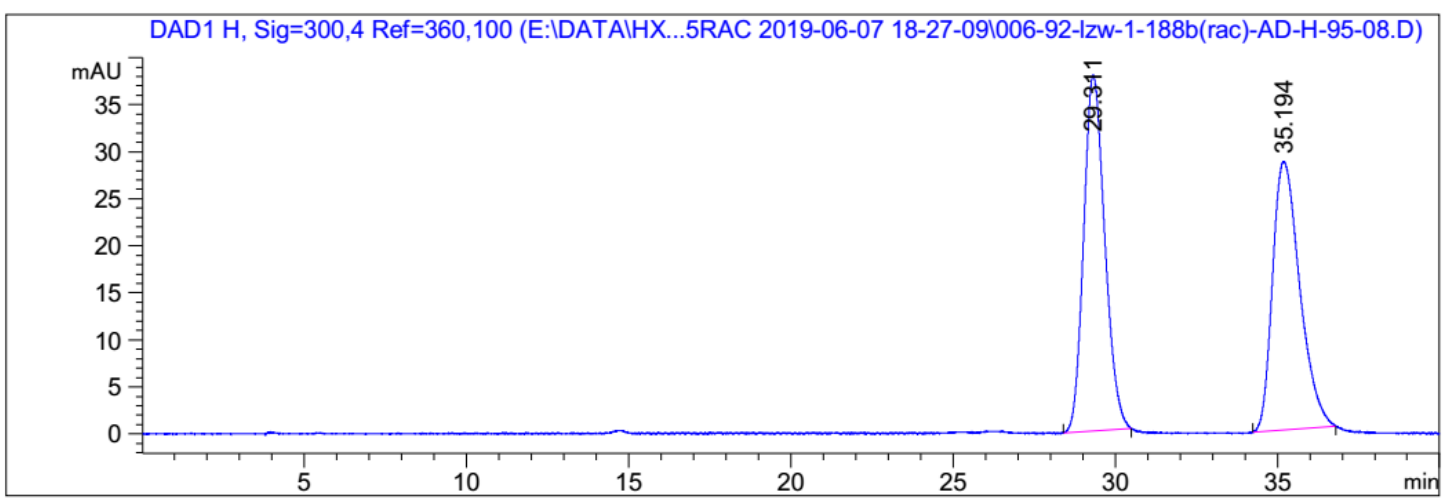

Signal 1: DAD1 H, Sig=300,4 Ref $=360,100$

\begin{tabular}{|c|c|c|c|c|c|}
\hline Peak & $\begin{array}{l}\text { RetTime Type } \\
\text { [min] }\end{array}$ & $\begin{array}{l}\text { Width } \\
\text { [min] }\end{array}$ & $\begin{array}{c}\text { Area } \\
{\left[\mathrm{mAU}^{*} \mathrm{~s}\right]}\end{array}$ & $\begin{array}{l}\text { Height } \\
{[\mathrm{mAU}]}\end{array}$ & $\begin{array}{c}\text { Area } \\
\%\end{array}$ \\
\hline & $-1-$ & & $1--2$. & & \\
\hline 1 & 29.311 BV R & 0.5322 & 1710.39282 & 37.81103 & 50.5842 \\
\hline 2 & 35.194 BВ & 0.6857 & 1670.88354 & 28.63570 & 49.4158 \\
\hline & & & 81 & .44674 & \\
\hline
\end{tabular}

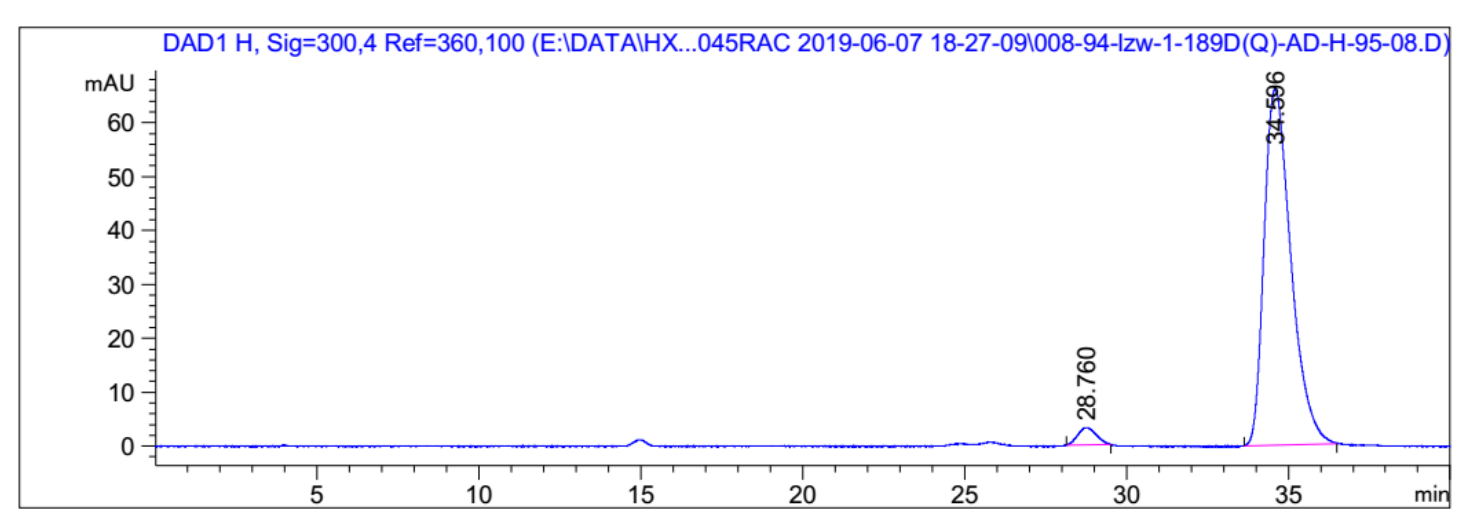

Signal 1: DAD1 H, Sig $=300,4$ Ref $=360,100$

\begin{tabular}{|c|c|c|c|c|c|}
\hline $\begin{array}{c}\text { Peak } \\
\quad \#\end{array}$ & $\begin{array}{l}\text { RetTime Type } \\
\text { [min] }\end{array}$ & $\begin{array}{l}\text { Width } \\
\text { [min] }\end{array}$ & $\begin{array}{c}\text { Area } \\
{\left[\mathrm{mAU}^{*} \mathrm{~s}\right]}\end{array}$ & $\begin{array}{l}\text { Height } \\
\text { [mAU] }\end{array}$ & $\begin{array}{c}\text { Area } \\
\%\end{array}$ \\
\hline-- & $\mid--$ & - & - & | & - \\
\hline 1 & 28.760 BВ & 0.4702 & 128.29205 & 3.21396 & 3.2606 \\
\hline 2 & 34.596 BB & 0.6772 & 3806.33228 & 66.21228 & 96.7394 \\
\hline Tota & $:$ & & 3934.62433 & 69.42624 & \\
\hline
\end{tabular}


<smiles>C=Cc1ccc(C2=C(c3ccccc3)C(=O)C(Br)(C(C)(Br)Br)CC2)cc1</smiles>

AD-H, 95\% HEX, $40 \mathrm{~min}, 0.8 \mathrm{~mL} / \mathrm{min}$

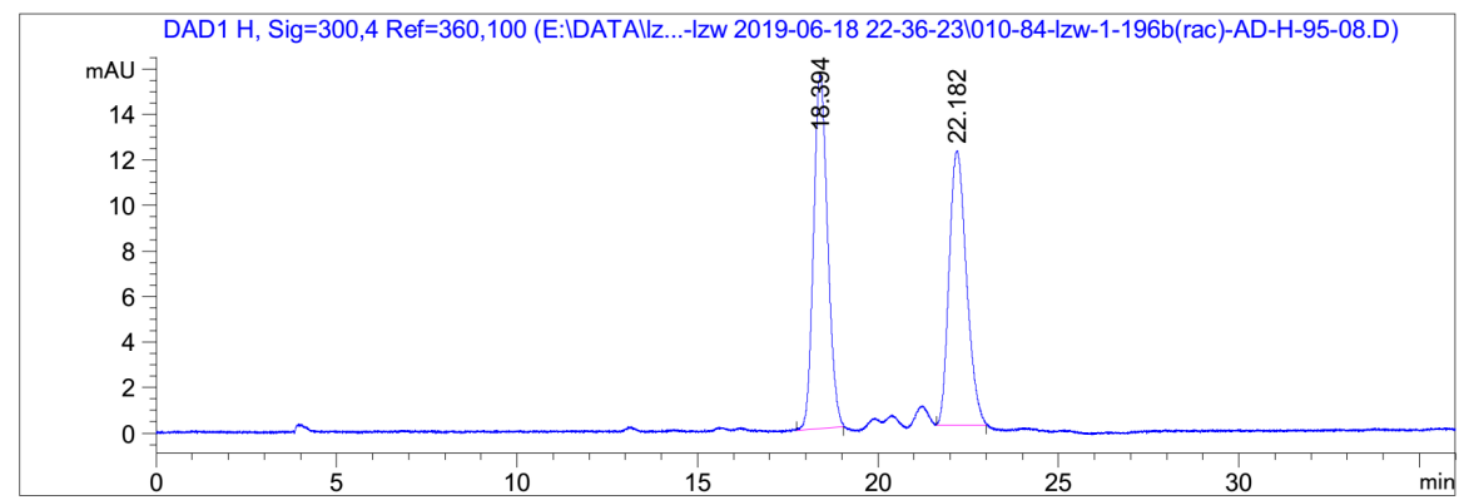

Signal 1: DAD1 H, Sig=300,4 Ref $=360,100$

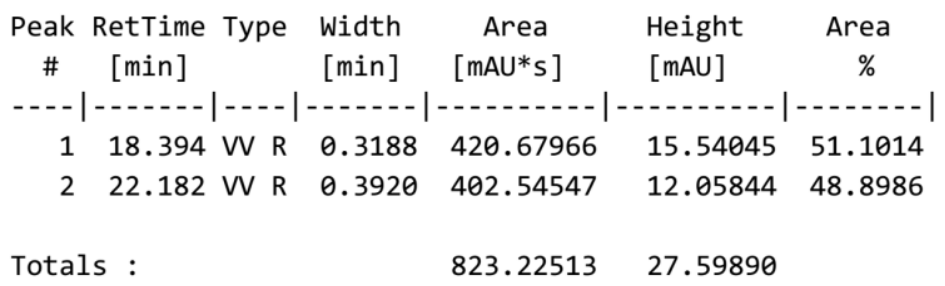

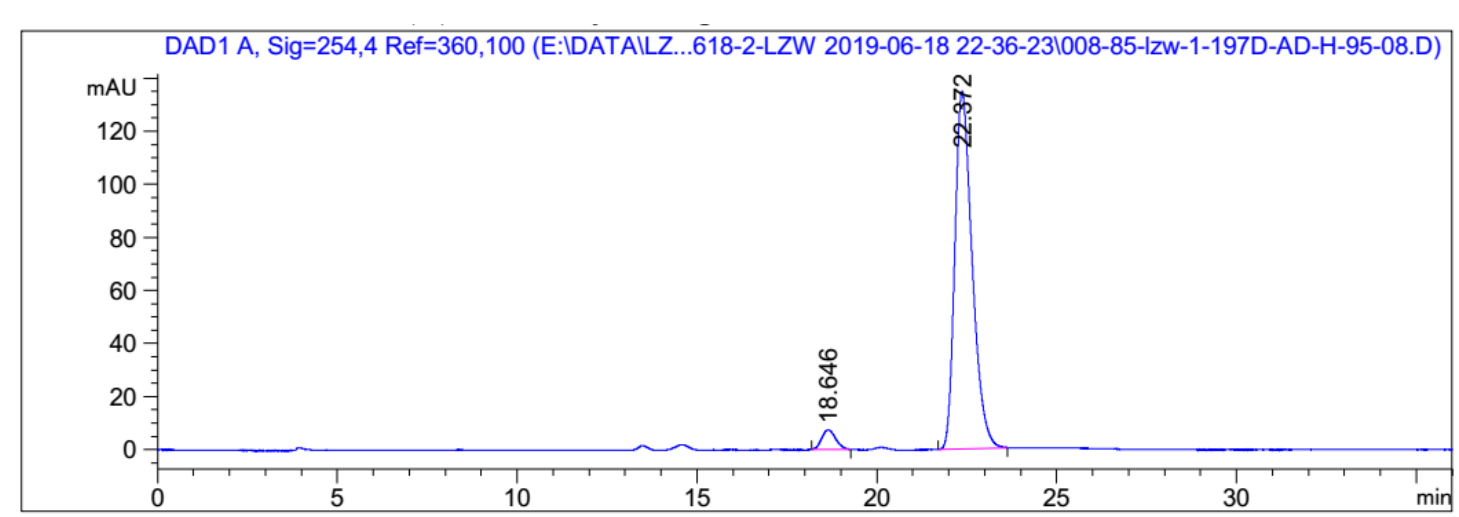

Signal 1: DAD1 A, Sig=254,4 Ref $=360,100$

\begin{tabular}{|c|c|c|c|c|c|c|}
\hline $\begin{array}{c}\text { Peak } \\
\#\end{array}$ & $\begin{array}{c}\text { RetTime } \\
\text { [min] }\end{array}$ & Type & $\begin{array}{l}\text { Width } \\
\text { [min] }\end{array}$ & $\begin{array}{c}\text { Area } \\
{\left[\mathrm{mAU}^{*} \mathrm{~s}\right]}\end{array}$ & $\begin{array}{l}\text { Height } \\
\text { [mAU] }\end{array}$ & $\begin{array}{c}\text { Area } \\
\%\end{array}$ \\
\hline & & & & $\mid--$. & --- & $------\mid$ \\
\hline 1 & 18.646 & BB & 0.3178 & 198.81889 & 7.37890 & 4.2257 \\
\hline 2 & 22.372 & BB & 0.4752 & 4506.12646 & 134.72676 & 95.7743 \\
\hline Tota. & Ls: & & & 4704.94536 & 142.10566 & \\
\hline
\end{tabular}


<smiles>N#CC1(Br)CCC(c2ccoc2)=C(c2ccccc2)C1=O</smiles>

3ap

AD-H, 95\% HEX, $40 \mathrm{~min}, 0.8 \mathrm{~mL} / \mathrm{min}$

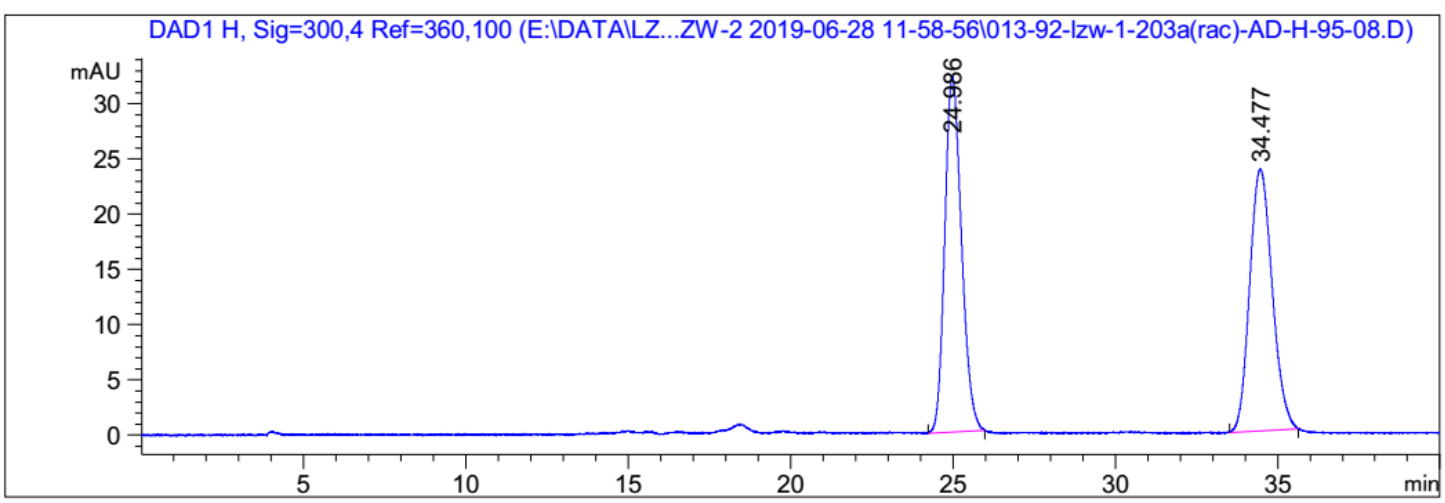

Signal 1: DAD1 H, Sig $=300,4$ Ref $=360,100$

\begin{tabular}{|c|c|c|c|c|c|}
\hline $\begin{array}{c}\text { Peak } \\
\#\end{array}$ & $\begin{array}{l}\text { RetTime Type } \\
\text { [min] }\end{array}$ & $\begin{array}{l}\text { Width } \\
\text { [min] }\end{array}$ & $\begin{array}{c}\text { Area } \\
{\left[\mathrm{mAU}^{*} \mathrm{~s}\right]}\end{array}$ & $\begin{array}{l}\text { Height } \\
\text { [mAU] }\end{array}$ & $\begin{array}{c}\text { Area } \\
\%\end{array}$ \\
\hline & 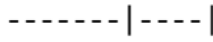 & & | - - - - - & ------ & | \\
\hline 1 & 24.986 VB $R$ & 0.4209 & 1153.29810 & 32.23925 & 50.2277 \\
\hline 2 & $34.477 \mathrm{BB}$ & 0.5662 & 1142.83997 & 23.71552 & 49.7723 \\
\hline Tot & : & & 2296.13806 & 55.95477 & \\
\hline
\end{tabular}

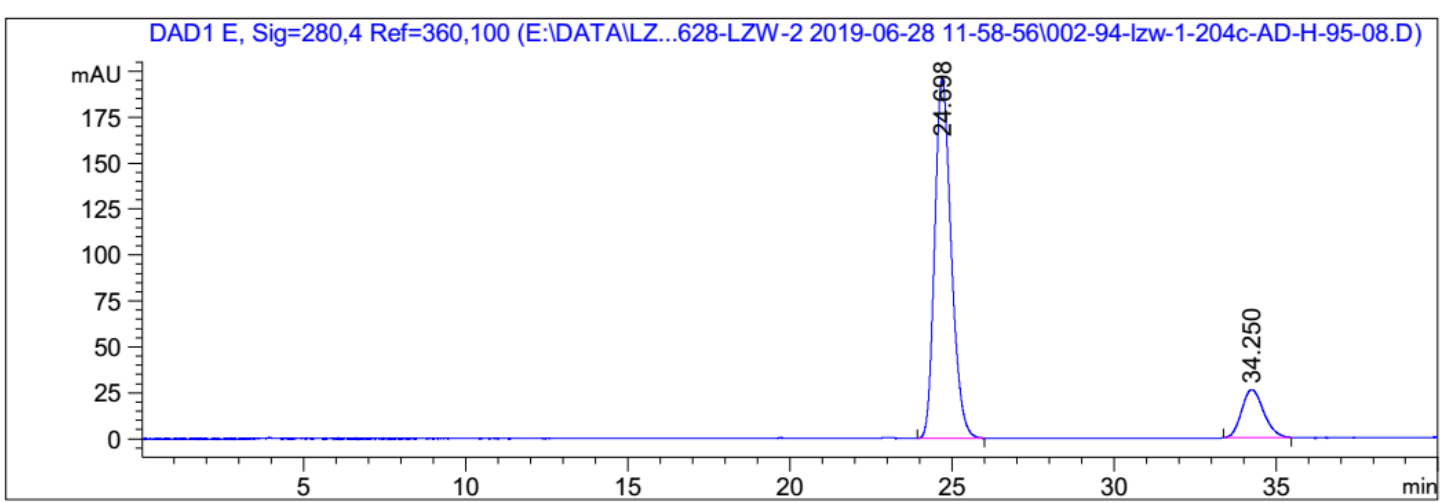

Signal 1: DAD1 E, Sig $=280,4$ Ref $=360,100$

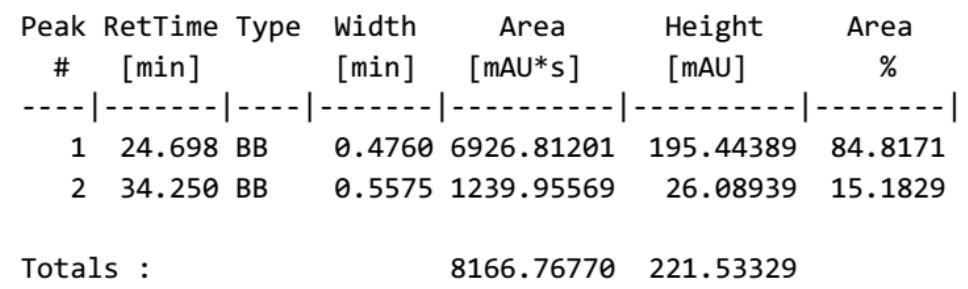


<smiles>N#CC1(Br)CCC(c2ccsc2)=C(c2ccccc2)C1=O</smiles>

3aq

$\mathrm{AD}-\mathrm{H}, 95 \% \mathrm{HEX}, 40 \mathrm{~min}, 0.8 \mathrm{~mL} / \mathrm{min}$

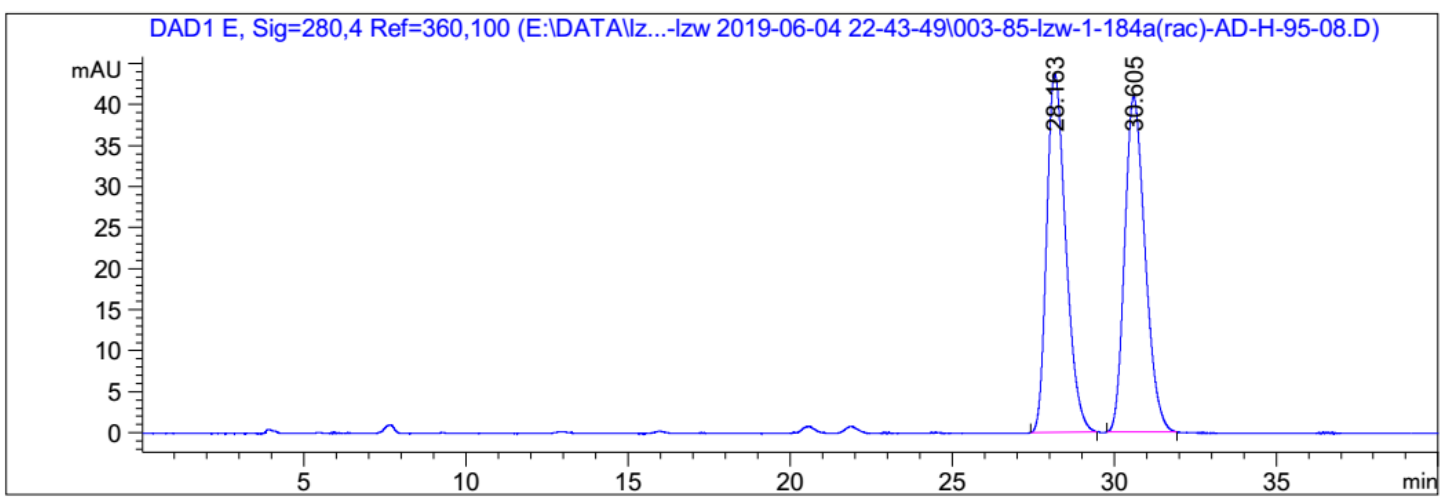

Signal 1: DAD1 E, Sig=280,4 Ref $=360,100$

\begin{tabular}{|c|c|c|c|c|c|c|}
\hline $\begin{array}{c}\text { Peak } \\
\#\end{array}$ & $\begin{array}{c}\text { RetTime } \\
\text { [min] }\end{array}$ & Type & $\begin{array}{l}\text { Width } \\
\text { [min] }\end{array}$ & $\begin{array}{c}\text { Area } \\
{\left[\mathrm{mAU}^{*} \mathrm{~s}\right]}\end{array}$ & $\begin{array}{l}\text { Height } \\
\text { [mAU] }\end{array}$ & $\begin{array}{c}\text { Area } \\
\%\end{array}$ \\
\hline 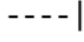 & 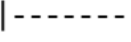 & & & 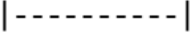 & 20 & - \\
\hline 1 & 28.163 & BB & 0.4833 & 1795.45129 & 43.66070 & 50.0000 \\
\hline 2 & 30.605 & BB & 0.5161 & 1795.45117 & 40.90427 & 50.0000 \\
\hline
\end{tabular}

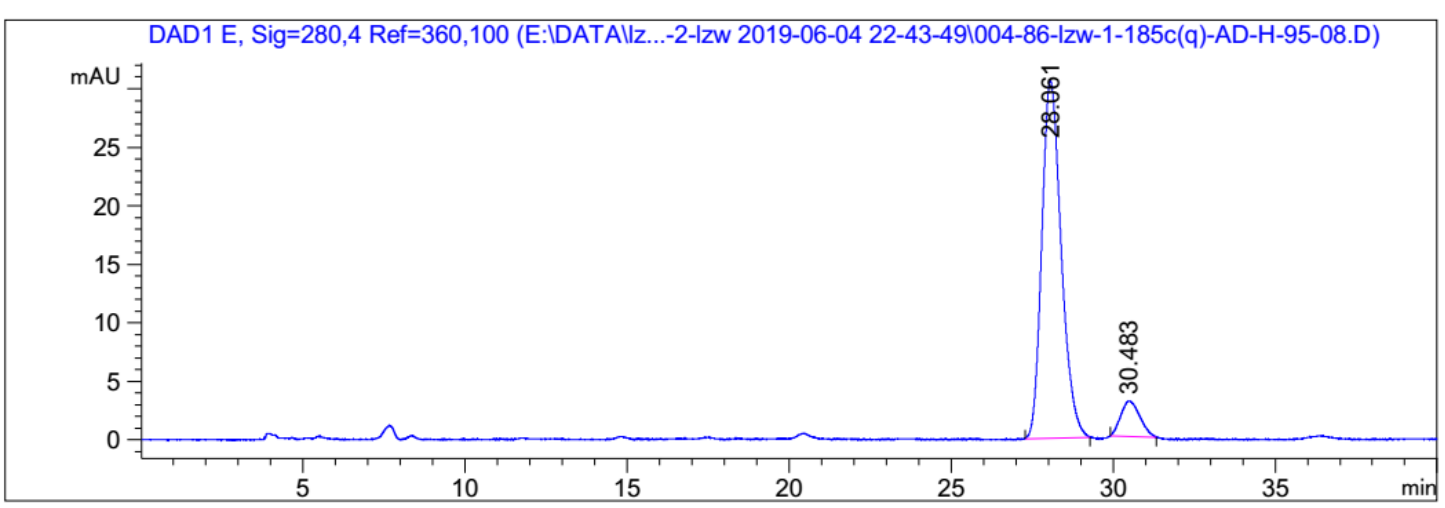

Signal 1: DAD1 E, Sig $=280,4$ Ref $=360,100$

\begin{tabular}{|c|c|c|c|c|c|}
\hline $\begin{array}{c}\text { Peak } \\
\text { \# }\end{array}$ & $\begin{array}{l}\text { RetTime Type } \\
\text { [min] }\end{array}$ & $\begin{array}{l}\text { Width } \\
\text { [min] }\end{array}$ & $\begin{array}{c}\text { Area } \\
{\left[\mathrm{mAU}^{*} \mathrm{~s}\right]}\end{array}$ & $\begin{array}{l}\text { Height } \\
{[\mathrm{mAU}]}\end{array}$ & $\begin{array}{c}\text { Area } \\
\%\end{array}$ \\
\hline & 1 & & - & & \\
\hline 1 & 28.061 BB & 0.4862 & 1268.49463 & 30.52991 & 91.0420 \\
\hline 2 & 30.483 вВ & 0.4808 & 124.81278 & 3.05098 & 8.9580 \\
\hline Total & : & & 1393.30741 & 33.58089 & \\
\hline
\end{tabular}




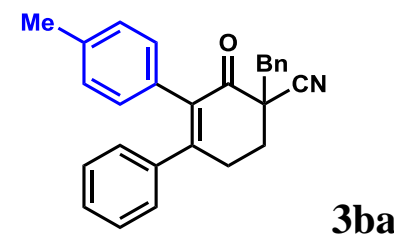

$\mathrm{AD}-\mathrm{H}, 95 \% \mathrm{HEX}, 40 \mathrm{~min}, 0.8 \mathrm{~mL} / \mathrm{min}$

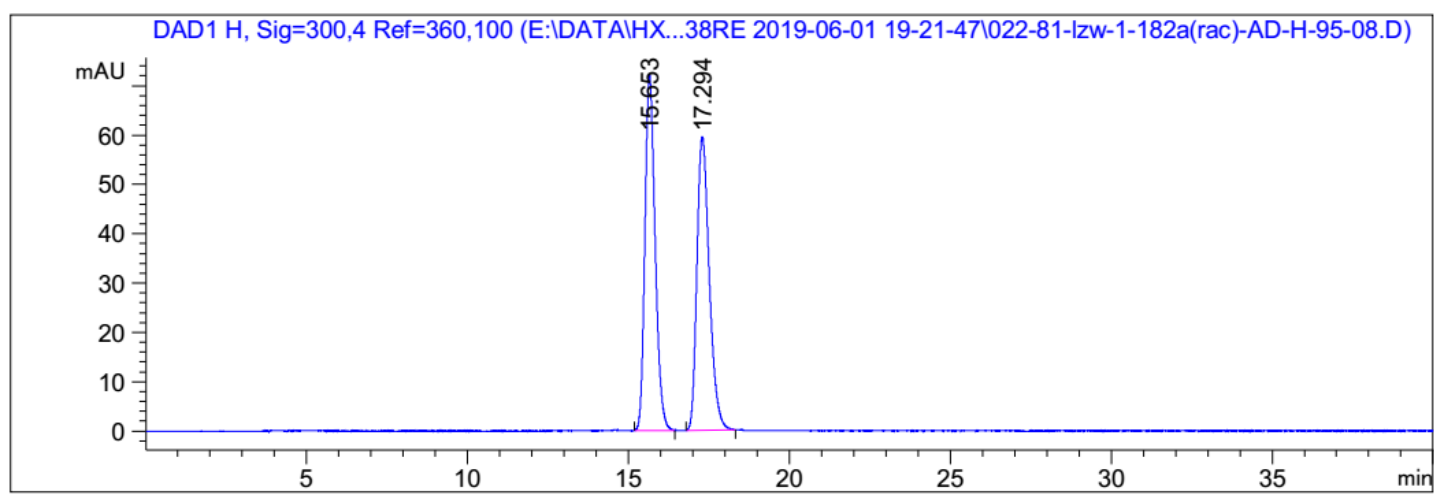

Signal 1: DAD1 H, Sig=300,4 Ref $=360,100$
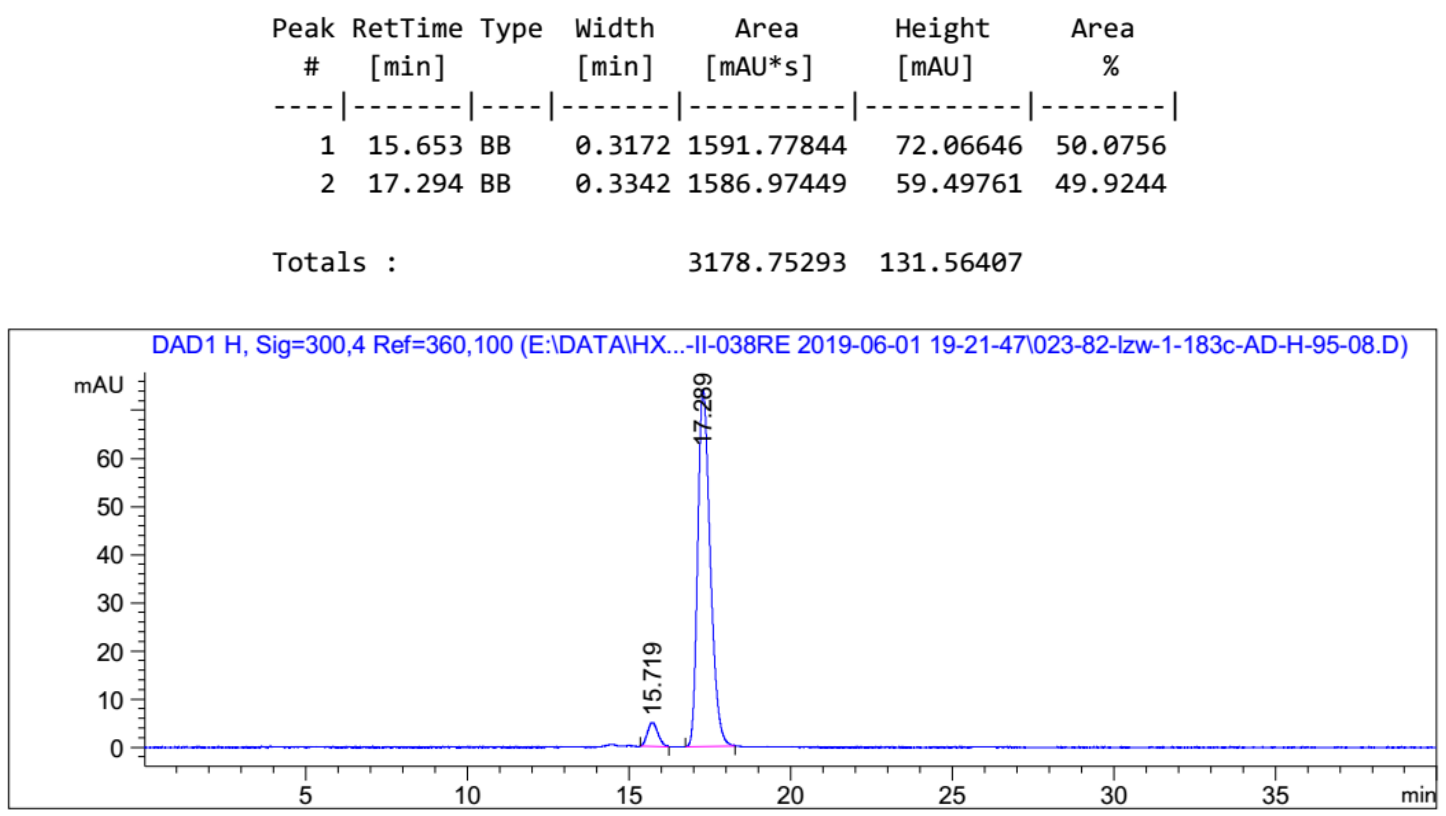

Signal 1: DAD1 H, Sig $=300,4$ Ref $=360,100$

\begin{tabular}{|c|c|c|c|c|c|}
\hline $\begin{array}{c}\text { Peak } \\
\quad \#\end{array}$ & $\begin{array}{l}\text { RetTime Type } \\
{[\mathrm{min}]}\end{array}$ & $\begin{array}{l}\text { Width } \\
{[\mathrm{min}]}\end{array}$ & $\begin{array}{c}\text { Area } \\
{[\mathrm{mAU} * \mathrm{~s}]}\end{array}$ & $\begin{array}{l}\text { Height } \\
\text { [mAU] }\end{array}$ & $\begin{array}{c}\text { Area } \\
\quad \%\end{array}$ \\
\hline$-\ldots$ & $\mid---1-1$ & ----1 & -------- & - & 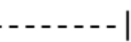 \\
\hline 1 & 15.719 ВВ & 0.2644 & 112.80389 & 5.00551 & 5.3282 \\
\hline 2 & 17.289 ВВ & 0.3650 & 2004.31091 & 74.12228 & 94.6718 \\
\hline Tot & : & & 2117.11480 & 79.12779 & \\
\hline
\end{tabular}




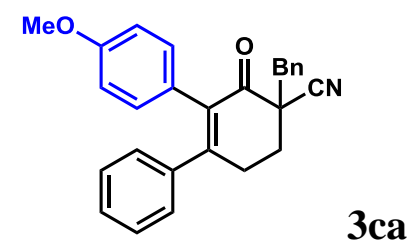

AS-H, 85\% HEX, $40 \mathrm{~min}, 1.0 \mathrm{~mL} / \mathrm{min}$

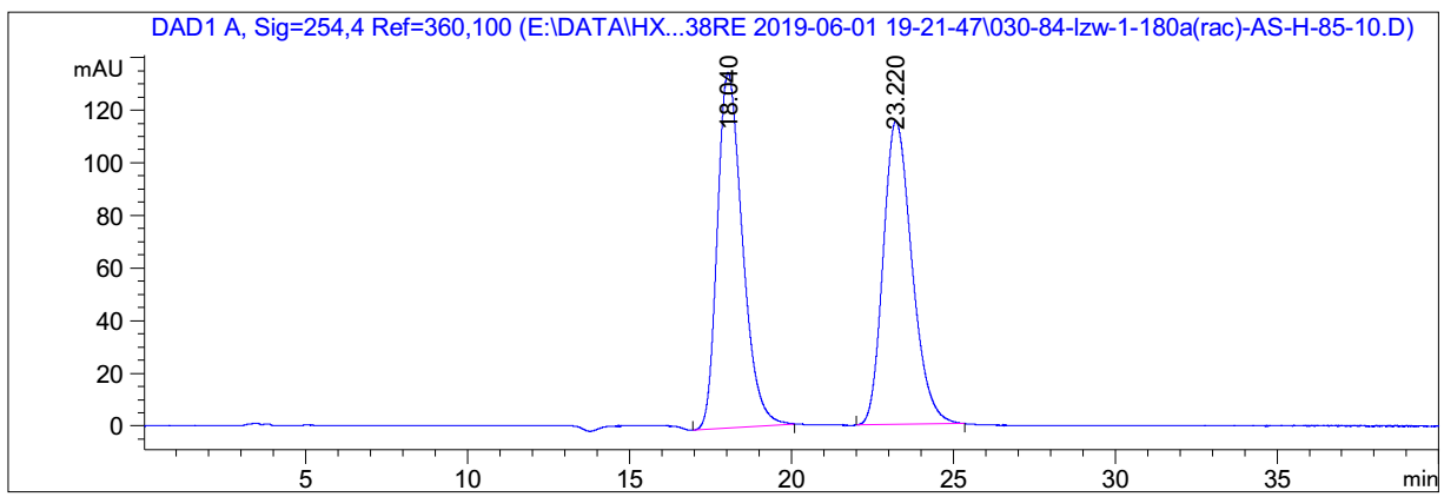

Signal 1: DAD1 A, Sig $=254,4$ Ref $=360,100$
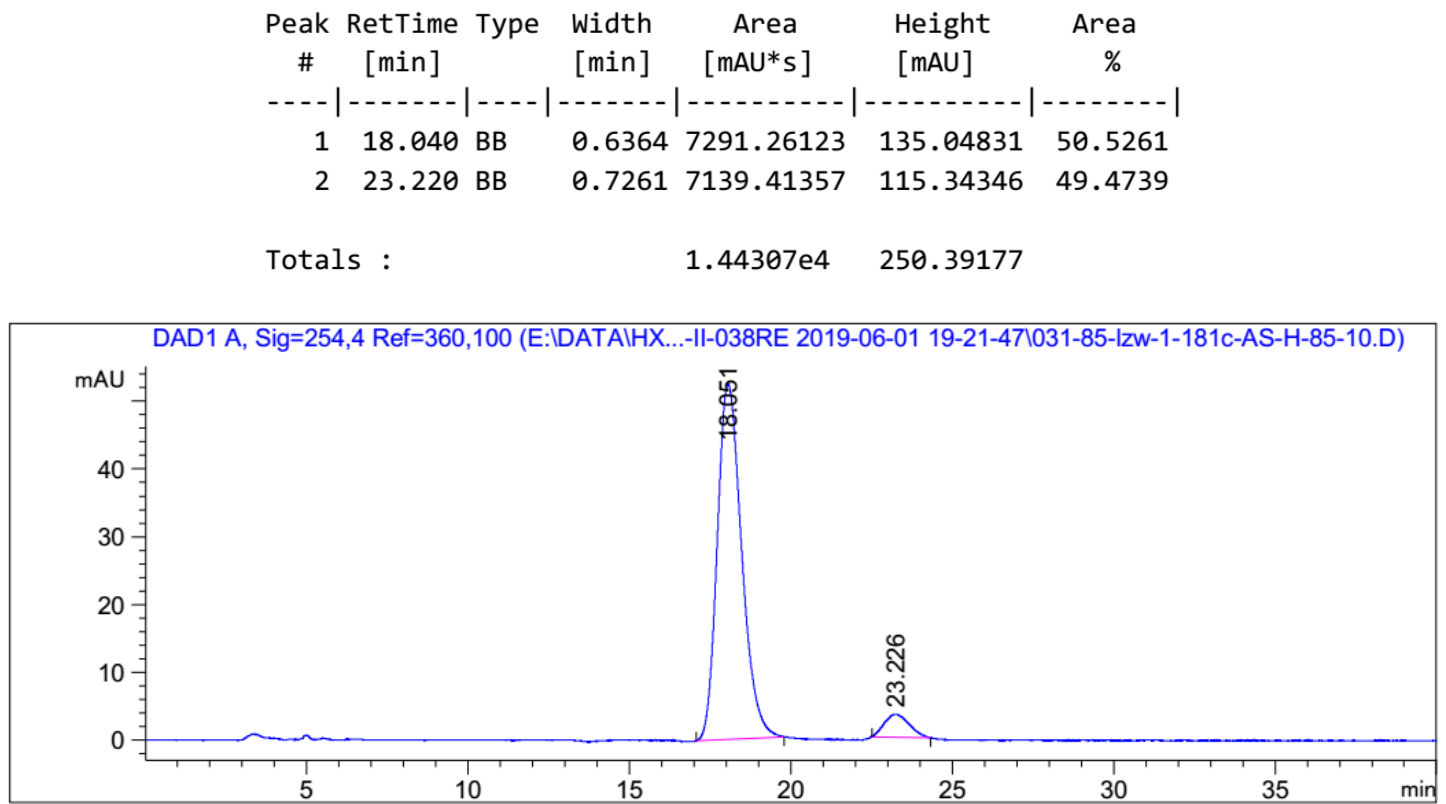

Signal 1: DAD1 A, Sig $=254,4$ Ref $=360,100$

\begin{tabular}{|c|c|c|c|c|c|}
\hline $\begin{array}{c}\text { Peak } \\
\#\end{array}$ & $\begin{array}{l}\text { RetTime Type } \\
\text { [min] }\end{array}$ & $\begin{array}{l}\text { Width } \\
\text { [min] }\end{array}$ & $\begin{array}{c}\text { Area } \\
{\left[\mathrm{mAU}^{*} \mathrm{~s}\right]}\end{array}$ & $\begin{array}{l}\text { Height } \\
{[\mathrm{mAU}]}\end{array}$ & $\begin{array}{c}\text { Area } \\
\%\end{array}$ \\
\hline-- & $--\mid--$ & - & $|--------|$ & 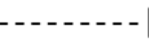 & $------\mid$ \\
\hline 1 & 18.051 ВВ & 0.6157 & 2748.34155 & 52.37619 & 93.7944 \\
\hline 2 & $23.226 \mathrm{BB}$ & 0.6251 & 181.83524 & 3.40187 & 6.2056 \\
\hline Tota & 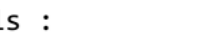 & & 2930.17679 & 55.77806 & \\
\hline
\end{tabular}




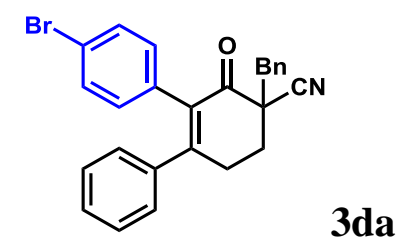

AS-H, 85\% HEX, $40 \mathrm{~min}, 1.0 \mathrm{~mL} / \mathrm{min}$

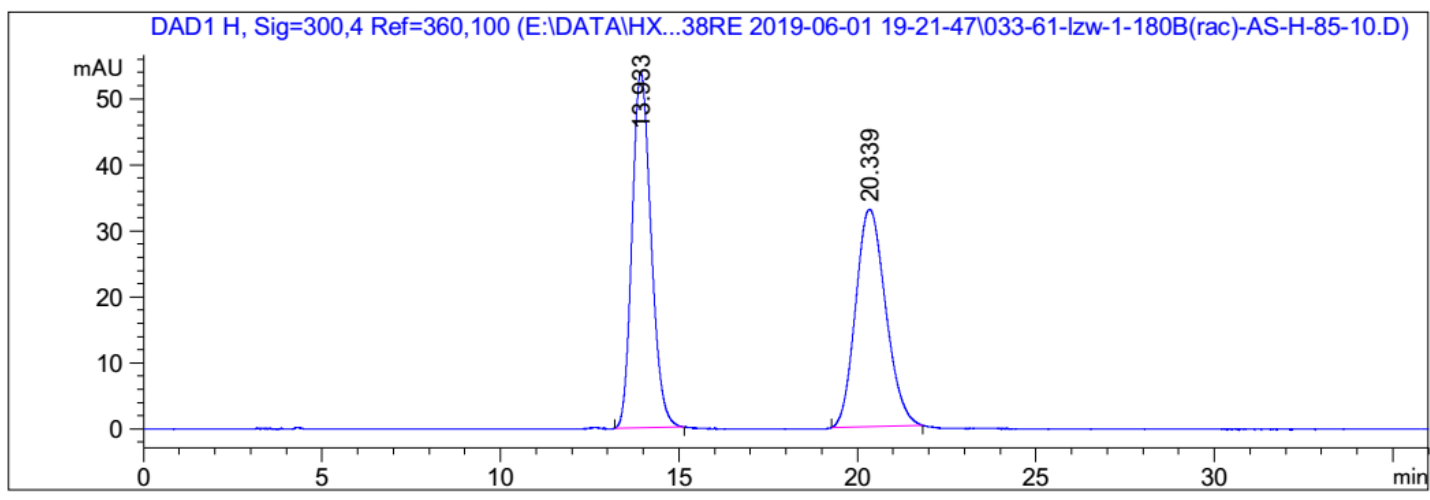

Signal 1: DAD1 H, Sig $=300,4$ Ref $=360,100$

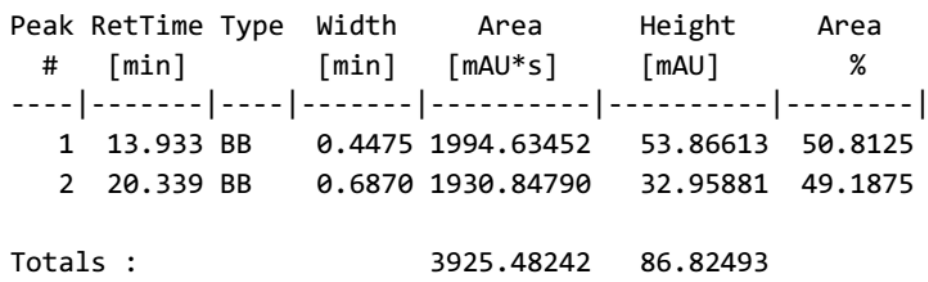

Totals :

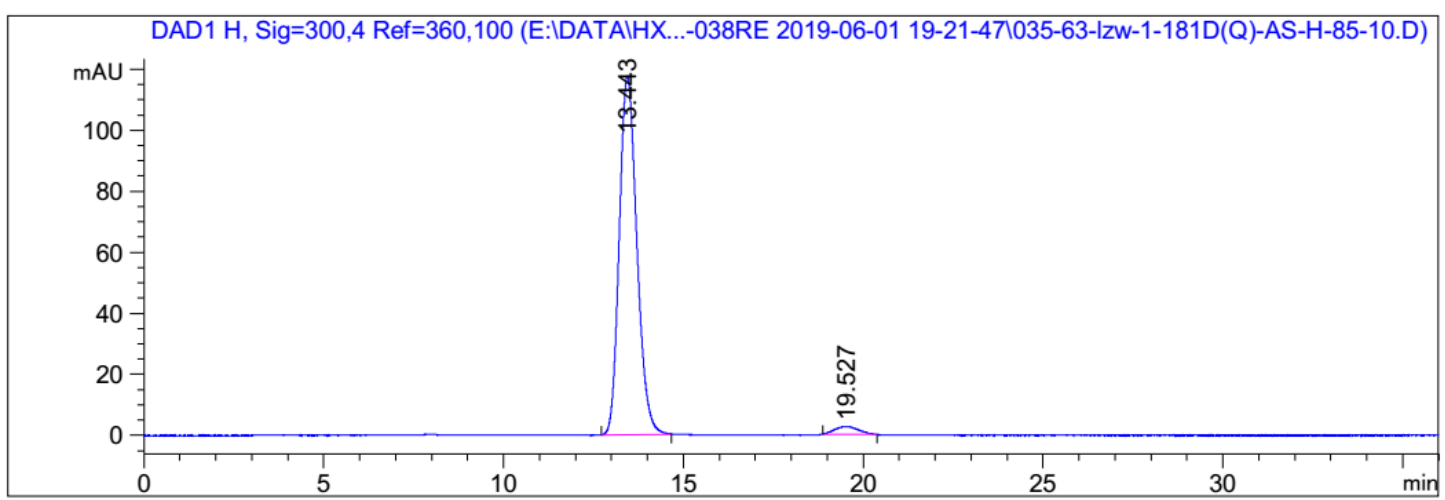

Signal 1: DAD1 H, Sig=300,4 Ref $=360,100$

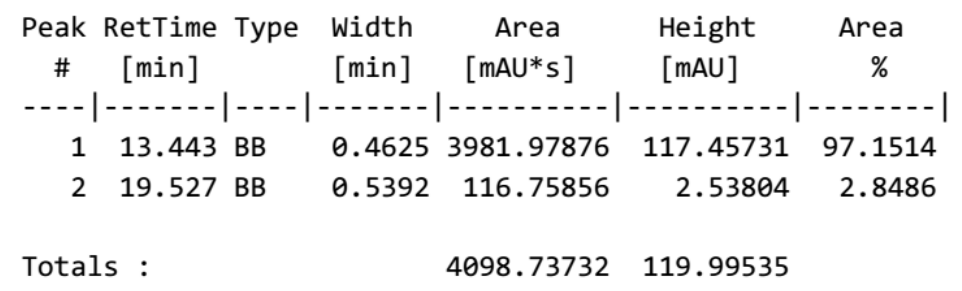




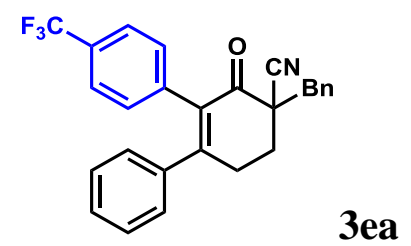

AS-H, 90\% HEX, $40 \mathrm{~min}, 0.8 \mathrm{~mL} / \mathrm{min}$

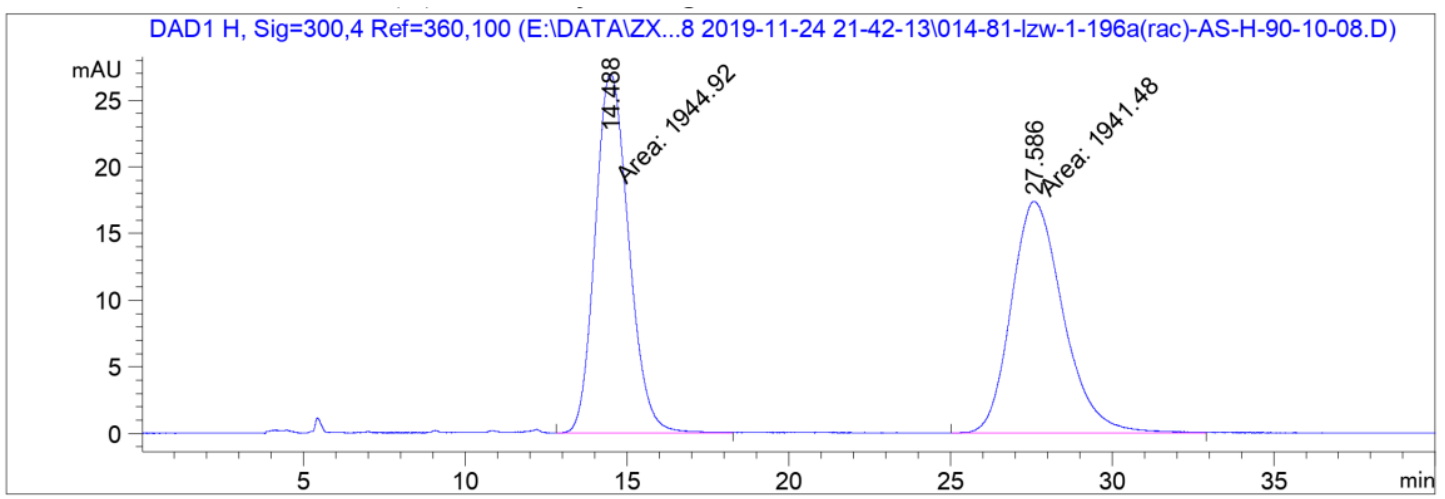

Signal 1: DAD1 H, Sig $=300,4$ Ref $=360,100$

\begin{tabular}{|c|c|c|c|c|c|}
\hline $\begin{array}{c}\text { Peak } \\
\text { \# }\end{array}$ & $\begin{array}{l}\text { RetTime Type } \\
\text { [min] }\end{array}$ & $\begin{array}{l}\text { Width } \\
\text { [min] }\end{array}$ & $\begin{array}{c}\text { Area } \\
{\left[\mathrm{mAU}^{*} \mathrm{~s}\right]}\end{array}$ & $\begin{array}{l}\text { Height } \\
\text { [mAU] }\end{array}$ & $\begin{array}{c}\text { Area } \\
\%\end{array}$ \\
\hline & | - - & & $\mid \cdots$ & & \\
\hline 1 & $14.488 \mathrm{MM}$ & 1.2052 & 1944.91760 & 26.89698 & 50.0442 \\
\hline 2 & $27.586 \mathrm{MM}$ & 1.8630 & 1941.48120 & 17.36906 & 49.9558 \\
\hline+ & & & 9880 & & \\
\hline
\end{tabular}

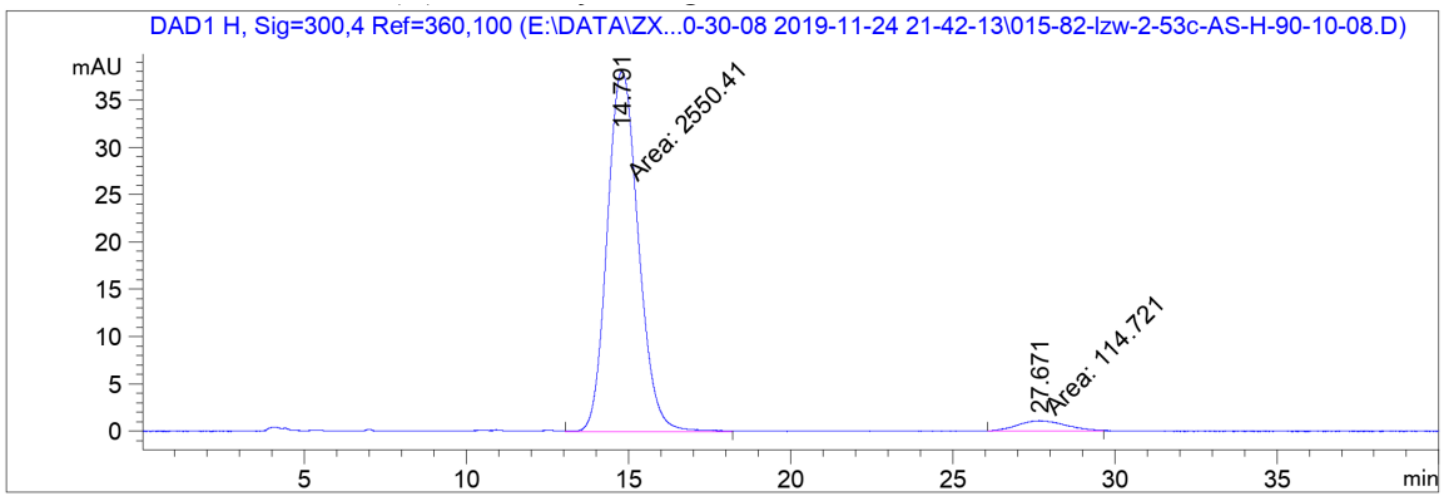

Signal 1: DAD1 H, Sig=300,4 Ref $=360,100$

\begin{tabular}{|c|c|c|c|c|c|}
\hline $\begin{array}{c}\text { Peak } \\
\quad \#\end{array}$ & $\begin{array}{l}\text { RetTime Type } \\
\text { [min] }\end{array}$ & $\begin{array}{l}\text { Width } \\
\text { [min] }\end{array}$ & $\begin{array}{c}\text { Area } \\
{\left[\mathrm{mAU}^{*} \mathrm{~s}\right]}\end{array}$ & $\begin{array}{l}\text { Height } \\
\text { [mAU] }\end{array}$ & $\begin{array}{c}\text { Area } \\
\%\end{array}$ \\
\hline ( & 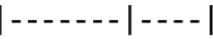 & 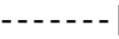 & $\mid--\infty-\infty$ & - & | \\
\hline 1 & $14.791 \mathrm{MM}$ & 1.1175 & 2550.41382 & 38.03856 & 95.6955 \\
\hline 2 & $27.671 \mathrm{MM}$ & 1.7506 & 114.72080 & 1.09220 & 4.3045 \\
\hline Tota] & ls : & & 2665.13462 & 39.13076 & \\
\hline
\end{tabular}




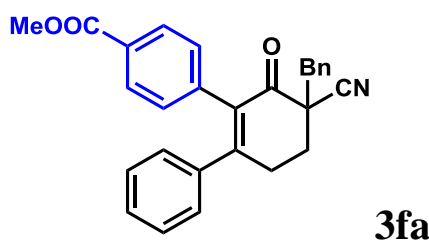

AS-H, 70\% HEX, 35min, $1 \mathrm{~mL} / \mathrm{min}$

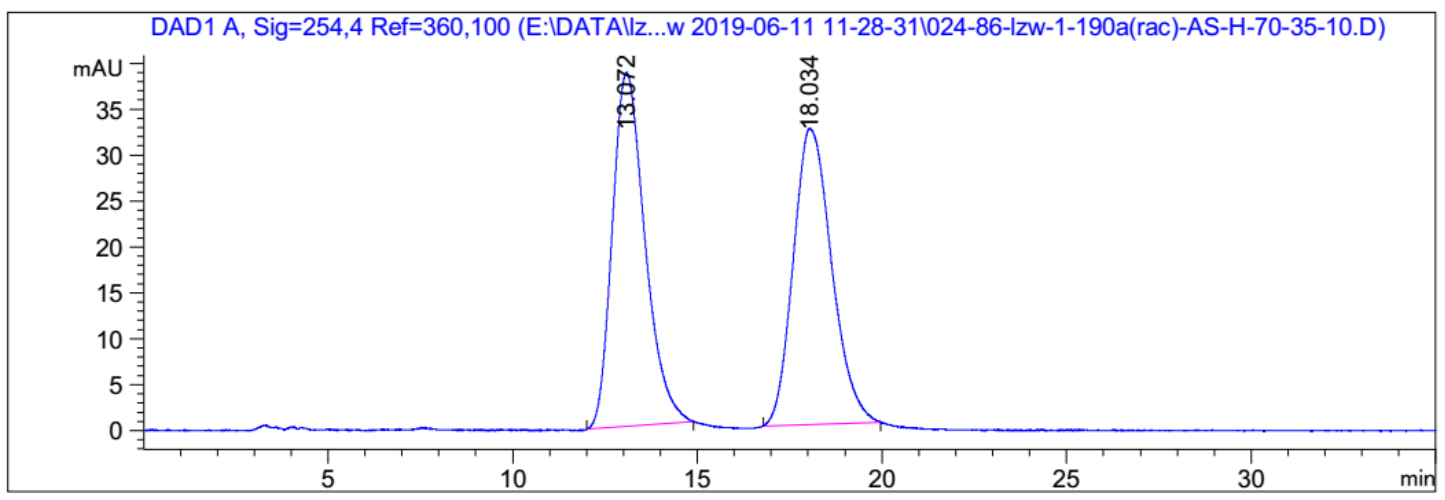

Signal 1: DAD1 A, Sig $=254,4$ Ref $=360,100$

\begin{tabular}{|c|c|c|c|c|c|}
\hline $\begin{array}{c}\text { Peak } \\
\#\end{array}$ & $\begin{array}{l}\text { RetTime Type } \\
\text { [min] }\end{array}$ & $\begin{array}{l}\text { Width } \\
\text { [min] }\end{array}$ & $\begin{array}{c}\text { Area } \\
{\left[\mathrm{mAU}^{*} \mathrm{~s}\right]}\end{array}$ & $\begin{array}{l}\text { Height } \\
{[\mathrm{mAU}]}\end{array}$ & $\begin{array}{c}\text { Area } \\
\%\end{array}$ \\
\hline & & & 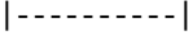 & & \\
\hline 1 & $13.072 \mathrm{BB}$ & 0.7208 & 2368.65723 & 38.49992 & 50.3967 \\
\hline 2 & $18.034 \mathrm{BB}$ & 0.8466 & 2331.37183 & 32.24146 & 49.6033 \\
\hline & & & 700.0290 & & \\
\hline
\end{tabular}

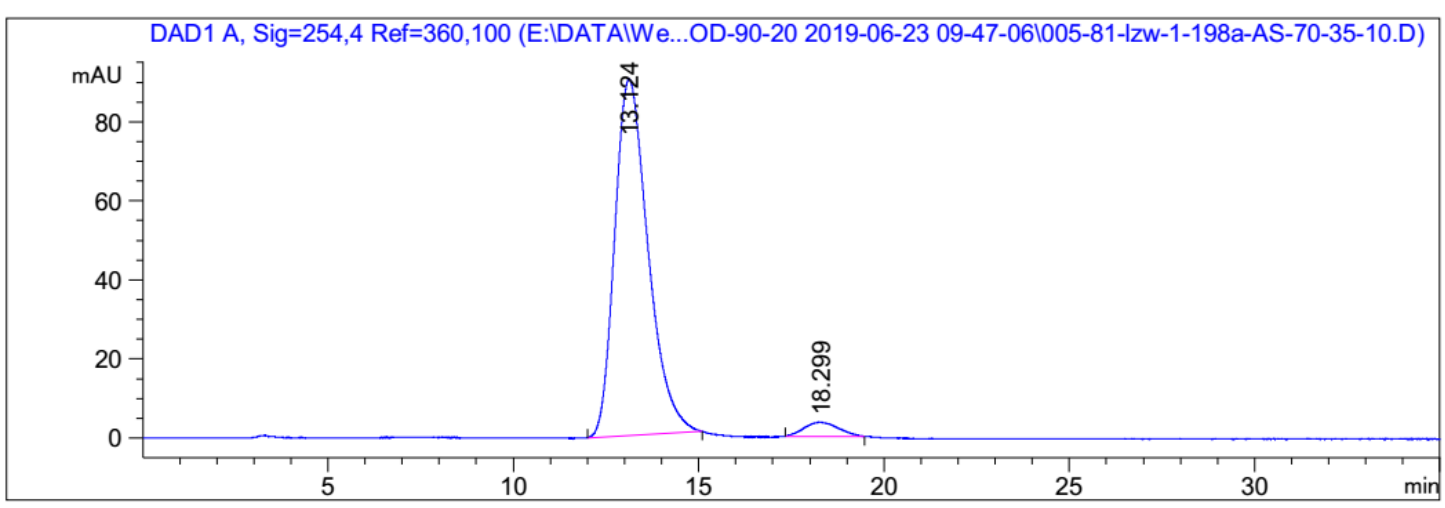

Signal 1: DAD1 A, Sig=254,4 Ref $=360,100$

\begin{tabular}{|c|c|c|c|c|c|}
\hline $\begin{array}{c}\text { Peak } \\
\text { \# }\end{array}$ & $\begin{array}{l}\text { RetTime Type } \\
{[\text { min] }}\end{array}$ & $\begin{array}{l}\text { Width } \\
{[\mathrm{min}]}\end{array}$ & $\begin{array}{c}\text { Area } \\
{\left[\mathrm{mAU}^{*} \mathrm{~s}\right]}\end{array}$ & $\begin{array}{l}\text { Height } \\
{[\mathrm{mAU}]}\end{array}$ & $\begin{array}{c}\text { Area } \\
\%\end{array}$ \\
\hline-1 & -1 & & $--1-1$ & $\ldots$ & | \\
\hline 1 & 13.124 ВВ & 0.7314 & 5638.76611 & 90.18690 & 96.1085 \\
\hline 2 & 18.299 BВ & 0.7566 & 228.31694 & 3.53482 & 3.8915 \\
\hline Total & Is : & & 5867.08305 & 93.72172 & \\
\hline
\end{tabular}




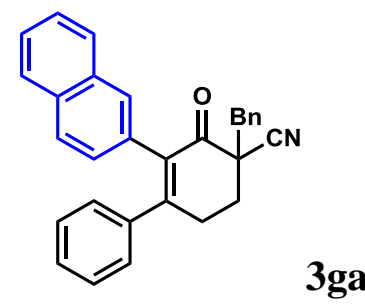

OD-H, 80\% HEX, $40 \mathrm{~min}, 1 \mathrm{~mL} / \mathrm{min}$

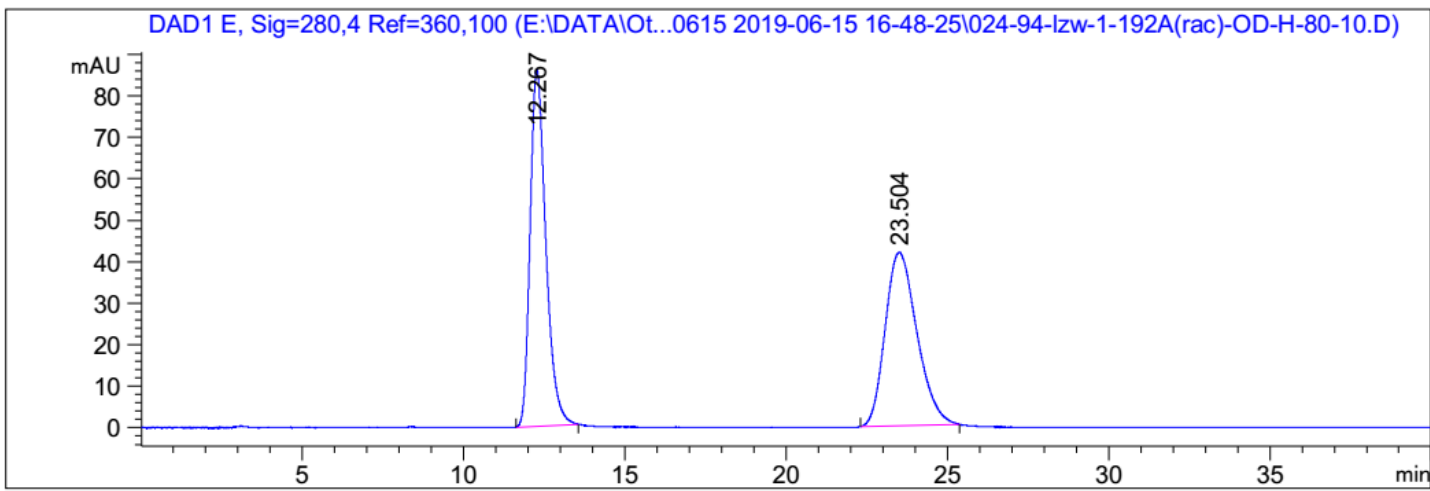

Signal 1: DAD1 E, Sig $=280,4$ Ref $=360,100$

\begin{tabular}{cccccc}
$\begin{array}{c}\text { Peak RetTime Type } \\
\text { \# } \\
\text { [min] }\end{array}$ & $\begin{array}{c}\text { Width } \\
{[\mathrm{min}]}\end{array}$ & $\begin{array}{c}\text { Area } \\
{\left[\mathrm{mAU}{ }^{*} \text { ] }\right.}\end{array}$ & $\begin{array}{c}\text { Height } \\
{[\mathrm{mAU}]}\end{array}$ & $\begin{array}{c}\text { Area } \\
\%\end{array}$ \\
\hdashline 1 & 12.267 BB & 0.4632 & 2903.87549 & 85.94072 & 50.3353 \\
2 & 23.504 BB & 0.8017 & 2865.19385 & 41.82721 & 49.6647
\end{tabular}

Totals :

$5769.06934 \quad 127.76793$

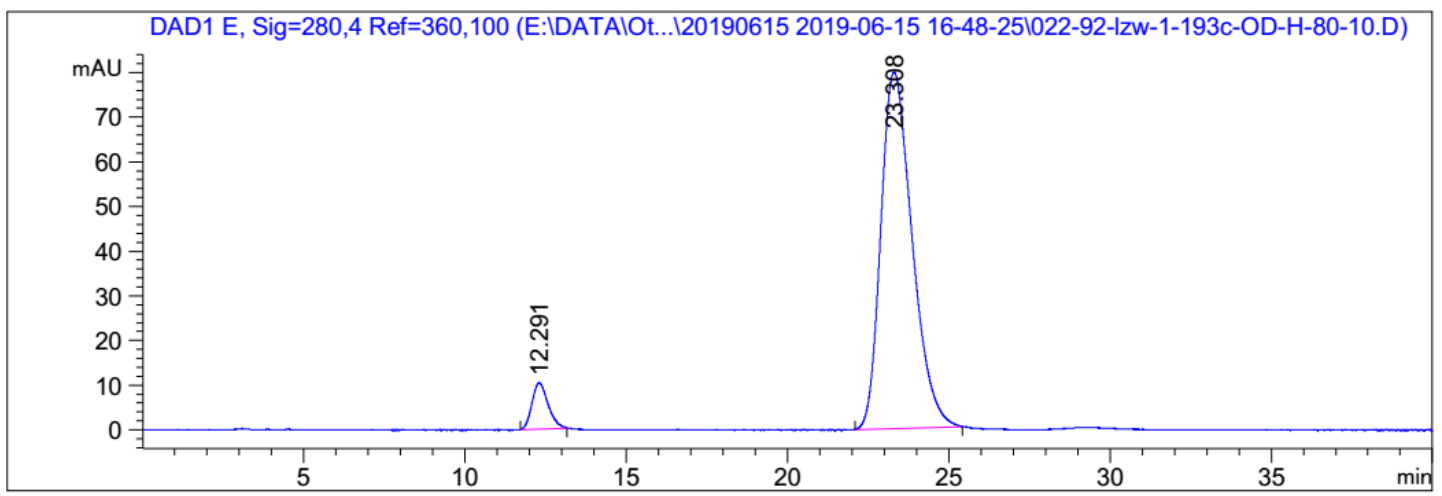

Signal 1: DAD1 E, Sig $=280,4$ Ref $=360,100$

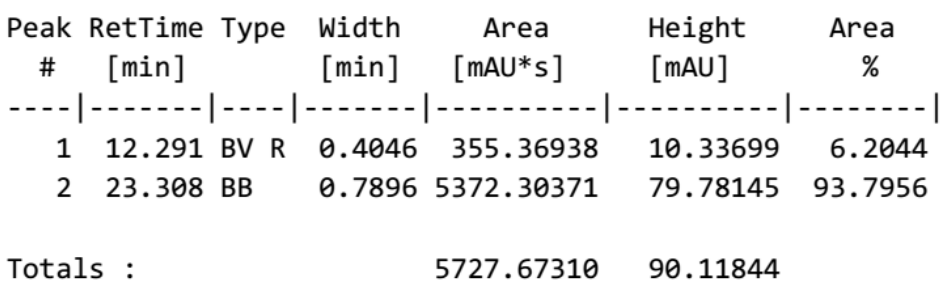




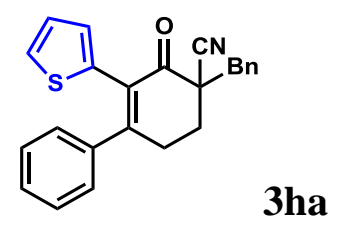

AD-H, 95\% HEX, $40 \mathrm{~min}, 0.8 \mathrm{~mL} / \mathrm{min}$

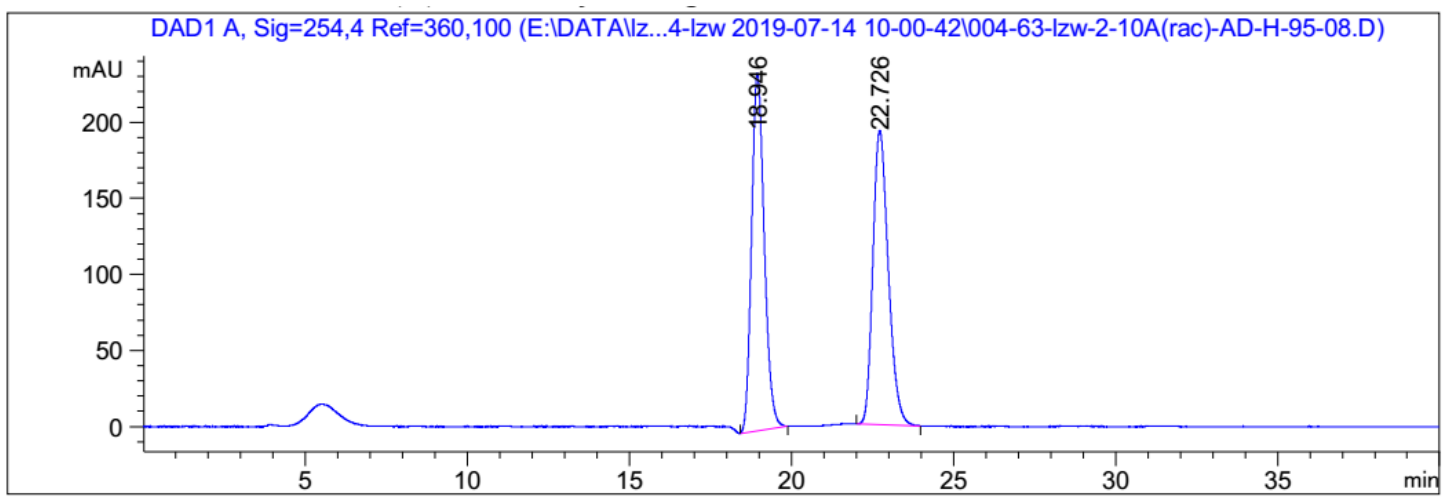

Signal 1: DAD1 A, Sig=254,4 Ref $=360,100$

\begin{tabular}{|c|c|c|c|c|c|c|}
\hline $\begin{array}{c}\text { Peak } \\
\quad \#\end{array}$ & $\begin{array}{c}\text { RetTime } \\
\text { [min] }\end{array}$ & Type & $\begin{array}{l}\text { Width } \\
\text { [min] }\end{array}$ & $\begin{array}{c}\text { Area } \\
{\left[\mathrm{mAU}^{*} \mathrm{~s}\right]}\end{array}$ & $\begin{array}{l}\text { Height } \\
{[\mathrm{mAU}]}\end{array}$ & $\begin{array}{c}\text { Area } \\
\%\end{array}$ \\
\hline & & & & & & \\
\hline 1 & 18. & R & 0.4018 & 6372.35303 & 234.70654 & 49.8978 \\
\hline 2 & 22.726 & BB & 0.4574 & 6398.45361 & 193.43410 & 50.1022 \\
\hline
\end{tabular}

$\begin{array}{lll}\text { Totals : } & 1.27708 \mathrm{e} 4 & 428.14064\end{array}$

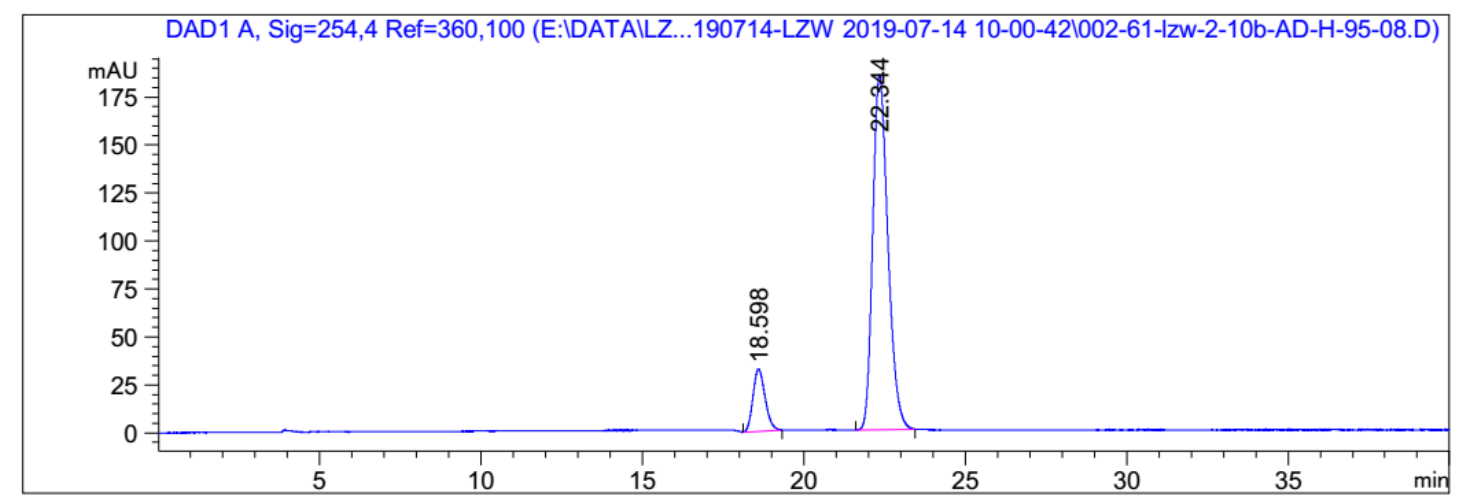

Signal 1: DAD1 A, Sig $=254,4$ Ref $=360,100$

\begin{tabular}{|c|c|c|c|c|c|c|}
\hline $\begin{array}{c}\text { Peak } \\
\#\end{array}$ & $\begin{array}{c}\text { RetTime } \\
\text { [min] }\end{array}$ & Type & $\begin{array}{l}\text { Width } \\
{[\mathrm{min}]}\end{array}$ & $\begin{array}{c}\text { Area } \\
{\left[\mathrm{mAU}^{*} \mathrm{~s}\right]}\end{array}$ & $\begin{array}{l}\text { Height } \\
{[\mathrm{mAU}]}\end{array}$ & $\begin{array}{c}\text { Area } \\
\%\end{array}$ \\
\hline & & & & & & \\
\hline 1 & 18.598 & B & 113 & 840.55292 & 32.43810 & 12.2140 \\
\hline 2 & 22.344 & BB & 0.4289 & 6041.34570 & 184.78969 & 87.7860 \\
\hline
\end{tabular}

Totals :

$6881.89862 \quad 217.22779$ 


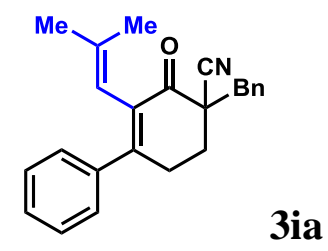

AD-H, 95\% HEX, $30 \mathrm{~min}, 0.5 \mathrm{~mL} / \mathrm{min}$

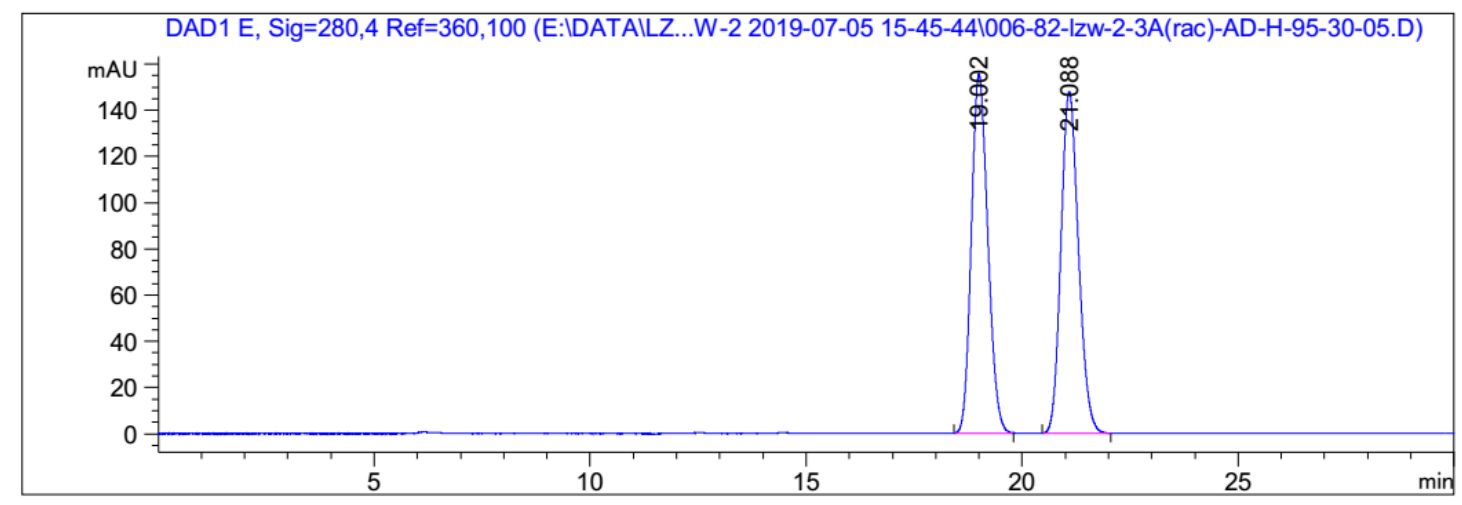

Signal 1: DAD1 E, Sig $=280,4$ Ref $=360,100$
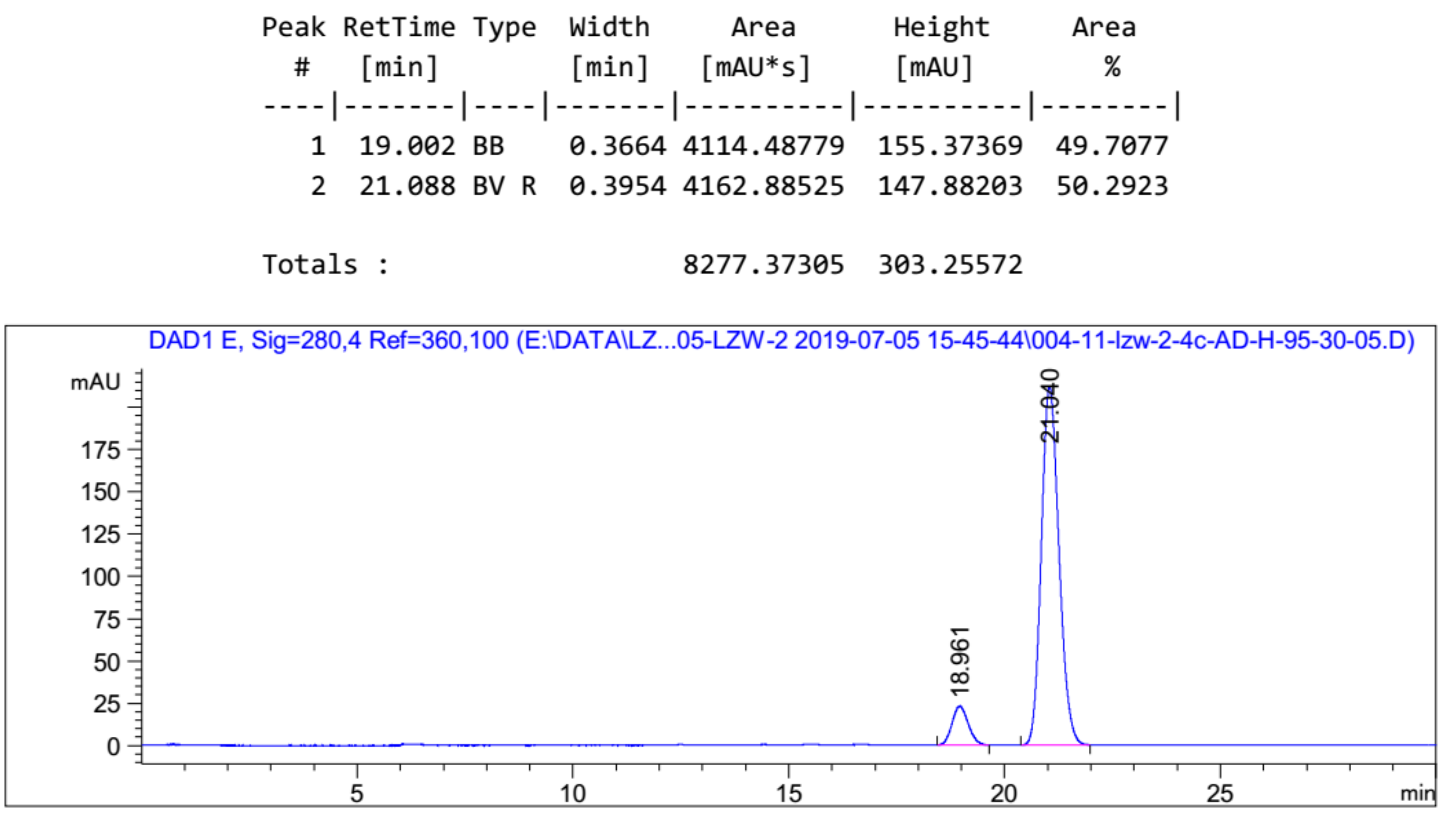

Signal 1: DAD1 E, Sig=280,4 Ref=360,100

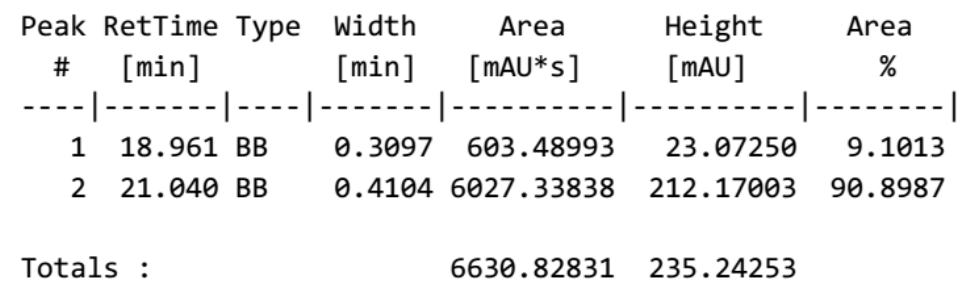




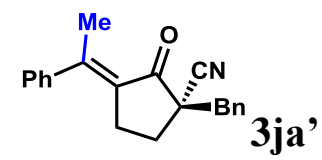

AD-H, 95\% HEX, 40 min, 0.8 mL/min

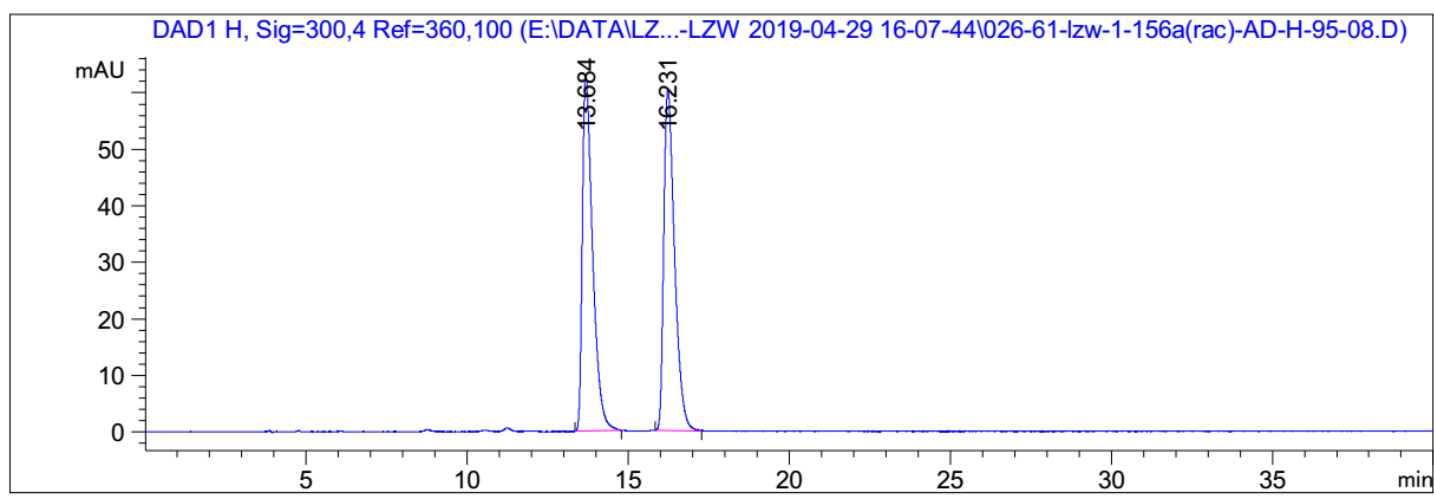

Signal 1: DAD1 H, Sig $=300,4$ Ref $=360,100$

\begin{tabular}{|c|c|c|c|c|c|}
\hline $\begin{array}{c}\text { Peak } \\
\quad \#\end{array}$ & $\begin{array}{l}\text { RetTime Type } \\
\text { [min] }\end{array}$ & $\begin{array}{l}\text { Width } \\
\text { [min] }\end{array}$ & $\begin{array}{c}\text { Area } \\
{[\mathrm{mAU} * \mathrm{~s}]}\end{array}$ & $\begin{array}{l}\text { Height } \\
{[\mathrm{mAU}]}\end{array}$ & $\begin{array}{c}\text { Area } \\
\quad \%\end{array}$ \\
\hline & & & & & \\
\hline 1 & $13.684 \mathrm{BB}$ & 0.3241 & 1398.03271 & 63.07200 & 49.9728 \\
\hline 2 & 16.231 BВ & 0.3409 & 1399.55640 & 60.28011 & 50.0272 \\
\hline & & & 2797.58911 & 123.35211 & \\
\hline
\end{tabular}

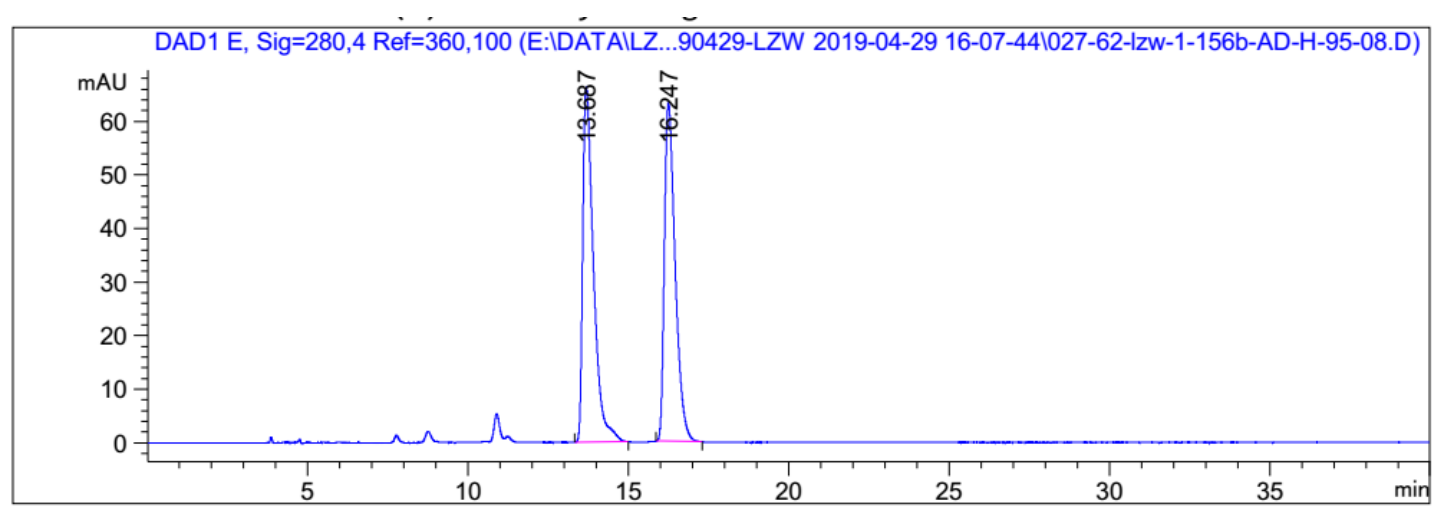

Signal 1: DAD1 E, Sig $=280,4$ Ref $=360,100$

\begin{tabular}{|c|c|c|c|c|c|}
\hline $\begin{array}{c}\text { Peak } \\
\quad \#\end{array}$ & $\begin{array}{l}\text { RetTime Type } \\
\text { [min] }\end{array}$ & $\begin{array}{l}\text { Width } \\
\text { [min] }\end{array}$ & $\begin{array}{c}\text { Area } \\
{\left[\mathrm{mAU}^{*} \mathrm{~s}\right]}\end{array}$ & $\begin{array}{l}\text { Height } \\
{[\mathrm{mAU}]}\end{array}$ & $\begin{array}{c}\text { Area } \\
\%\end{array}$ \\
\hline & & & & & \\
\hline 1 & $13.687 \mathrm{BB}$ & 0.3360 & 1544.18420 & 66.22587 & 51.1576 \\
\hline 2 & 16.247 BB & 0.3348 & 1474.29993 & 63.17260 & 48.8424 \\
\hline otal & & & 3018.48413 & 129.39847 & \\
\hline
\end{tabular}




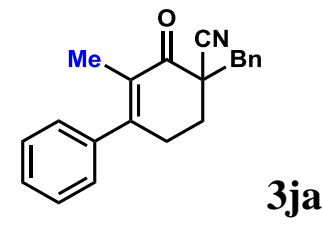

IC, $95 \% \mathrm{HEX}, 40 \mathrm{~min}, 0.5 \mathrm{~mL} / \mathrm{min}$

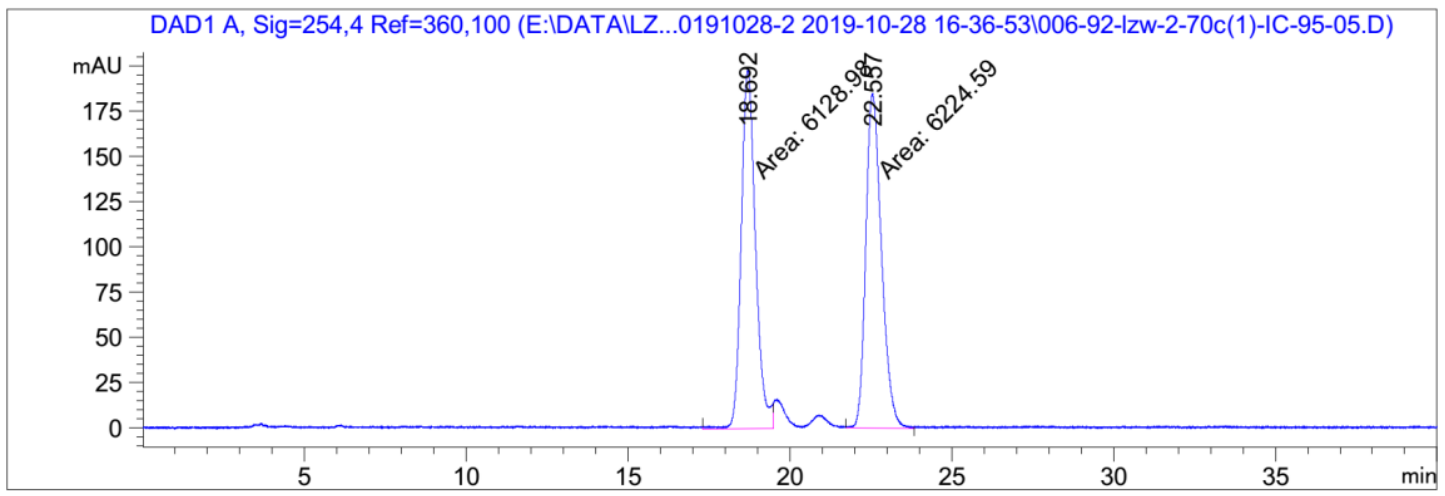

Signal 1: DAD1 A, Sig=254, 4 Ref $=360,100$

\begin{tabular}{cccccc}
$\begin{array}{c}\text { Peak RetTime Type } \\
\text { \# } \\
{[\text { min] }}\end{array}$ & $\begin{array}{c}\text { Width } \\
{[\text { min] }}\end{array}$ & $\begin{array}{c}\text { Area } \\
{[\text { mAU*s] }}\end{array}$ & $\begin{array}{c}\text { Height } \\
{[\mathrm{mAU}]}\end{array}$ & $\begin{array}{c}\text { Area } \\
\%\end{array}$ \\
\hdashline 1 & 18.692 MM & 0.5156 & 6128.97559 & 198.13519 & 49.6130 \\
2 & 22.557 MM & 0.5608 & 6224.58789 & 185.00330 & 50.3870
\end{tabular}

Totals : $\quad 1.23536 \mathrm{e} 4 \quad 383.13849$

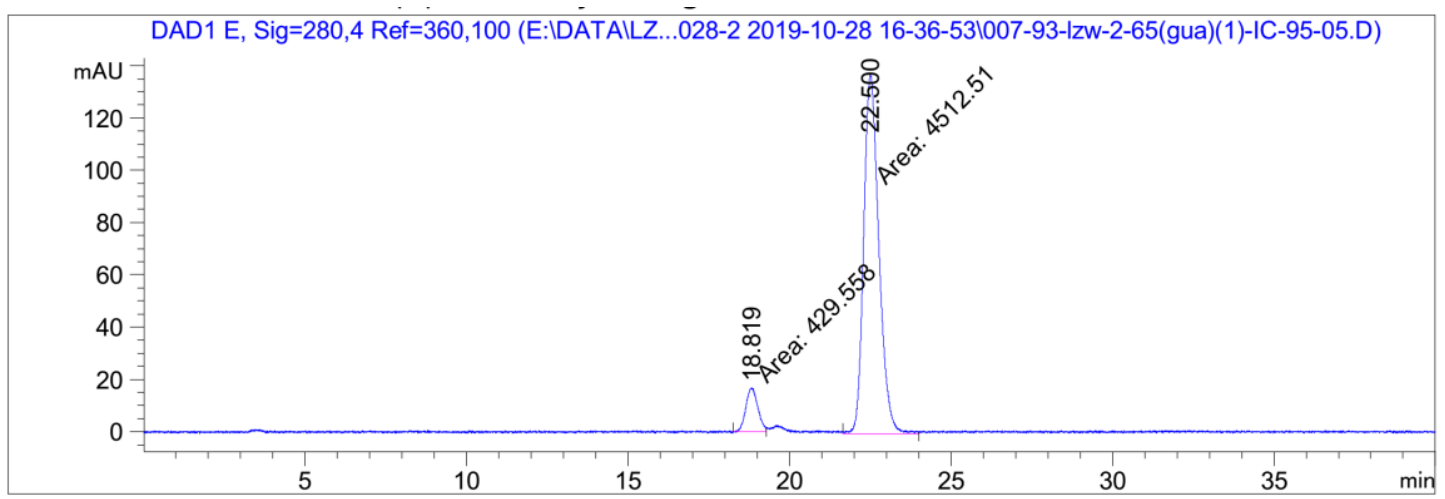

Signal 1: DAD1 E, Sig=280,4 Ref $=360,100$

\begin{tabular}{|c|c|c|c|c|c|c|}
\hline $\begin{array}{c}\text { Peak } \\
\#\end{array}$ & $\begin{array}{c}\text { RetTime } \\
\text { [min] }\end{array}$ & Type & $\begin{array}{l}\text { Width } \\
\text { [min] }\end{array}$ & $\begin{array}{c}\text { Area } \\
{\left[\mathrm{mAU}^{*} \mathrm{~s}\right]}\end{array}$ & $\begin{array}{l}\text { Height } \\
{[\mathrm{mAU}]}\end{array}$ & $\begin{array}{c}\text { Area } \\
\%\end{array}$ \\
\hline---1 & | - - - & & --- & |--.-- & - & 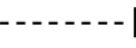 \\
\hline 1 & 18.819 & MM & 0.4264 & 429.55762 & 16.78945 & 8.6919 \\
\hline 2 & 22.500 & MM & 0.5482 & 4512.51172 & 137.19781 & 91.3081 \\
\hline \multicolumn{4}{|c|}{ Totals : } & 4942.06934 & 153.98727 & \\
\hline
\end{tabular}


<smiles>[SeH2]</smiles>

$\mathrm{AD}-\mathrm{H}, 95 \% \mathrm{HEX}, 40 \mathrm{~min}, 0.8 \mathrm{~mL} / \mathrm{min}$

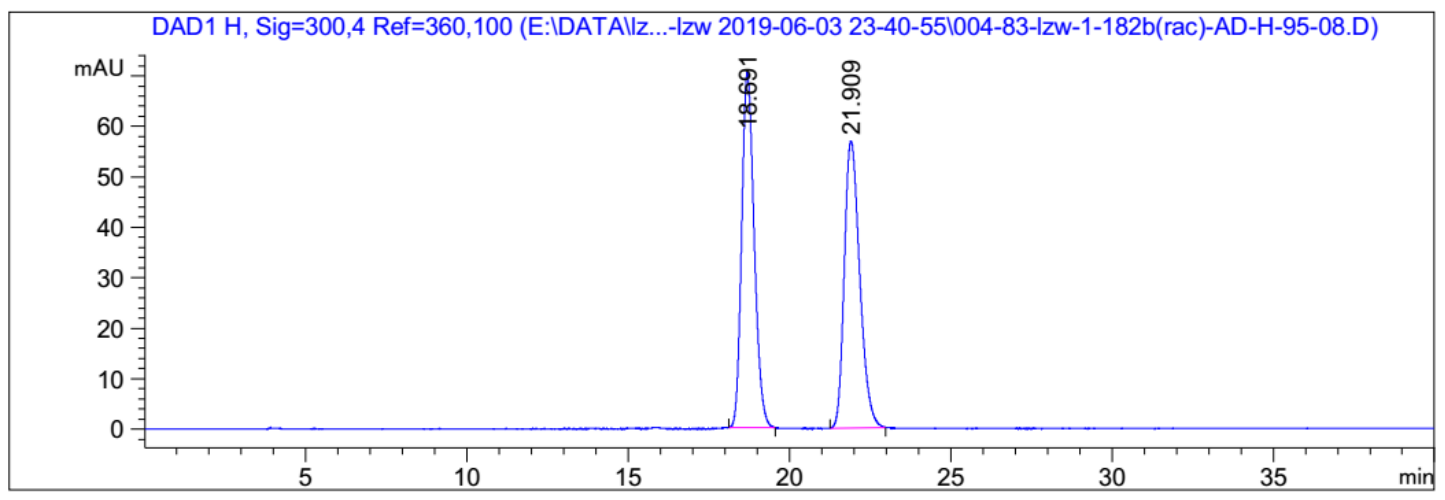

Signal 1: DAD1 H, Sig=300,4 Ref=360,100

\begin{tabular}{|c|c|c|c|c|c|c|}
\hline $\begin{array}{c}\text { Peak } \\
\#\end{array}$ & $\begin{array}{c}\text { RetTime } \\
\text { [min] }\end{array}$ & Type & $\begin{array}{l}\text { Width } \\
\text { [min] }\end{array}$ & $\begin{array}{c}\text { Area } \\
{\left[\mathrm{mAU}^{*} \mathrm{~s}\right]}\end{array}$ & $\begin{array}{l}\text { Height } \\
{[\mathrm{mAU}]}\end{array}$ & $\begin{array}{c}\text { Area } \\
\%\end{array}$ \\
\hline & & & & |--.-. & ---1 & ------ \\
\hline 1 & 18.691 & BB & 0.3632 & 1897.14404 & 70.43356 & 50.1004 \\
\hline 2 & 21.909 & BB & 0.3975 & 1889.53748 & 56.89539 & 49.8996 \\
\hline ota & Is & & & 3786.68152 & 32895 & \\
\hline
\end{tabular}

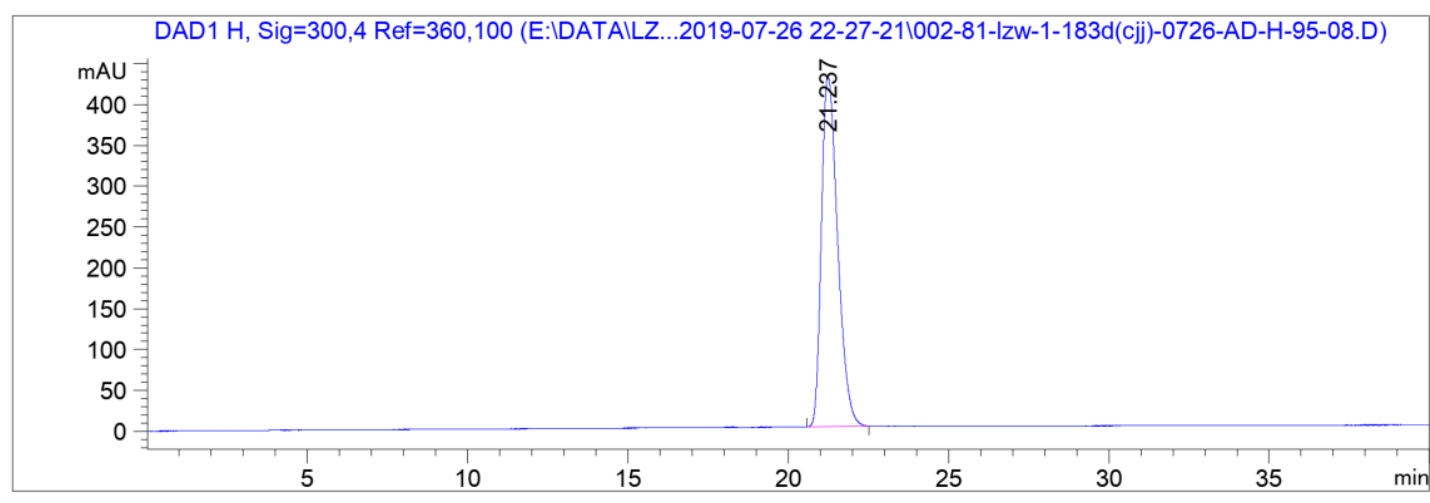

Signal 1: DAD1 H, Sig=300, 4 Ref $=360,100$

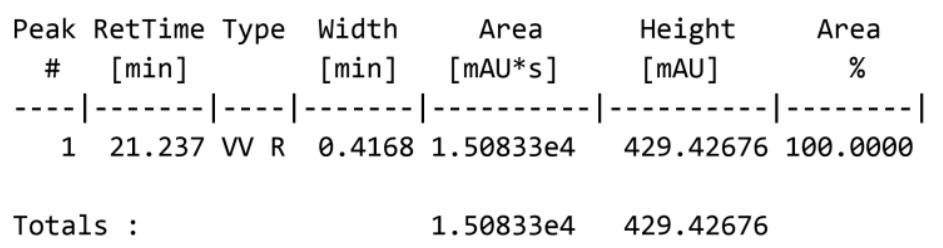




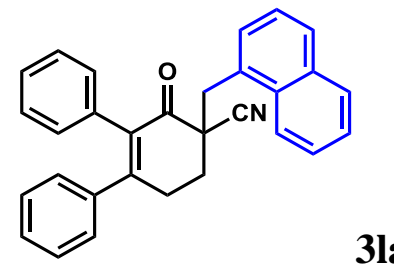

IB, $90 \%$ HEX, $40 \mathrm{~min}, 0.8 \mathrm{~mL} / \mathrm{min}$

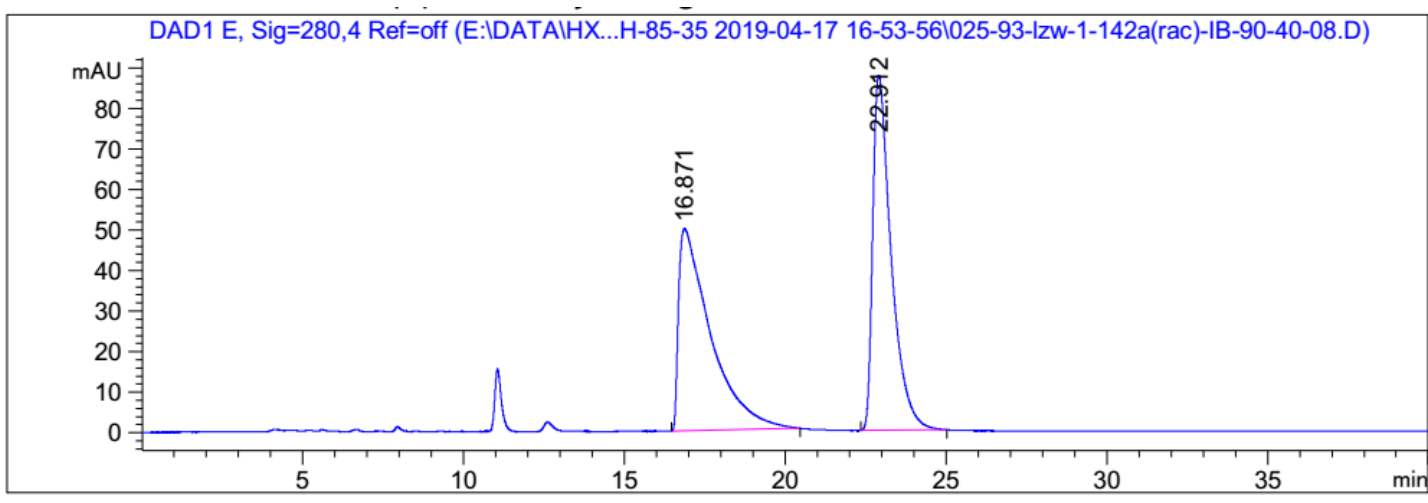

Signal 1: DAD1 E, Sig=280,4 Ref=off
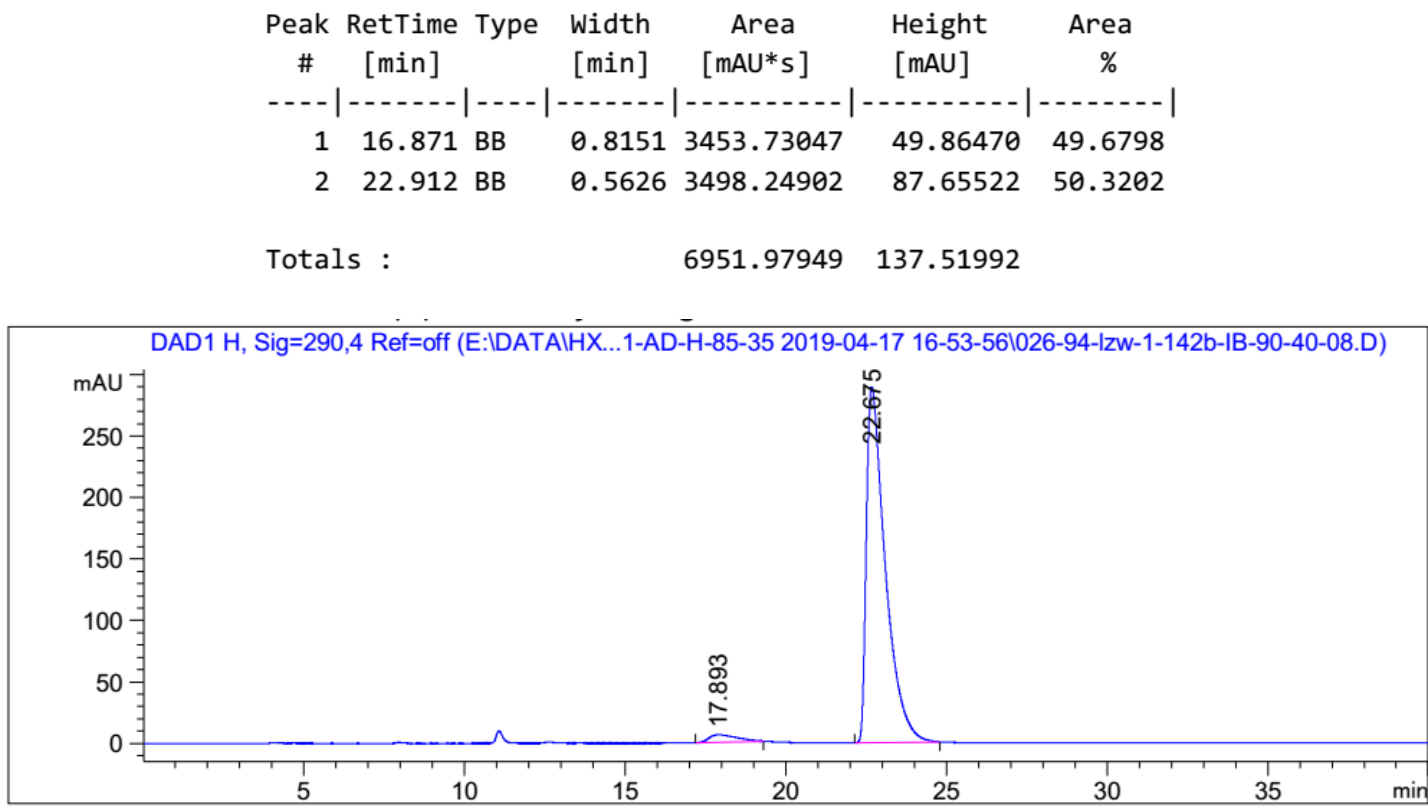

Signal 1: DAD1 H, Sig=290,4 Ref=off

\begin{tabular}{|c|c|c|c|c|c|}
\hline $\begin{array}{c}\text { Peak } \\
\#\end{array}$ & $\begin{array}{l}\text { RetTime Type } \\
\text { [min] }\end{array}$ & $\begin{array}{l}\text { Width } \\
\text { [min] }\end{array}$ & $\begin{array}{c}\text { Area } \\
{\left[\mathrm{mAU}^{*} \mathrm{~s}\right]}\end{array}$ & $\begin{array}{l}\text { Height } \\
{[\mathrm{mAU}]}\end{array}$ & $\begin{array}{c}\text { Area } \\
\%\end{array}$ \\
\hline & & & 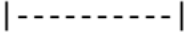 & $-x_{-1}$ & \\
\hline 1 & 17.893 ВВ & 0.7060 & 377.20358 & 6.26030 & 3.0811 \\
\hline 2 & 22.675 BB & 0.5557 & $1.18652 \mathrm{e} 4$ & 289.45868 & 96.9189 \\
\hline & & & $22424 \mathrm{e} 4$ & 1898 & \\
\hline
\end{tabular}




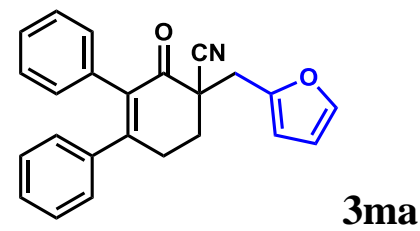

$\mathrm{AD}-\mathrm{H}, 95 \% \mathrm{HEX}, 40 \mathrm{~min}, 0.8 \mathrm{~mL} / \mathrm{min}$

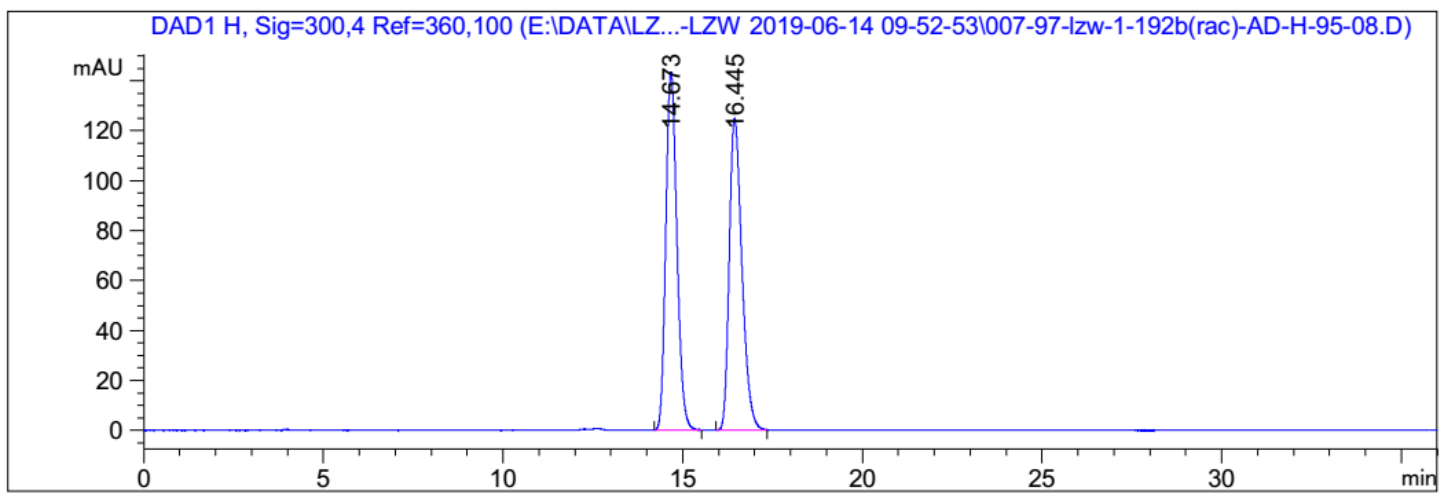

Signal 1: DAD1 H, Sig=300,4 Ref $=360,100$

\begin{tabular}{|c|c|c|c|c|c|c|}
\hline $\begin{array}{c}\text { Peak } \\
\quad \#\end{array}$ & $\begin{array}{c}\text { RetTime } \\
\text { [min] }\end{array}$ & Type & $\begin{array}{l}\text { Width } \\
\text { [min] }\end{array}$ & $\begin{array}{c}\text { Area } \\
{\left[\mathrm{mAU}^{*} \mathrm{~s}\right]}\end{array}$ & $\begin{array}{l}\text { Height } \\
{[\mathrm{mAU}]}\end{array}$ & $\begin{array}{c}\text { Area } \\
\%\end{array}$ \\
\hline$-\ldots$ & $\mid--$ & & - & 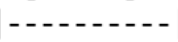 & 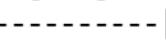 & $------\mid$ \\
\hline 1 & 14.673 & BB & 0.3048 & 3077.80933 & 143.44453 & 50.1822 \\
\hline 2 & 16.445 & BB & 0.3526 & 3055.46143 & 124.80907 & 49.8178 \\
\hline Tota & 5 & & & 6133.27075 & 268.25361 & \\
\hline
\end{tabular}

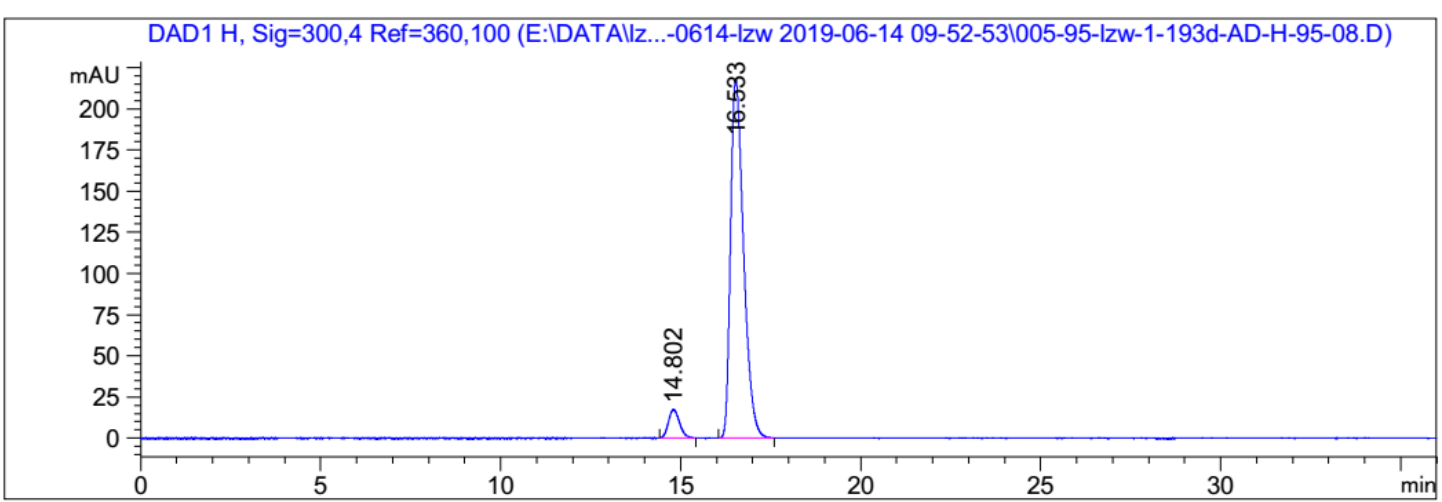

Signal 1: DAD1 H, Sig $=300,4$ Ref $=360,100$

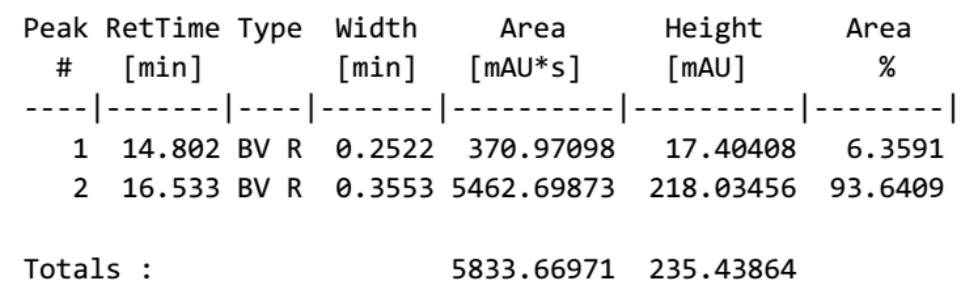


<smiles>CC(C)=CCC1(C)CCC(c2ccccc2)=C(c2ccccc2)C1=O</smiles>

\section{3na}

AD-H, 95\% HEX, $40 \mathrm{~min}, 0.8 \mathrm{~mL} / \mathrm{min}$

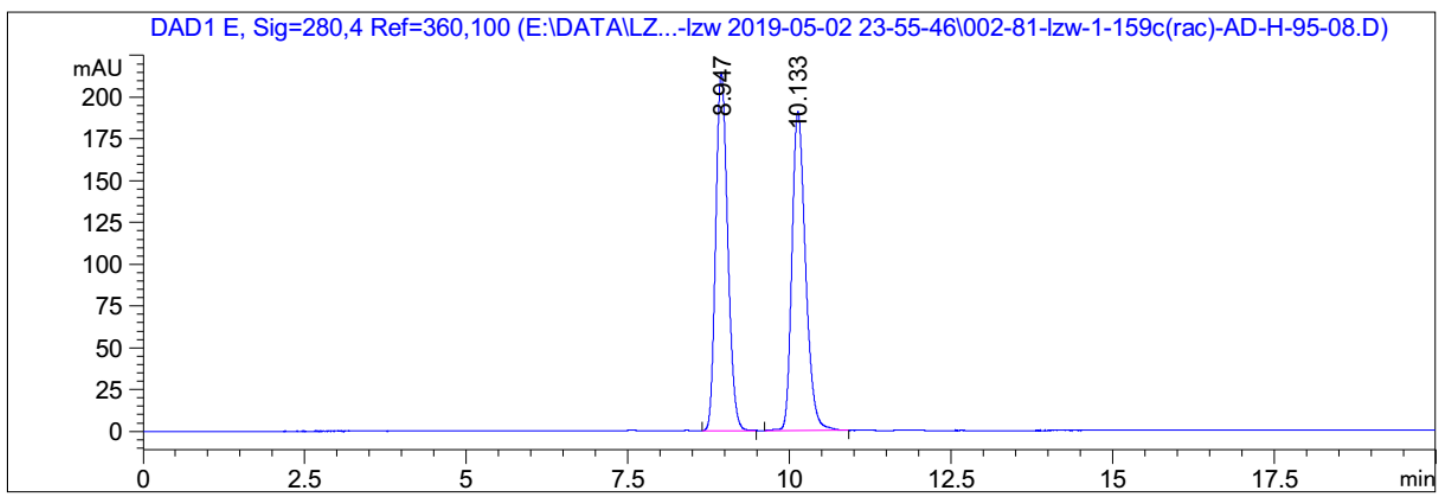

Signal 1: DAD1 E, Sig $=280,4$ Ref $=360,100$

\begin{tabular}{|c|c|c|c|c|c|c|}
\hline $\begin{array}{c}\text { Peak } \\
\#\end{array}$ & $\begin{array}{c}\text { RetTime } \\
{[\text { min] }}\end{array}$ & Type & $\begin{array}{l}\text { Width } \\
\text { [min] }\end{array}$ & $\begin{array}{c}\text { Area } \\
{\left[\mathrm{mAU}^{*} \mathrm{~s}\right]}\end{array}$ & $\begin{array}{l}\text { Height } \\
{[\mathrm{mAU}]}\end{array}$ & $\begin{array}{c}\text { Area } \\
\%\end{array}$ \\
\hline & & & & 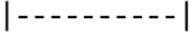 & & \\
\hline 1 & 8.947 & BB & 0.2006 & 2757.15210 & 214.28464 & 49.6323 \\
\hline 2 & 10.133 & BB & 0.2255 & 2798.00391 & 190.88585 & 50.3677 \\
\hline & & & & .56 & & \\
\hline
\end{tabular}

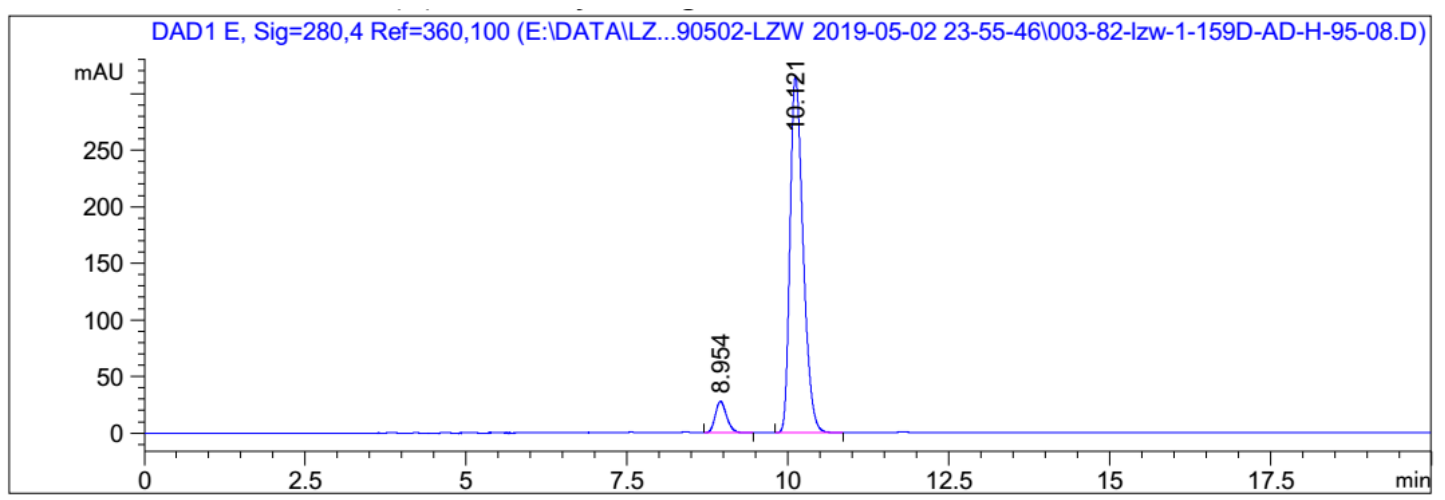

Signal 1: DAD1 E, Sig=280,4 $\operatorname{Ref}=360,100$

\begin{tabular}{|c|c|c|c|c|c|}
\hline $\begin{array}{c}\text { Peak } \\
\#\end{array}$ & $\begin{array}{l}\text { RetTime Type } \\
\text { [min] }\end{array}$ & $\begin{array}{l}\text { Width } \\
\text { [min] }\end{array}$ & $\begin{array}{c}\text { Area } \\
{[\mathrm{mAU} * \mathrm{~s}]}\end{array}$ & $\begin{array}{l}\text { Height } \\
\text { [mAU] }\end{array}$ & $\begin{array}{c}\text { Area } \\
\%\end{array}$ \\
\hline-1 & 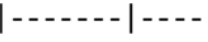 & $\cdots$ & 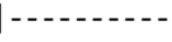 & 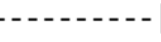 & ------ \\
\hline 1 & $8.954 \mathrm{BB}$ & 0.1874 & 342.46606 & 27.85466 & 7.1447 \\
\hline 2 & $10.121 \mathrm{BB}$ & 0.2193 & 4450.82031 & 315.10382 & 92.8553 \\
\hline Tota & : & & 4793.28638 & 342.95848 & \\
\hline
\end{tabular}




\section{$30 a$}

$\mathrm{AD}-\mathrm{H}, 95 \% \mathrm{HEX}, 30 \mathrm{~min}, 0.5 \mathrm{~mL} / \mathrm{min}$

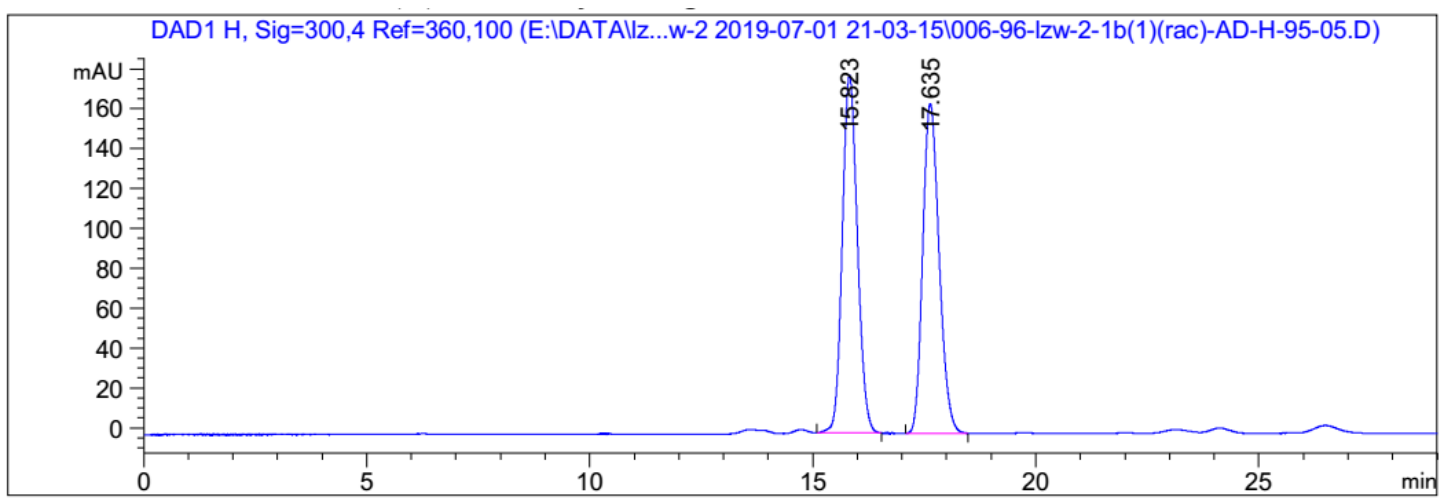

Signal 1: DAD1 H, Sig $=300,4$ Ref $=360,100$
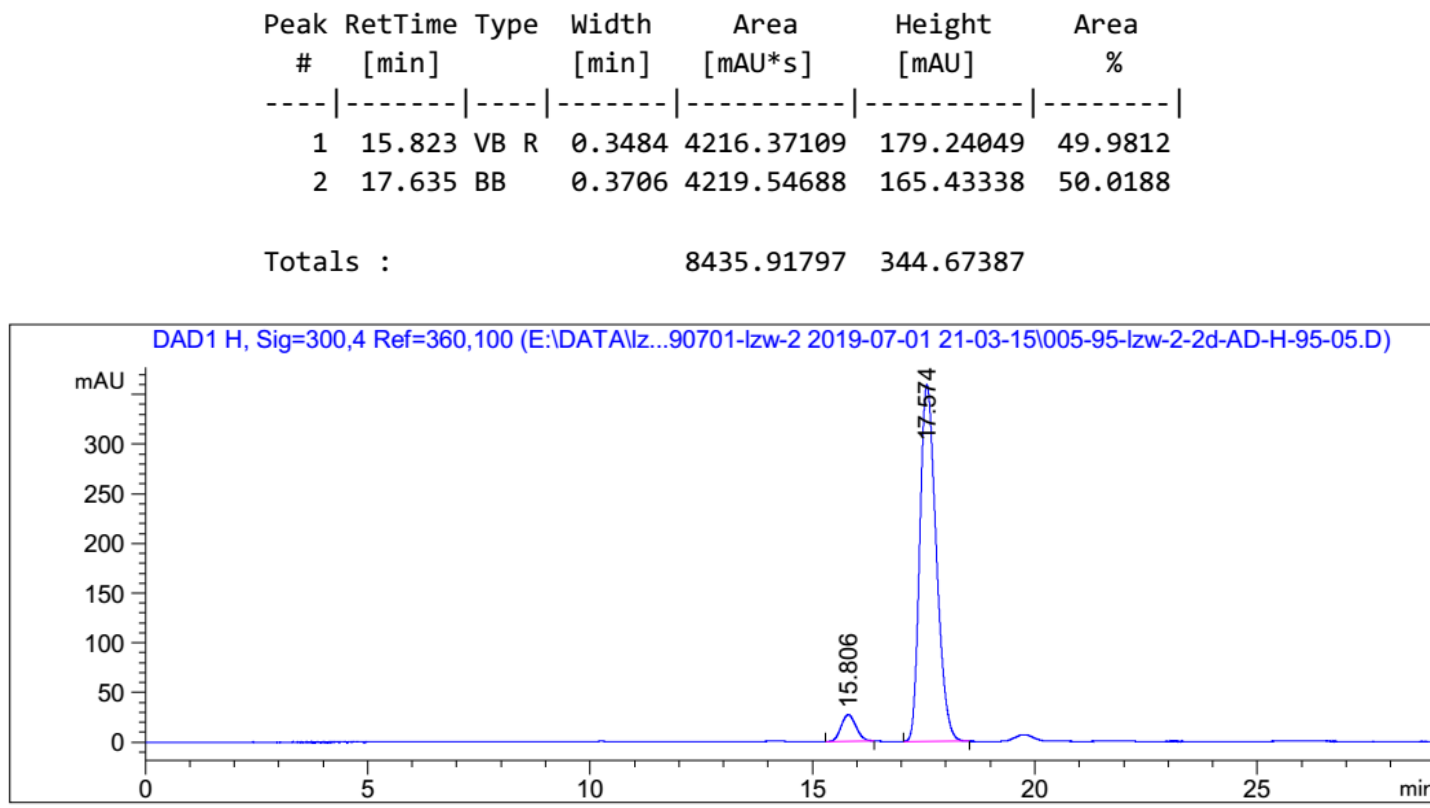

Signal 1: DAD1 H, Sig $=300,4$ Ref $=360,100$

\begin{tabular}{|c|c|c|c|c|c|c|}
\hline $\begin{array}{c}\text { Peak } \\
\#\end{array}$ & $\begin{array}{c}\text { RetTime } \\
\text { [min] }\end{array}$ & Type & $\begin{array}{l}\text { Width } \\
\text { [min] }\end{array}$ & $\begin{array}{c}\text { Area } \\
{\left[\mathrm{mAU}^{*} \mathrm{~s}\right]}\end{array}$ & $\begin{array}{l}\text { Height } \\
\text { [mAU] }\end{array}$ & $\begin{array}{c}\text { Area } \\
\%\end{array}$ \\
\hline--- & $\mid--$ & & -- & $\mid--$ & | & $-----\mid$ \\
\hline 1 & 15.806 & BB & 0.2730 & 628.40015 & 27.00057 & 6.3835 \\
\hline 2 & 17.574 & BV $R$ & 0.3797 & 9215.70508 & 358.76007 & 93.6165 \\
\hline Tot & : & & & 9844.10522 & 385.76064 & \\
\hline
\end{tabular}


<smiles>CC(=O)CCC1(C)CCC(c2ccccc2)=C(c2ccccc2)C1=O</smiles>

\section{3pa}

AD-H, 95\% HEX, $40 \mathrm{~min}, 0.8 \mathrm{~mL} / \mathrm{min}$

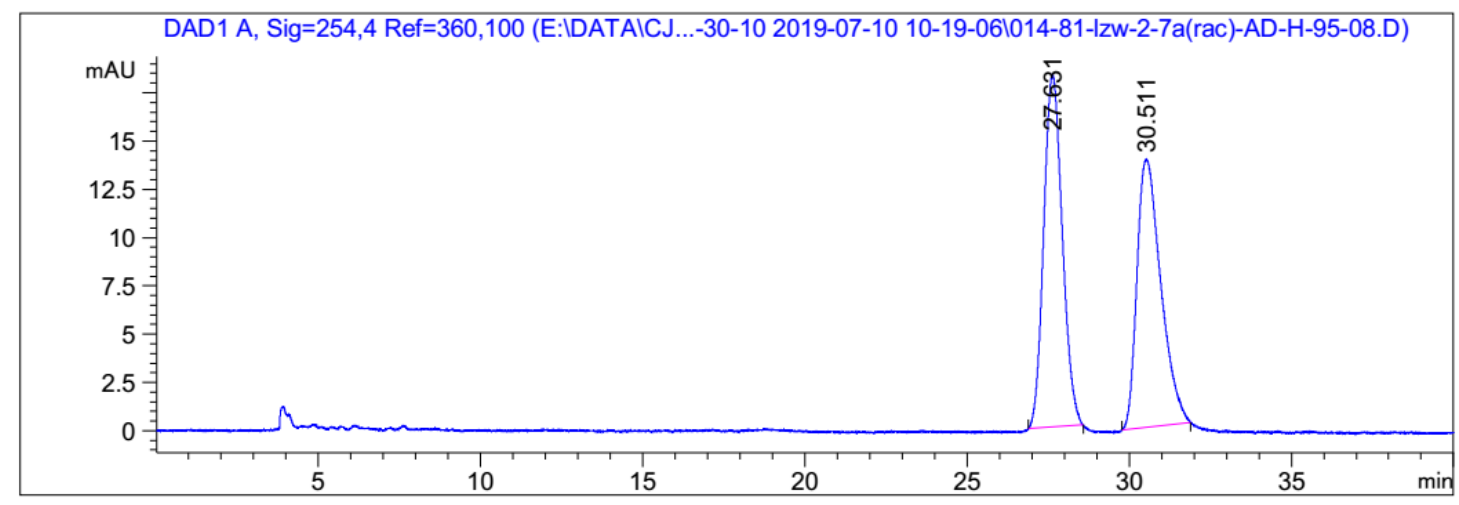

Signal 1: DAD1 A, Sig=254,4 Ref=360,100

\begin{tabular}{|c|c|c|c|c|c|}
\hline $\begin{array}{c}\text { Peak } \\
\text { \# }\end{array}$ & $\begin{array}{l}\text { RetTime Type } \\
\text { [min] }\end{array}$ & $\begin{array}{l}\text { Width } \\
\text { [min] }\end{array}$ & $\begin{array}{c}\text { Area } \\
{[\mathrm{mAU} * \mathrm{~s}]}\end{array}$ & $\begin{array}{l}\text { Height } \\
{[\mathrm{mAU}]}\end{array}$ & $\begin{array}{c}\text { Area } \\
\%\end{array}$ \\
\hline & 1 & -- & $-\cdots$ & 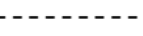 & $-\cdots$ \\
\hline 1 & 27.631 BB & 0.4670 & 727.03290 & 18.28029 & 50.6172 \\
\hline 2 & $30.511 \mathrm{BB}$ & 0.5992 & 709.30273 & 13.89058 & 49.3828 \\
\hline Tota & : & & 1436.33563 & 32.17087 & \\
\hline
\end{tabular}

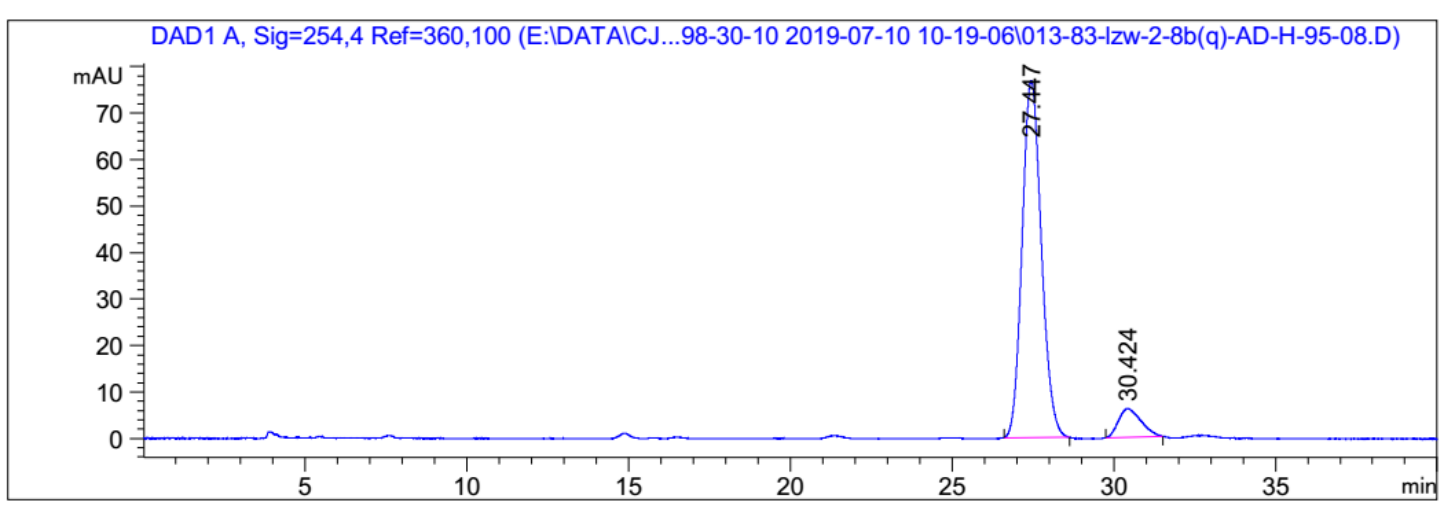

Signal 1: DAD1 A, Sig=254,4 Ref $=360,100$

\begin{tabular}{|c|c|c|c|c|c|c|}
\hline $\begin{array}{c}\text { Peak } \\
\#\end{array}$ & $\begin{array}{c}\text { RetTime } \\
\text { [min] }\end{array}$ & Type & $\begin{array}{l}\text { Width } \\
{[\mathrm{min}]}\end{array}$ & $\begin{array}{c}\text { Area } \\
{\left[\mathrm{mAU}^{*} \mathrm{~s}\right]}\end{array}$ & $\begin{array}{l}\text { Height } \\
\text { [mAU] }\end{array}$ & $\begin{array}{c}\text { Area } \\
\%\end{array}$ \\
\hline-- & - & & $-\cdots$ & |- & | & $-------\mid$ \\
\hline 1 & 27.447 & BB & 0.4857 & 3173.65234 & 76.79109 & 91.3254 \\
\hline 2 & 30.424 & BB & 0.5710 & 301.44986 & 6.19147 & 8.6746 \\
\hline Tota & : & & & 3475.10220 & 82.98256 & \\
\hline
\end{tabular}




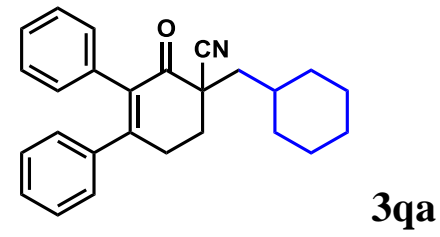

$\mathrm{AD}-\mathrm{H}, 95 \% \mathrm{HEX}, 30 \mathrm{~min}, 0.8 \mathrm{~mL} / \mathrm{min}$

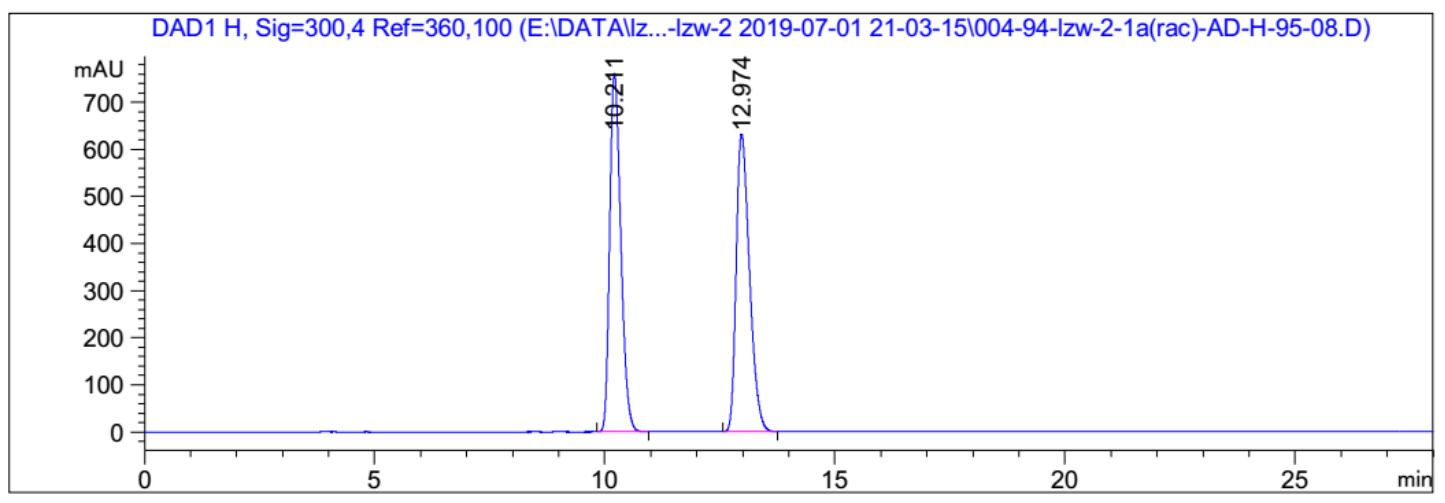

Signal 1: DAD1 H, Sig $=300,4$ Ref $=360,100$

Peak RetTime Type Width Area Height Area

\begin{tabular}{cccccc}
$\#$ & {$[\mathrm{~min}]$} & {$[\mathrm{min}]$} & {$[\mathrm{mAU} * \mathrm{~s}]$} & {$[\mathrm{mAU}]$} & $\%$ \\
\hline 1 & 10.211 BB & 0.2586 & $1.27376 \mathrm{e} 4$ & 759.88861 & 50.0355
\end{tabular}

$\begin{array}{lllllll}2 & 12.974 \text { вB } & 0.3022 & 1.27196 \mathrm{e} 4 & 632.34576 & 49.9645\end{array}$

Totals :

$2.54572 \mathrm{e} 4 \quad 1392.23438$

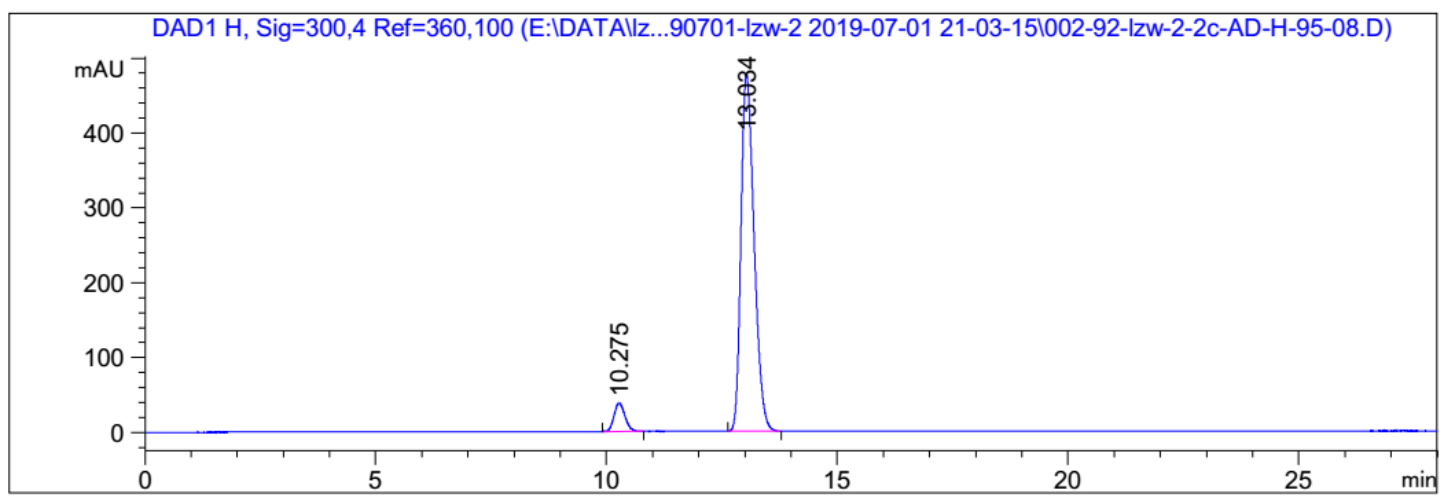

Signal 1: DAD1 H, Sig $=300,4$ Ref $=360,100$

\begin{tabular}{|c|c|c|c|c|c|}
\hline $\begin{array}{c}\text { Peak } \\
\#\end{array}$ & $\begin{array}{l}\text { RetTime Type } \\
\text { [min] }\end{array}$ & $\begin{array}{l}\text { Width } \\
\text { [min] }\end{array}$ & $\begin{array}{c}\text { Area } \\
{[\mathrm{mAU} * \mathrm{~s}]}\end{array}$ & $\begin{array}{l}\text { Height } \\
{[\mathrm{mAU}]}\end{array}$ & $\begin{array}{c}\text { Area } \\
\%\end{array}$ \\
\hline 1 & 10.275 & 54 & 623.40448 & 89106 & 6.2193 \\
\hline 2 & 13.034 вВ & 0.2986 & 9400.36133 & 477.57712 & 93.7807 \\
\hline
\end{tabular}

Totals :

$1.00238 \mathrm{e} 4 \quad 515.46818$ 
<smiles>CC1(C)CCC(c2ccccc2)=C(c2ccccc2)C1=O</smiles>

3ra

AD-H, 95\% HEX, $30 \mathrm{~min}, 0.8 \mathrm{~mL} / \mathrm{min}$

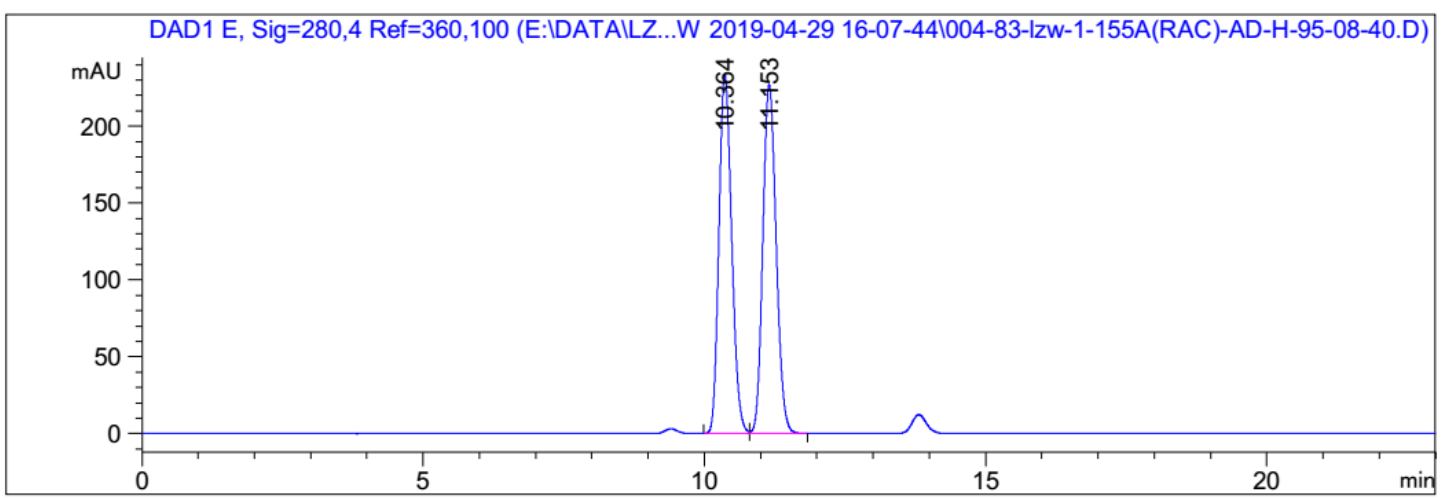

Signal 1: DAD1 E, Sig $=280,4$ Ref $=360,100$
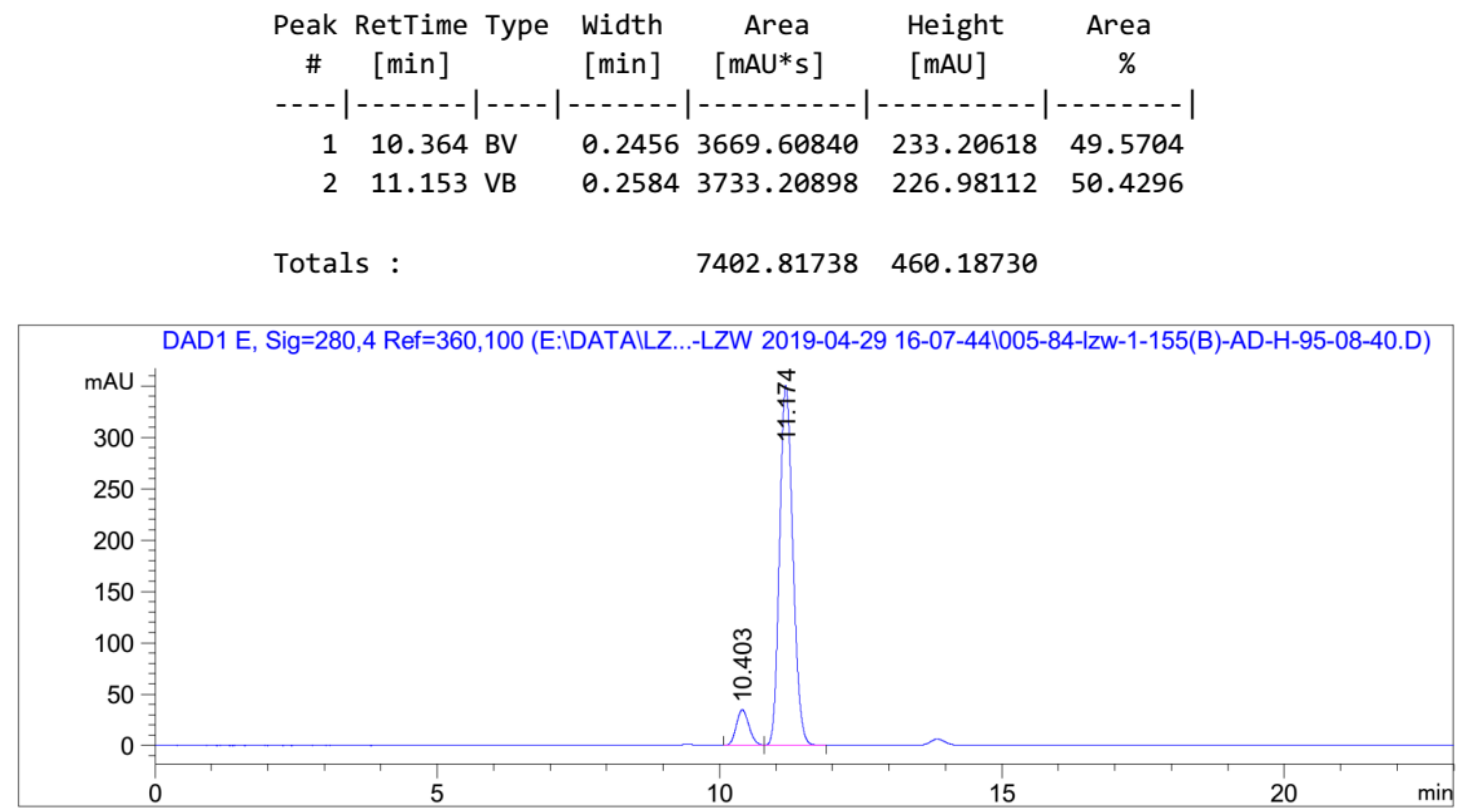

Signal 1: DAD1 E, Sig $=280,4$ Ref $=360,100$

\begin{tabular}{|c|c|c|c|c|c|c|}
\hline $\begin{array}{c}\text { Peak } \\
\#\end{array}$ & $\begin{array}{c}\text { RetTime } \\
\text { [min] }\end{array}$ & Type & $\begin{array}{l}\text { Width } \\
\text { [min] }\end{array}$ & $\begin{array}{c}\text { Area } \\
{[\mathrm{mAU} * \mathrm{~s}]}\end{array}$ & $\begin{array}{l}\text { Height } \\
{[\mathrm{mAU}]}\end{array}$ & $\begin{array}{c}\text { Area } \\
\%\end{array}$ \\
\hline- & & & & $\cdots$ & --- & ----1 \\
\hline 1 & 10.403 & BB & 0.2366 & 536.35229 & 34.54954 & 8.5537 \\
\hline 2 & 11.174 & BB & 0.2541 & 5734.07666 & 350.01135 & 91.4463 \\
\hline Tota & ls : & & & 6270.42896 & 384.56089 & \\
\hline
\end{tabular}


3sa

OD-H, 75\% HEX, $35 \mathrm{~min}, 0.5 \mathrm{~mL} / \mathrm{min}$

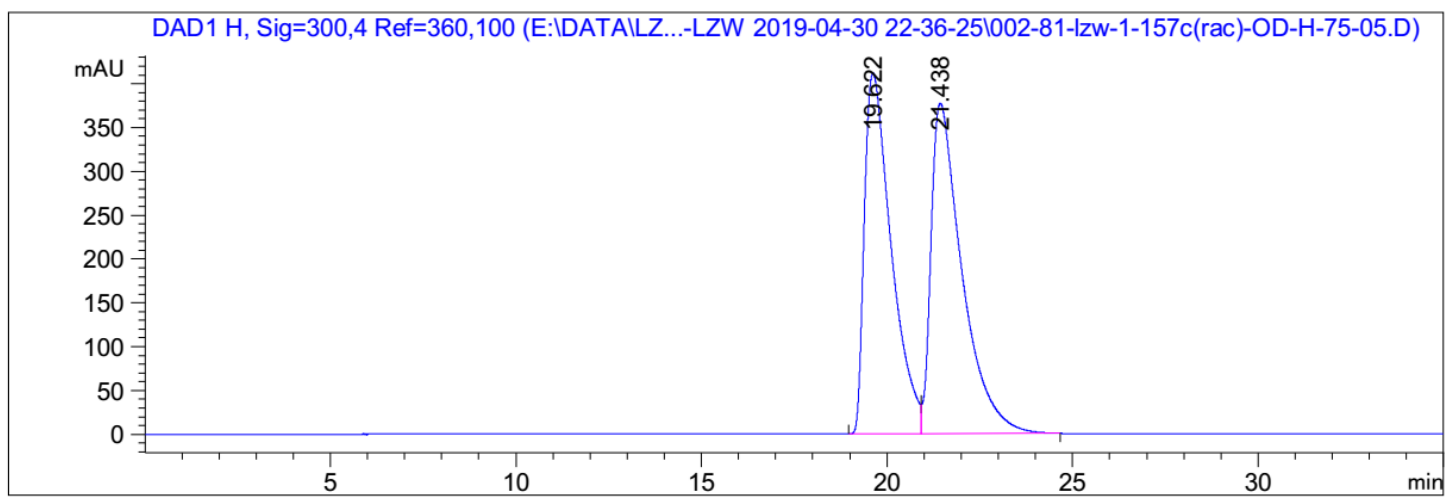

Signal 1: DAD1 H, Sig $=300,4$ Ref $=360,100$

\begin{tabular}{cccccc}
$\begin{array}{c}\text { Peak RetTime Type } \\
\text { \# } \\
{[\text { min] }}\end{array}$ & $\begin{array}{c}\text { Width } \\
{[\mathrm{min}]}\end{array}$ & $\begin{array}{c}\text { Area } \\
{[\mathrm{mAU} \text { s }]}\end{array}$ & $\begin{array}{c}\text { Height } \\
{[\mathrm{mAU}]}\end{array}$ & $\begin{array}{c}\text { Area } \\
\%\end{array}$ \\
\hline 1 & 19.622 BV & 0.6938 & $2.07191 \mathrm{e} 4$ & 411.55576 & 48.5514 \\
2 & 21.438 VB & 0.7989 & $2.19555 \mathrm{e} 4$ & 377.27142 & 51.4486 \\
& & & & \\
Totals : & & $4.26746 \mathrm{e} 4$ & 788.82718 &
\end{tabular}

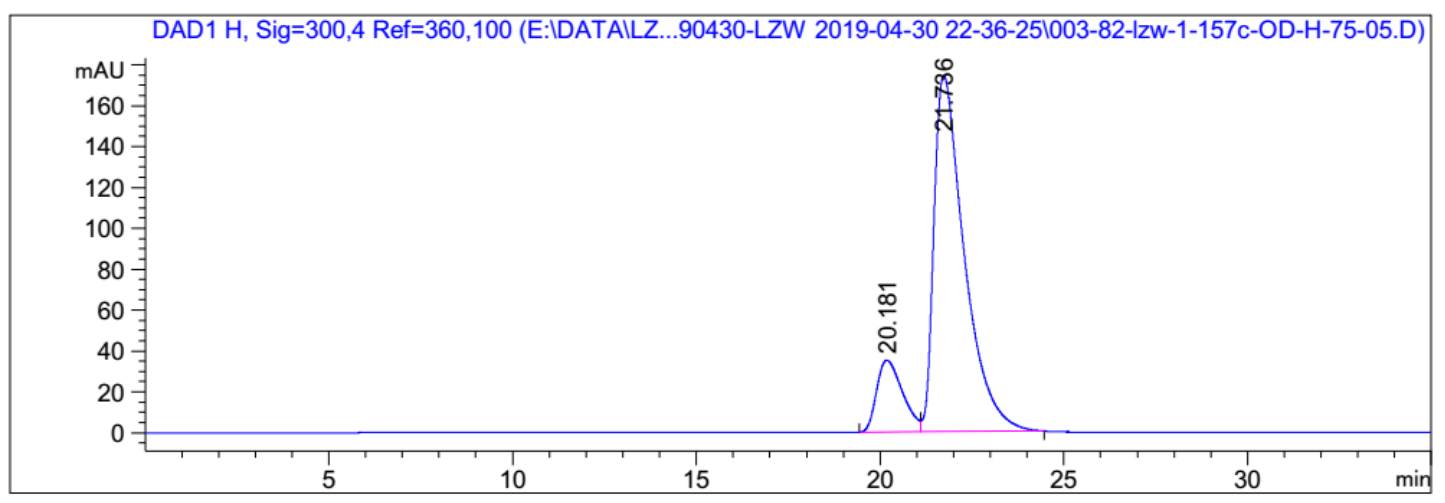

Signal 1: DAD1 H, Sig=300,4 Ref $=360,100$

\begin{tabular}{llllll}
$\begin{array}{c}\text { Peak RetTime Type } \\
\text { W } \\
{[\mathrm{min}]}\end{array}$ & $\begin{array}{c}\text { Width } \\
{[\mathrm{min}]}\end{array}$ & $\begin{array}{c}\text { Area } \\
{[\mathrm{mAU} * \mathrm{~s}]}\end{array}$ & $\begin{array}{c}\text { Height } \\
{[\mathrm{mAU}]}\end{array}$ & $\begin{array}{c}\text { Area } \\
\%\end{array}$ \\
\hline 1 & 20.181 BV & 0.5745 & 1719.94446 & 35.07787 & 14.6348 \\
2 & 21.736 VB & 0.6953 & $1.00325 \mathrm{e} 4$ & 174.23419 & 85.3652 \\
& & & & \\
Totals : & & $1.17524 \mathrm{e} 4$ & 209.31207 &
\end{tabular}




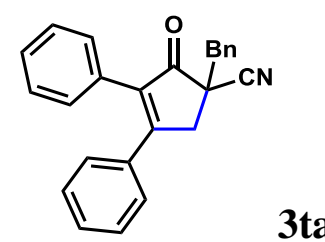

$\mathrm{AD}-\mathrm{H}, 80 \% \mathrm{HEX}, 30 \mathrm{~min}, 0.3 \mathrm{~mL} / \mathrm{min}$

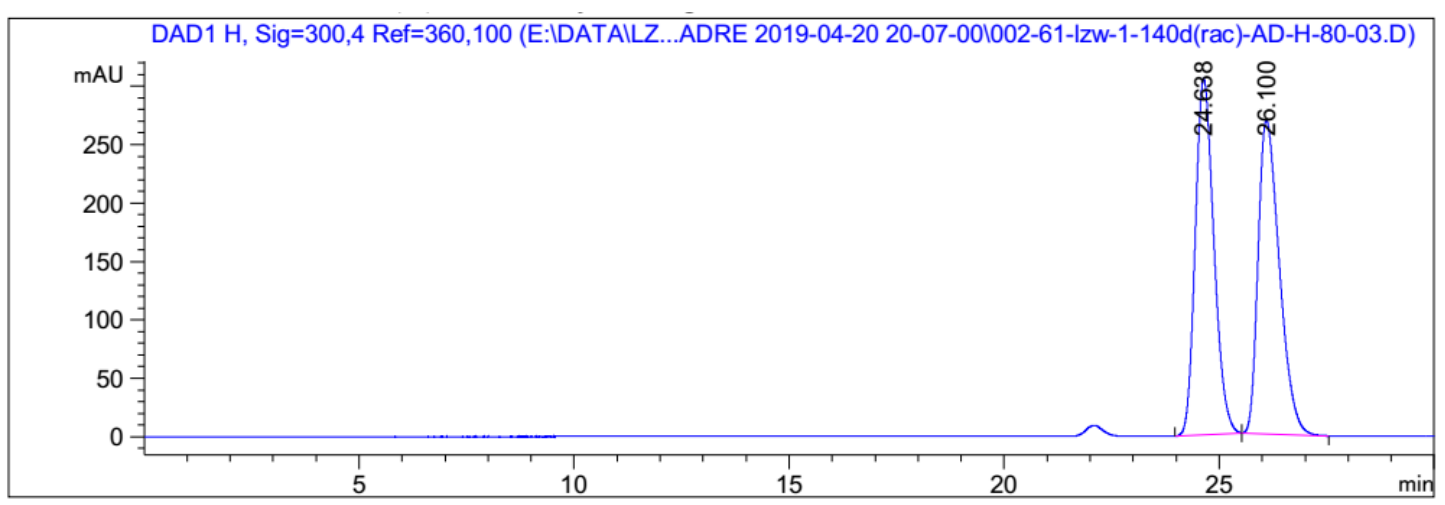

Signal 1: DAD1 H, Sig=300, 4 Ref $=360,100$
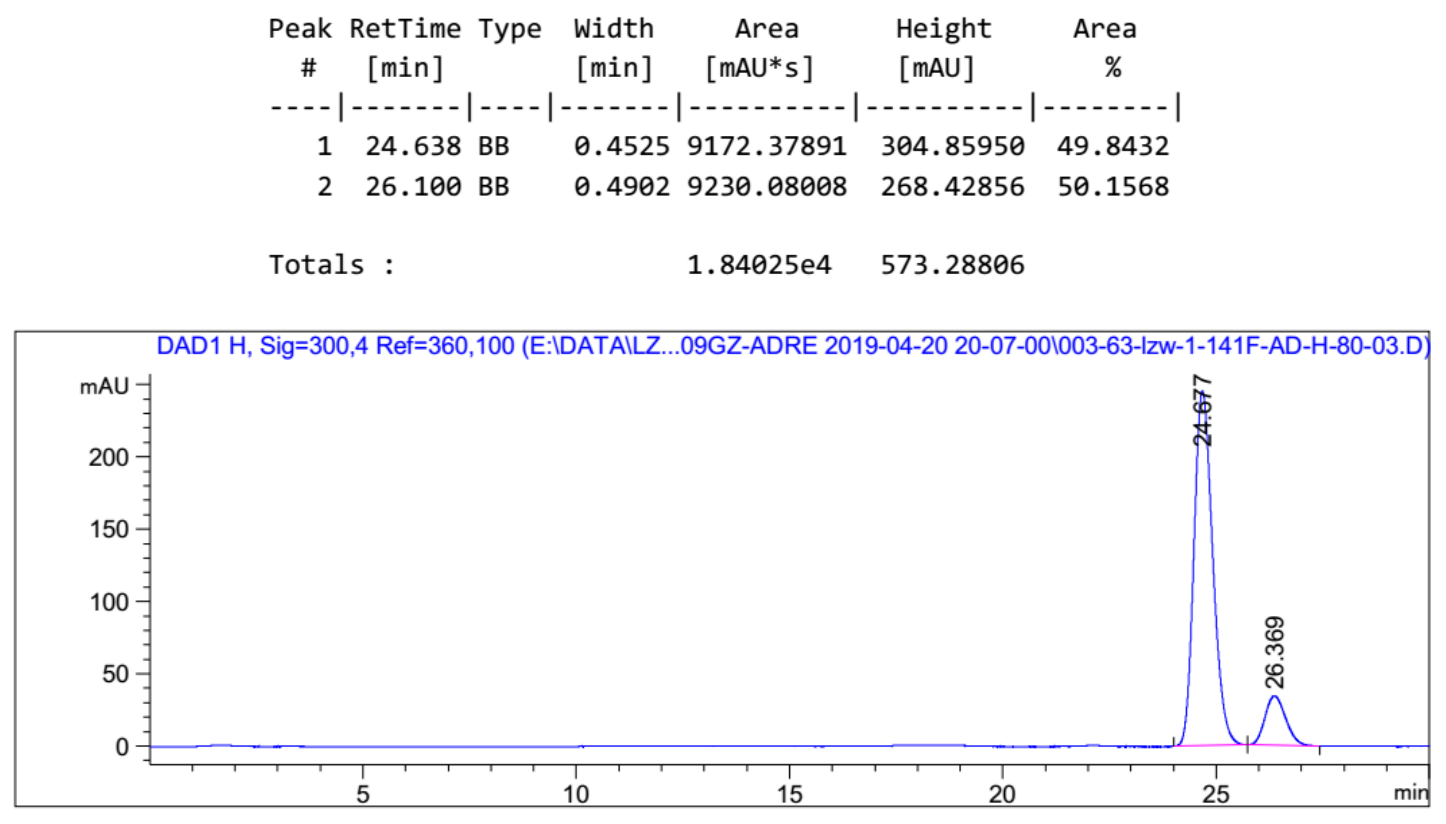

Signal 1: DAD1 H, Sig $=300,4$ Ref $=360,100$

\begin{tabular}{|c|c|c|c|c|c|}
\hline $\begin{array}{c}\text { Peak } \\
\quad \#\end{array}$ & $\begin{array}{l}\text { RetTime Type } \\
\text { [min] }\end{array}$ & $\begin{array}{l}\text { Width } \\
\text { [min] }\end{array}$ & $\begin{array}{c}\text { Area } \\
{[\mathrm{mAU} * \mathrm{~s}]}\end{array}$ & $\begin{array}{l}\text { Height } \\
{[\mathrm{mAU}]}\end{array}$ & $\begin{array}{c}\text { Area } \\
\%\end{array}$ \\
\hline---1 & $\mid--$ & $-\ldots$ & -..- & | & | \\
\hline 1 & 24.677 BB & 0.4519 & 7488.54248 & 245.13234 & 86.6573 \\
\hline 2 & 26.369 ВВ & 0.4073 & 1153.02185 & 34.19915 & 13.3427 \\
\hline Tota & : & & 8641.56433 & 279.33149 & \\
\hline
\end{tabular}




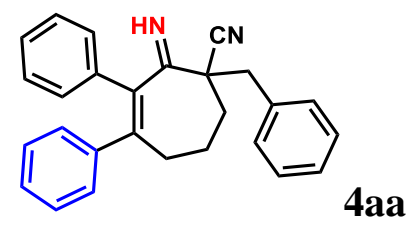

$\mathrm{AD}-\mathrm{H}, 95 \% \mathrm{HEX}, 30 \mathrm{~min}, 0.8 \mathrm{~mL} / \mathrm{min}$

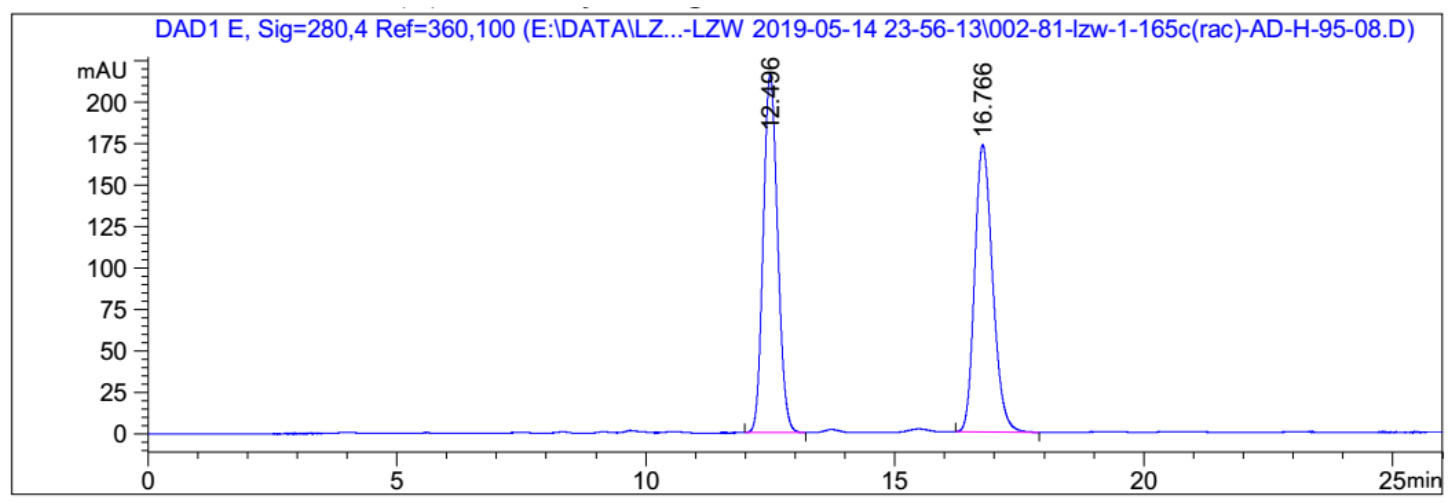

Signal 1: DAD1 E, Sig $=280,4$ Ref $=360,100$
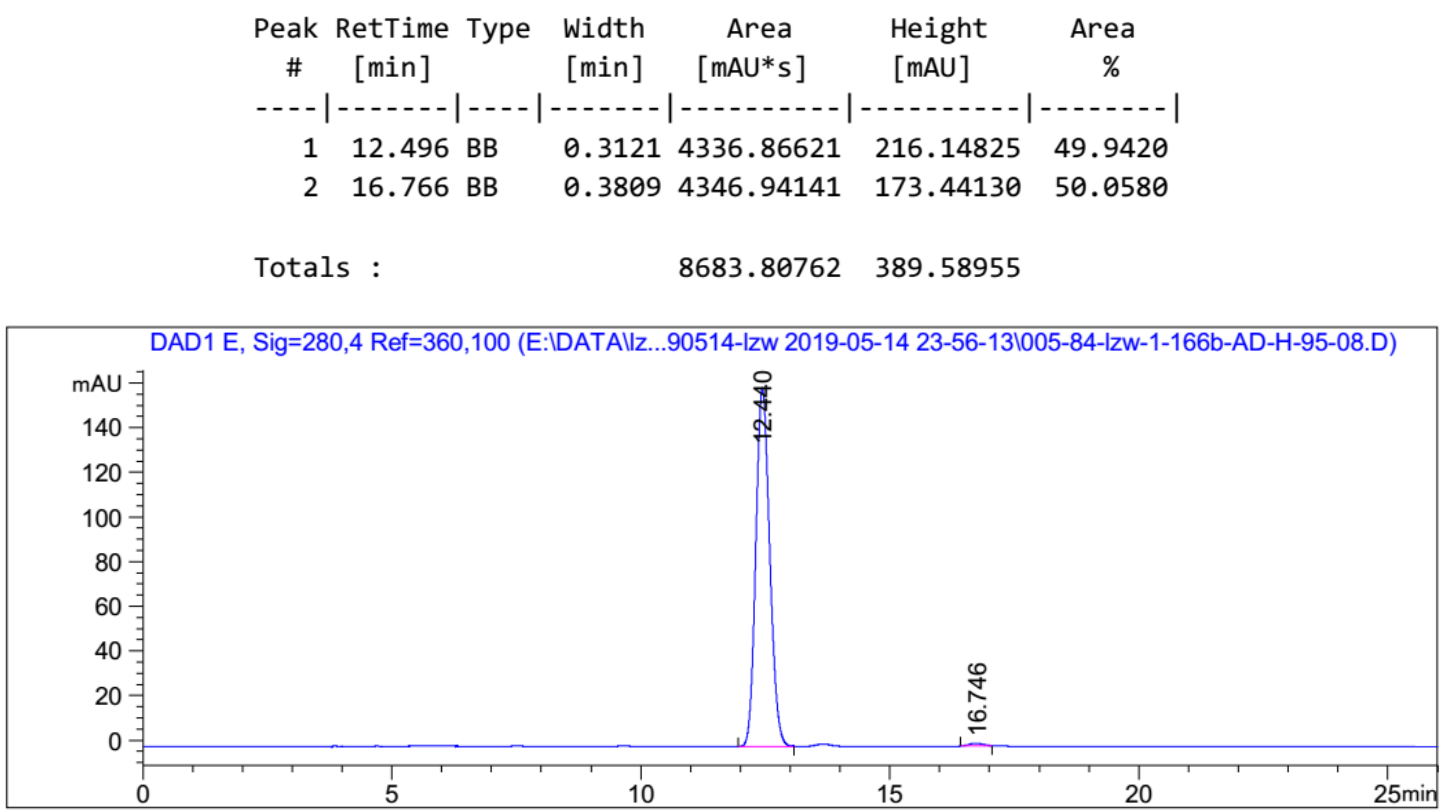

Signal 1: DAD1 E, Sig $=280,4$ Ref $=360,100$

\begin{tabular}{|c|c|c|c|c|c|}
\hline $\begin{array}{c}\text { Peak } \\
\#\end{array}$ & $\begin{array}{l}\text { RetTime Type } \\
\text { [min] }\end{array}$ & $\begin{array}{l}\text { Width } \\
\text { [min] }\end{array}$ & $\begin{array}{c}\text { Area } \\
{[\mathrm{mAU} * \mathrm{~s}]}\end{array}$ & $\begin{array}{l}\text { Height } \\
{[\mathrm{mAU}]}\end{array}$ & $\begin{array}{c}\text { Area } \\
\%\end{array}$ \\
\hline$-\ldots$ & -- & 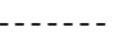 & |---.-- - - & | & | \\
\hline 1 & $12.440 \mathrm{BB}$ & 0.2974 & 3117.53906 & 160.68239 & 99.2703 \\
\hline 2 & 16.746 BB & 0.2358 & 22.91737 & 1.15107 & 0.7297 \\
\hline Tot & : & & 3140.45643 & 161.83346 & \\
\hline
\end{tabular}




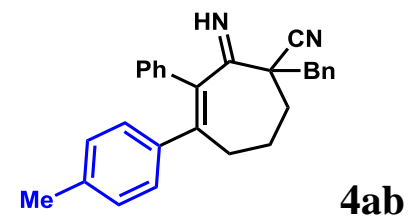

$\mathrm{AD}-\mathrm{H}, 95 \% \mathrm{HEX}, 20 \mathrm{~min}, 1.0 \mathrm{~mL} / \mathrm{min}$

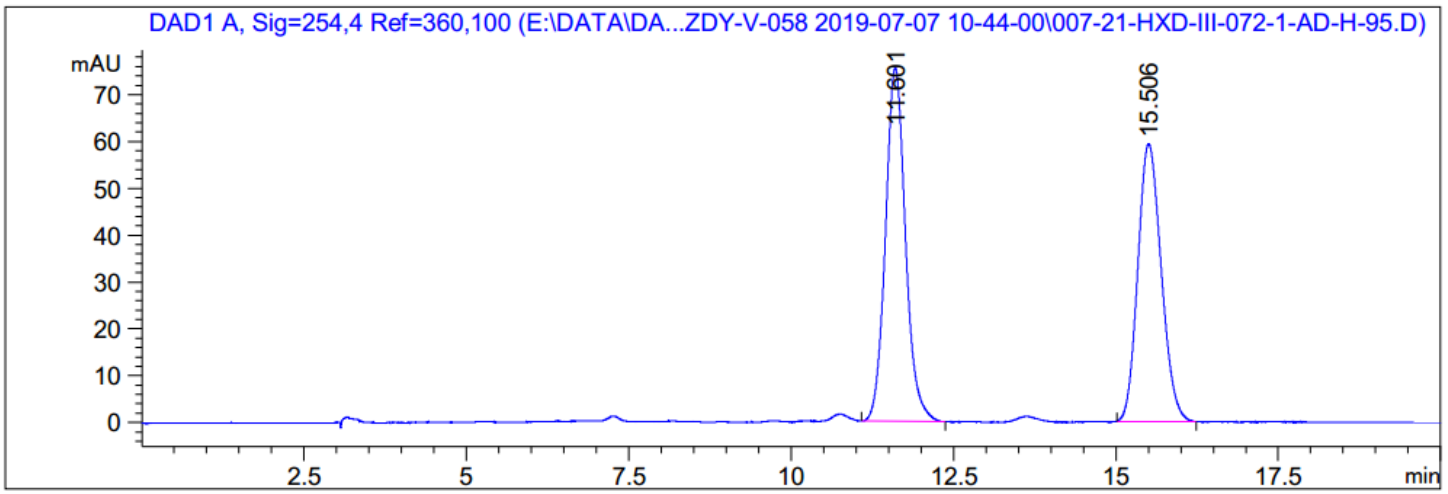

Signal 1: DAD1 A, Sig=254,4 Ref $=360,100$
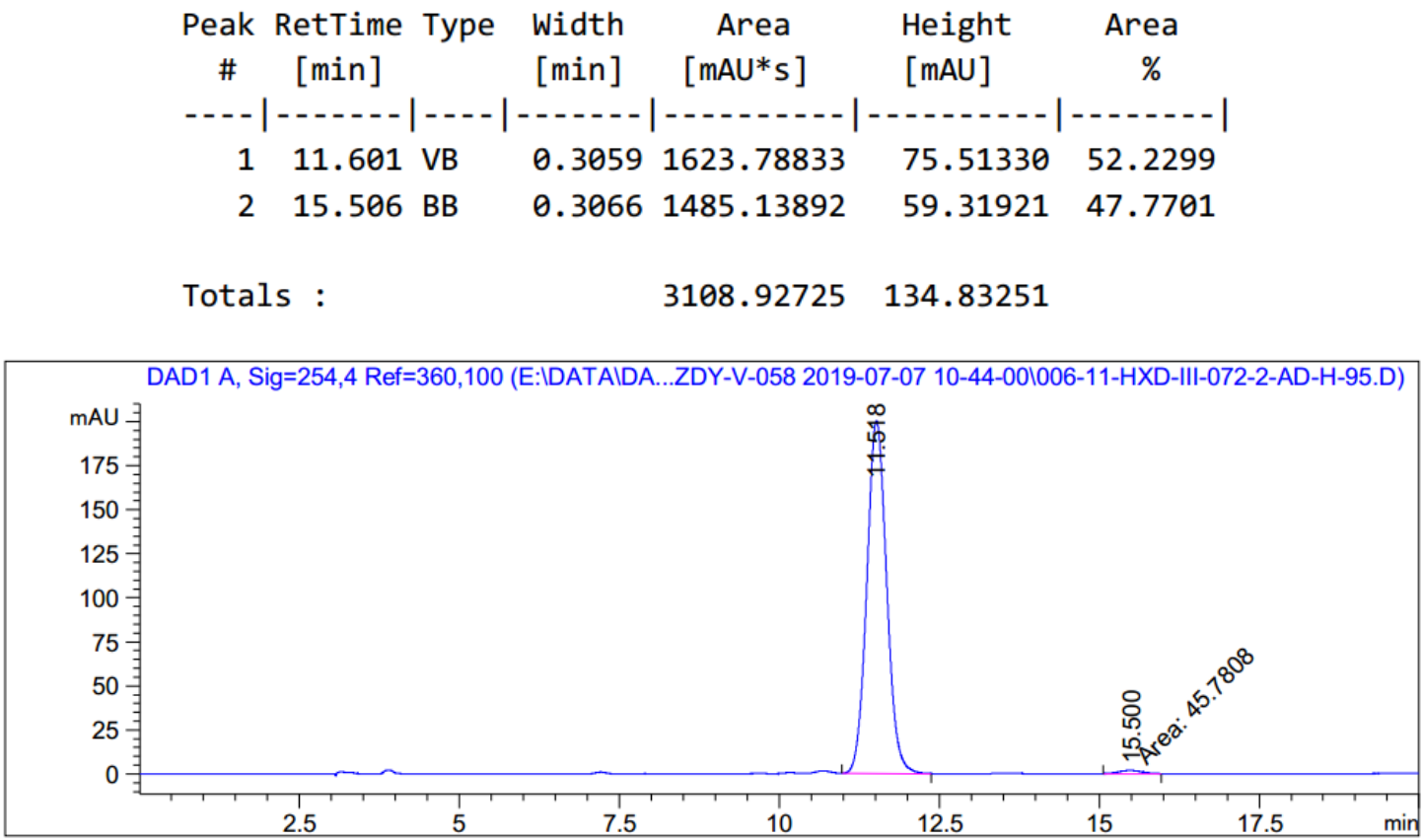

Signal 1: DAD1 A, Sig=254,4 Ref $=360,100$

\begin{tabular}{cccccc}
$\begin{array}{c}\text { Peak RetTime Type } \\
\text { W }\end{array}$ Width & $\begin{array}{c}\text { Area } \\
\text { [min] }\end{array}$ & $\begin{array}{c}\text { Height } \\
{[\mathrm{mAU} \text { s }]}\end{array}$ & $\begin{array}{c}\text { Area } \\
{[\mathrm{mAU}]}\end{array}$ & $\%$ \\
\hline 1 & 11.518 BB & 0.3183 & 4309.16553 & 200.01472 & 98.9488 \\
2 & 15.500 MM & 0.4013 & 45.78077 & 1.90130 & 1.0512
\end{tabular}

Totals :

$4354.94630 \quad 201.91602$ 
<smiles>Cc1cccc(C2=C(c3ccccc3)CCC(C#N)(C#N)C2=N)c1</smiles>

$\mathrm{AD}-\mathrm{H}, 95 \% \mathrm{HEX}, 20 \mathrm{~min}, 1.0 \mathrm{~mL} / \mathrm{min}$

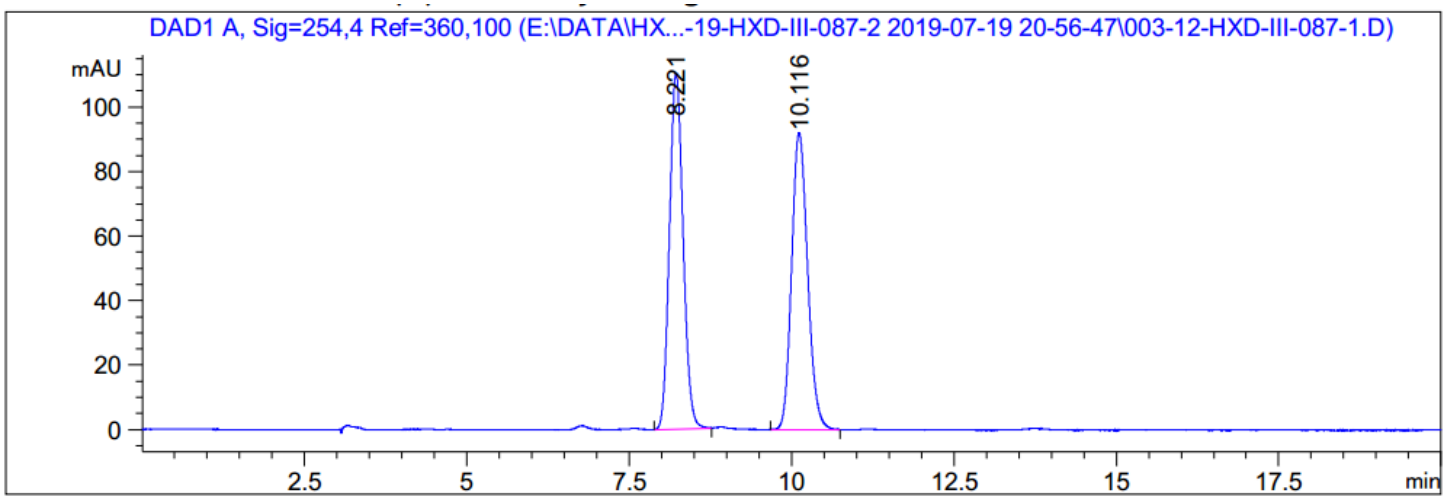

Signal 1: DAD1 A, Sig=254,4 Ref $=360,100$

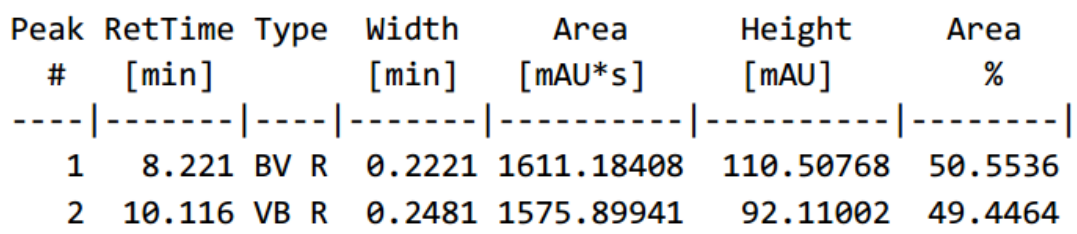

Totals :

$3187.08350 \quad 202.61770$

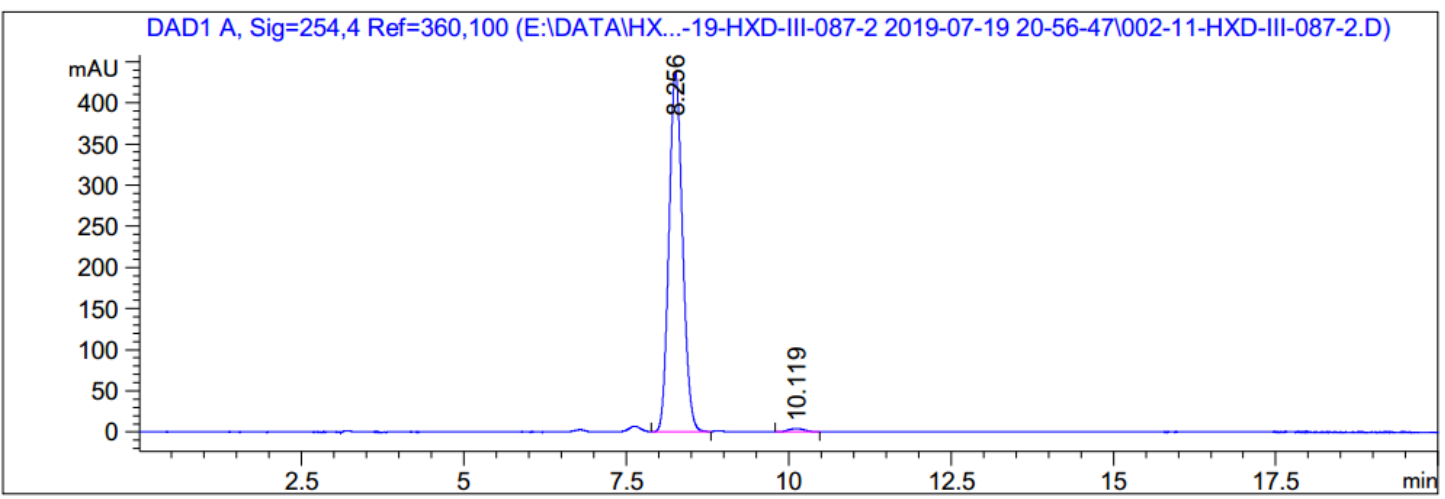

Signal 1: DAD1 A, Sig=254,4 Ref=360,100

\begin{tabular}{cccccc}
$\begin{array}{c}\text { Peak RetTime Type } \\
\text { \# } \\
\text { [min] }\end{array}$ & $\begin{array}{c}\text { Width } \\
{[\mathrm{min}]}\end{array}$ & $\begin{array}{c}\text { Area } \\
{[\mathrm{mAU} \text { *s }]}\end{array}$ & $\begin{array}{c}\text { Height } \\
{[\mathrm{mAU}]}\end{array}$ & $\begin{array}{c}\text { Area } \\
\%\end{array}$ \\
\hdashline 1 & 8.256 BB & 0.2249 & 6352.46924 & 436.15042 & 98.9208 \\
2 & 10.119 BV R & 0.1970 & 69.30149 & 4.16851 & 1.0792
\end{tabular}

Totals :

$6421.77073 \quad 440.31893$ 


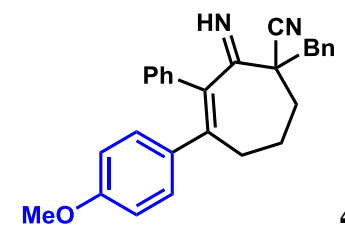

4ad

$\mathrm{AD}-\mathrm{H}, 95 \% \mathrm{HEX}, 30 \mathrm{~min}, 1.0 \mathrm{~mL} / \mathrm{min}$

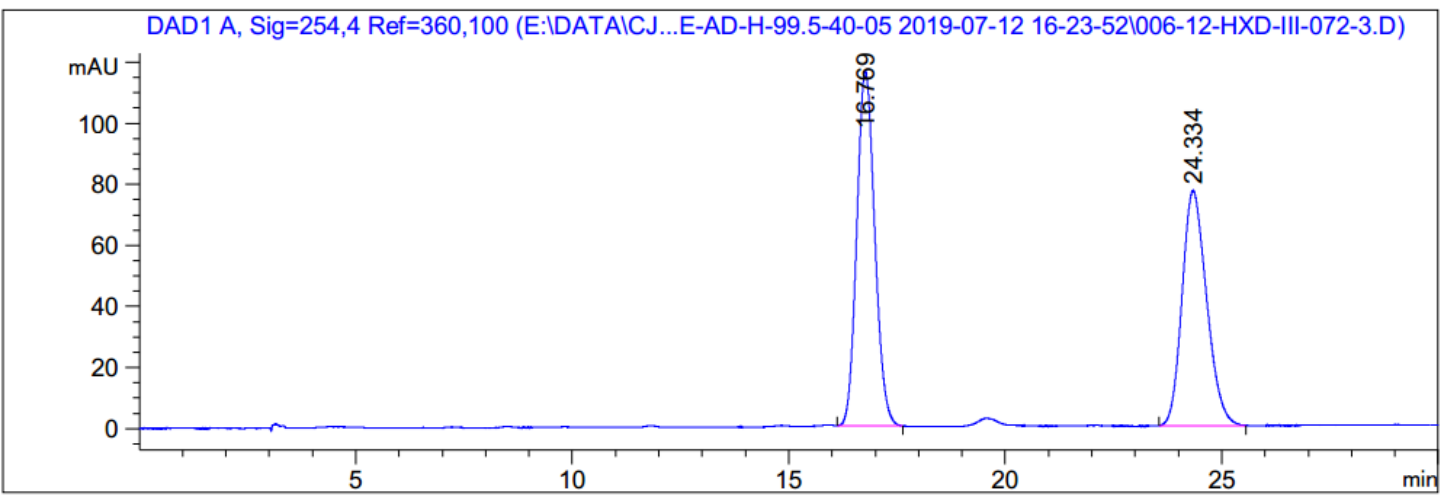

Signal 1: DAD1 A, Sig=254,4 Ref $=360,100$

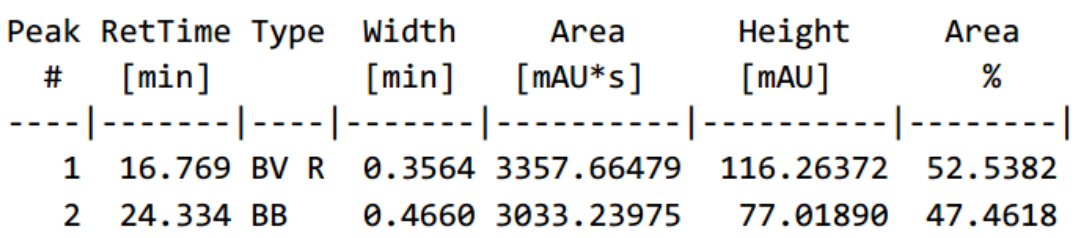

Totals :

$6390.90454 \quad 193.28262$

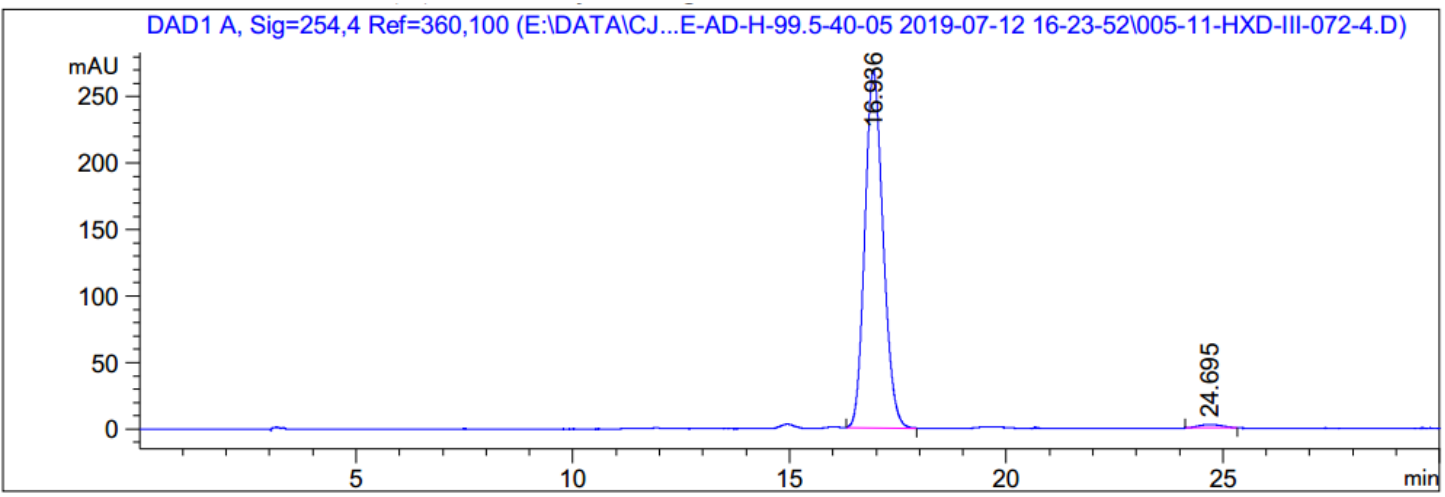

Signal 1: DAD1 A, Sig=254,4 Ref $=360,100$

\begin{tabular}{|c|c|c|c|c|c|c|}
\hline $\begin{array}{c}\text { Peak } \\
\quad \#\end{array}$ & $\begin{array}{c}\text { RetTime } \\
\text { [min] }\end{array}$ & Type & $\begin{array}{l}\text { Width } \\
\text { [min] }\end{array}$ & $\begin{array}{c}\text { Area } \\
{\left[\mathrm{mAU}^{*} \mathrm{~s}\right]}\end{array}$ & $\begin{array}{l}\text { Height } \\
{[\mathrm{mAU}]}\end{array}$ & $\begin{array}{c}\text { Area } \\
\%\end{array}$ \\
\hline - & & & & |--. & $\mid$ & | \\
\hline 1 & 16.936 & BB & 0.4088 & 7868.52393 & 268.94754 & 98.7389 \\
\hline 2 & 24.695 & BB & 0.4262 & 100.49467 & 2.77017 & 1.2611 \\
\hline & & & & 7969.01859 & 71 & \\
\hline
\end{tabular}


<smiles>N#CC1(C#N)CCC(c2ccccc2)=C(c2ccc(F)cc2)C1=N</smiles>

$\mathrm{AD}-\mathrm{H}, 95 \% \mathrm{HEX}, 30 \mathrm{~min}, 1.0 \mathrm{~mL} / \mathrm{min}$

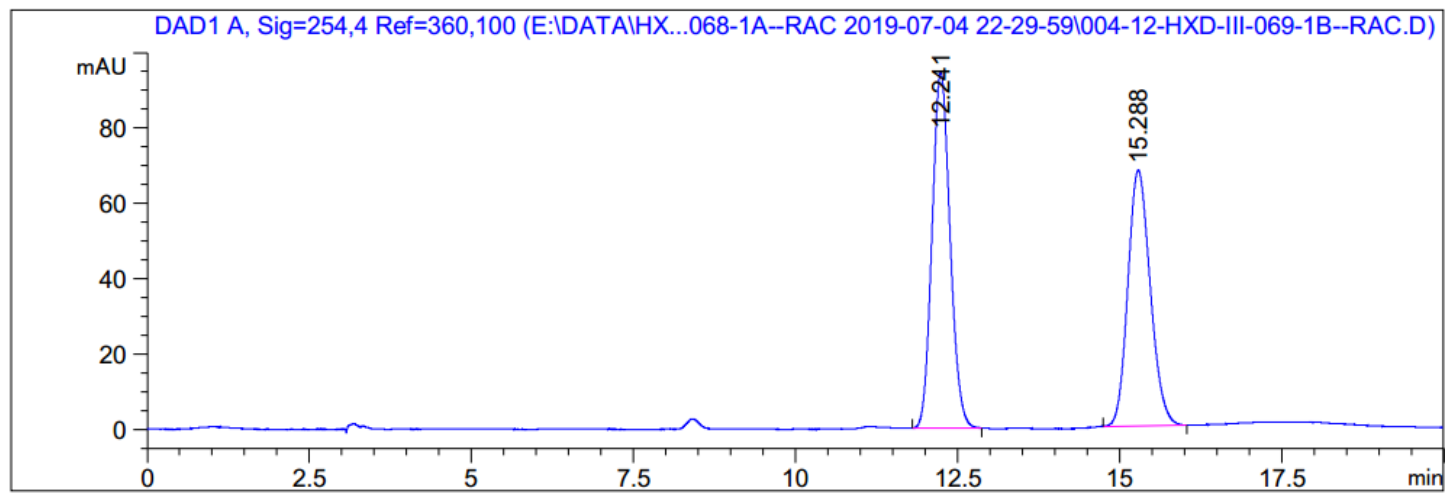

Signal 1: DAD1 A, Sig $=254,4$ Ref $=360,100$

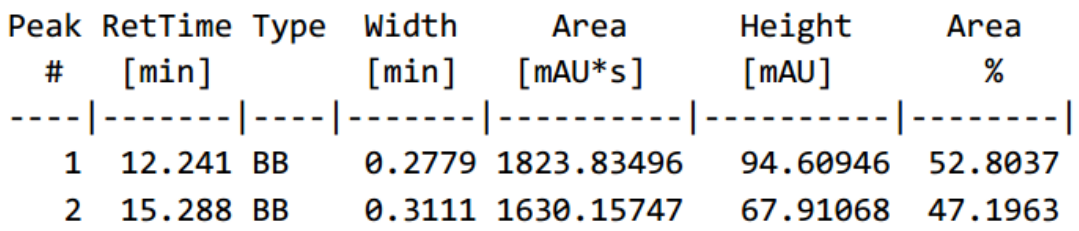

Totals :

$3453.99243 \quad 162.52014$

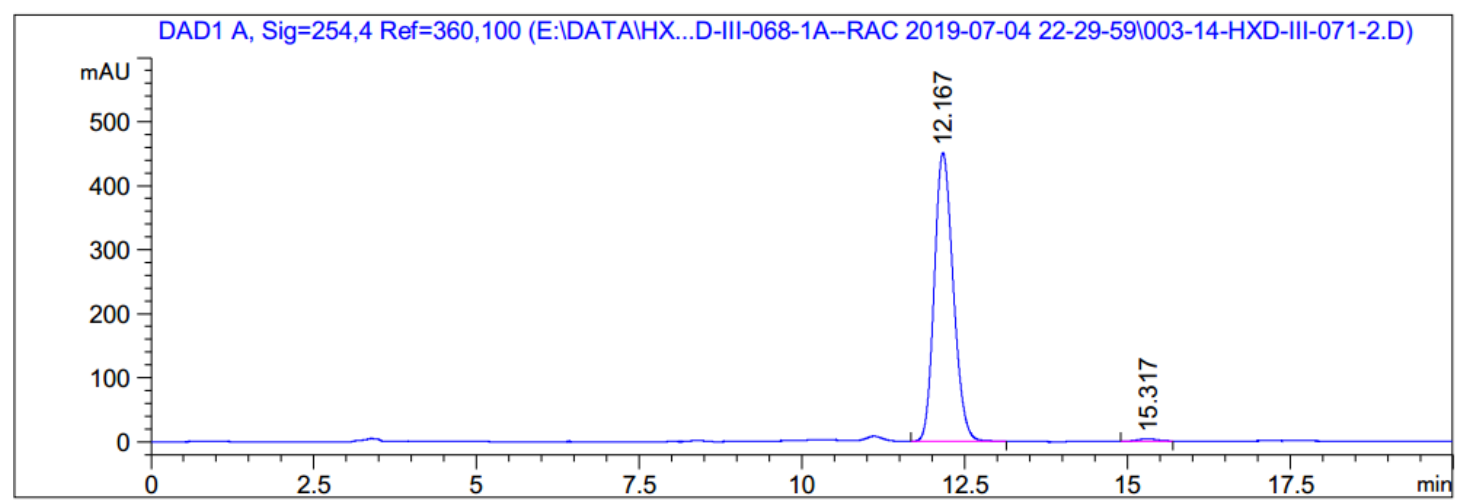

Signal 1: DAD1 A, Sig $=254,4$ Ref $=360,100$

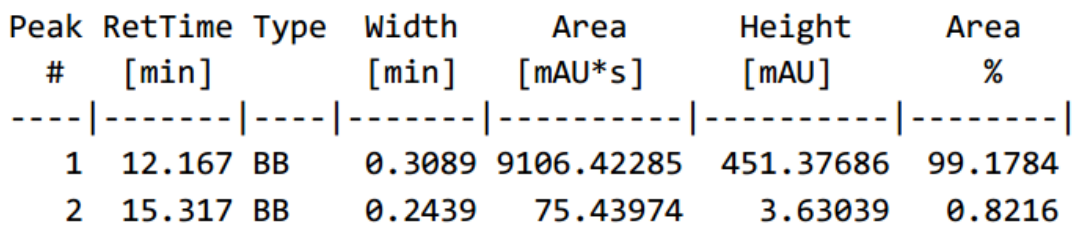

Totals :

$9181.86259 \quad 455.00726$ 


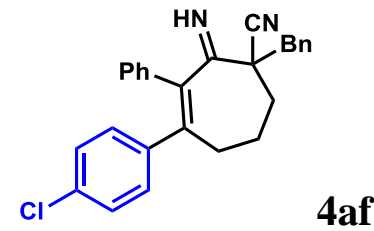

AD-H, 95\% HEX, $30 \mathrm{~min}, 1.0 \mathrm{~mL} / \mathrm{min}$

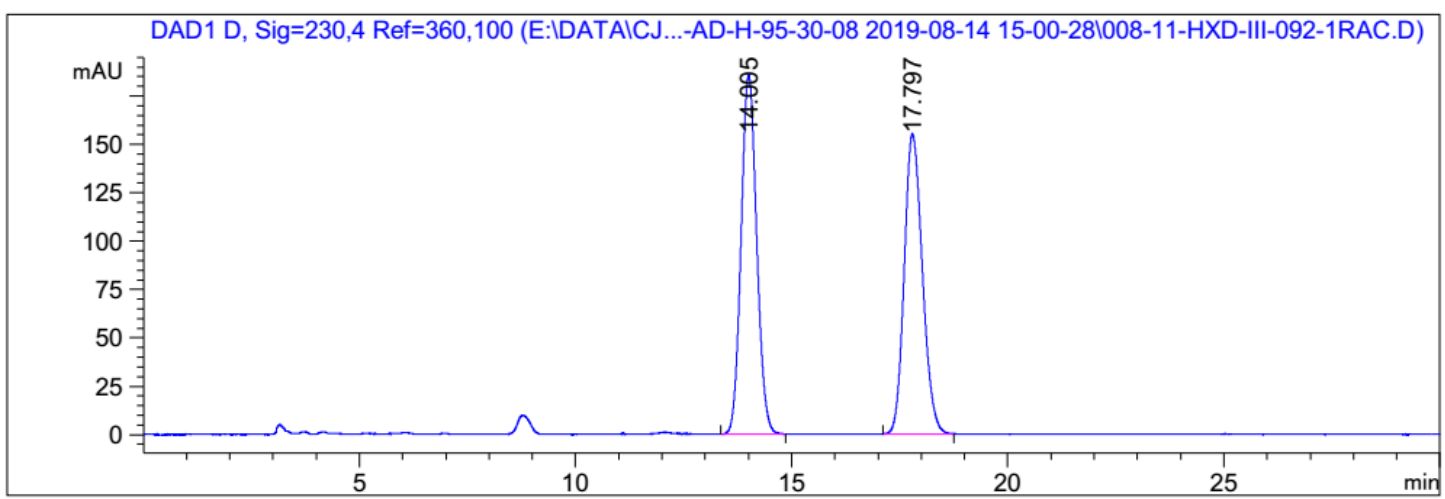

Signal 1: DAD1 D, Sig=230,4 Ref=360,100

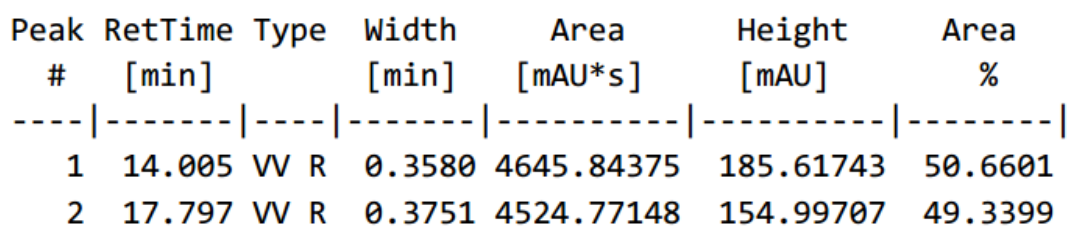

Totals :

$9170.61523 \quad 340.61450$

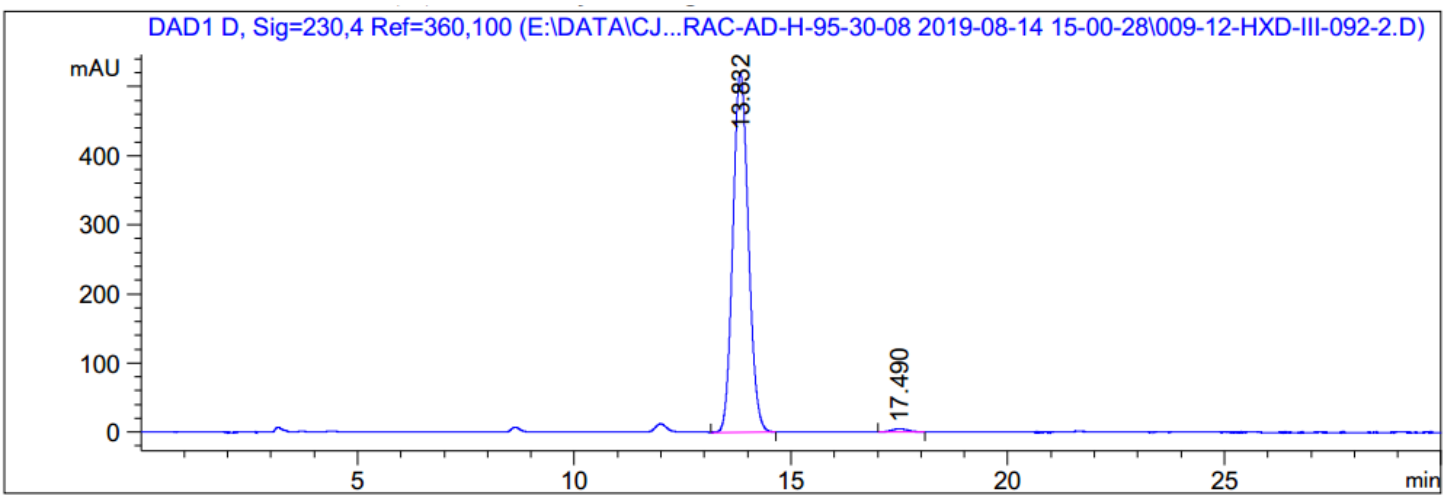

Signal 1: DAD1 D, Sig=230,4 Ref=360,100

\begin{tabular}{cccccc}
$\begin{array}{c}\text { Peak RetTime Type } \\
\text { \# } \\
{[\text { min] }}\end{array}$ & $\begin{array}{c}\text { Width } \\
{[\text { min] }}\end{array}$ & $\begin{array}{c}\text { Area } \\
{[\text { mAU*s }}\end{array}$ & $\begin{array}{c}\text { Height } \\
{[\text { mAU] }}\end{array}$ & $\begin{array}{c}\text { Area } \\
\%\end{array}$ \\
\hline 1 & 13.832 BV R & 0.3596 & $1.29556 e 4$ & 521.05444 & 98.9700 \\
2 & 17.490 VV R & 0.3200 & 134.82475 & 4.94654 & 1.0300
\end{tabular}

Totals :

$1.30904 \mathrm{e} 4526.00099$ 


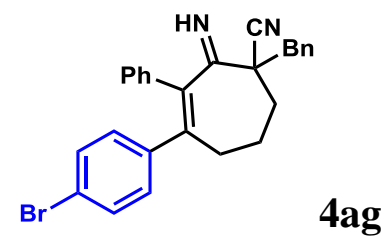

AD-H, 95\% HEX, $30 \mathrm{~min}, 1.0 \mathrm{~mL} / \mathrm{min}$

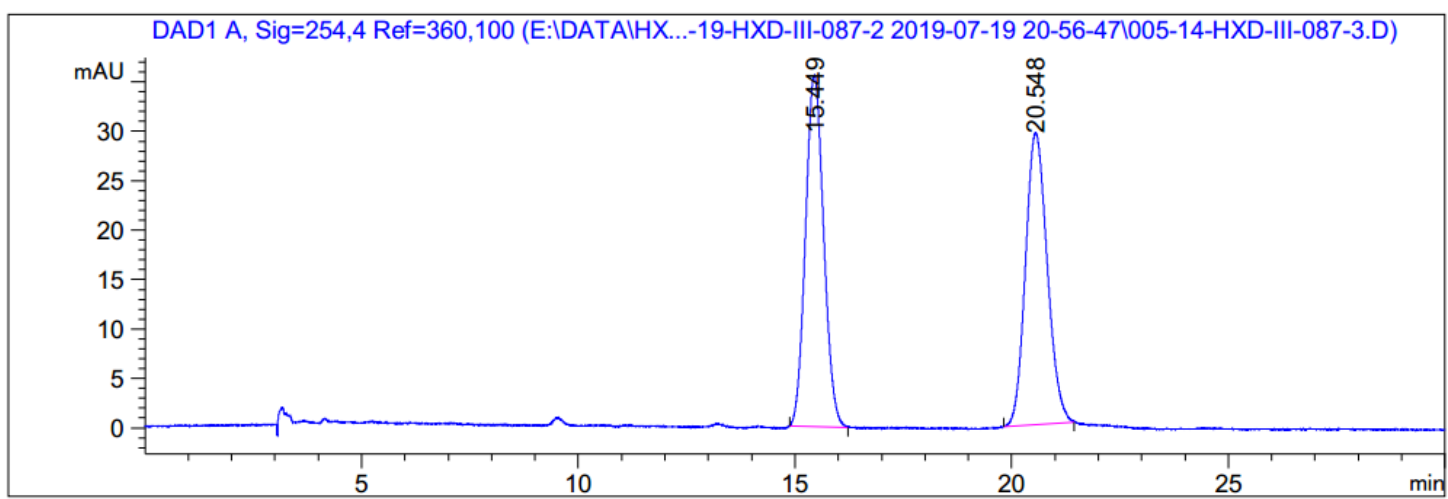

Signal 1: DAD1 A, Sig=254,4 Ref=360,100

\begin{tabular}{|c|c|c|c|c|c|c|}
\hline eak & $\begin{array}{c}\text { RetTime } \\
\text { [min] }\end{array}$ & ype & $\begin{array}{l}\text { Width } \\
\text { [min] }\end{array}$ & $\begin{array}{c}\text { Area } \\
{\left[\mathrm{mAU}^{*} \mathrm{~s}\right]}\end{array}$ & $\begin{array}{l}\text { Height } \\
{[\mathrm{mAU}]}\end{array}$ & $\begin{array}{c}\text { Area } \\
\%\end{array}$ \\
\hline & & & & & & \\
\hline- & & $\mathrm{V} R$ & & 1036 & 37 & 50 \\
\hline & & & & 1029.28296 & 52224 & 49 \\
\hline
\end{tabular}

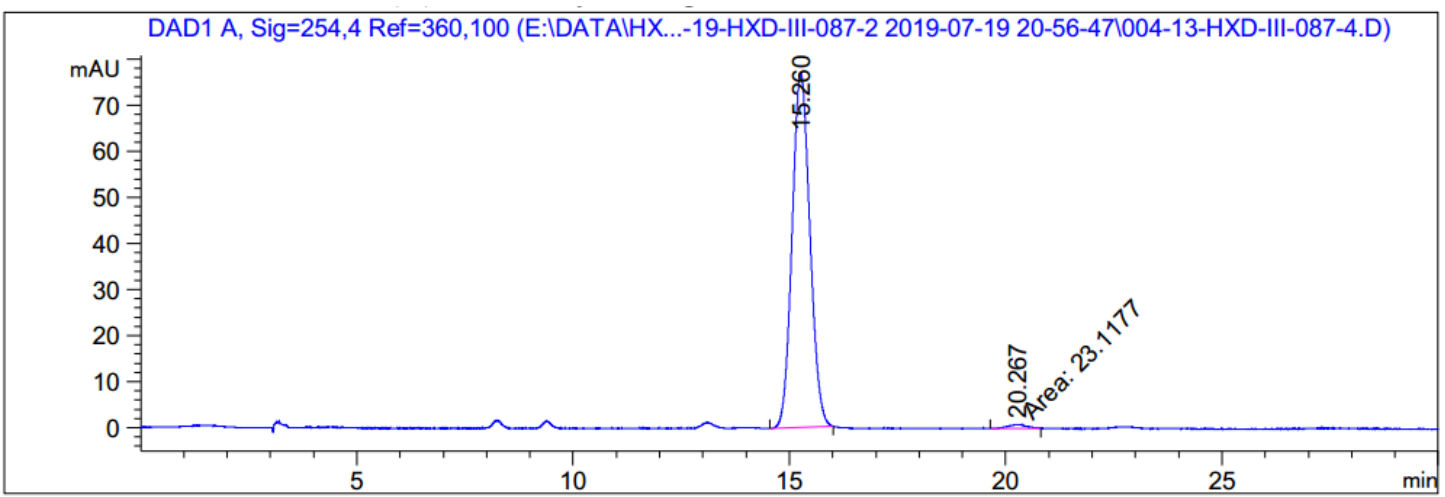

Signal 1: DAD1 A, Sig=254,4 Ref=360,100

\begin{tabular}{|c|c|c|c|c|c|c|}
\hline eak & $\begin{array}{c}\text { RetTime } \\
\text { [min] }\end{array}$ & ype & $\begin{array}{l}\text { Width } \\
\text { [min] }\end{array}$ & $\begin{array}{c}\text { Area } \\
{\left[\mathrm{mAU}^{*} \mathrm{~s}\right]}\end{array}$ & $\begin{array}{l}\text { Height } \\
{[\mathrm{mAU}]}\end{array}$ & $\begin{array}{c}\text { Area } \\
\%\end{array}$ \\
\hline & & & & & & \\
\hline 1 & & & & 2200. & & \\
\hline & & & & 23.11775 & $6 e-1$ & \\
\hline
\end{tabular}

Totals :

$2223.95564 \quad 77.78456$ 


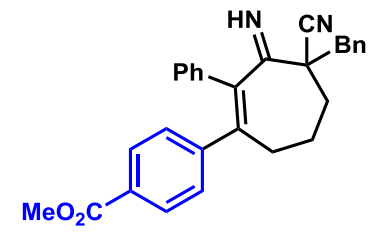

4ah

$\mathrm{AD}-\mathrm{H}, 85 \% \mathrm{HEX}, 30 \mathrm{~min}, 1 \mathrm{~mL} / \mathrm{min}$

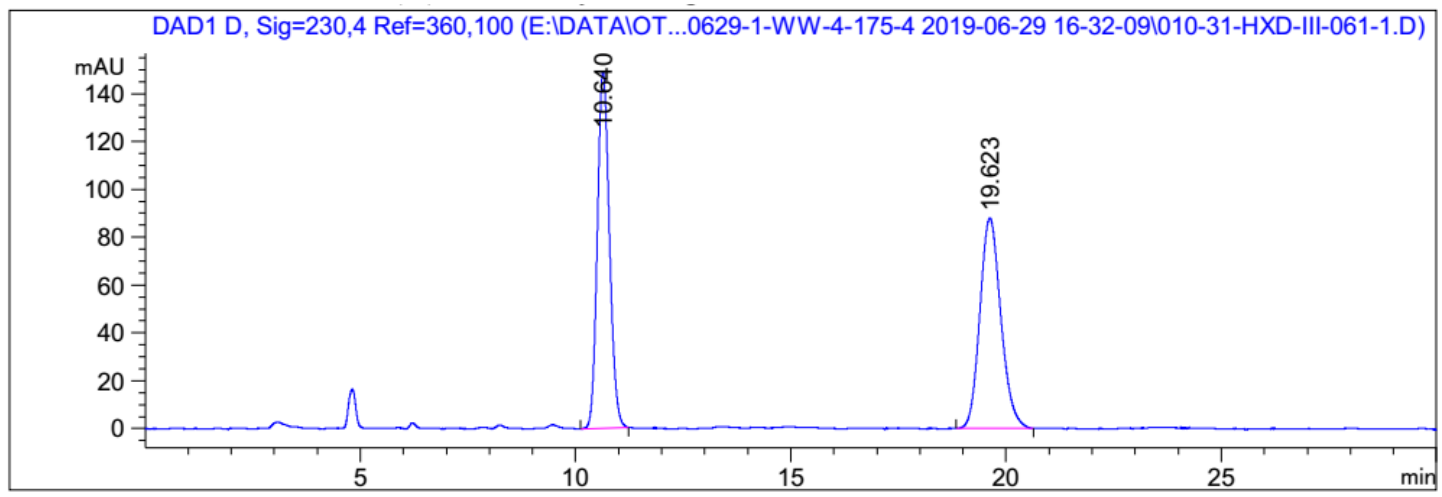

Signal 1: DAD1 D, Sig=230,4 Ref $=360,100$

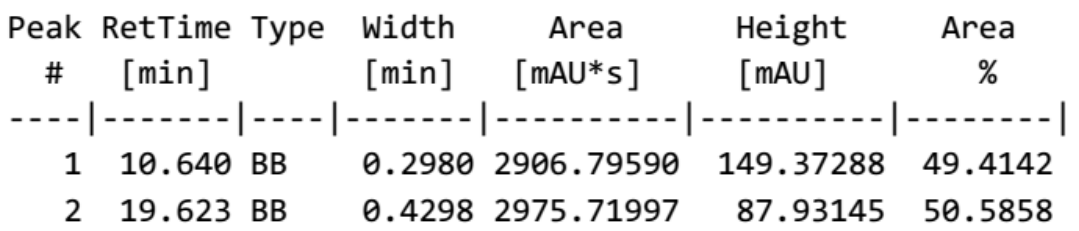

Totals :

$5882.51587 \quad 237.30433$

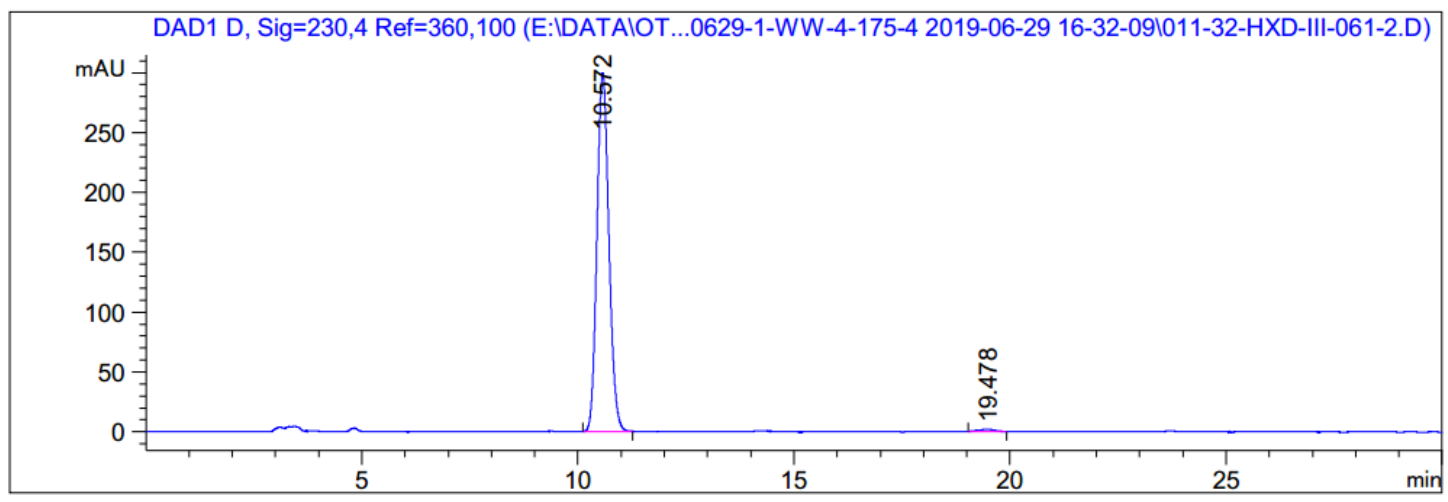

Signal 1: DAD1 D, Sig=230,4 Ref $=360,100$

\begin{tabular}{|c|c|c|c|c|c|c|}
\hline $\begin{array}{l}\text { ak } \\
\#\end{array}$ & $\begin{array}{c}\text { RetTime } \\
\text { [min] }\end{array}$ & Type & $\begin{array}{l}\text { Width } \\
\text { [min] }\end{array}$ & $\begin{array}{c}\text { Area } \\
{\left[\mathrm{mAU}^{*} \mathrm{~s}\right]}\end{array}$ & $\begin{array}{l}\text { Height } \\
{[\mathrm{mAU}]}\end{array}$ & $\begin{array}{c}\text { Area } \\
\%\end{array}$ \\
\hline & & & & & & \\
\hline 1 & & & & 5742 & 299 & 99 \\
\hline 2 & 8 & & & 49.88938 & .76402 & 0.8613 \\
\hline
\end{tabular}

Totals :

$5792.23704 \quad 301.62592$ 
<smiles>N#CC1([18O])CCCC(c2ccccc2)=C(c2ccccc2)C1=O</smiles>

AD-H, 90\% HEX, $20 \mathrm{~min}, 1 \mathrm{~mL} / \mathrm{min}$

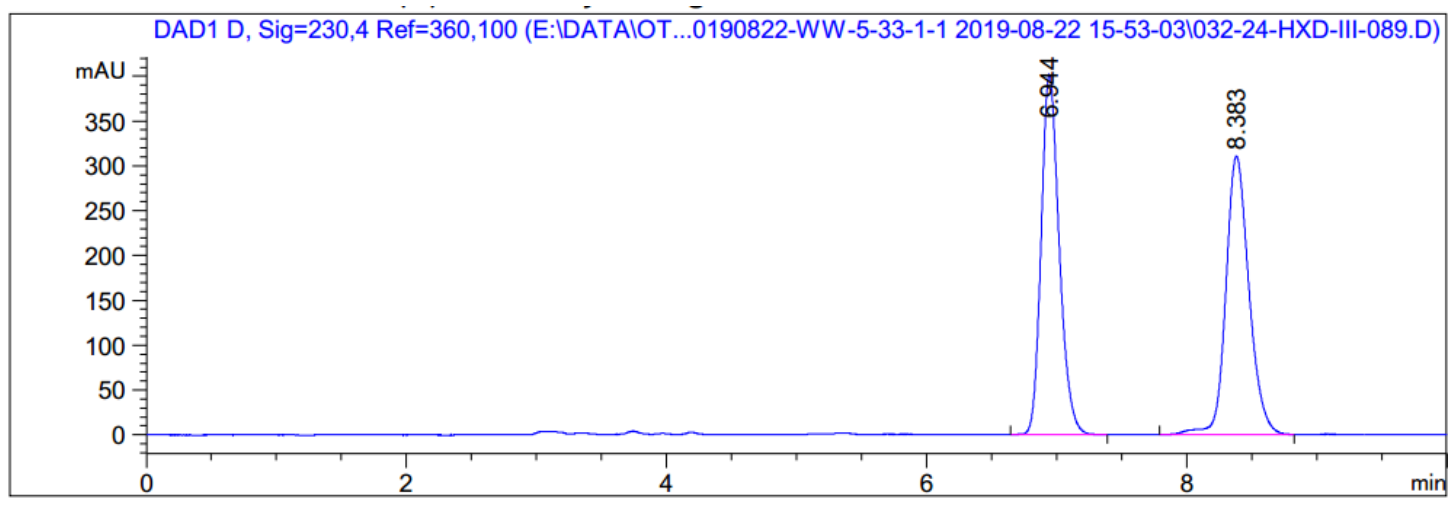

Signal 1: DAD1 D, Sig=230,4 Ref=360,100
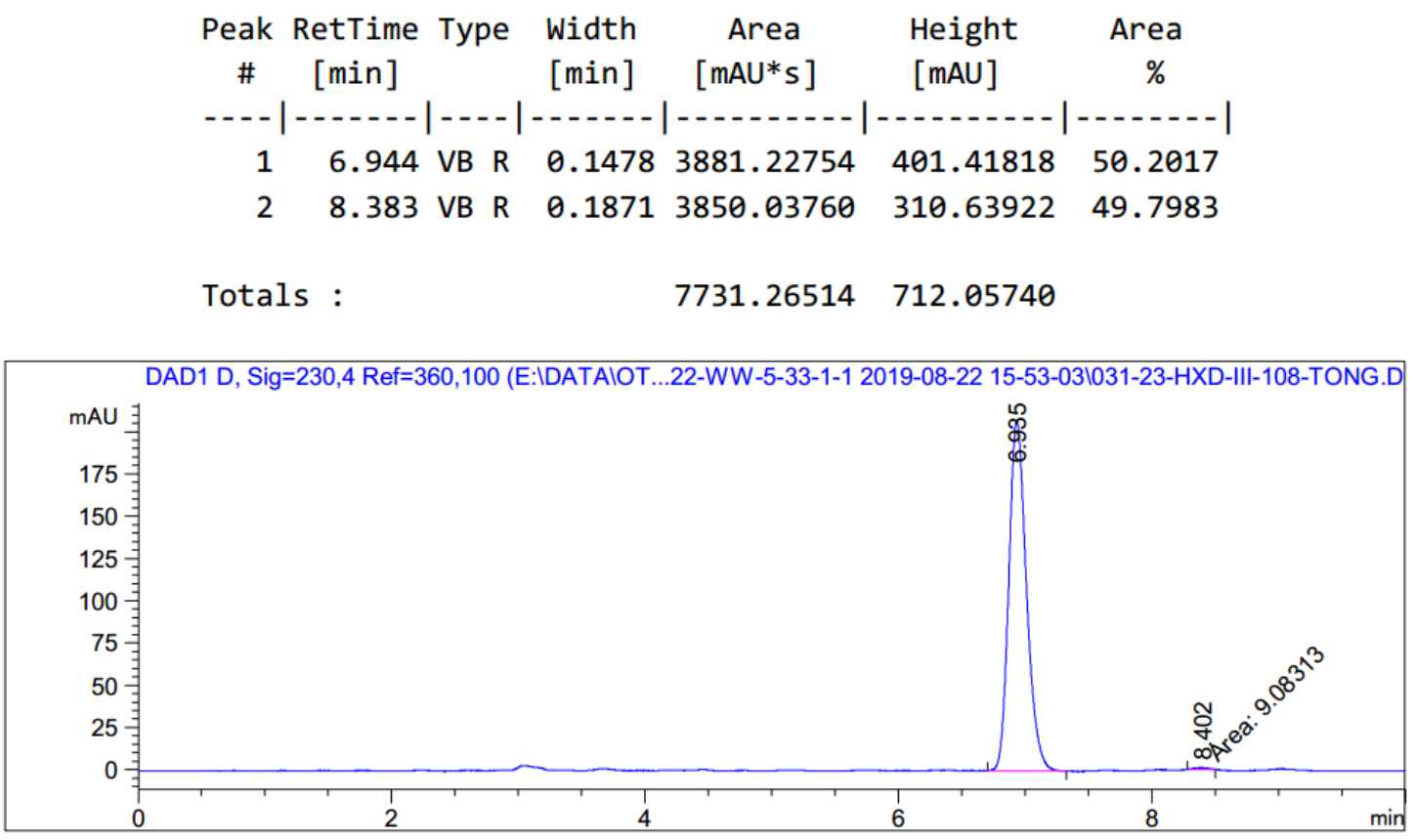

Signal 1: DAD1 D, Sig=230,4 Ref $=360,100$

\begin{tabular}{|c|c|c|c|c|c|c|}
\hline $\begin{array}{c}\text { Peak } \\
\#\end{array}$ & $\begin{array}{c}\text { RetTime } \\
\text { [min] }\end{array}$ & Type & $\begin{array}{l}\text { Width } \\
\text { [min] }\end{array}$ & $\begin{array}{c}\text { Area } \\
{[\mathrm{mAU} * \mathrm{~s}]}\end{array}$ & $\begin{array}{l}\text { Height } \\
{[\mathrm{mAU}]}\end{array}$ & $\begin{array}{c}\text { Area } \\
\%\end{array}$ \\
\hline & & & & 1 & & \\
\hline 1 & 6.935 & $3 V R$ & 0.1478 & 2005.14575 & 207.32063 & 99.5491 \\
\hline 2 & 8.402 & MM & 0.1333 & 9.08313 & 1.13574 & 0.4509 \\
\hline
\end{tabular}

Totals :

$2014.22889 \quad 208.45637$ 


\section{NMR Spectra}
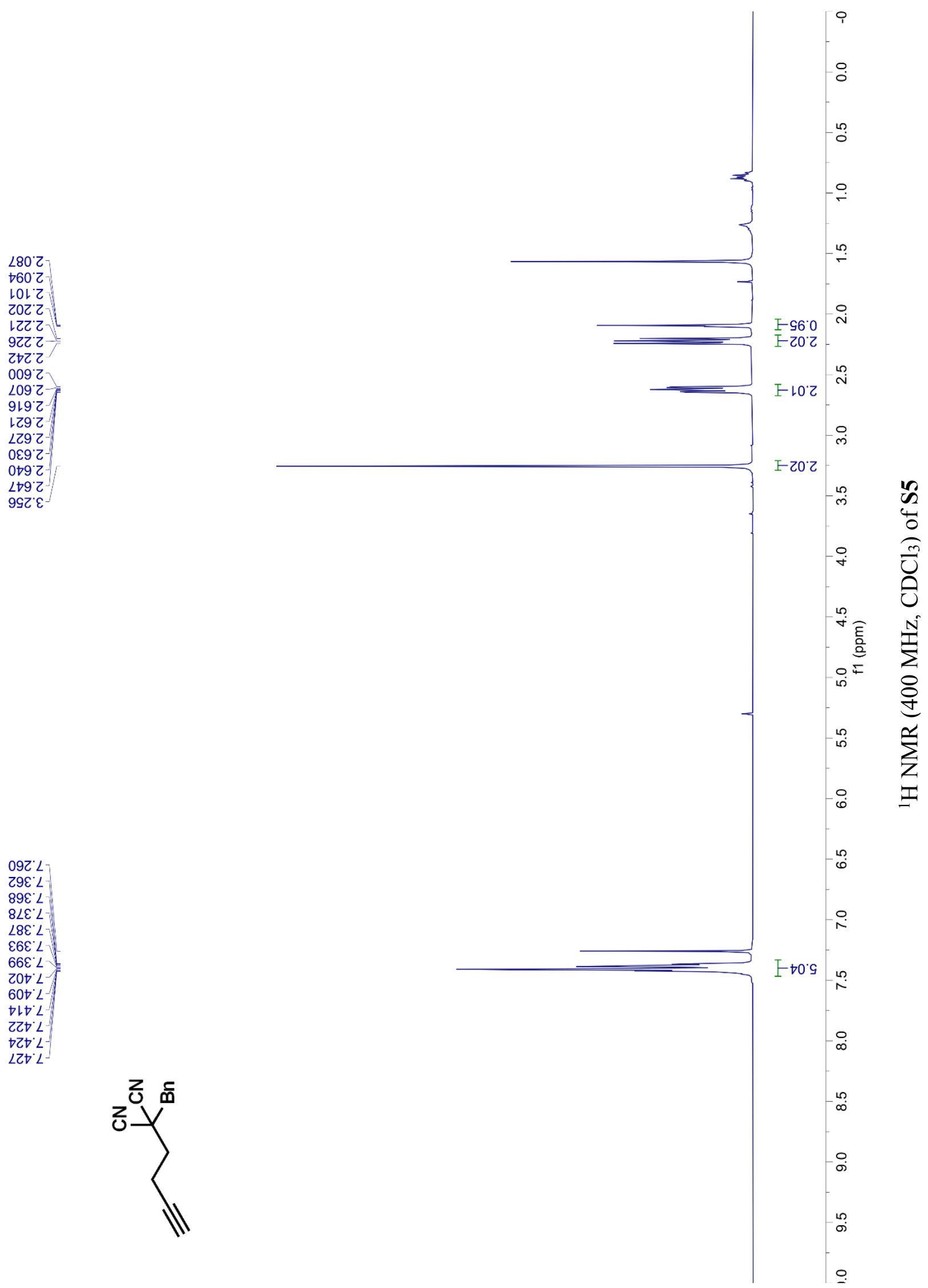
L9.

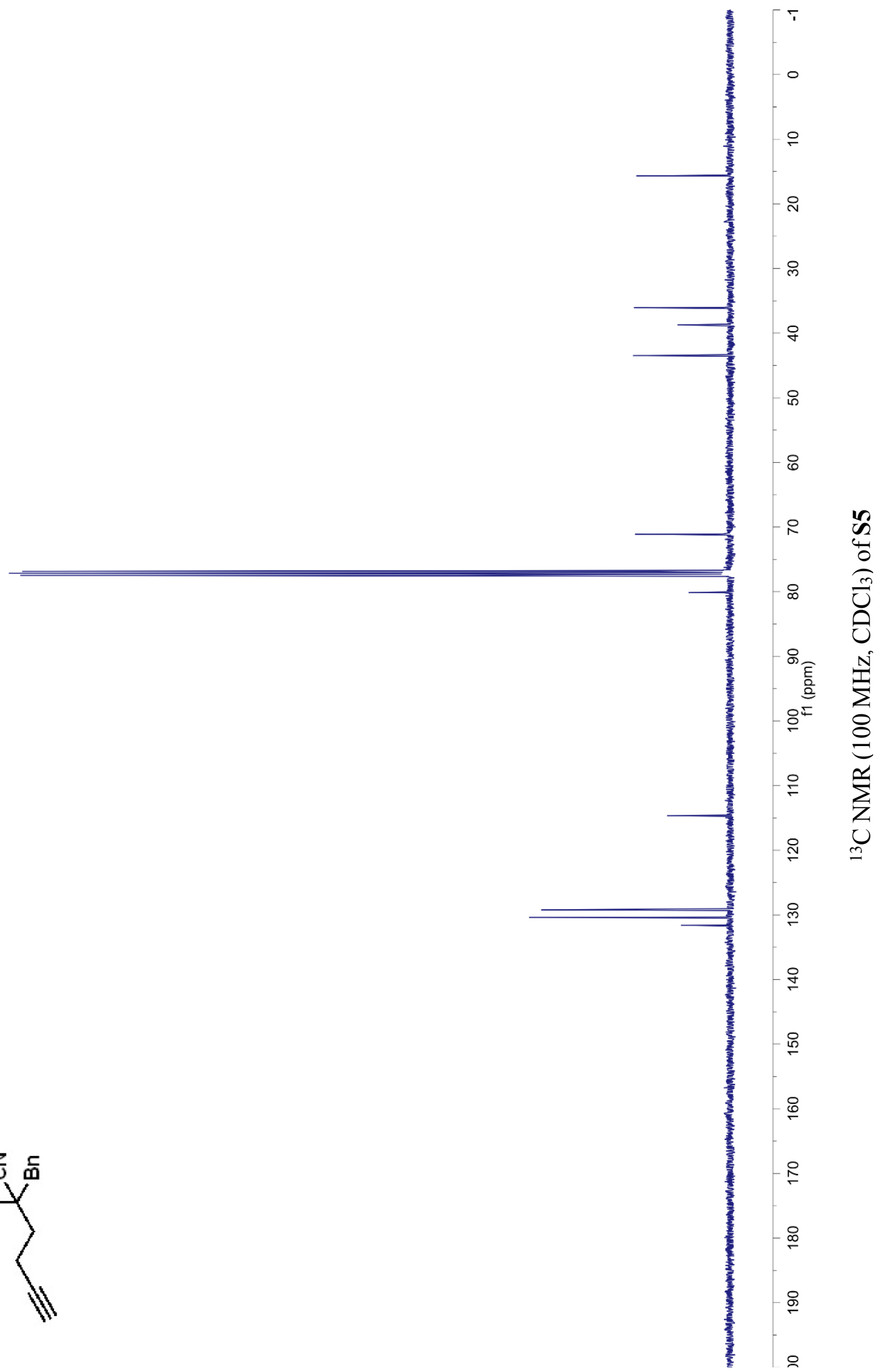

ZL'6ZL

ZZ 6ZL-
$8 \varepsilon^{\circ} 0 \varepsilon\llcorner-$

Z9. LE

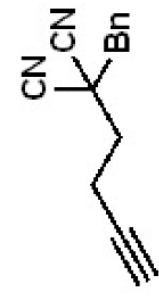




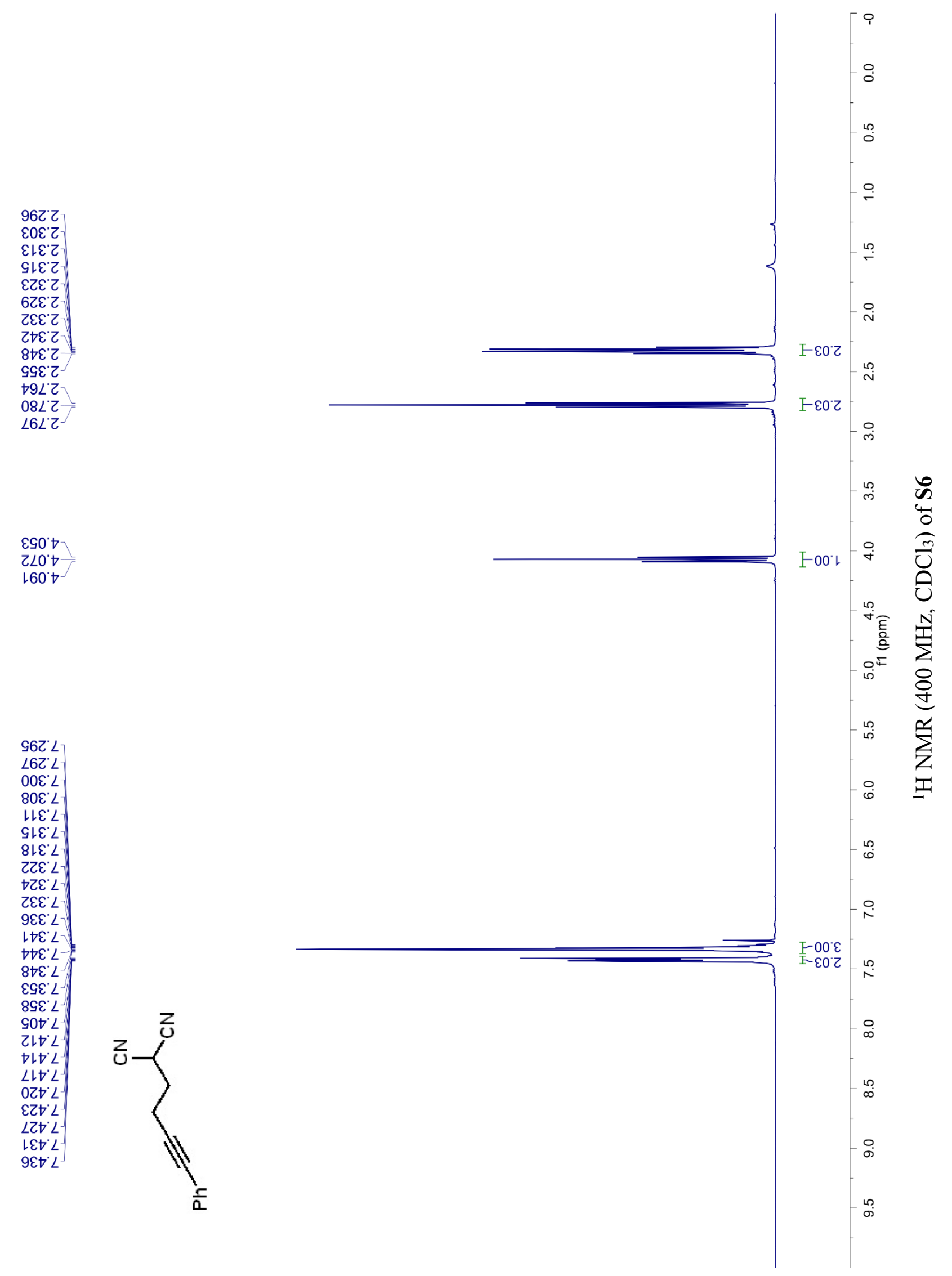


90 L -

ISL-

$\varepsilon\llcorner 0 \varepsilon$

$81 \cdot 78$

$\angle \varepsilon^{\circ} \triangleright 8$

ยどてレー

6ガててレー

IS $82 \mathrm{~L}$

†9.8Z

$8 L L E L$

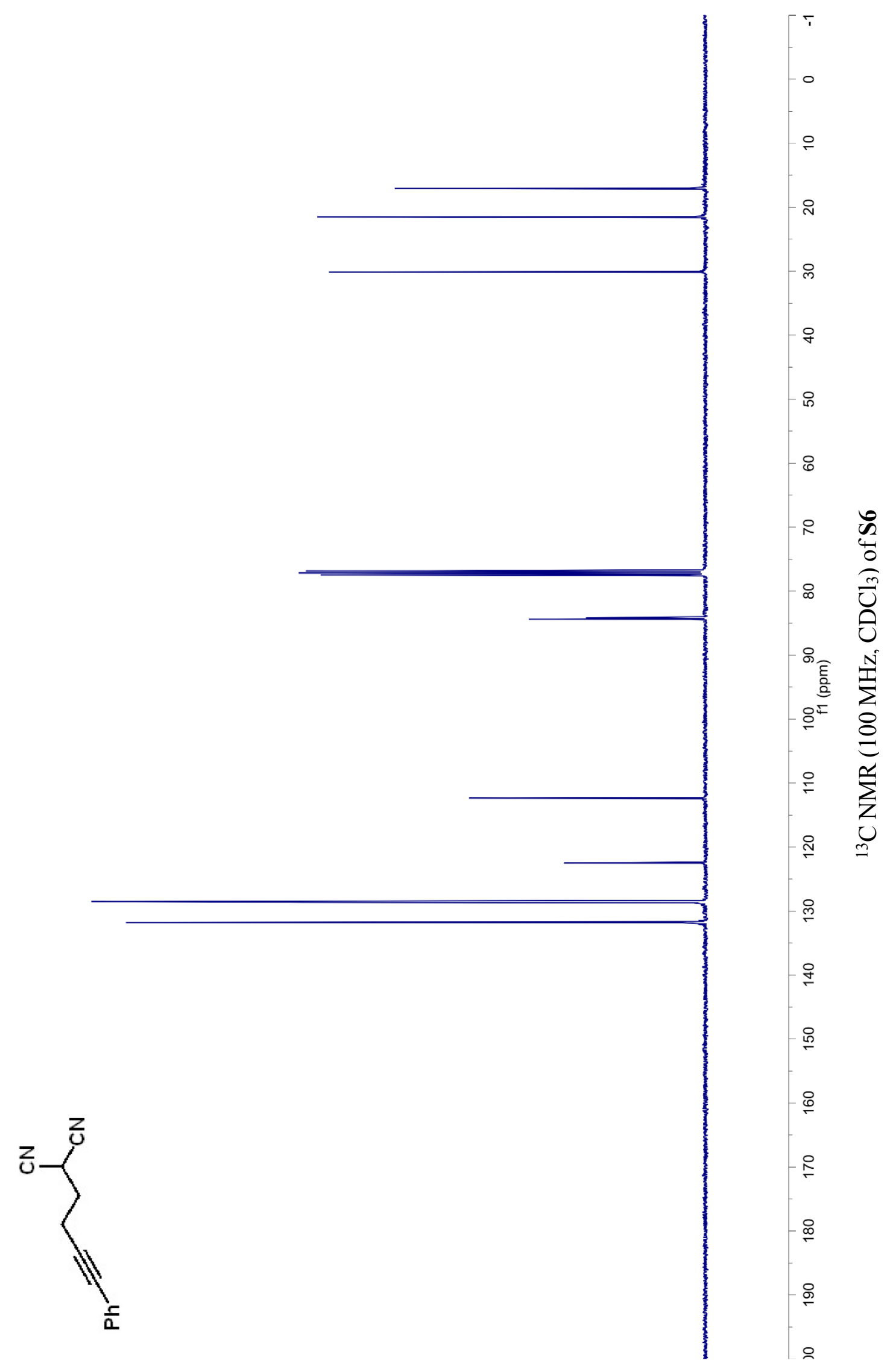


s90.

$690^{\circ} \mathrm{L}$

2901

$760^{\circ}$ L

$860^{\circ} \mathrm{L}$

จレレ

6ll

StL'

$89 L^{\circ}$.

9Lᄂᄂ

L9Z'

SLZ'

เ6己 ᄂ

$66 乙 レ N$

LOE'

ZZE'L-

LE⿱口:-

$099^{\circ}$ L

S99.

$699^{\circ}$

$\varepsilon 99^{\circ} \mathrm{L}$

$899^{\circ}$

89

$89^{\circ}$

$\checkmark 69^{\circ} \mathrm{L}$

$669^{\circ}$ L

ZOL'

OLL'

9LL'

OZL'

9ZL'

6ZL'

SEL'L

$8 \varepsilon L$ L

$\nabla D L '$

$8 \nabla L$

ZSLL

8SL'

I $9 L^{\circ}$

L9LL

.

乙E8.

E68 ᄂ

$68^{\circ}$

206.

S06.

$806^{\circ} \mathrm{L}$

216.

$66^{\circ} \mathrm{L}$

8Z6' -

乙E6 เ

$\neg \varepsilon 6^{\circ}$ '

$8 \varepsilon 6^{\circ}$ '

276.

$8 \angle 0^{\circ} \mathrm{Z}$

$980^{\circ} \mathrm{Z}$

$89 l^{\circ} \mathrm{Z}$

$\angle L L ' Z$

781 '

$88 L^{\prime} \mathrm{Z}$

E6 ' 2

$00 \mathrm{Z} Z$

$80 Z^{\prime} Z$

GG'

99' $\mathrm{Z}$

$\angle S^{\circ} \mathrm{Z}$

$8 \angle S^{\prime} Z$

789

889

$169^{\prime} \mathrm{Z}$

†09 $\mathrm{Z}$

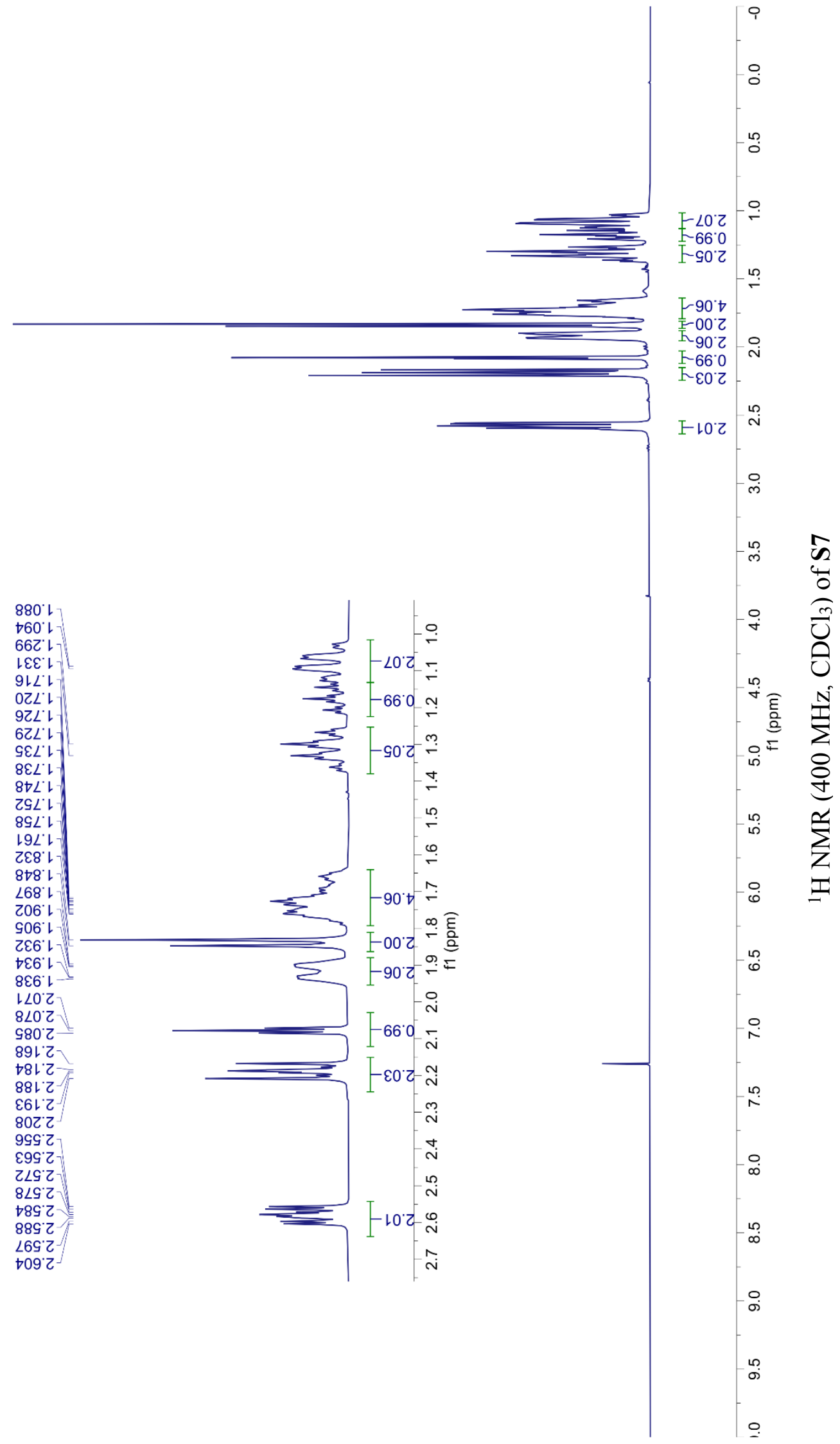


6ย'ง -

$6 \varepsilon^{\prime} \varepsilon \mathcal{E}$

$\angle Z ' S E$

Z $29^{\circ} \mathrm{E}^{-}$

29 t巾

98 $02-$

LZOS-

ธะ์งเレ-

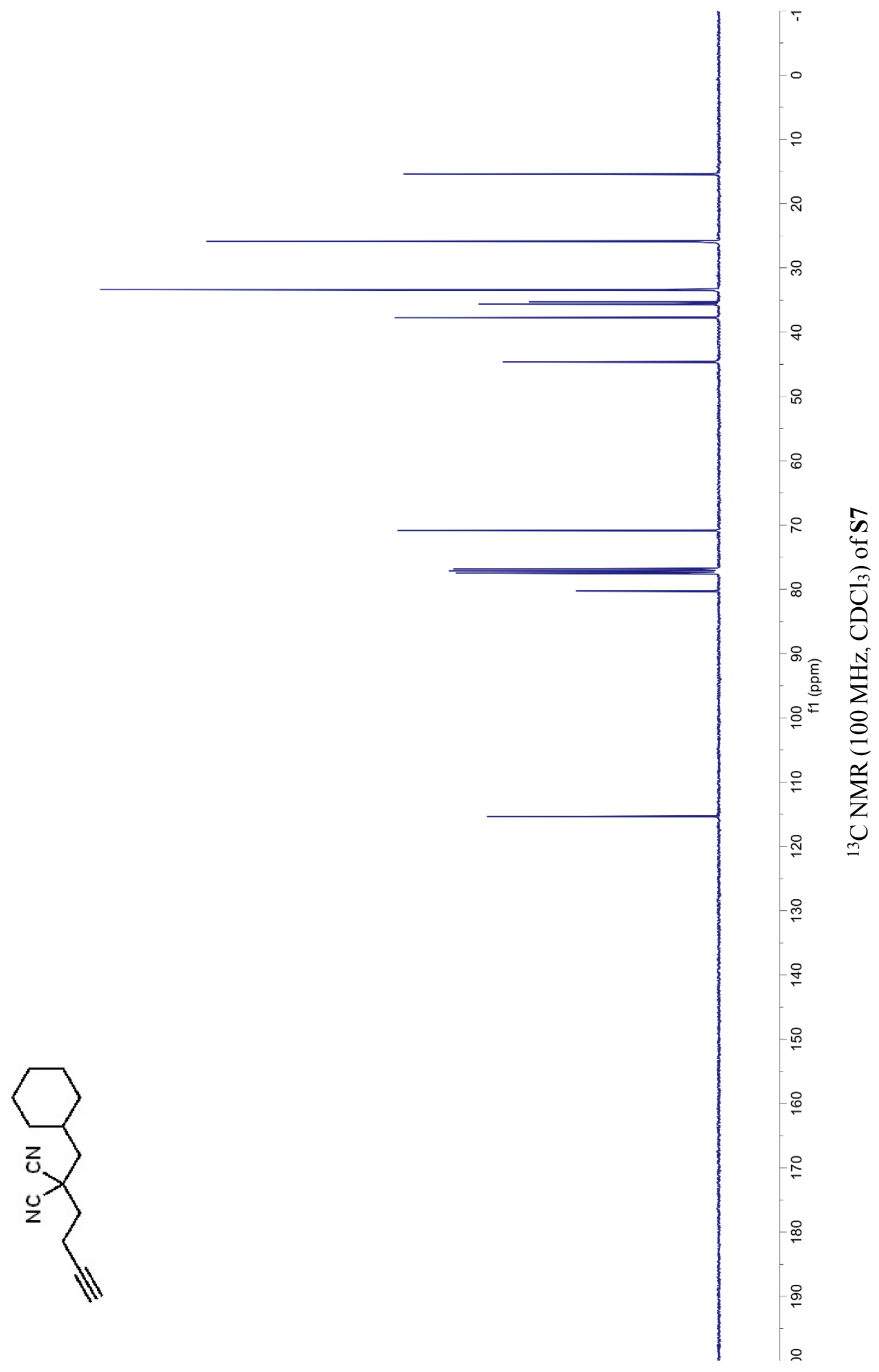


LLZ Z

98Z'

† 乙 $\mathrm{Z}$

96乙 乙

$66 乙$ 乙

$\angle 0 \varepsilon^{\prime} 乙$

9เ $\varepsilon^{\prime} \mathrm{Z}$

8ह8'乙

G98'

898.7

198' $\mathrm{Z}$

8L8'

$\varepsilon 6 乙^{\circ} \varepsilon$
ह8ح L

S8Z L

$88 Z^{\circ} \mathrm{L}$

$76{ }^{\prime} L$

$\angle 6 Z$ ' $L$

$66 Z^{\prime} L$

GOE'L

แE ${ }^{\prime} L$

9เE'L

6เE'L

เ乙E'L

$08 \varepsilon^{\circ} L$

$68 \varepsilon^{\circ}$

$\varepsilon 6 \varepsilon^{\prime} L$

$86 \varepsilon^{\circ} L-$

$00 t L$

SOt L

L $\nabla L$

จレ゙ L

9เV'L

レナレ

EZち L

GZt'L

6Zち'L

$\varepsilon \varepsilon \nabla^{\circ} L$

$9 \varepsilon t^{\circ} L$

8Et $L$

เカt'L

$9 \nabla \nabla L$

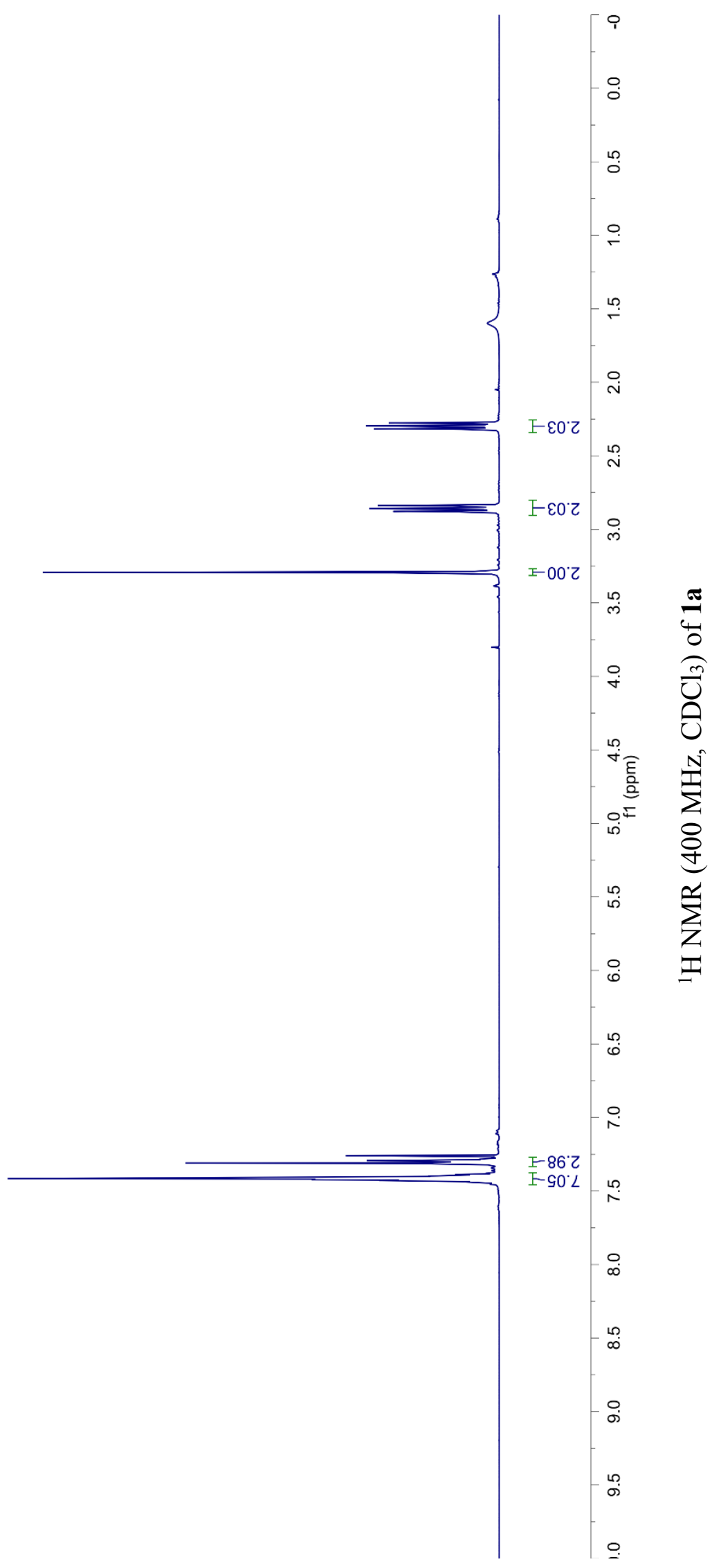


99.91-
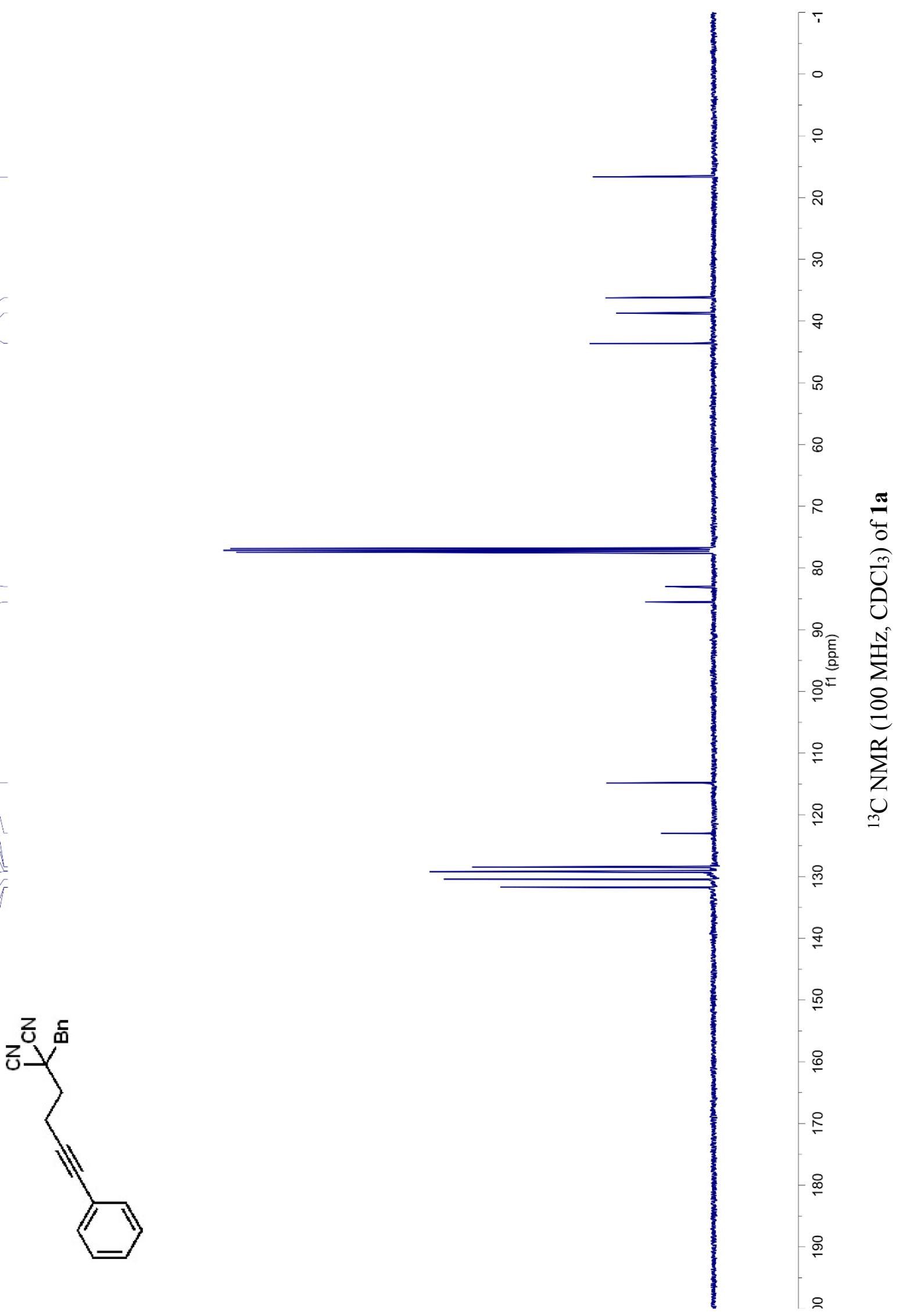


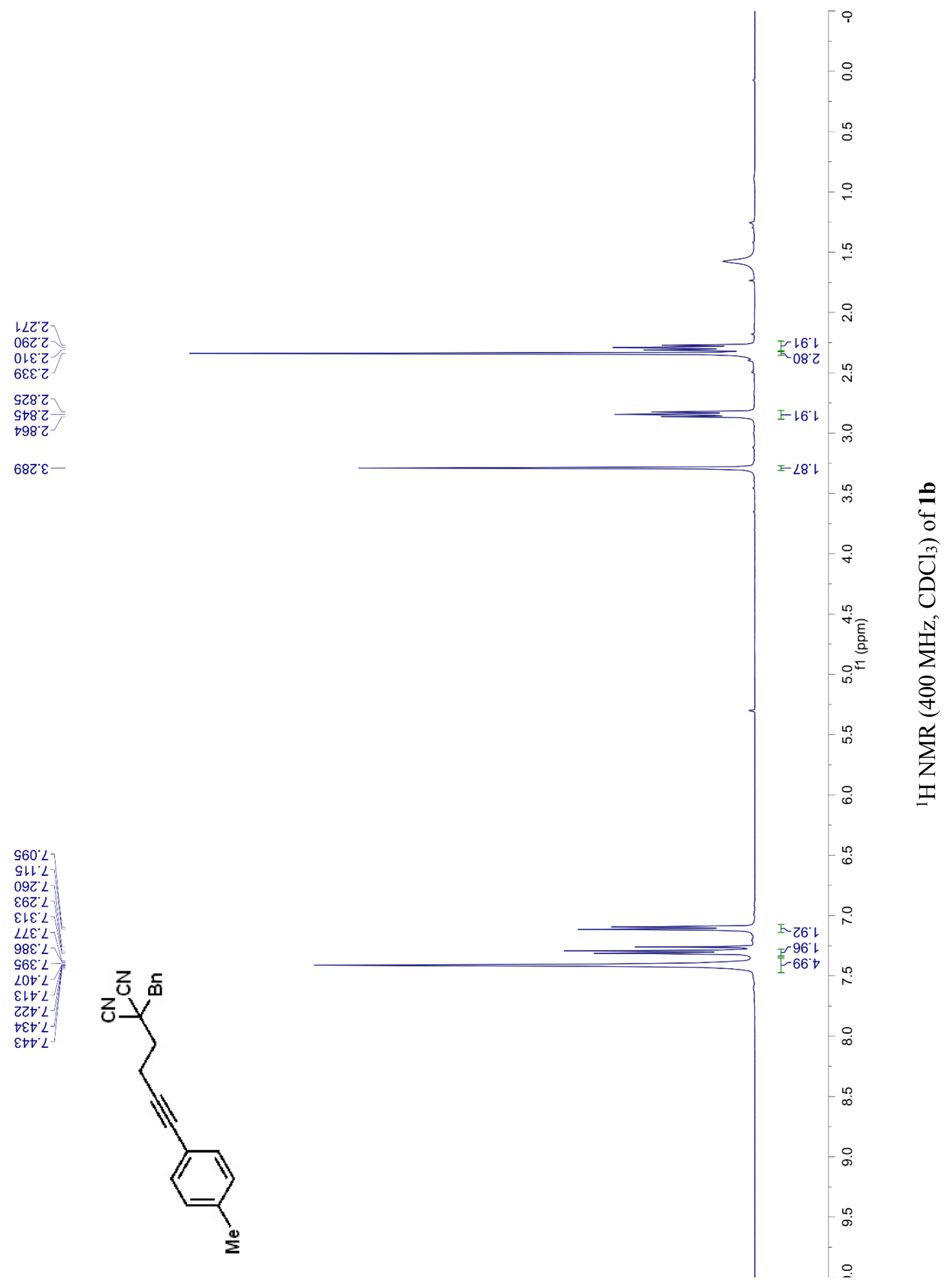


89.91-

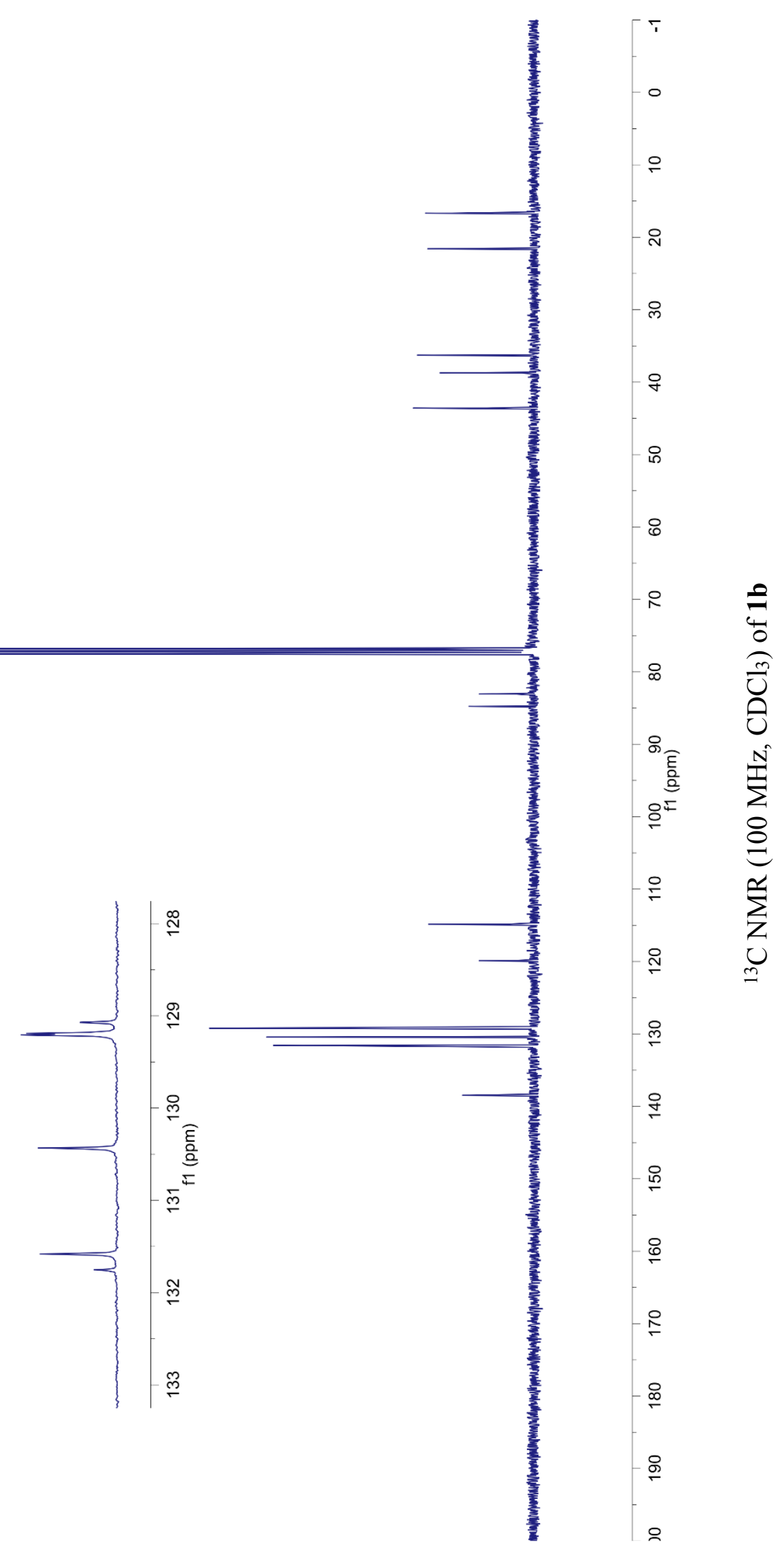

09.12

$8{ }^{\circ} 9 \varepsilon$
$\varepsilon L^{\circ} 8 \varepsilon$

$6 c^{\circ} \varepsilon t-$

\section{.}
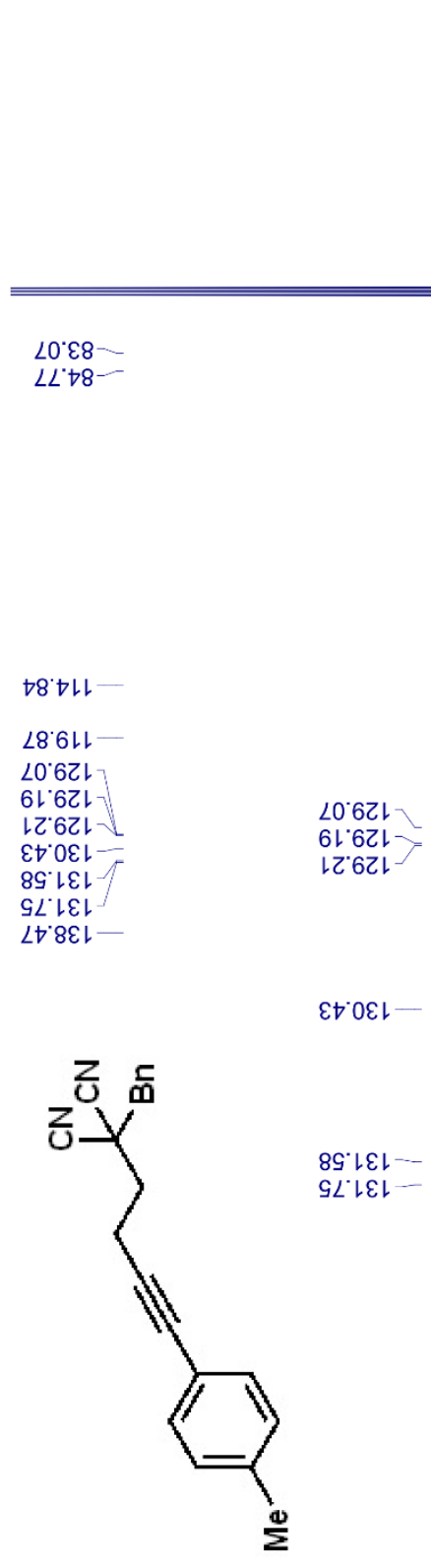

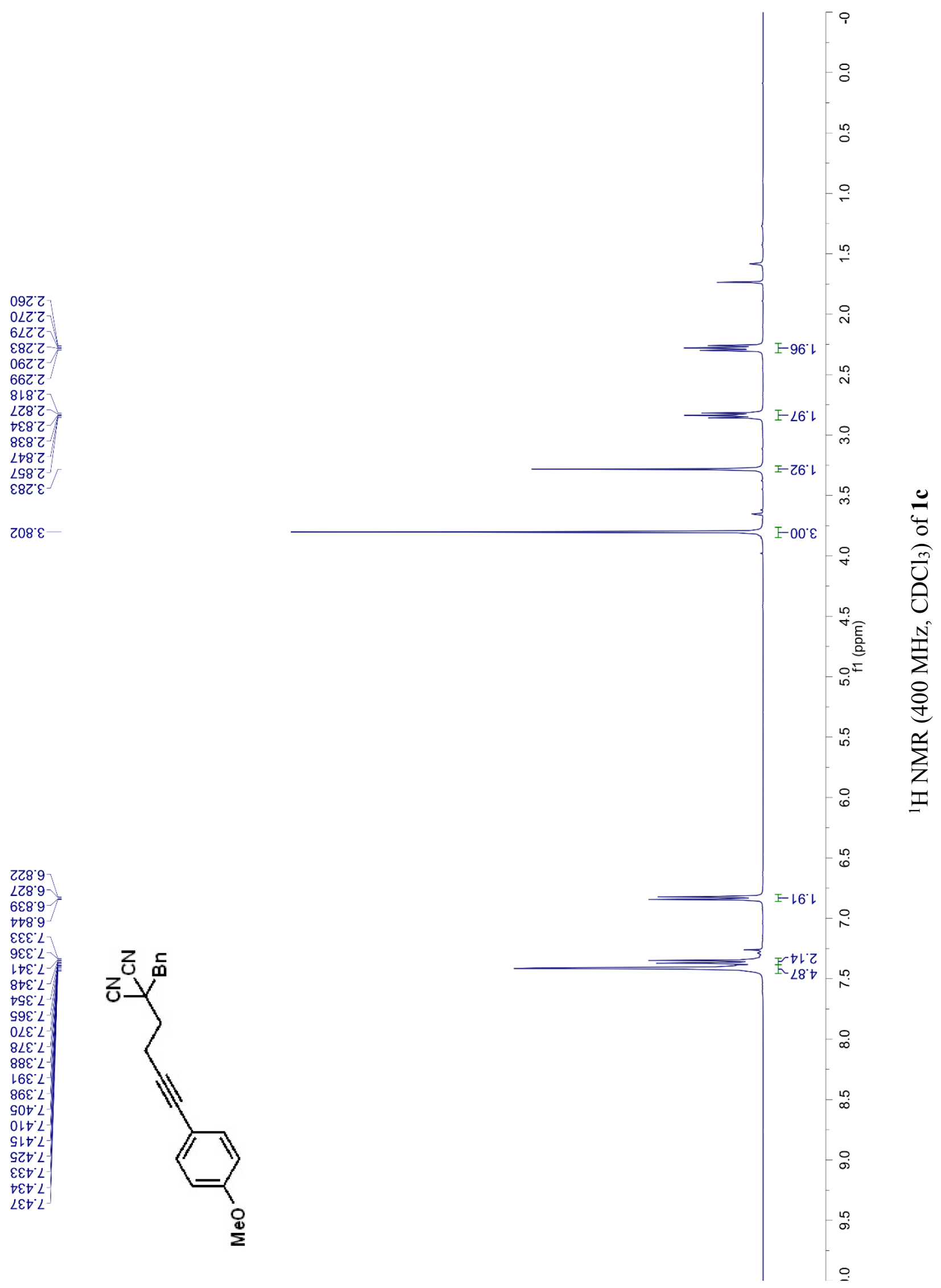
$\succ 9^{\circ} 9$ เ

LZ'9E

$89^{\circ} 8 \varepsilon$

$99^{\prime} \varepsilon \supset$

$8 \varepsilon^{\prime} \mathrm{S}-$

ह8' २8-

$20{ }^{\circ} \triangleright 8$

90"tレ

ง8"ヤレー-

$80^{\circ}$ SIL

ع0.6ZL

sl.6Zl

เ๋ 0 L

8L'LL

$60^{\circ} \varepsilon \varepsilon$

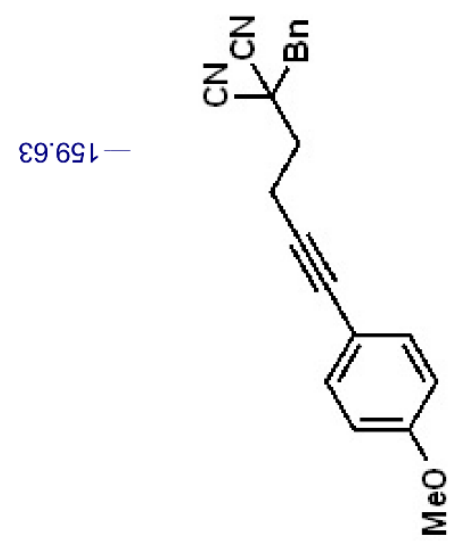

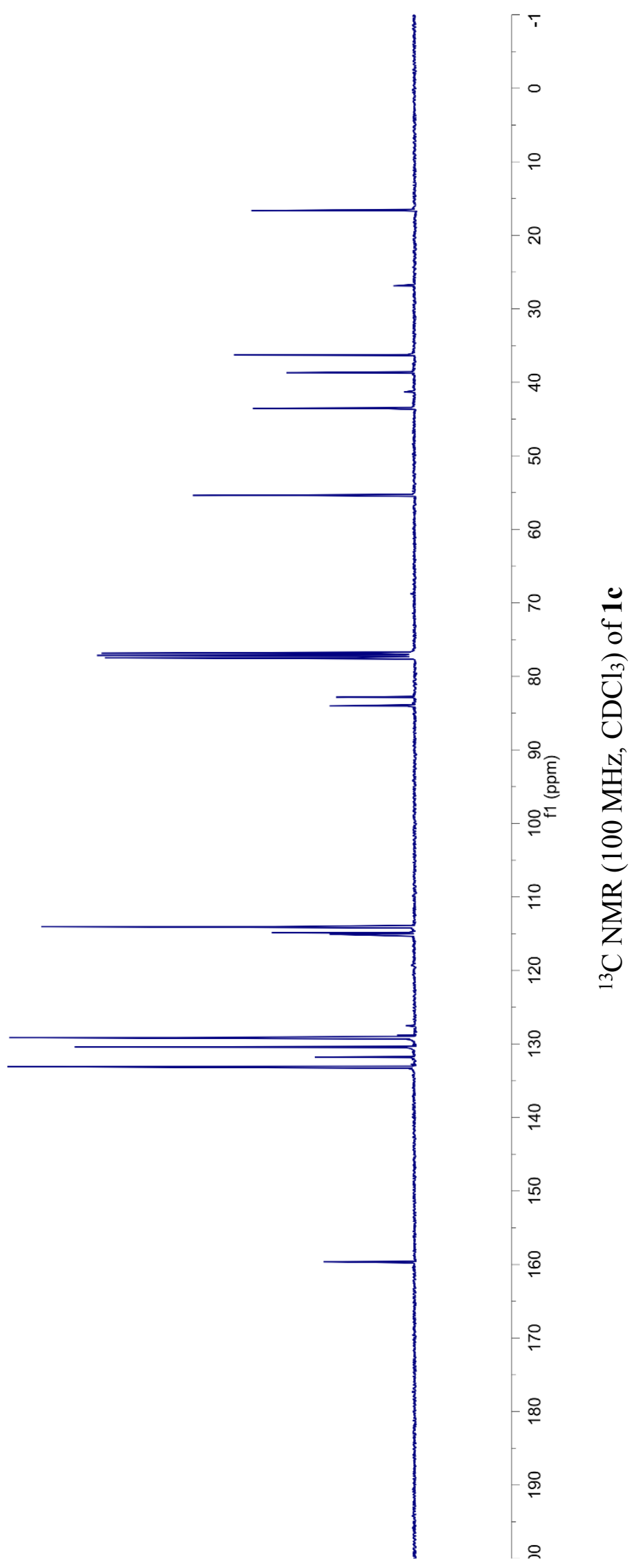

S132 

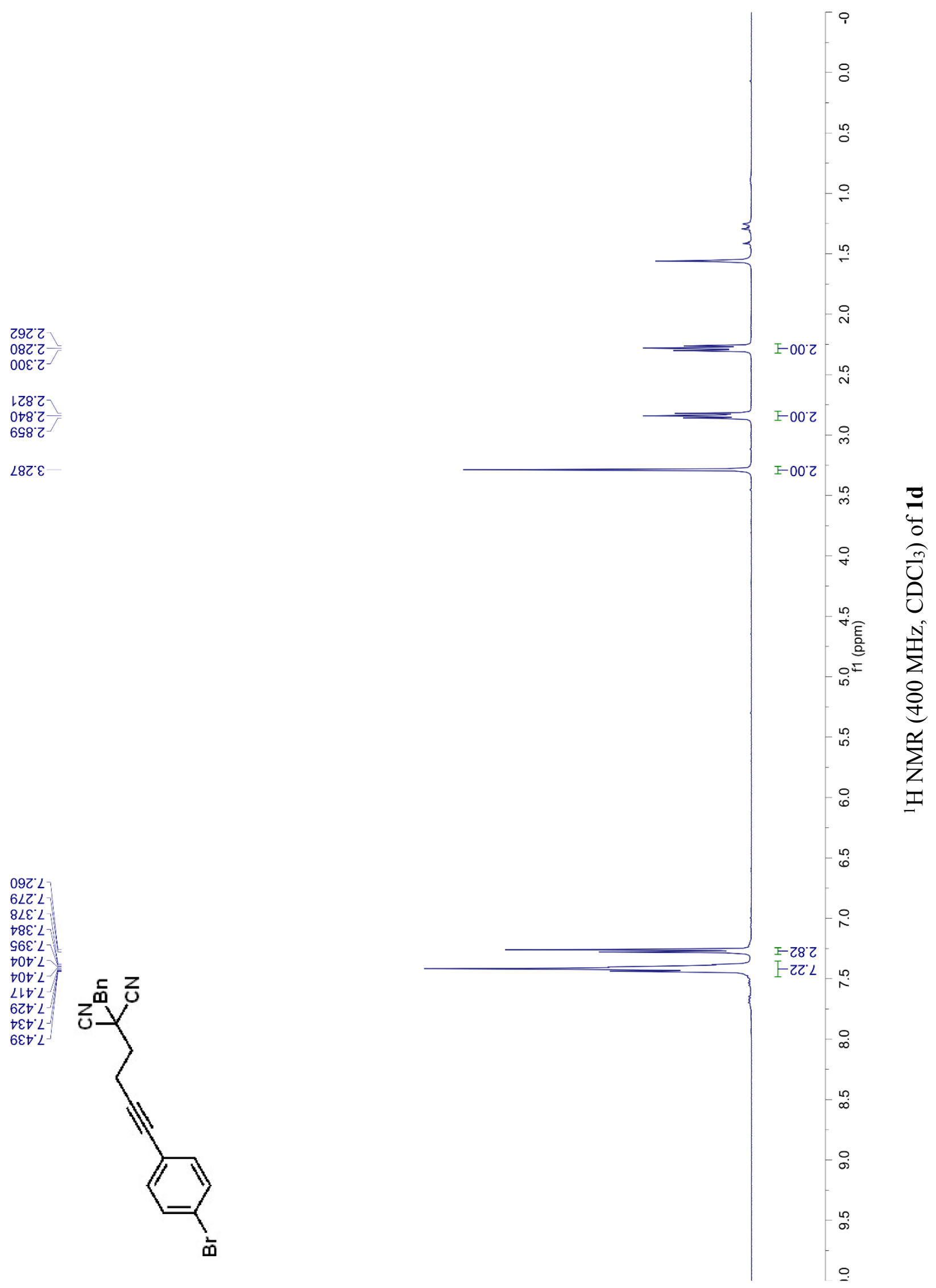
$0 L 91-$
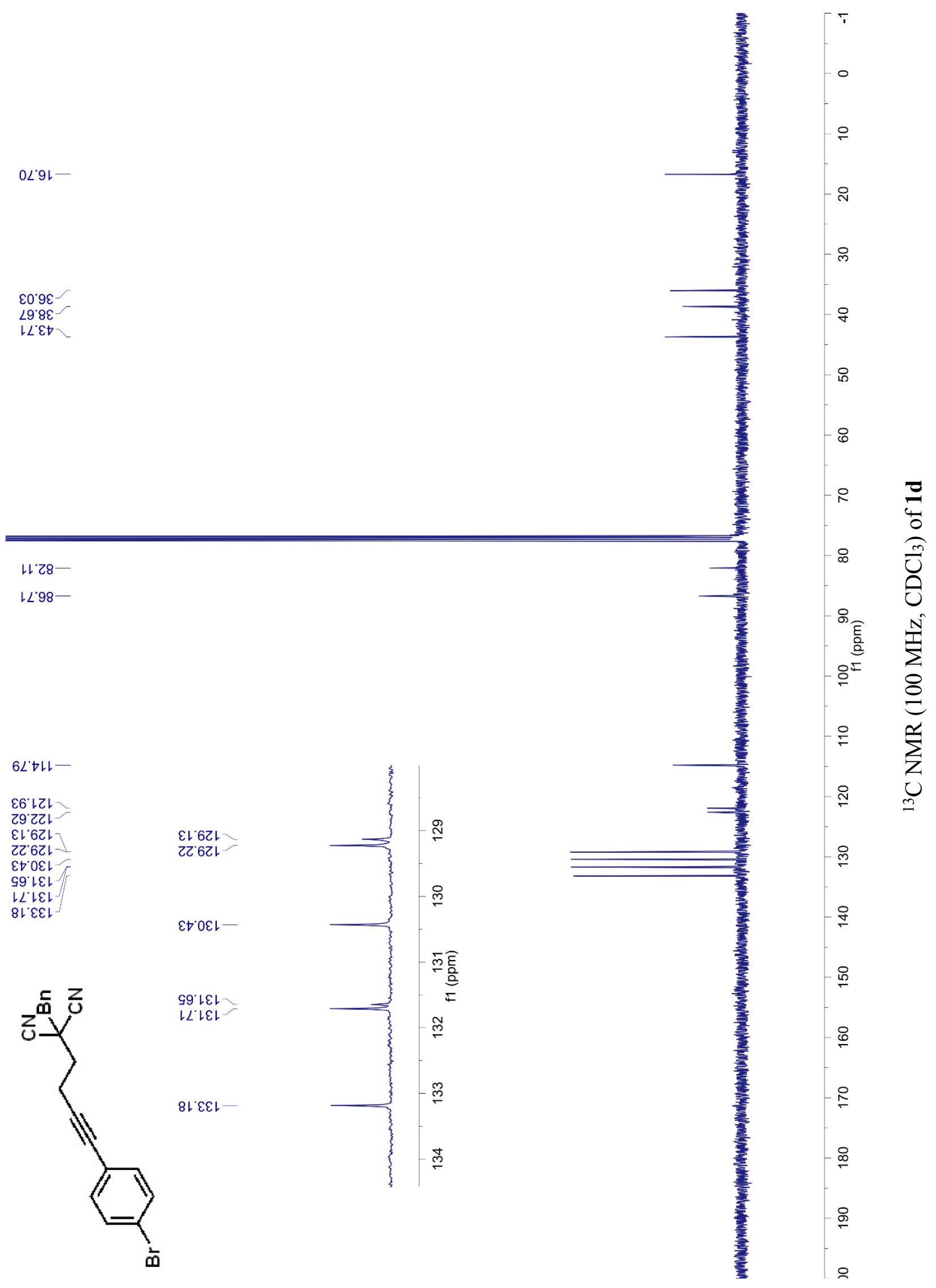
$1091-$

$08 Z 2$
$66 Z 2$

6LE'

$198 \cdot 2$

$188^{\circ} \mathrm{Z}$

$668^{\circ} \mathrm{Z}$

५6乙 $\varepsilon-$

09Z' L

$16 \varepsilon^{\circ} L$

LOt L

ILt 2

LZ' $L$
$9 Z \nabla^{\circ} L$

$\forall \varepsilon t^{\circ} L$

$6 \varepsilon \nabla^{\circ}\llcorner$ 作

OSt L $L$

†OG 2

$\nabla Z S^{\prime} L$

$87 G^{\circ} \mathrm{L}$

$699^{\circ} L$
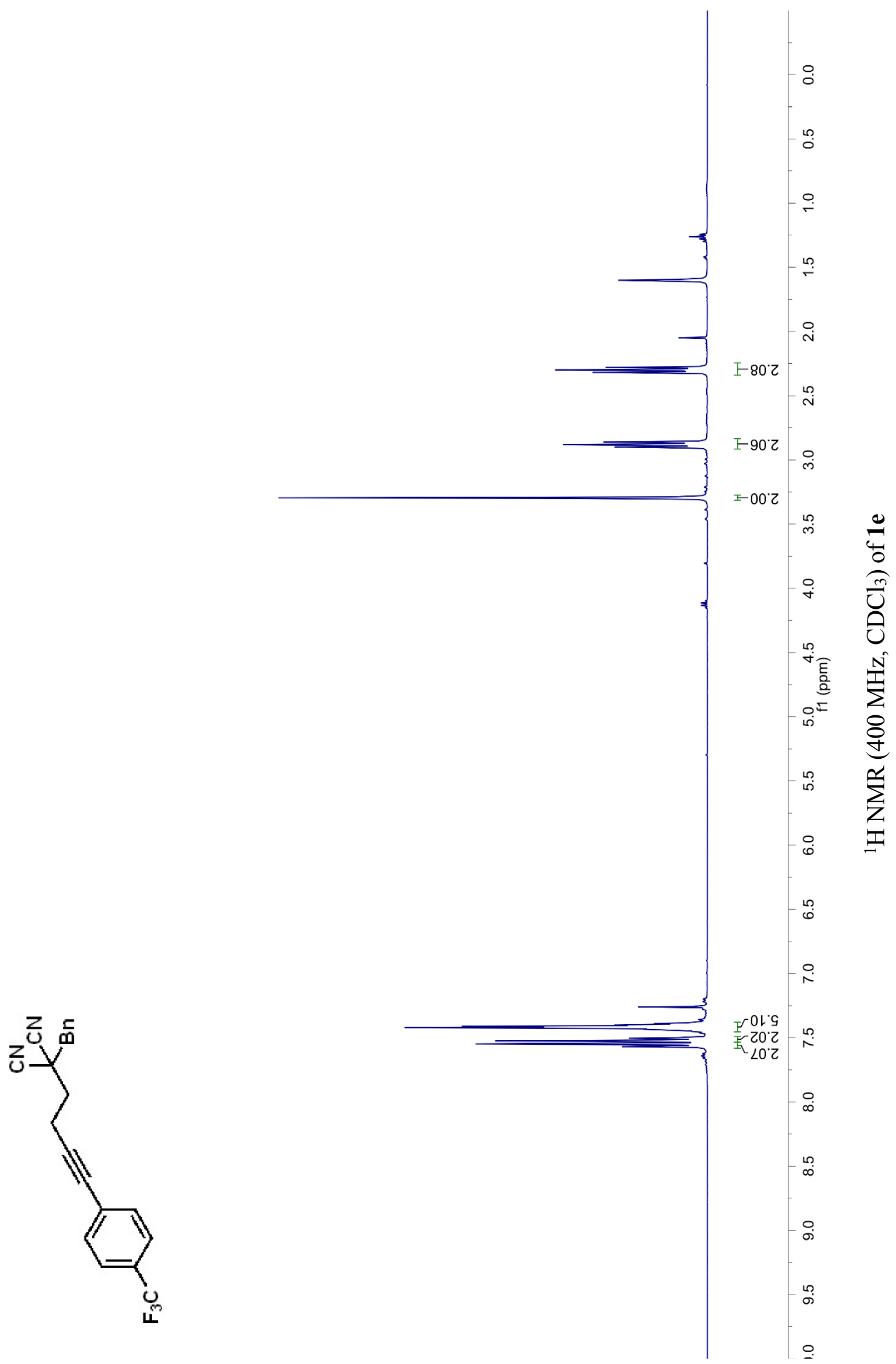

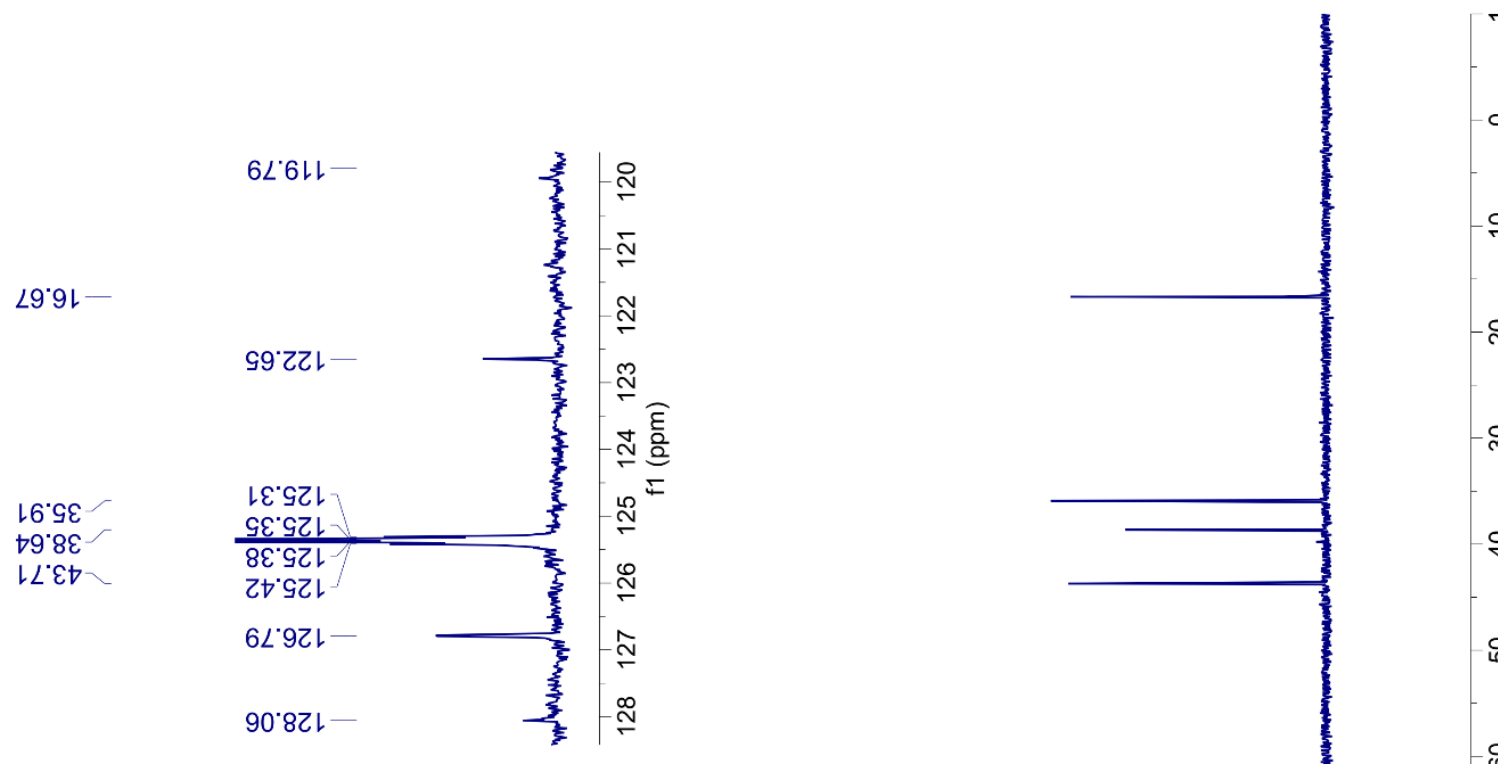

$16.18-$

$01 \cdot 88$

9L'tルL

62 6L

S9'ZZL

เE'GZL

GE'GZL

$8 \varepsilon^{\prime} \mathrm{GZL}$

Z† GZL

6L'9ZL

$90^{\circ} 8 Z L$

tr.6Zl

ZZ6ZL

89.6Zl

Z6 6ZL

$\nabla Z^{\prime} 0 \varepsilon \downarrow$

เナ0Eレ

$\angle G^{\circ} 0 \varepsilon^{-}$

$09^{\circ} L E$ -

$86^{\circ} L E L^{-}$

zo
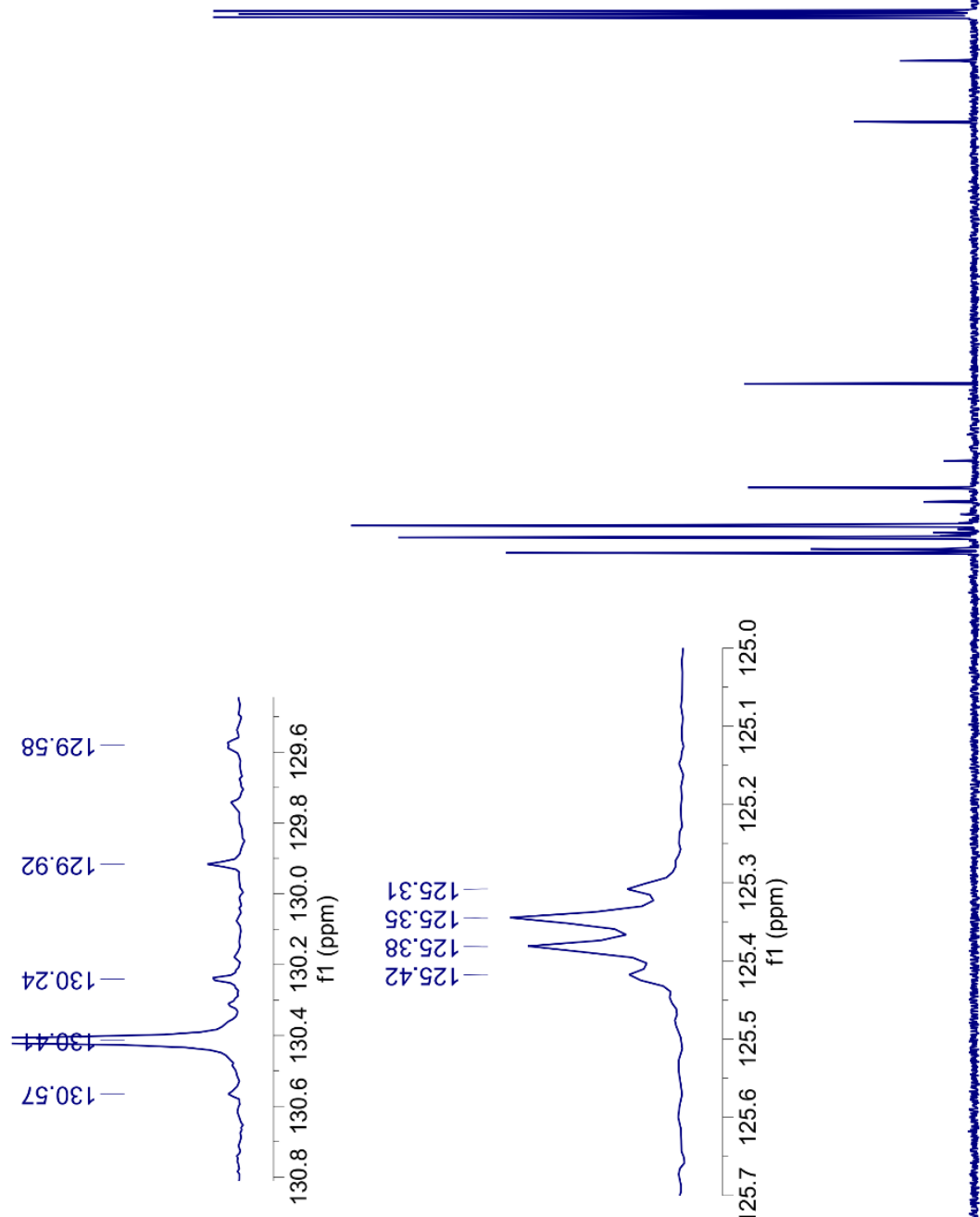

욜

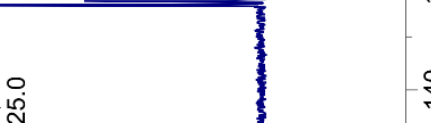

$\stackrel{9}{\circ}$

욤

$\stackrel{\circ}{\circ}$

운

$\stackrel{\circ}{\stackrel{\infty}{\infty}}$

\langle\rangle

-

ำ

ํํㄹ

突

ํำ

这

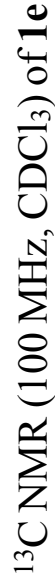

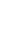

음

ํㅗํ

응 


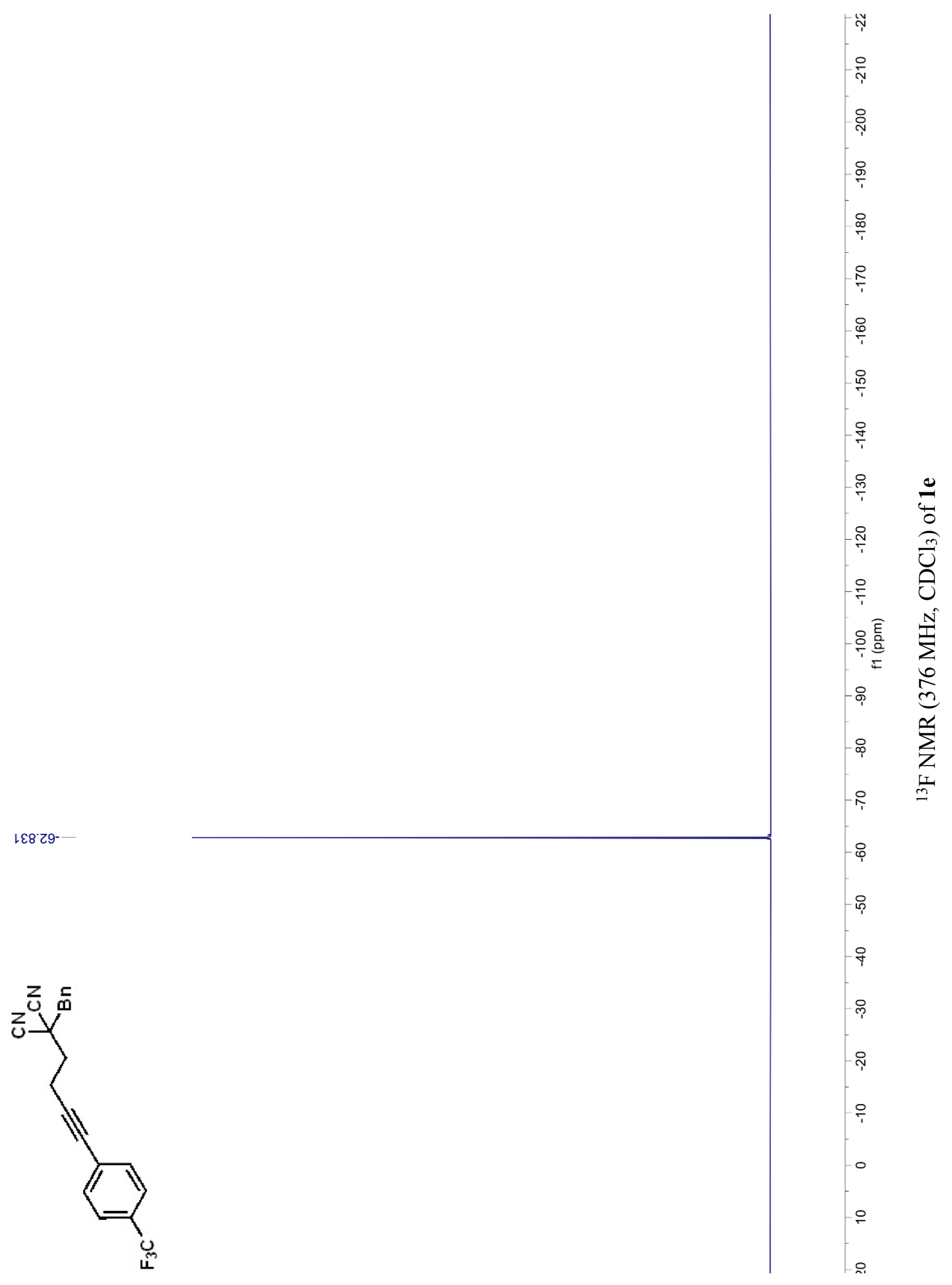




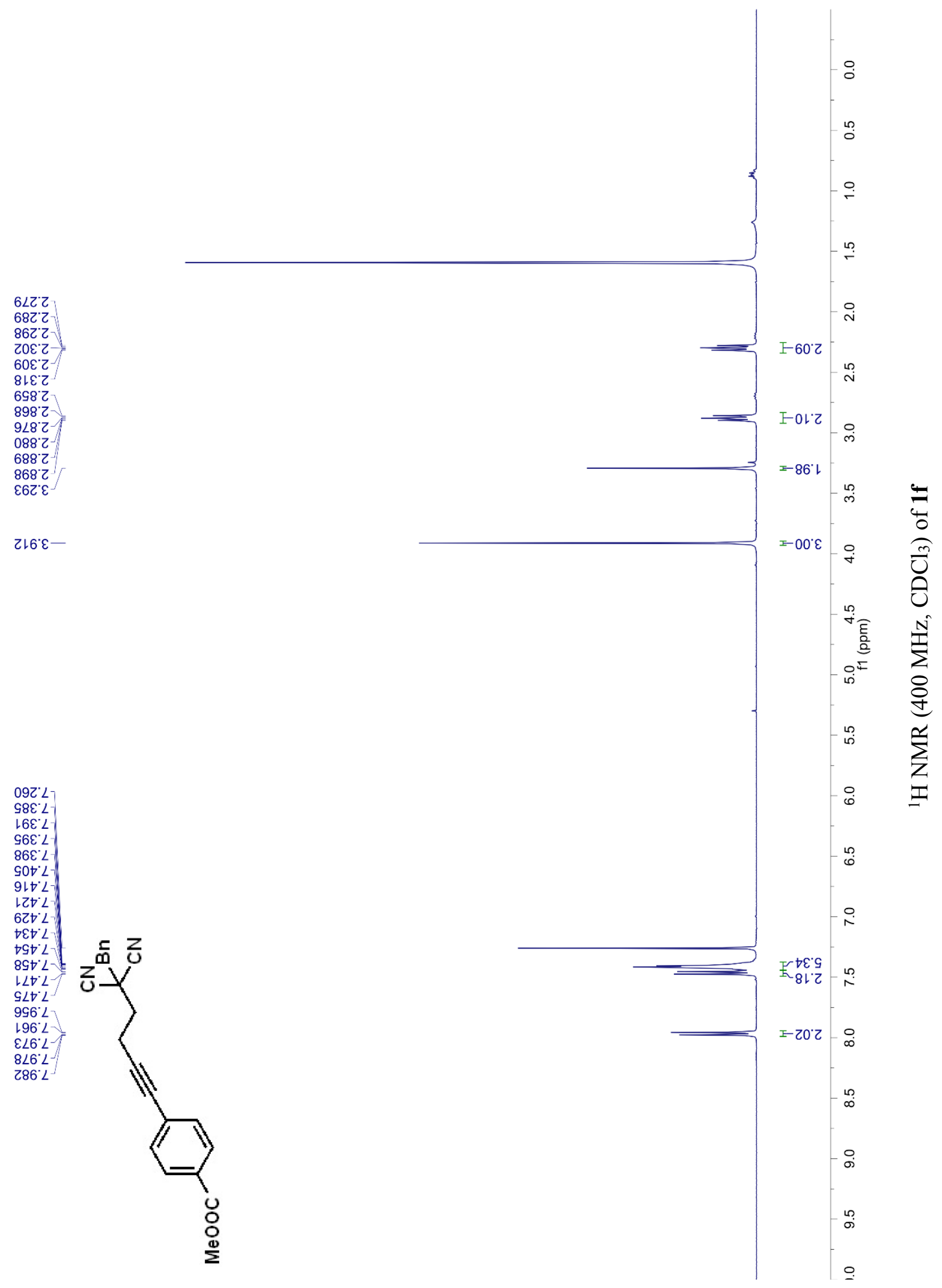




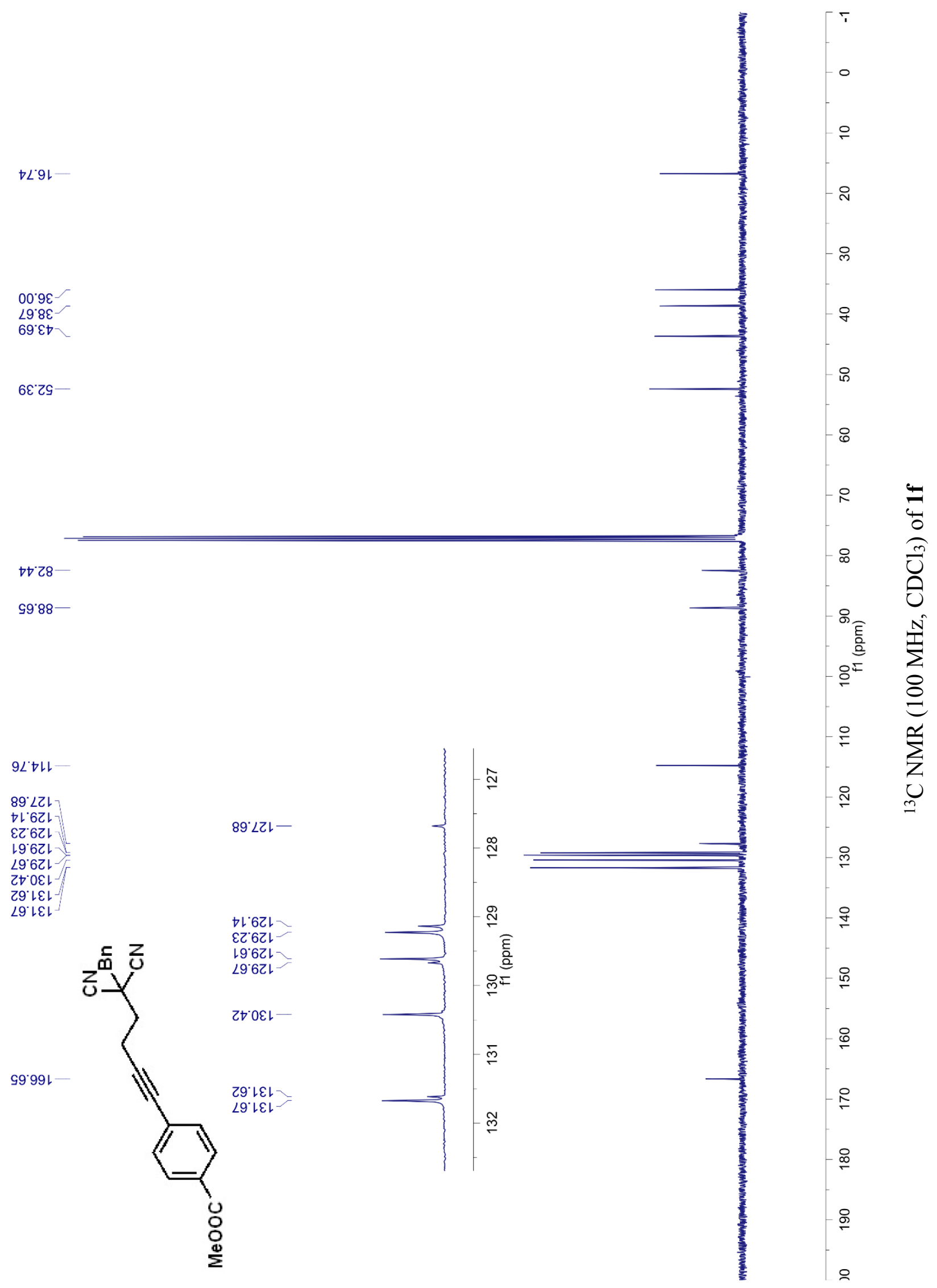



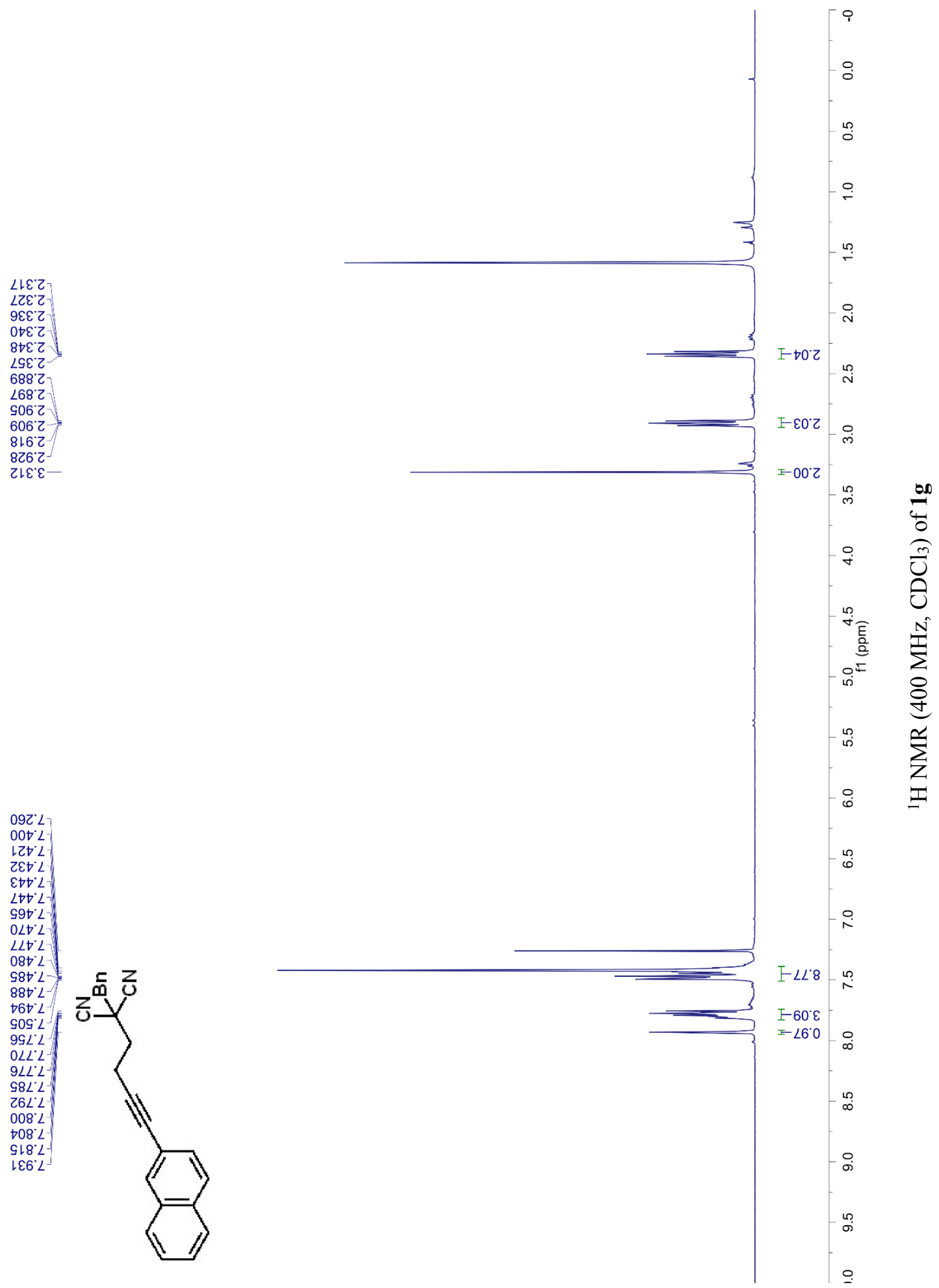
$8 L 91-$
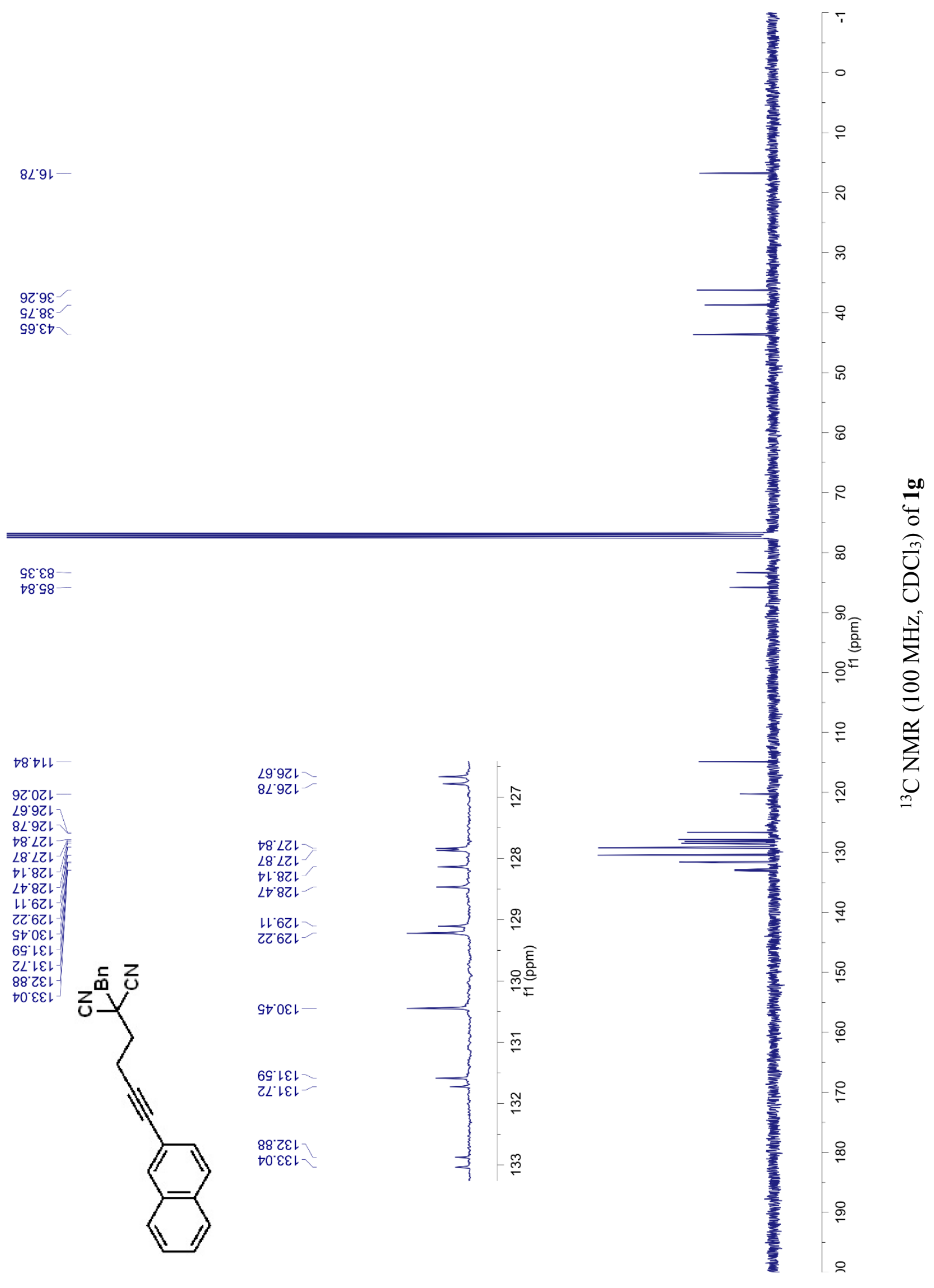

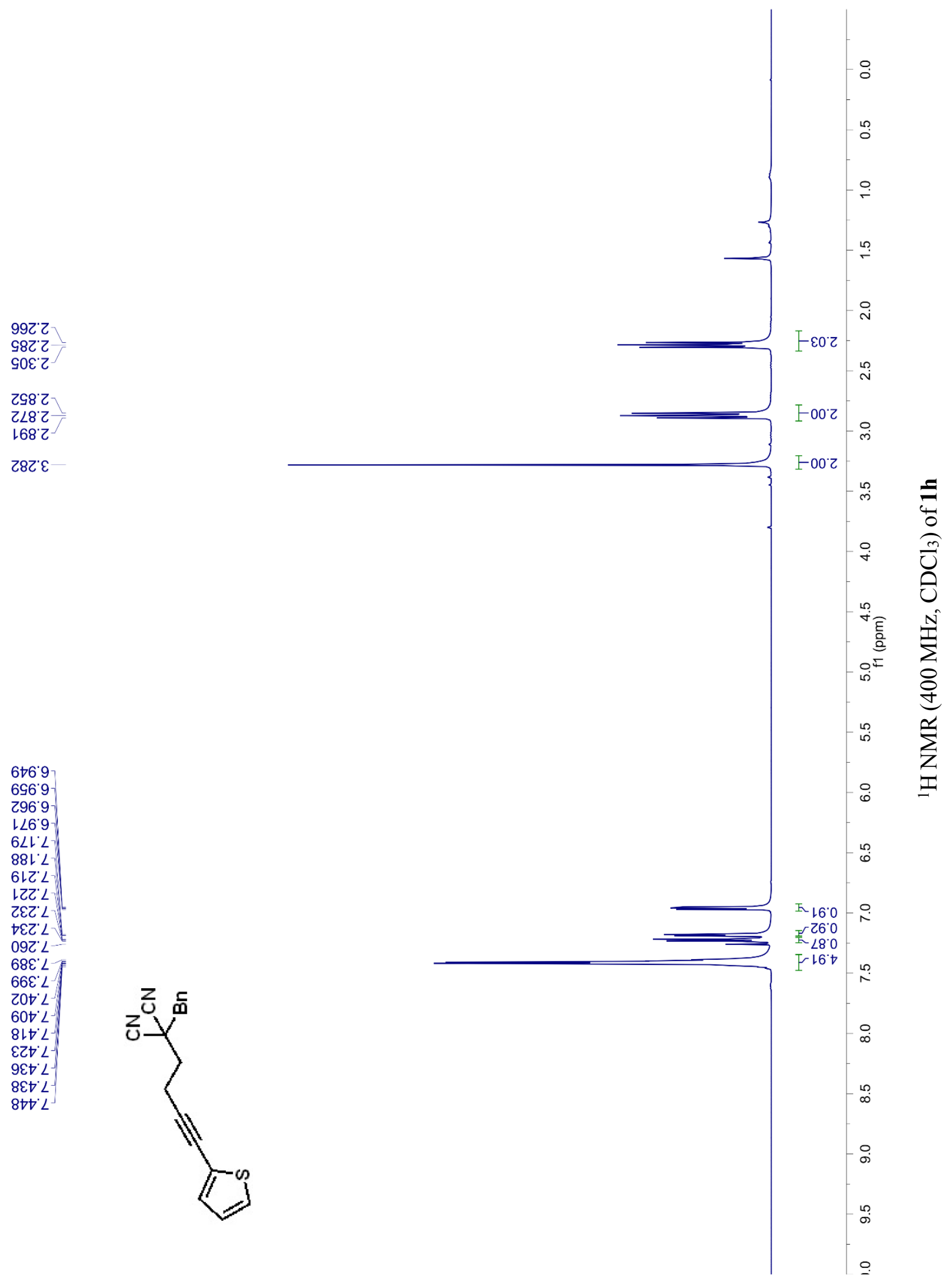
$89^{\circ} L E L$

Z०'ZहL-
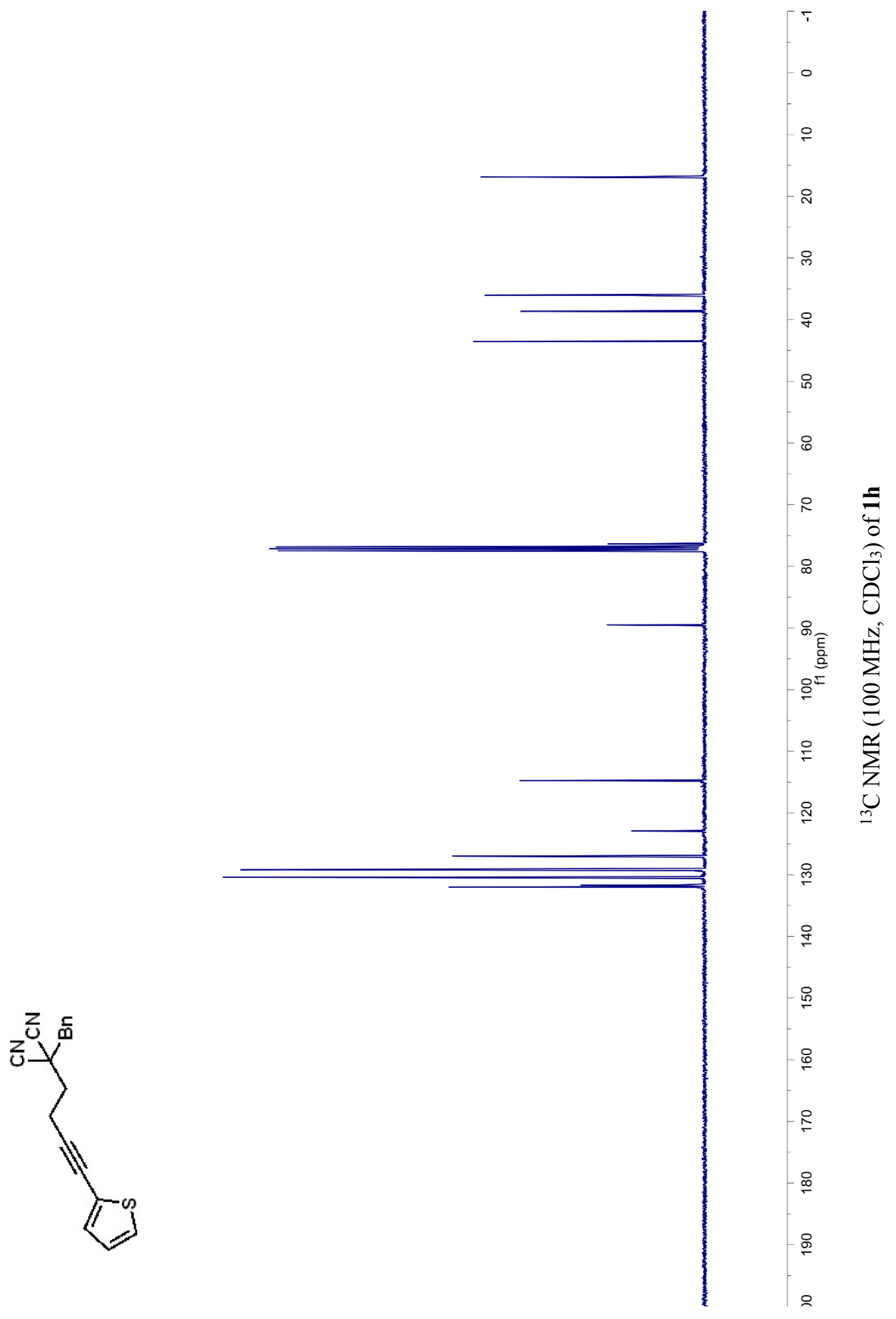
CIL

$88 L^{\circ} \mathrm{Z}$

$\nabla 6 L{ }^{\prime} Z$

89ट $\varepsilon$

ยเฉ S

LLZG

ZZZ
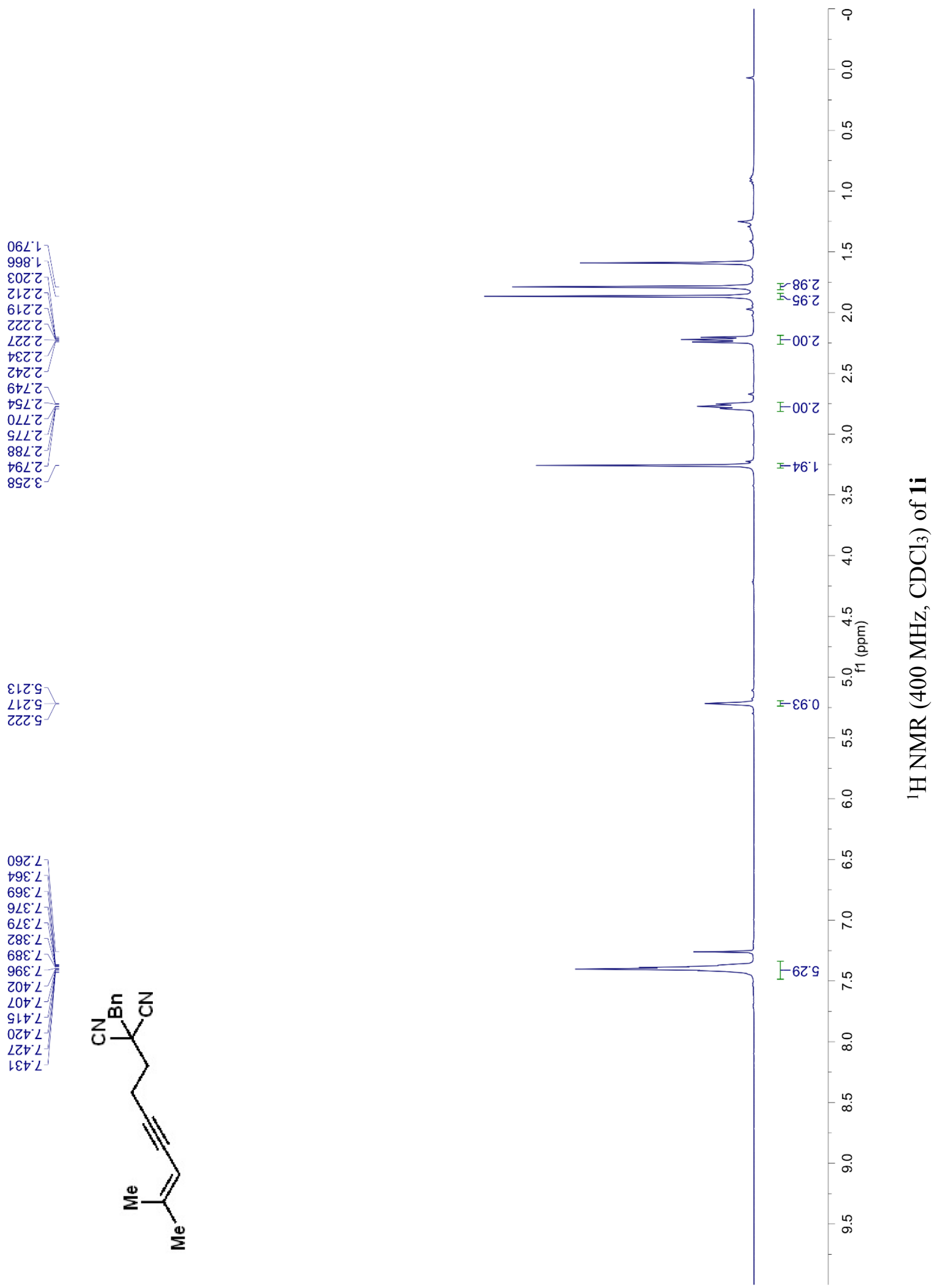
$\nabla L 9 L$
$\angle 0^{\circ} L$

$98^{\circ} \textrm{ }$

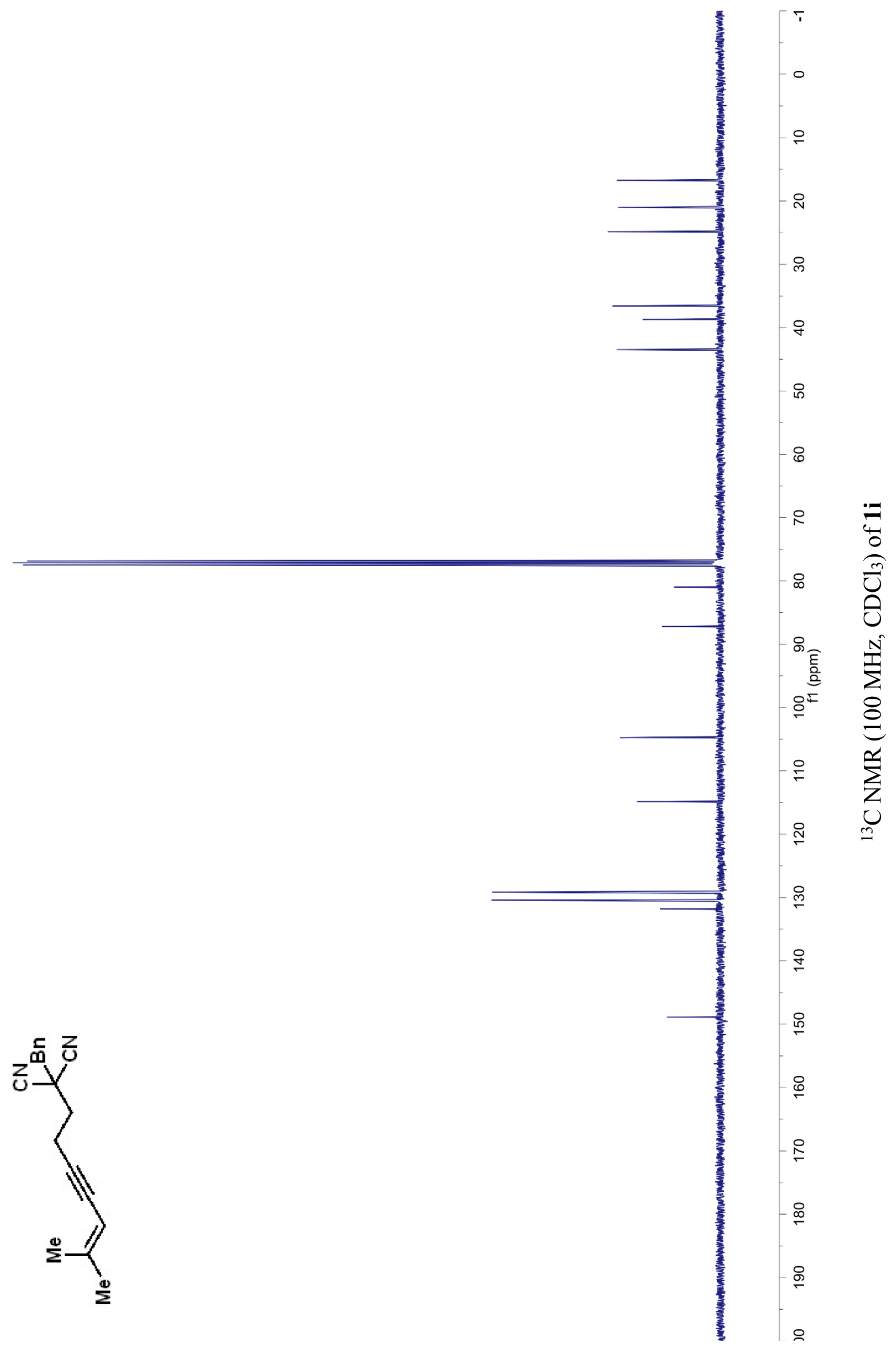

$706 \mathrm{LL}$

LL6ZL

$6 L$ 'L

68・8ㄴ-

乙L. $8 \varepsilon$

OS'E

†L'DOL-

ย8เレー 
9LL'

Z8LL

$88 L$

6El

$\angle G L ' Z$

$8 \angle L ' Z$

GES'

$1+S^{\prime} Z$

ISG'Z

999'乙

$199^{\circ} \mathrm{Z}$

$\nabla \angle G^{\prime} Z$

$089^{\prime} Z^{\prime}$

$\nabla \nabla Z^{\prime} \varepsilon$

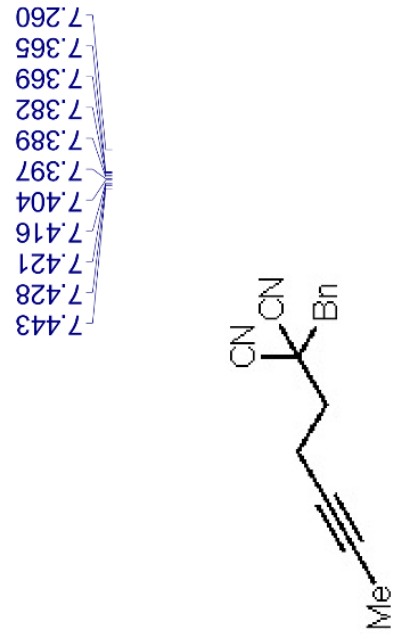

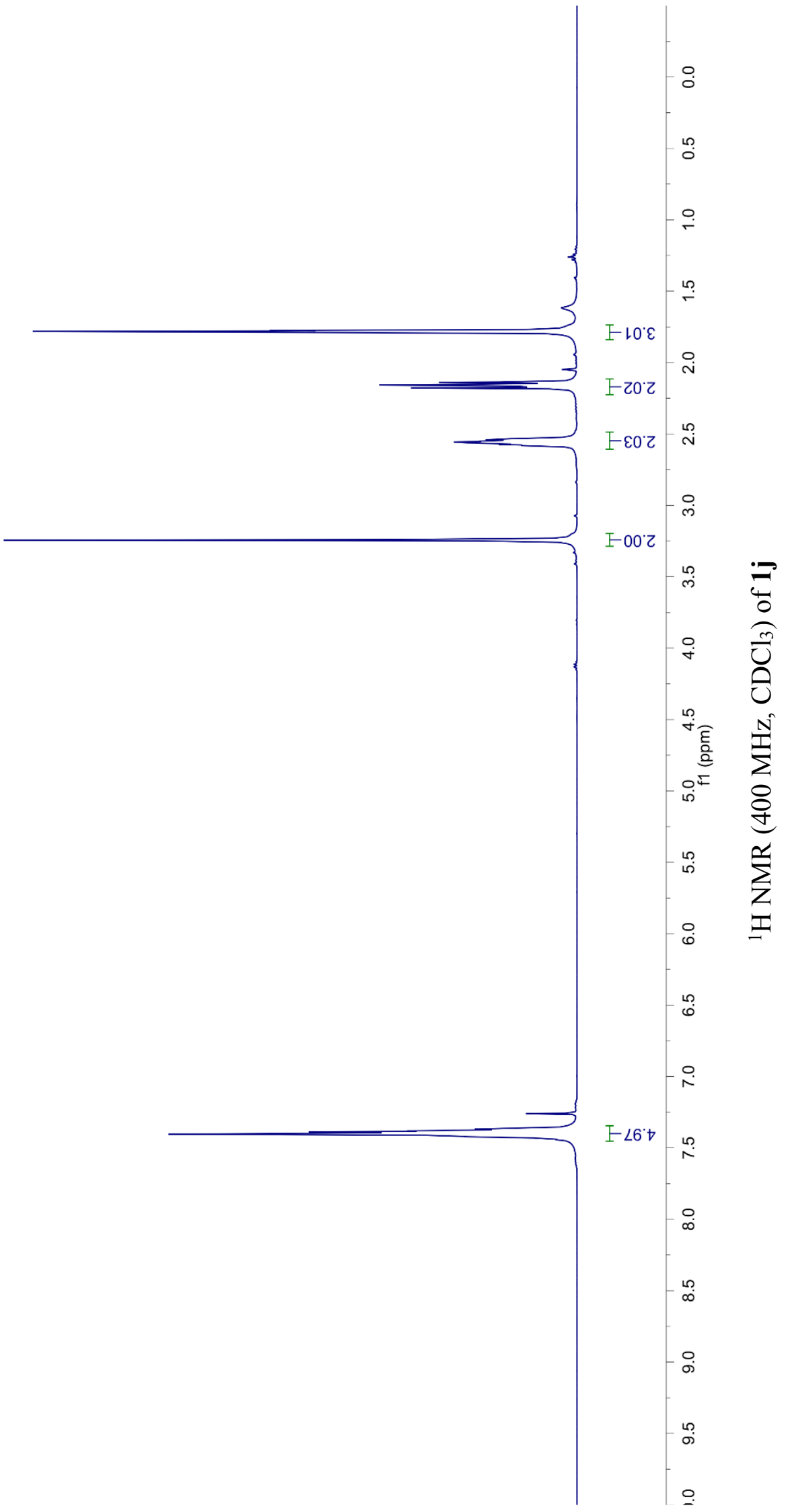

S146 
99' $\varepsilon-$

G6'G

$67^{\circ} 9 \varepsilon$
$\varepsilon 9.8 \varepsilon$

ISED

$80^{\circ} S L-$ $08^{\circ} 8 L$

ட8จルレー

0062 -

El'6Zl-

0† 0हL

L8. LE

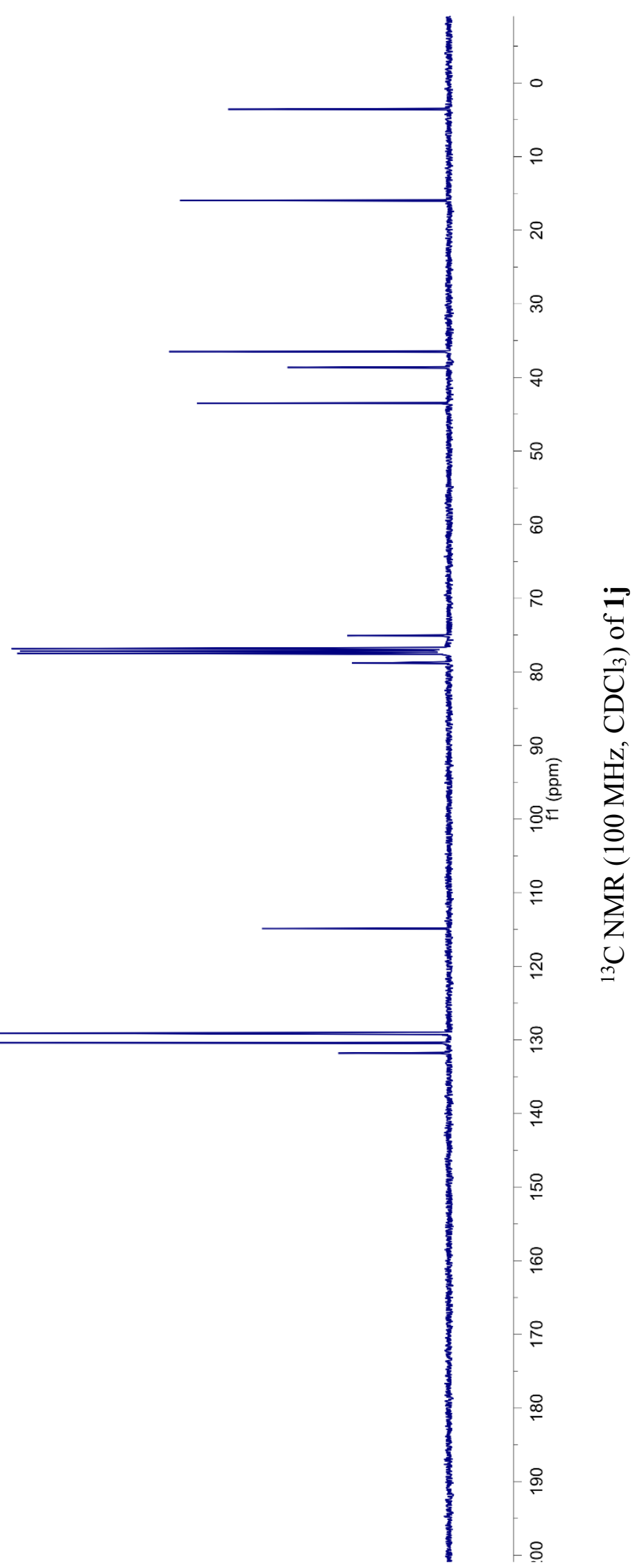

S147 
เナ8

$198^{\circ} \mathrm{Z}$

$088^{\circ} \mathrm{Z}^{-}$

$8 \nabla \tau^{\circ} \varepsilon-$

092: L

LLZ $L$

$9 \angle Z^{\circ} L$
$98 Z^{\circ} L$

Z62' 2

$66 \mathrm{C}^{\circ} \mathrm{L}$

$\angle O E^{\circ} L$

ZLE'L

ᄂ乙E'L

LOT L

Oレナ゙ L

OZ

G乙† L

OSG $L^{\circ}$

SGS' $L$

999. $L$

L $\angle S^{\circ} L$

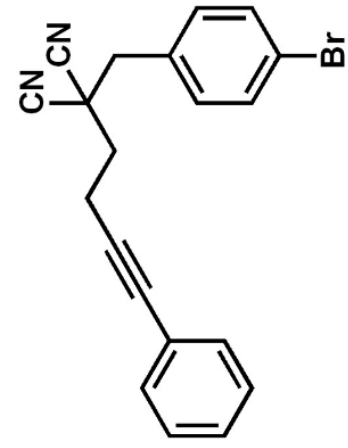



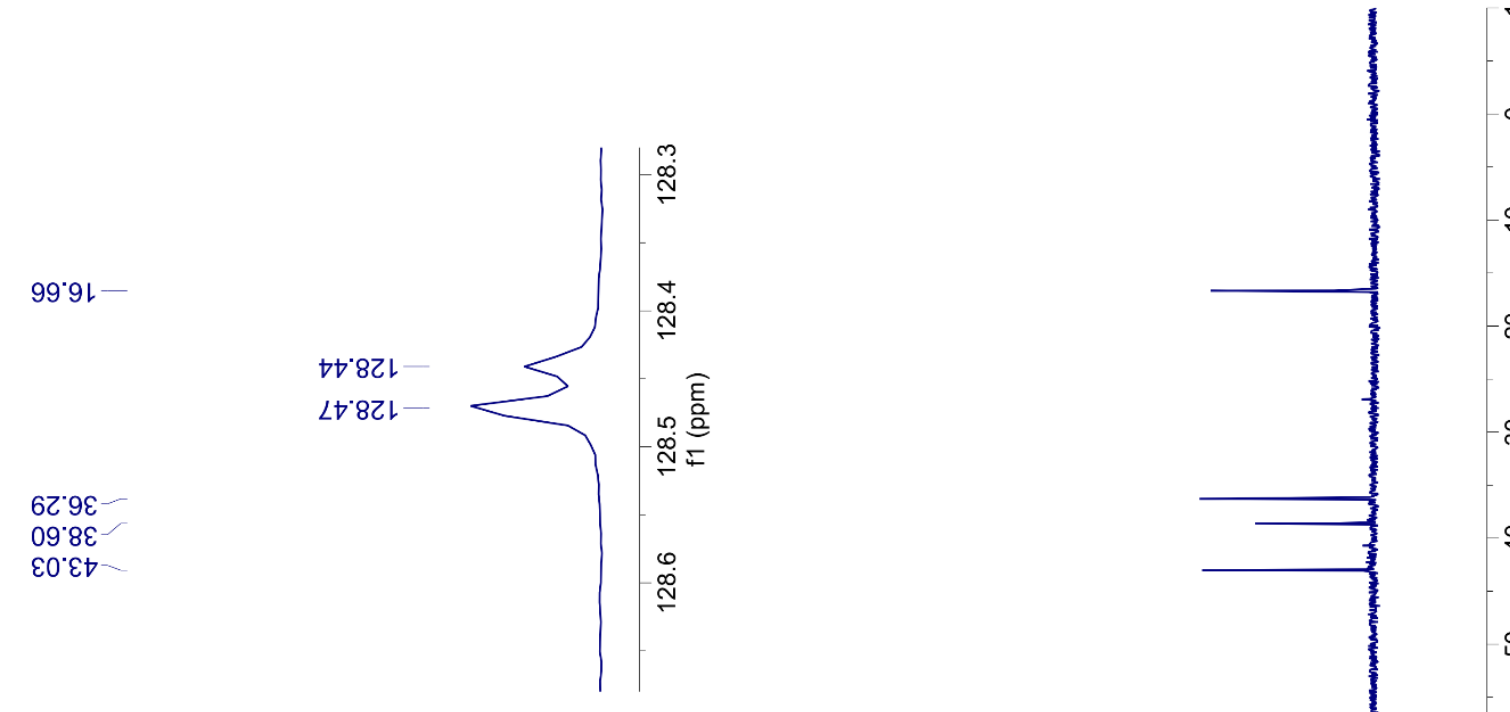

0乙' 8

乙E'
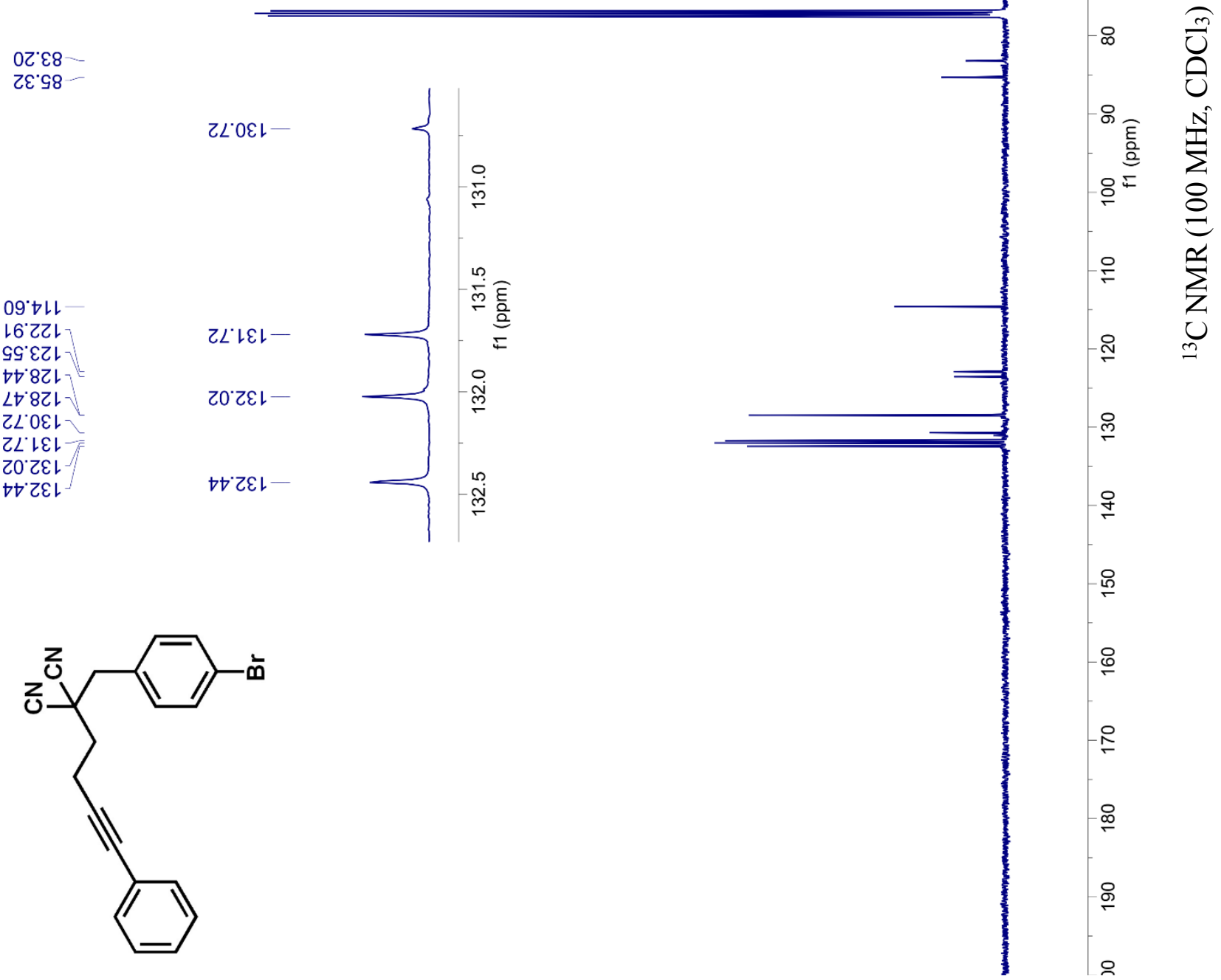
G99'レ-

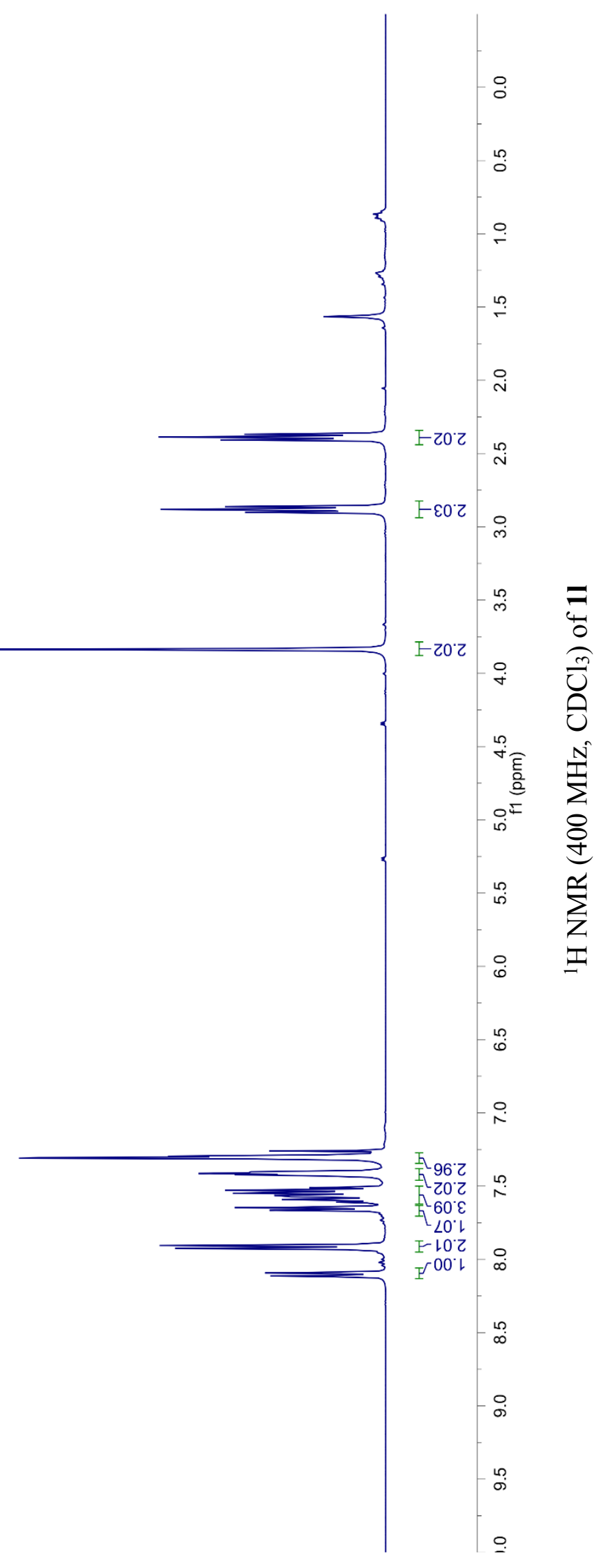

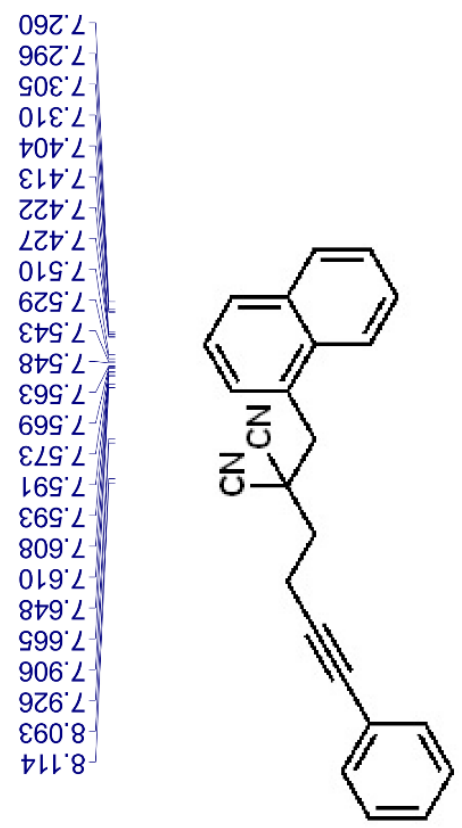

L8E' 乙

86E'乙

LOt'

Z98' $\mathrm{Z}$

乙88 乙

$006^{\circ} 乙$

$\angle \mathcal{L} 8^{\circ} \mathcal{E}-$ 
18.91-

$99^{\circ} 9 \varepsilon$

$8 \mathrm{~S}^{\circ} 8 \varepsilon$

2l.6E

ร०० $88-$

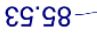

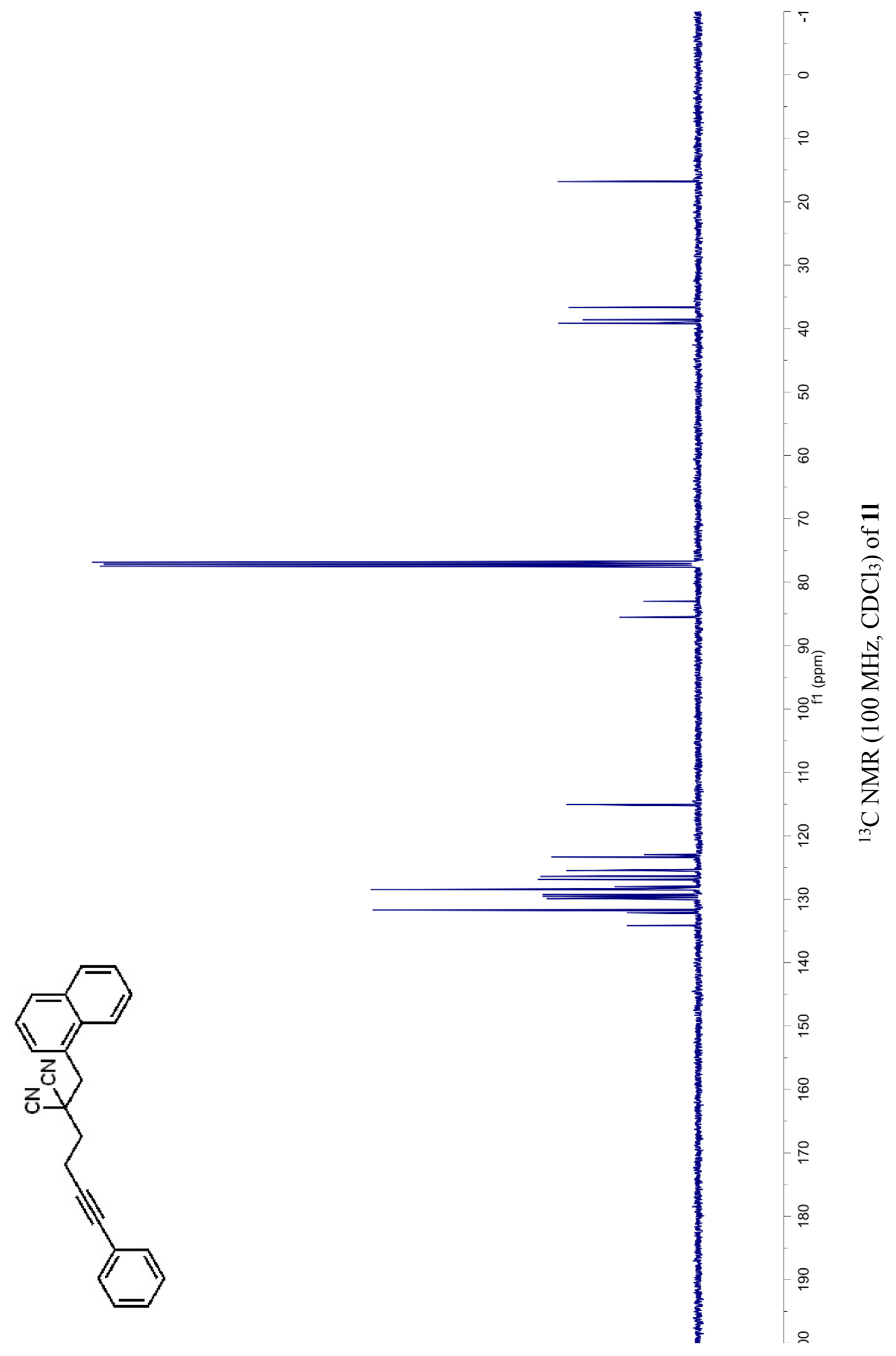

80 '

$\angle 6{ }^{\circ} \mathrm{ZZL}$

ธE'EZL

9† GZL

$6 \varepsilon^{\circ} 92$

$\angle 8.9 Z 1$

L0'8Z

$\angle \varepsilon^{\circ} 8 Z \mathrm{~L}$

tt' $8 Z \mathrm{~L}$

LZ6ZL

89'6ZL

L6 62L

ZL'LEL

9 ' $Z E L$

Gl' $\nabla \varepsilon L$ 

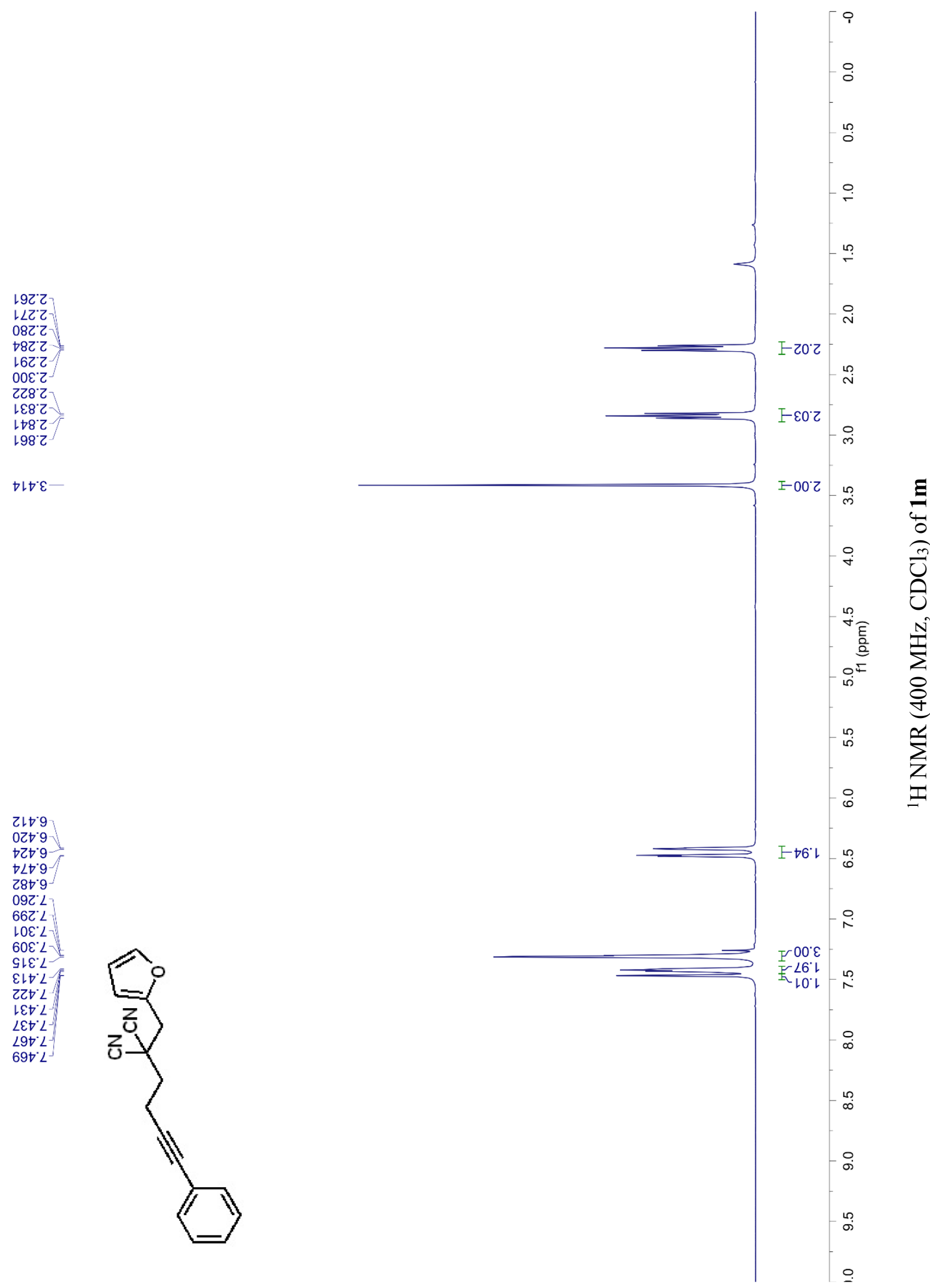


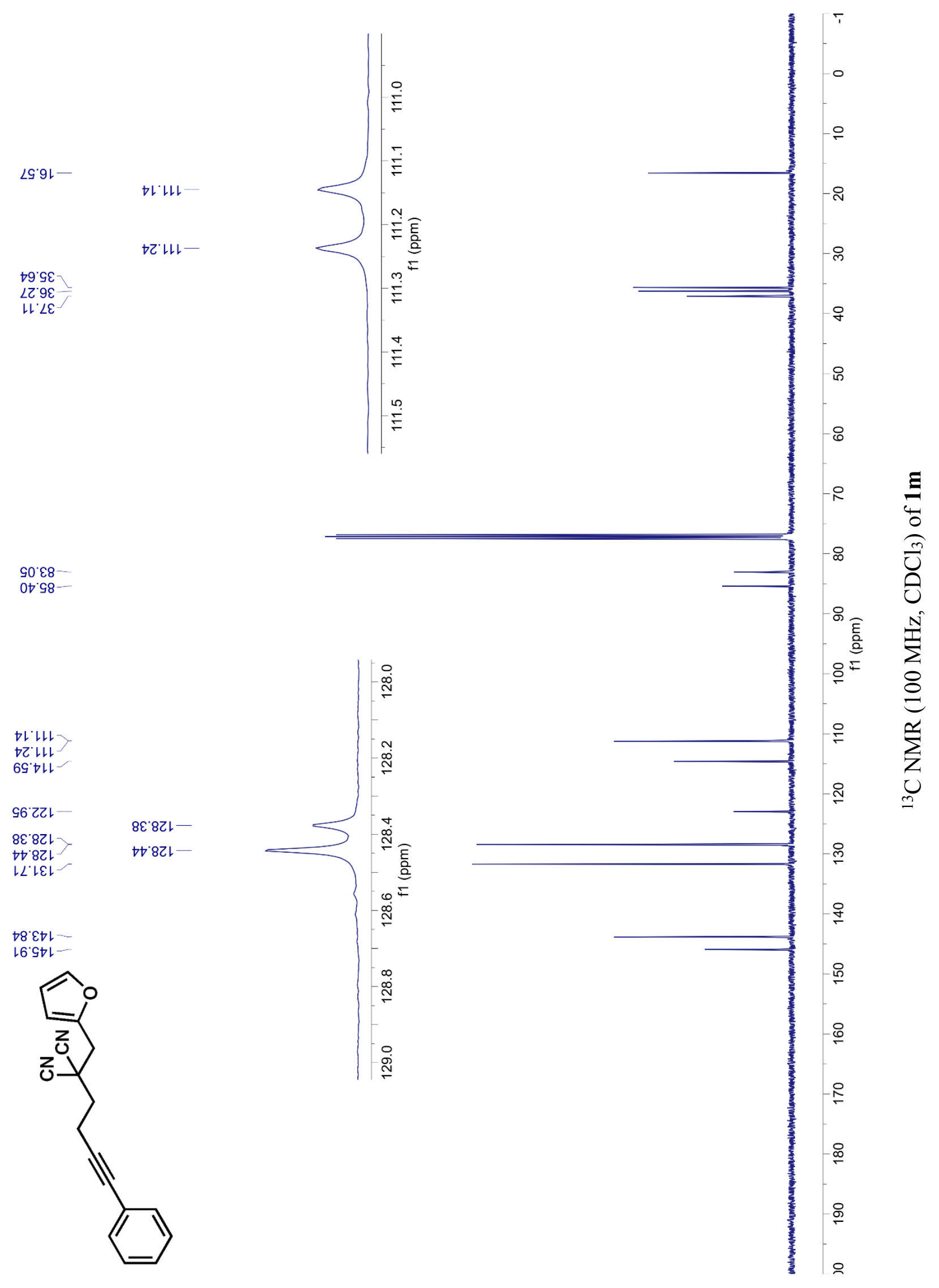


LEL'L-

乙๖乙 乙

乙G乙 乙

192'

G9Z'乙

$\varepsilon L Z^{\circ} 乙$

$182 \mathrm{Z}$

$Z \square L Z$

$19 \angle 2$

$86 L^{\circ} Z$

s.8.

$618^{\circ} \mathrm{Z}$

878 $\mathrm{Z}$

$8 \varepsilon 8^{\circ} \mathrm{Z}$

982 9

$\mathrm{S} 0 \mathcal{E}^{\circ} \mathrm{G}$
$\rightarrow Z \mathcal{E}^{*} \mathrm{G}$

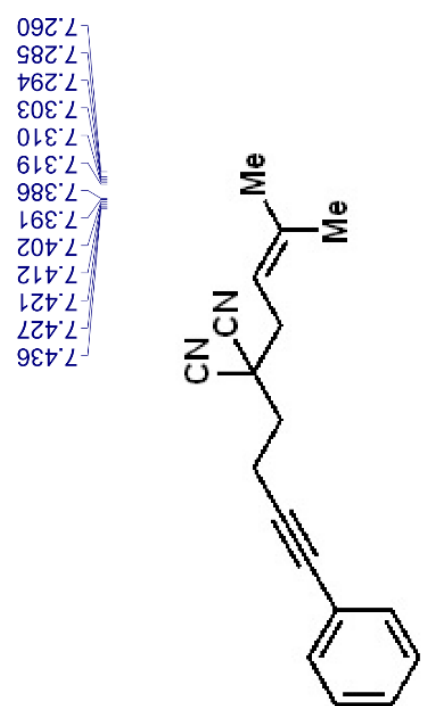

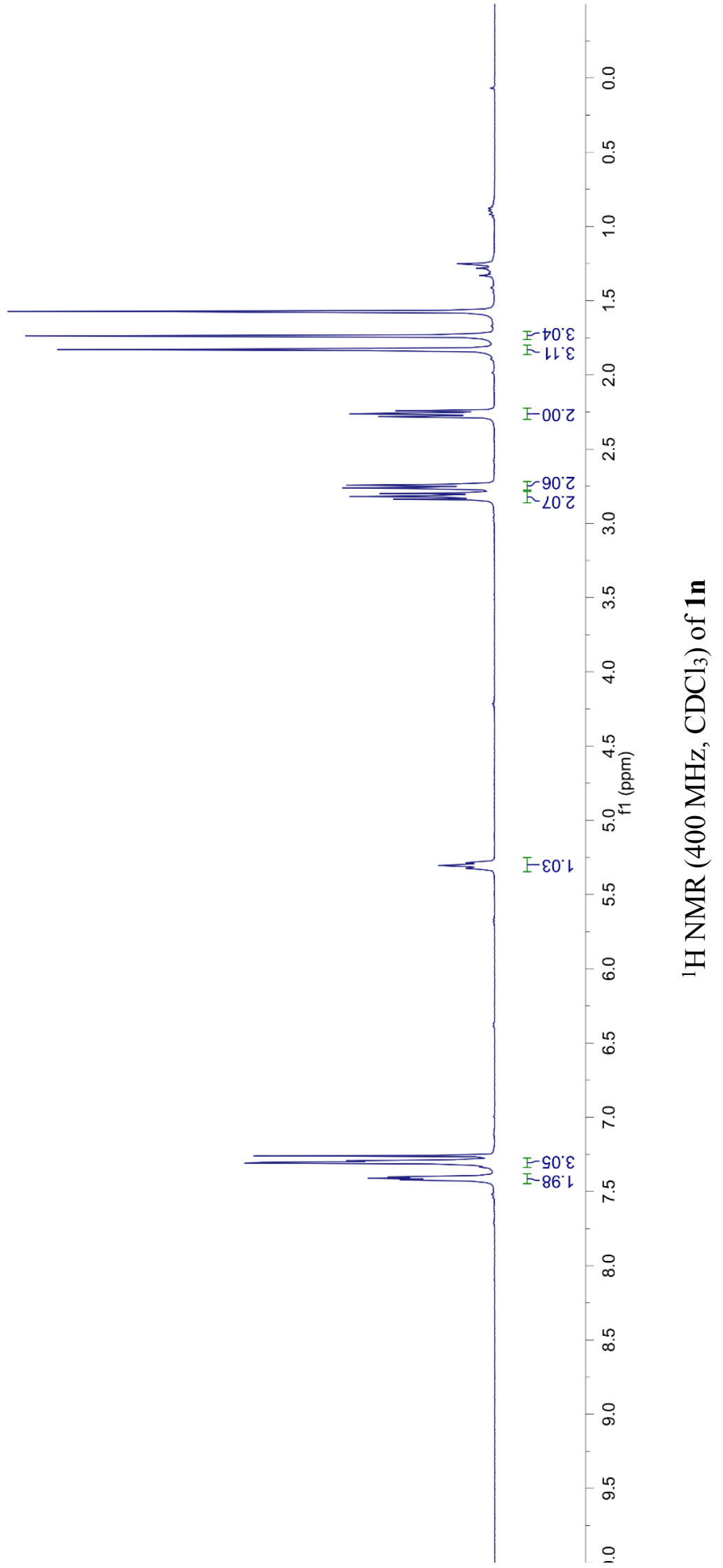



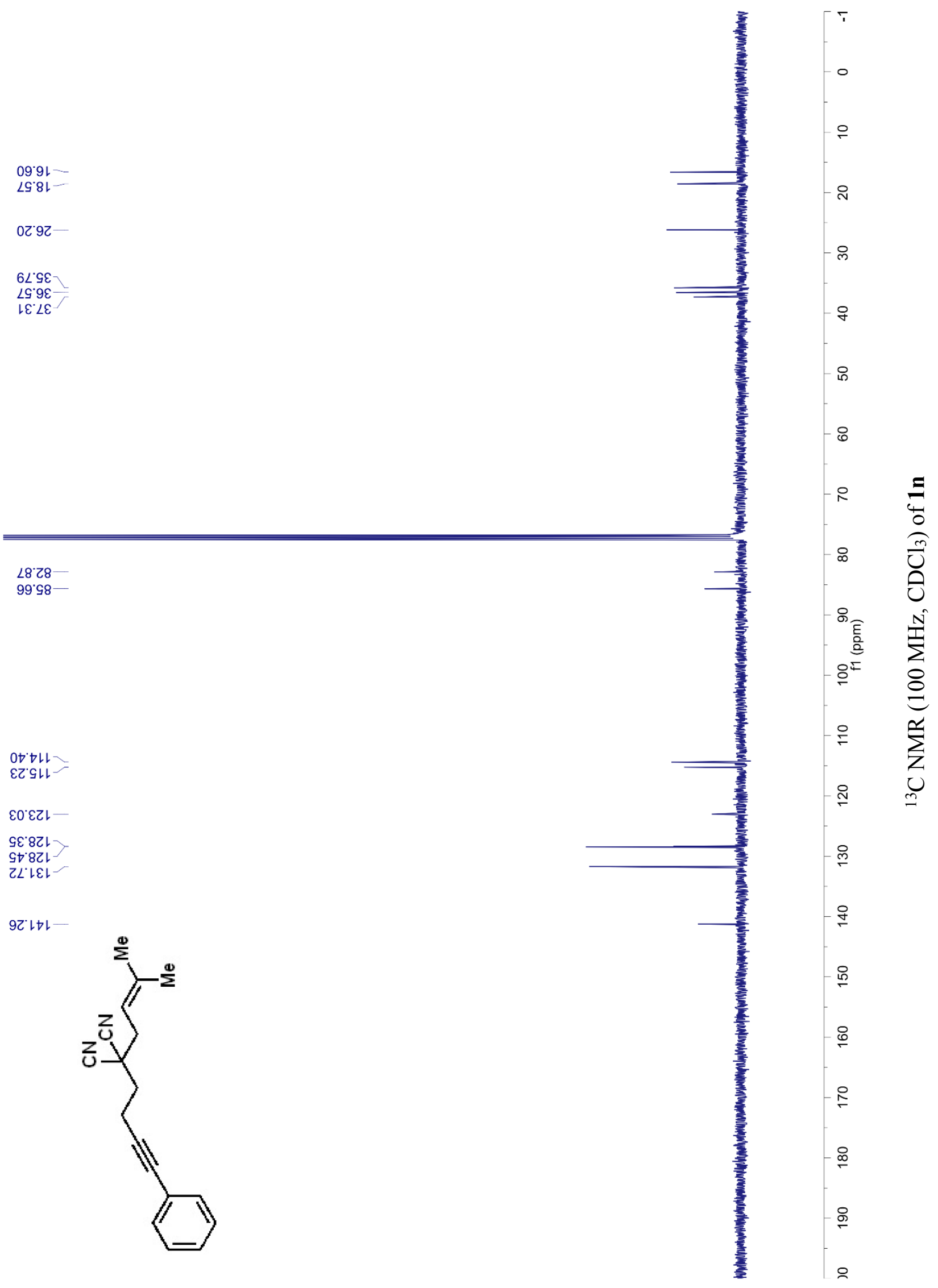


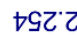

ย6乙'乙

乙9L乙

0827

แL乙

828'

ZE8'乙

098'

ELV'G

9เt's

6เt'

乙Z†

ZSt

sGt'

8St'

197' 9

s9t'

SLV'G

$8 \angle \nabla^{\circ} G=$

$08 \nabla^{\circ}$

LL8'G

$688^{\circ} \mathrm{G}$

968' 9

$\angle 06$ 'G

ㄴ.6'G

เE6. 9

$8 \varepsilon 6.9$

676.9

996.9

SL6' 9

S8Z L

$\angle 8 C^{\prime} L$

$06 Z^{\prime} L$

G6Z' L

$86 \mathrm{C}^{\circ} \mathrm{L}$

$00 \varepsilon^{\circ} L$

$90 \varepsilon^{\circ} L$

ZIE'L

$\angle L E^{\circ} L$

IZE'L

$\neg 6 \varepsilon^{\circ} L$

$90 t^{\circ} L$

$60 \nabla^{\circ} L$

ZLtL

SIt $L$

8Lt'L

†乙ち L

OEt $L$

$6 \varepsilon \nabla^{\circ} L$
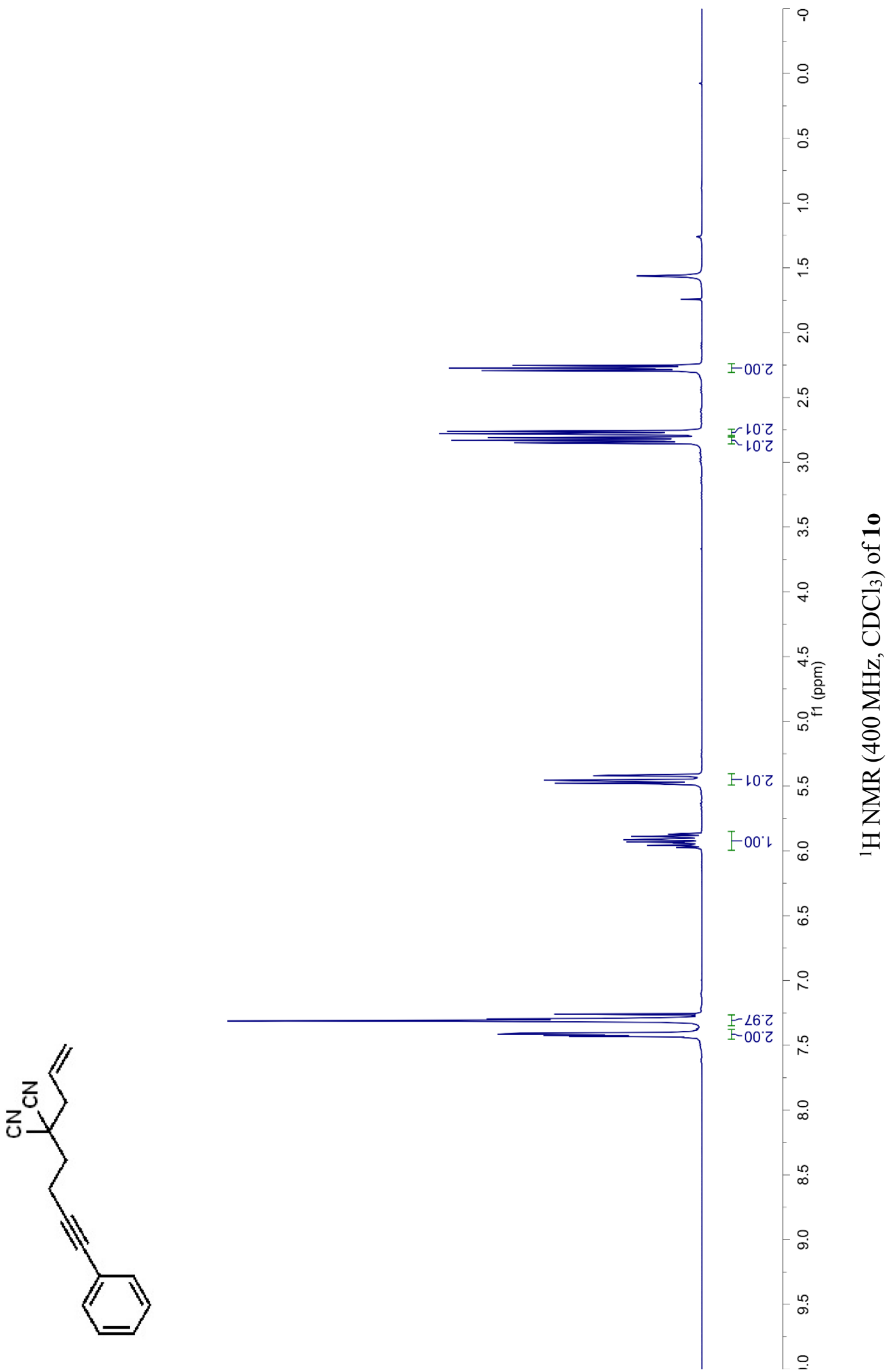


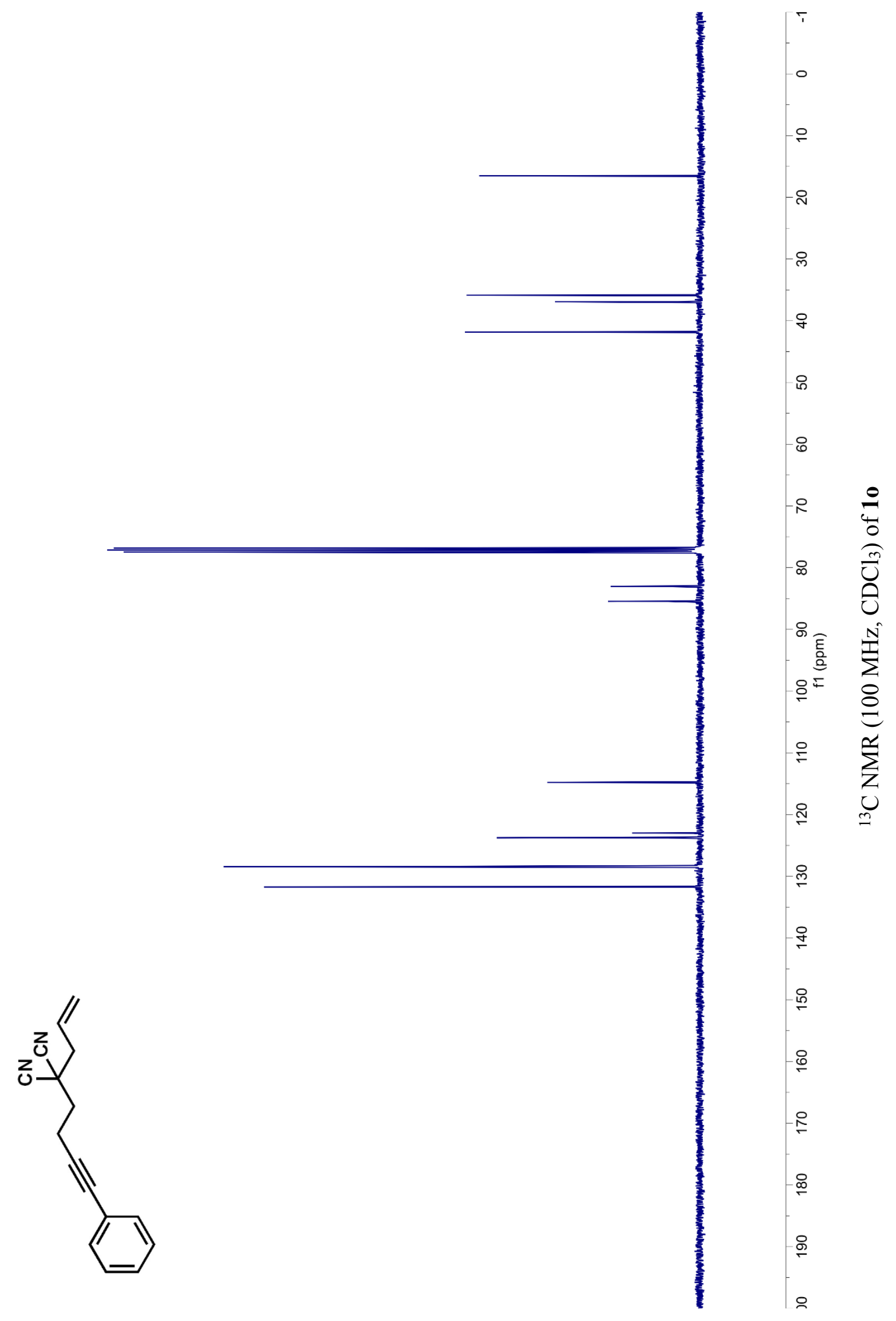

LLth

66'ZZL

9LEZL-

GE' $8 Z \mathrm{~L}$

SE'8ZL-

ZL $L E L$ 
$788^{\circ} 乙$

$688^{\circ} 乙$

乙68'乙

Z06 2

$806^{\circ} \mathrm{Z}$
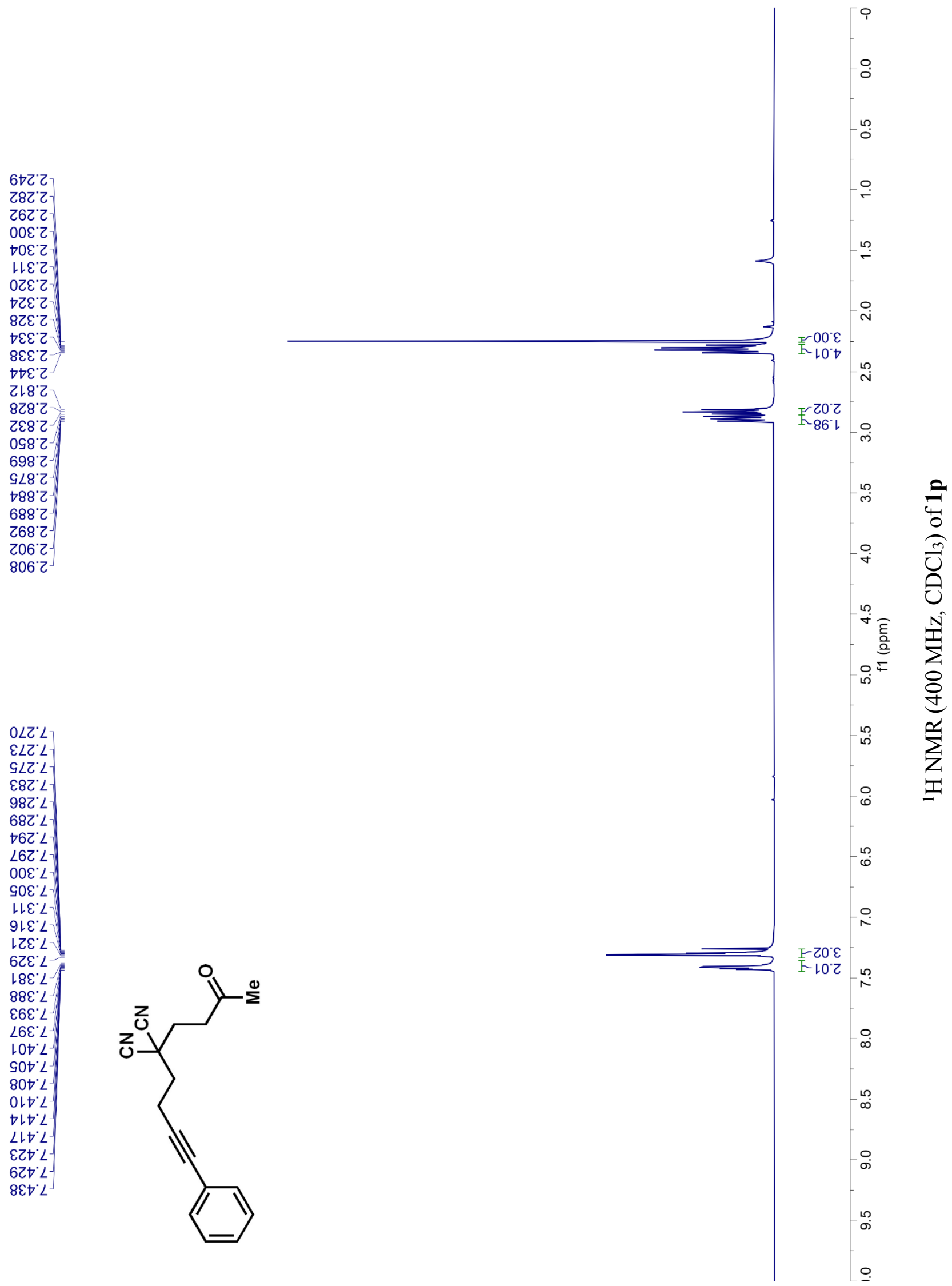
9G.91-

$9 L^{\circ} 0 \varepsilon$

18.18

$8 \varepsilon^{\prime} 9 \varepsilon$

$69^{\circ} 9 \varepsilon$

เE $6 \varepsilon$

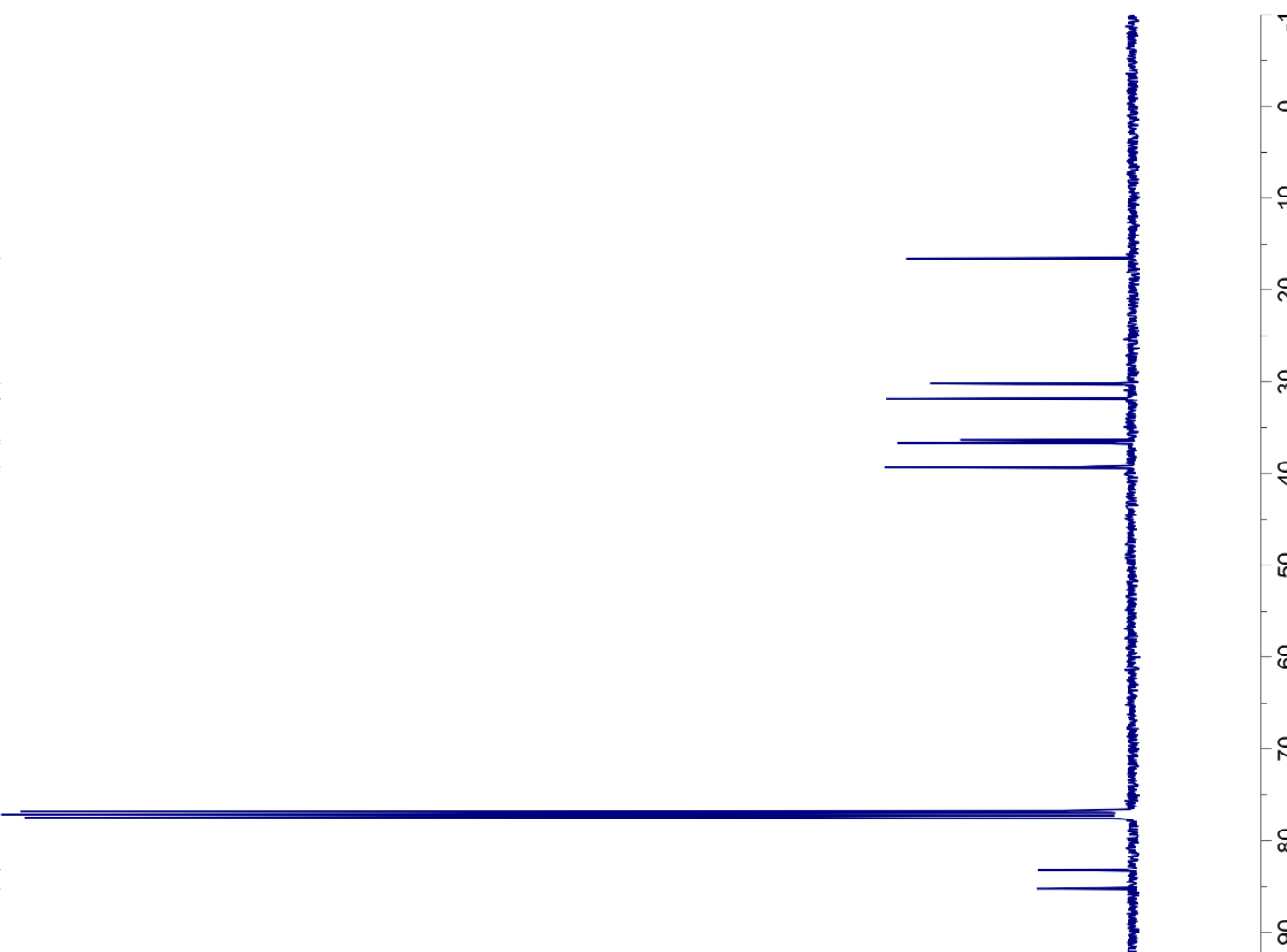

8L゙ทルー

26.221

เท'8ZL

9t'8ZL

ZL'LEL-
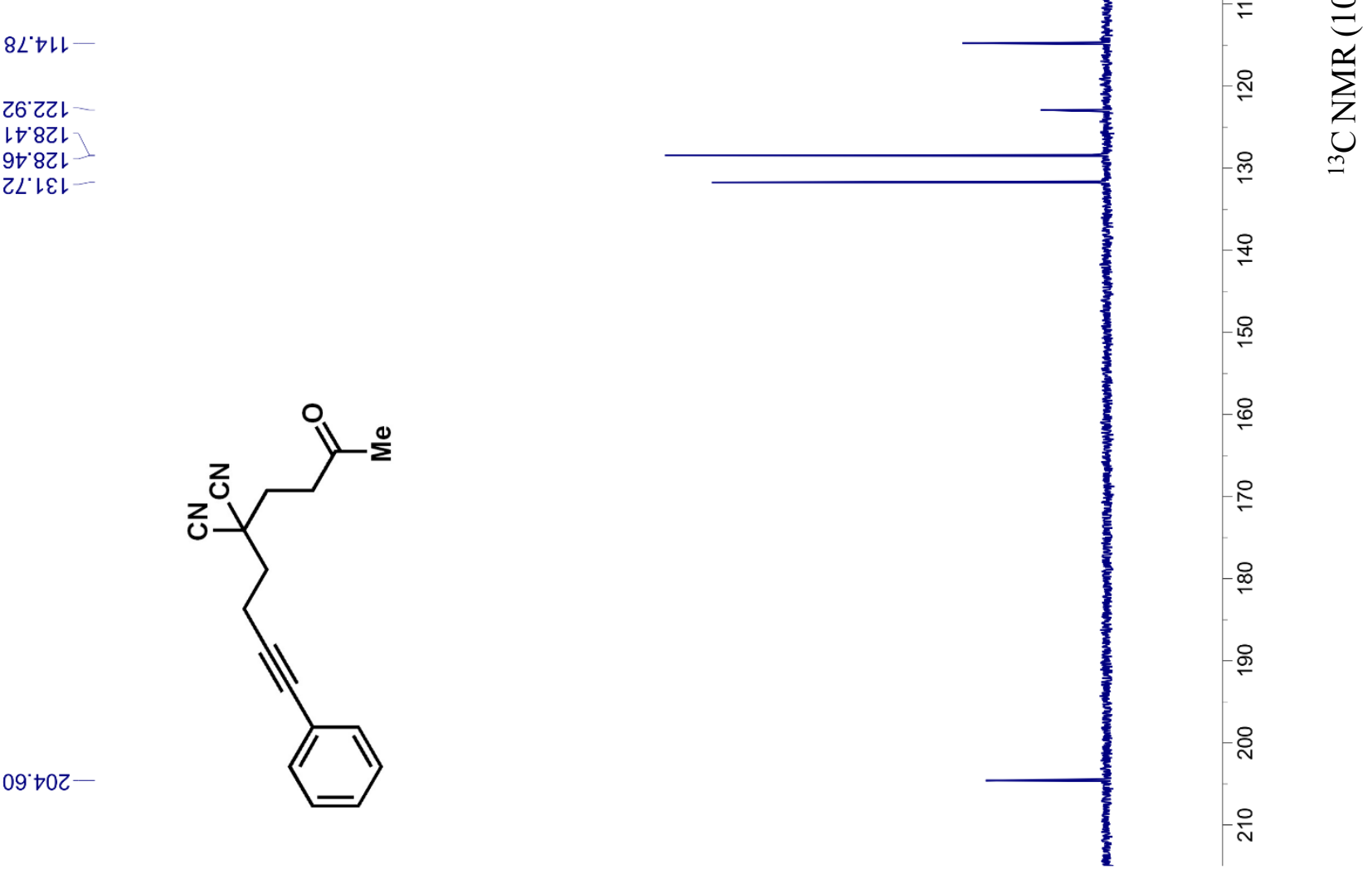
$180^{\circ} \mathrm{L}$

060"

난

8レ゙レ

しレレ

Zヤト

OSL

09L

เ6L'

ㄴ. $\varepsilon^{\circ}$

8เE"เ

9乙E' ᄂ

เฤย'เ

OSE'เ

$89 \varepsilon^{\prime \prime} \mathrm{L}$

EL9'

GOL"L

$\varepsilon \varepsilon L\llcorner$

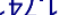

OSL L

GLL

09L'

G9L'L

8921

SLL'L

$\rightarrow 8 L^{\circ} L$

E6L

E88. ᄂ

$668^{\circ}$

216

ZZ6.

$926^{\circ} \mathrm{L}$

8Z6"

乙E6.

ตE6.

$8 \varepsilon 6^{\circ}$

$2 \downarrow 6^{\circ}$

$896^{\circ} \mathrm{L}$

$696^{\circ}$

$\varepsilon 96^{\circ} \mathrm{L}$

$\angle 96^{\circ}$ '

ZL6.

9G乙 乙

G9乙 乙

ELZ"Z

9L乙"乙

6LZ"Z

$\angle 8 Z^{\circ} 乙$

$96 \mathrm{C}^{\circ} \textrm{ }$

†08 2

0Z8'乙

†२८

LZ8'Z

$\checkmark \nabla 8^{\circ}$ '

G82' L

$88 Z^{\circ} L$

E6乙"L

96乙" L

$66 Z^{\circ} \mathrm{L}$

†० $\mathcal{E}^{\circ} \mathrm{L}$

OLE'L

GLE"L

$0 Z E^{\circ} L$

$96 \varepsilon^{\circ} L$

$66 \varepsilon^{\circ} /$

EOt $L$

SOt" $L$

Zレナ゙

8เだ

†乙ヤ'

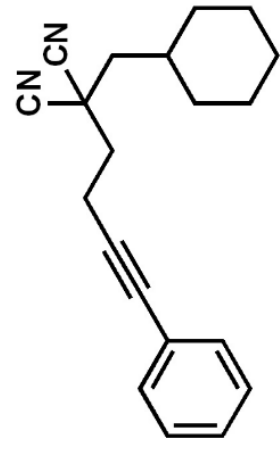

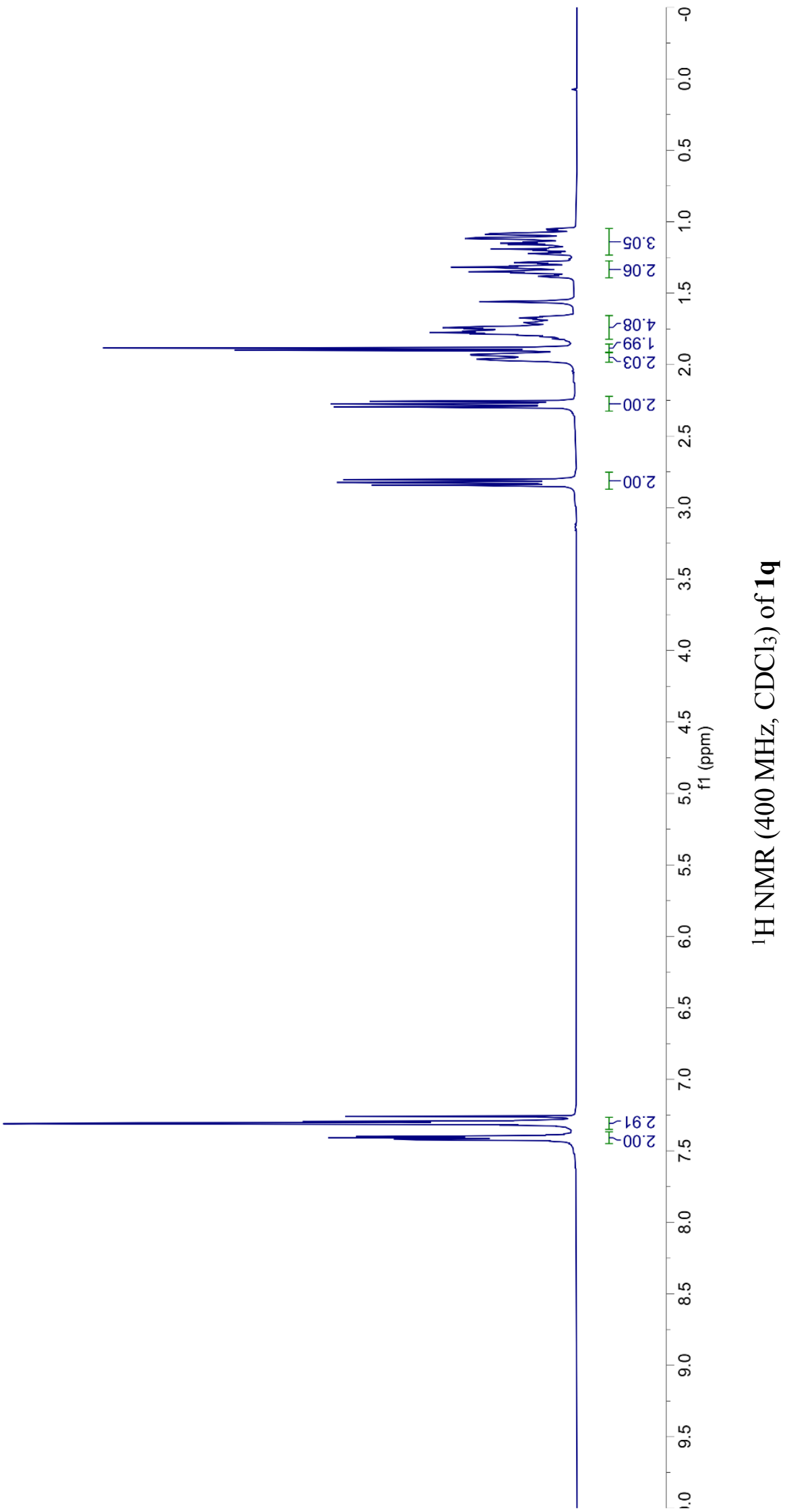


tเ 9 -

${ }^{\circ} \mathrm{G} Z-$

$0 \mathcal{G}^{\circ} \varepsilon \varepsilon-$

$\downarrow \varepsilon \cdot G \varepsilon$

$89^{\circ} \mathrm{G} \varepsilon$

$\angle 6^{\circ} \angle \varepsilon^{-}$

$\varepsilon 8^{\circ} \downarrow \downarrow$

†8 28

$\angle 9^{\circ} 98$

GG'SIL-

90とてレ-

$\neg \varepsilon^{\prime} 8 Z \mathrm{~L}$

†七 8ZL

LLLEL -

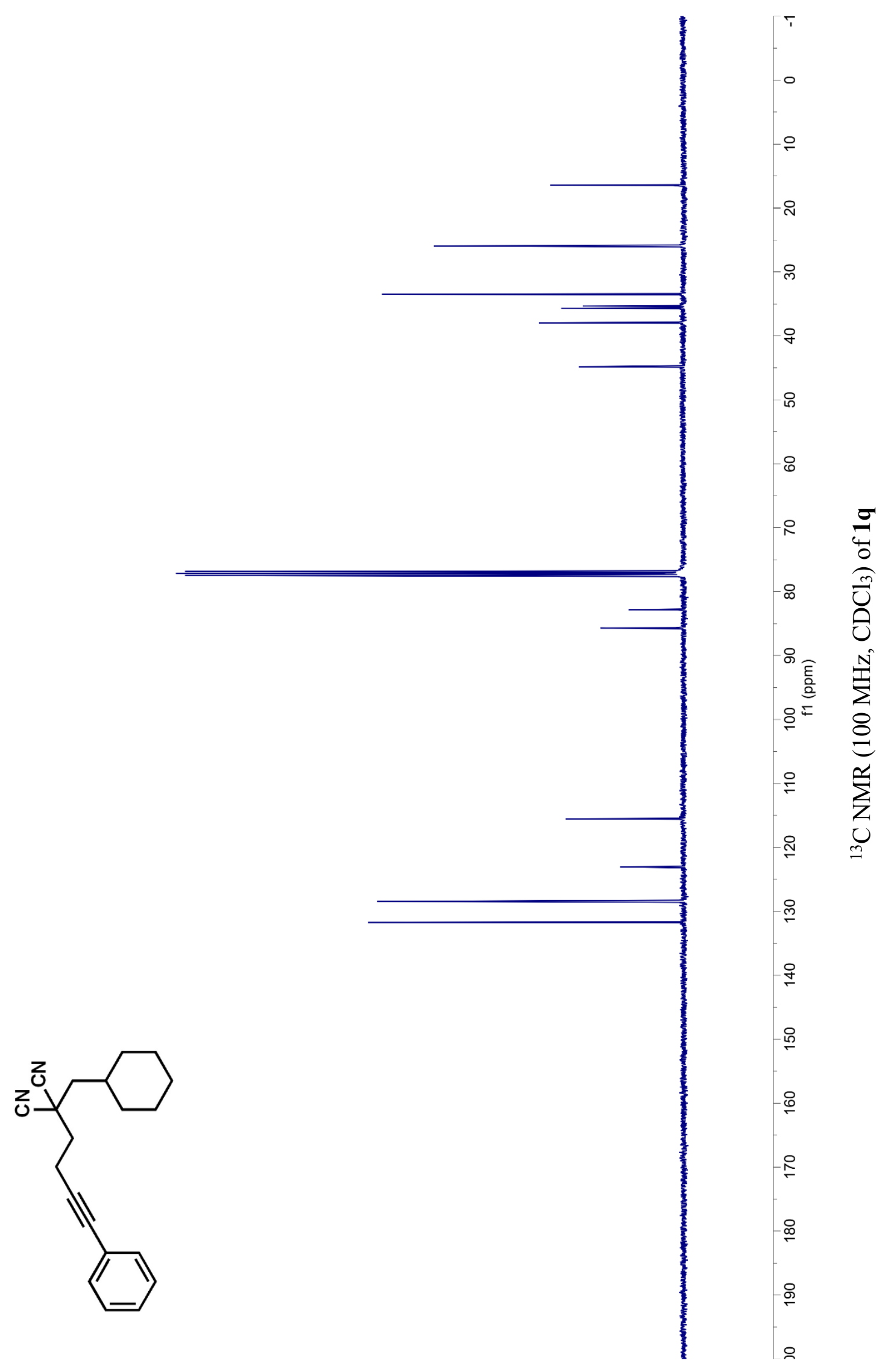

S161 

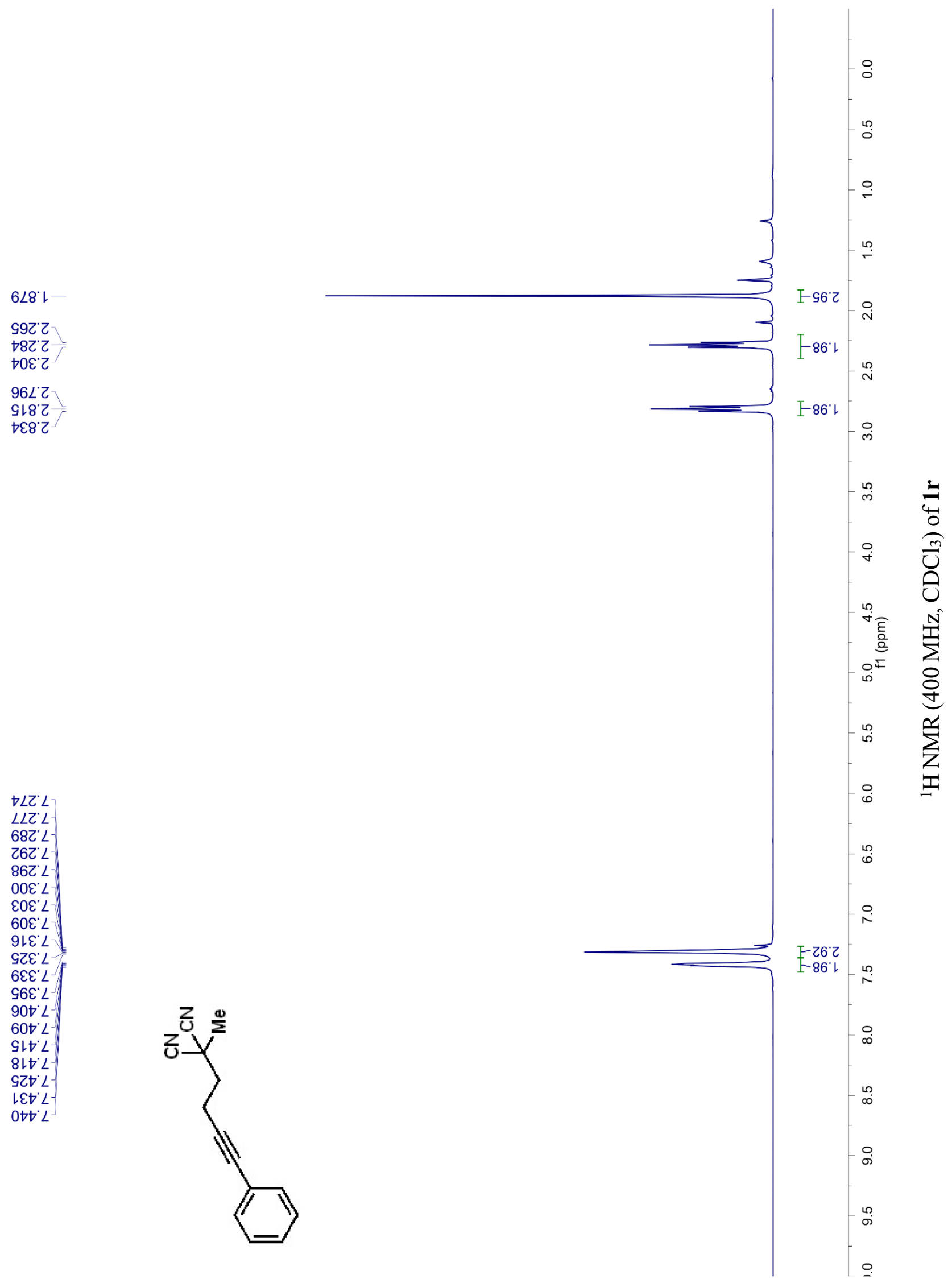
ZG'91

$20^{\circ} \mathrm{Z}$

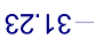

$\varepsilon \varepsilon^{\circ}\llcorner\mathcal{E}$

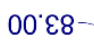

$\angle E^{\circ} 98-$

OL'GL

E6'ZZL-

$9 \varepsilon^{\circ} 8 Z$ -

ह7.8Z -

$\angle 9^{\circ} L E L-$

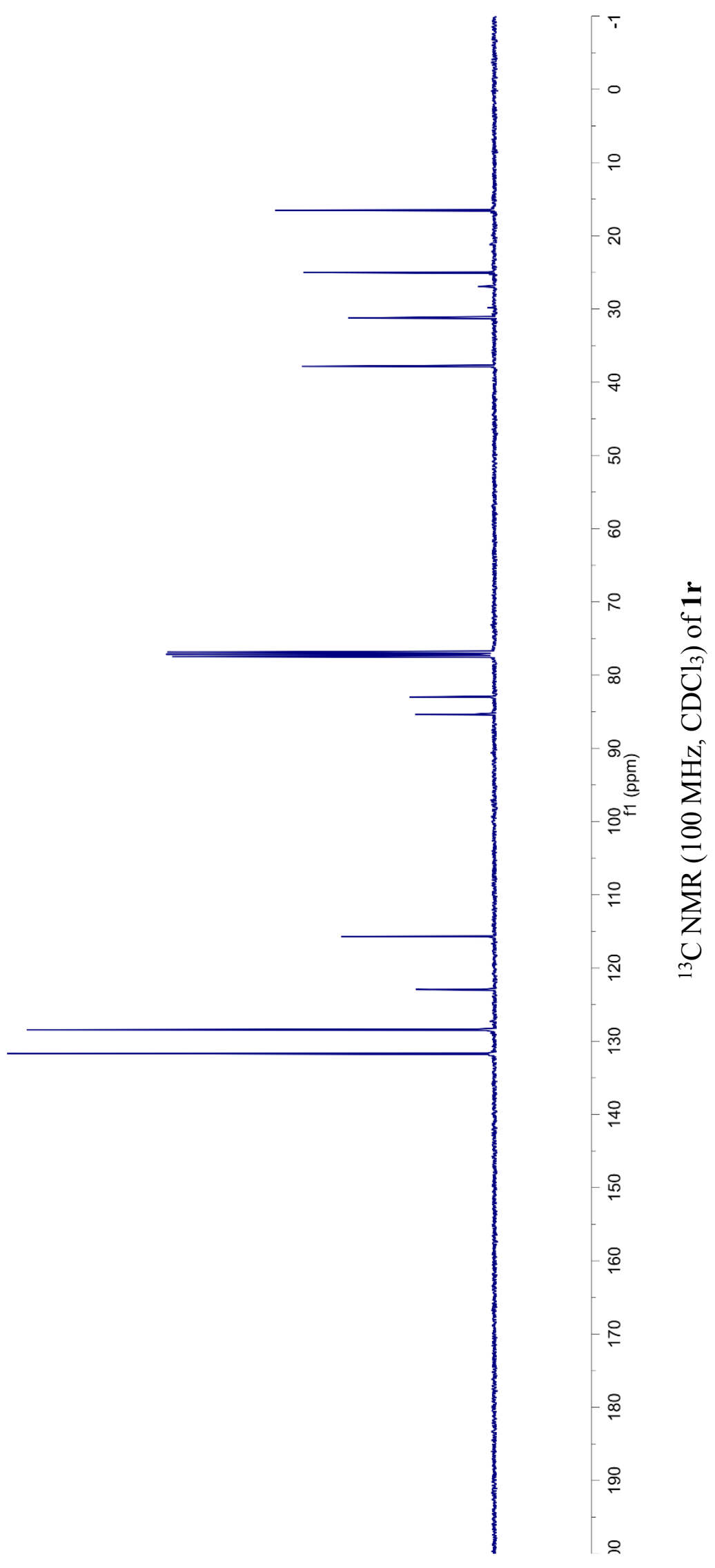

S163 
69G'乙

699 乙

$\angle L 9^{\circ} \mathrm{Z}$

† $9^{\circ} \mathrm{Z}$

เ69'乙

869'乙

OLL'

$\angle L L Z$

$\checkmark \mathrm{CL}$

LEL $Z$

$6 \nabla L ' 乙$
ZLZ L

Z8С ' L

$98 Z^{\circ} \mathrm{L}$

$\nabla 6 Z^{\circ} \mathrm{L}$

00Е'L

$80 \varepsilon^{\circ} \mathrm{L}$

$89 \varepsilon^{\circ} L$

$\angle L \varepsilon^{\circ} L$

$98 \varepsilon^{*} L$

เ $6 \varepsilon^{\circ} L$

$\nabla 6 \varepsilon^{\circ} L$

G9t $L$

$697^{\circ} \mathrm{L}$

$\nabla 8 \nabla^{\circ} L$

$16 \nabla^{\circ} L$

$96 \nabla^{\circ} L$

LOS. $L$

$\rightarrow 0 G^{\circ} L$

8IS'L

EZS $L$

EES $L$

$6 E G^{\circ} L$

GtS'L

169'L

$86 \mathrm{~S}^{\circ} \mathrm{L}$

$209^{\circ} \mathrm{L}$

$909^{\circ} \mathrm{L}$

EL ${ }^{\circ} L$

$819^{\circ} \mathrm{L}$

EZ9. $\mathrm{L}$

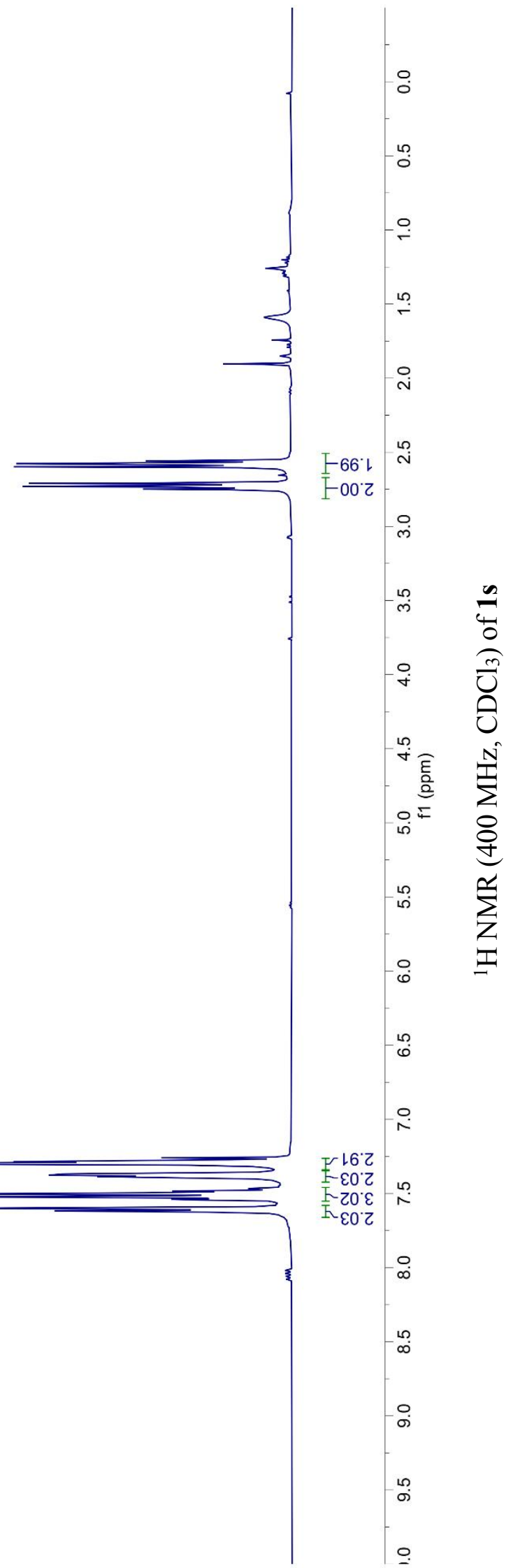


เง'9เ

sL:L

$09^{\circ} L t$

$86^{\circ} \mathrm{Z8}$

ย乙"ต8-

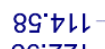

G6 ZZL

ह6" GZL

$\varepsilon \varepsilon^{\prime} 8 Z L$

乙† 8ZL

$000^{\circ} 0 \varepsilon$

乙E' $0 \varepsilon \downarrow$

$9 \nabla^{\circ}\llcorner\varepsilon L$

$89^{\circ} L E$ -

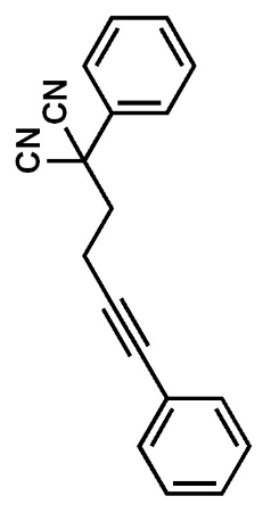

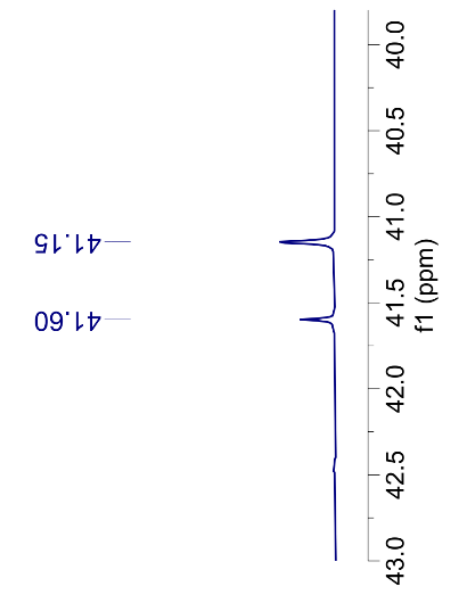

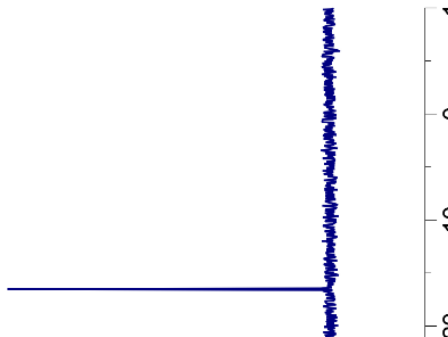

요

이

우

요

$-8$

오

อับ

흘 Nㅗㄹ

든

읃

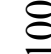

ำ 는

옴

암

욤

$\stackrel{8}{-}$

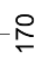

$\stackrel{\circ}{\stackrel{\infty}{\infty}}$

$\stackrel{8}{9}$

89.ยน-

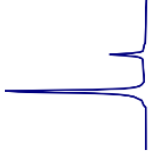

임

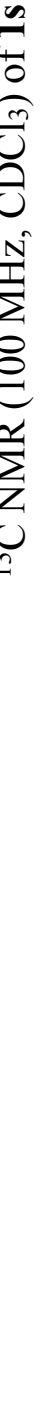


oเน' $\varepsilon$

8เเ๋

เยะ 2

ธย์ $L$

$6 \varepsilon \varepsilon^{\circ} L$

$\varepsilon \sqcup \varepsilon^{\circ} L$

$\angle \nabla \varepsilon^{\circ} L$

$\varepsilon \subseteq \varepsilon^{\circ} L$

$\downarrow S \varepsilon^{\circ} L$

$89 \varepsilon^{\circ} L$

$Z 9 \varepsilon^{\circ} L$

$\angle 9 \varepsilon^{\circ} \angle$

ZLE'L

$8 \angle \varepsilon^{\circ} L$

$08 \varepsilon^{\circ} L$

$\angle 8 \varepsilon^{\circ} \angle$

$06 \varepsilon^{\circ} L$

EOt' $L$

$\angle O D^{\circ} L$

OLt'L

$\angle L{ }^{\circ} L$

IZt 2

GZt ${ }^{\circ} L$

GEt ${ }^{\circ} L$

$0+t^{\circ} L$

6tt 2

ESt L

EOS $L$

$809^{\circ} L$

ELS.L

LIG'L

6 LG $^{\circ} \mathrm{L}$

ZZS $L$

$\angle Z G^{\circ} L$

EES $L$

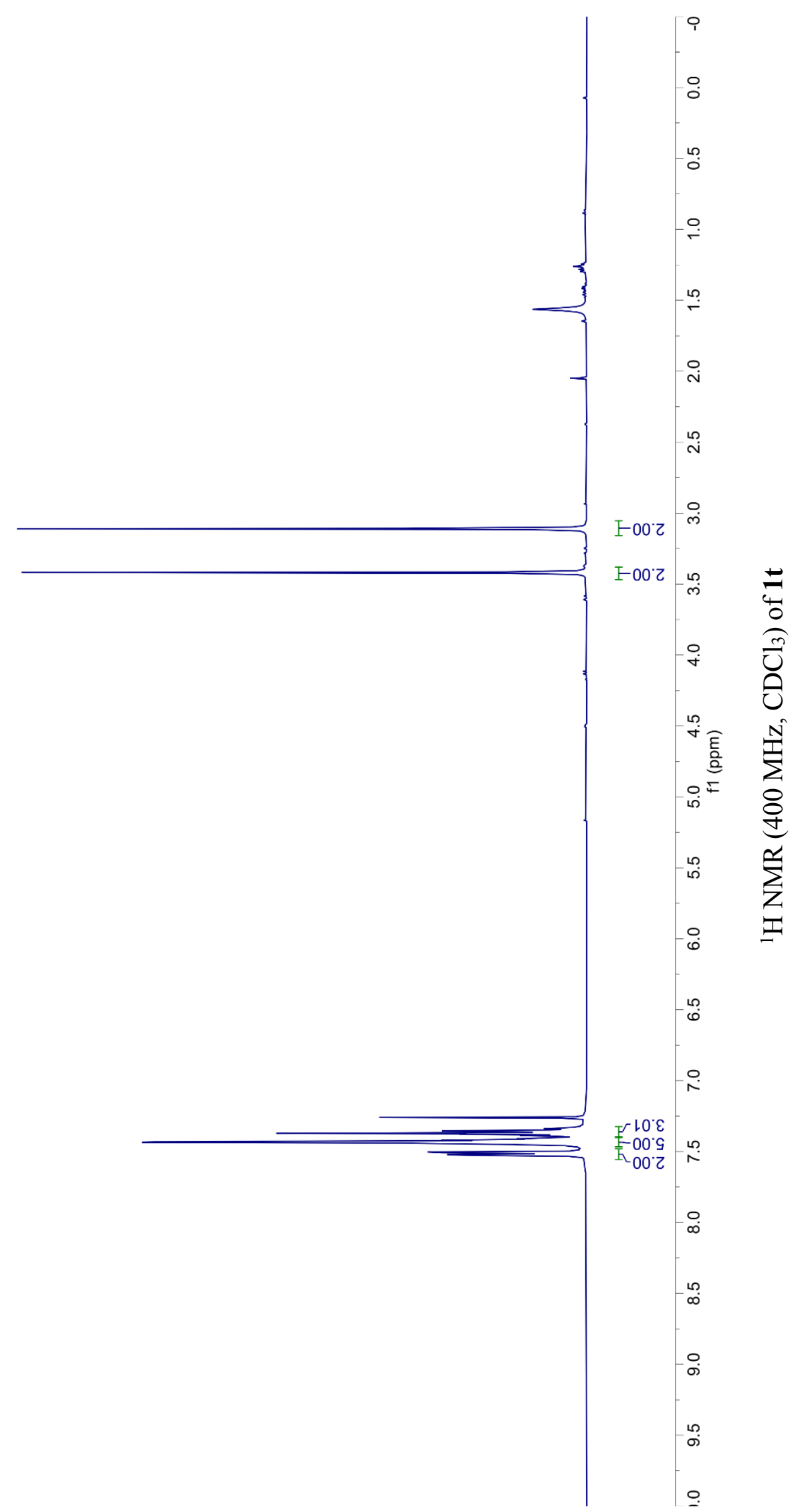


$89.82-$

$6 \varepsilon^{\circ} 8 \varepsilon$

98.レ

ง9๋ルー

เ8เてL

$09^{\circ} 8 \mathrm{LL}$

$91^{\circ} 6 \mathrm{ZL}$

GZ6ZL

$9 t^{\circ} 0 \varepsilon L$

$6 \rightarrow เ \varepsilon L$

Oเ'ZEL

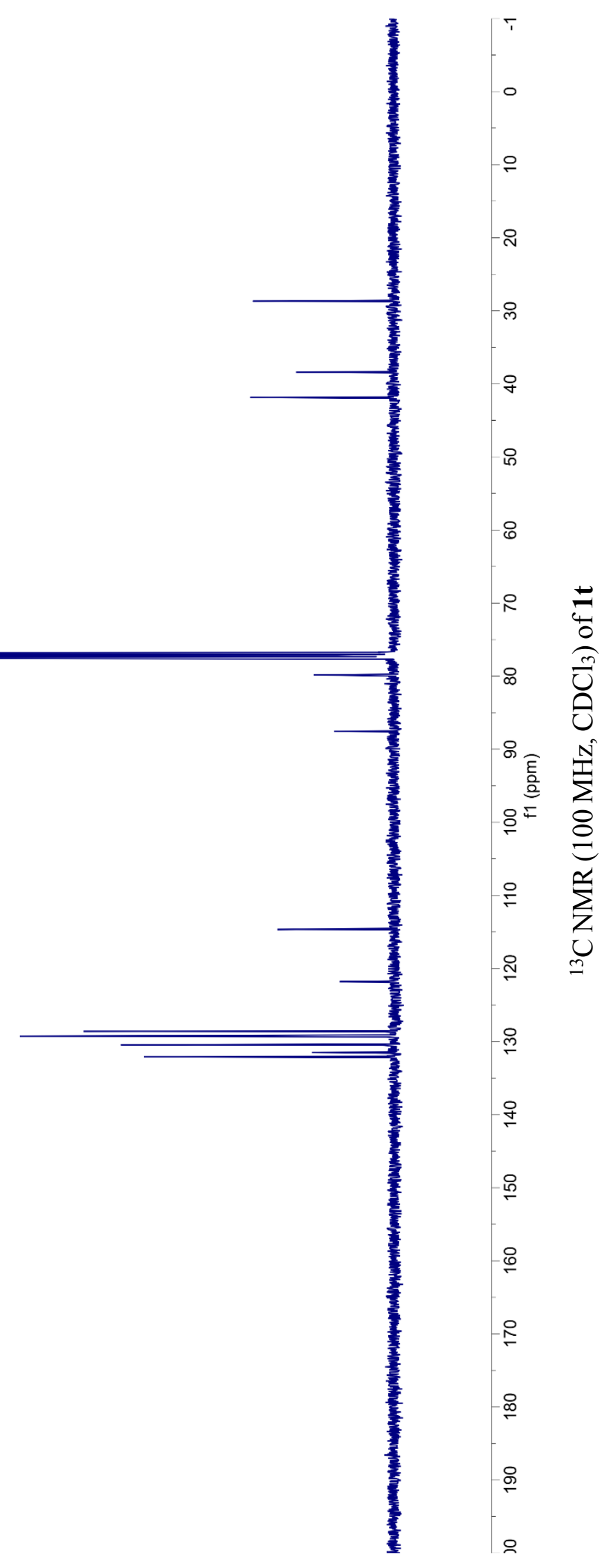



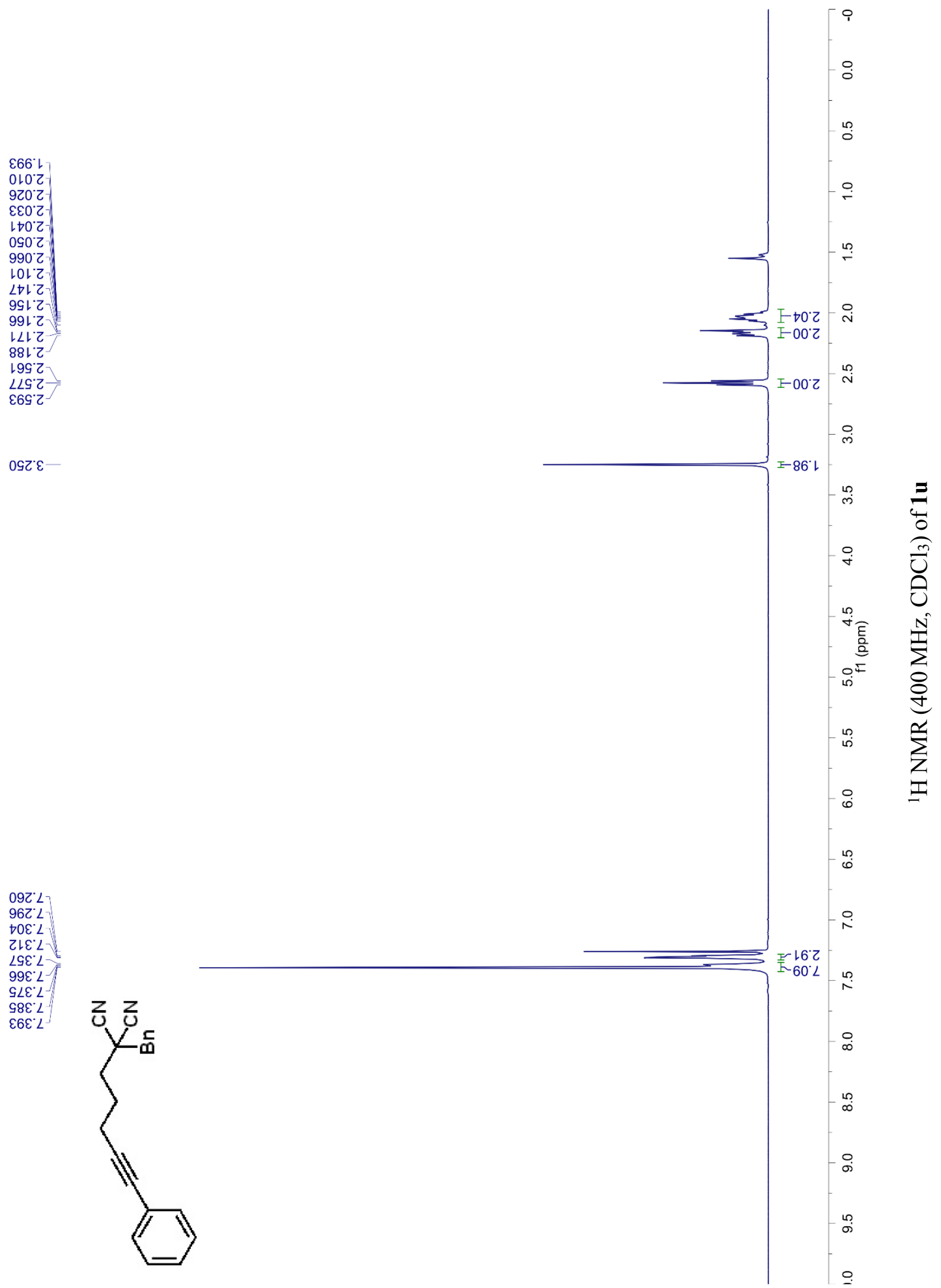


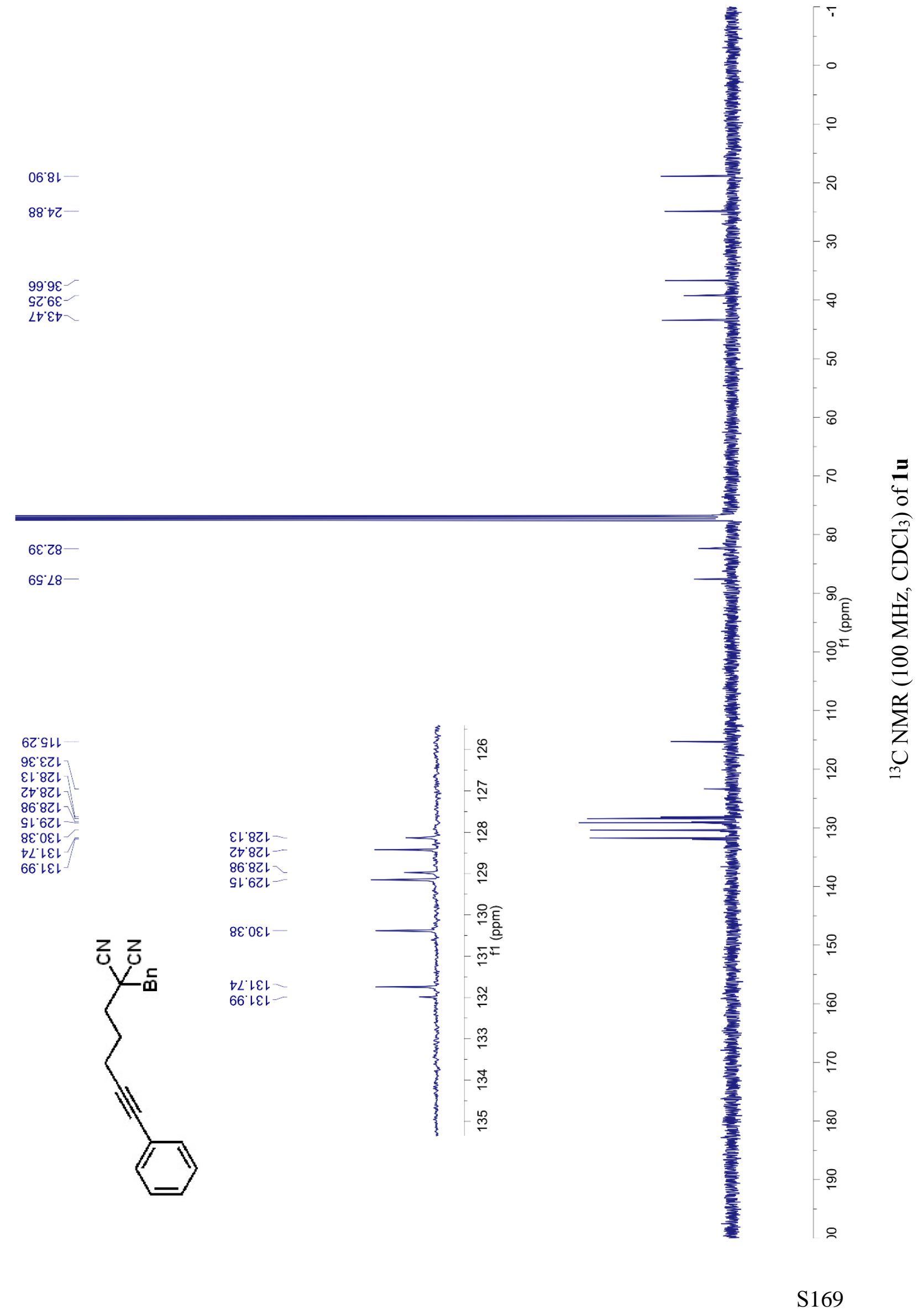



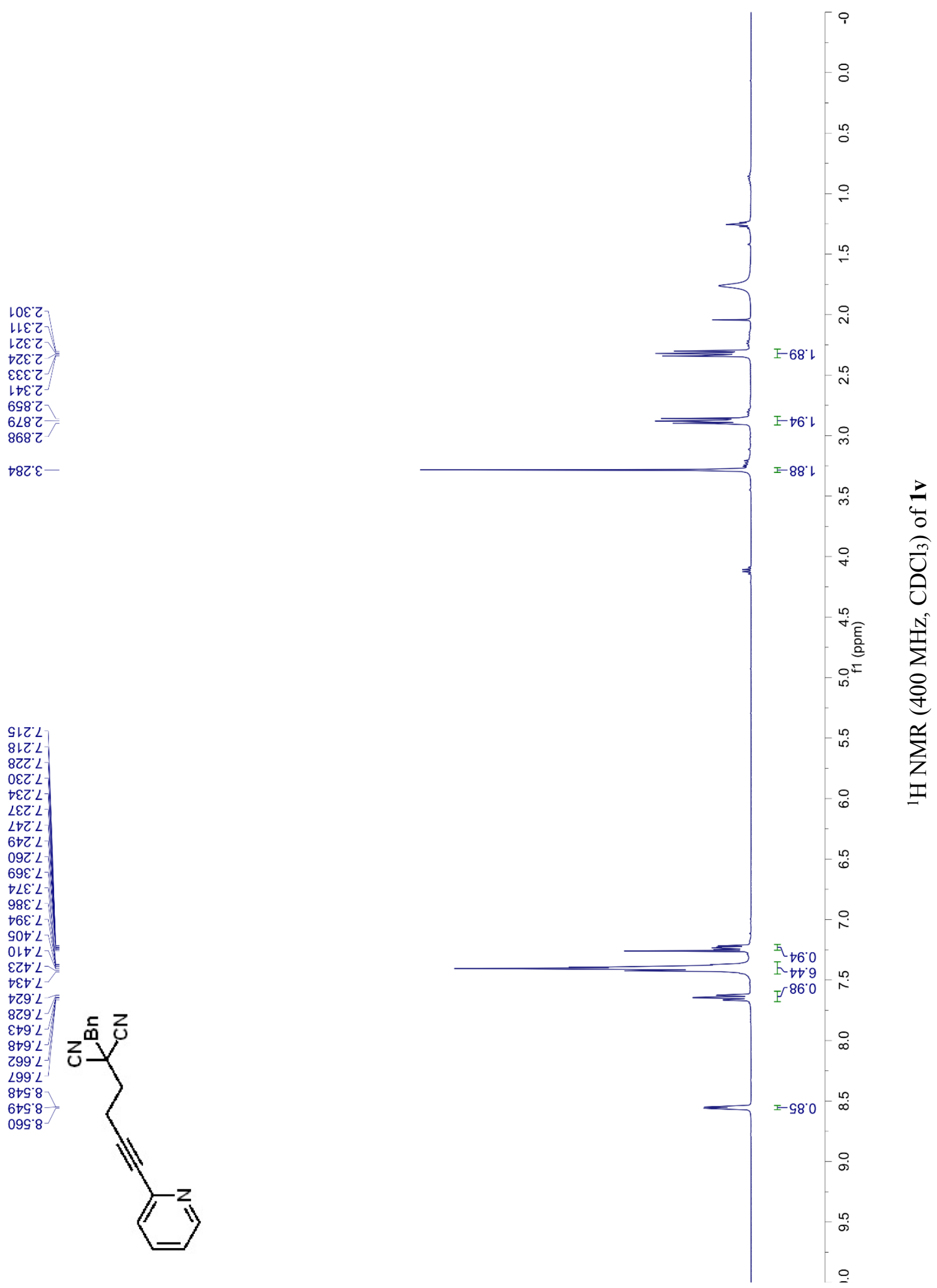


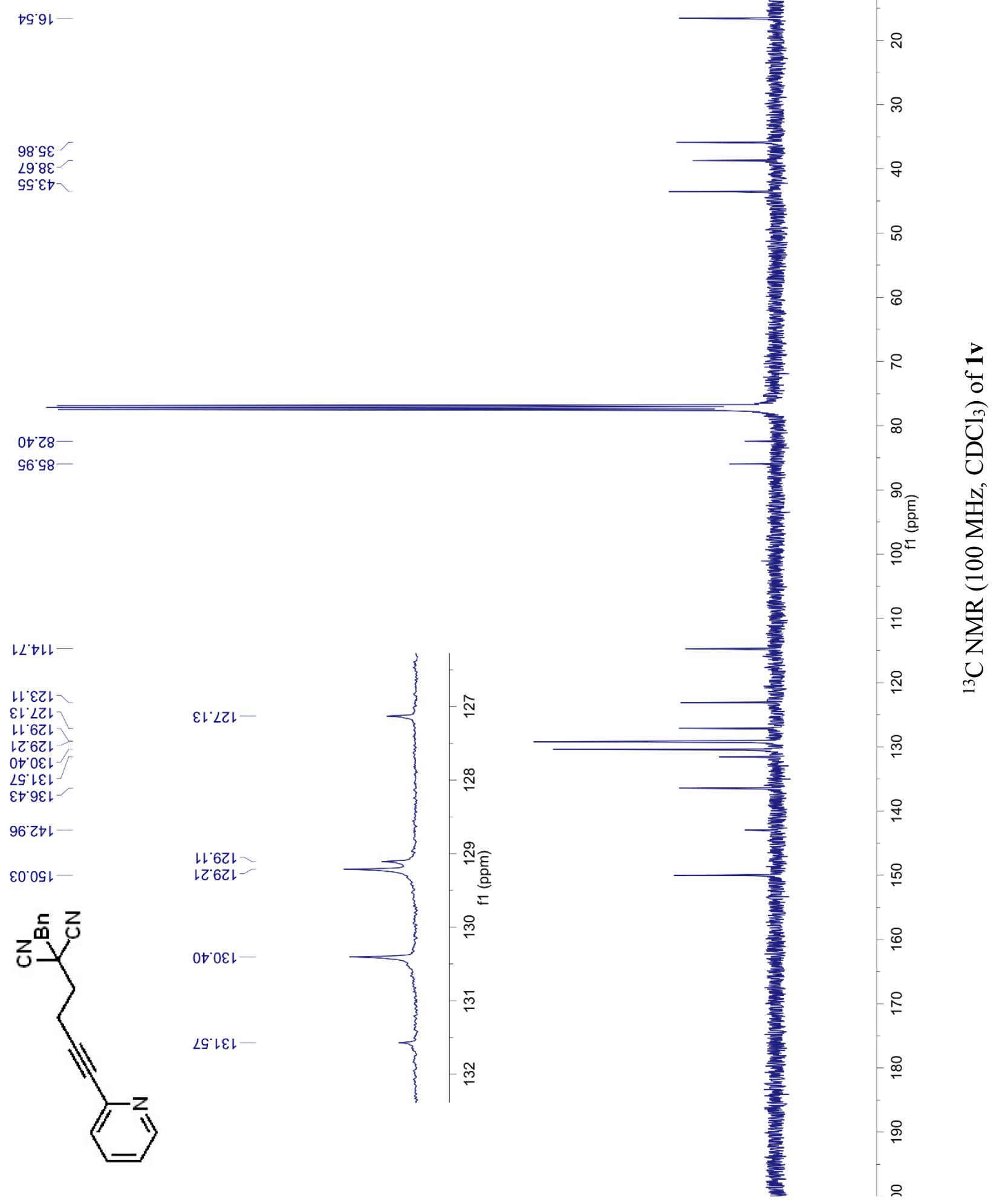



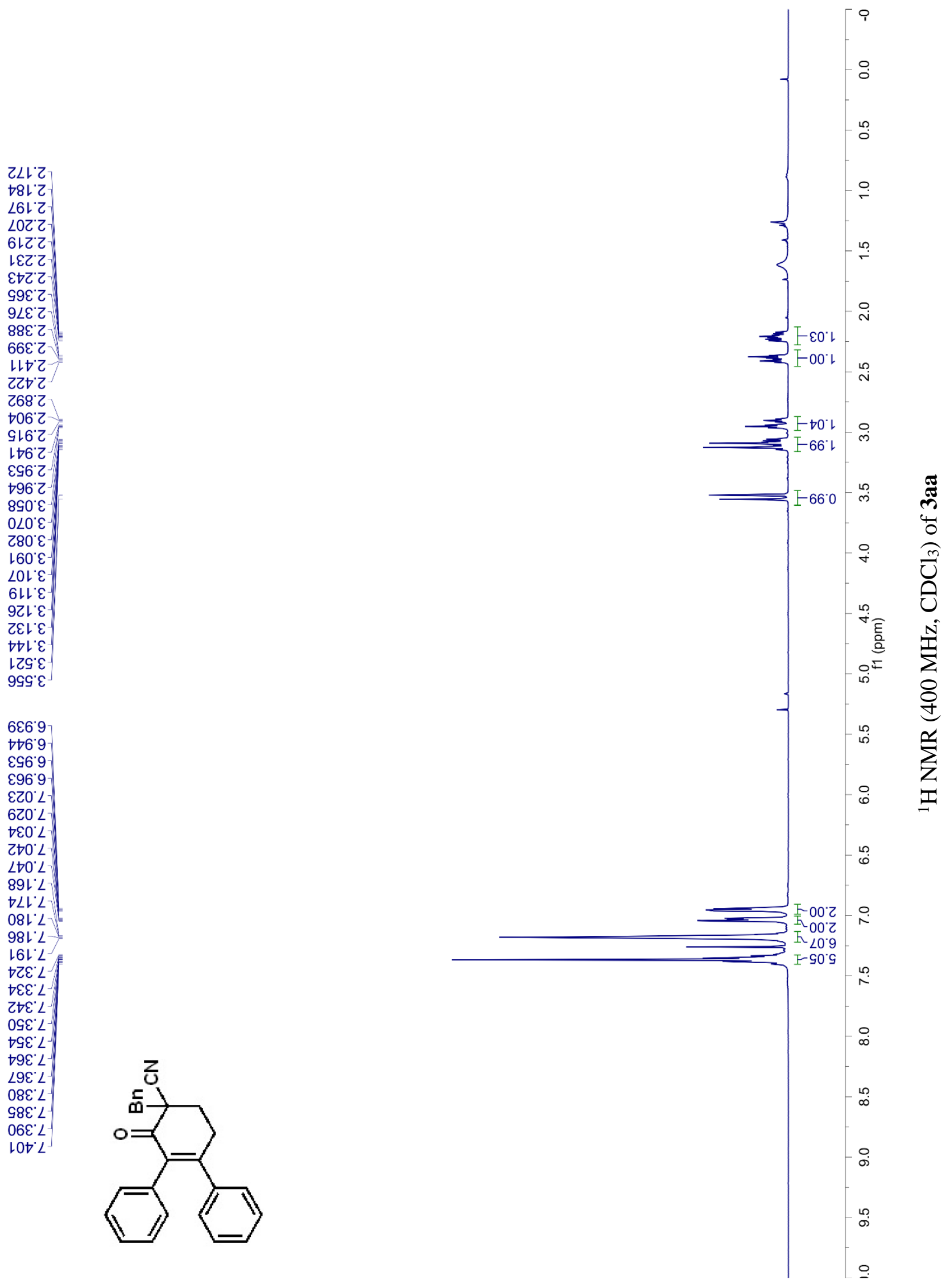
s9. $6 \varepsilon-$

$s 9^{\circ} \angle t$

ZL'8H

$8 D^{\circ} \angle Z L$

$6 L \angle Z L$

$\checkmark 6 \angle Z L$

0Z 8ZL

七乙 $8 Z \mathrm{~L}$

$\angle 9)^{\circ} 8 \mathrm{~L}$

$\angle L ' 8 Z L$

s9.0\&

เ6.0\&

LG' $\downarrow \varepsilon L$

$18^{\circ} \downarrow \varepsilon L$

9L'GEL-

$\varepsilon 9^{\circ} 6 \varepsilon \mathrm{L}$

$87^{\circ} 8 \mathrm{~L}$
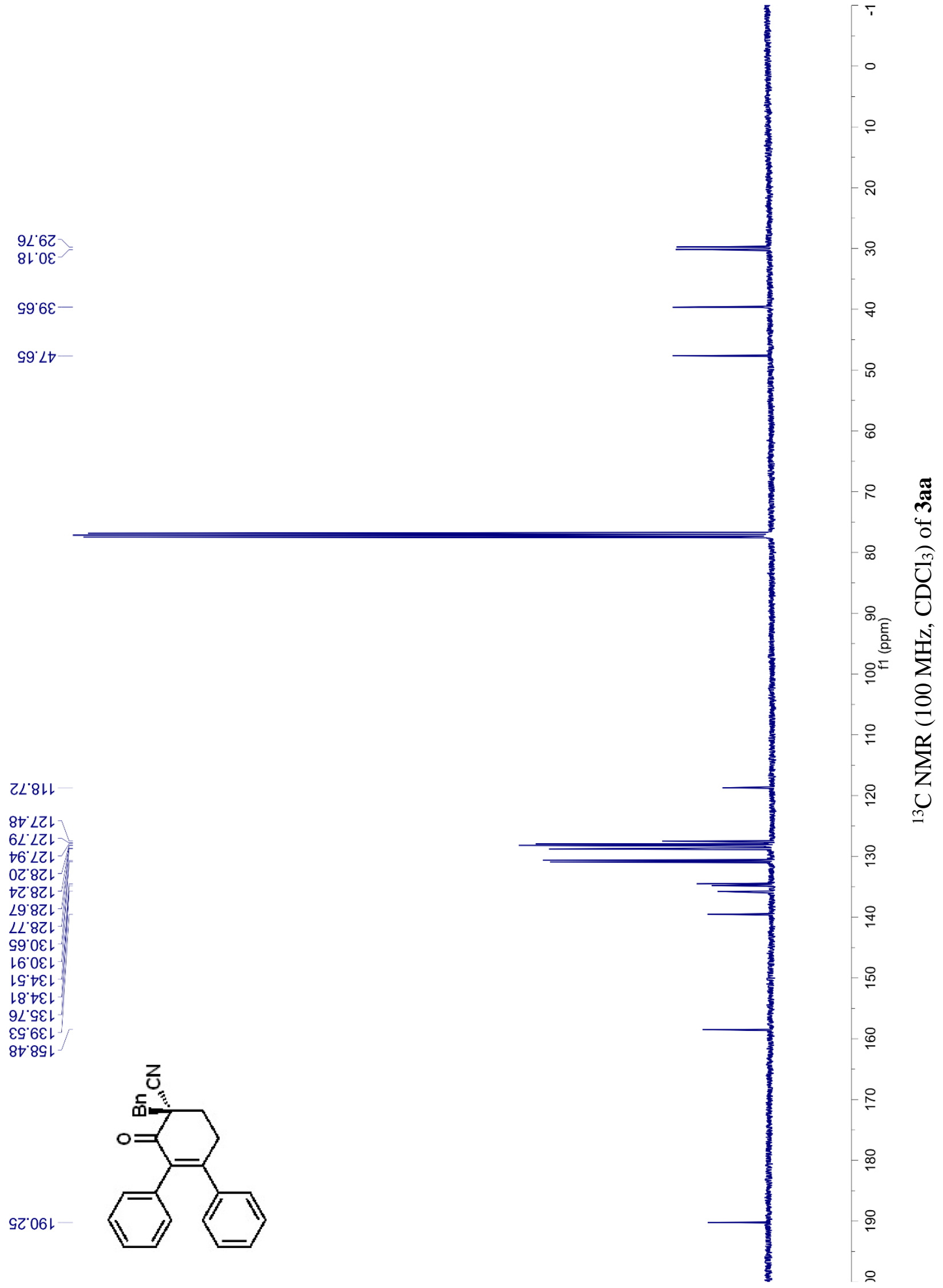
9Gレ'

89l' 2

08L乙

L6L'z

乙0乙 乙

†レ乙

9乙乙乙

89乙'乙

I $9 \varepsilon^{\prime} 乙$

乙9ध'乙

$\neg L \varepsilon^{\prime} Z$

G8E' $\mathrm{Z}$

$96 \varepsilon^{\prime} 乙$

80t'

$888^{\prime} \mathrm{Z}$

$668^{\circ} Z$

レ6 乙

$9 \varepsilon 6^{\circ} 乙$

$8 \nabla 6{ }^{\circ} 2$

$096{ }^{\circ} 乙$

$8 \varepsilon 0^{\circ} \varepsilon$

$090^{\circ} \varepsilon$

乙90. $\varepsilon$

$\downarrow \angle 0^{\circ} \varepsilon$

G80' $\varepsilon$

$660^{\circ} \varepsilon$

แルE

OZL $\varepsilon$

9เG'

ISG' $\mathcal{E}$

126.9

เ 6.9

\&G6. 9

896.9

$\angle 966^{\circ} 9$

$\angle L 66^{\circ} 9$

$886^{\circ} 9$

$\mathrm{Z8L} \mathrm{L}$

$16 \mathrm{~L}^{\circ} \mathrm{L}$

$\angle 6 L '-$

GOZ' L

$60 Z^{\circ} \mathrm{L}$

$6 \mathrm{LC} L$

$8 \forall \varepsilon^{\circ} L$

乙GE'L

G9E'L

$08 \varepsilon^{\circ} L$
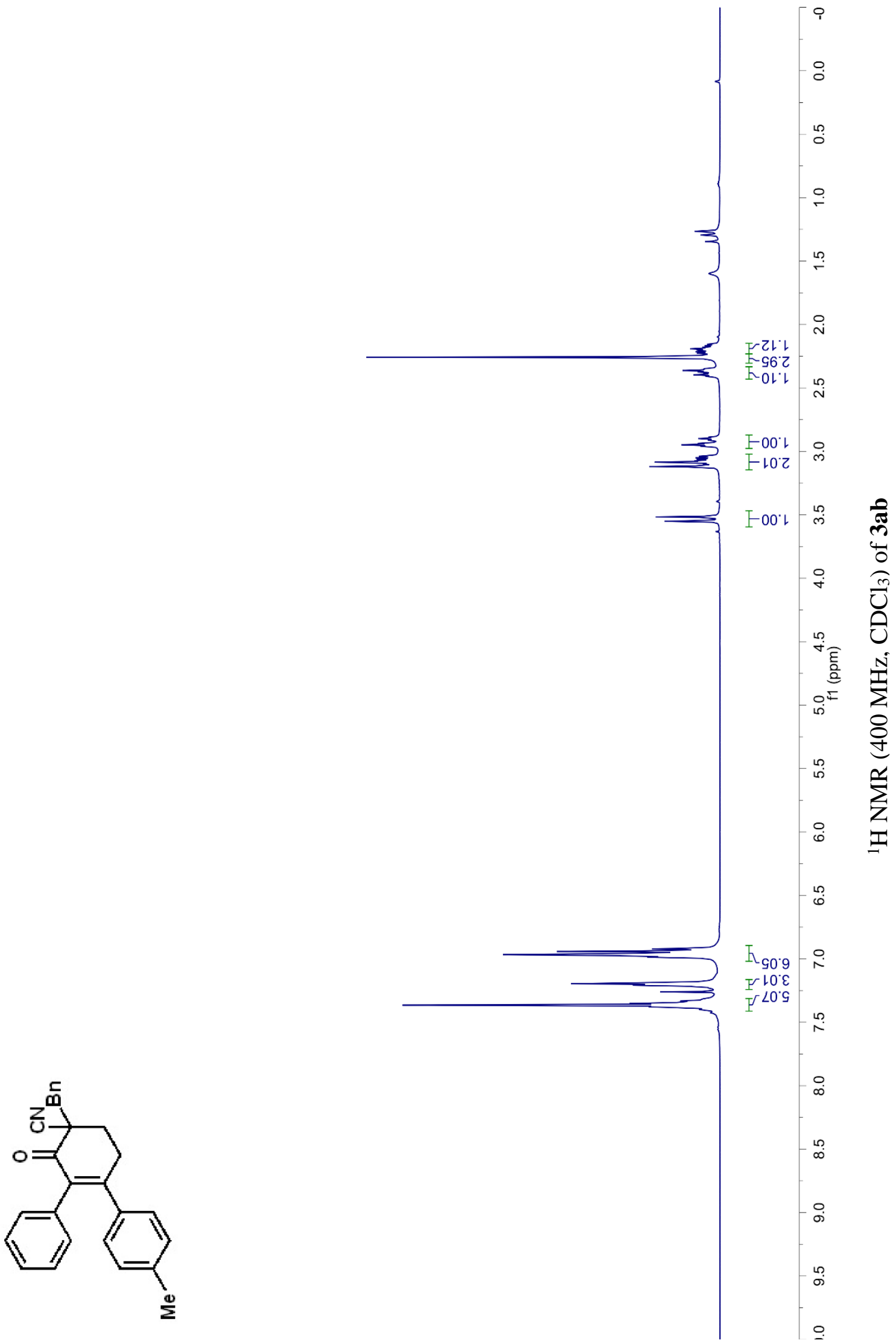


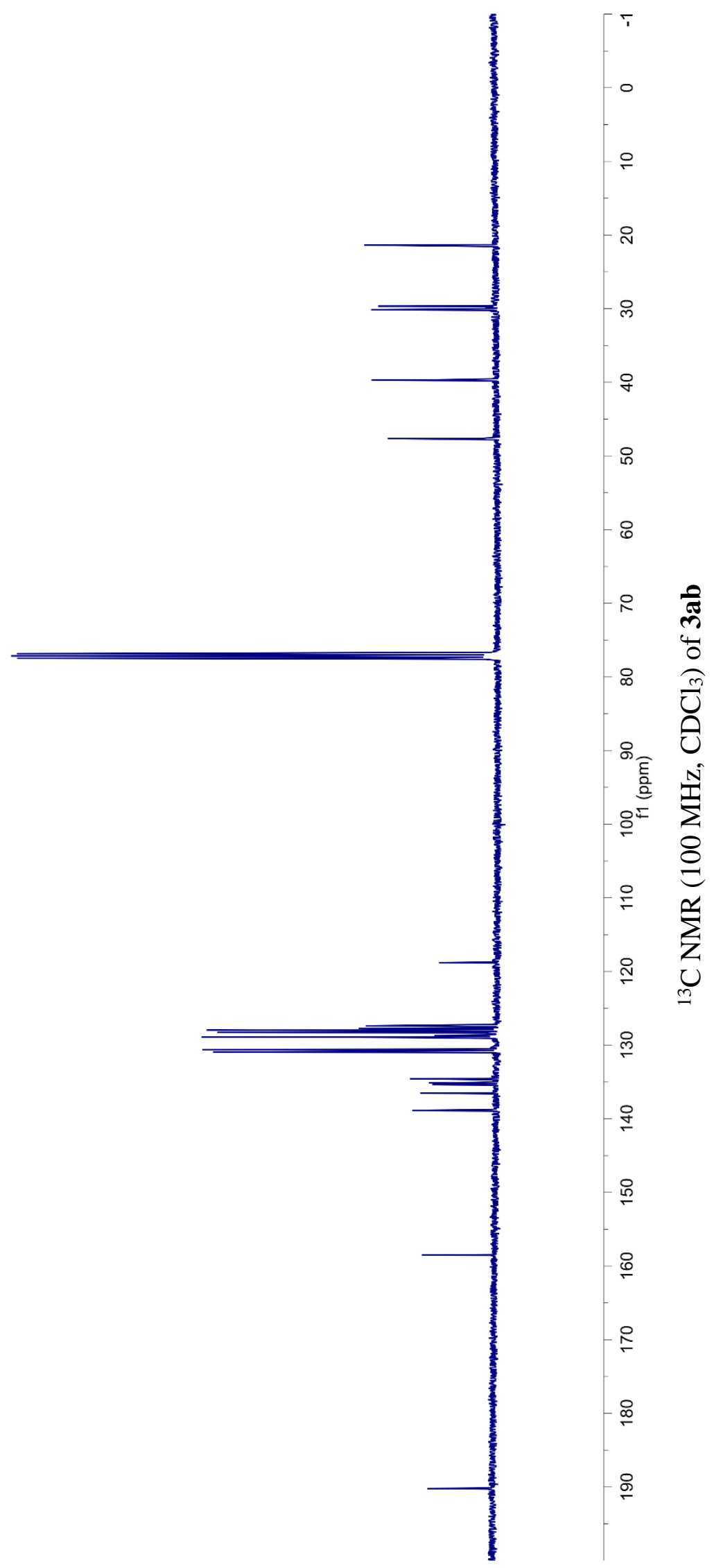


てカレて

$\rightarrow$ G'

99よ'

$\angle L L ' Z$

$881 ' 2$

レ๐乙

ยL乙て

乙乙乙 乙

レレと

乙GE'乙

† $9 \varepsilon^{\prime} Z$

GLE'Z

$98 \varepsilon^{\prime} 乙$

$86 \varepsilon^{\prime} Z$

$868^{\prime} \mathrm{Z}$

606

126'Z

9†6'乙

896

$696^{\circ} \mathrm{Z}$

ᄂEO $\mathcal{E}$

¿ 70

५ $90^{\circ} \varepsilon$

$890^{\circ} \mathcal{E}$

$8 \angle 0^{\circ} \varepsilon$

EเL

ZIG' $\varepsilon$

$\angle \forall G^{\prime} \varepsilon$

$9 \varepsilon L \mathcal{E}$

$1 \angle 9 \cdot 9$

$9 \angle 9^{\circ} 9$

$\angle 89^{\circ} 9$

Z69.9

096.9

s96. 9

$\angle L 66^{\circ} 9$

†86. 9

669

$666^{\circ} 9$

$\varepsilon L L^{\circ} L$

$\angle L L^{\circ} L$

$68 \mathrm{~L} L$

E6L'L

$\varepsilon O 乙 L$

90Z L

ZLZ'L

IZZ L

OE乙 L

† ट L

$\varepsilon \nabla Z L$

$\angle O E^{\circ} L$

ELE'L

8LE'L

$8 Z \varepsilon^{\circ} L$

$\angle E E^{\prime} L$

$\nabla \nabla \varepsilon^{\circ} L$

$8 \sqcup \varepsilon^{\circ} L$

I $9 \varepsilon^{\circ} L$

$G L E^{\circ} L$

$6 \angle E^{\circ} L$

$96 \varepsilon^{\circ} L$

$66 \varepsilon^{\circ} L$
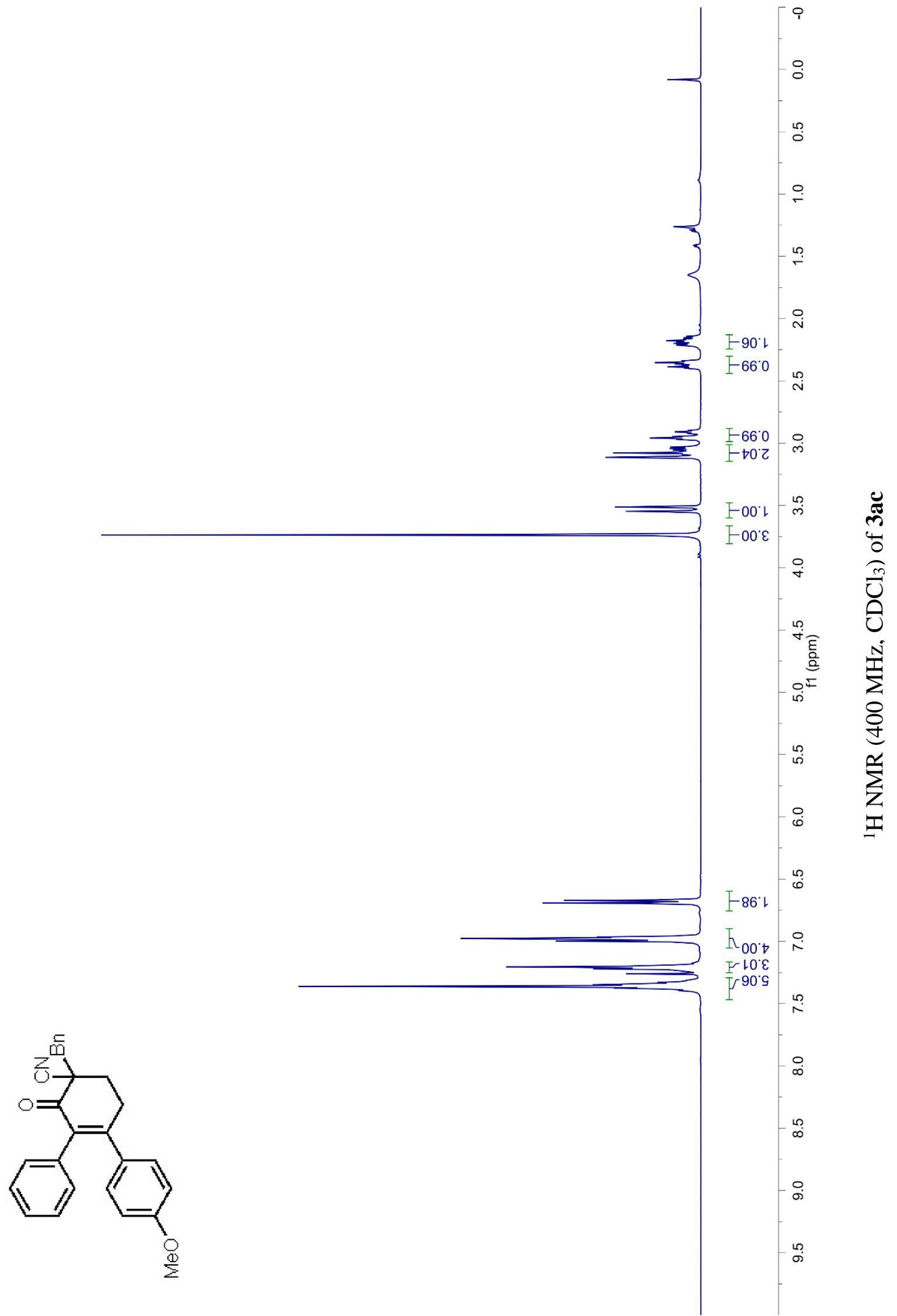
$09^{\circ} \nabla \varepsilon L$

$96 " \nabla \varepsilon L$

$8 \varepsilon^{\prime} \mathrm{GL}$
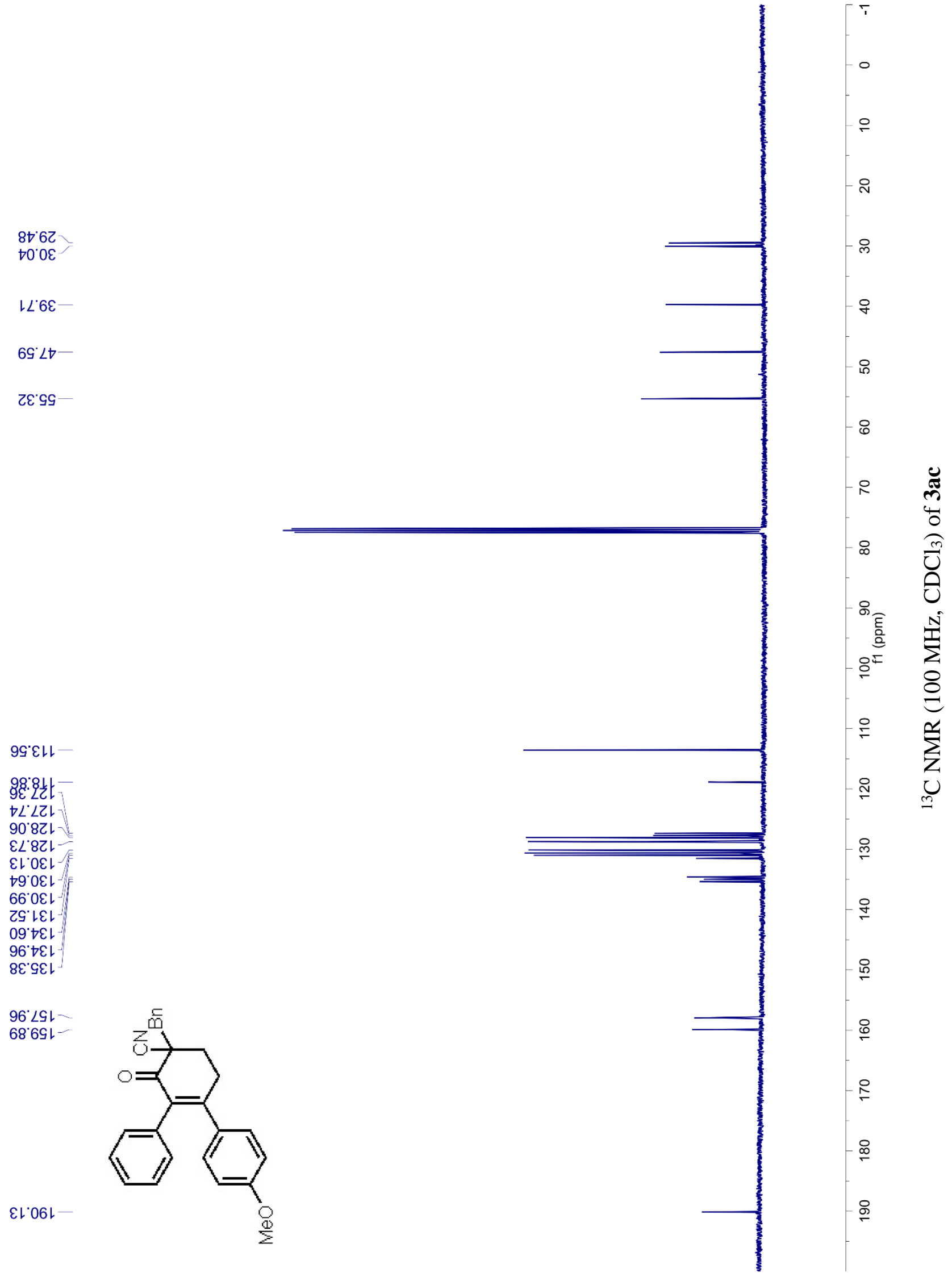
LGL'Z

$\mathrm{ZBL} 2$

Z6L'Z

† 6 เ 2

†OZ'

$\angle L Z Z$

6乙乙乙

99E'乙

$89 \varepsilon^{\prime} 乙$

$6 \angle \varepsilon^{\prime} 乙$

เ6ع'乙

ZOt 2

$\varepsilon 1 \nabla 2$

898'乙

$698^{\circ} \mathrm{Z}$

$188^{\circ} \mathrm{Z}$

$\angle 06^{\circ} \mathrm{Z}$

816.2

$0 \varepsilon 6$ 乙

$\angle \varepsilon 0^{\circ} \varepsilon$

$6 \triangleright 0^{\circ} \varepsilon$

乙90. $\varepsilon$

$\neg \angle 0^{\circ} \varepsilon$

$980^{\circ} \varepsilon$

$860^{\circ} \varepsilon^{-}$

ルレย

OZL'

ILG'E

$9 \nabla \mathcal{G}^{\prime} \varepsilon$

$\angle \mathcal{E} 8^{\circ} 9$

ह†8:9

† $98^{\circ} 9$

$698^{\circ} 9$

†98. 9

$\angle 98^{\circ} 9$

SL8. 9

188.9

926.9

เE6.9

$8 \varepsilon 6.9$

เ 6.9

St6. 9

096.9

$200^{\circ} \mathrm{L}$

$\angle 00^{\circ} \mathrm{L}$

SLO L

$0 Z 0^{\circ} \mathrm{L}$

$\rightarrow 20 \%$

¿EO $\mathrm{L}$

EO 2

$S 8 L^{\circ} L$

S6L'L

IOZ L

$\angle O Z Z^{\prime}$

$60 Z \angle$

ZIZ'L

LIZ L

OZZ L

GLE'L

IZE'L

เEE L

$\downarrow \varepsilon \varepsilon^{\circ}\llcorner$

$0 † \varepsilon^{\circ} L$

G† $\varepsilon^{\circ} L$

$6 \forall \varepsilon^{\circ} L$

$99 \varepsilon^{\circ} L$

乙9E'L

$08 \varepsilon^{\circ} L$

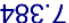

$\angle 6 \varepsilon^{\circ} L$

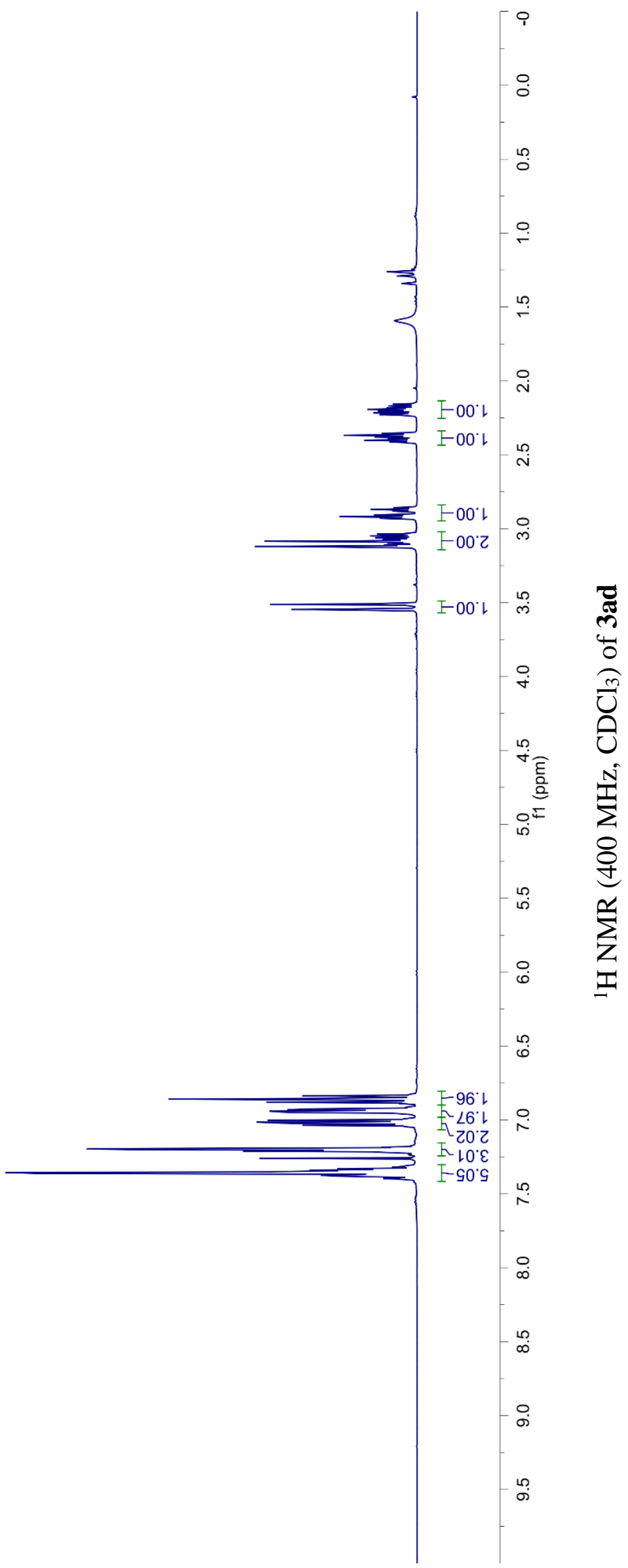




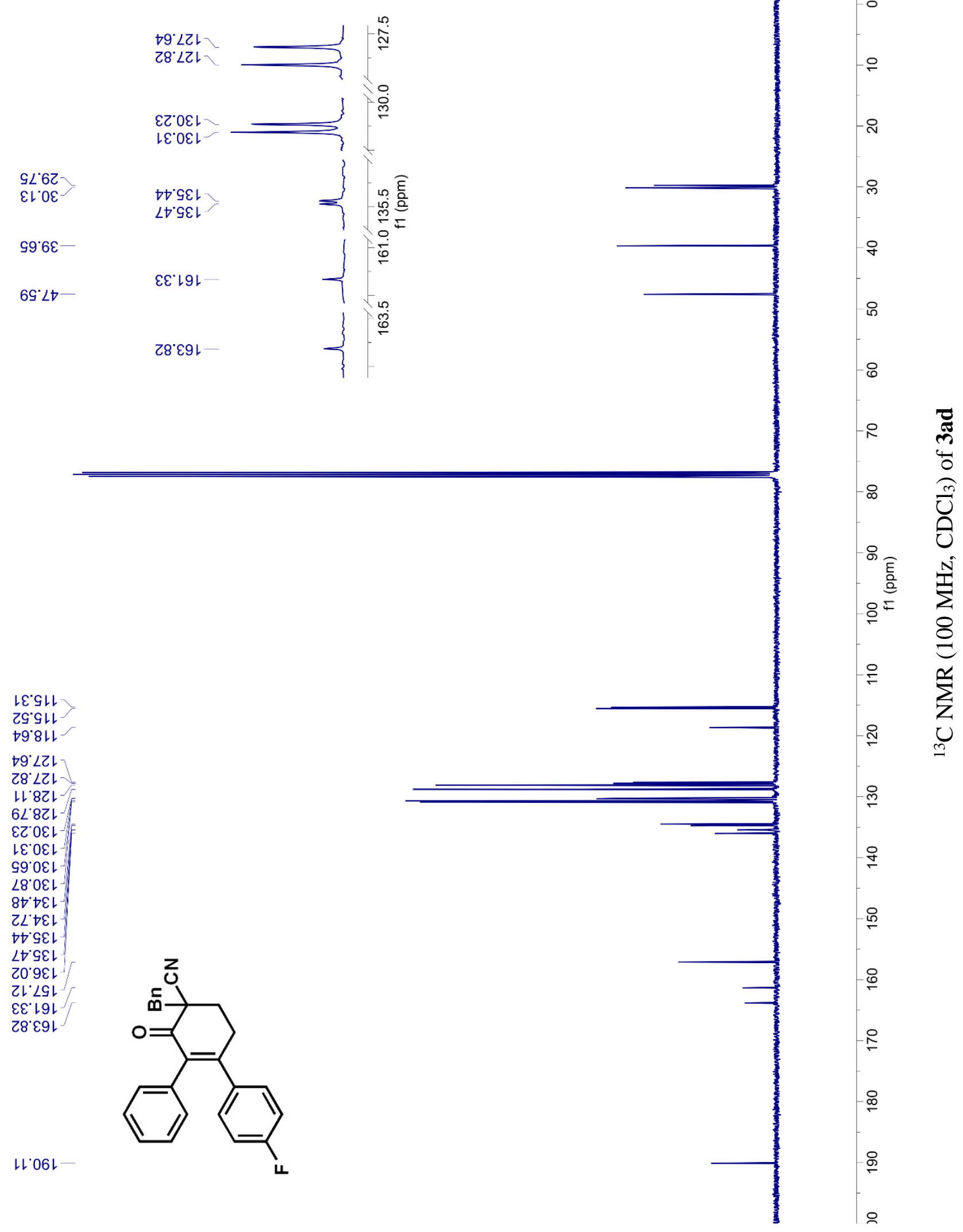




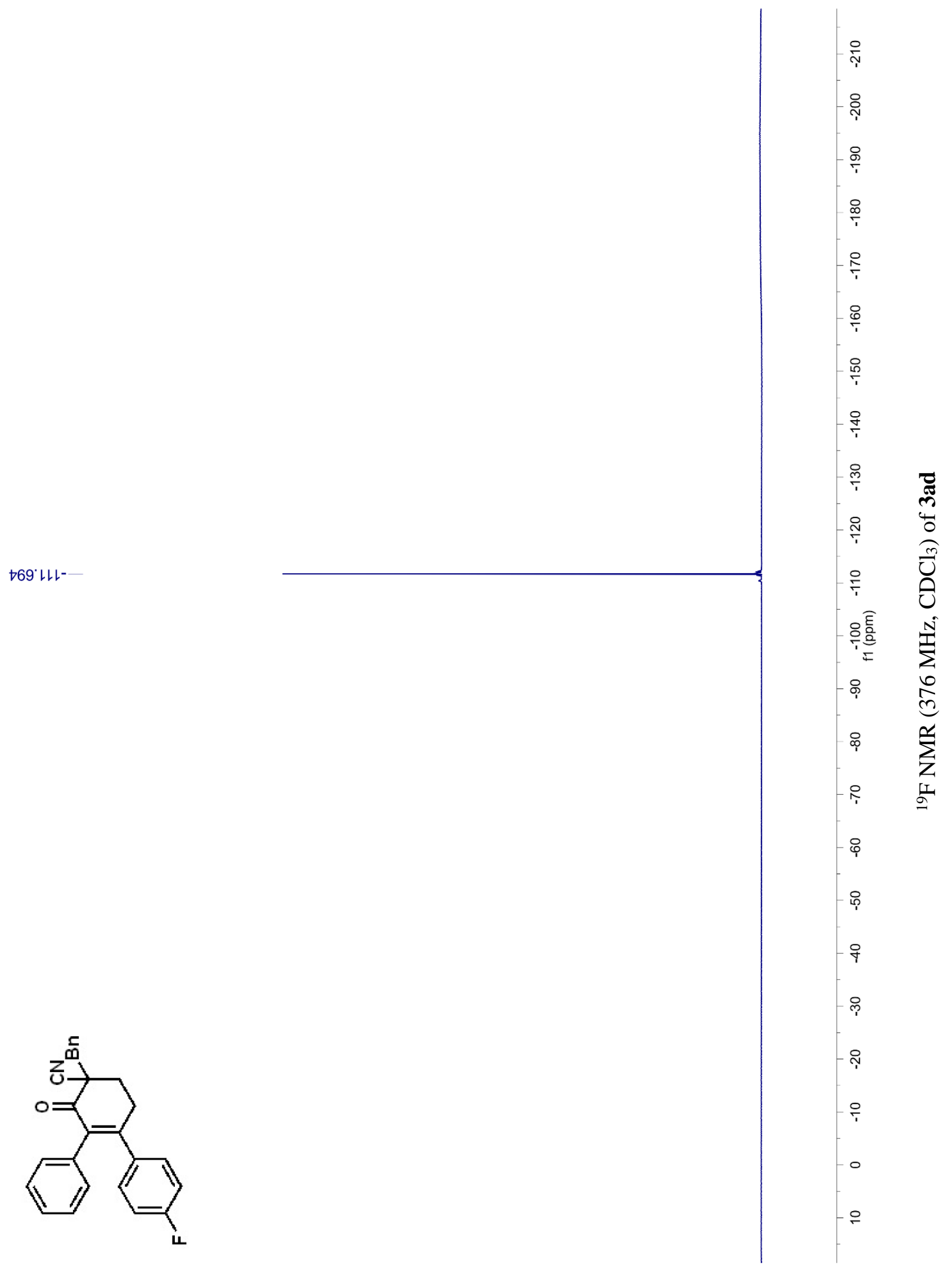




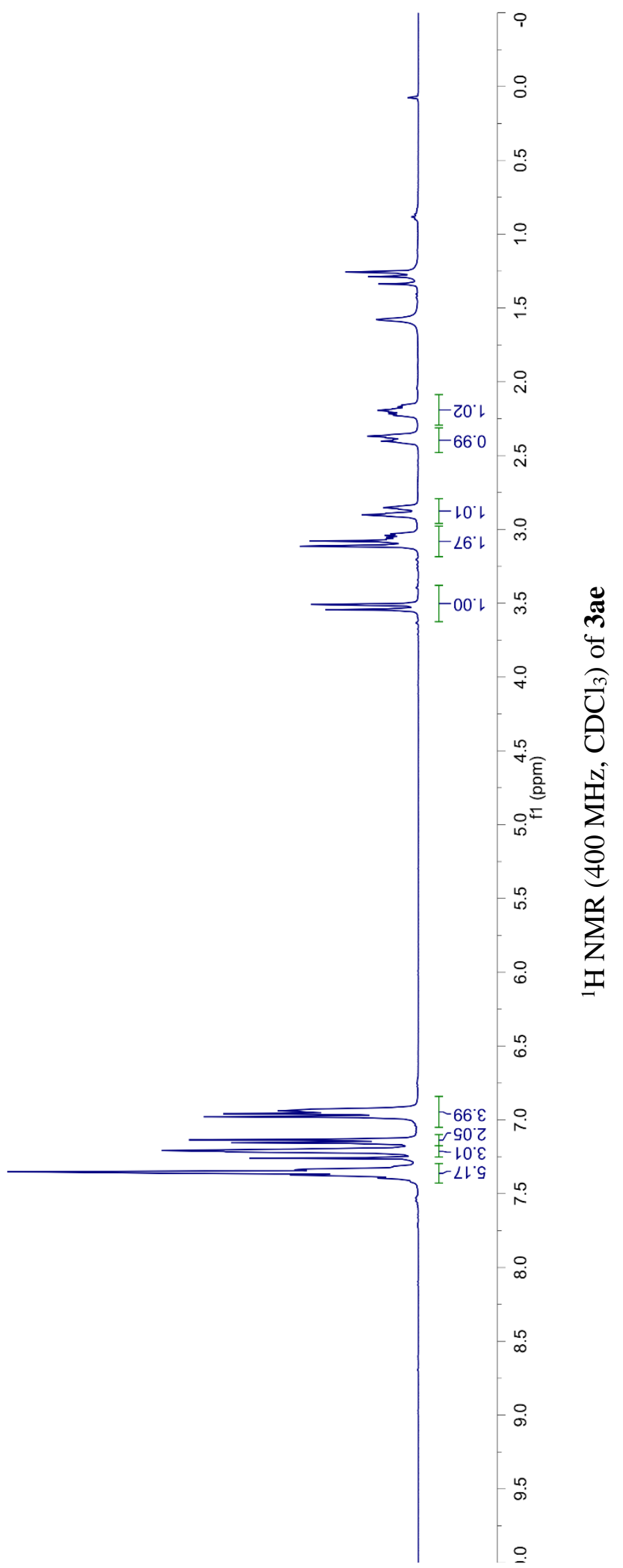




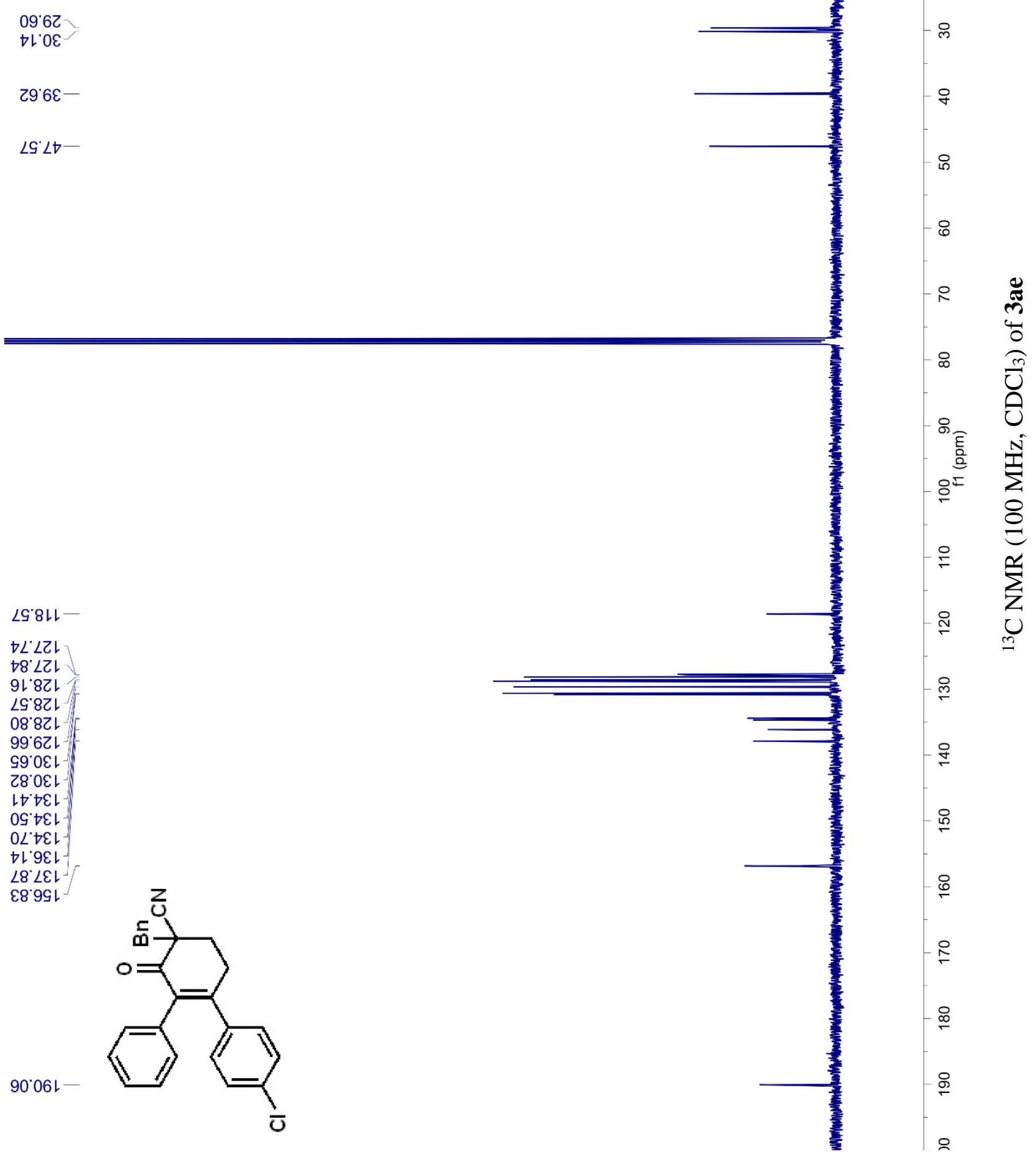



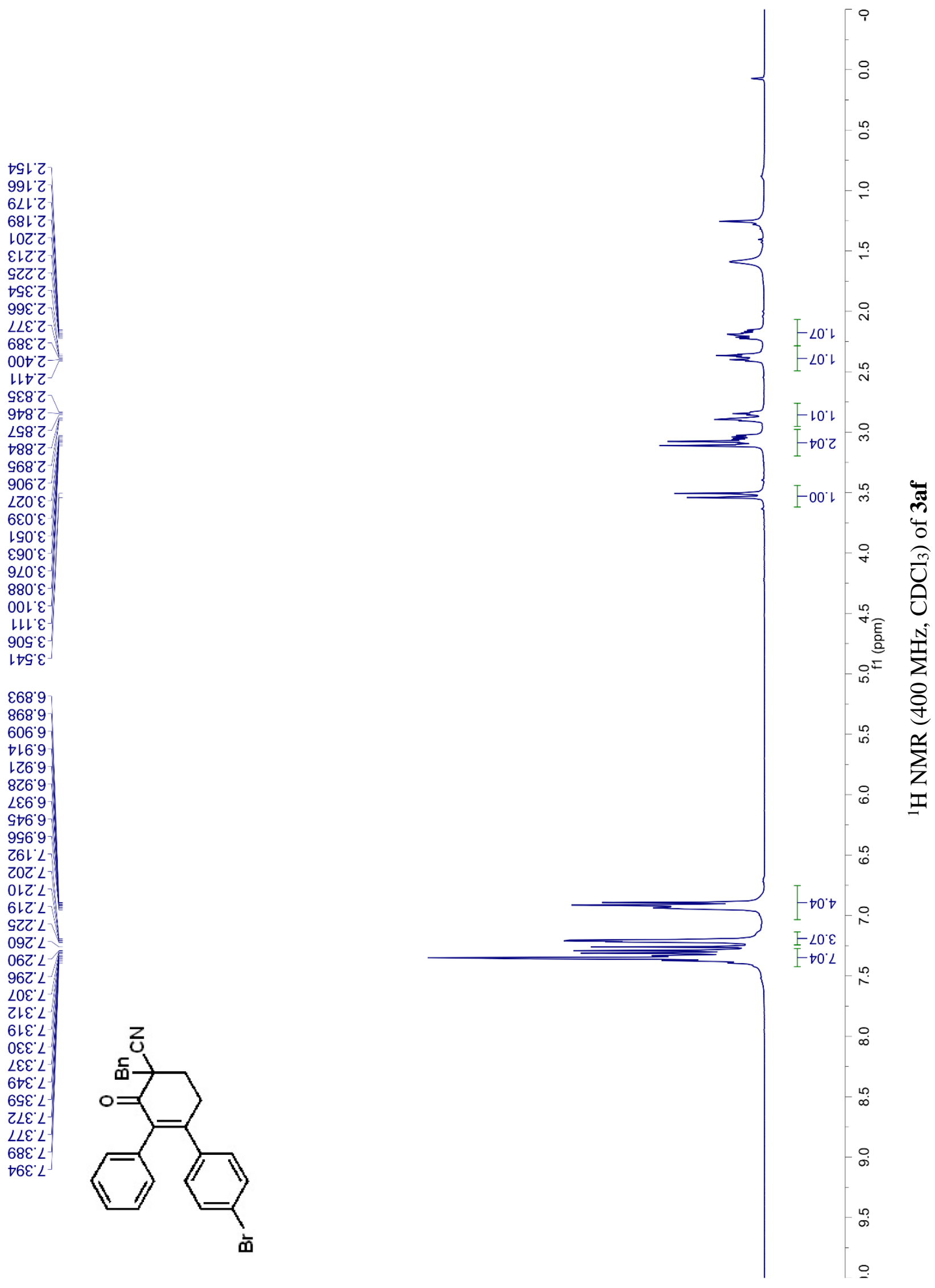
$29^{\circ} 6 \varepsilon-$

$\angle S^{\circ} \angle b-$

99:8th

66'ZZL

$98 . \angle Z L$

$81.82 \mathrm{~L}$

$08^{\circ} 8 \mathrm{ZL}$

$68^{\circ} 62 \mathrm{~L}$ -

s9.0ع

L.0EL

$\varepsilon S^{\circ}\llcorner\varepsilon L-$

เナ๋EL

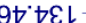

$\mathrm{Zl} \cdot 9 \varepsilon \mathrm{-}$

५ $\varepsilon^{\circ} 8 \varepsilon \downarrow$

\&8.99เ -
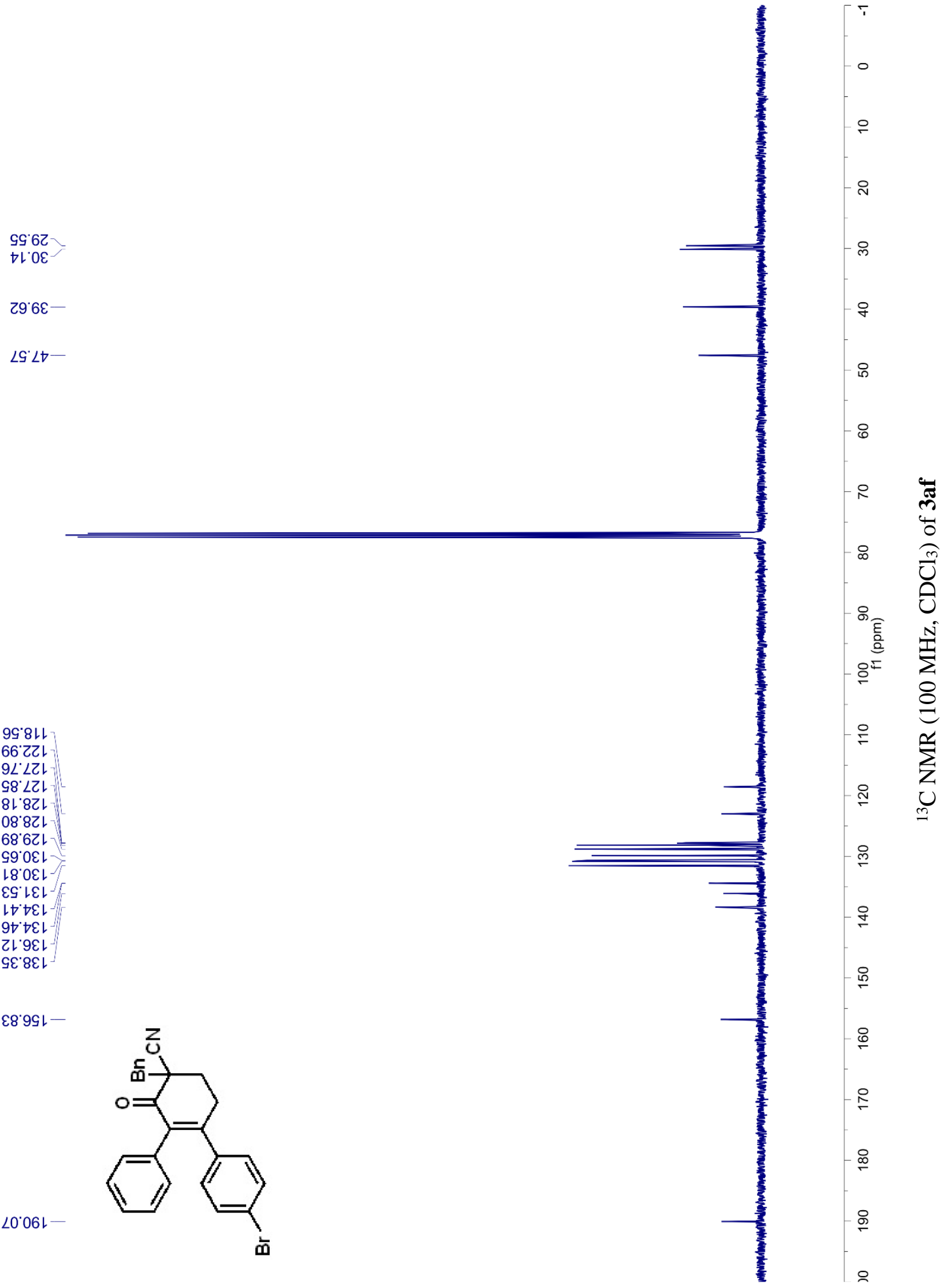
$98 r^{\prime} Z$

$86 \mathrm{~L} Z$

ll2

乙๕乙 乙

G†でて

$\angle G Z$ '

乙8E'乙

$6 \varepsilon 乙$

จ०७乙

9เท'

Z 2

$6 \varepsilon t^{\prime} Z$

Gจ8'

998'

$898^{\circ} \mathrm{Z}$

$68^{\circ} \mathrm{Z}$

S06.

$\angle 16{ }^{\circ} 2$

$090^{\circ} \varepsilon$

ZLO

$\mathrm{s} 80^{\circ} \mathcal{E}$

$960^{\circ} \varepsilon$

$60 L^{\prime} \varepsilon$

レレ'

เEL' $\varepsilon$

9ㄴ' $\varepsilon$

6 L'E

$\nabla G \mathcal{G}^{\circ} \mathcal{E}$

†06. 9

$9\llcorner 6.9$

Gट6. 9

$\downarrow \varepsilon 6.9$

$0 เ 6.9$

676.9

Iㄴㄴ는

$19 L^{\circ} \mathrm{L}$

Z8L'L

$98 \mathrm{~L} \mathrm{~L}$

$76 L^{\circ} L$

EOZ L

OLZ L

OZZ L

GLE'L

OZE $L$

G乙E' $L$

$9 \varepsilon \varepsilon^{\prime} L$

O† $\varepsilon^{\circ} L$

G†E'L

$99 \varepsilon^{\circ} L$

$99 \varepsilon^{\circ} L$

$8 \angle E^{\circ} L$

$\varepsilon 8 \varepsilon^{\circ} /$

$96 \varepsilon^{\circ} L$

LOT'L

GOt'L

GZV L

Stti L

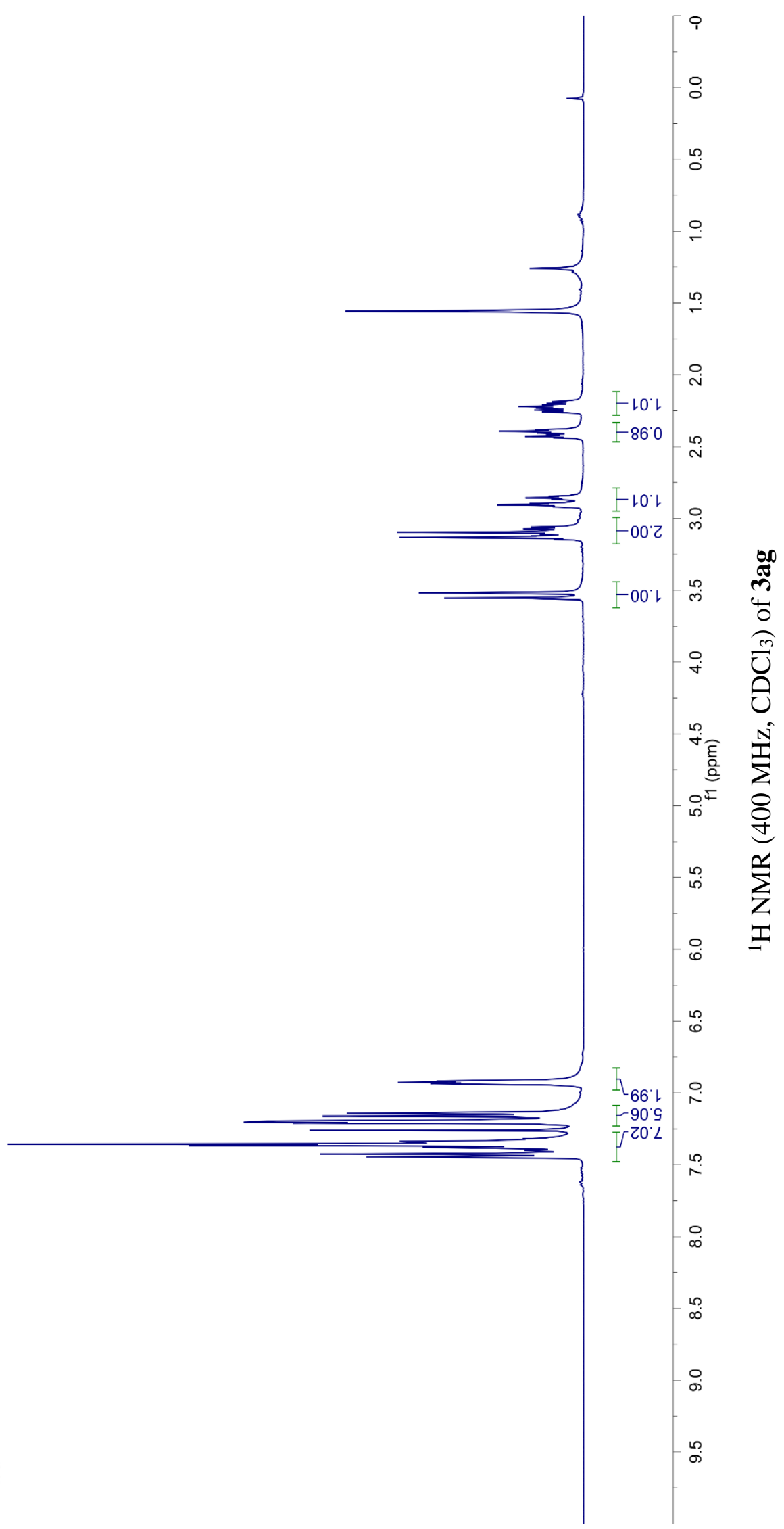



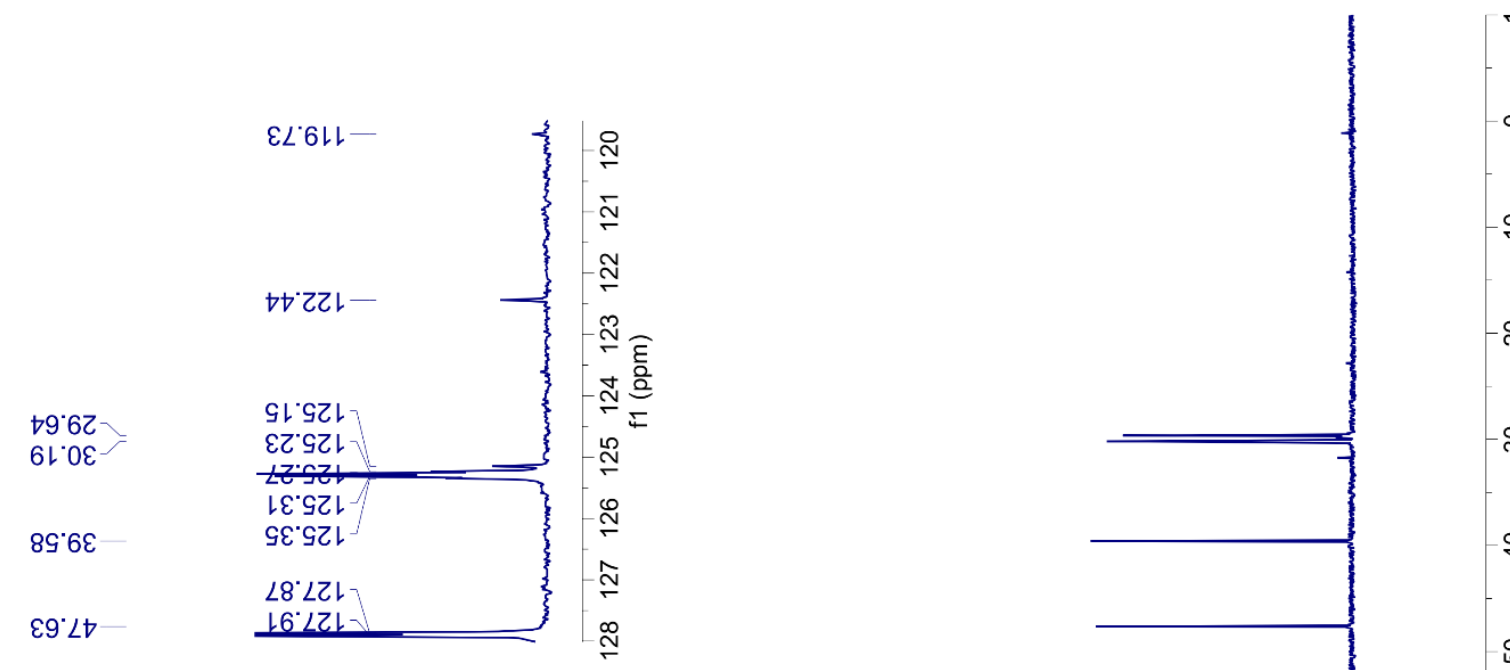

レガ8レレ

$\varepsilon L “ 6$ เ

Gl GZL

हट"GटL

L乙G乙L

เ

GE'G乙L-

$\angle 8 \angle Z L$ -

16 LZL

GL'8ZL

EG'8ZL

เ8.8Zレ

76.62 ᄂ

$\angle Z{ }^{\circ} 0 \varepsilon$.

6S.0EL

ع9.0EL

$\neg L^{\circ} 0 \varepsilon L$

乙6. 0 \&

$80^{\circ} \nabla \varepsilon L-$

$\varepsilon \varepsilon^{\circ} \nabla \varepsilon\llcorner$

$89^{\circ} 9 \varepsilon \mathrm{L}$

งเEเレ-

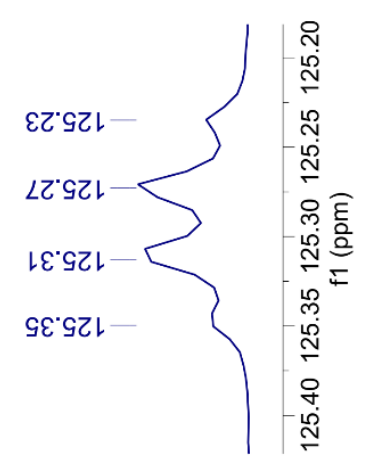

เナ9ดレー
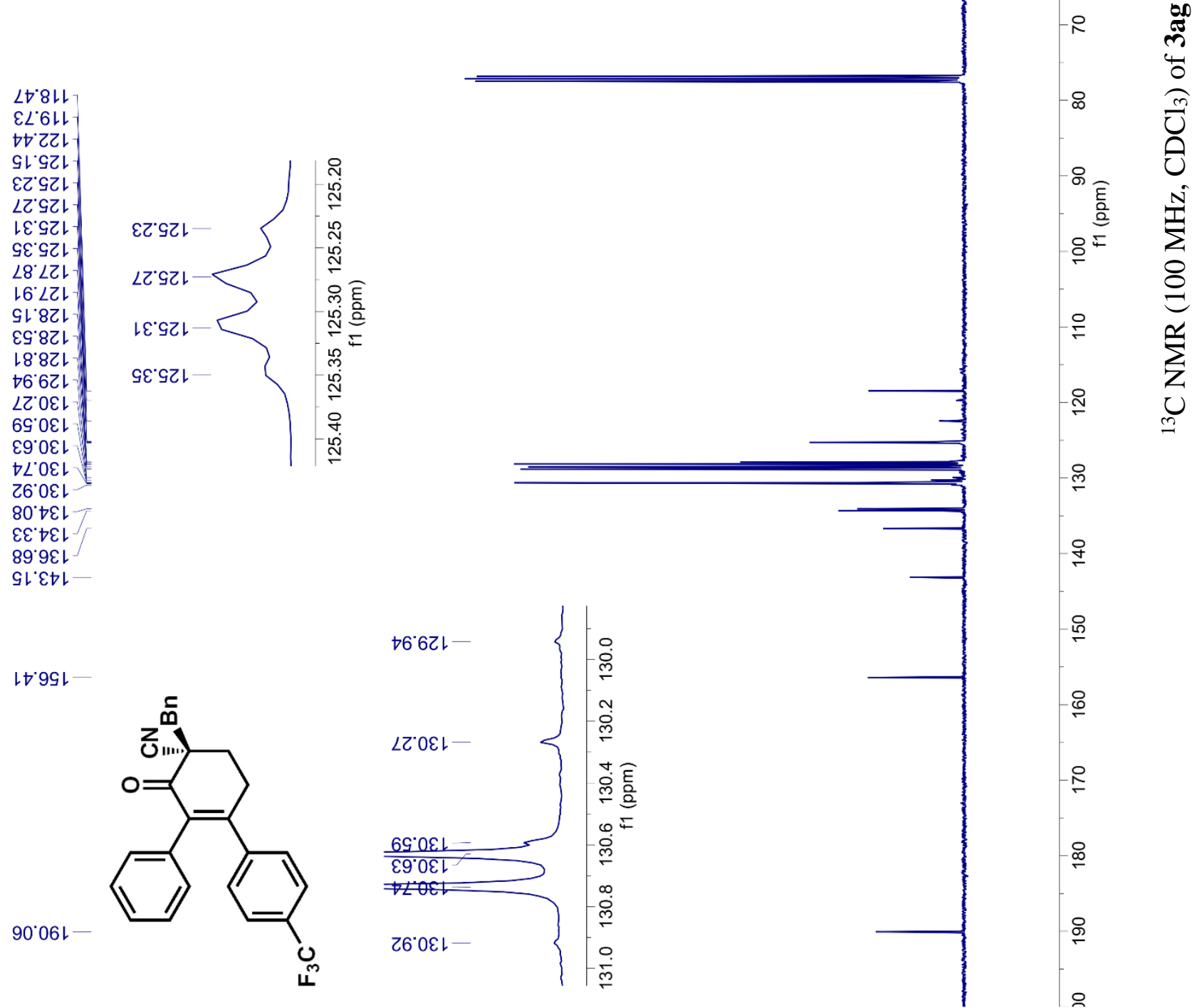


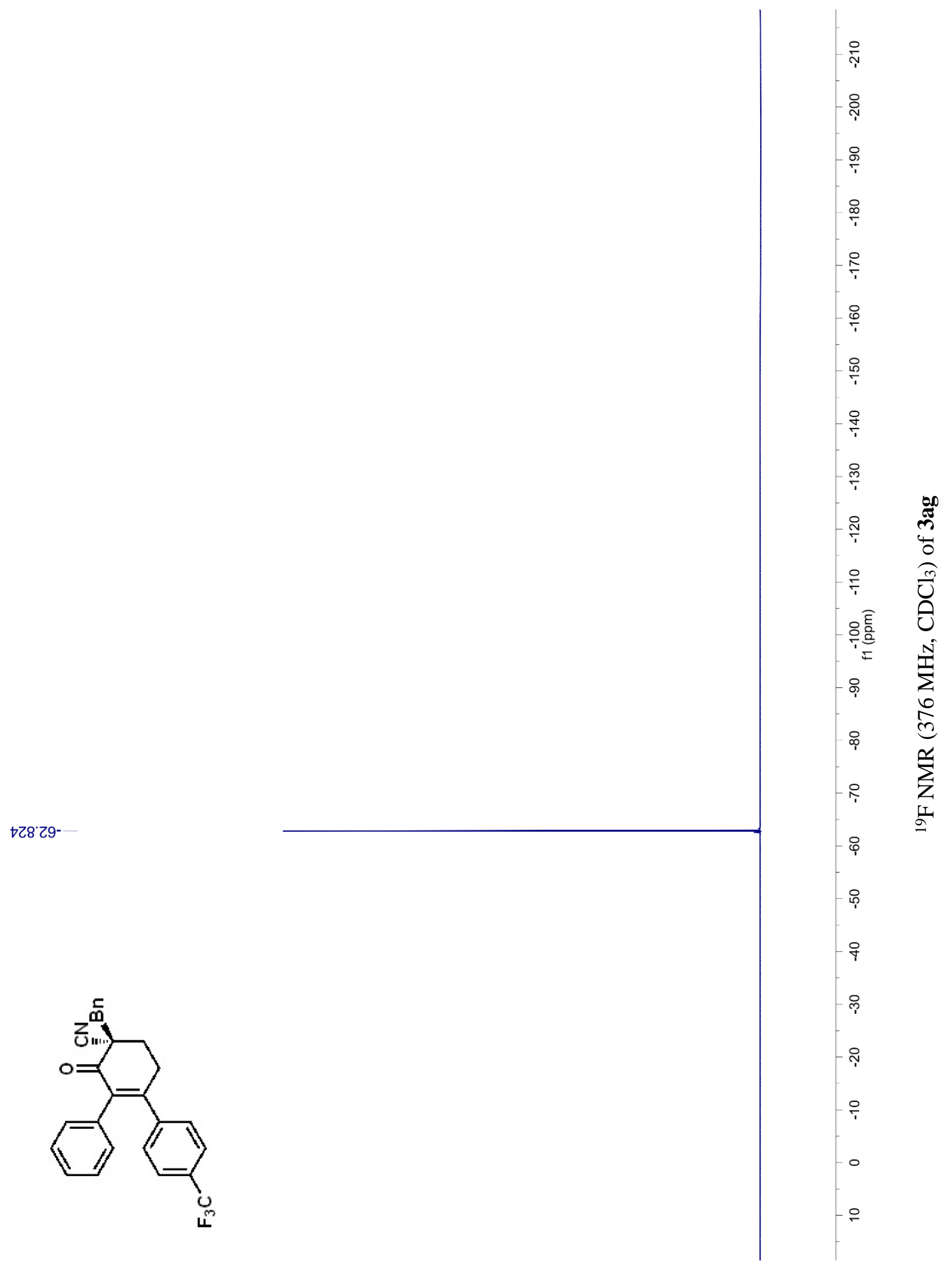


GLL'Z

L8L'

$00 Z Z$

ルでる

乙Z乙'乙

†ย乙'乙

9†乙 乙

乙LE'乙

$\varepsilon 8 \varepsilon^{\prime} 乙$

५6ย'乙

$\angle O t^{\circ} Z$

8レナて

6てヤ て

乙98'

$\varepsilon \angle 8^{\circ} Z$

$788^{\prime} 乙$

เᄂ $6^{\circ} \mathrm{Z}$

ZZ6'乙

$\varepsilon \varepsilon 6.2$

$8 \neg 0^{\circ} \varepsilon$

$090^{\circ} \varepsilon$

Z $\angle 0^{\circ} \varepsilon$

$680^{\circ} \varepsilon$

$860^{\circ} \varepsilon$

$60 L^{\circ} \varepsilon$

$\rightarrow 乙 L^{\circ} \varepsilon$

†

OZG' $\varepsilon$

$\mathcal{G G S}^{\circ} \mathcal{E}$

$826^{\circ} 9$

५ะ6. 9

Et6. 9

เS6. 9

$900^{\circ} \mathrm{L}$

$\angle 2 O^{\circ} \mathrm{L}$

$290^{\circ} \mathrm{L}$

$880^{\circ} \mathrm{L}$

LOZ'L

$80 Z$ L

9LCL

E乙E'L

8حध 'L

$6 \varepsilon \varepsilon^{\circ} L$

$\angle \nabla \varepsilon^{\circ} L$

乙9E ' $L$

$69 \varepsilon^{\circ}\llcorner$

เ8E' $L$

$\angle 8 \varepsilon^{\circ} \angle$

tOt $L$

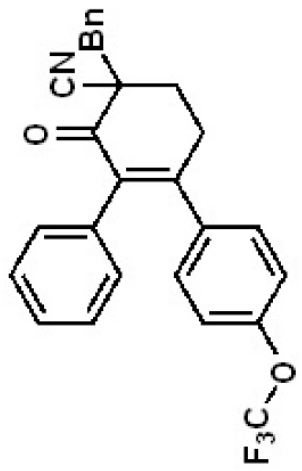

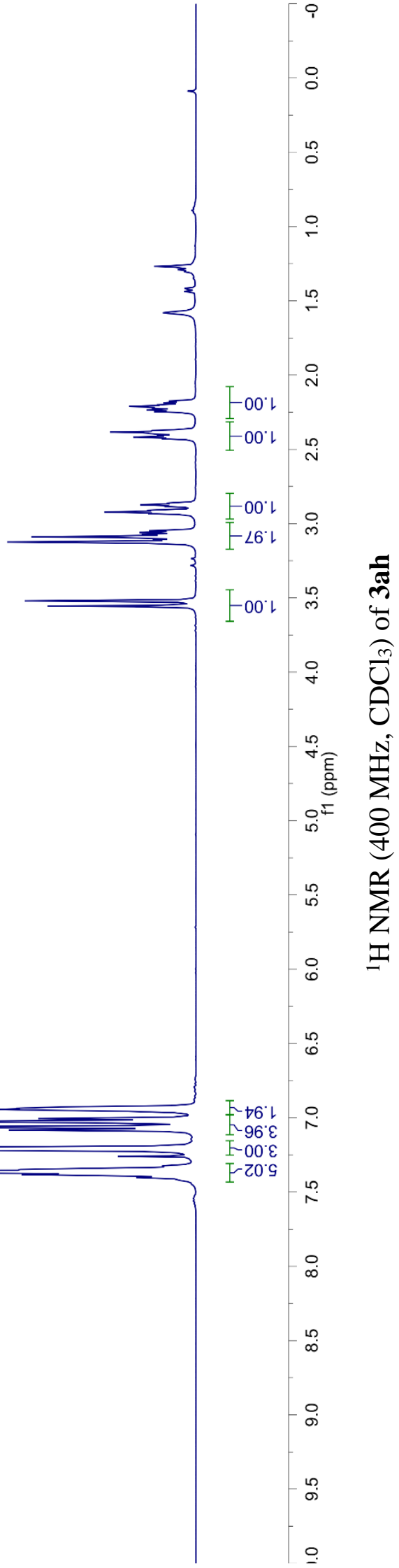


79.62

OL"OE

$89^{*} 6 \varepsilon$

$89^{\circ} \angle t-$

E9.9เ

SG“8レレ

$60^{\circ} 6 \mathrm{LL}$

IS'0ZL

99レてレ

¿乙一

8L'LZL

๑8 LZL

ZL'8ZL

6L'8ZL-

06.6ZL

\&9.0ㄴ

$08^{\circ} 0 \varepsilon$ ᄂ

$8 \varepsilon^{\prime} \nabla \varepsilon\llcorner$

$6 \mathrm{C}^{\prime} 9 \varepsilon \mathrm{L}$

$96^{\circ} \angle \mathcal{L}$.

806 t -

89.99レ-

20.06 L -
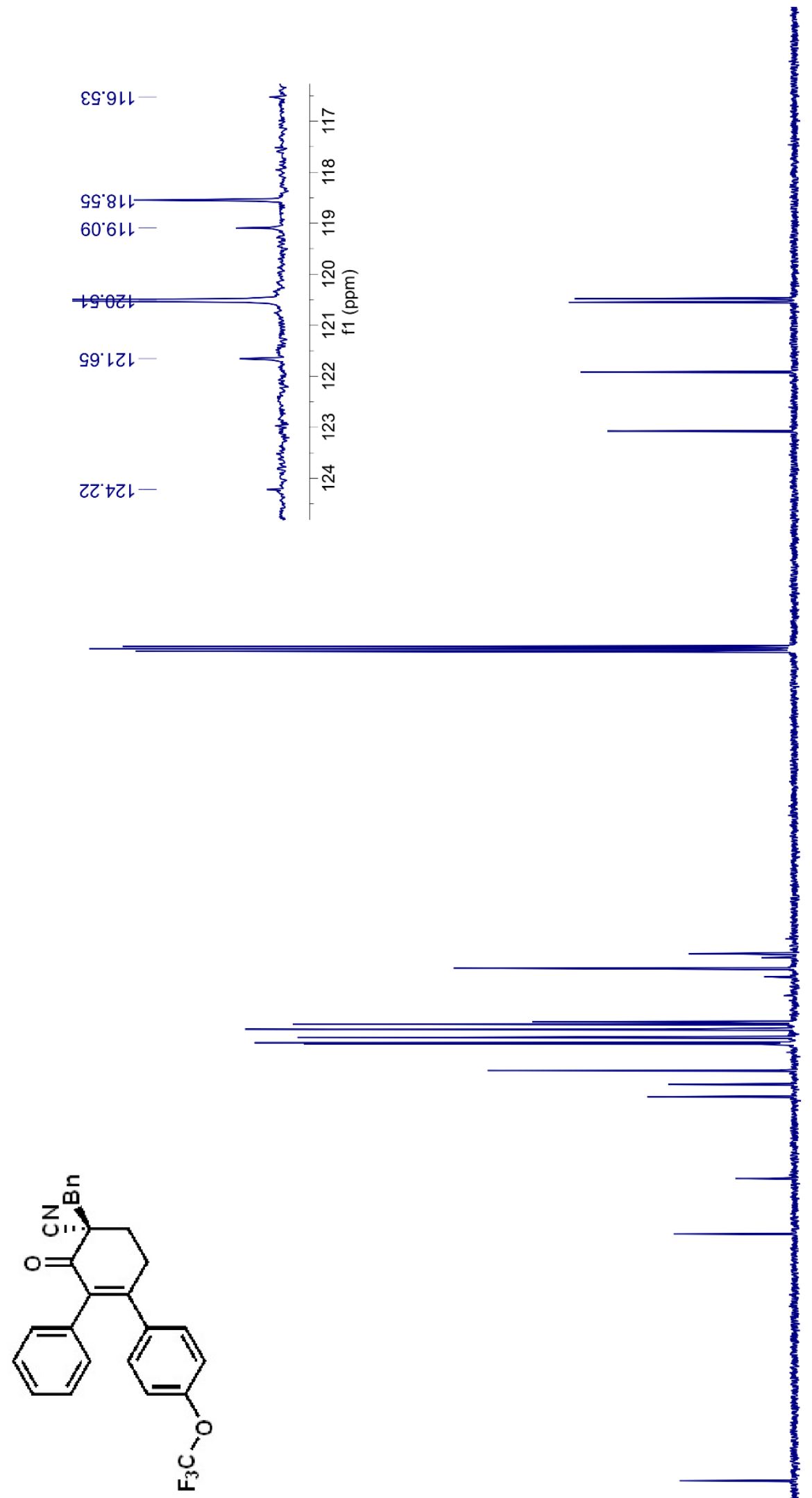

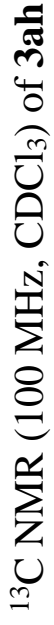

요

요

요

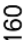

$\stackrel{9}{?}$

$\stackrel{\infty}{\leftarrow}$

옹

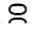




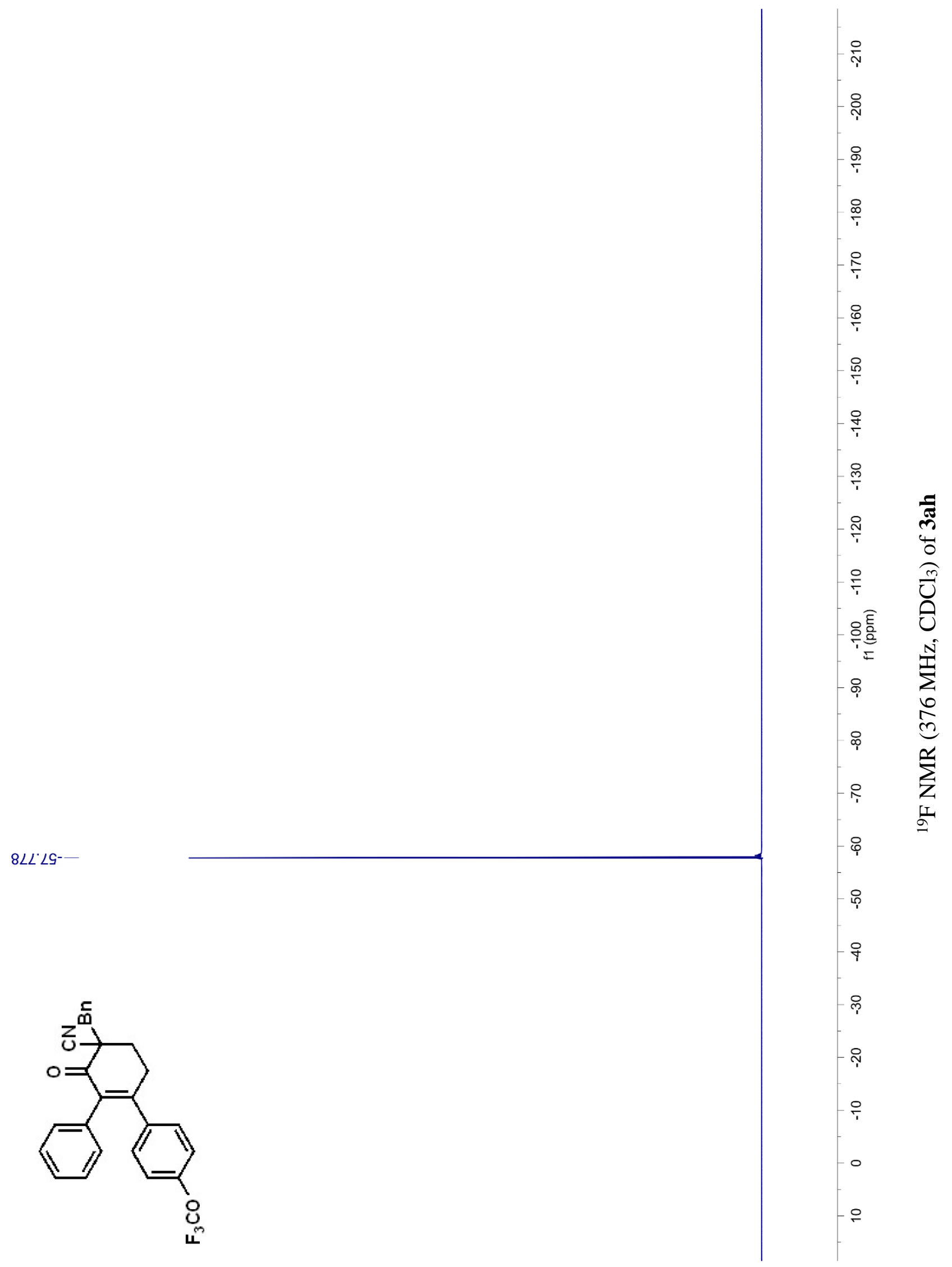



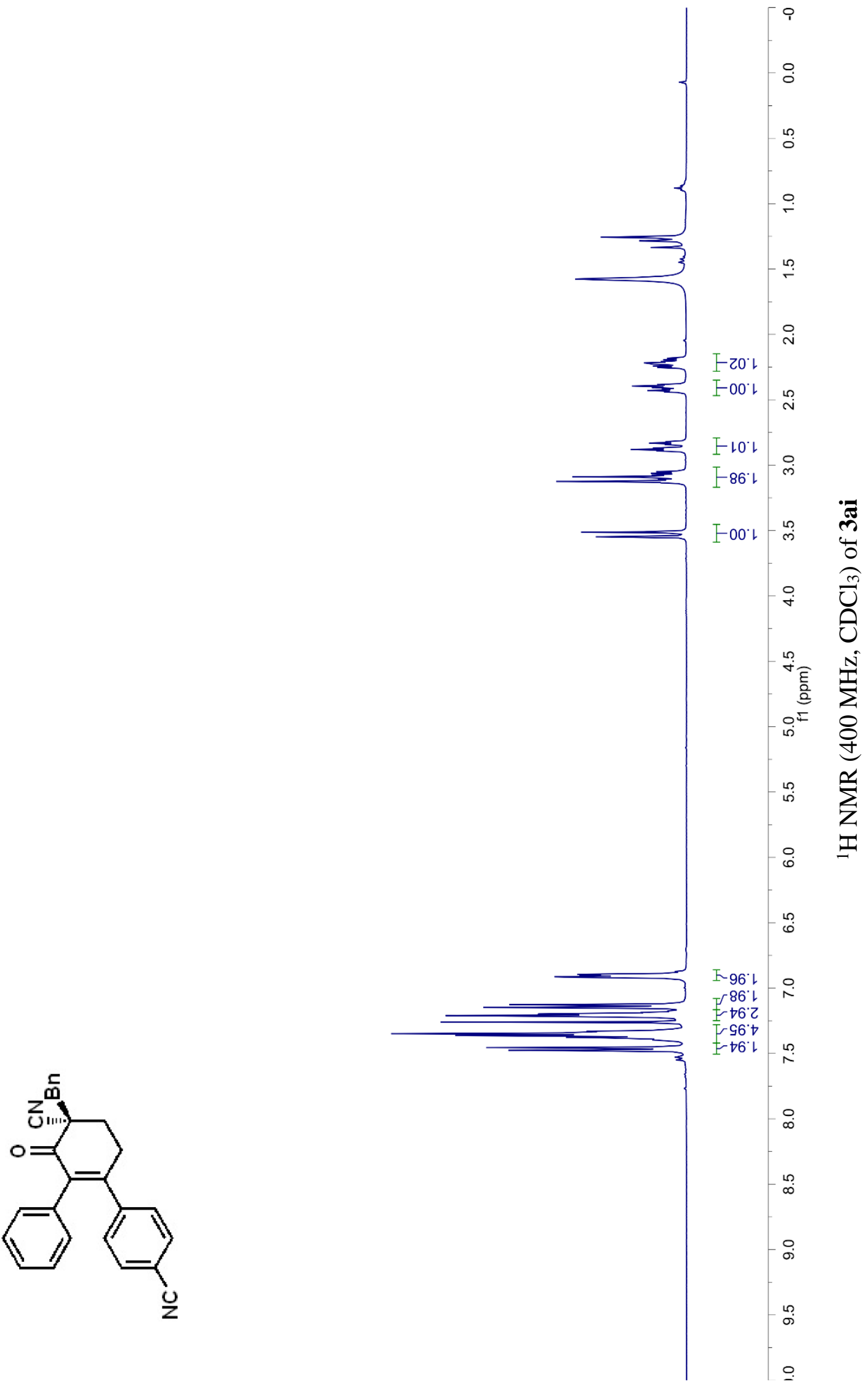
2762

$81^{\circ} 0 \varepsilon$

\&ง $6 \varepsilon-$

$9 S^{\circ} \angle b-$

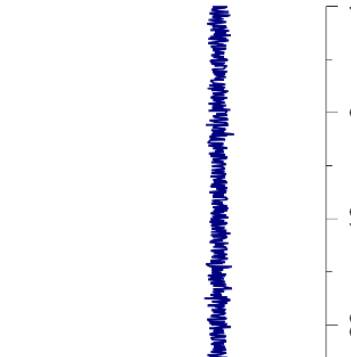

ลิ

이

우

오

8

$\varepsilon L \cdot 8 Z L$

9Z'8ZL

$\checkmark 8.82 \mathrm{~L}$

$68.8 \mathrm{ZL}$

$\rightarrow 9^{\circ} 0 \varepsilon$

$69^{\circ} 0 \varepsilon \downarrow$

乙レ'ZعL

6L $\varepsilon \varepsilon\llcorner$

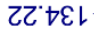

66.9ع

9l.tol.

89.งตเ-

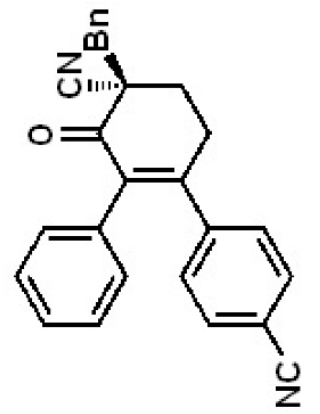

를

8

등



$\stackrel{2}{=}$



음

요

$\stackrel{\circ}{\underline{0}}$

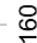

온

$\stackrel{\infty}{\infty}$

$\stackrel{\circ}{9}$

○ 

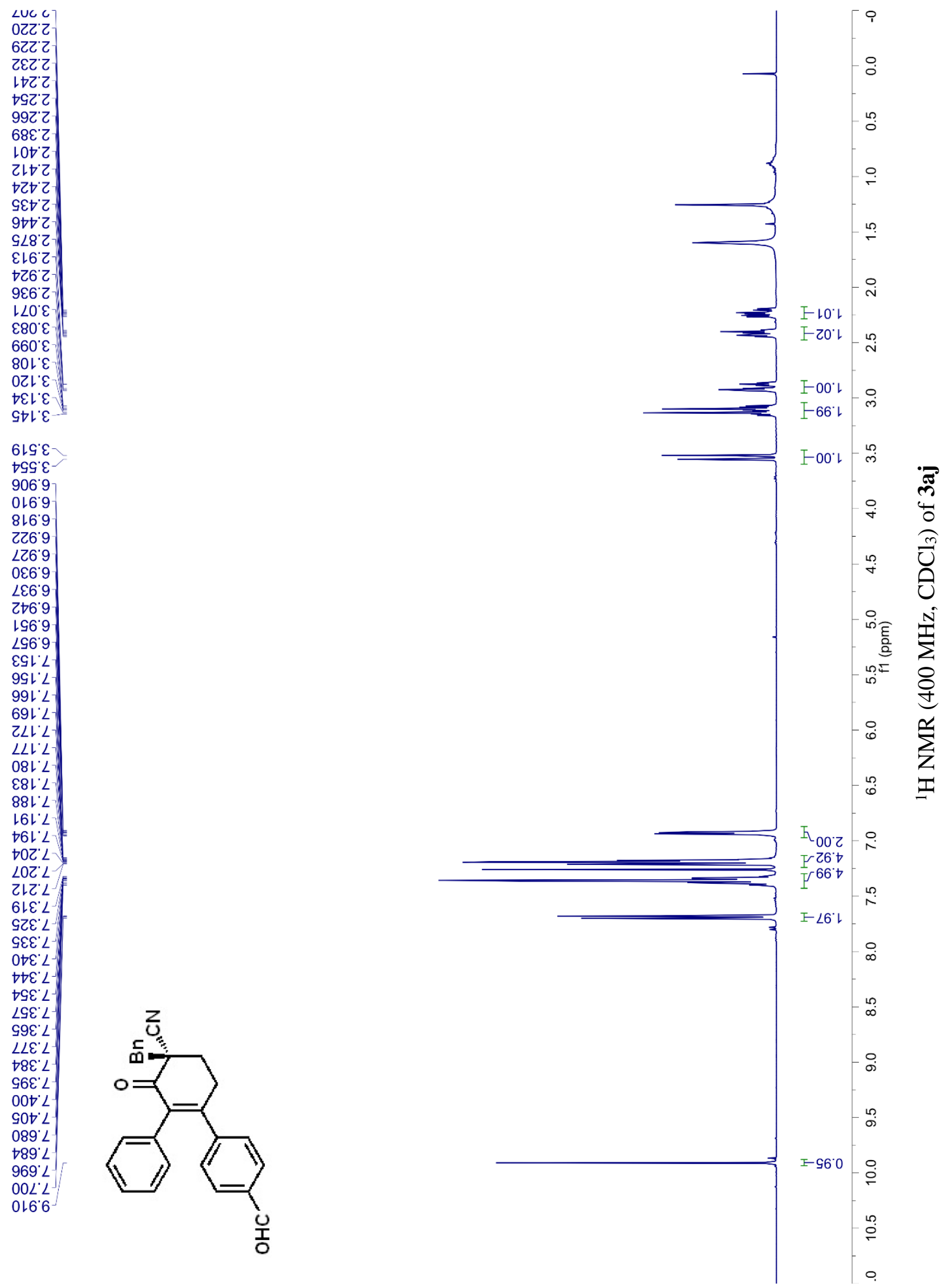
9G.6己

$9 Z^{\circ} 0 \varepsilon^{-}$

$09^{\circ} 6 \varepsilon$

$99^{\circ} \angle t$

งt8レ

$06^{\circ} \angle Z$ L

$\angle 6^{\circ} \angle Z L$

9レ'8Z

$78{ }^{\circ} 8 \mathrm{~L}$

$19^{\circ} 6 \mathrm{ZL}$

$99^{\circ} 0 \varepsilon$ L $^{-}$

$9 L^{\circ} 0 \varepsilon L$

Oレ๋ $\nabla \varepsilon$ -

$\varepsilon \varepsilon^{*} \nabla \varepsilon\llcorner$.

98. $G \mathcal{L} L$

$9 \angle \cdot 9 \varepsilon L$

99'Gヤレ

Z9.99l

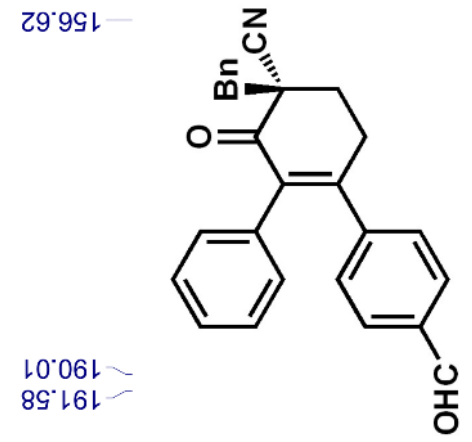

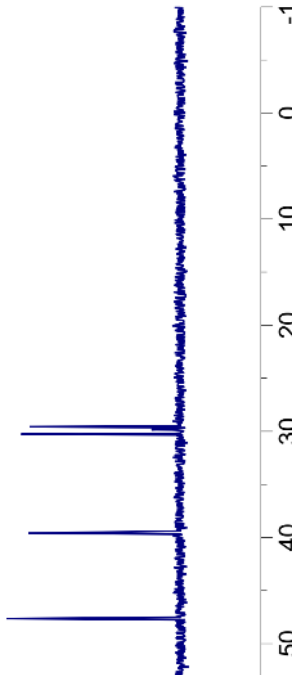

\%

$\overbrace{0}^{0}$

己

음

o 8

당

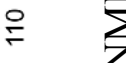

인 
L8L'

$66 \mathrm{~L}^{\prime} \mathrm{C}$

ZL乙'Z

IZZZ

¿乙乙

$\nabla \varepsilon 乙 乙$

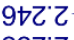

8S乙乙

$6 \angle \varepsilon^{\prime} 乙$

เ $6 \varepsilon^{\prime} 乙$

乙O† 乙

カレナて

G乙† 乙

\& 2

हZS'乙

198' $\mathrm{Z}$

$\varepsilon 88^{\circ} 乙$

0เ6'乙

126乙

乙ย6 乙

५५0 $\varepsilon$

$\angle 90^{\circ} \varepsilon$

$080^{\circ} \varepsilon$

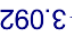

$960^{\circ} \varepsilon^{-}$

†०น' $\varepsilon$

$9 น \mathcal{E}$

เEL' $\varepsilon$

เจレ

GLG'

$0 \mathcal{S G}^{\prime} \varepsilon$

6เ6.9

26 9

५ट6. 9

$826^{\circ} 9$

เE6.9

† 6. 9

$0 \triangleright 6.9$

76.9

$91 L L$

ICLL

9ZL'L

$8 \varepsilon L^{\circ} L$

Z†L'L

$\angle D L ' \angle$

$\angle 9 L^{\circ} \mathrm{L}$

ILL'L

$6 \angle L L$

$\angle 8 L^{\circ} L$

$\checkmark 6 \mathrm{~L}^{\circ} \mathrm{L}$

$\supset 0 Z$ ' $L$

9เ $\varepsilon^{\circ} L$

乙乙E $L$

乙EE $L$

O†E $L$

$\nabla \nabla \varepsilon^{\prime} L$

IGE L

GSE' $L$

乙9E'L

$\neg L E^{\prime} L$

$\angle L E^{\circ} L$

L $8 \varepsilon^{\circ} L$

$\neg 8 \varepsilon^{\circ} L$

$\varepsilon 6 \varepsilon^{\circ} L$

$\angle 6 \varepsilon^{\circ} \angle$

$0 t L L$

$S \forall L L$

$0 G L$

$Z 9 L L$

$99 \angle 2 L$

ILLL

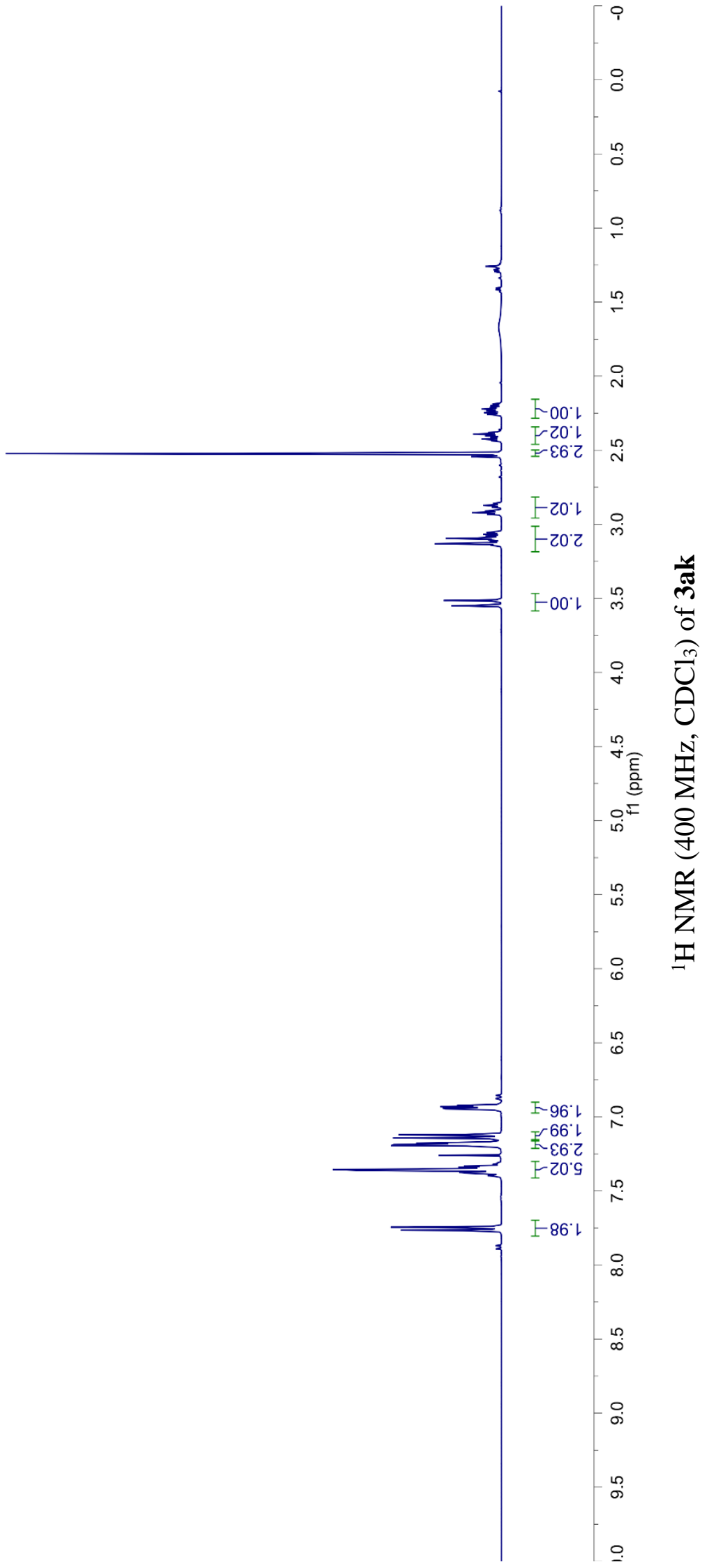




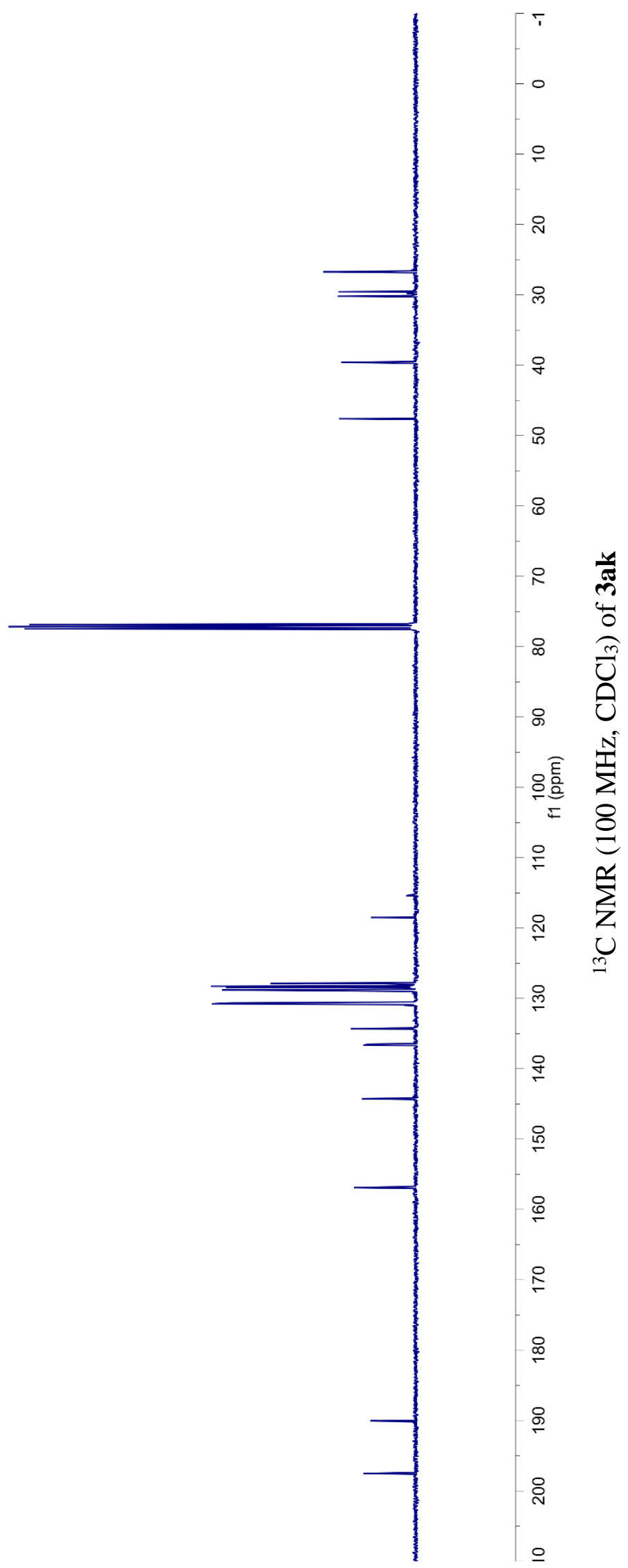

09.8เ

S8 $L Z L$

乙L'8ZL

9乙'8Zレ

S7 82L

08 '8Z

$\varepsilon 9^{\circ} 0 \varepsilon \mathrm{L}$

9L'OEL -

$86^{\circ} 0 \varepsilon$ L

乙๐ $\nabla \varepsilon L$

$\varepsilon \varepsilon^{\circ} \nabla \varepsilon\llcorner-$

6ฑ $9 \varepsilon$ L

†9.9ع -

〔でャレー

06.99ட-

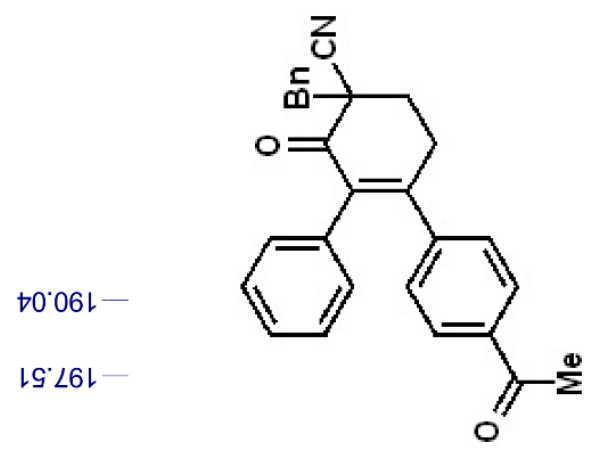



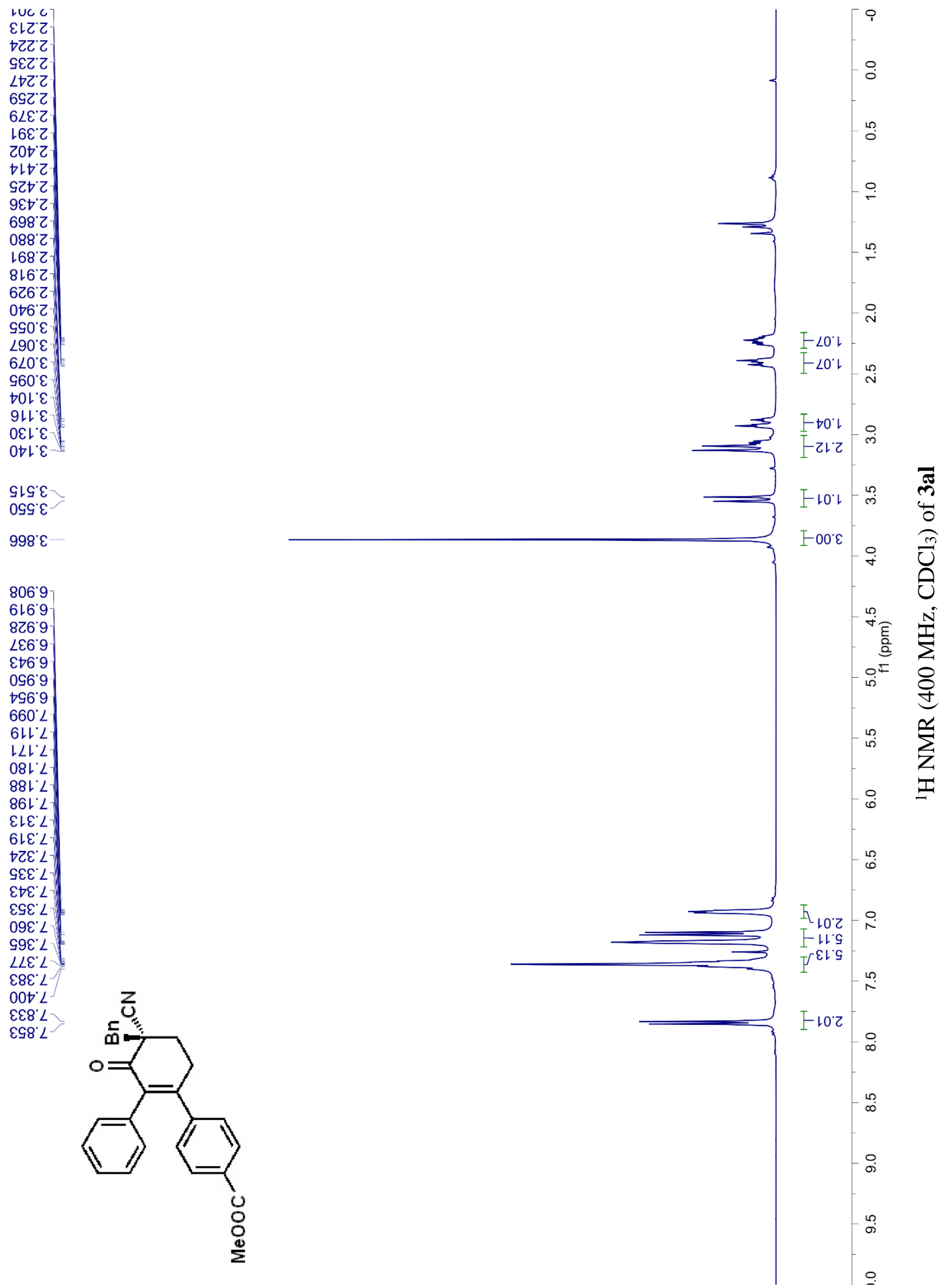
s. $6 \varepsilon-$

$\varepsilon 9^{\circ} \angle t$

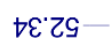

LG'8H

$6 L^{\circ} \angle Z L$

E8. LZL

$90^{\circ} 8 Z L$

$6 L^{\circ} 8 \mathrm{LL}$

$8 L^{\circ} 8 Z \mathrm{~L}$

6t6ZL

96.6ZL

$19^{\circ} 0 \varepsilon L$

$\nabla L^{\circ} 0 \varepsilon L$

$0 Z$ ' $\downarrow \varepsilon L-$

$\varepsilon \varepsilon^{\circ} \triangleright \varepsilon\llcorner$

乙† $9 \varepsilon L$

80เทレ-

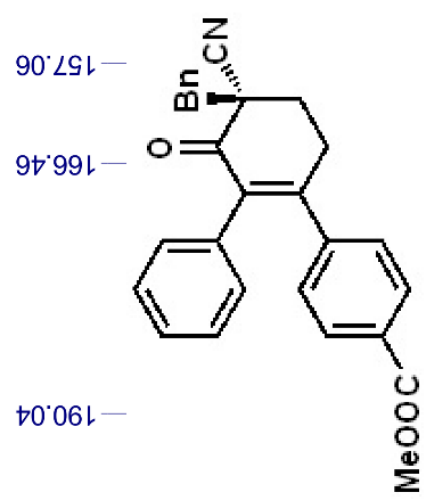

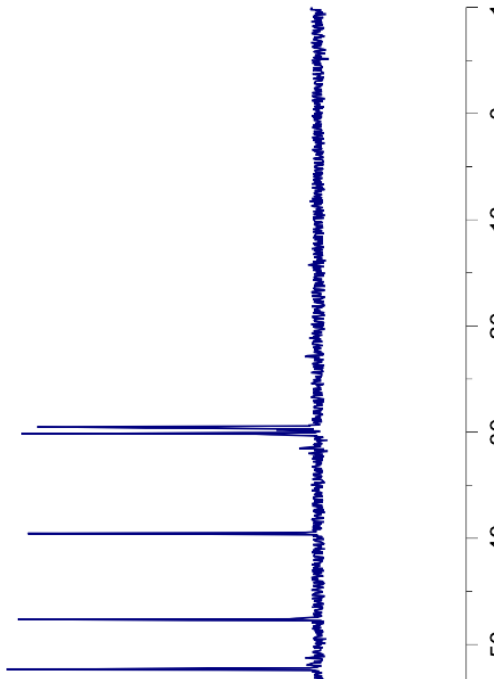

오

8

․ ڤँ

๑ิ

은

ᄋ

을

- Z

음

g

옹

$\stackrel{8}{\circ}$

운

$\stackrel{\infty}{\infty}$

옴 
ชனเc

902'

LLZ'Z

$0 \varepsilon Z \cdot Z$

乙৩乙'乙

19E'乙

$\varepsilon L \varepsilon^{\prime} Z$

๑8$: 乙$

96ย'乙

LOt'Z

s98 $\mathrm{Z}$

$\angle \angle 8^{\circ} \mathrm{Z}$

$888^{\circ} \mathrm{Z}$

†เ6'

926"Z

$\angle \varepsilon 6^{\circ} \mathrm{C}$

$196^{\circ} \mathrm{Z}$

$\varepsilon \varepsilon 0^{\circ} \varepsilon$

$\mathrm{S}^{\circ} 0^{\circ} \varepsilon$

$890^{\circ} \varepsilon$

$0 \angle 0^{\circ} \varepsilon$

$\varepsilon 80^{\circ} \varepsilon$

$\neg 60^{\circ} \varepsilon$

$\angle 0 L^{\circ} \varepsilon$

8เレ'

ZOS ${ }^{\circ} \varepsilon$

$\angle \mathcal{L} \mathcal{G}^{\circ} \mathcal{E}$

806"9

2L6.9

8เ6"9

๕२6"9

५ट6. 9

26. 9

† 6.9

乙†6"9

ES6.9

$\checkmark 90^{\circ} \mathrm{L}$

$\nabla \angle 0^{\circ} \mathrm{L}$

เDL'L

$\angle \nabla L^{\circ} L$

EGL'L

$\angle G L^{\circ} L$

† L $^{\circ} \mathrm{L}$

$\varepsilon L L^{\circ} L$

$6 \angle L^{\circ} \mathrm{L}$

ह8L $L$

ZレでL

乙E乙 'L

$882^{\circ} \mathrm{L}$

$\nabla 6 乙 2$

ZOE' L

$80 \varepsilon^{\circ} L$

$\varepsilon L \varepsilon^{\circ} L$

ह乙E. $L$

$0 \varepsilon \varepsilon^{\circ} L$

GE $\varepsilon^{\circ} L$

$0 \succ \varepsilon^{\circ} L$

$\downarrow \nabla \varepsilon^{\circ} L$

$0 G \varepsilon^{\circ} L$

GSE.L

$\angle 9 \varepsilon^{\circ} L$

$\varepsilon \angle \varepsilon^{\circ} L$

$\angle L E^{\circ} L$

S8E"L

$68 \varepsilon^{\circ} L$

$\varepsilon 6 \varepsilon^{\circ} L$

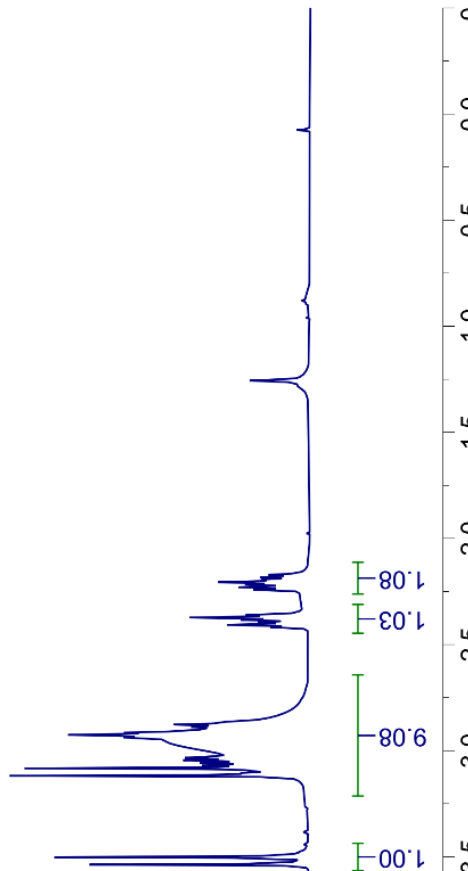

@

$\stackrel{ }{\circ}$

$\stackrel{n}{-}$

오

$\ddot{m}$

$-00$.

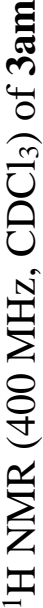

$\stackrel{\circ}{\circ}$

-502
$1-00^{\circ} z$

- $90^{\circ} \varepsilon$

ก20.9

ก

$\stackrel{\circ}{\infty}$

$\infty$

ஸे

คి

움. 
GS.6Z

レレ 0 -

$\angle S^{*} 6 \mathcal{E}$

$29^{\circ} \angle \nabla^{\circ}$

89'8ルレ-

$\angle 0 \angle Z L$

$99^{\circ} \mathrm{LZL}$

$6 \angle L Z$ -

$66^{\circ} \mathrm{LZL}$

ZZ 8ZL

†L'8Zレ

$6 S^{\circ} 0 \varepsilon L$

$18^{\circ} 0 \varepsilon \mathrm{L}$ -

$8 \varepsilon^{\circ} \nabla \varepsilon\llcorner$

ฤヤ゙レ

9l.9ع

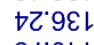

GLOOL
$6 Z \angle G L$

6ट 29

$08^{\circ} 0 \angle L$

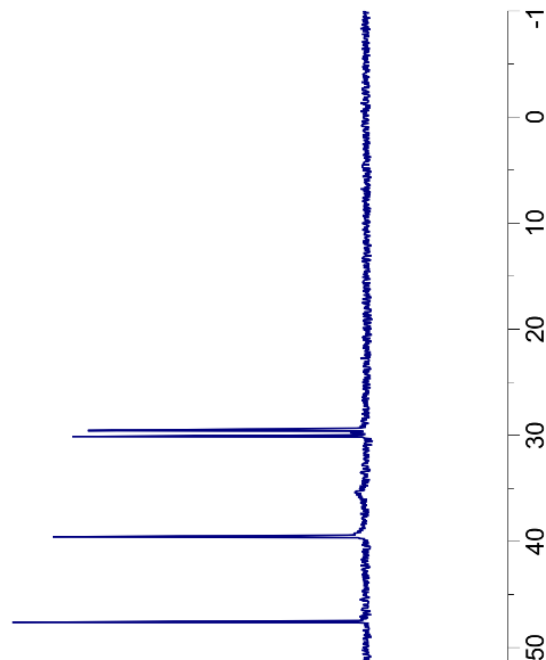

in

:

○ 节

-

\&ิ

\& 8

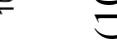

을

Z

요

온

요

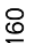

요

$\stackrel{\infty}{\infty}$

옹

ㅇ 
6ㄴ. $\varepsilon$

LIG'

乙G. $\mathcal{~}$

$8 \angle 8^{\circ} \varepsilon$

เE6. 9

LE6.9

$\nabla \nabla 6.9$

$9 \rightarrow 6.9$

096.9

\&96. 9

996.9

$\mathrm{LOL} L$

ULL

GHLL

LCL'L

LEL L

GEL L

$19 L^{\circ} \mathrm{L}$

$0 \angle L L$

$9 \angle \mathrm{L} \angle$

Z8L'L

G81'L

$\angle 8 \mathrm{~L}^{\circ} \mathrm{L}$

$76 L^{\circ} L$

ELC L

6เE'L

†乙E' \llcorner

$\downarrow \varepsilon \varepsilon^{\circ} L$

乙† $L$

$\angle \forall \varepsilon^{\circ} L$

乙GE L

GSE. L

$\varepsilon 9 \varepsilon^{\circ} L$

$\angle 9 \varepsilon^{\circ} L$

$6 \angle E^{\prime} L$

$\neg 8 \varepsilon^{\circ} L$

$88 \varepsilon^{\circ} L$

LOt $L$

¿ह8. $L$

$8 \varepsilon 8^{\circ} L$

乙†8 L

$\forall \forall 8^{\circ} \mathrm{L}$

$878^{\circ} L$

Z98 $\mathrm{L}$

$\varepsilon 98^{\circ} \mathrm{L}$

$\angle 98^{\circ} \mathrm{L}$

I $\angle 8^{\circ} L$
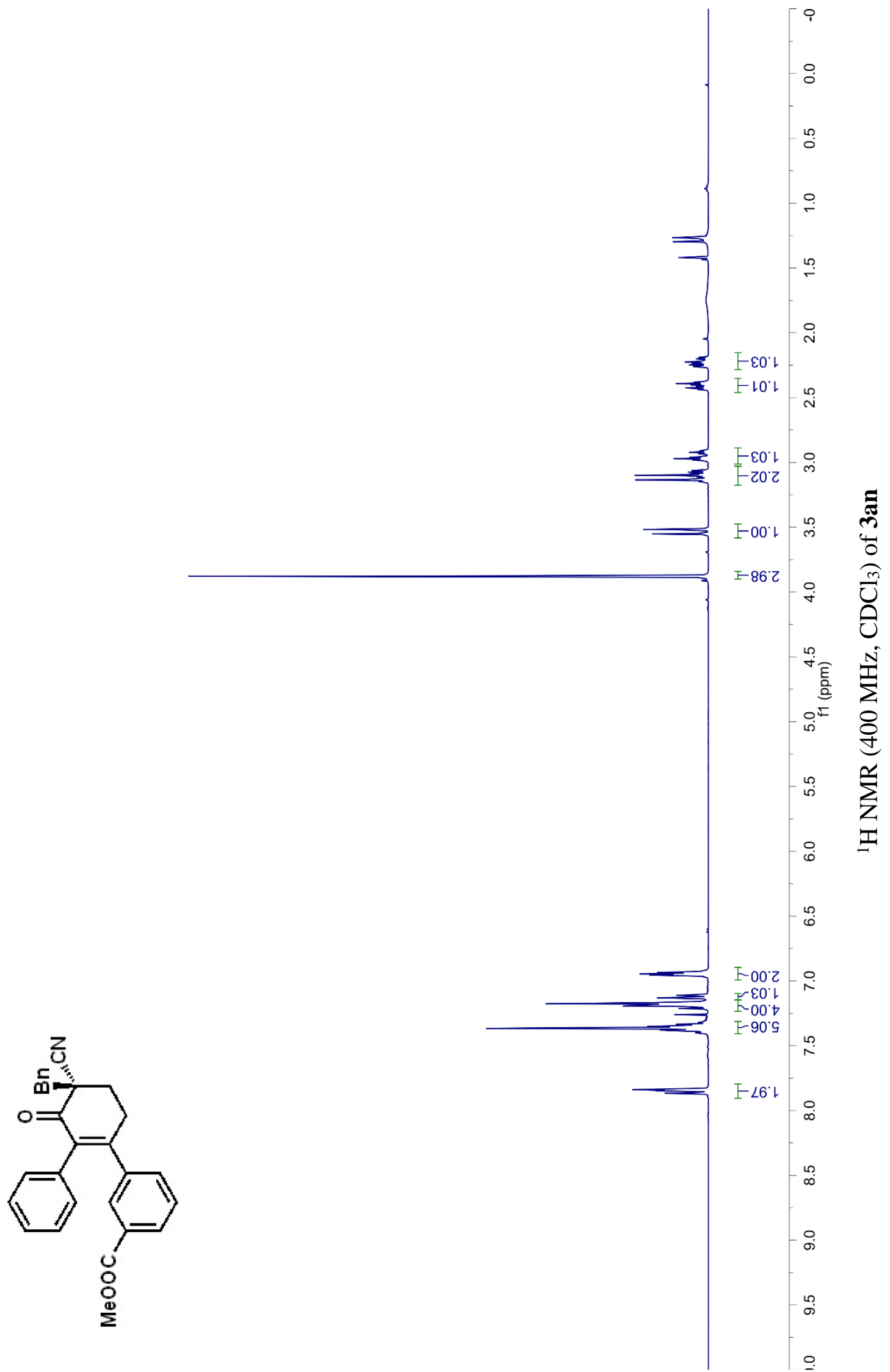
$\angle S^{\circ} 62$

†レ'OE-

$\angle \mathcal{G}^{\prime} 6 \mathcal{E}-$

$\checkmark 9^{\circ} \angle \nabla$

$\angle E^{\prime} Z S$

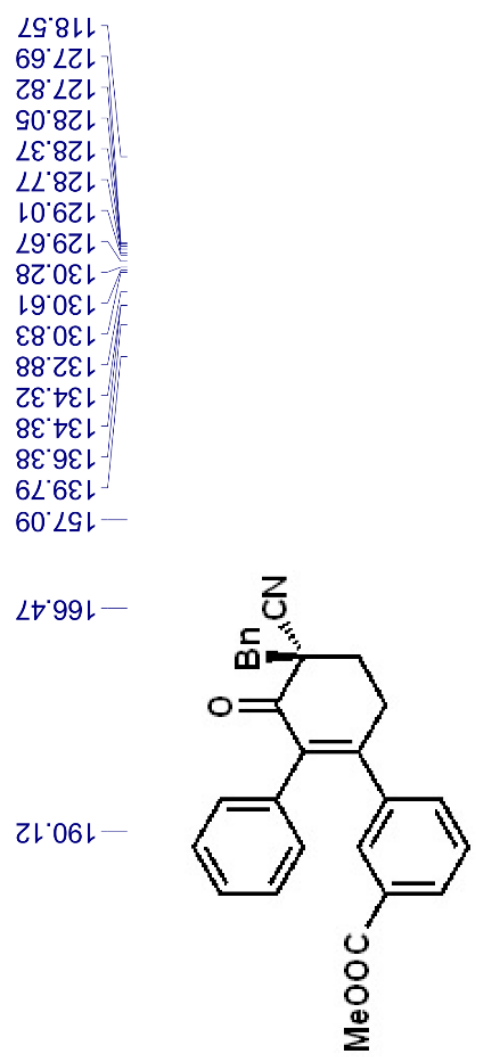

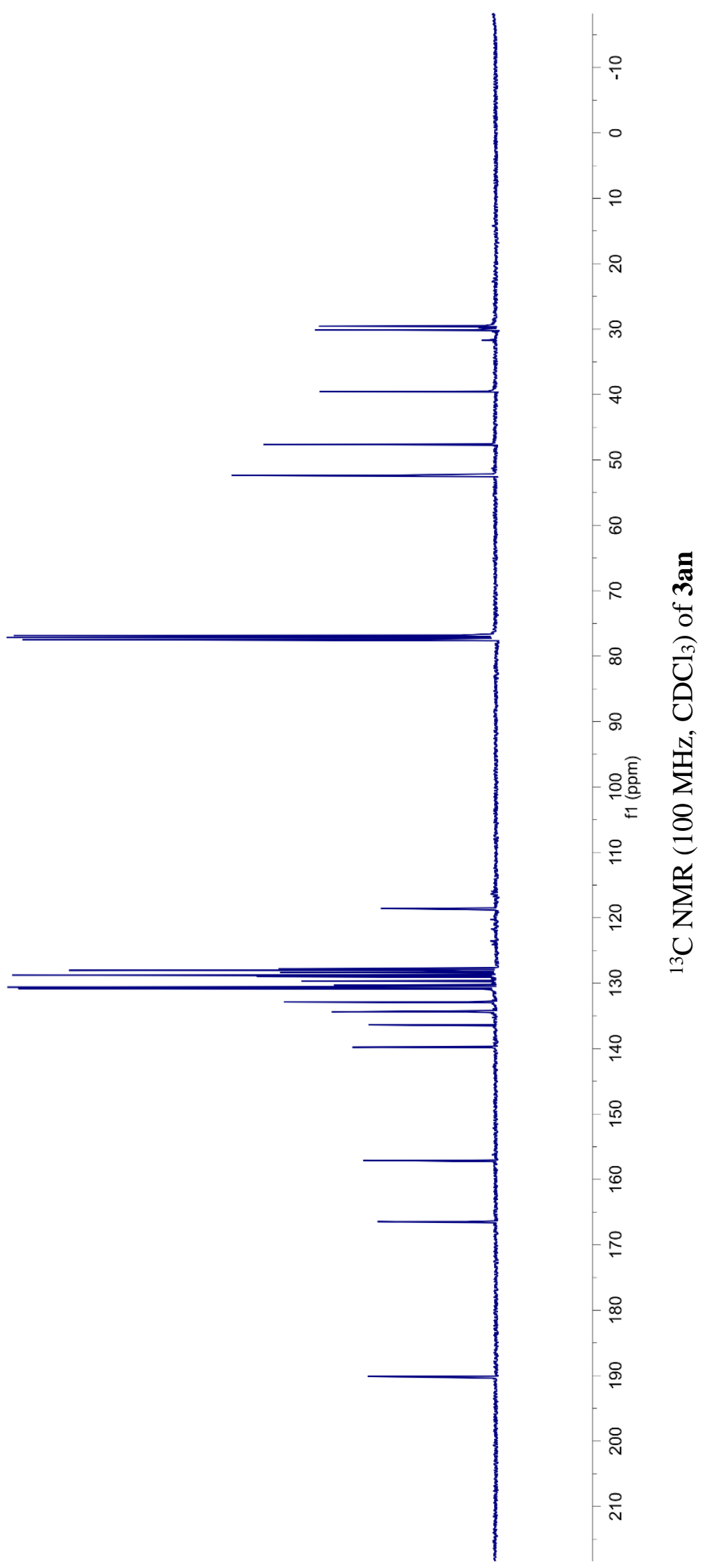


乙8E' $L$

$98 \varepsilon^{\circ} L$
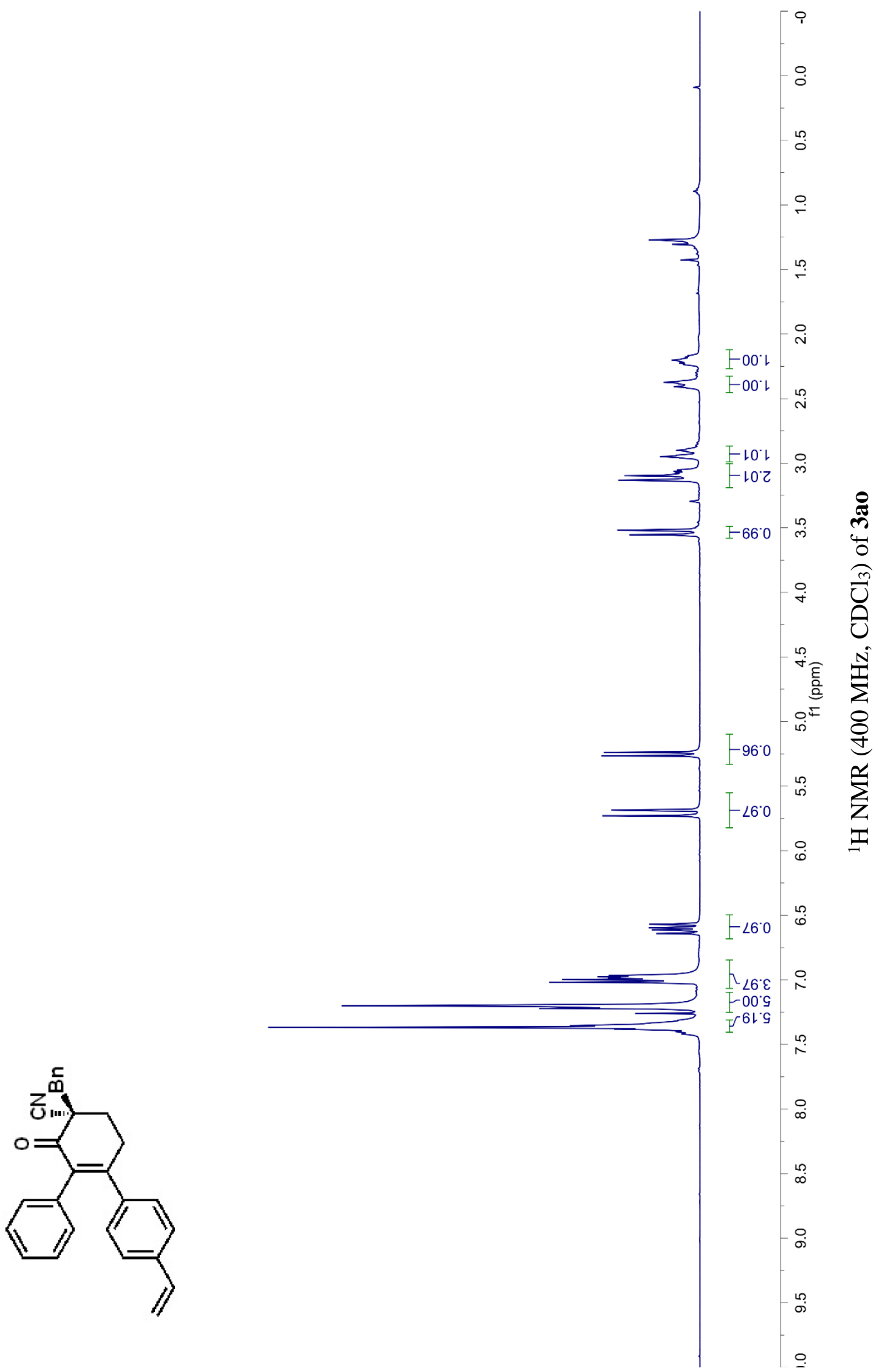
$69^{\circ} 6 \mathcal{E}$

$99^{\circ} \angle \nabla$

89.8Z -

9L8ZL

†9 0 \&

乙6.0 0 L

$\varepsilon G^{*} \nabla \varepsilon L$

Z6 $\triangleright \varepsilon\llcorner-$

$\neg 9^{\circ} \mathrm{SEL}$

Go.9ع .

98 ' $\angle E L$

ट8. $8 \mathrm{EL}$

$\angle 8^{\circ} \angle G$ L

9l.06L-

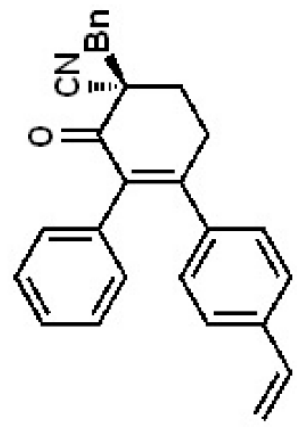




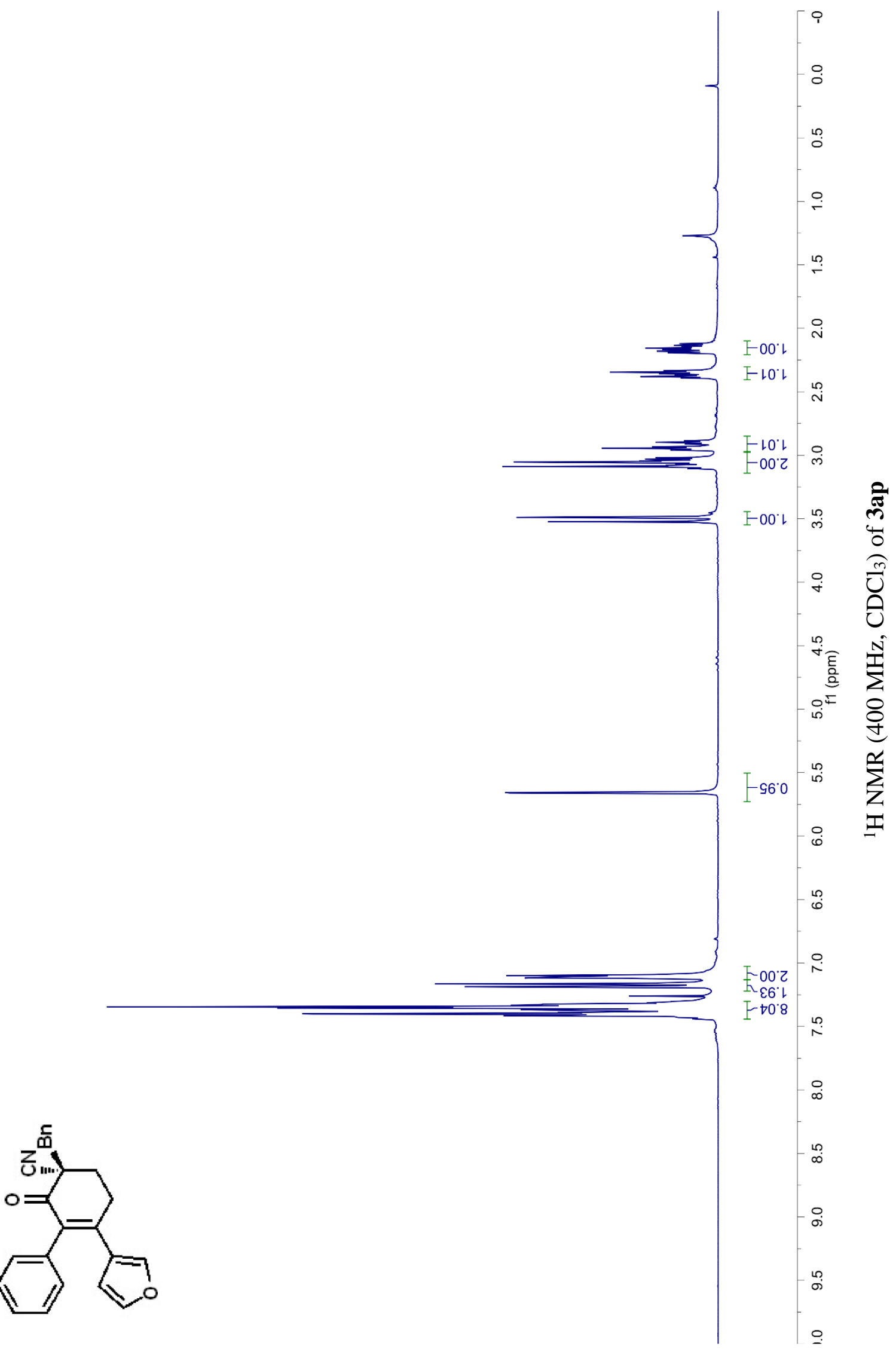


$500^{\circ} \angle Z-$

$9 L^{\circ} 62$

${ }^{\circ} 6 \varepsilon-$

$9 \nabla^{\circ} \angle t-$

$66^{\circ} 601-$

08.8ル -

$\angle 9^{\circ} \nabla Z L$

$\forall L \angle Z L$

$0 \varepsilon 8 Z$ -

$\varepsilon L^{\circ} 8 Z L$

$76{ }^{\circ} 8 \mathrm{ZL}$

E6 62l-

$09^{\circ} 0 \varepsilon \mathrm{L}$

$10 \circ \varepsilon L^{\circ}$

$s s^{\circ} \nabla \varepsilon-$

$\angle 0^{\circ} 9 \varepsilon L$

レでャレ

8ガทtレ

$L \angle \angle T L$

เ8 68 L-

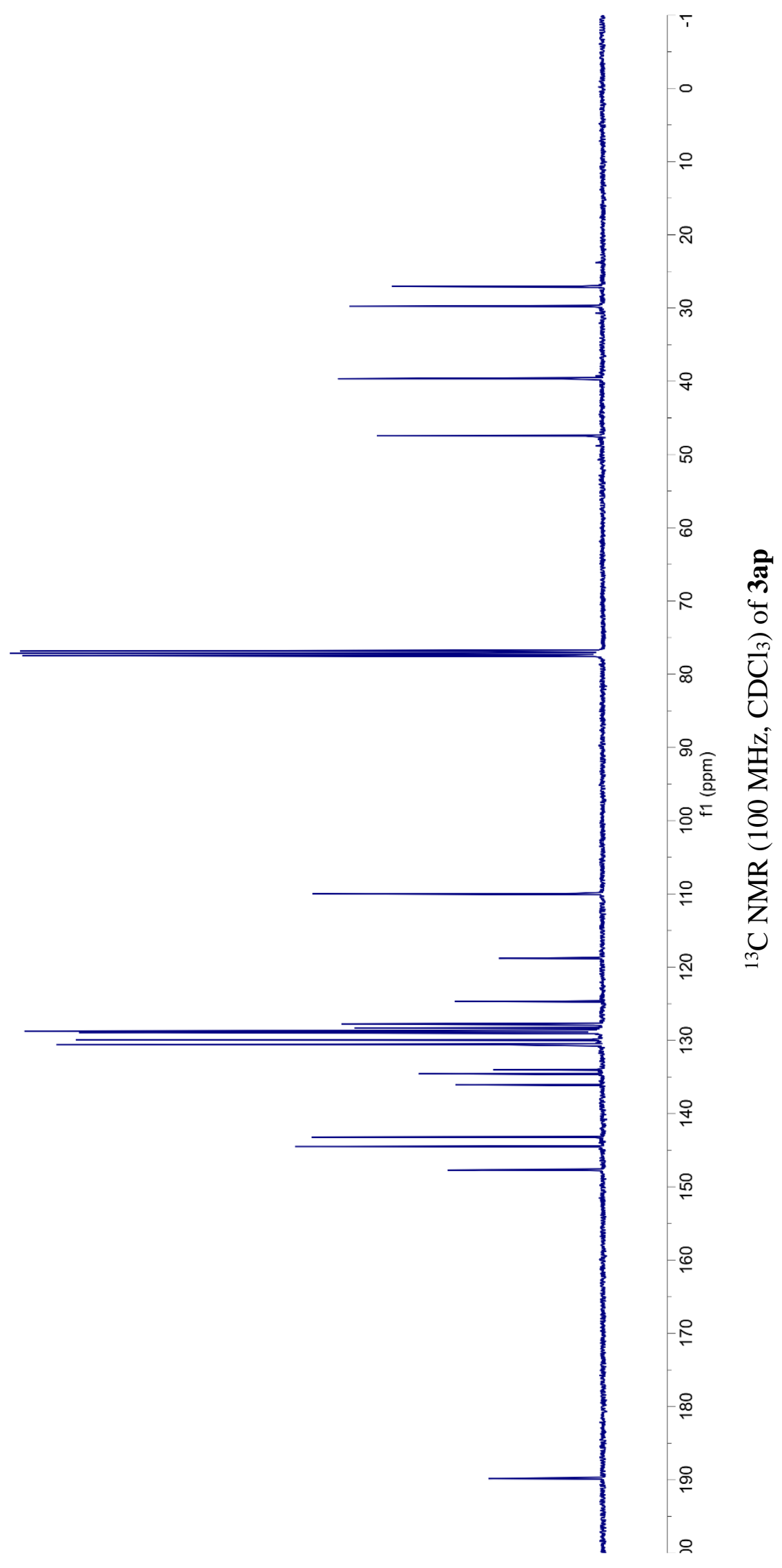



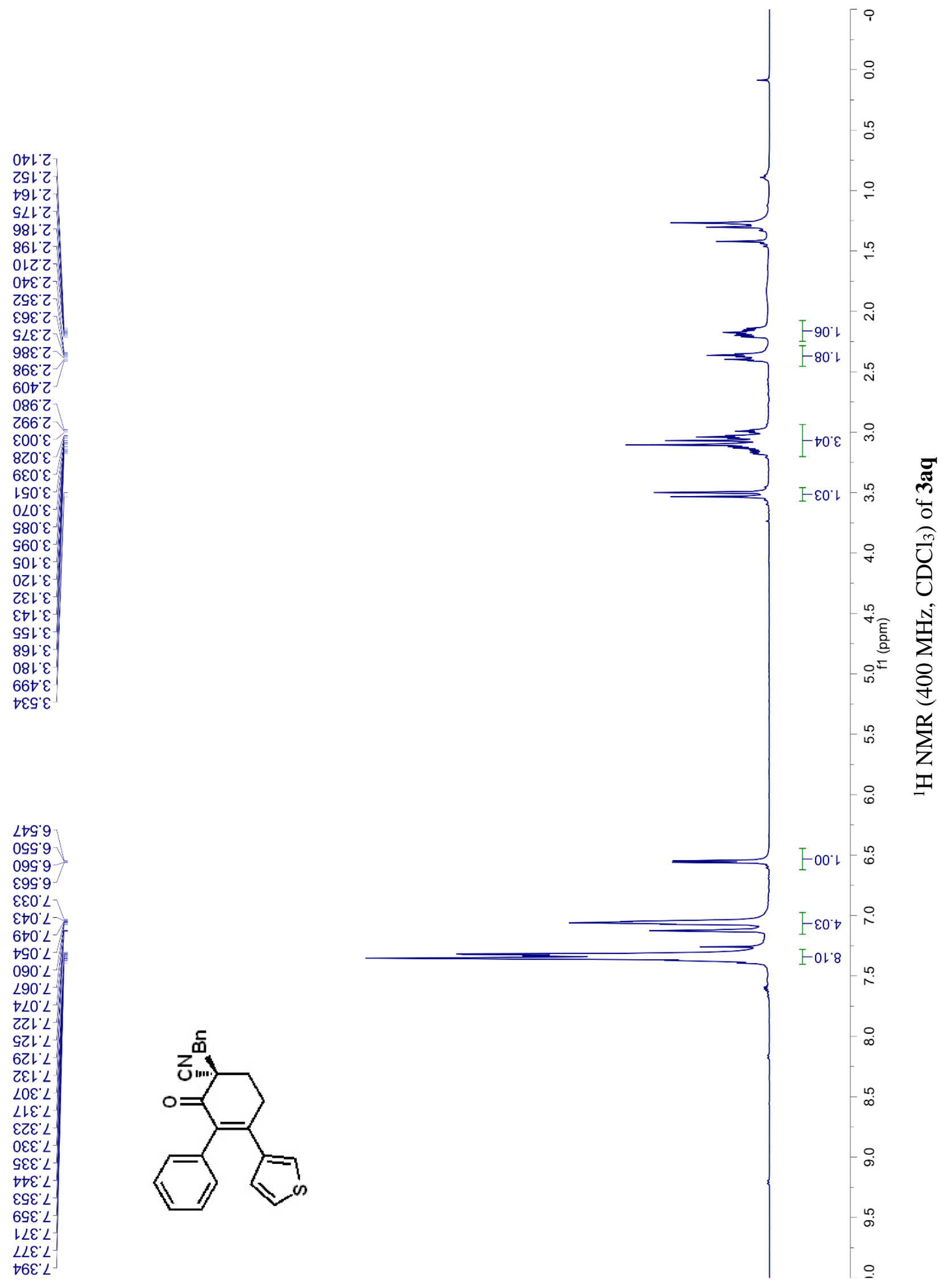
$99^{\circ} \nabla \varepsilon L$

L8.SEL

S96ع

E6.09ᄂ

9Z'06เ
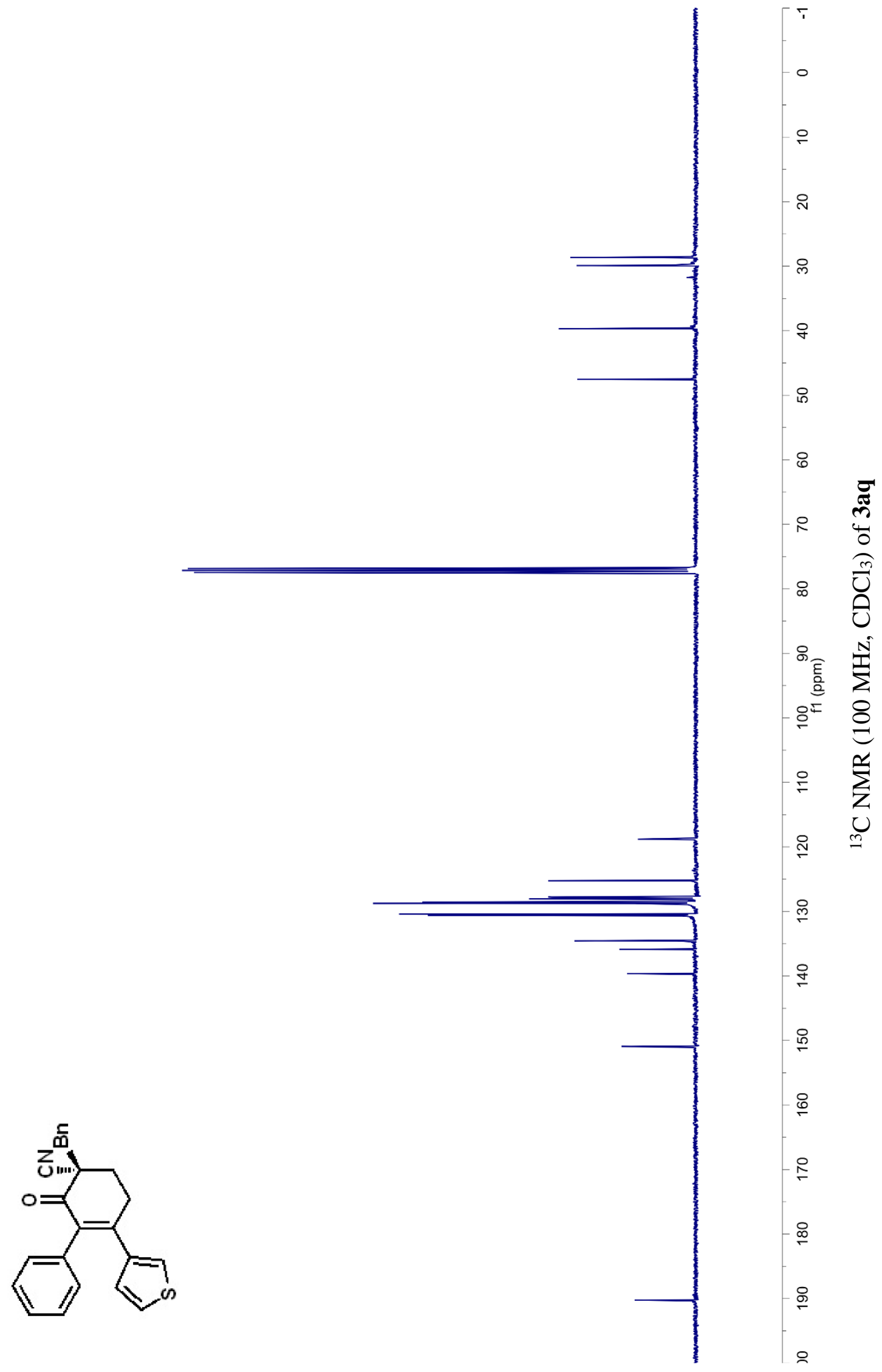

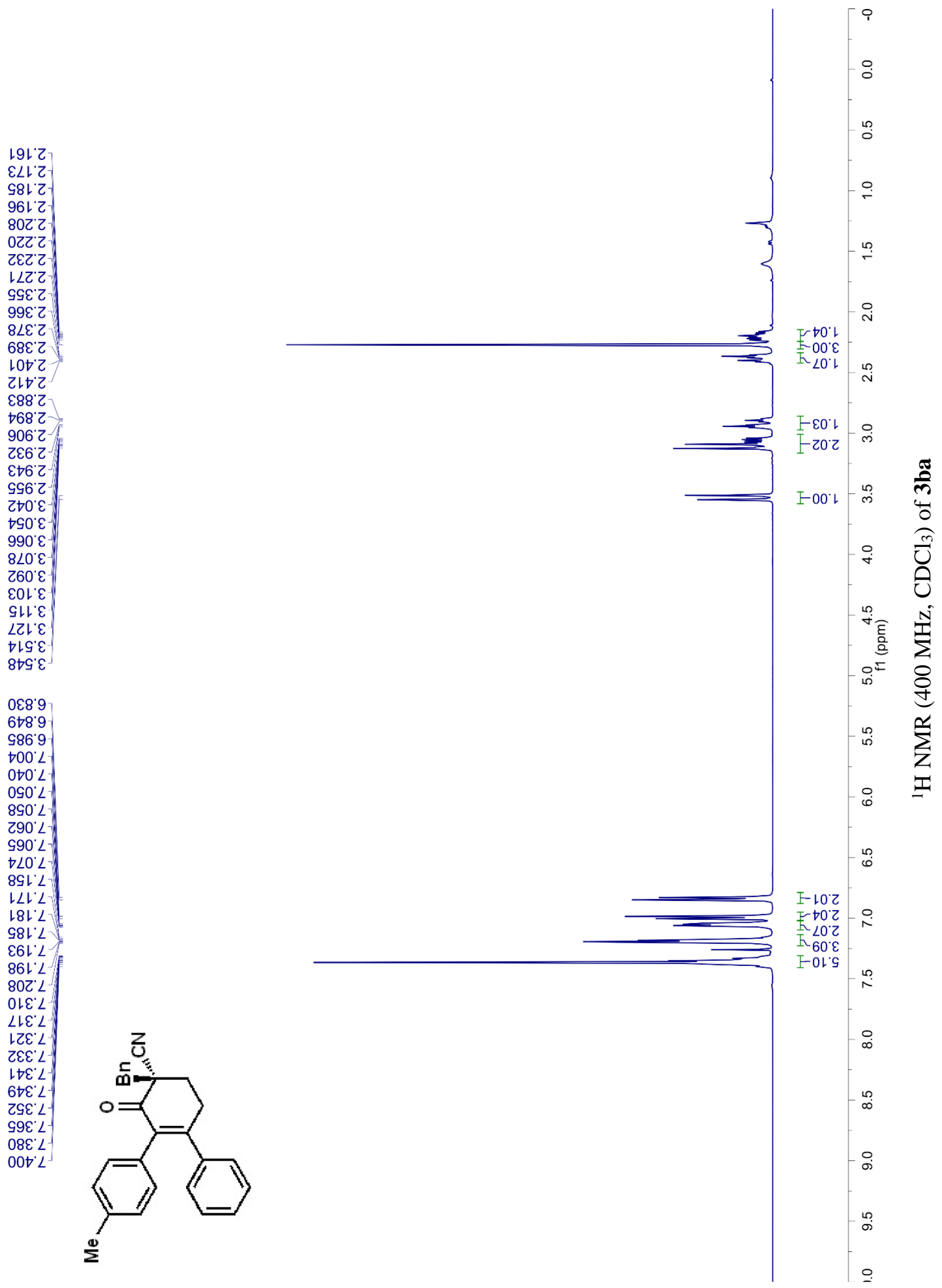
SE'L-

SL' 62

Sl.0E-

t9.6ع-

$\neg 9^{\circ} \angle t$

8L'8L-

SL'LZL

ZZ'8ZL

EZ $8 Z$ -

99.8ZL

LL'8ZL

$\checkmark L \cdot 8 Z L$

G9.0ع

$\nabla L L^{\circ} 0 \varepsilon L$

$\varepsilon L \cdot L E L$

9G' $\nabla \varepsilon L$

S9. $9 E$

SL $\angle E L$

LL6EL

Lo $8 \mathrm{SL}$

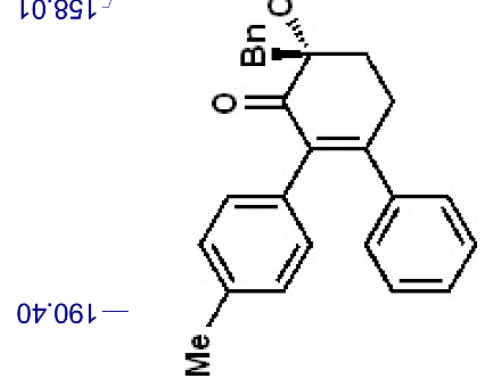

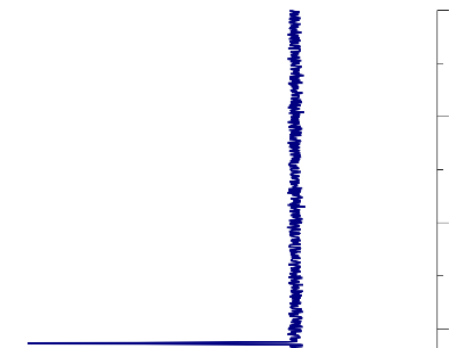

ำ

이

우

오

8

० ले

$\therefore \quad$

$\therefore \quad$

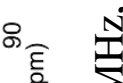

은

을

ㄴ

ติ

g

옹

$\stackrel{\circ}{\circ}$

운

$\stackrel{\infty}{\sim}$

옴

○ 
$9 \nabla L^{\prime} \varepsilon$

90L' 9

OLL'9

ZZL'9

$\angle Z L \cdot 9$

EL 9

GEL' 9

$\angle E L ' 9$ -

$\angle \nabla 8 \cdot 9$

† 9

698: 9

†98. 9

$0 \angle 8^{\circ} 9$

9L8' 9

088.9

$\angle 88^{\circ} 9$

IEO 2

SEO L

$0 \rightarrow 0^{\circ} L$

$S \rightarrow 0^{\circ} L$

$670^{\circ} \mathrm{L}$

$290^{\circ} \mathrm{L}$

$990^{\circ} \mathrm{L}$

$690^{\circ} \mathrm{L}$

$\angle 90^{\circ} \mathrm{L}$

$\mathrm{Z8L}$ L

$06 \mathrm{~L}^{\circ} \mathrm{L}$

L6L'L

$00 Z$ ' $\angle$

แE'L

GIE.L

9ZE L $L$

GEE'L

$8 \varepsilon \varepsilon^{\circ} L$

$\varepsilon \forall \varepsilon^{\circ} L$

$\angle \nabla \varepsilon^{\prime} \angle$

$09 \varepsilon^{\circ} L$

$\varepsilon \angle \varepsilon^{\circ} L$

$G \angle E^{\circ} L$

$\varepsilon 6 \varepsilon^{\circ} L$

$\angle 6 \varepsilon^{\prime} L$

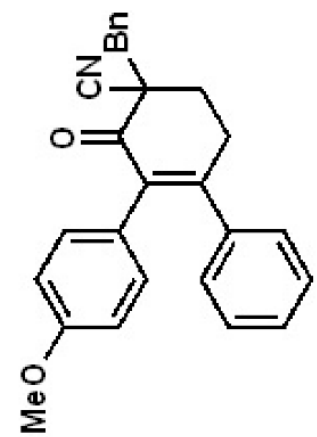




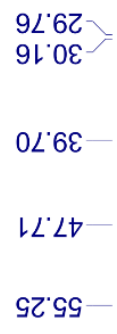

8จ゙ยレー

L8: 8 th

$8 L \angle Z L$

9Z 8ZL

เE'8ZL

9G'8ZL

LL'8ZL

$99^{\circ} 0 \varepsilon\llcorner$

GL'ZEL

6G' $\nabla \varepsilon L$

9ट'GEL

乙8. $6 \varepsilon$ ᄂ
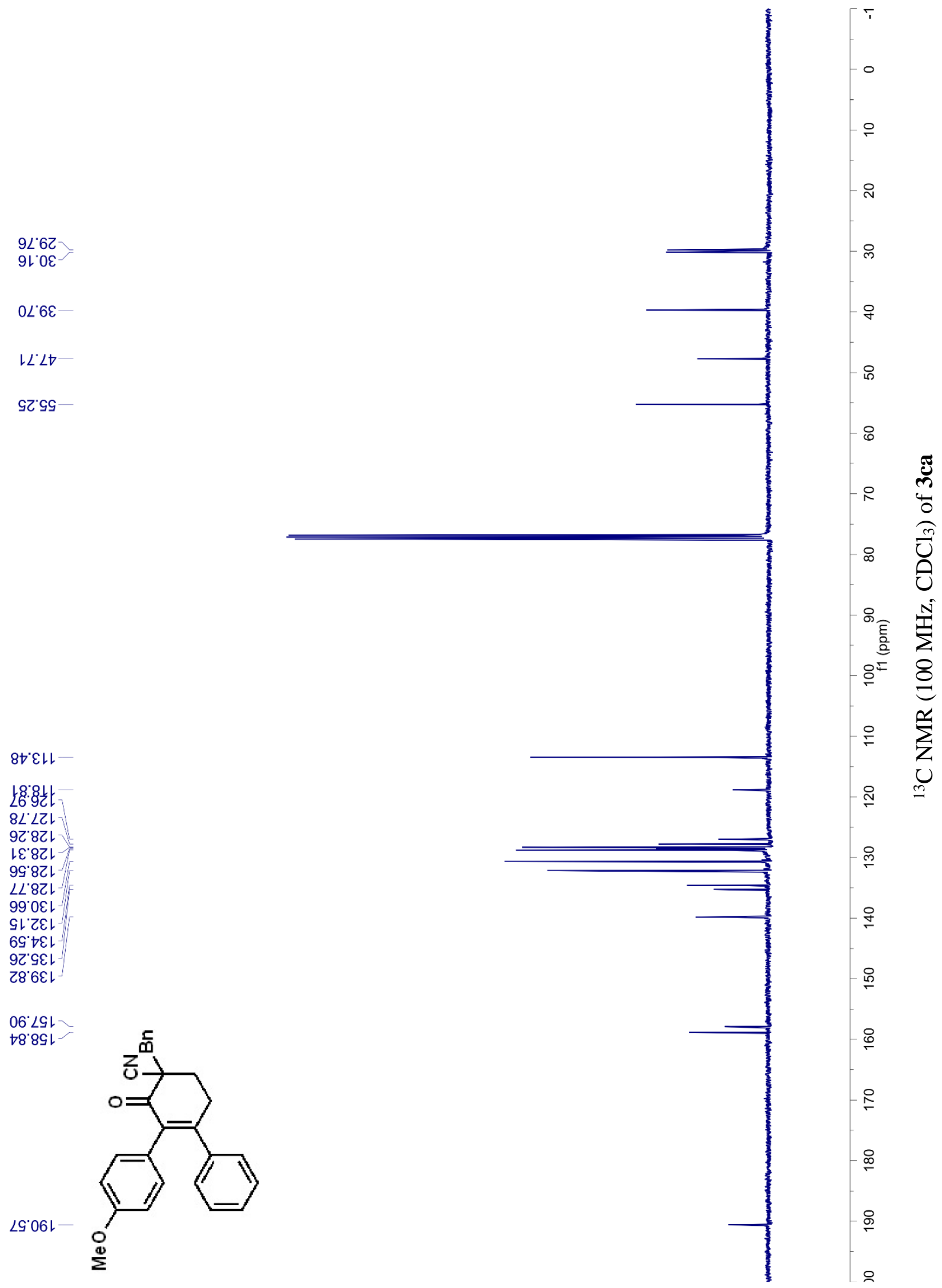

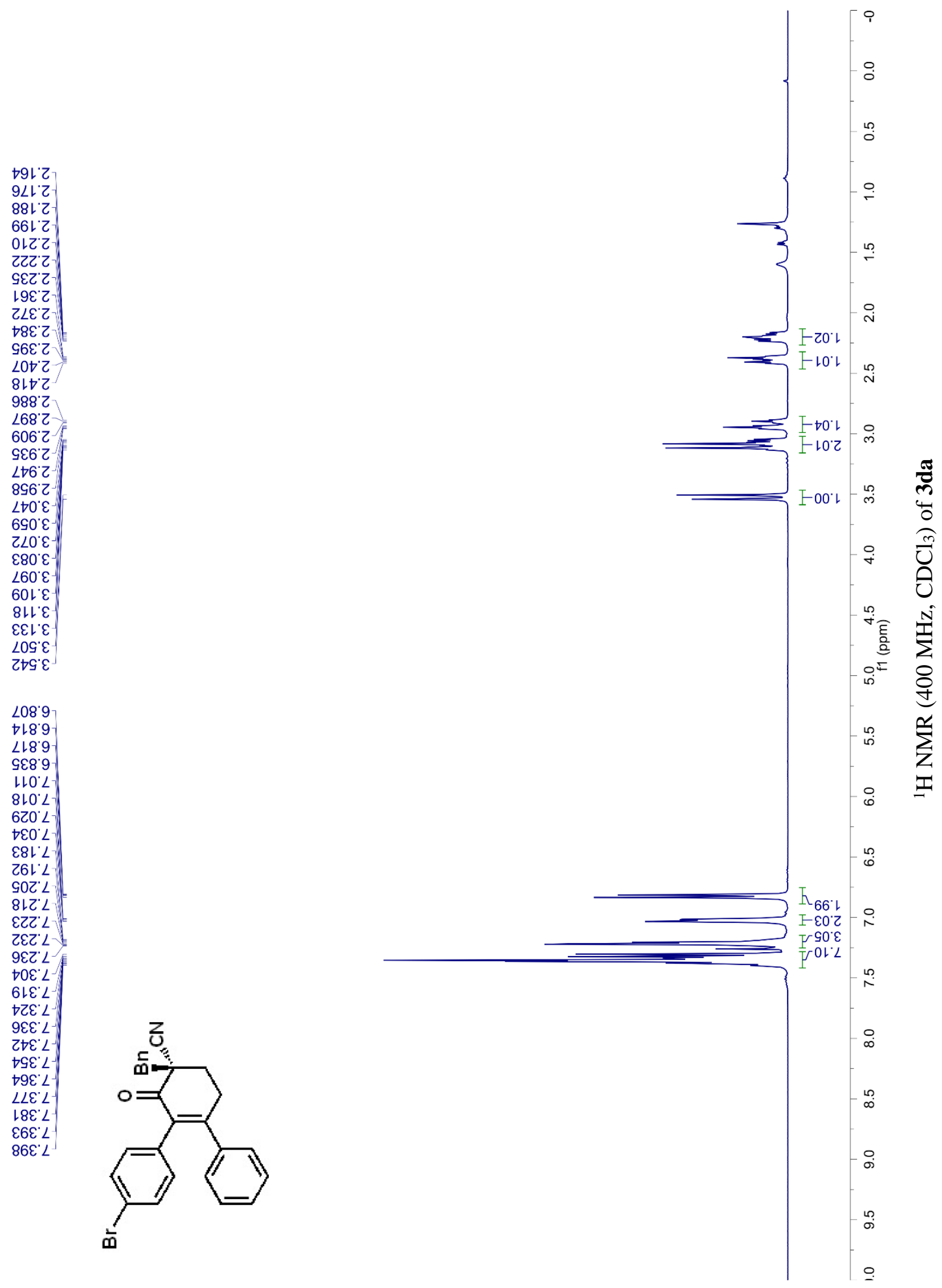
$09^{\circ} 6 \varepsilon$

$6 G^{\circ} \angle \nabla-$

L98ーレ-

LLレZL-

G8'LZL

ル゚ス

87.8Zレ

08.8Z

96.8Zレ

$29^{\circ} 0 \varepsilon \mathrm{L}$

$\angle L^{\circ} L E$ -

Z9'乙E -

$\neg L \mathcal{E} \varepsilon$ -

$9 \varepsilon^{\circ} \triangleright \varepsilon\llcorner$.

$\varepsilon 9^{\circ} \nabla \varepsilon\llcorner$

$9 L^{\circ} 6 \varepsilon L^{-}$

6เ'6sᄂ
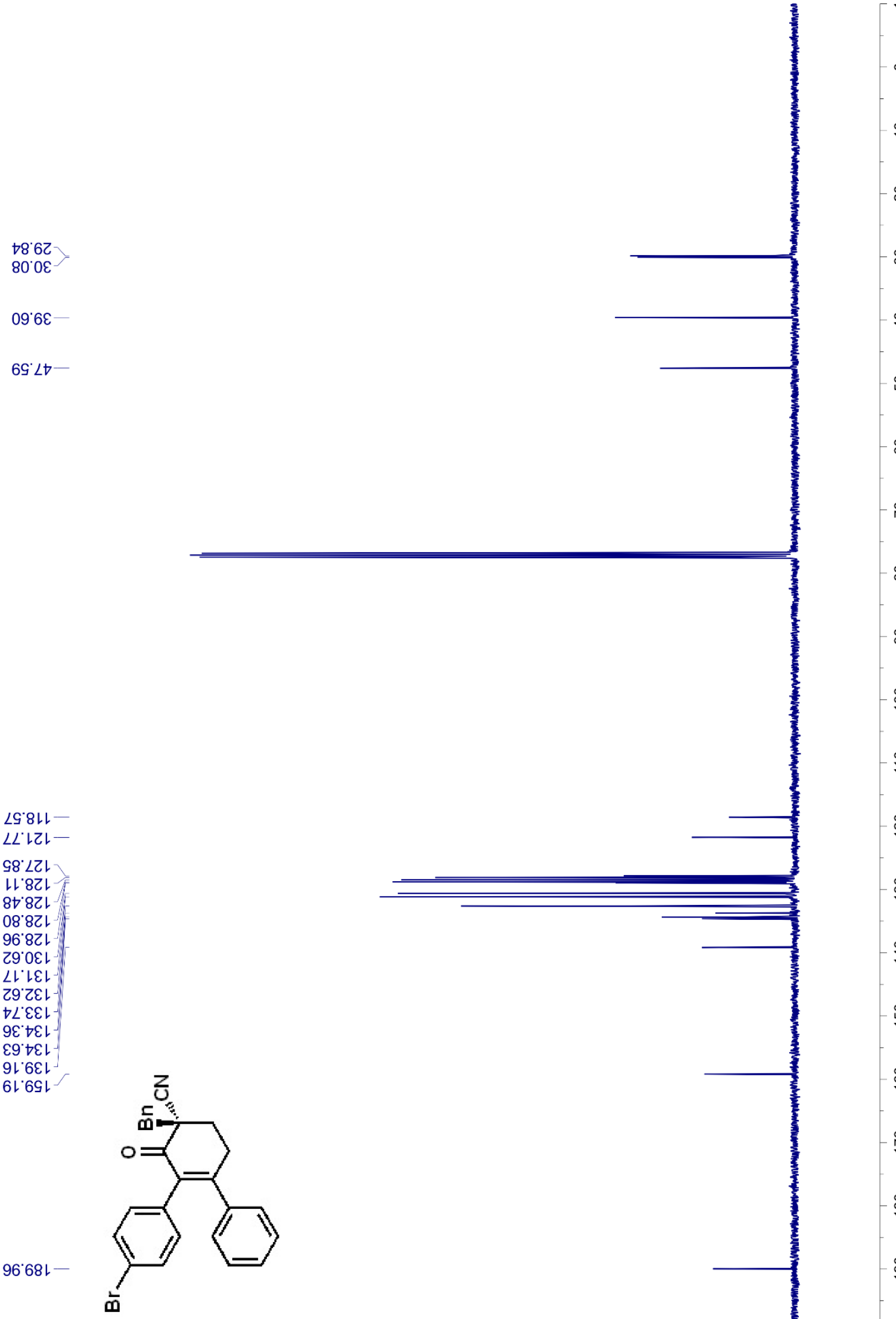
西々

90Z乙

8L乙'

6己乙'乙

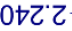

乙G乙 乙

S9Z'Z

L8E乙

$86 \varepsilon^{\prime} 乙$

$60 \nabla^{\prime} Z$

ᄂ 2

乙Et

t大t

626' 2

$0+6.2$

$\angle 96^{\circ} \mathrm{Z}$

$8 \angle 6^{\prime} Z$

$066^{\circ} \mathrm{Z}$

$\left.980^{\circ} \varepsilon\right\urcorner$

$\angle 60^{\circ} \mathcal{E}$

ZOL $\varepsilon$

0 느

乙乙ட $\varepsilon$

$\angle \varepsilon L^{\circ} \varepsilon$

$\angle \nabla L \varepsilon$

6GL

LLI $\varepsilon$

6 LG'

$\supset G \mathcal{G}^{\prime} \varepsilon$

$666^{\circ} 9$

$200^{\circ} \mathrm{L}$

$\angle 00^{\circ} \mathrm{L}$

$\mathrm{ZLO} \mathrm{L}$

$\angle 1 O^{\circ} \mathrm{L}$

$0 Z 0^{\circ} \mathrm{L}$

$\mathrm{EZO}^{\circ} \mathrm{L}$

$920^{\circ} \mathrm{L}$

$\varepsilon \varepsilon 0^{\circ} \mathrm{L}$

$890^{\circ} \mathrm{L}$

$880^{\circ} \mathrm{L}$

GLL'L

$08 L^{\circ} L$

$\varepsilon 8 \mathrm{~L} L$

$\angle 8 L^{\circ} \mathrm{L}$

$06 \mathrm{~L}^{\circ} \mathrm{L}$

$86 \mathrm{~L}^{\circ} \mathrm{L}$

ZOZ'L

$90 Z$ L

ル乙 L

9l乙L

LZZ L

†ZZ L

เEZ L-

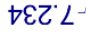

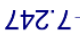

9ZE'L

เEE $L$

L $\mathcal{E}^{\circ} L$

$9 \nabla \varepsilon^{\prime} L$

IGE'L

I $9 \varepsilon^{\circ} L$

$\varepsilon 9 \varepsilon^{\circ}\llcorner$

L $\angle E^{\prime} L$

$\varepsilon 8 \varepsilon^{\circ} L$

$68 \varepsilon^{\circ} L$

$\varepsilon 6 \varepsilon^{\circ} L$

LOt $L$

$90 t L$

OLV L

SEt L

GSt L

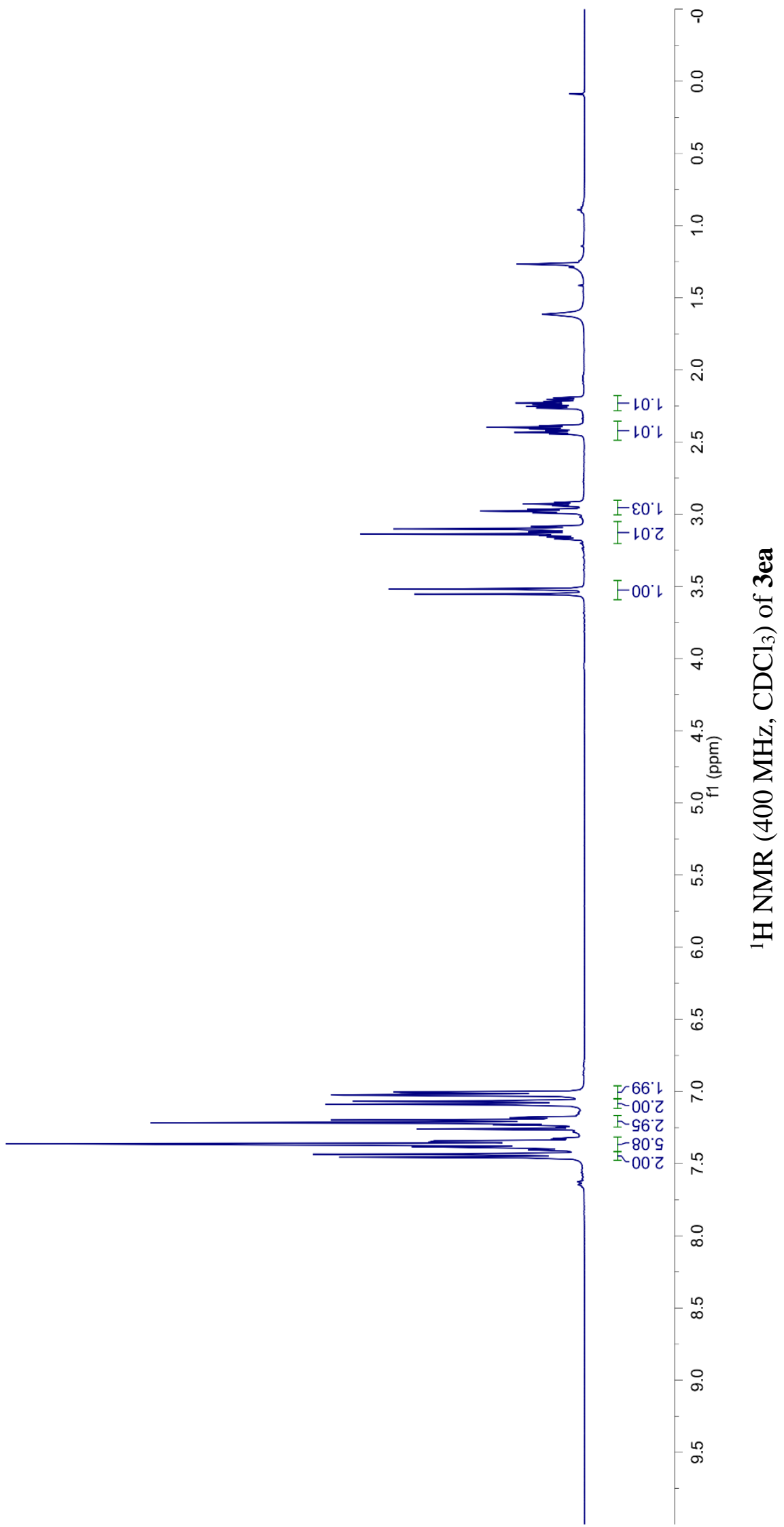



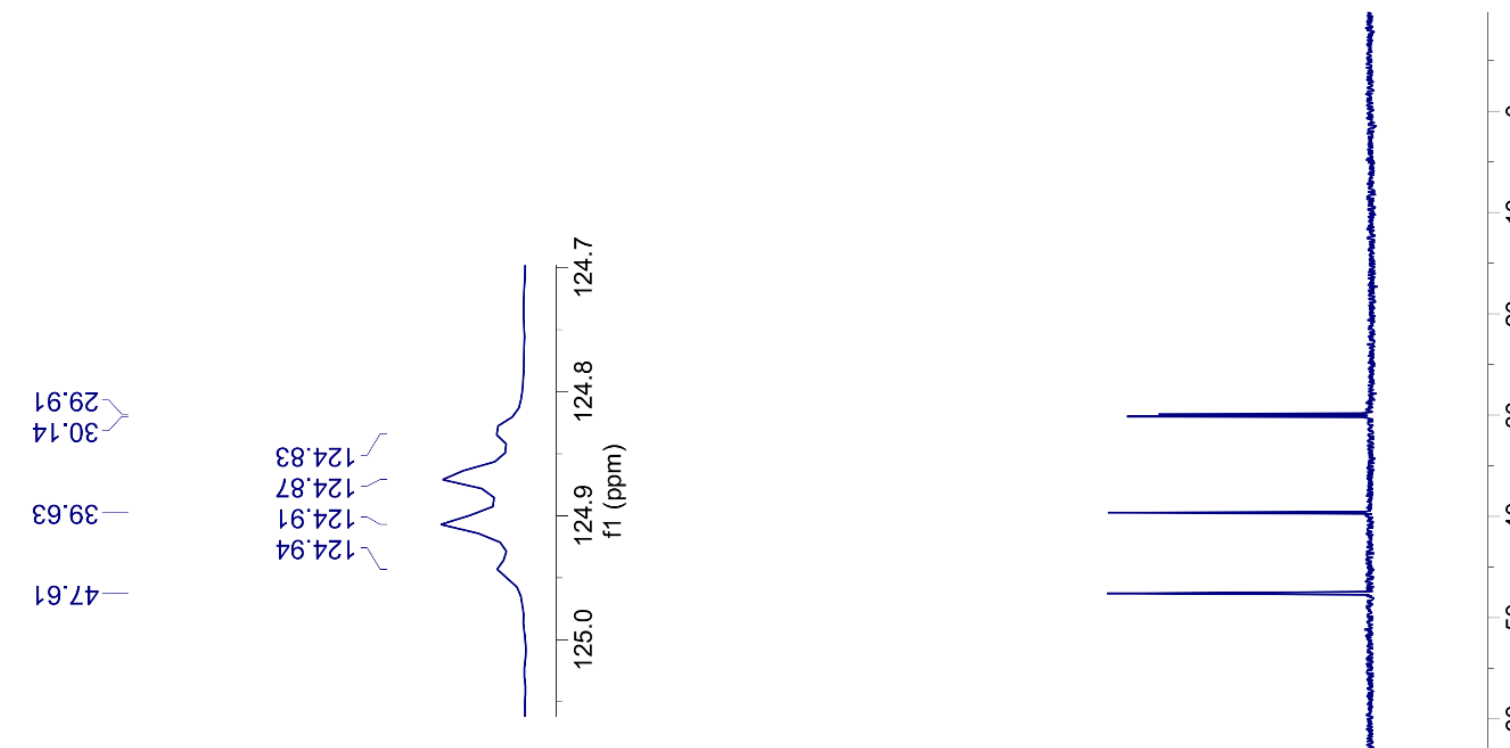

Z9'8เ

$60^{\circ}$ 0ZL

6LててL

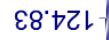

$\angle 8 \nabla Z L$

レ6ってレ

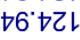

OG'GZL

$06^{\circ} \angle Z L$

80.8ZL.

L'8ZL

ZG'8ZL

ع8.8Zレ

E0 6ZL -

Gl'6ZL-

与ะ'6Zレ

L96ZL.

$00^{\circ} 0 \varepsilon^{\circ}$.

$\succ 9^{\circ} 0 \varepsilon$ L

$9 \varepsilon^{\circ}\llcorner\varepsilon\llcorner$

乙Eं $\nabla \varepsilon\llcorner$ -

乙9. $\nabla \varepsilon\llcorner-$

L $\angle 8 L$.

乙6.8\&

Z6.69l-
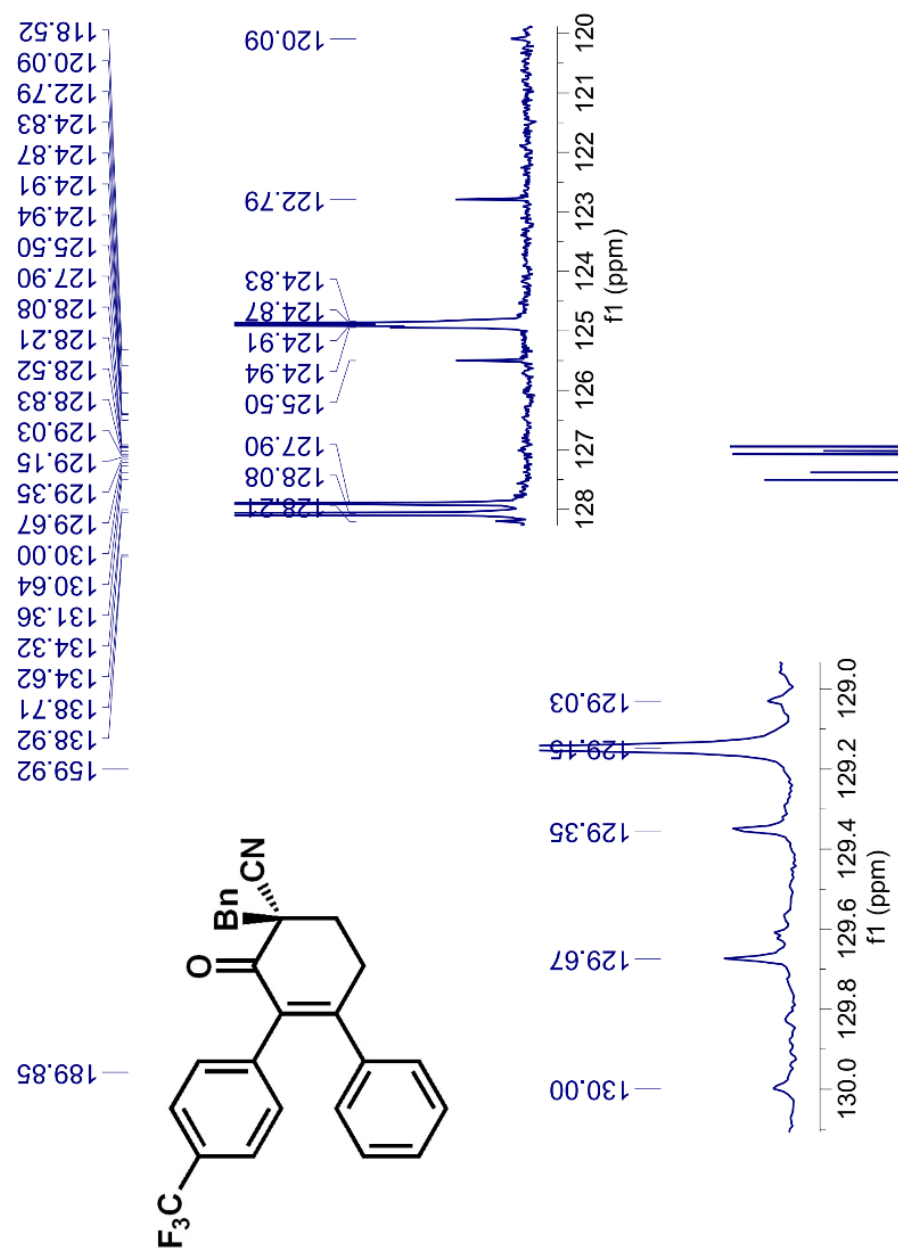


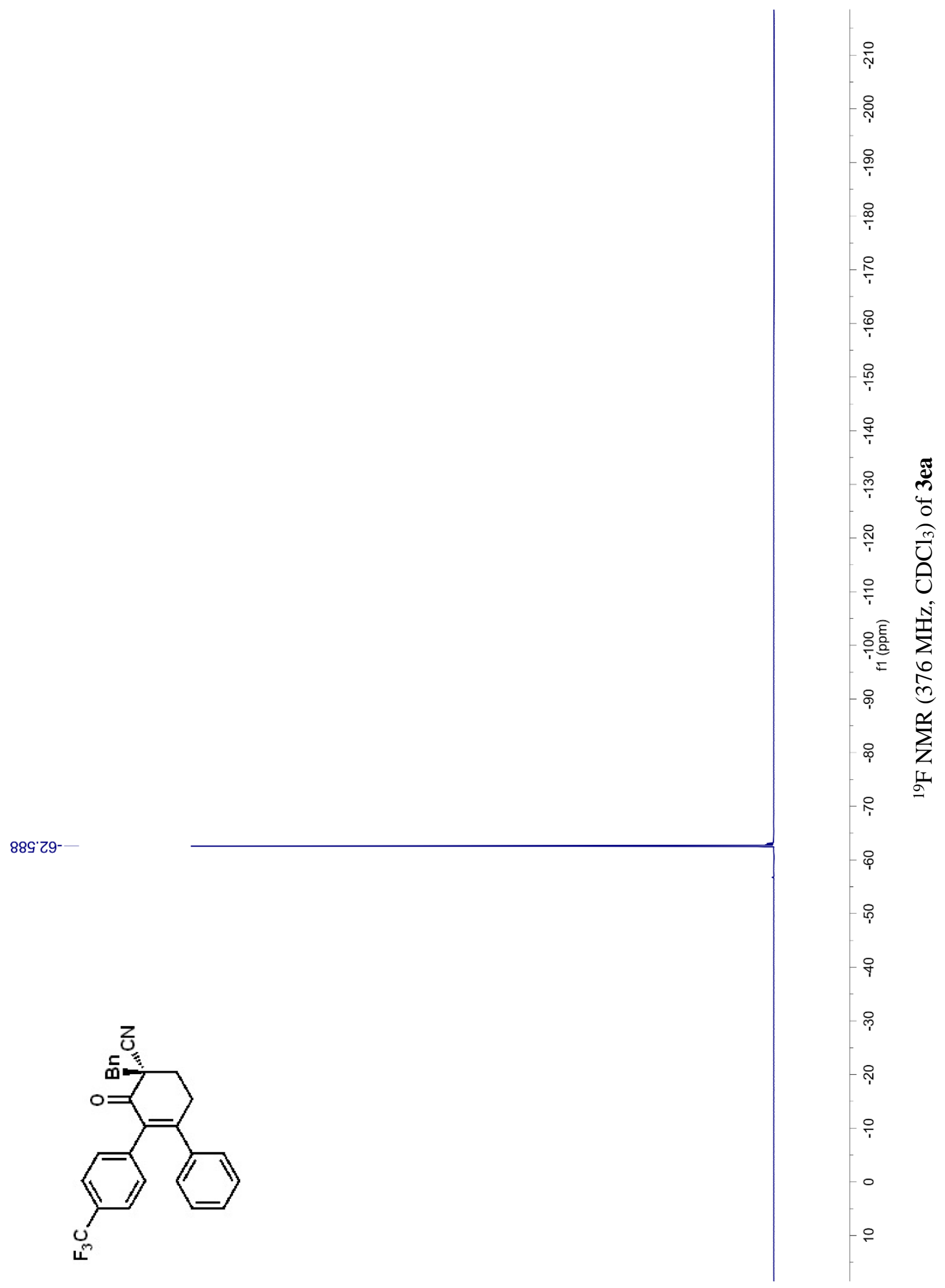



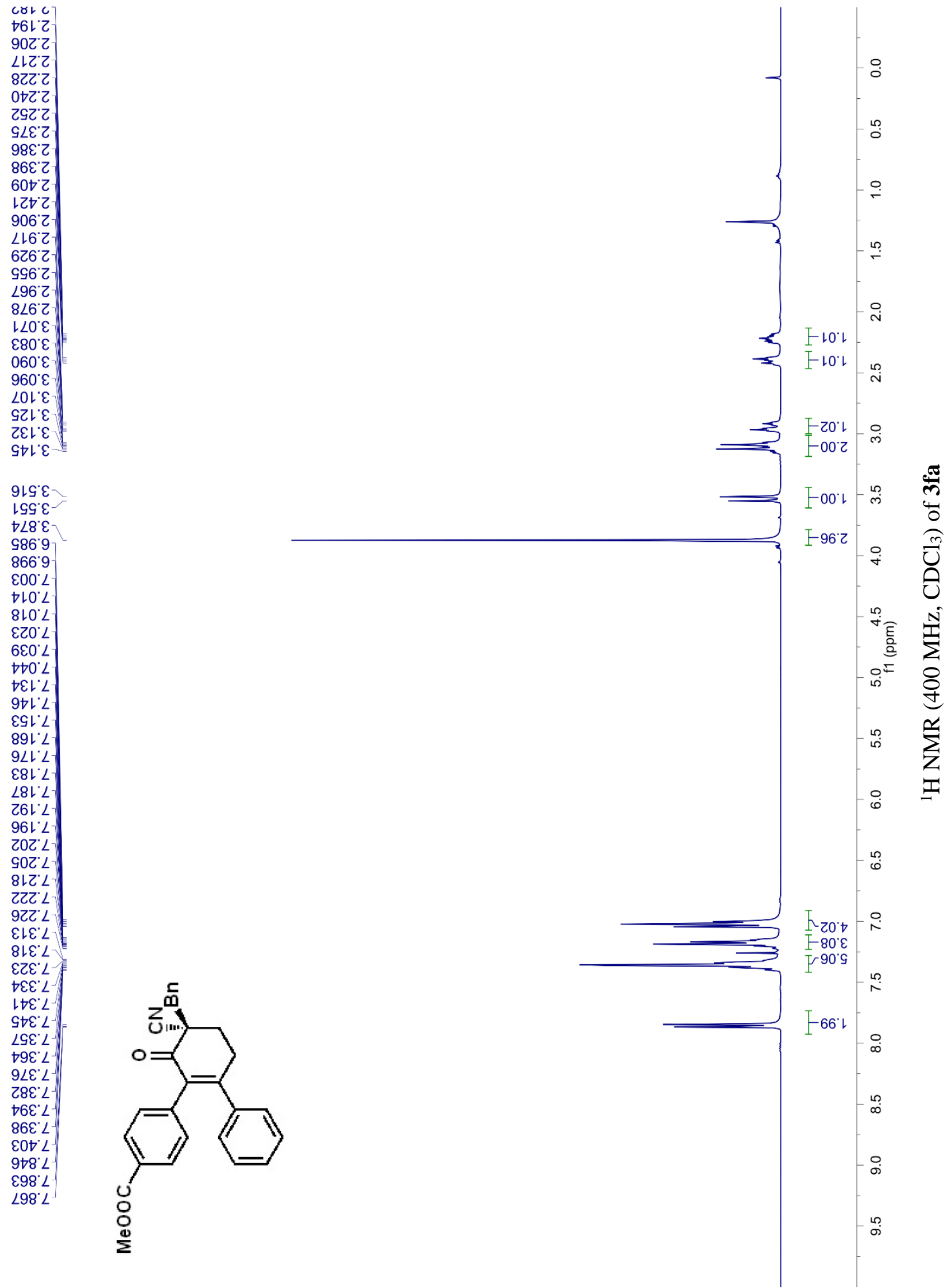

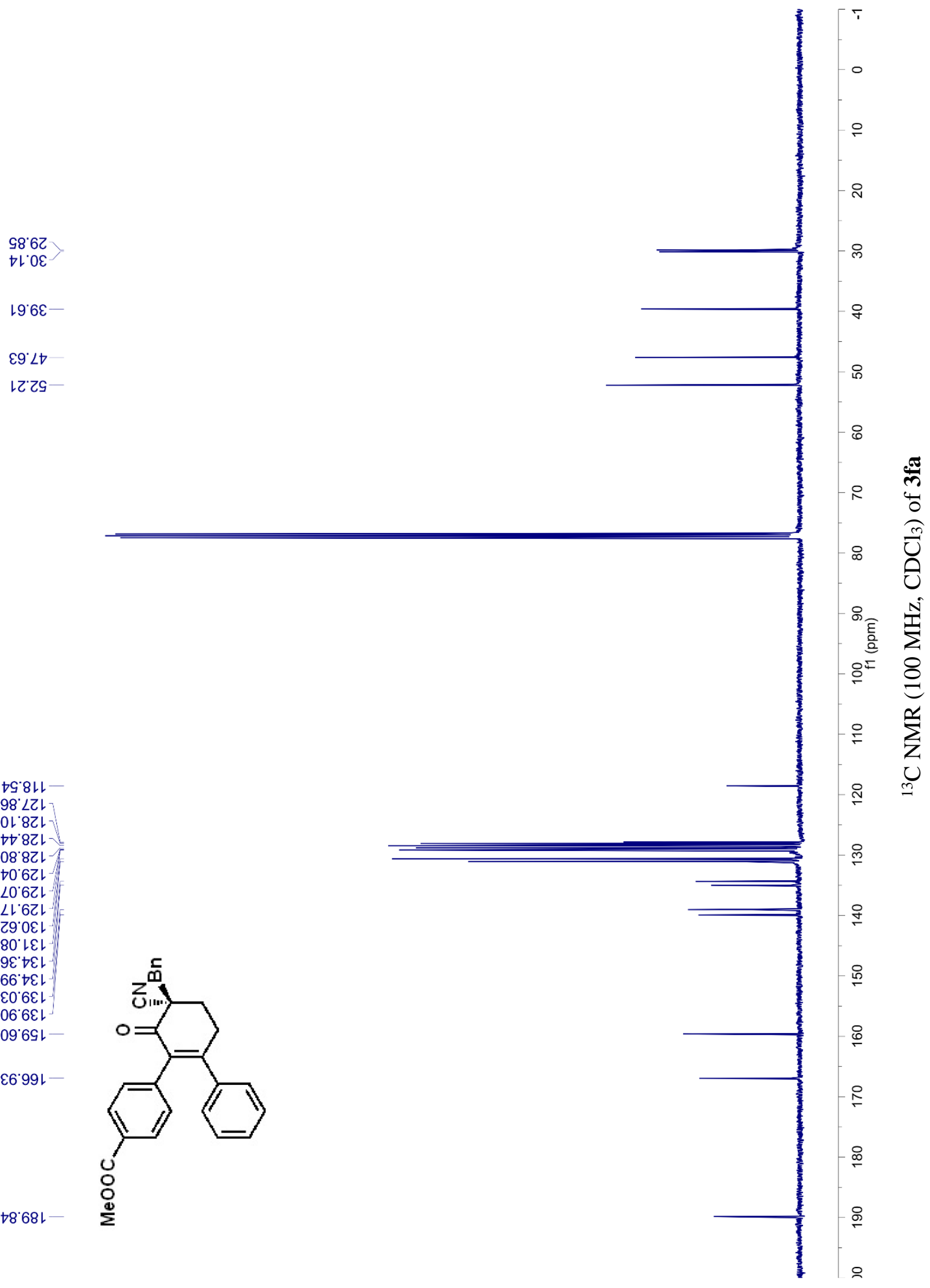

tG.8L-

$98 \angle Z L$

$0{ }^{\circ} 8 Z \mathrm{~L}$

t七'8ZL

$0882 \mathrm{~L}$

$706 \mathrm{~L}$

$\angle 0^{\circ} 6 Z L$

$\angle L^{\prime} 62 L$

Z9.0عL

$80^{\circ} L E L$

$9 \varepsilon^{\prime} \triangleright \varepsilon\llcorner$
$66^{\circ} \triangleright \varepsilon \downarrow$

ع० $6 \varepsilon\llcorner$

$06.6 \varepsilon \mathrm{L}$

09 6S -

E6.99l-

$7868 \mathrm{~L}-$ 

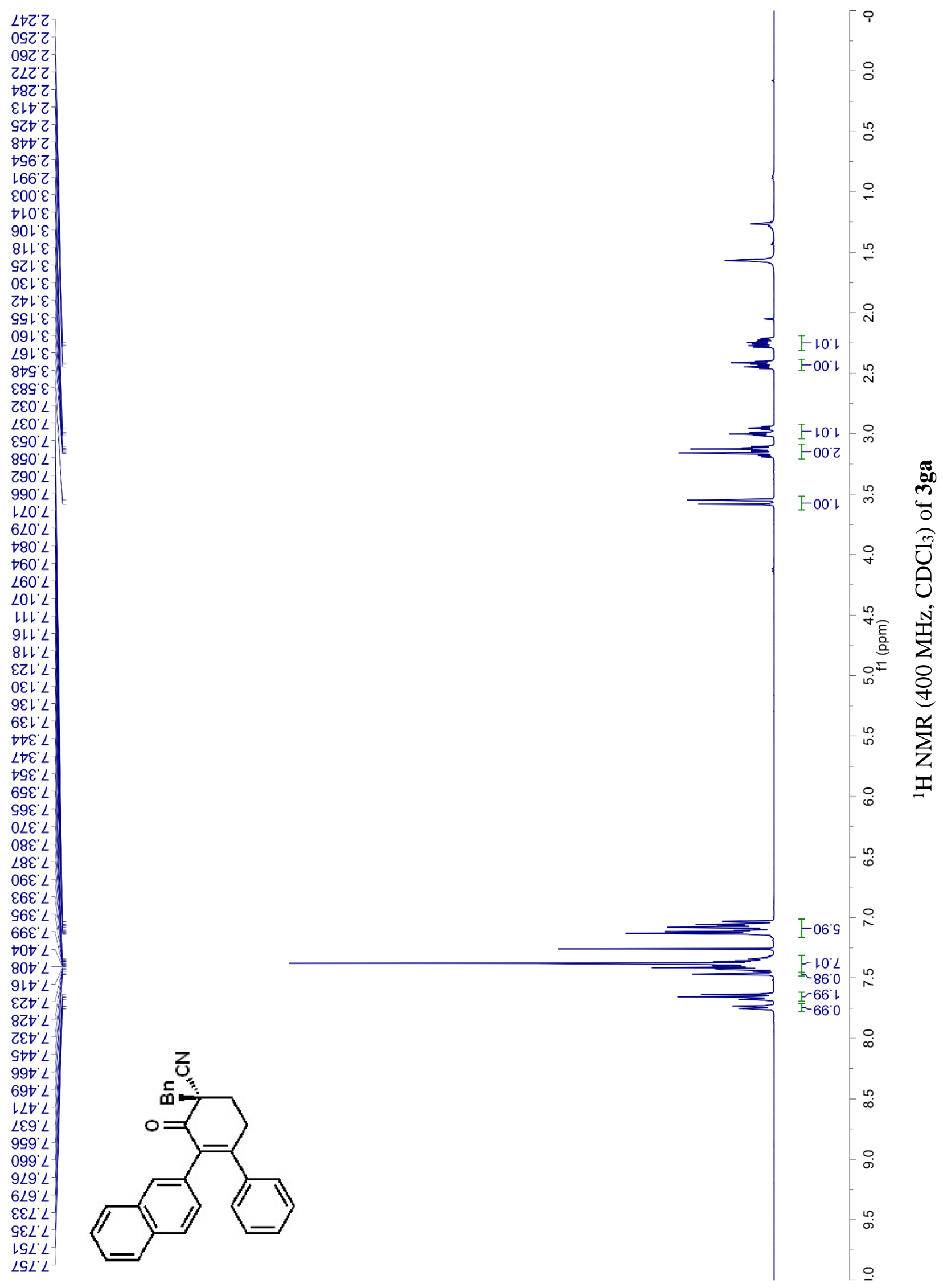
$\angle 0^{\circ} \varepsilon \varepsilon L-$

$99^{\circ} \nabla \varepsilon L$

†9 9 \&L-

09.6ع -

6L'8GL.

てヤ゙06レ-
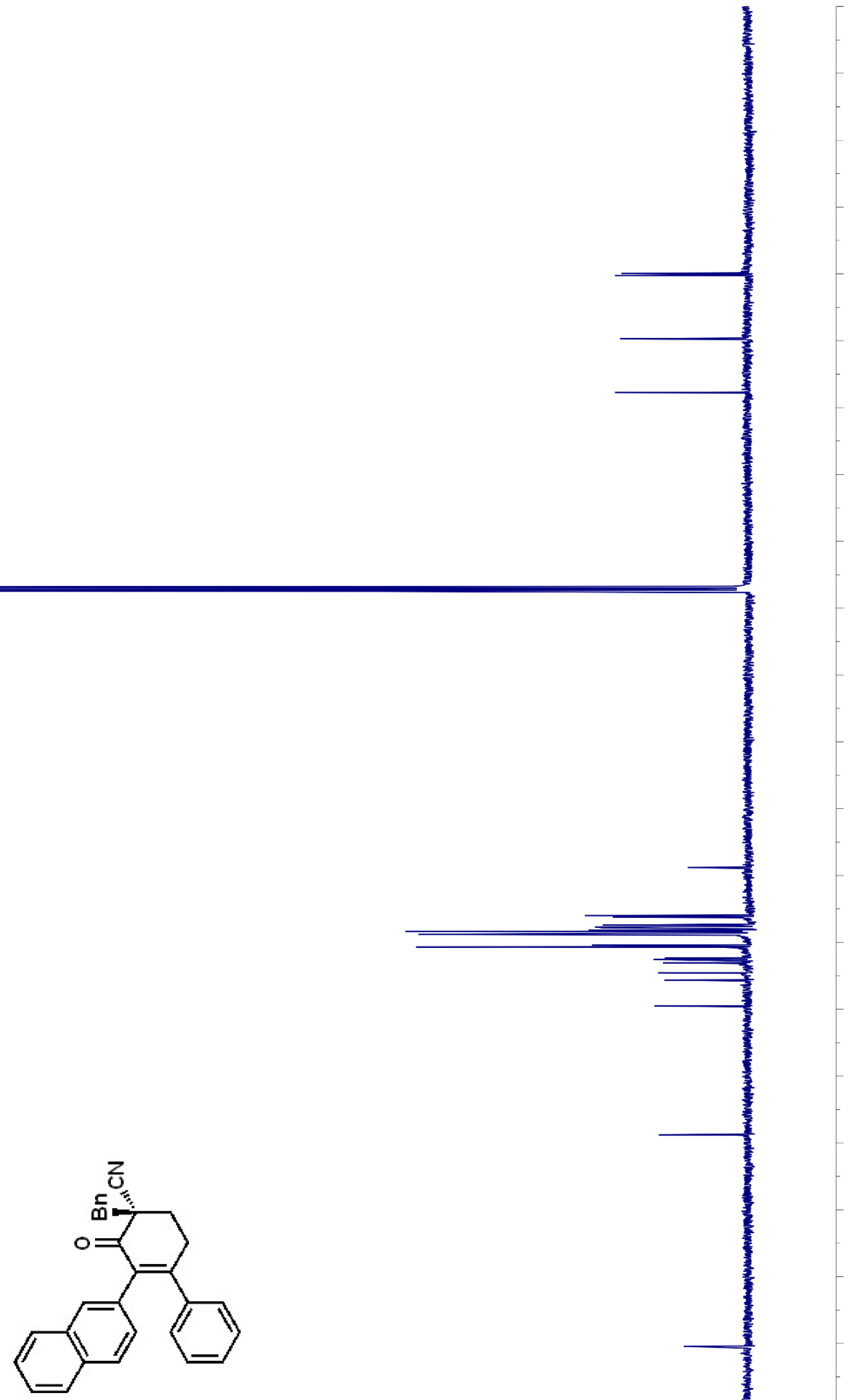
Z8L'Z

E6L'Z

GOZ'Z

9レで

8टて

0†乙 乙

9SE'

$89 \varepsilon 乙$

$08 \varepsilon^{\prime} Z$

$06 \varepsilon^{\prime} 乙$

ZOt 2

เレナて

เレ6.

EG6'

†96'乙

$9 \angle 6^{\circ} \mathrm{Z}$

$70 \varepsilon$

$890^{\circ} \mathcal{E}$

$690^{\circ} \varepsilon$

乙80 $\varepsilon$

$\varepsilon 60^{\circ} \varepsilon$

$6 เ \mathcal{6}$

8टा' $\varepsilon$

乙Eเ' $\varepsilon$

$0 \supset \mathcal{G}^{\circ} \varepsilon$

GLG'

$9 \angle 9^{\circ} 9$

$629^{\circ} 9$

ต89.9

889.9

५ट8'9

†८०: 9

8\&8: 9

$\angle \neg 89$

เVLL

t大t:

9ti:

$6+1 ' L$

ZGL'L

9GL'L

$09 L^{\circ} \mathrm{L}$

G9L'L

EEZ L

9E乙 L

StZ'L

8†Z: L

09Z L

$\checkmark 9 Z 2$

$\angle 9 Z$ ' L

$69 Z$ L

ZLC' $L$

$\nabla L Z^{\prime} L$

18Z L

$98 Z^{\circ} L$

乙6乙 ' L

$9 \varepsilon \varepsilon^{\circ}\llcorner$

$6 \varepsilon \varepsilon^{\prime} L$

$\nabla \nabla \varepsilon^{\prime} L$

$9 \nabla \varepsilon^{\circ} L$

$0 G \varepsilon^{\circ} L$

$\varepsilon S \varepsilon^{\circ} L$

GGE'L

เ $9 \varepsilon^{\circ} \mathrm{L}$

$\angle 9 \varepsilon^{\circ} L$

$\nabla L E^{\circ} L$

9LE'L

8LE'L

$08 \varepsilon^{\circ} L$

乙8E $L$

S8E $L$

$88 \varepsilon^{\circ} L$

$00 t^{\circ} L$

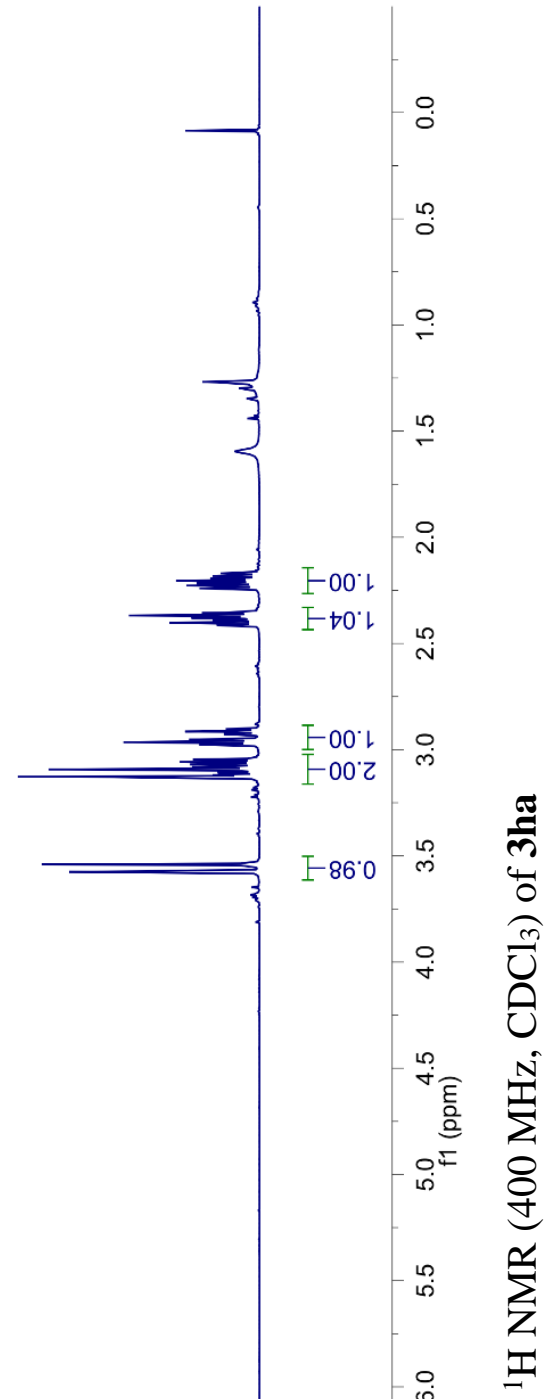

0

F $26^{\circ} 0$

工 $\nabla 6^{\circ} 0$

$66^{\circ}-N$

$66^{\circ} 0$

$\downarrow 0^{\circ} \varepsilon$

80.9

$\stackrel{ก}{ก}$

$\infty$

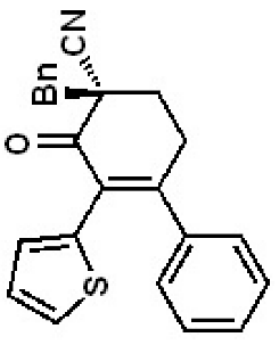


EL'LZL

S8 LZL

$\angle 8^{\circ} \angle Z L$

99.8ZL-

28.8Z

98.8Z

90.6ZL

レ๐OEL

乙9. 0 \&

$8 \varepsilon^{\circ} \nabla \varepsilon L$

$\angle L ' \nabla \varepsilon L$

98.6ع -

29.69เ

\&9.68ᄂ-
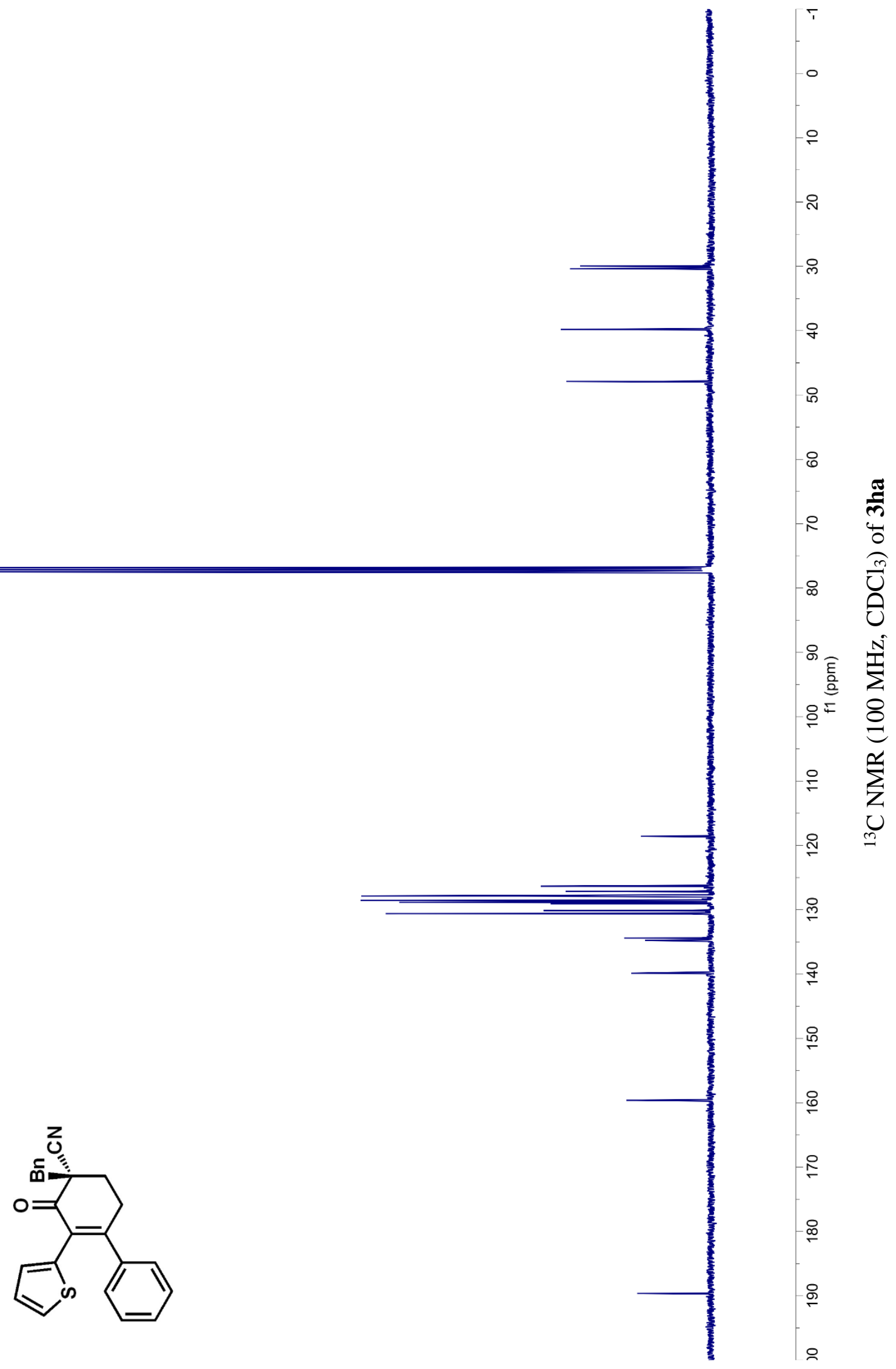
$v v \cup c$
$990^{\circ} Z$

$890^{\circ} \mathrm{Z}$

$6 \angle 0^{\circ} \mathrm{Z}$

$060^{\circ} \mathrm{Z}$

Z0เ'

จル乙

$\angle 9 Z^{\circ} Z$

$6 L Z^{\prime} Z$

เ6乙'

乙0६'乙

$\varepsilon\left\llcorner\varepsilon^{\prime} 乙\right.$

GटE'乙

86L'

$\mathrm{Z08} \mathrm{Z}$

9ع8'

$0 \triangleright 8^{\circ} Z$

$\angle \nabla 8^{\circ} Z$

เ98'Z

698'乙

Z96'乙

$896^{\circ} 2$

s96'Z

ᄂ $\angle 6^{\circ} \mathrm{Z}$

† $0^{\circ} \varepsilon$

$6 \triangleright 0^{\circ} \varepsilon$

$\varepsilon \angle \nabla^{\circ} \varepsilon$

$809^{\circ} \varepsilon$

$\downarrow \rightarrow 9^{\circ} G$

† $Z^{\circ} \mathrm{L}$

$69 Z^{\circ} L$

$\varepsilon L Z^{\prime} L$

$6 L Z^{\prime} L$

$\neg 8 Z^{\circ} L$

$\dashv 0 \varepsilon^{\prime} L$

$80 \varepsilon^{\circ} L$

OLE'L

จLE'L

LLE'L

OZE'L

$\neg 乙 \varepsilon 2$

8ZЕ"L

乙EE'L

$\nabla \varepsilon \varepsilon^{*} L$

$8 \varepsilon \varepsilon^{\circ} L$

$0 \nabla \varepsilon^{\circ} L$

$9 \nabla \varepsilon^{\circ} L$

$99 \varepsilon^{\circ} L$

$89 \varepsilon^{\circ} L$

$09 \varepsilon^{\circ} L$

$\neg 9 \varepsilon^{\circ} L$

$\angle 9 \varepsilon^{\circ} \angle$

เ8E 2

G8E $L$

$60 \nabla^{\circ} L$

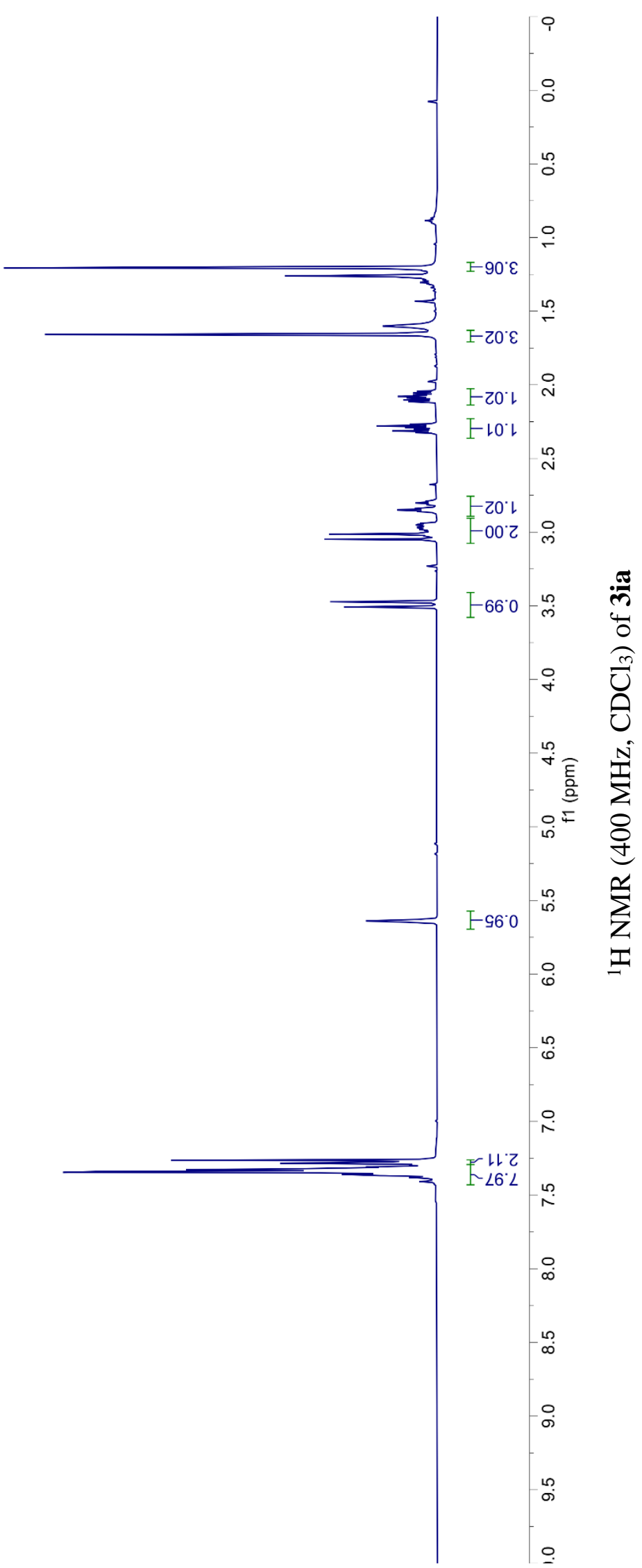




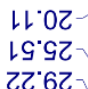

8ट.0ह-

$\varepsilon 9^{\circ} 6 \varepsilon-$

$19^{\circ} \angle t$
เZ"8ルL

†8'8 L

$\varepsilon L \angle Z L$

$\nabla 6$ LCL

$\varepsilon Z$ 8ZL

$\varepsilon\llcorner 8 Z$ L

$\varepsilon 8.8 Z$ L

เ. $0^{\circ} 0 \varepsilon$

$0 \varepsilon^{\prime}$ 乙 $\varepsilon$

$\neg 9^{\circ} \nabla \varepsilon L$

GL $8 E L$

ह己'0t।

$89^{\circ} \angle G L-$

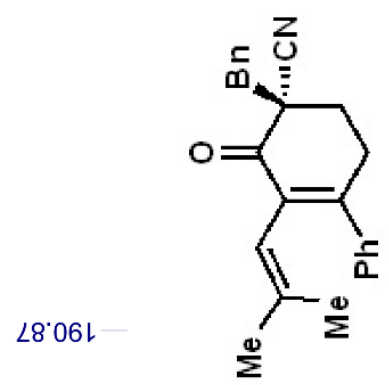

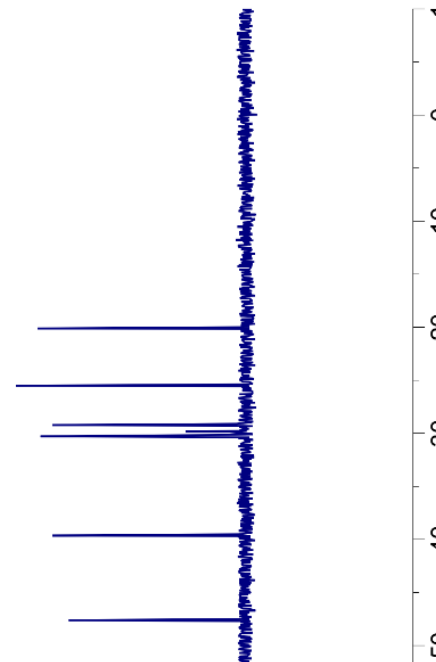

오

8

․

:

을 볼

OF

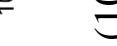

음

Z

은

요

o

옹

ঃ

운

$\stackrel{\infty}{\circ}$

용

음 
$86 L$ L

E08'

808'

$\angle E O^{\circ} Z$

$670 \mathrm{Z}$

$090^{\circ} \mathrm{Z}$

L $\angle 0^{\circ} \mathrm{Z}$

$\varepsilon 80 \mathrm{Z}$

$7600^{\circ} Z$

$90 \mathrm{~L} Z$

$6 \nabla 乙 \cdot 2$

1927

$\varepsilon L 乙 \backslash 乙$

$\varepsilon 8 乙^{\prime} 乙$

५6乙'乙

66乙' 2

$\angle 0 \varepsilon^{\prime} Z$

$0 \angle 9^{\circ} Z$

$\nabla \angle 9^{\circ} \mathrm{Z}$

$90 \angle Z$

ULZ

ㄴL乙

6LC

ह乙L '

$\angle Z L ' Z$

LEL $Z$

898' 2

$798^{\circ} \mathrm{Z}$

$0 \angle 8^{\circ} 乙$

GL8' $\mathrm{Z}$

$188^{\prime} \mathrm{Z}$

$\angle 88^{\prime} \mathrm{Z}$

006.2

$\varepsilon\left\llcorner 0^{\circ} \varepsilon\right.$

$8 \supset 0^{\circ} \varepsilon$

$\varepsilon 9 \nabla^{\circ} \varepsilon$

$867^{\circ} \mathcal{E}$

$88 L^{\circ} \mathrm{L}$

$76 L^{\circ} \mathrm{L}$

$\angle 6 L^{\circ} \mathrm{L}$

ZOZ L

$60 Z$ L

†LC'L

$6 \mathrm{LZ} L$

G8Z" L

I6Z'L

96乙 L

$00 \varepsilon^{\circ} L$

$90 \varepsilon^{\circ} L$

IIE L

GLE'L

OZE'L

8乙E $L$

$0 \varepsilon \varepsilon^{\circ} L$

$\varepsilon \nabla \varepsilon^{\prime} L$

乙GE L

$\angle G E^{\prime} L$

เ9E. L

$99 \varepsilon^{\prime} L$

ZLE'L

$6 \angle \varepsilon^{\circ} L$

$\varepsilon 8 \varepsilon^{\circ} L$

$\angle 8 \varepsilon^{\circ} L$

$96 \varepsilon^{\circ} L$

ZOt' L

$90 t^{\circ} \mathrm{L}$

9เt'L

เZt

9Zち L

$\varepsilon \varepsilon \nabla^{\circ} L$

8Et L

乙†t L

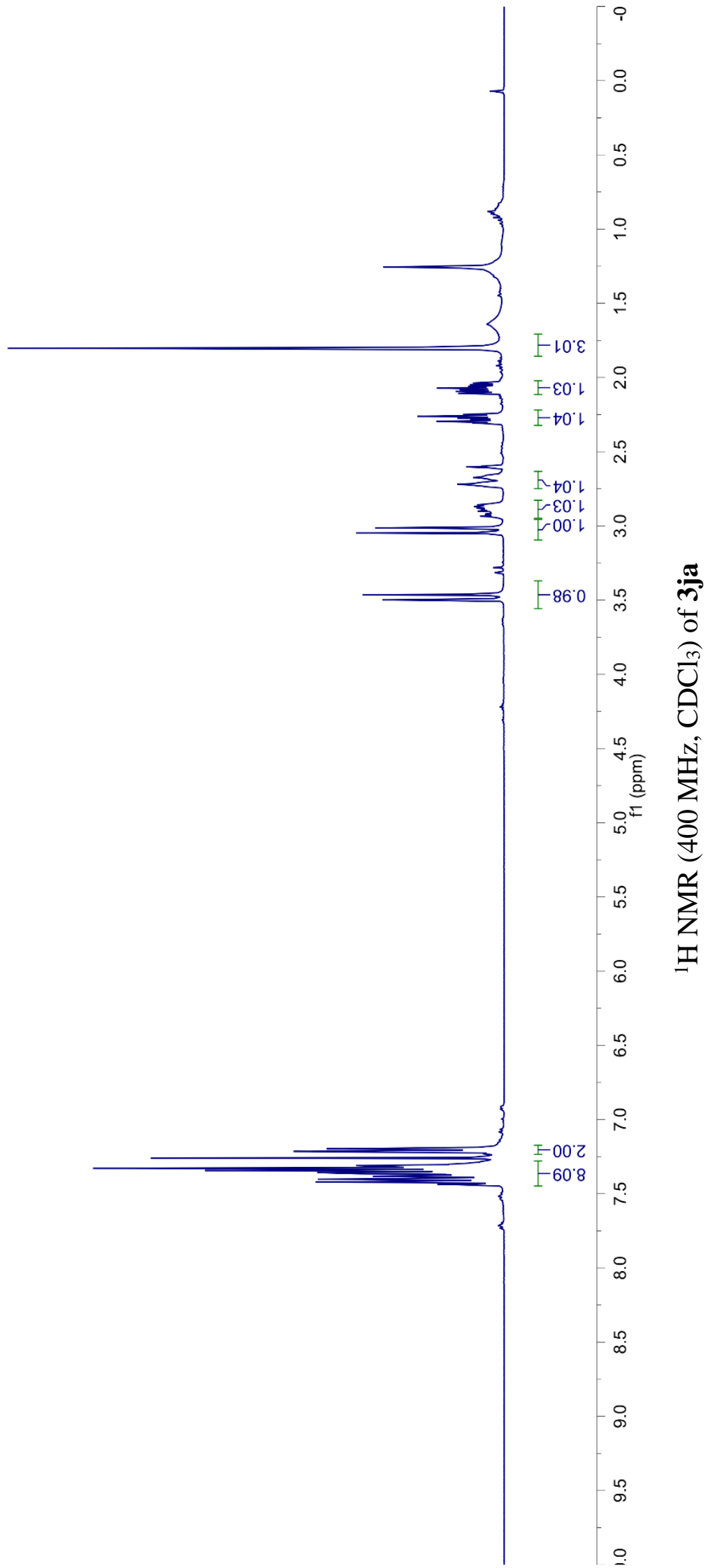


$\angle 8^{\circ} \varepsilon$

$\varepsilon<6 z$

$\varepsilon \nabla 0 \varepsilon$

$9 L 6 \varepsilon-$

$s t<b-$

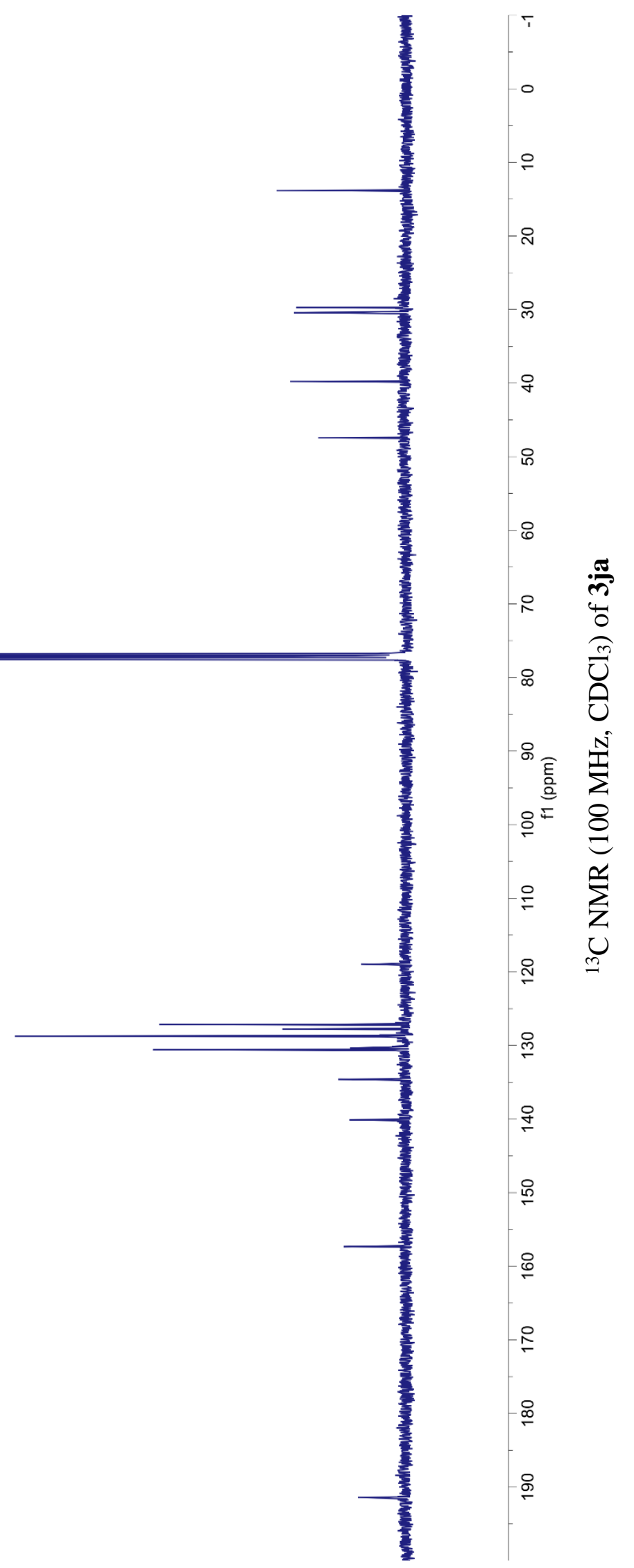

$6 \varepsilon เ\llcorner$ เ

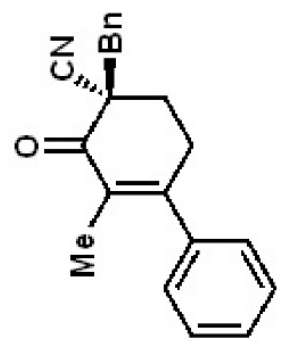


(udd)

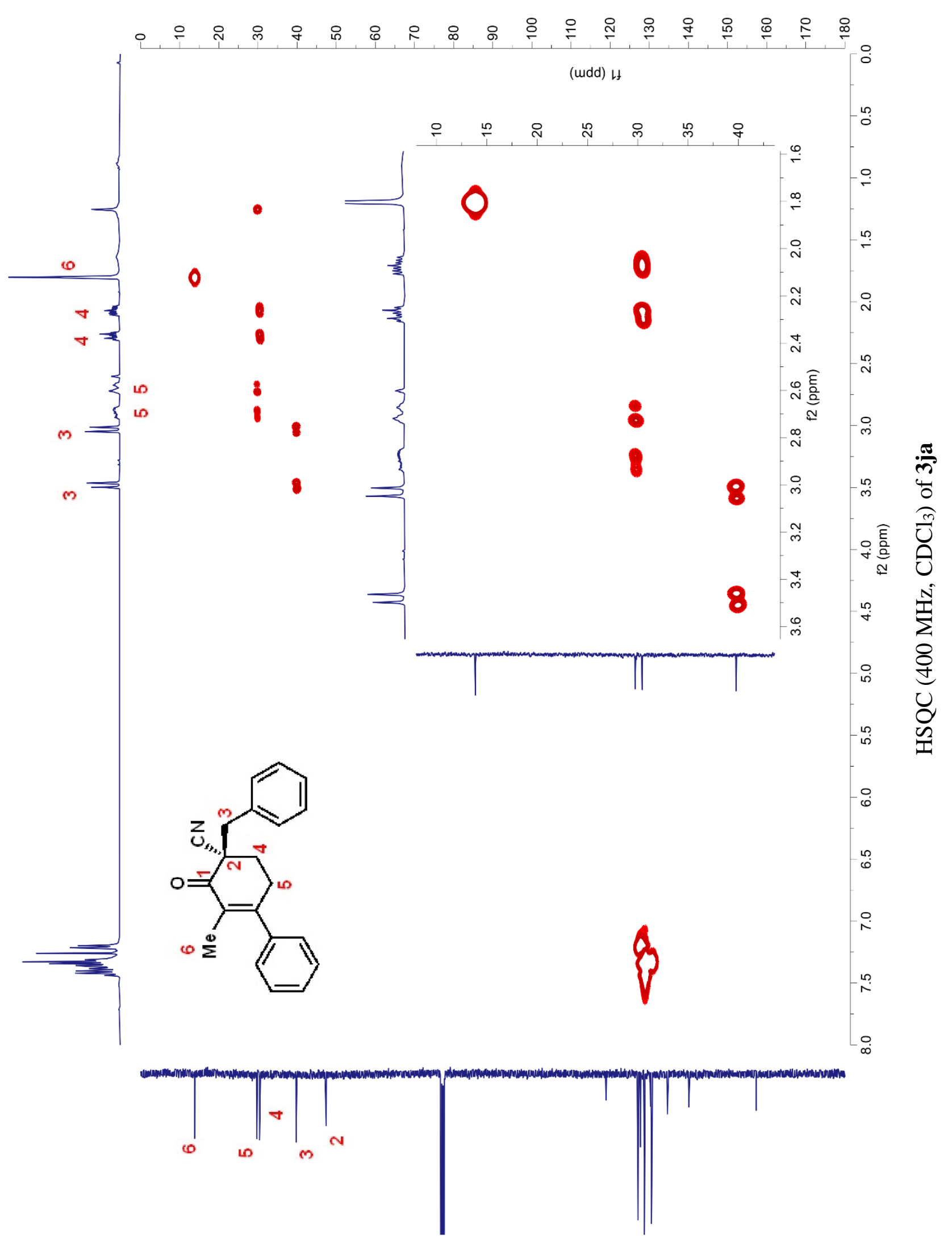


(udd) bl

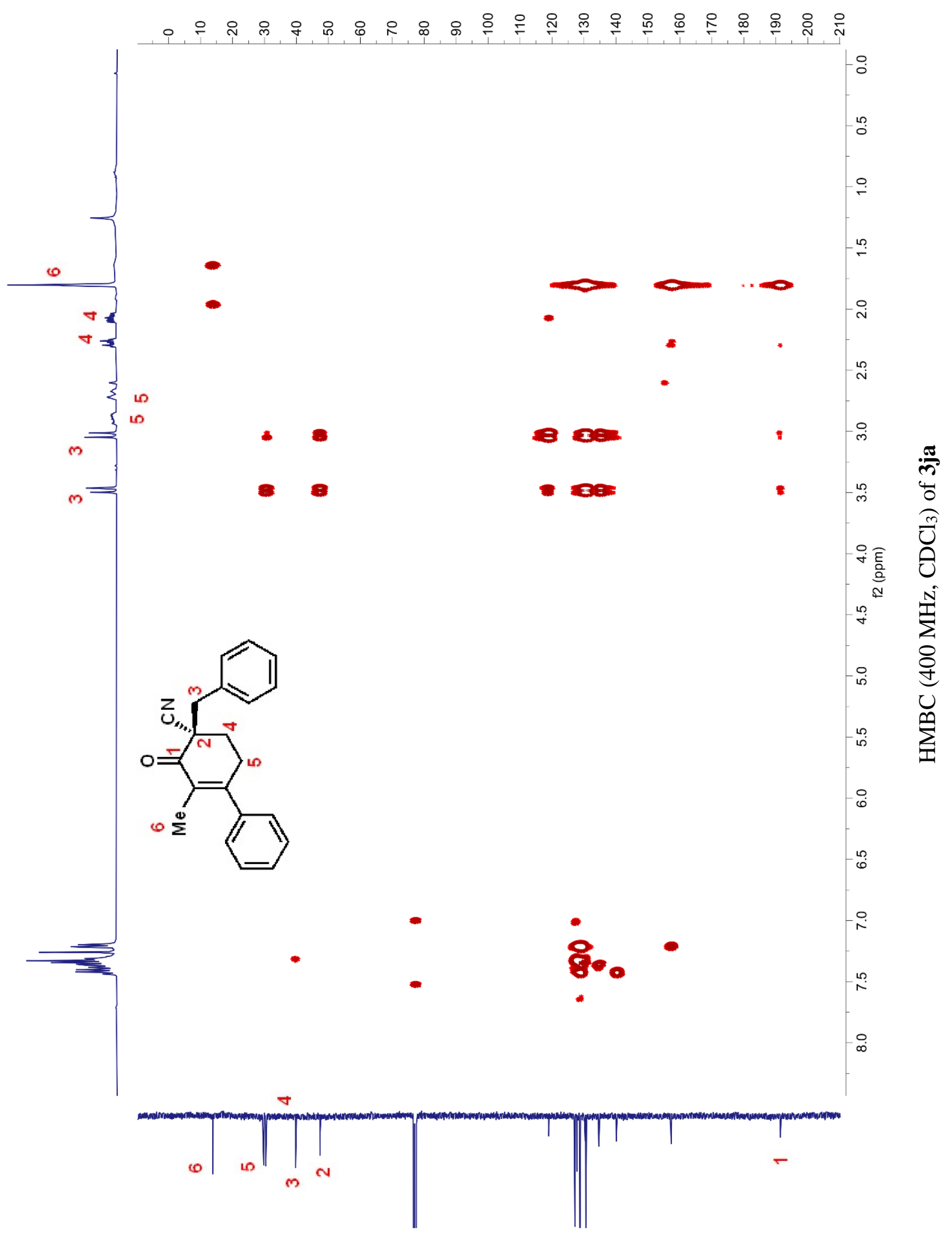


200'乙

乙20 乙

与\&0'Z

$\mathrm{Z} \supset 0^{\circ} Z$

$\mathrm{sSO} Z$

SLO'

$8 \angle L Z$

$\varepsilon \varepsilon 乙 乙$

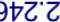

† ¿'乙

99乙乙

$08 Z^{2} Z$

$\angle 8 Z$ '

00ध'

$\nabla \angle 9^{\circ} Z$
$8 \angle 9^{\circ} Z$

969'Z

669 ' $\mathrm{Z}$

$\varepsilon 0 L ' Z$

$80 \angle Z$

ZLL

$9 L \angle Z$

$0 Z L Z$

$\varepsilon 乙 L 乙$

乙EL'乙

$9 \varepsilon L Z$

96L'乙

G08'

918'

เZ8'

Gट8'

6ع8' $Z$

898' $\mathrm{Z}$

ह98' $ح$

乙9. $\varepsilon$

$\angle 6 L^{\circ} \varepsilon$

$19 L^{\circ} \mathrm{L}$

$89 L^{\circ} \mathrm{L}$

ELL'L

$6 \angle L^{\circ} L$

$98 \mathrm{~L} L$

$\mathrm{C} 6 \mathrm{~L}^{\circ} \mathrm{L}$

60Z L

IDZ'L

$G \nabla Z L$

OSZ L

09Z L

S9Z" L

$\angle L Z \angle$

I8Z L

G8Z" L

$88 Z^{\prime} \mathrm{L}$

$66 Z^{\circ} \mathrm{L}$

$\angle O E^{\circ} L$

เレE L

9เE'L

เ乙E' $L$

9ZE' $L$

GEE' $L$

$0 \succ \varepsilon^{\prime} L$

$9 \nabla \varepsilon^{\circ} L$

GGE'L

乙9E'L

ZLE'L

$9 \angle E^{\circ} L$

$18 \varepsilon^{\circ} L$

$\angle 8 \varepsilon^{\circ} \angle$

L6E' $L$

$00 t \angle$

†Ot L

$60 \nabla^{\circ} L$

ELtL

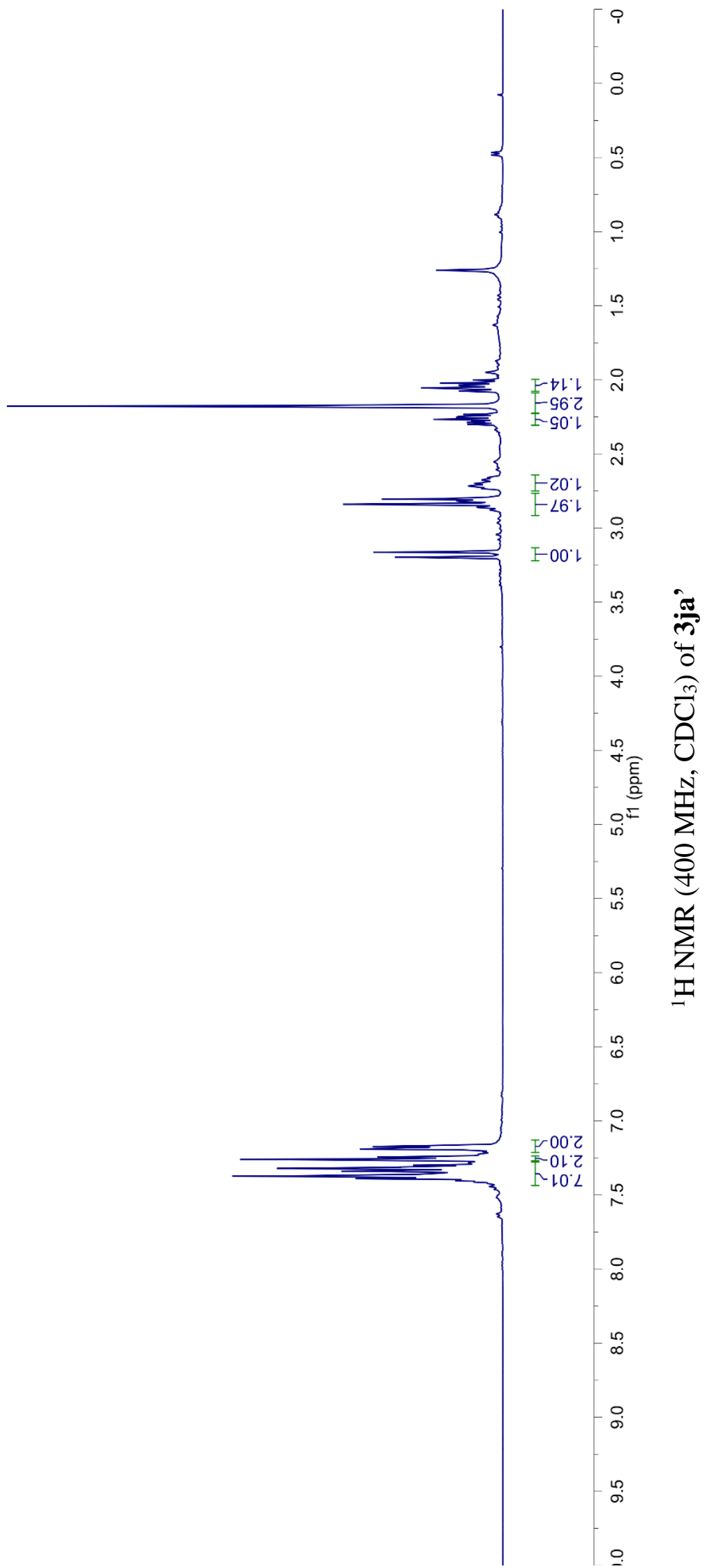


乙๖:
レ 92

乙L $6 \mathrm{C}^{-}$

$89^{\circ} 6 \varepsilon$

89'IS-

ع96ル

$0 Z$ LZL

$\varepsilon L \angle Z L$

St 6 CLL

$98^{\circ} 8 \mathrm{ZL}$

9 는

$06^{\circ} \downarrow \varepsilon L$

69'0tᄂ

00 เงเ-

$9 \varepsilon^{*} \ni 6\llcorner$

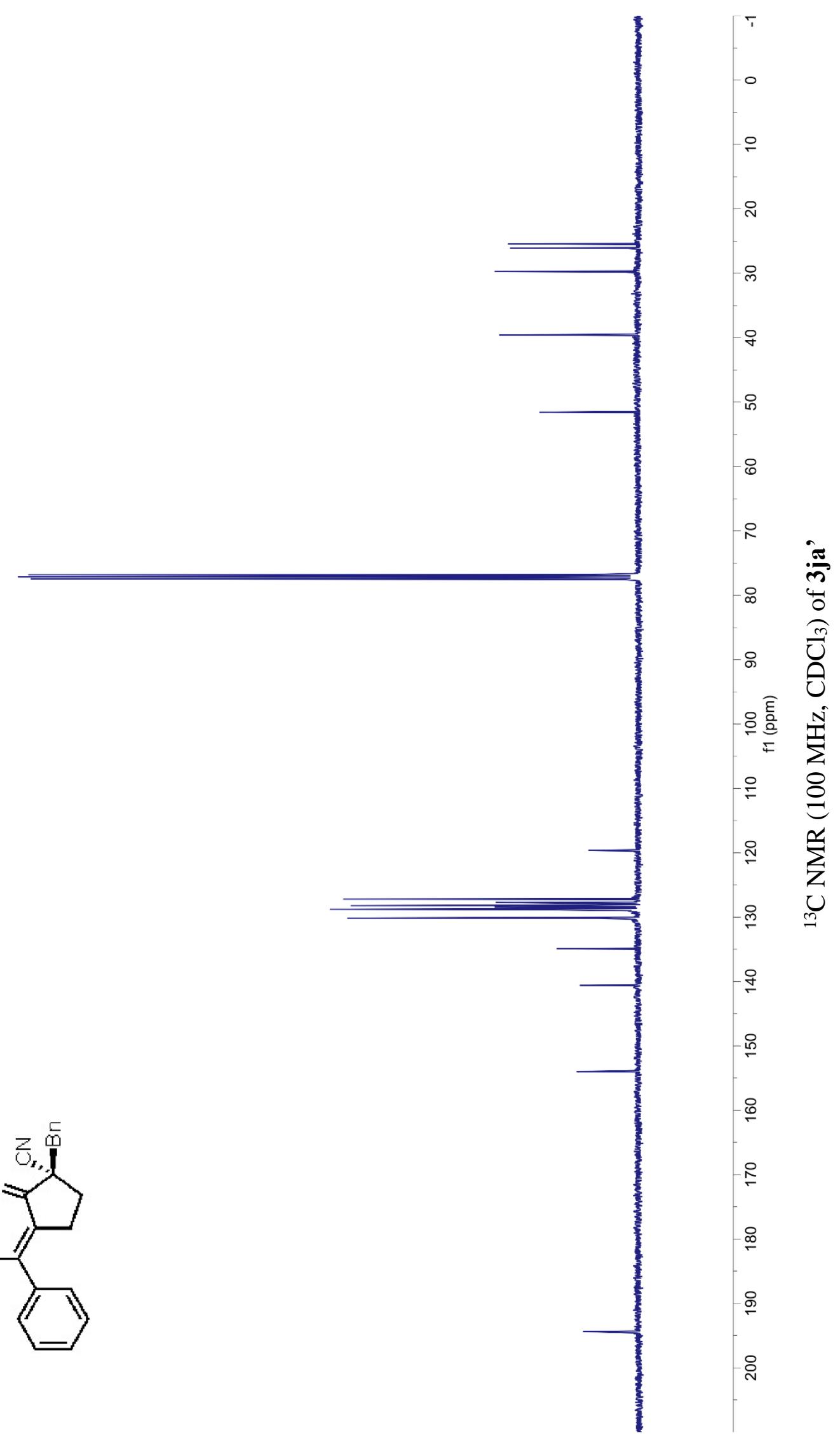


(udd)

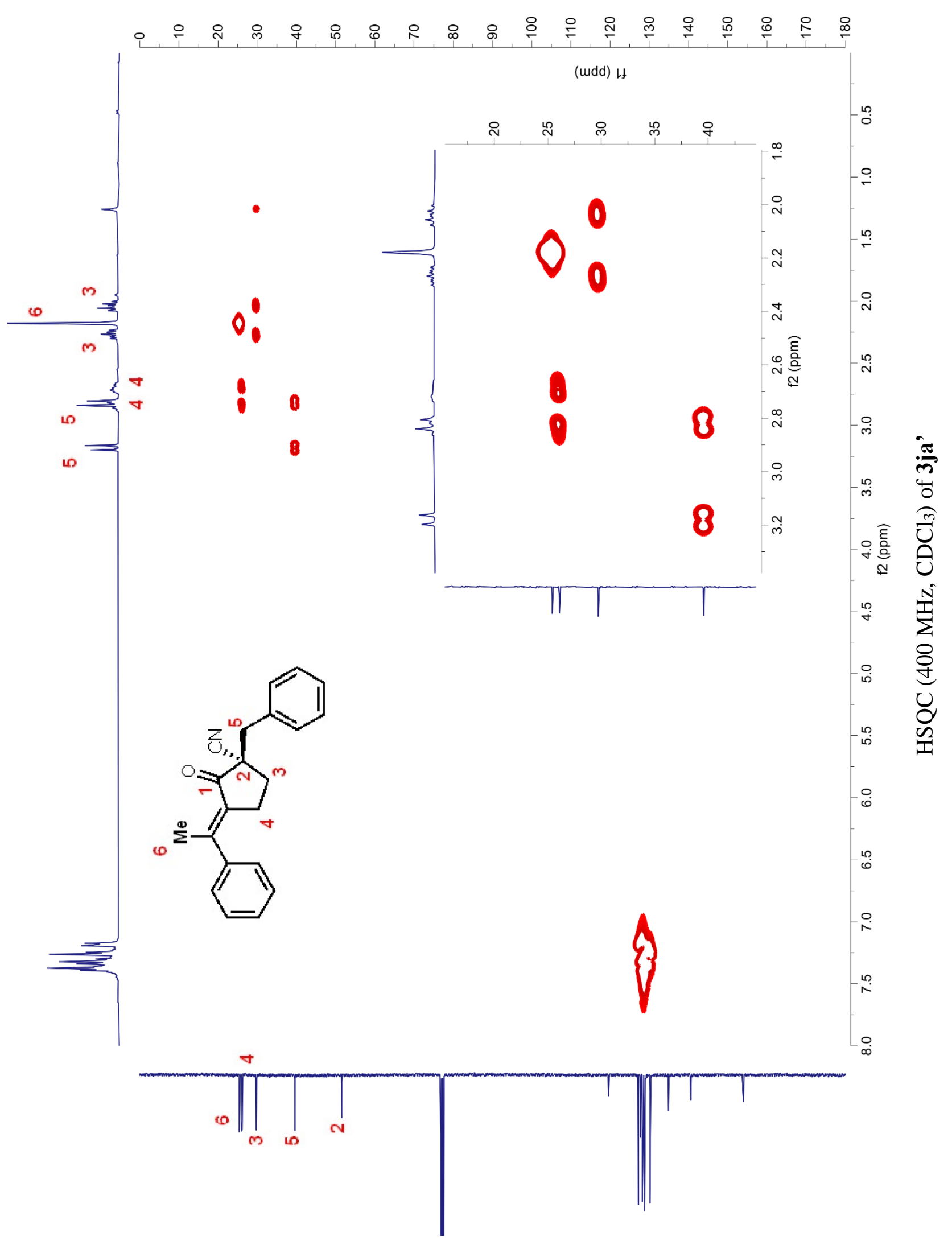


(mdd) bf

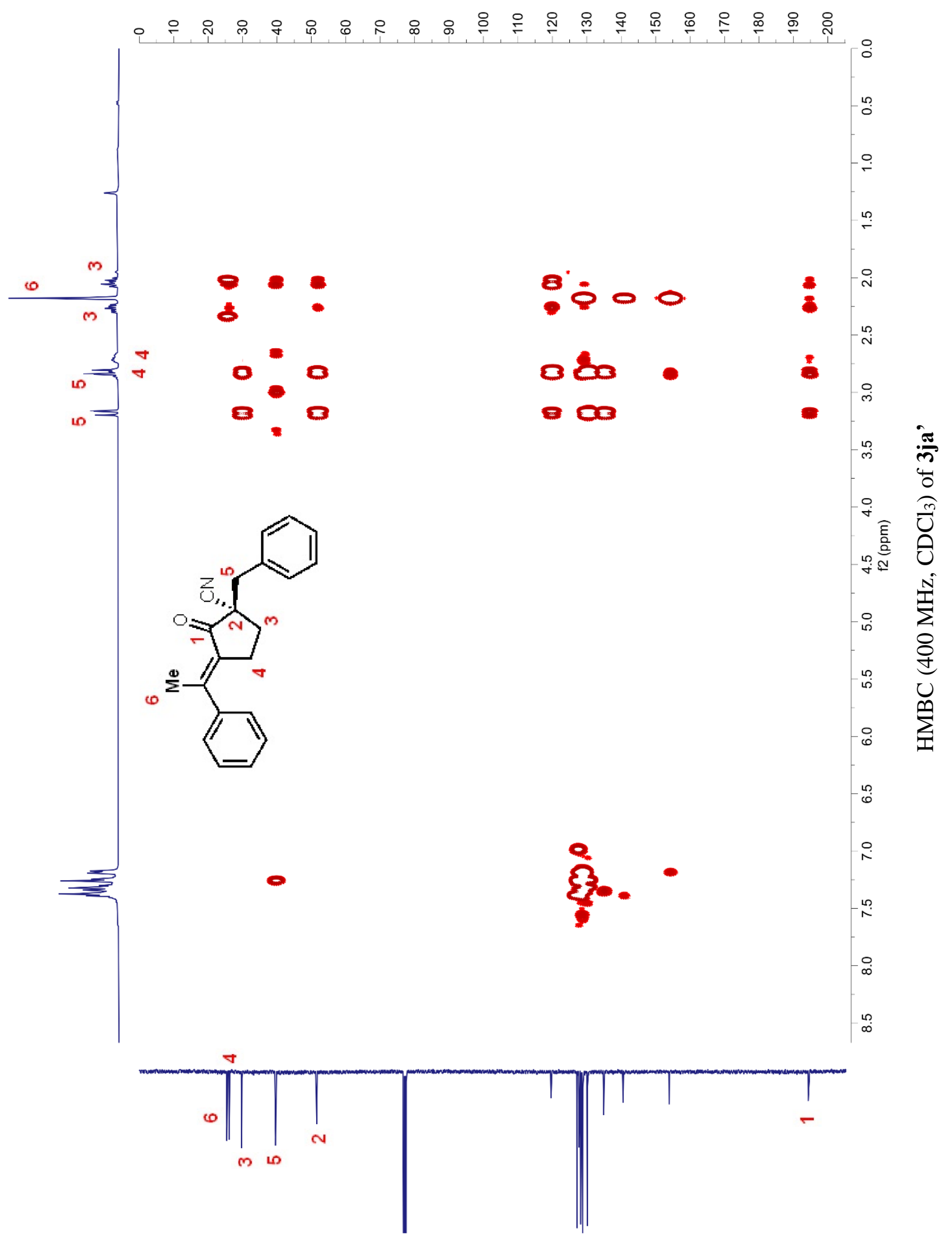


(udd) If

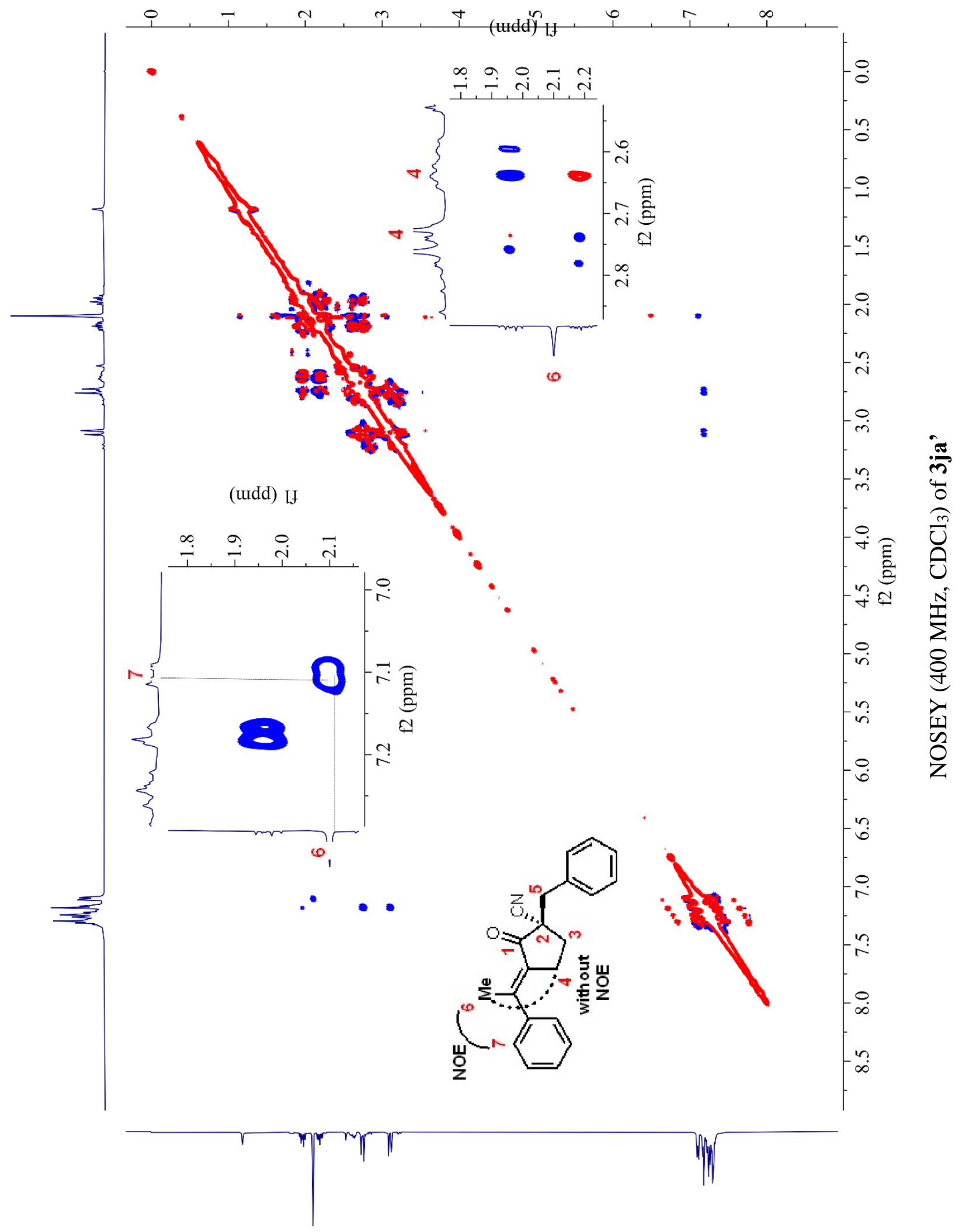


cytc

s8l'

96เ'

$60 乙 ' 乙$

เ乙Z'

9†ย'乙

$\angle G E^{\prime} Z$

$89 \varepsilon^{\prime} 乙$

08ध 乙

เ6ย'乙

Z0t'

606' 2

026 '

$8 \nabla 6 \cdot$

8962

696 ' 2

$690^{\circ} \varepsilon$

$9200^{\circ} \varepsilon$

$880^{\circ} \varepsilon$

$\neg 60^{\circ} \varepsilon$

ZOL' $\varepsilon$

हLเ'

9ZL' $\varepsilon$

$8 \varepsilon L^{\prime} \varepsilon$

เGL'E-

乙9॰ $\varepsilon$

$\angle 6 \nabla^{\circ} \varepsilon$

\&२6.9

乙ะ6. 9

$8 \varepsilon 6.9$

$\angle \nabla 6 \cdot 9$

996.9

896.9

$\varepsilon \angle 6.9$

$\triangle Z 0^{\circ} L$

$0 E 0^{\circ} \mathrm{L}$

$980^{\circ} \mathrm{L}$

$\triangle \nabla 0^{\circ} \mathrm{L}$

$870^{\circ} \mathrm{L}$

$990^{\circ} \mathrm{L}$

$890^{\circ} \mathrm{L}$

6난

EGL $L$

$09 L^{\circ} L$

LLLL

$9 \angle L^{\circ} L$

$\mathrm{Z8L} \mathrm{L}$

G8.'

$06 L^{\circ} \mathrm{L}$

L6L'L

IOZ'L

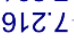

$\varepsilon Z Z^{\prime L} L$

8ZZ'L

$6 \varepsilon Z^{\circ} L$

$\nabla \nabla Z^{\prime} L$

Z8t'L

$06 \nabla^{\circ} L$

S6t'L

SOS. $L$

OLG'L

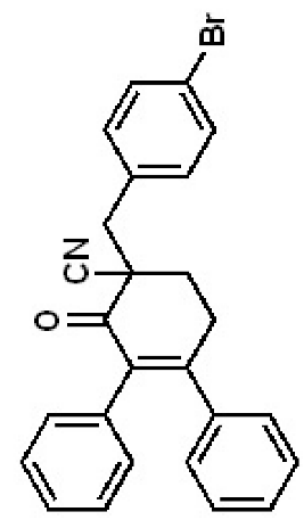

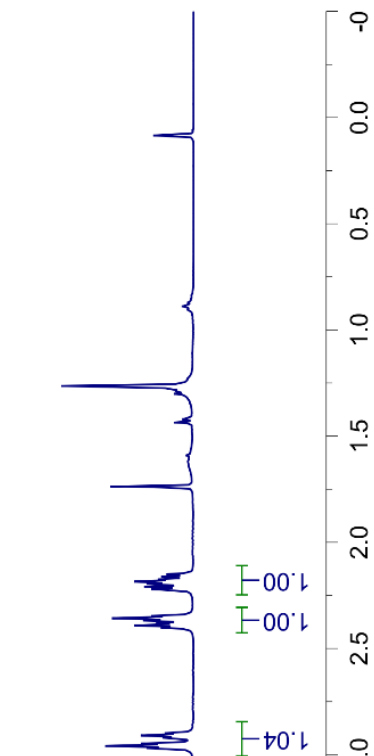

ल

क ल

लै

$\circ$

+ึ

OF

นึำ

-

F-66.t

-

FE⿺ $\mathrm{z}-\stackrel{n}{N}$

$\stackrel{\infty}{\infty}$

$\infty$

क․

?

๕ั 

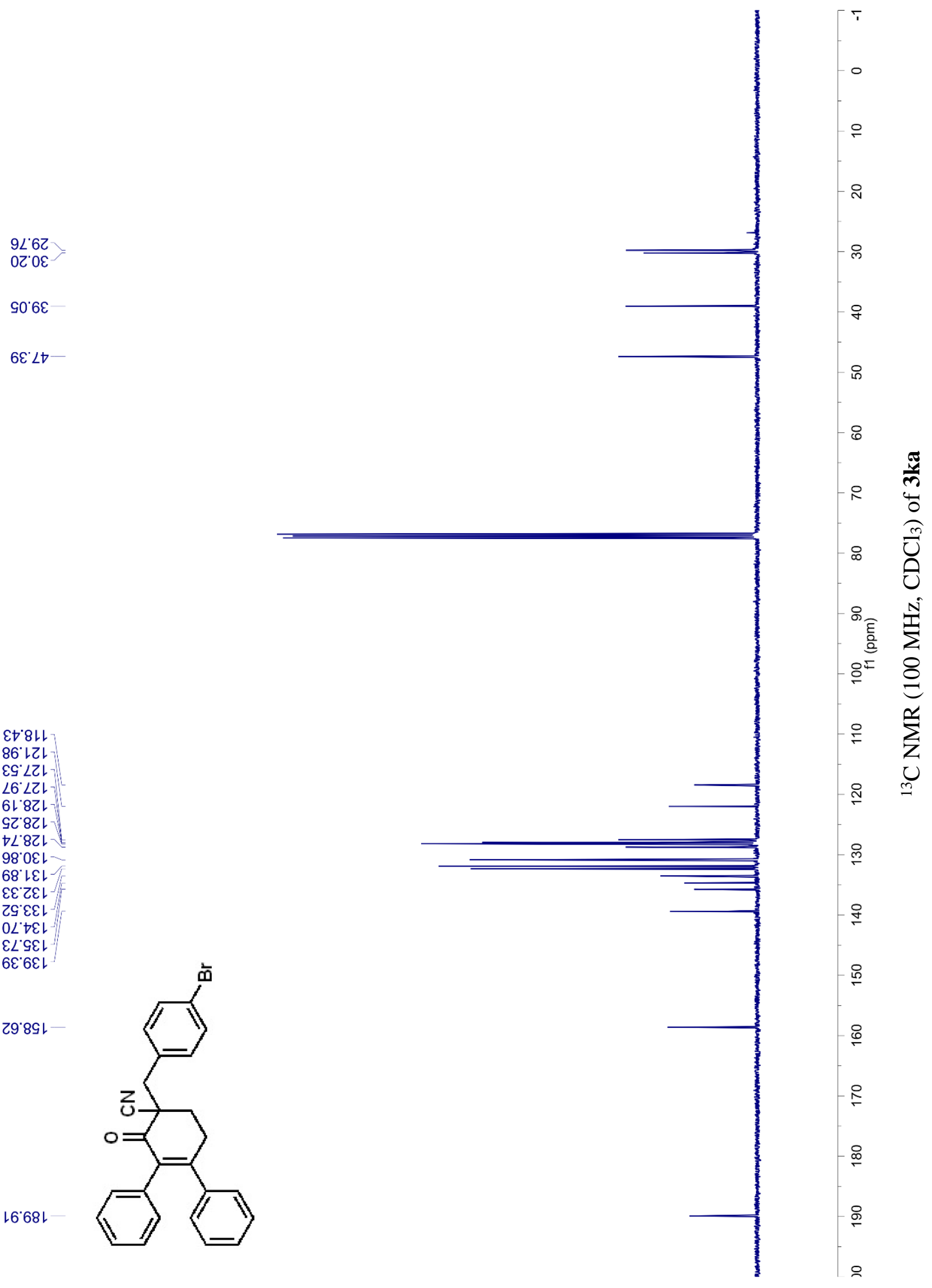

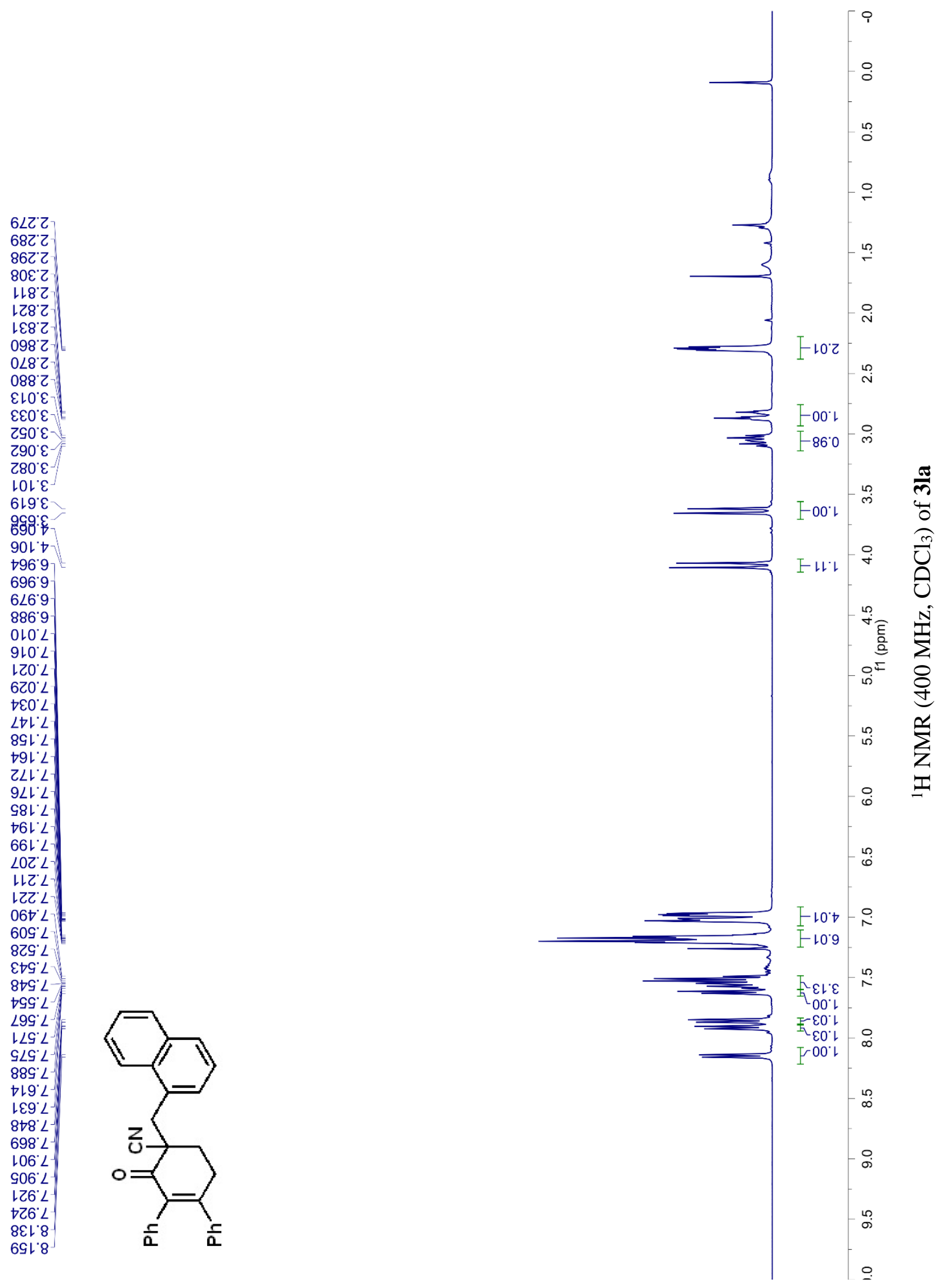
Z6" †६ -

$08^{\circ} \mathrm{GEL}$

$87^{\circ} 6 \varepsilon \mathrm{L}$

69'8S -
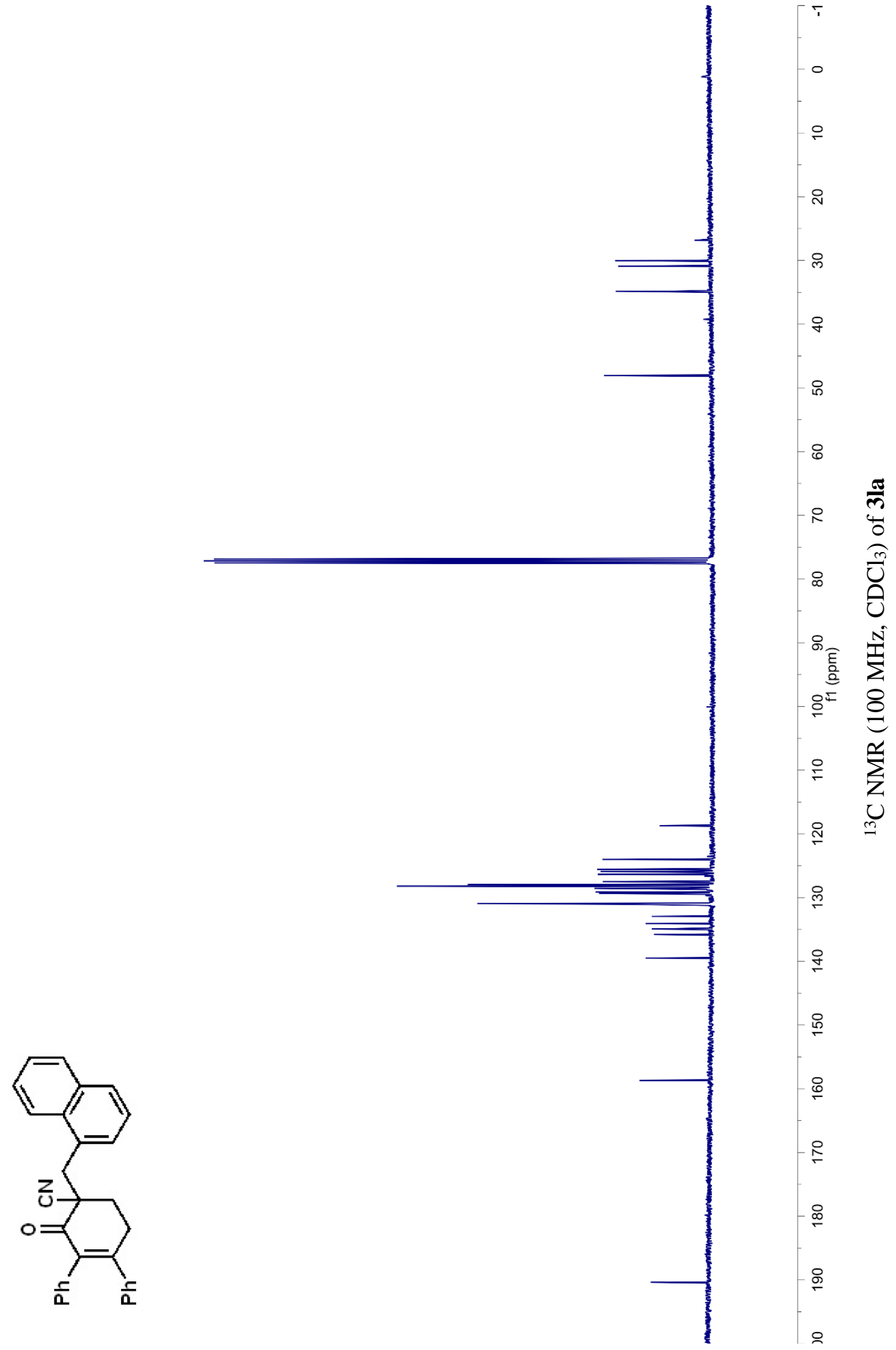

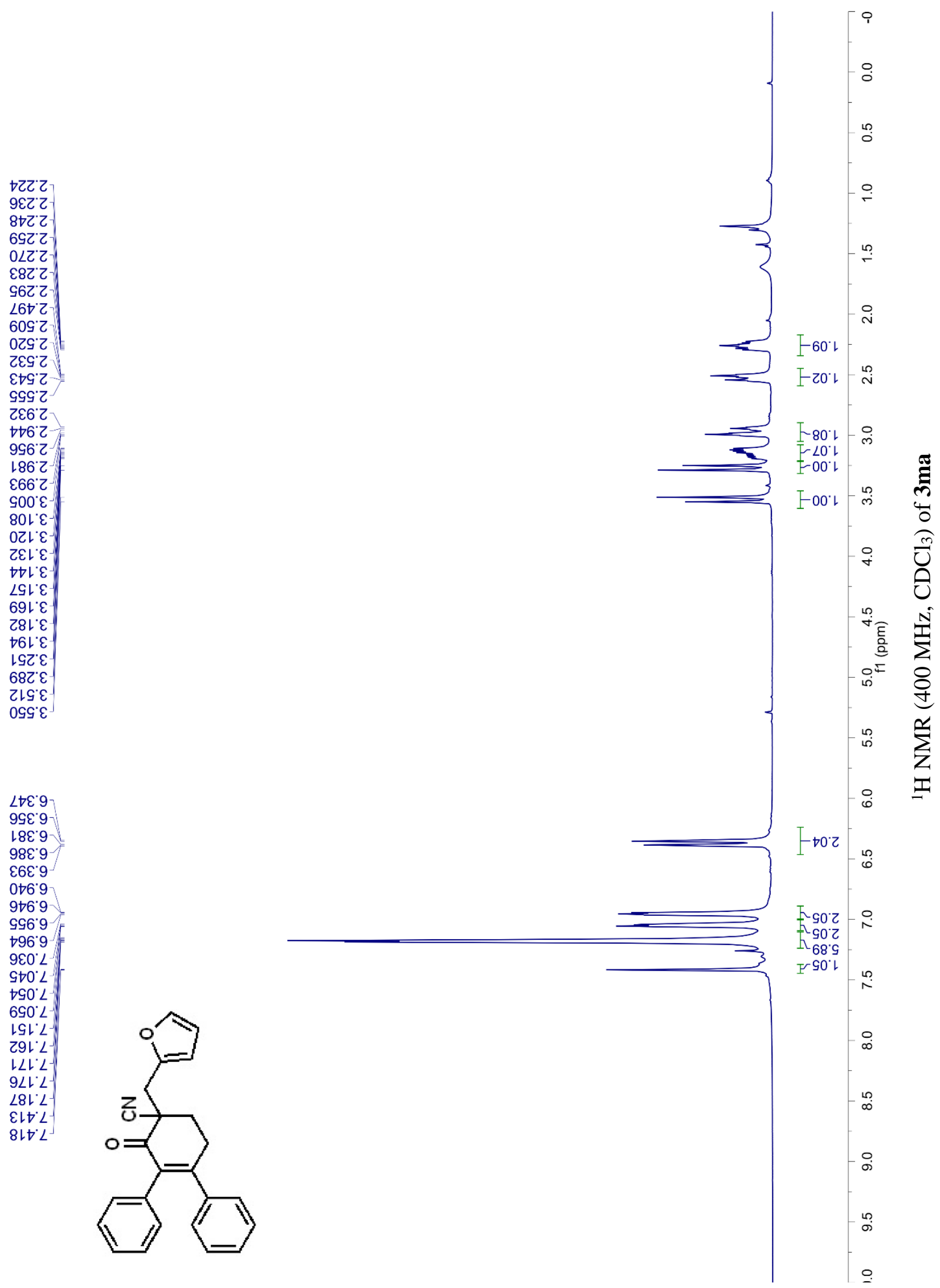
$0 L 6 Z$
${ }^{\circ} \cdot 0 \mathcal{E}^{\circ}$

¿' $ح \varepsilon^{-}$

$0 Z \angle \nabla-$

6L60L

26.0レ-

978เ

$9 \nabla^{\circ} \angle Z L$

L6 $\angle Z L$

LL8ZL

LC 8Z

$\angle 9 \mathrm{CL}^{\circ}$

$\angle 8{ }^{\circ} 0 \mathcal{L}^{-}$

$\varepsilon\llcorner\cdot \nabla \varepsilon\llcorner-$

$\angle G$ 'SEL

0G'6ع -

ย9 て†

เレ6เレ -
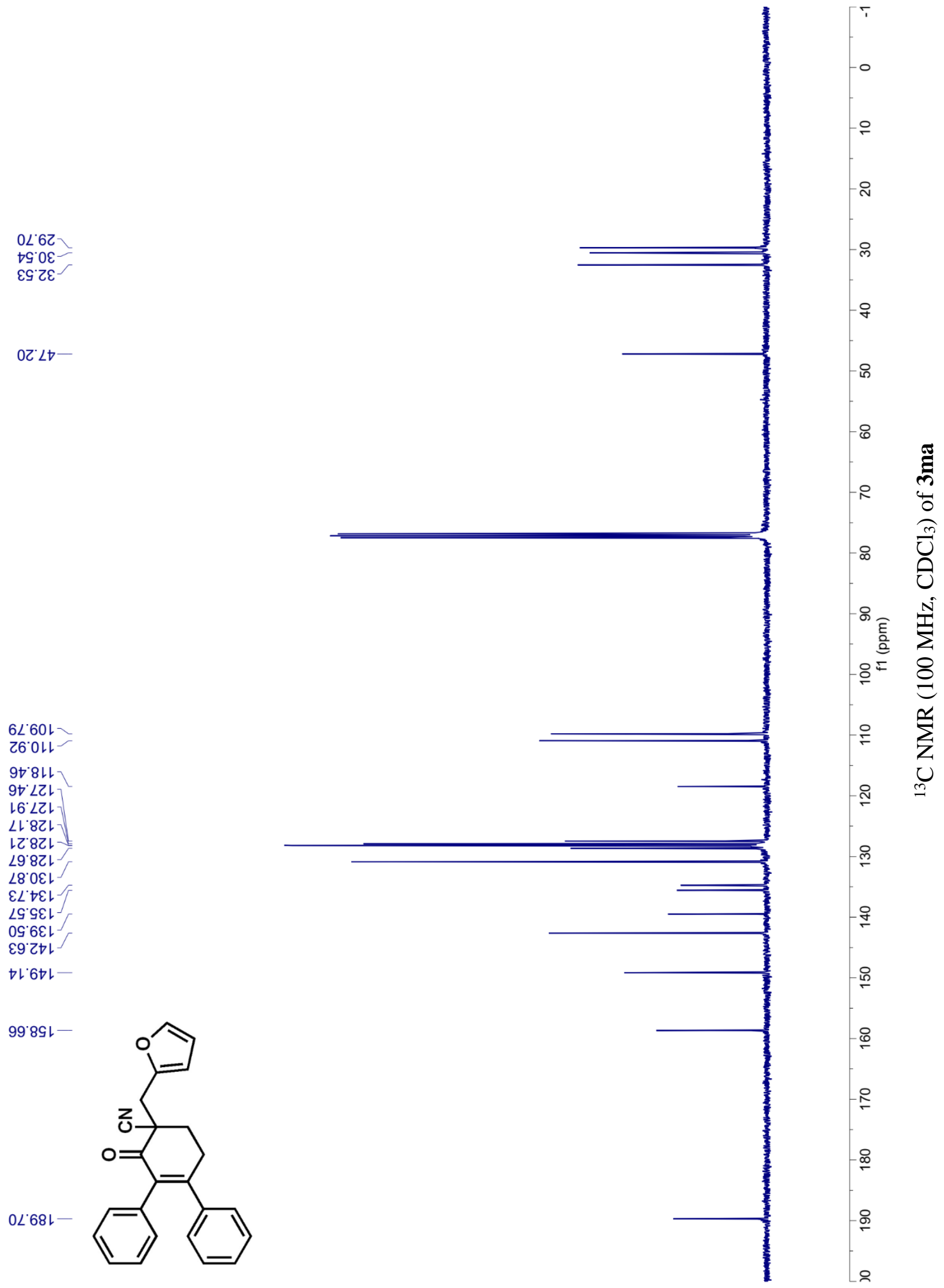
8LL'

乙08 1

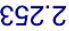

G9Z'

9L乙乙

88乙 乙

0०ह'乙

0เE'ح

乙乙ย'乙

6ZG'乙

†EG'乙

乙† '

$\downarrow G G^{\prime} Z$

$\nabla 99^{\circ} \mathrm{Z}$

$0 \angle G^{\prime} Z$

9LG'

689' $\mathrm{C}^{-}$

$188^{\circ} \mathrm{Z}$

$888^{\circ}$ '

$006^{\prime} Z$

จเ6'

6เ6'

$\angle \varepsilon \sigma^{\prime} Z$

096'乙

Z96'乙

乙60' $\varepsilon$

†०เ' $\varepsilon$

จレ'E

$\angle Z L^{\prime} \varepsilon$

เทเ์

$\nabla G L^{\prime} \varepsilon$

$\nabla 9 L^{\circ} \varepsilon$

$9 \angle L^{\circ}$

เ8Z 9

$00 \varepsilon^{\prime} \mathrm{S}$

$6 เ \varepsilon^{\prime} \mathrm{s}$

616.9

† 6. 9

$\varepsilon \varepsilon 6.9$

$\varepsilon \forall 6.9$

$0 \varepsilon 0^{\circ} \mathrm{L}$

$0 \rightarrow 0^{\circ} \mathrm{L}$

$670^{\circ} \mathrm{L}$

$\checkmark 90^{\circ} \mathrm{L}-\mathrm{L}$

$\checkmark G L L^{-}$

$09 L^{\circ} \mathrm{L}$

$\angle 9 L^{\circ} \mathrm{L}$

ILL'L

$9 \angle L L$

$\varepsilon 8 L^{\circ} \mathrm{L}$

$68 \mathrm{~L}^{\circ} \mathrm{L}$

09Z L

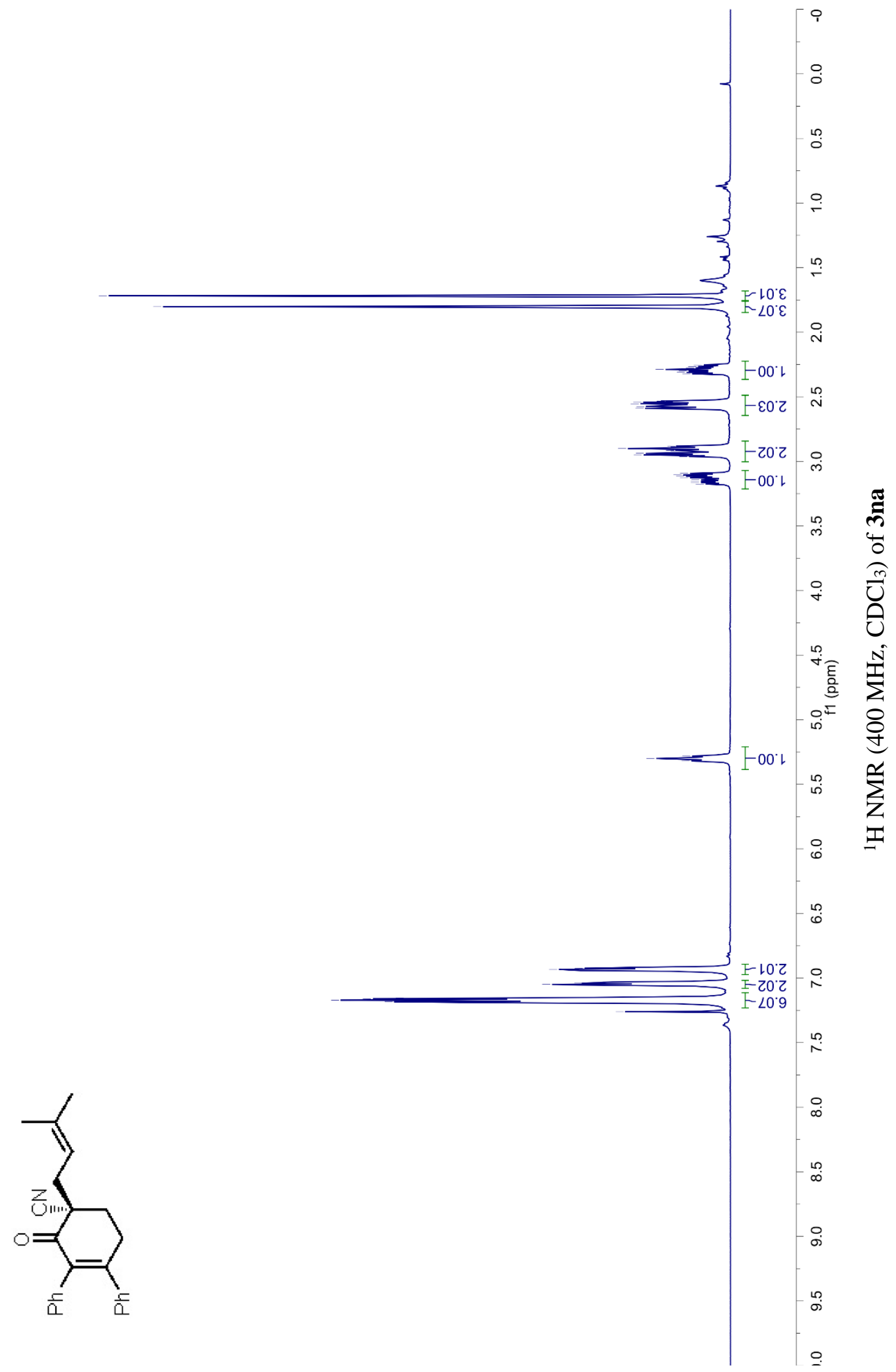




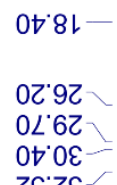

حG'

$0 Z \angle \nabla$

$78.9 L$

$9 L^{\circ} L L=$

68.9レ

Gレ6レ-

$6 \varepsilon^{\prime}$ LZL

$\angle 8^{\circ} \angle Z$ Z

6L8ZL

ह乙'8Zレ

69'8Z

乙6. 0 \&

$\neg 8^{\circ} \nabla \varepsilon$ L

$\angle 9^{\circ} \mathrm{SEL}$

S8 $\angle E L$

E9.6ع -
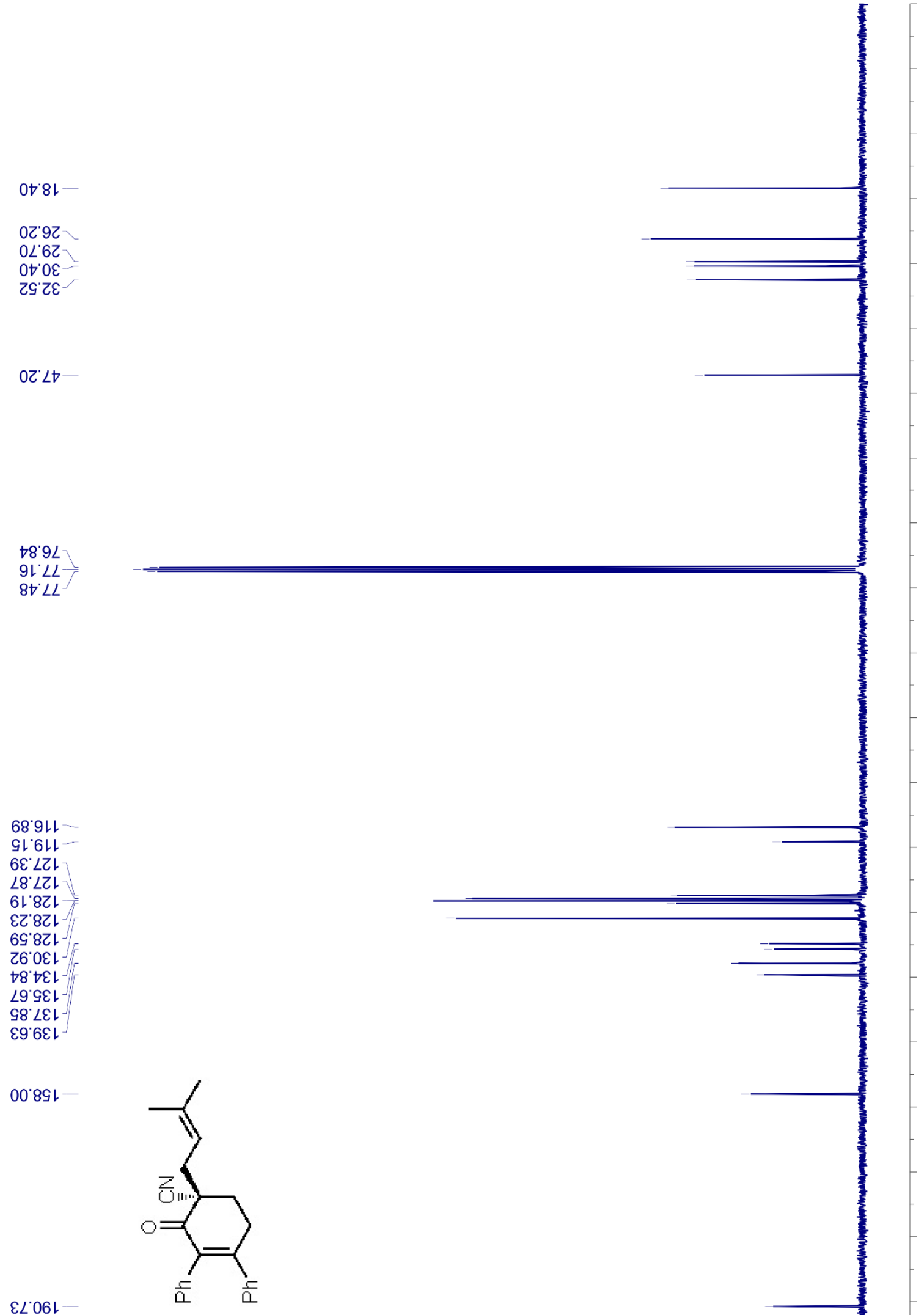


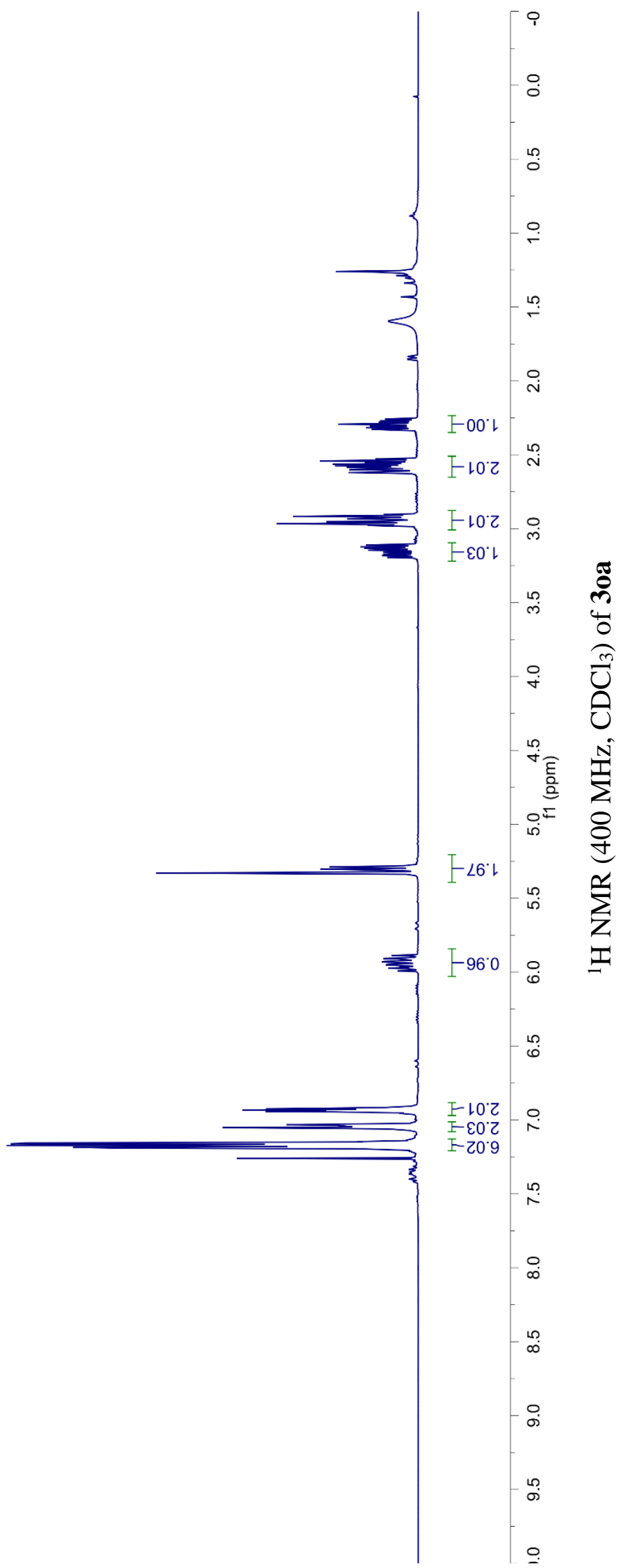



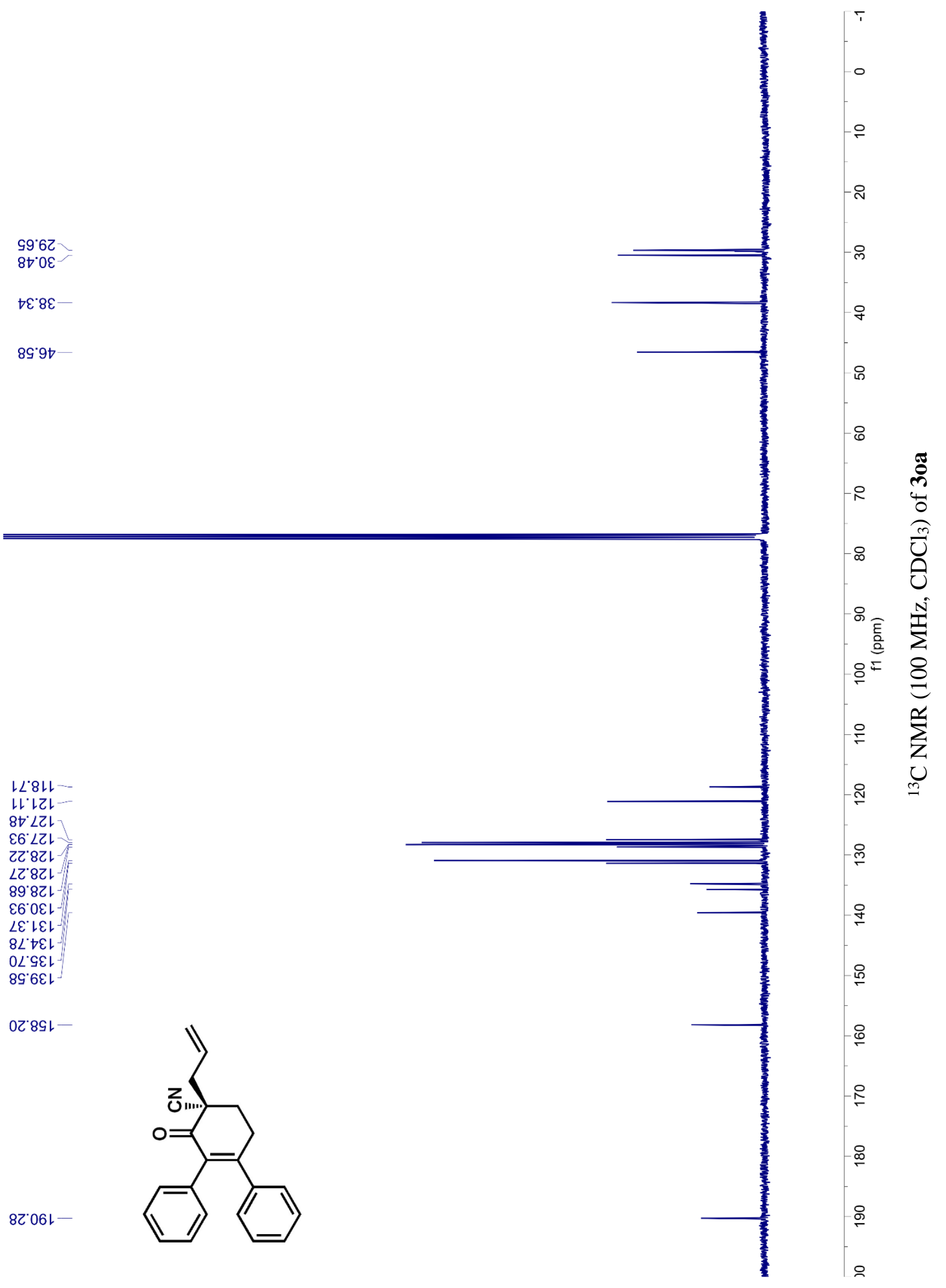
LLL'

6เレ'

LEL'

เレL'

GSL

$60 乙$ '

$\angle \forall \varepsilon^{\prime} Z$

$0 \subseteq \varepsilon^{\prime} 乙$

$\varepsilon 9 \varepsilon^{\prime} 乙$

$69 \varepsilon^{\prime} Z$

Lレ๐

LEt 2

レナて

Est?

sSt?

$\angle L D^{\circ} Z$

เ67 2

L8G'Z

ह09.

L29 2

$\varepsilon 6 L ' Z$

$\angle 08^{\circ} \mathrm{Z}$

$\angle 18^{\circ} Z^{-}$

LE8 $Z^{\prime}$

$8 \angle 8^{\circ} Z$

$\varepsilon 68$ '

$\varepsilon 06.2$

$\angle 16{ }^{\circ} \mathrm{C}$

$\supset 00^{\circ} \varepsilon$

LLO

$0 Z 0^{\circ} \varepsilon$

$\varepsilon \varepsilon 0^{\prime} \varepsilon$

$890^{\circ} \varepsilon$

$180^{\circ} \varepsilon$

$\angle 80^{\circ} \varepsilon$

$00 L^{\prime} \varepsilon$

†06. 9

606.9

ㄴ.6.9

\&เ6.9

$\angle L 6.9$

616.9

\&Z6. 9

9Z6. 9

676.9

เE6.9

GZO'L

$\angle Z O^{\circ} \mathrm{L}$

$0 \varepsilon 0^{\circ} \mathrm{L}$

ZE 2

$9 \varepsilon 0^{\circ} \mathrm{L}$

${ }^{8} 80^{\circ} \mathrm{L}$

$0 \rightarrow 0$ L

$S t 0.2$

$670^{\circ} \mathrm{L}$

290.2

EtL L

$87 L$

ZGL'L

$99 L^{\circ} L$

$69 L 2$

$79 L^{\circ} \mathrm{L}$

$\angle 9 L^{\circ} \mathrm{L}$

$69 \mathrm{~L}^{\circ} \mathrm{L}$

CLL'L

SLLL

$8 \angle L^{\circ} L$

$08 \mathrm{~L}^{\circ} \mathrm{L}$

S8L'L

$88 \mathrm{~L} L$

16L'L

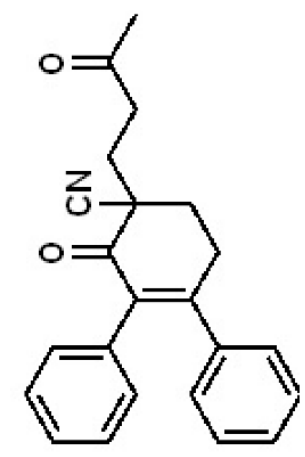

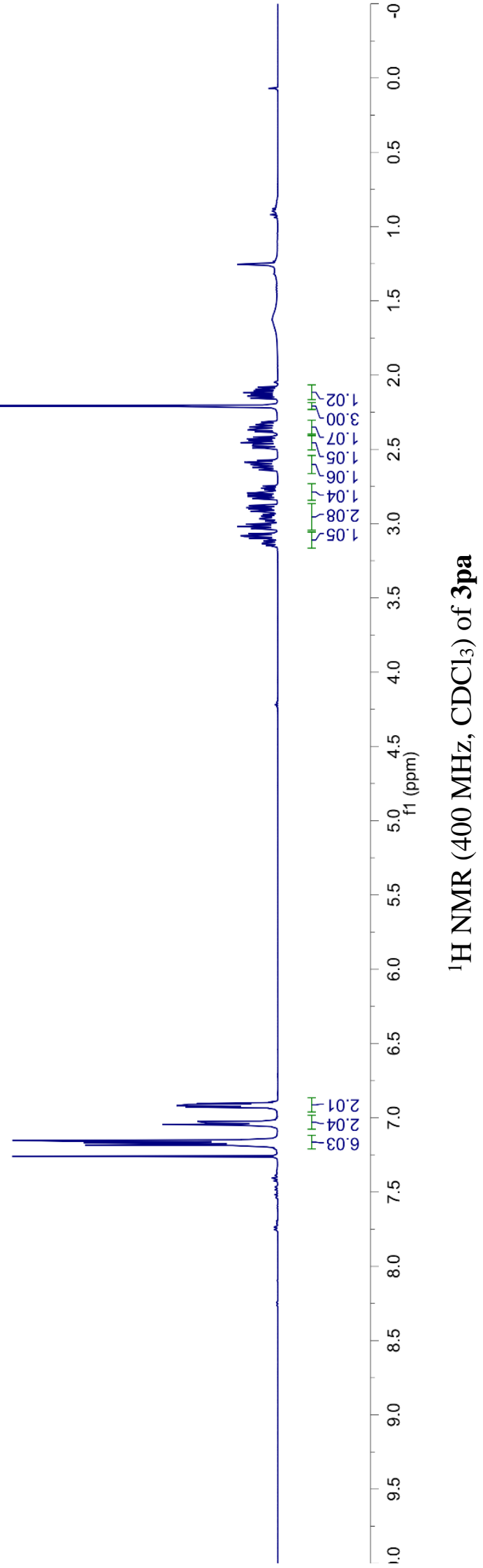


$9 L \cdot L Z$
$\forall \nabla^{\circ} 6 Z$
$\varepsilon Z^{\prime} 0 \varepsilon$
$\varepsilon 0^{\circ} 2 \varepsilon$
$8 \varepsilon^{\prime} 6 \varepsilon-$
$69^{\circ} 9 t-$

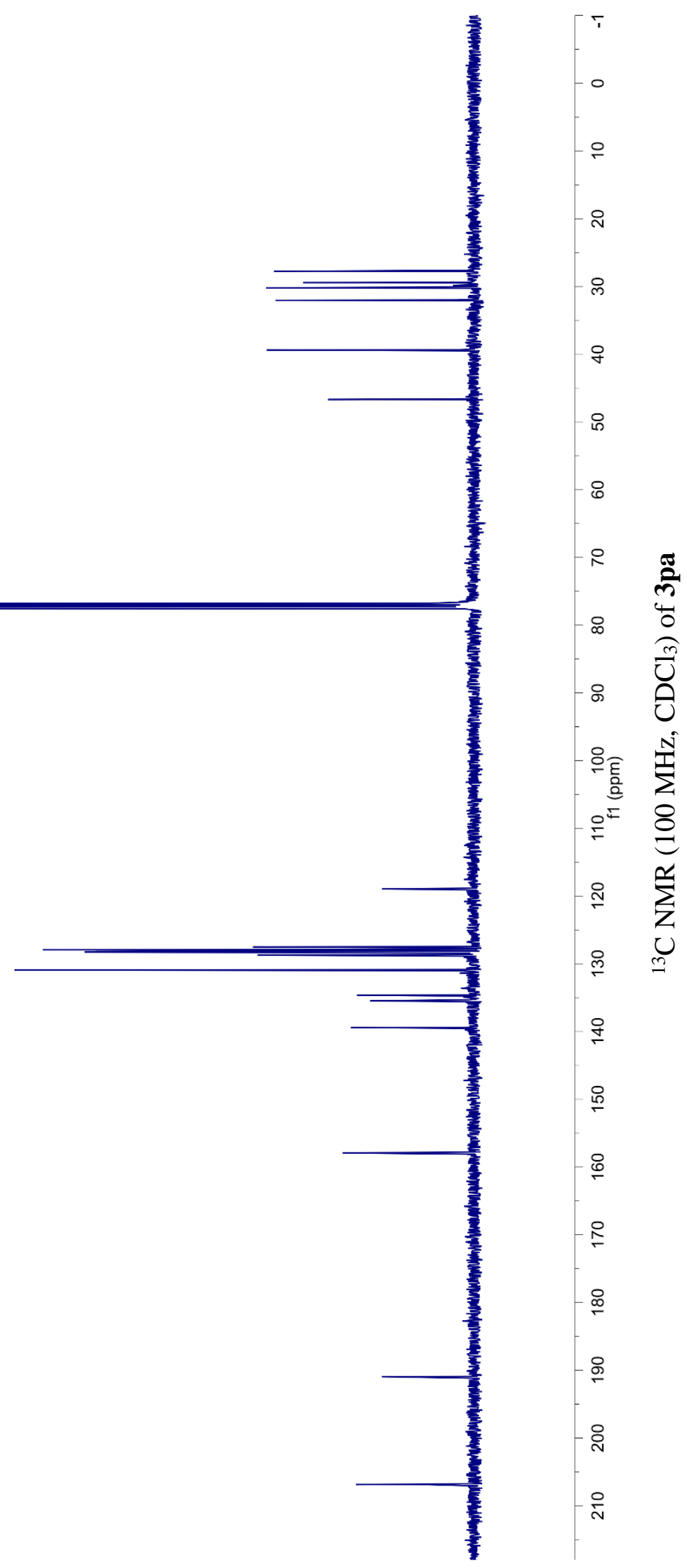

.

$96.8 \mathrm{~L}$

$0 S^{\circ} \angle Z L$

6L'8ZL-

$8 Z 8 Z \mathrm{~L}$

ZL'8ZL

L6.0EL

$Z 9^{\circ} \nabla \varepsilon L$

$97 \mathrm{~s} \varepsilon$

E6. LSL -

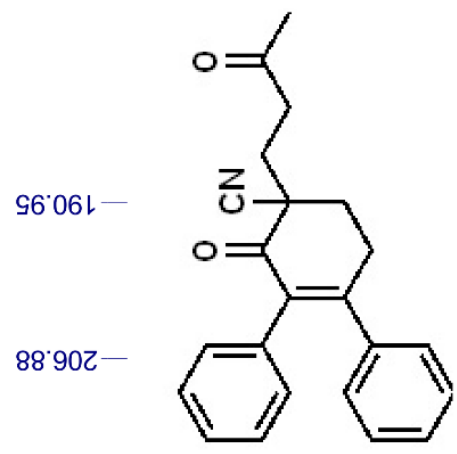




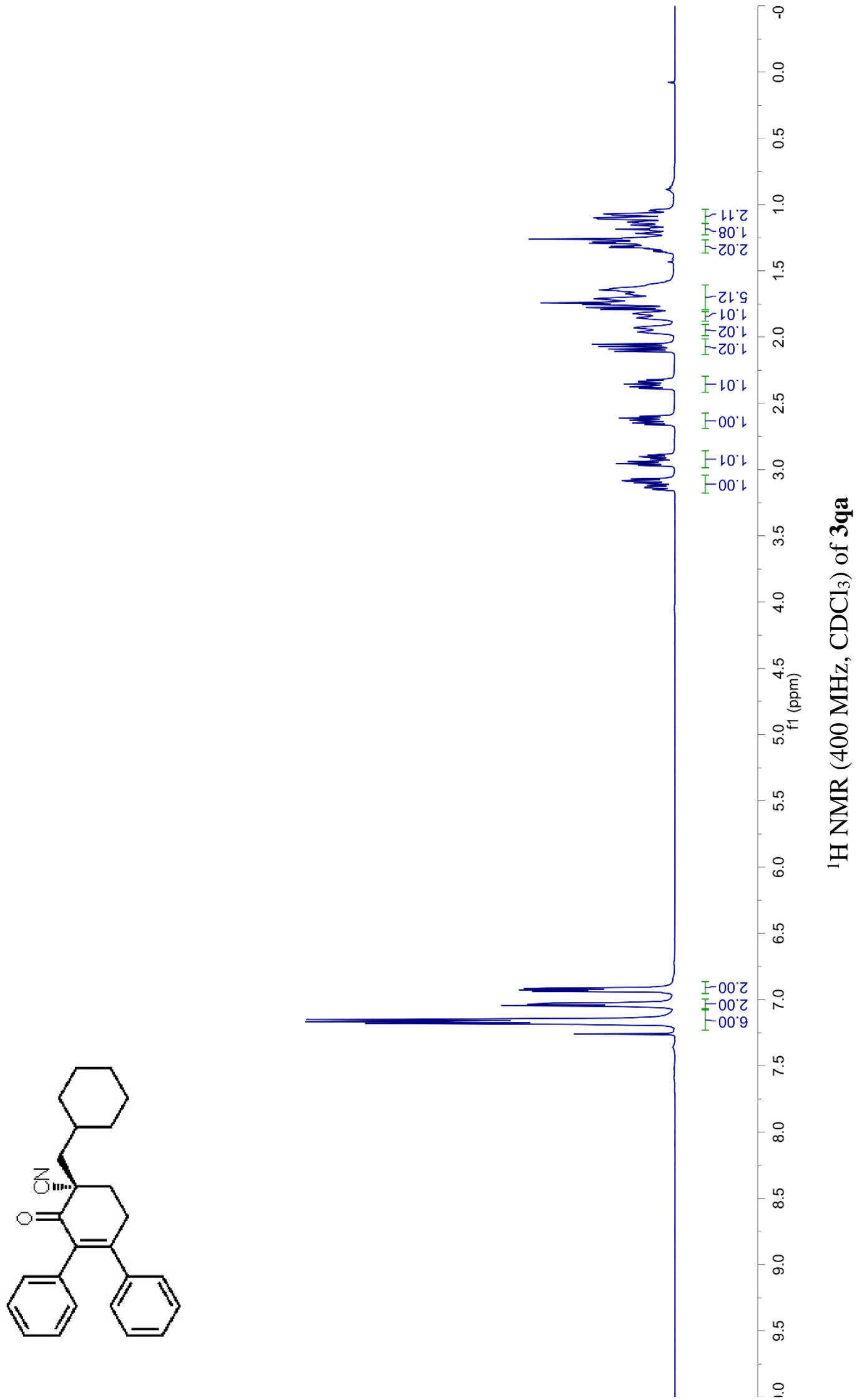



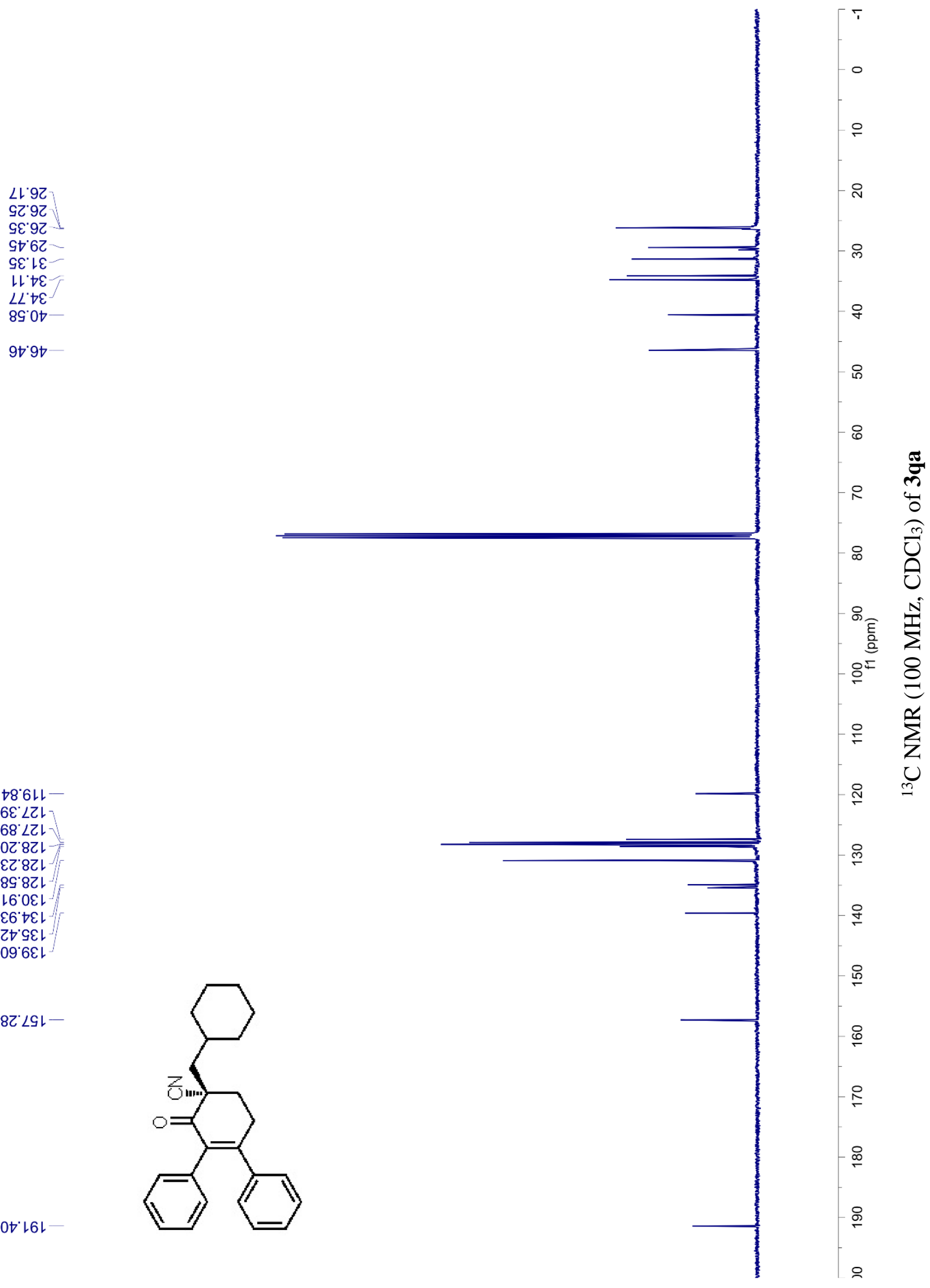


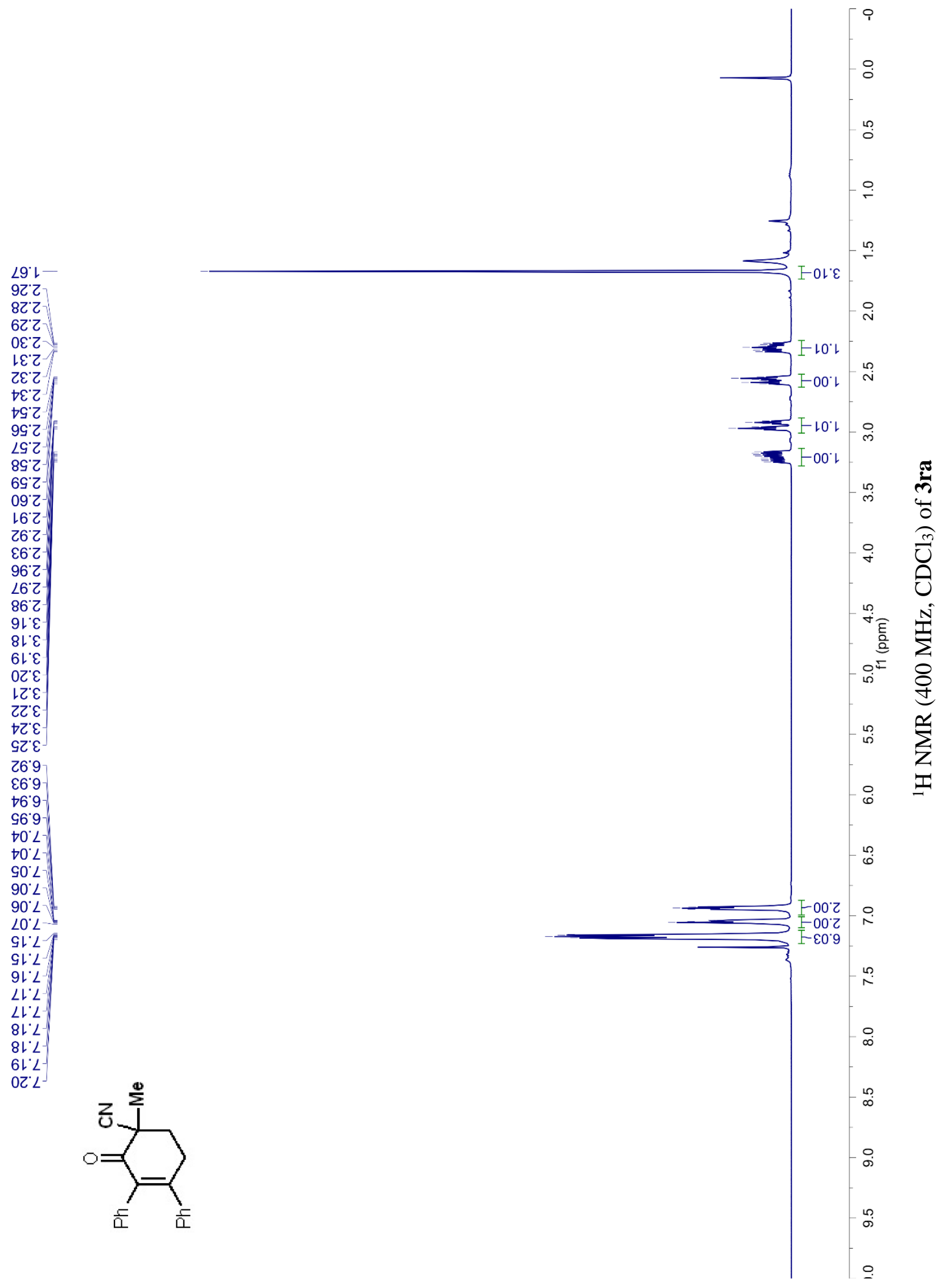




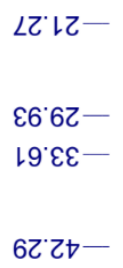

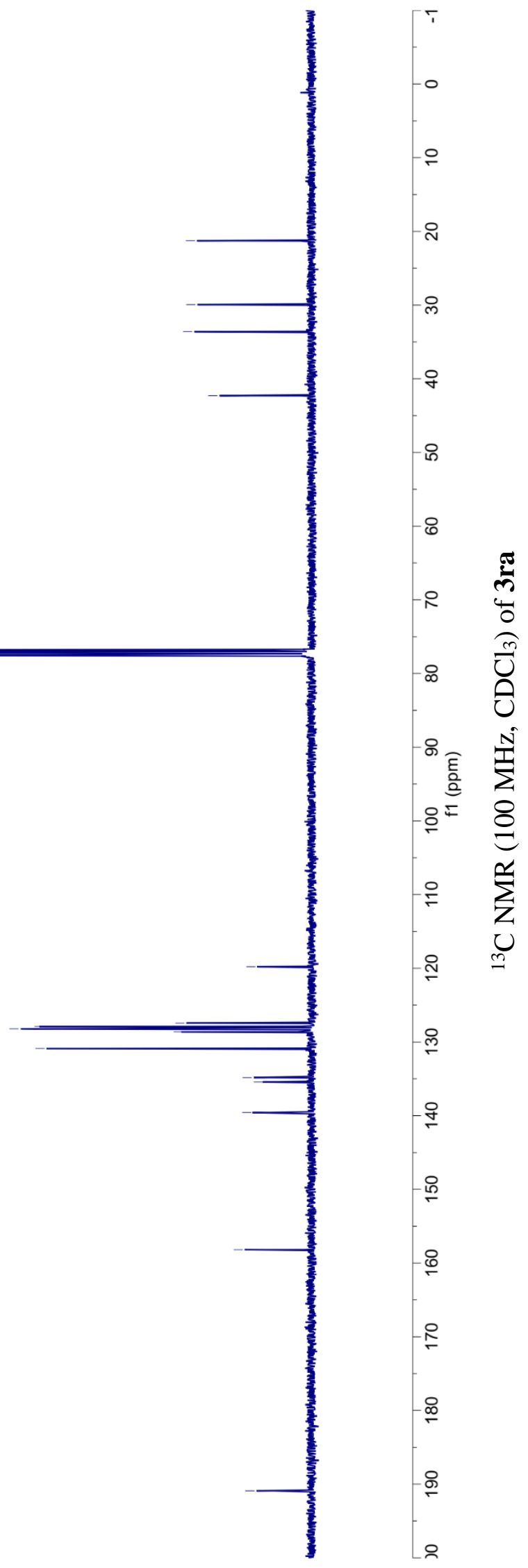

8L'6L-

$\varepsilon t^{\circ} \angle Z L$

$06 \angle Z L$

เZ8ZL

$\downarrow Z$ ८ZL

\&9.8ZL

$88^{\circ} 0 \mathrm{\varepsilon l}$

乙8 $\sqcup \varepsilon\llcorner$

$\varepsilon † \subseteq \varepsilon L^{-}$

$8 S^{\circ} 6 \varepsilon \mathrm{L}$

6เ.891- 
諨 $\varepsilon^{-}$

996. 9

$086^{\prime} 9$

$686^{\prime} 9$

†66. 9

$866^{\circ} 9$

$700^{\circ} \mathrm{L}$

ELO L

$810 \mathrm{~L}$

$\mathrm{ZZO} \mathrm{L}$

$620^{\circ} \mathrm{L}$

$6 \varepsilon 0^{\circ} \mathrm{L}$

GZL'L

GEL L

七大 ${ }^{\circ} L$

$8+L L$

$\angle G L L$

G9l'L

ILL'L

GLL'L

$181^{\circ} 2$

$68 \mathrm{~L}^{\circ} \mathrm{L}$

E6L'L

$\varepsilon O Z \angle$

SIC: $L$

gदर 2

$\neg 9 \varepsilon^{\circ} L$

$0 \angle E^{\circ} L$

$9 \angle \varepsilon^{\circ} L$

L8E $L$

$06 \varepsilon^{\circ} L^{-}$

G6E'L

EOt L

แナ้L

6เだ

EZt L

8Zt L

$\varepsilon \varepsilon \nabla \cdot L$

Zヤサ'L

SSt'L

$19 t^{\circ} \mathrm{L}$

$8 \angle t^{\circ} L$

$187^{\circ} \mathrm{L}$

18t

เ6t' $\mathrm{L}$

$\angle 0 \mathrm{~S}^{\circ} \mathrm{L}$

ZIS'L
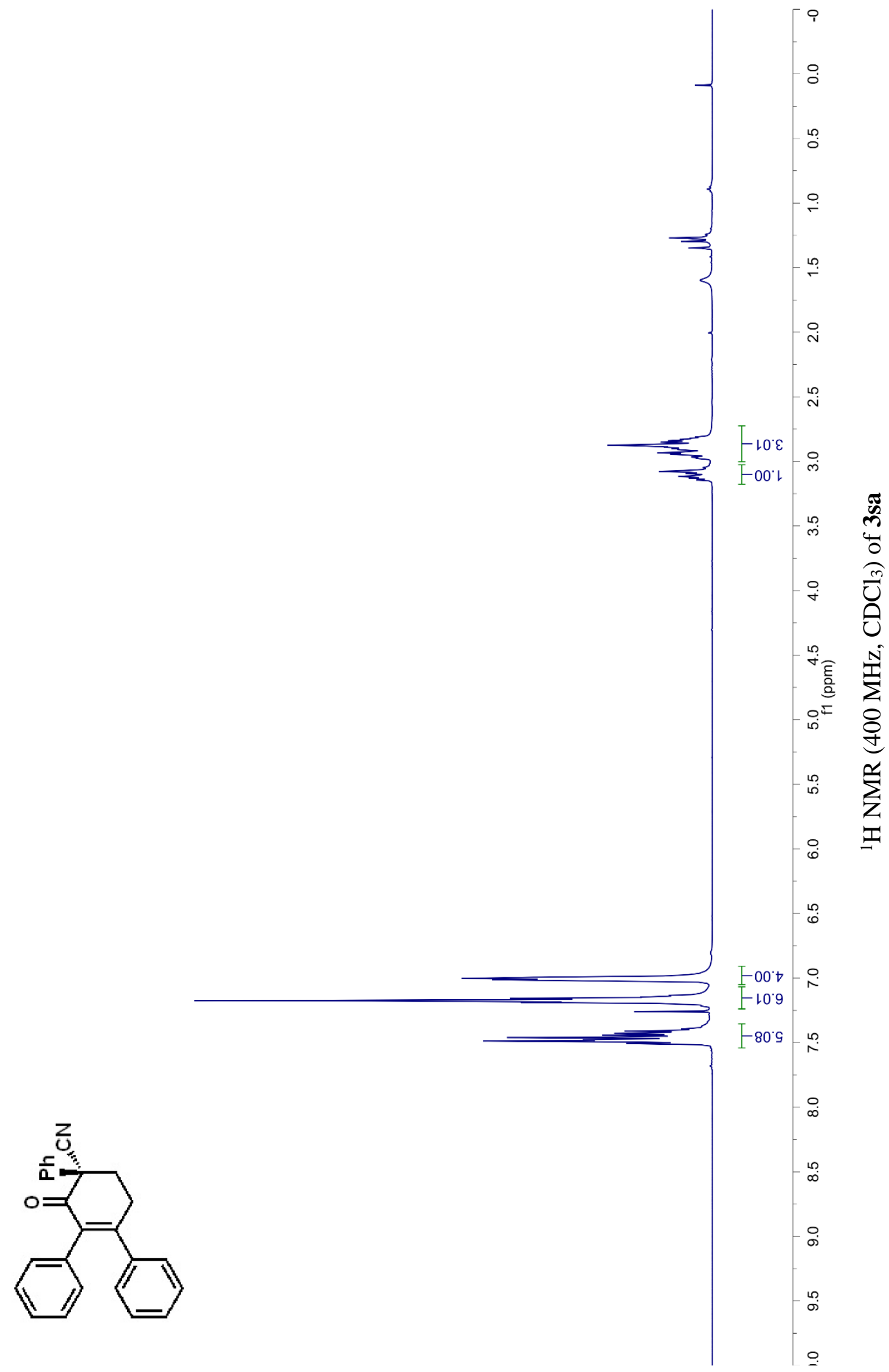


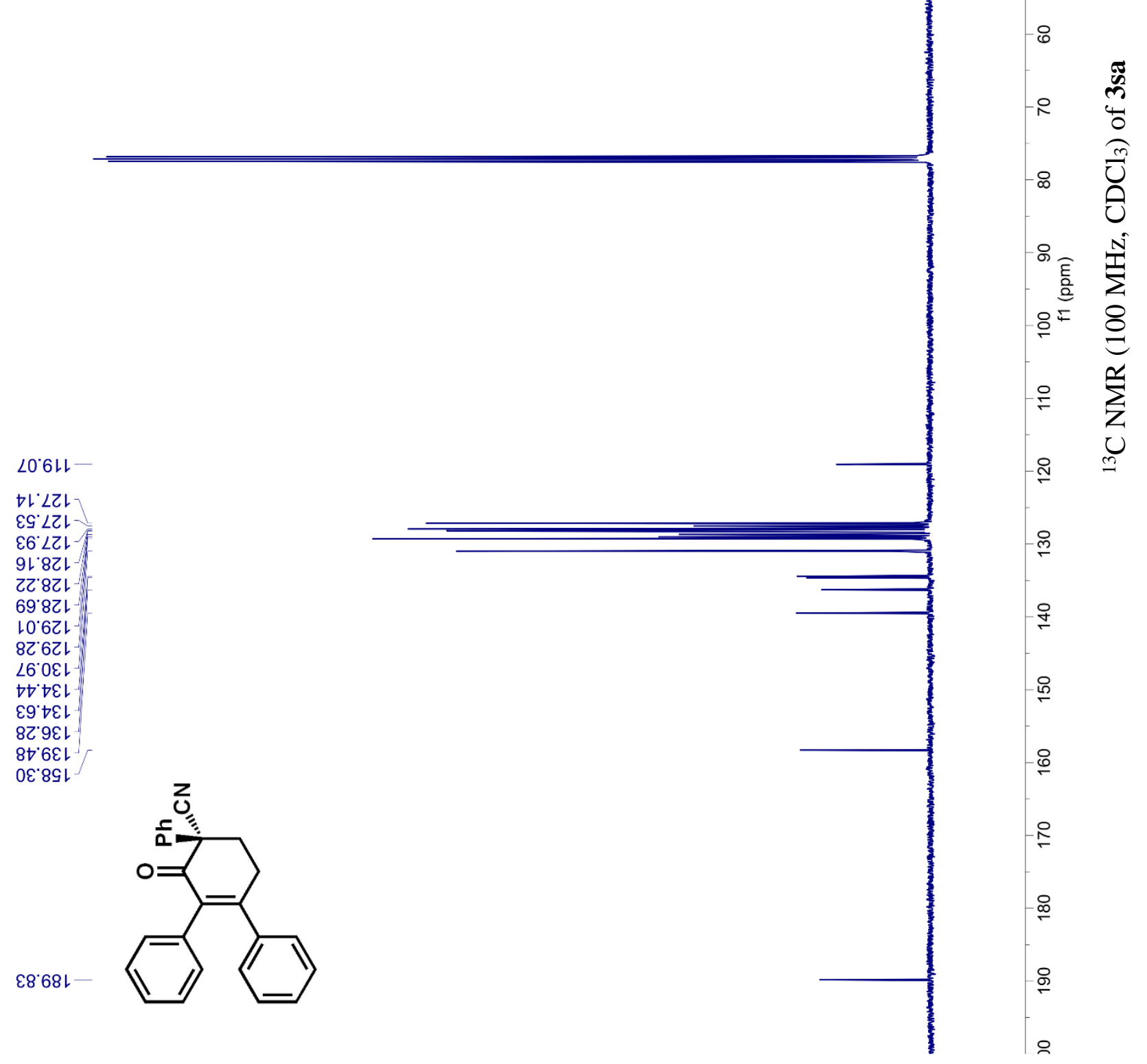


$69 \mathcal{C}^{\prime} \varepsilon$

$\varepsilon 0 \varepsilon \varepsilon$

9เE'

9เฑ $\varepsilon$

乙Eฑ $\varepsilon$

乙9॰ $\mathcal{~} \varepsilon$

OOL'L

$70 L^{\circ} \mathrm{L}$

$60 \mathrm{~L} L$

ELLL

$8 \mathrm{LL}$

$\triangle 2 L^{\circ} L$

LEL $L$

$\checkmark \varepsilon L L$

Z†L'L

StDL $L$

0912

6GL'L

E9L'L

$99 L^{\circ} \mathrm{L}$

LLL L

6SZ'L

$79 Z 2$

$8 L Z^{\prime} L$

$78 Z^{\prime} L$

$76 Z^{\prime} L$

$862 \mathrm{~L}$

$80 \varepsilon^{\circ} L$

乙IE L

6LE'L

$\angle Z E^{\circ} L$

LEE L

ธEع ' $L$

$6 \varepsilon \varepsilon^{\circ} L$

$\nabla \nabla \varepsilon^{\prime} L$

$6 \nabla \varepsilon^{\circ} L$

हG.

ह

09E'L

$\neg 9 \varepsilon^{\circ} L$

L $\angle E^{\circ} L$

$\angle L E^{\circ} \angle$

$\angle 8 E^{\circ} \angle$

$06 \varepsilon^{\circ} L$

$\rightarrow 6 \varepsilon^{\circ} L$

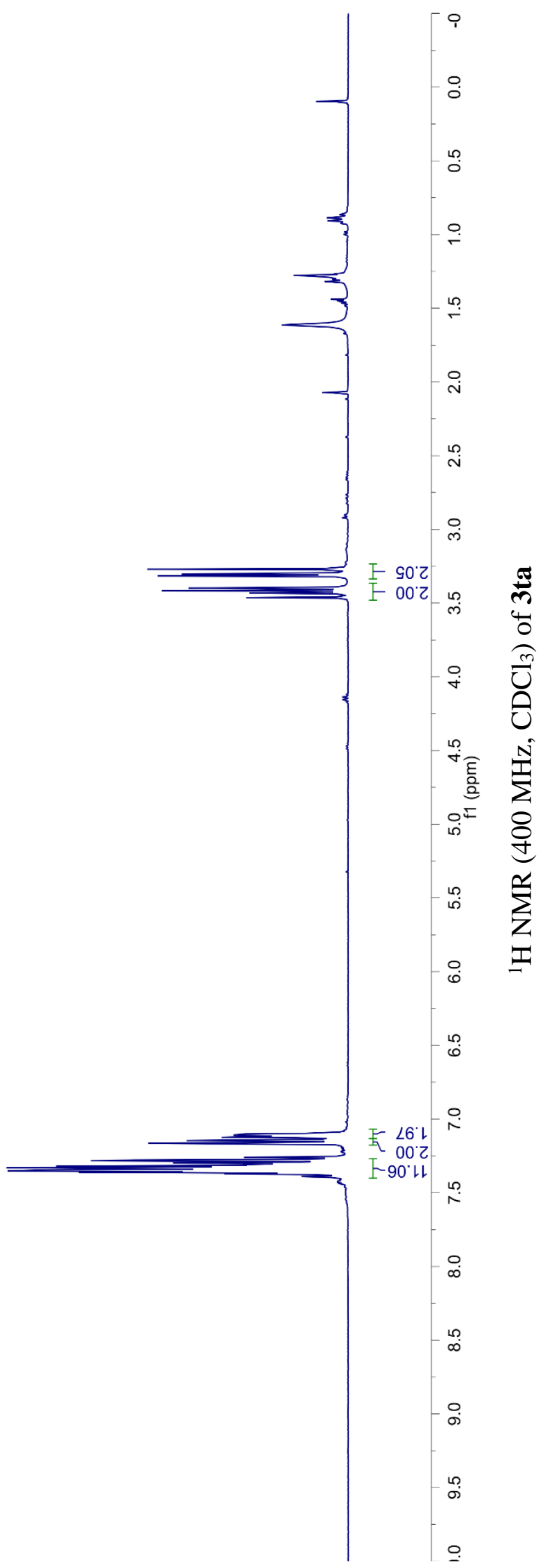



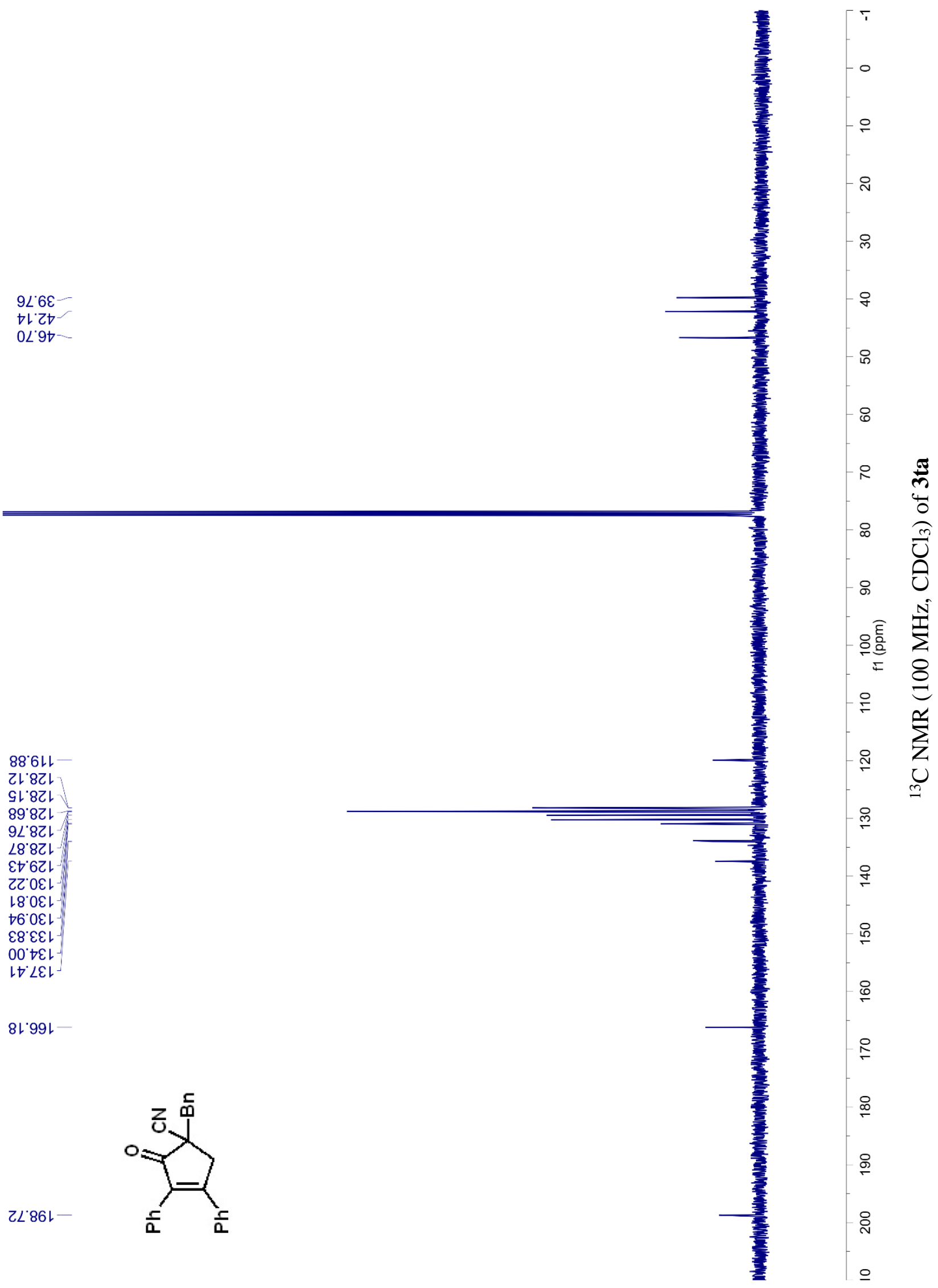

88*6レ-

乙L8ZL

Gl'8ZL

89.8ZL

9L'8ZL

L8'8Z

E७ 6ZL

乙乙'0ع

เ8.0ع

$76^{\circ} 0 \varepsilon\llcorner$

$\varepsilon 8^{\circ} \varepsilon \varepsilon-$

$00^{\circ} \nabla \varepsilon L-$

เ $\angle \varepsilon L$

8L'99L- 
ESLL

L9L'L

Z98.

Z88.

S06.

LLE'乙

$\neg 乙 \varepsilon ' 乙$

เ $\varepsilon \varepsilon^{\circ} 乙$

เจย 乙

乙9६"乙

69ع 乙

6LE'乙

L8E'乙

009'乙

GZS'Z

6عG'乙

จ9G'乙

$690^{\circ} \varepsilon$

$\varepsilon 0 L^{\circ} \varepsilon$

8ح9. $\varepsilon$

Z99' $\varepsilon$

†Z8'9

E†8:9

$678^{\circ} 9$

$\angle 98^{\circ} 9$

$\angle 98^{\circ} 9^{\circ}$

GL8.9

$088^{\circ} 9$

988:9-

689

$\angle 68^{\circ} 9$

$906^{\circ} 9$

เ 6 .9

हट6. 9

$8 Z 6.9$

\&E6.9-

$\angle E O^{\circ} \angle$

$0 \supset 0^{\circ} L$

$\triangle \nabla 0^{\circ} \mathrm{L}$

$190^{\circ} \mathrm{L}$

$990^{\circ} \mathrm{L}$

$190^{\circ} \mathrm{L}$

6レL

$9 \varepsilon L$

09L'L

$6 L^{\circ} L$

087" L

009 $L$

IEL 6

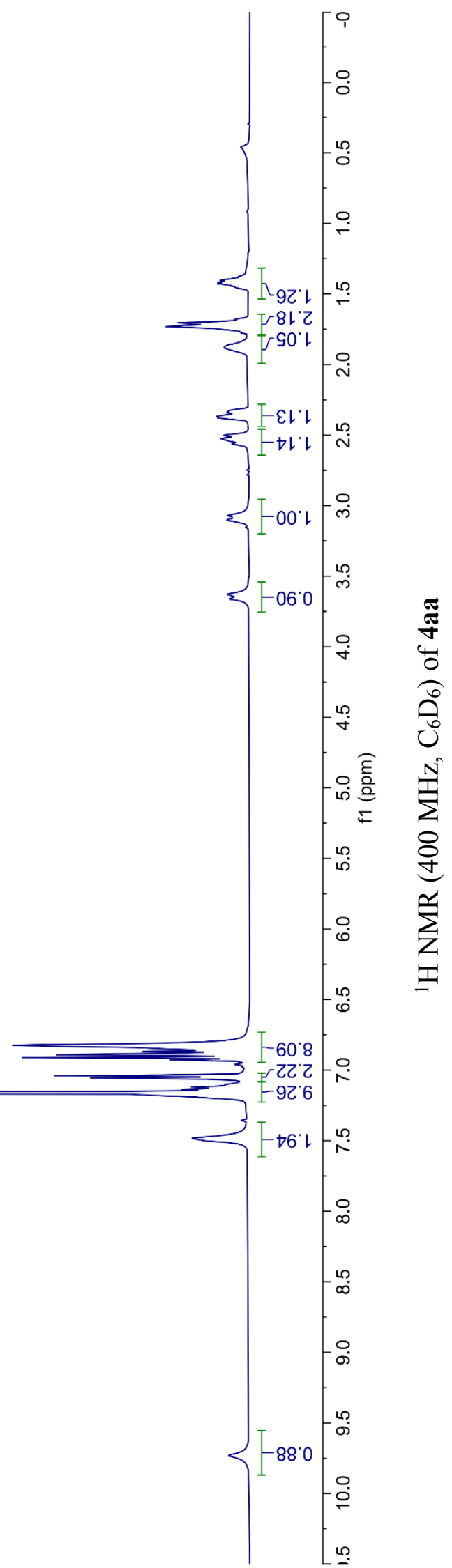


๑๐:乙-

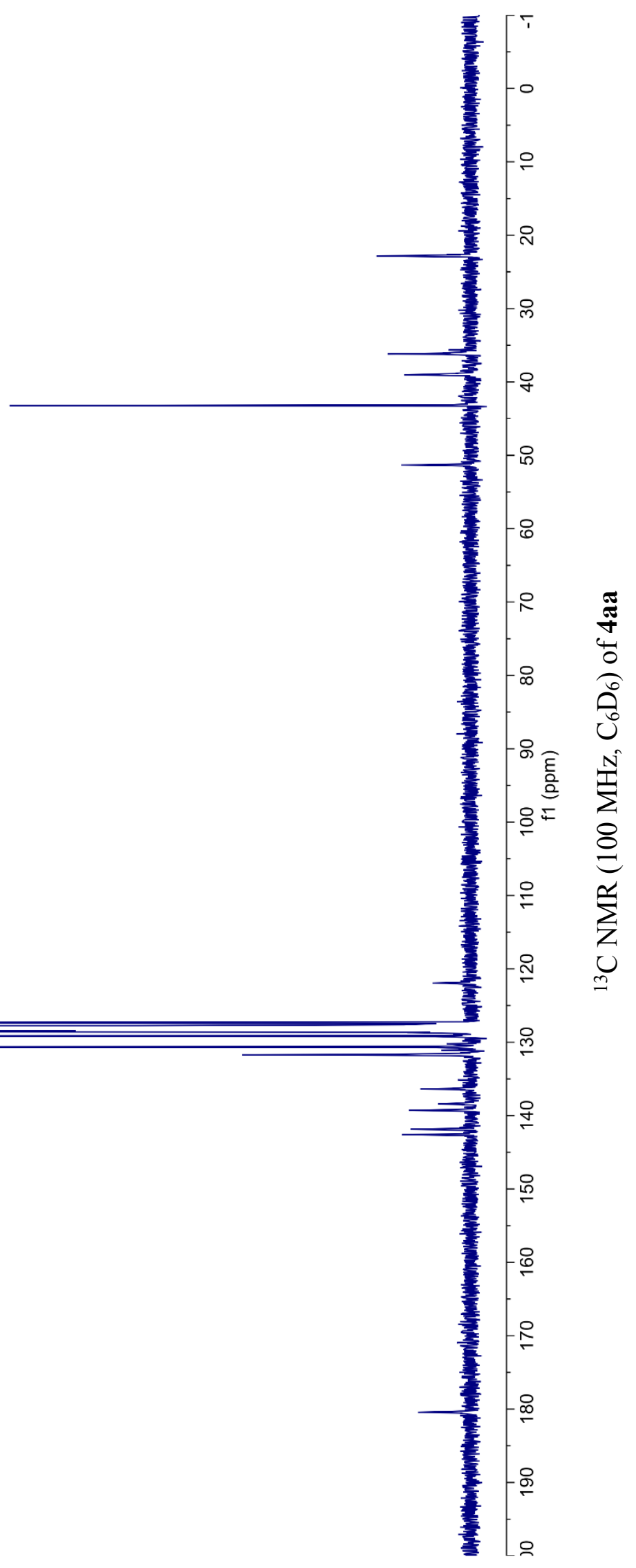

$\forall 6 . L Z L$

$\left.\begin{array}{r}9 \\ { }^{\circ} \angle Z L\end{array}\right]$

Z8 LZL

$90.8 Z \mathrm{~L}$

$0 \varepsilon^{\circ} 8 Z \mathrm{~L}$

09.8ZL

$9 l^{\circ} 6 \mathrm{ZL}$

99.0ह

$8 \varepsilon^{\prime} 9 \varepsilon \downarrow$ -

Z† $8 \varepsilon \downarrow$

$8 Z^{\prime} 6 \varepsilon 1$ -

98・十ト

09'Z†L

st.08เ-

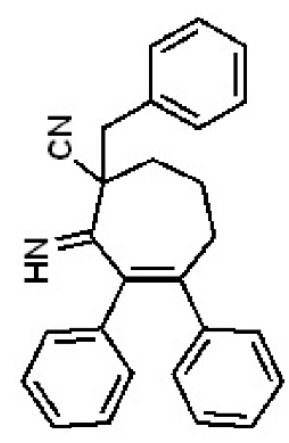




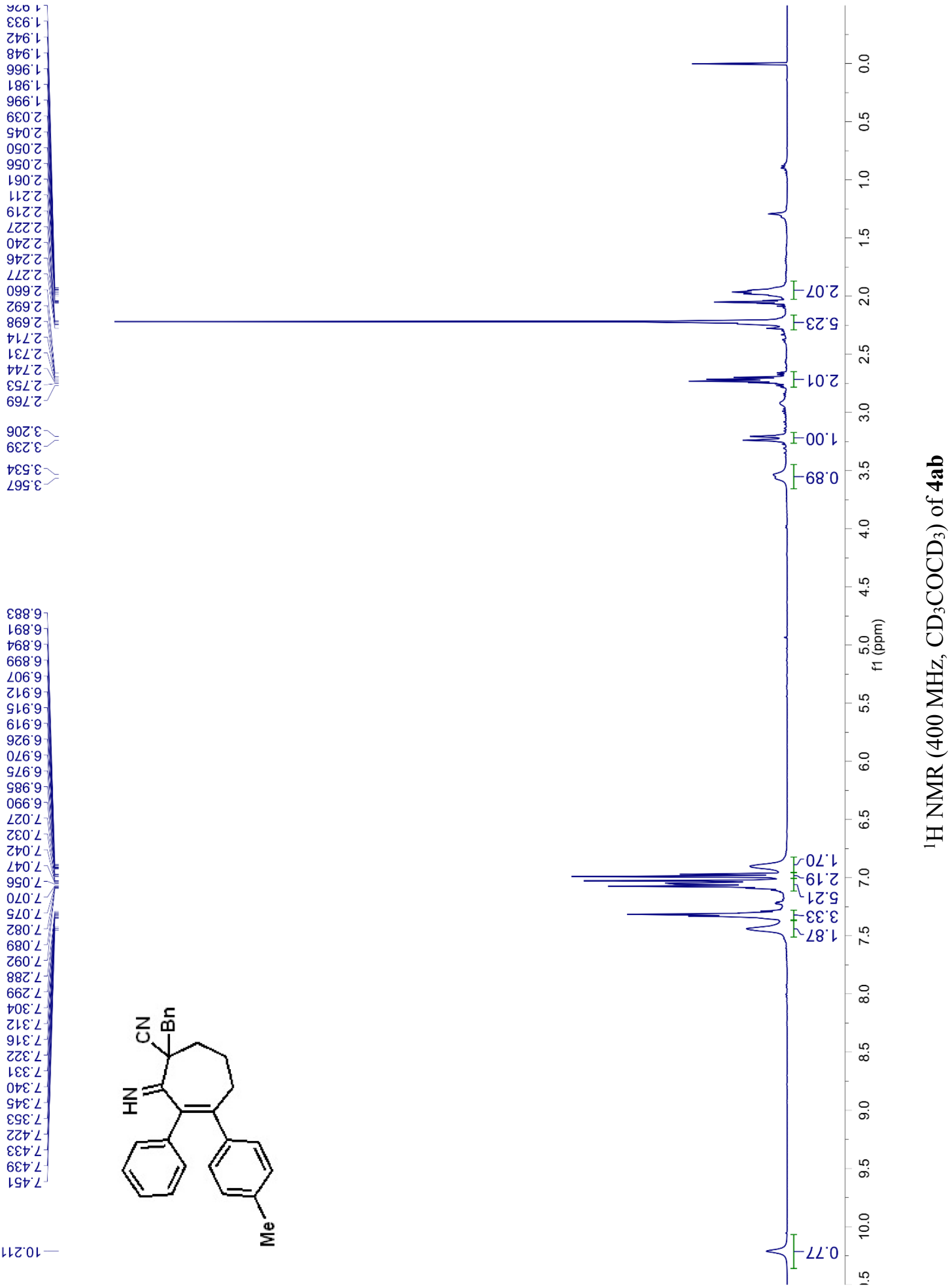




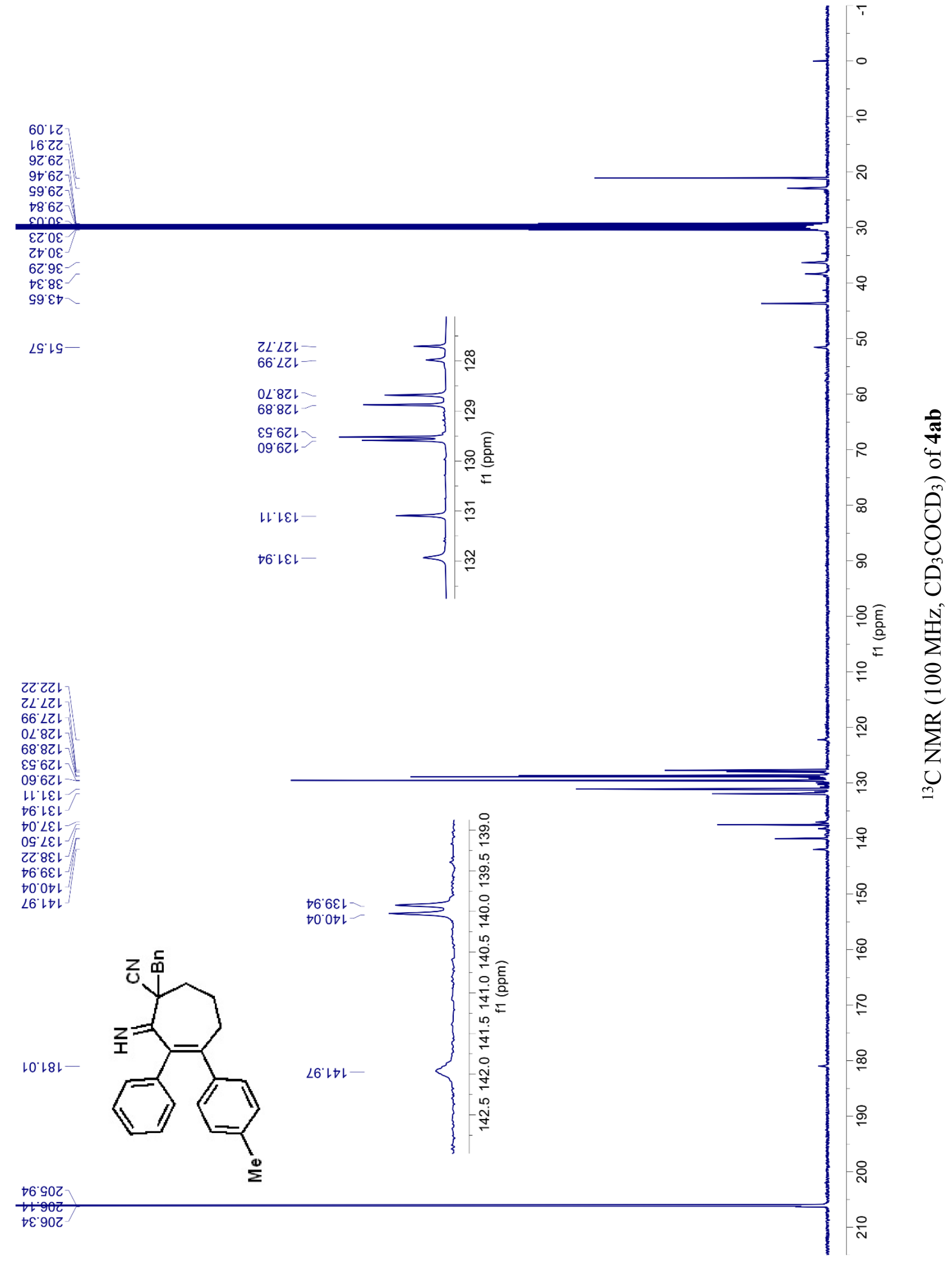


8Z6"

$\nabla \varepsilon 6^{\circ}$ -

$0+66^{\circ} \mathrm{L}$

976.

$096^{\circ} \mathrm{L}$

Z96.

996.

$896^{\circ} \mathrm{L}$

796 L

$18 L^{\circ} 2$

S6L' 2

OLZ'Z

s99'

$199^{\circ} \mathrm{Z}$

$999^{\circ}$

$\nabla \angle 9^{\circ} \mathrm{Z}$

$089^{\circ} Z$

$\angle 89^{\circ} \mathrm{Z}$

$169^{\circ} \mathrm{Z}$

Eงเ' $\varepsilon$

$\angle 8)^{\circ} \varepsilon$

$\neg 8 \nabla^{\circ} \varepsilon$

$\angle L G^{\prime} \varepsilon$

\&96"9

896.9

乙L6"9

ELO $L$

$910^{\circ} \mathrm{L}$

乙EO ${ }^{\circ}$

$670^{\circ} \mathrm{L}$

$290^{\circ} \mathrm{L}$

$090^{\circ} \mathrm{L}$

S90 $\mathrm{L}$

GLO $L$

$060^{\circ} \mathrm{L}$

$760^{\circ} \mathrm{L}$

$660^{\circ} \mathrm{L}$

EOL L

$60 L^{\circ} \mathrm{L}$

ZIL'L

6ZL L

EOE ' $L$

$80 \varepsilon^{\circ} L$

GLE $L$

OZE $L$

9ZE $L$

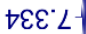

$\angle \nabla \varepsilon^{\circ} \angle$

GSE 'L

G8E' $L-$

$86 \varepsilon^{\circ} L$

$90 t^{\circ} L$

OLOOL-

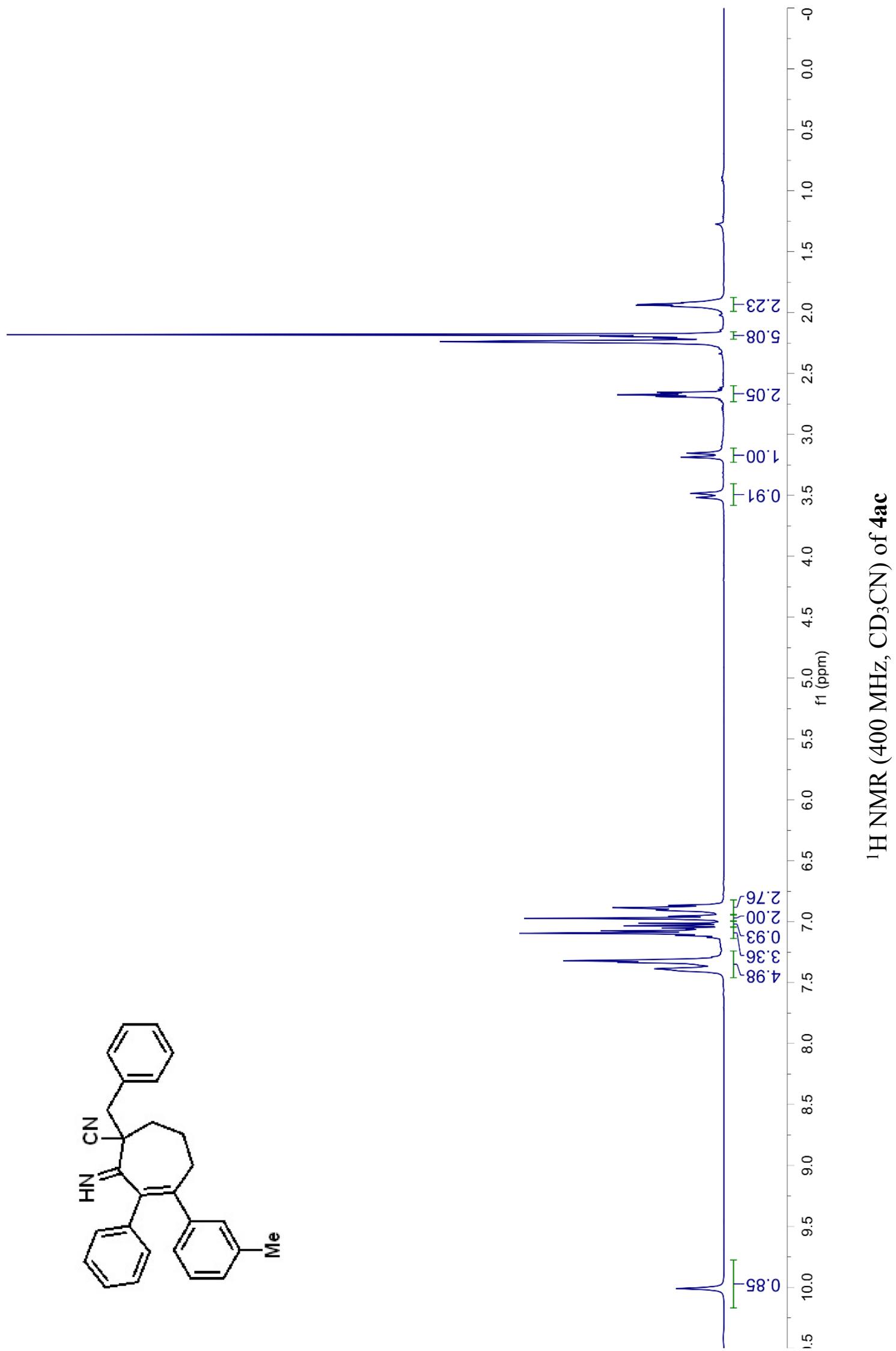




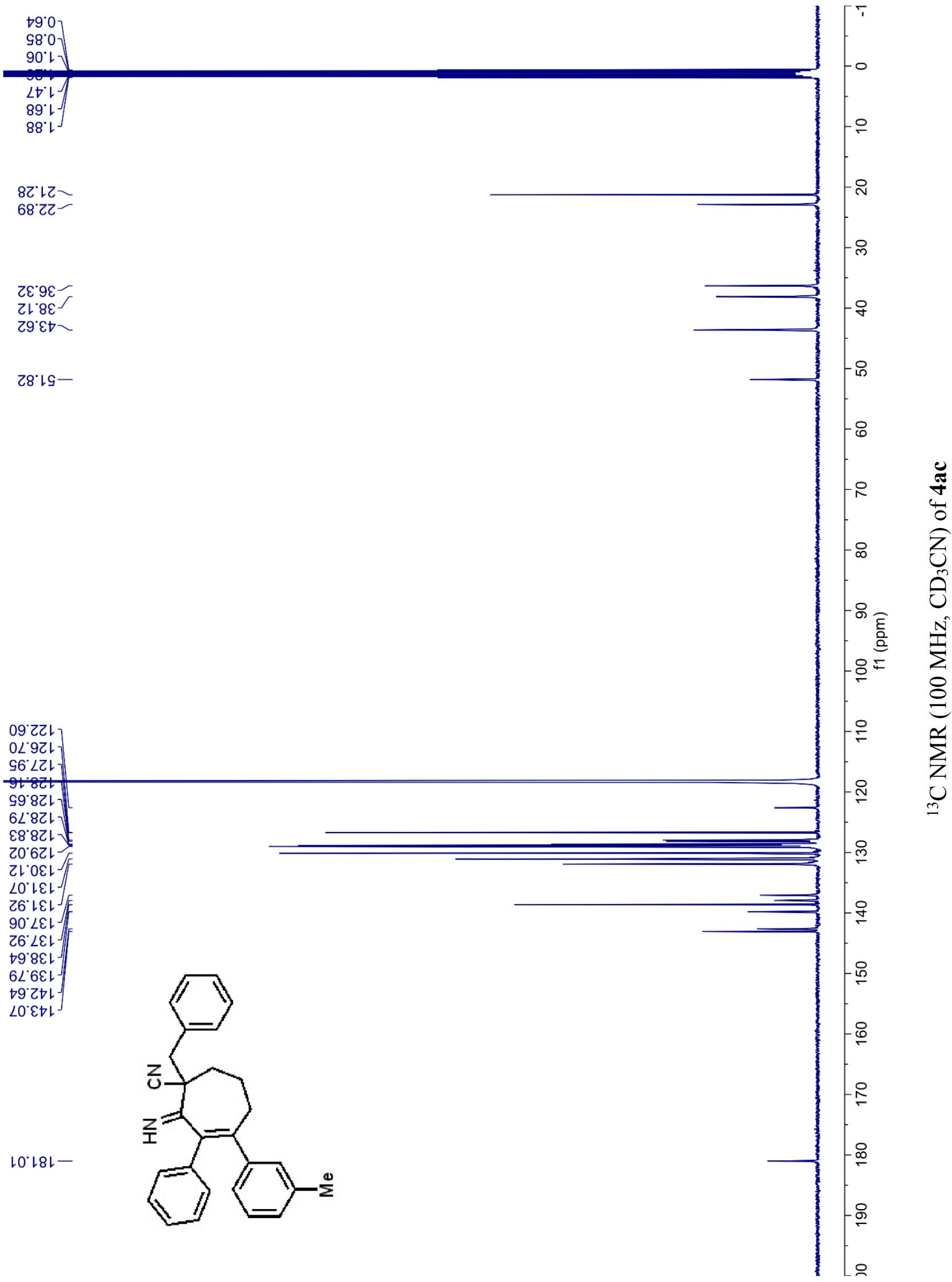




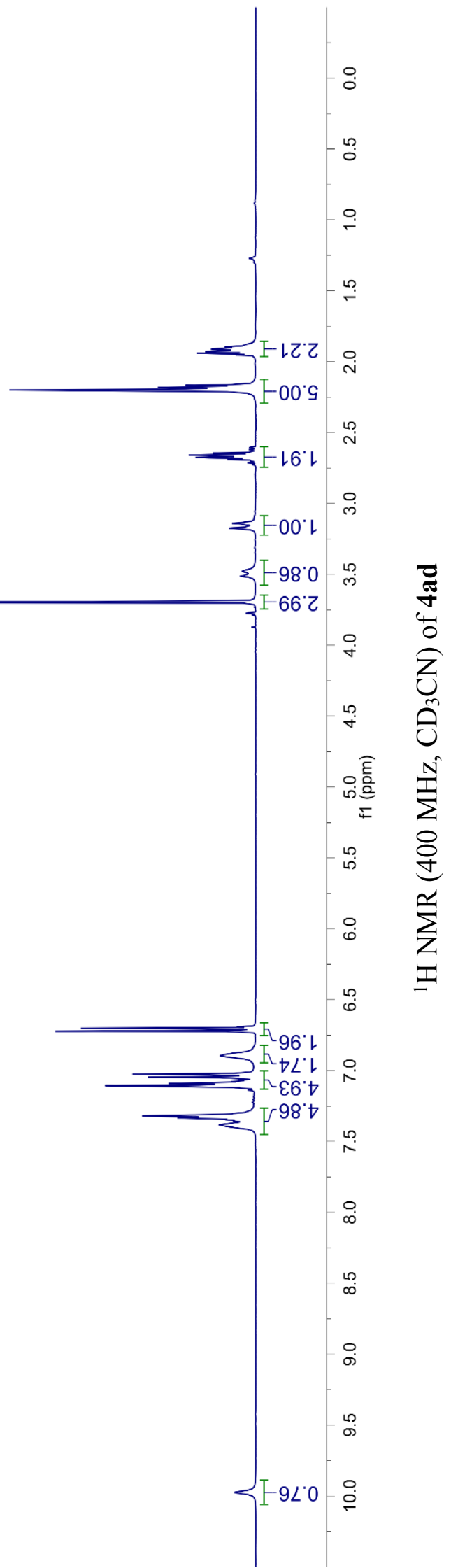




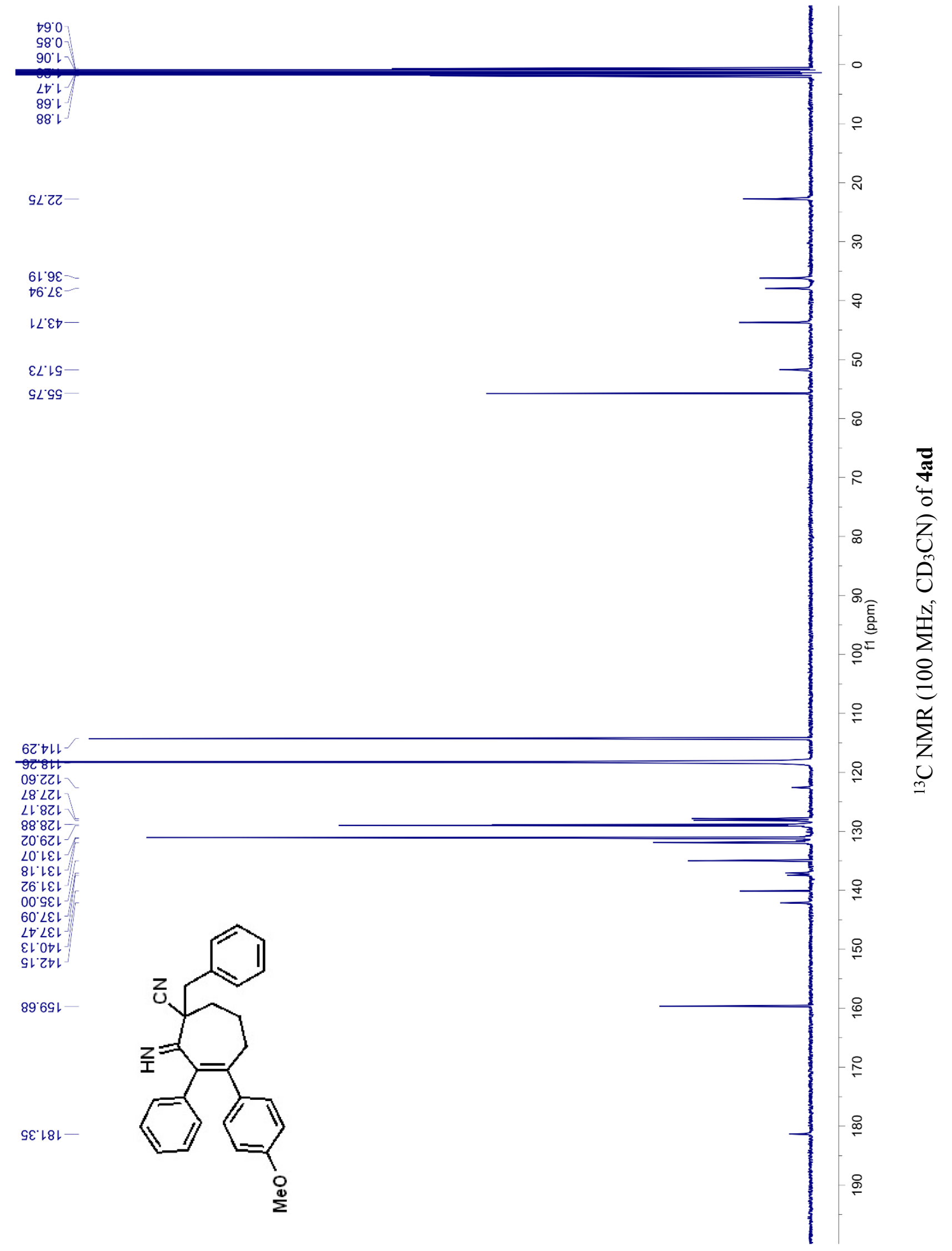




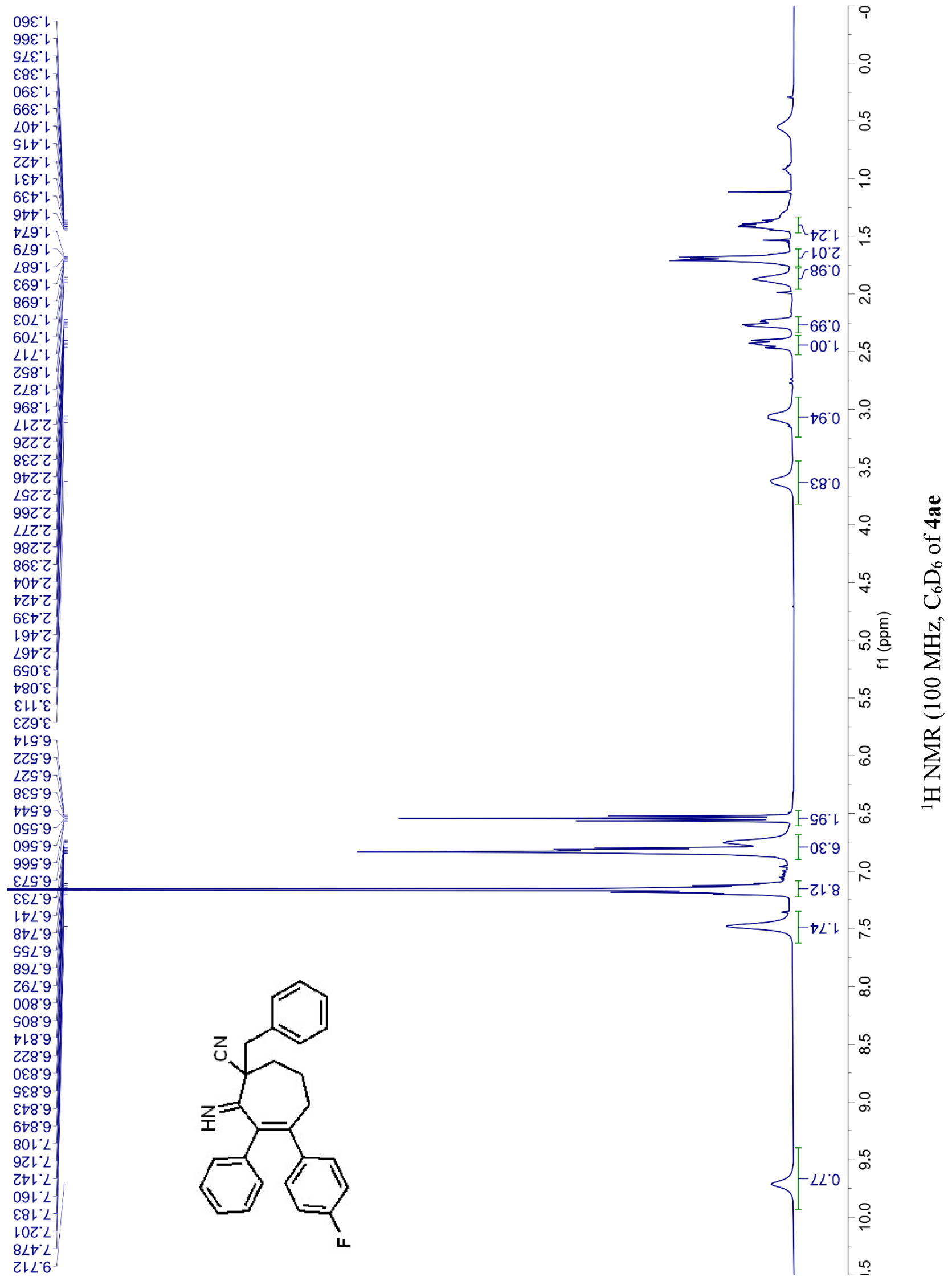




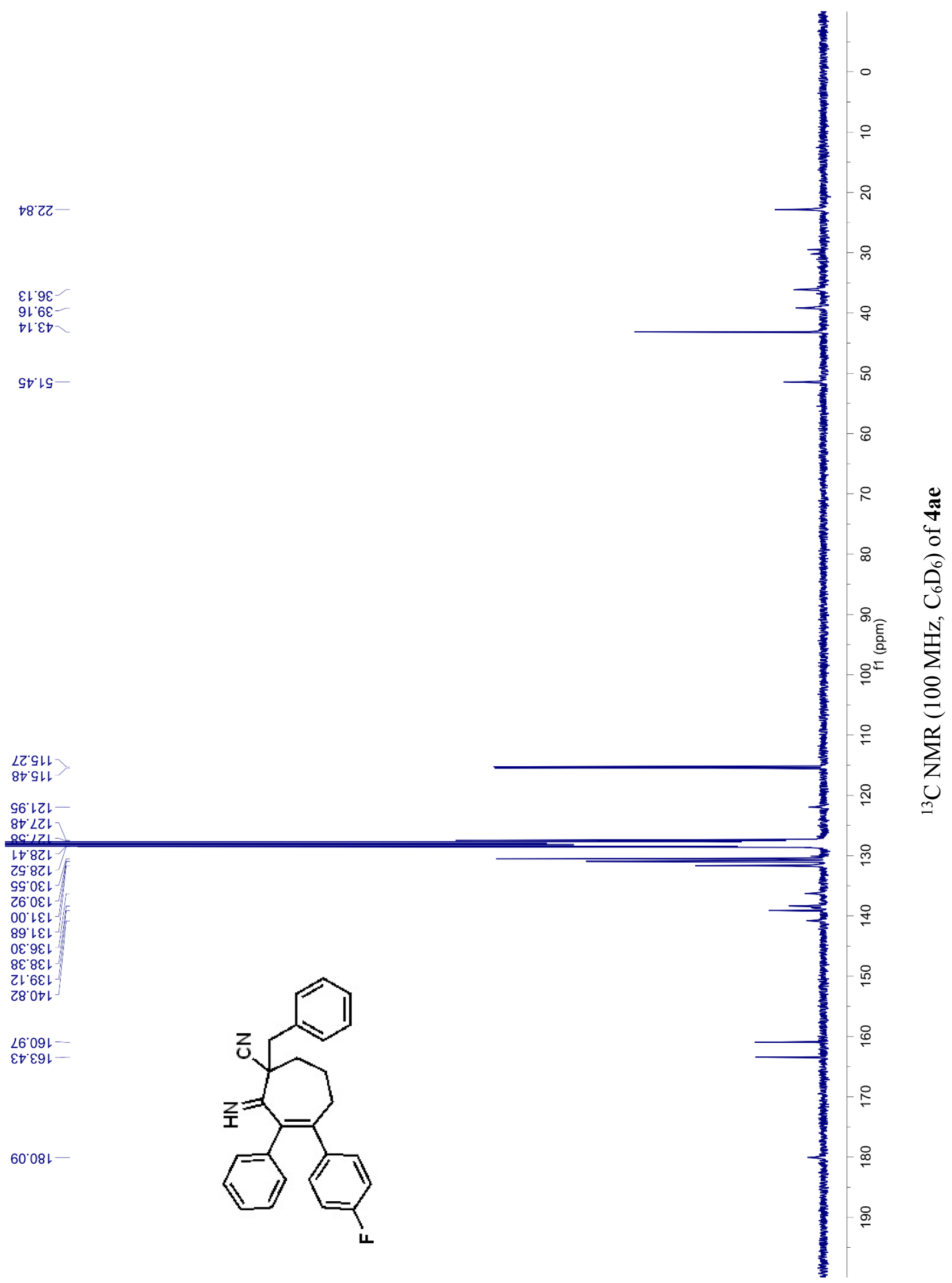




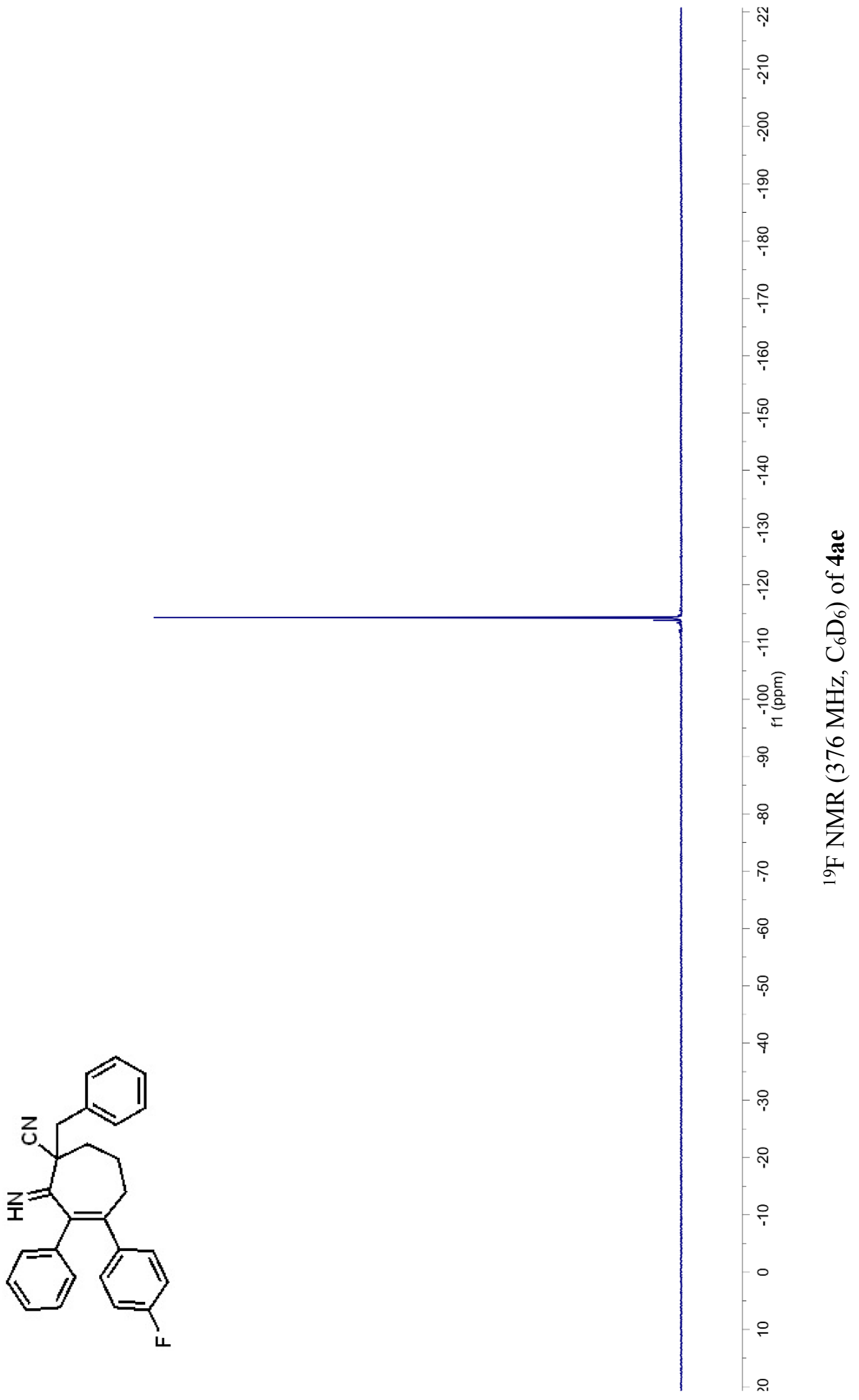


†E6.

0†6 เ

976 เ

296.'

$996^{\circ} \mathrm{L}$

L $\angle 6^{\circ} \mathrm{L}$

$\angle L L^{\circ} \mathrm{Z}$

$98 \mathrm{~L}^{\circ} \mathrm{C}$

乙6レ'

10Z'乙

0レ乙'

GLZ'Z

LEZ Z

乙99'乙

099'乙

999'乙

9L9'乙

$789^{\circ} \mathrm{Z}$

$069^{\circ} 乙$

6 날

$\varepsilon 8 L^{\circ} \varepsilon$

เ8t $\varepsilon$

SIG'

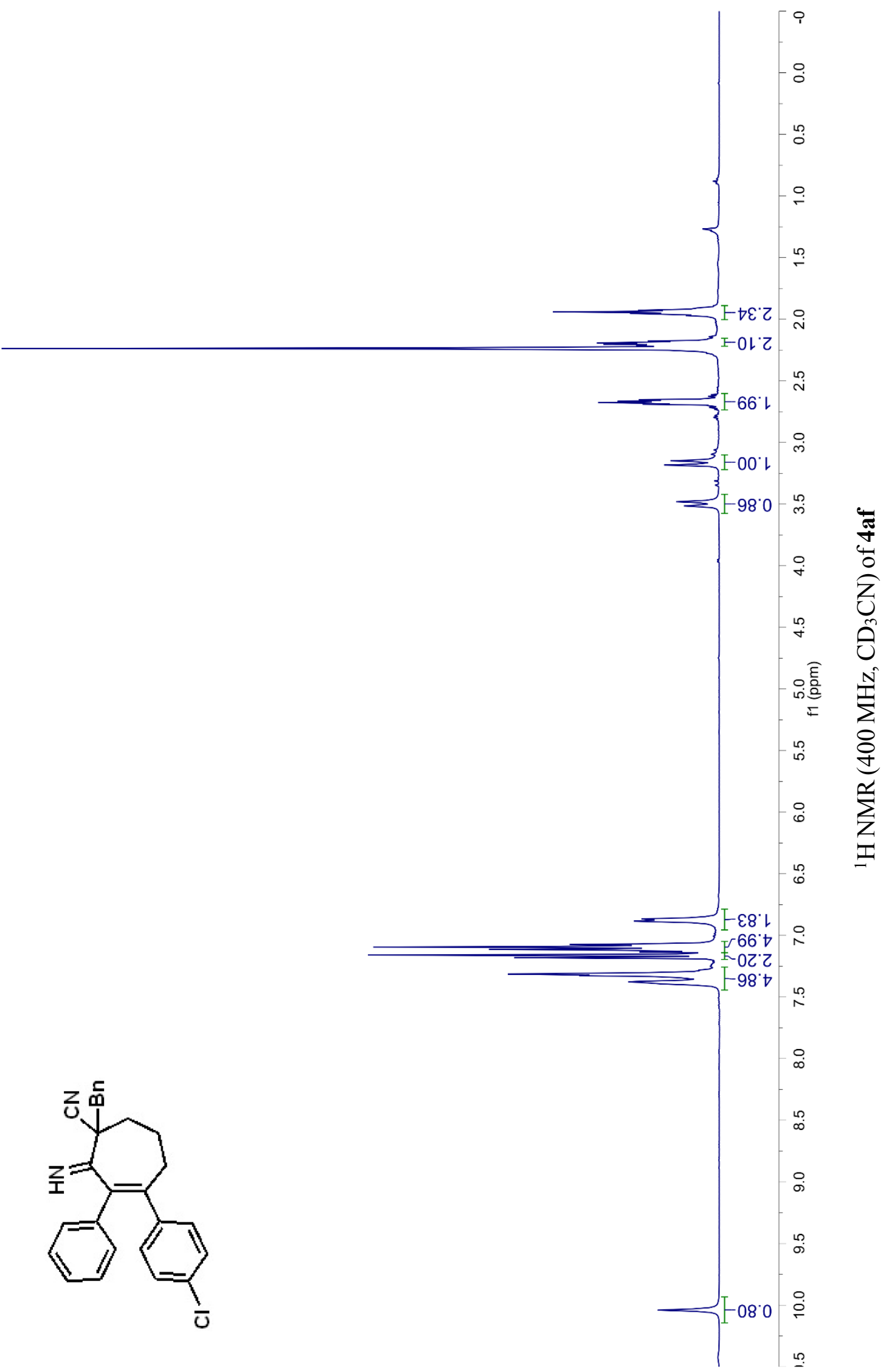


t9.0

S8.0

$90^{\circ}$ ᄂ

$\angle D^{\circ}-$

$29^{\circ}$

$88^{\circ} \mathrm{L}$

จ6 22

$\varepsilon Z^{\prime} 9 \varepsilon$

$9 \varepsilon^{\prime} 8 \varepsilon^{-}$

$\varepsilon \nabla^{\circ} \varepsilon \nabla^{-}$

86.19-

92'8L

$09^{\circ} \mathrm{ZZ}$

8l.8ZL

प्र०8टt

00621

20 $6 \mathrm{ZL}$

$80^{\circ} L E L$

$\angle E^{\prime} L E L$

เ6.

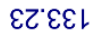

$\varepsilon 0 . \angle \varepsilon L$

$9 L 8 \varepsilon \mathrm{L}$

St $6 \varepsilon 1$

8ナレเレ

06เทレ

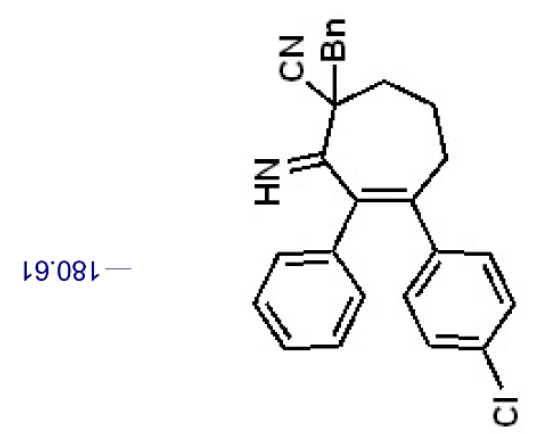



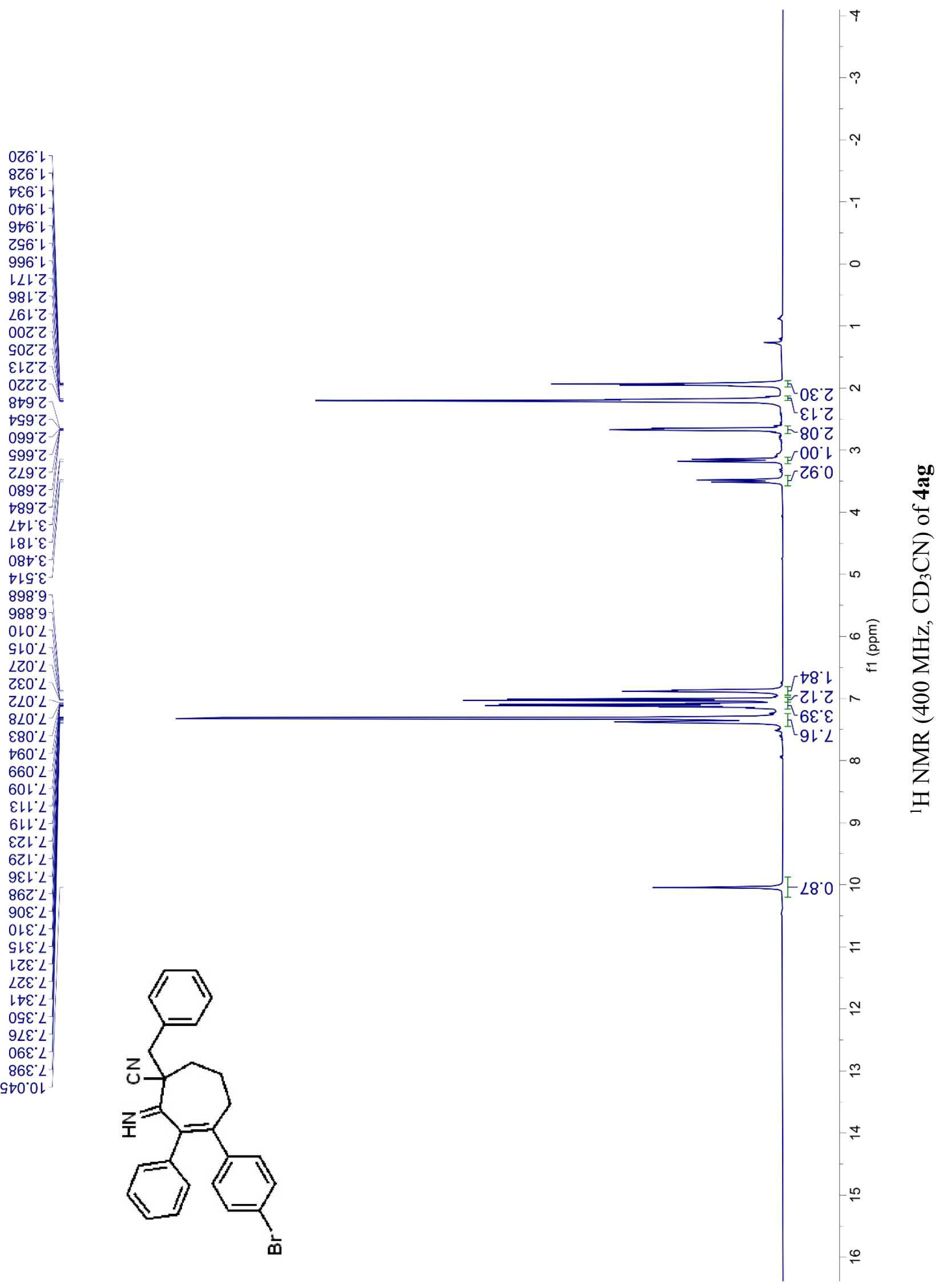


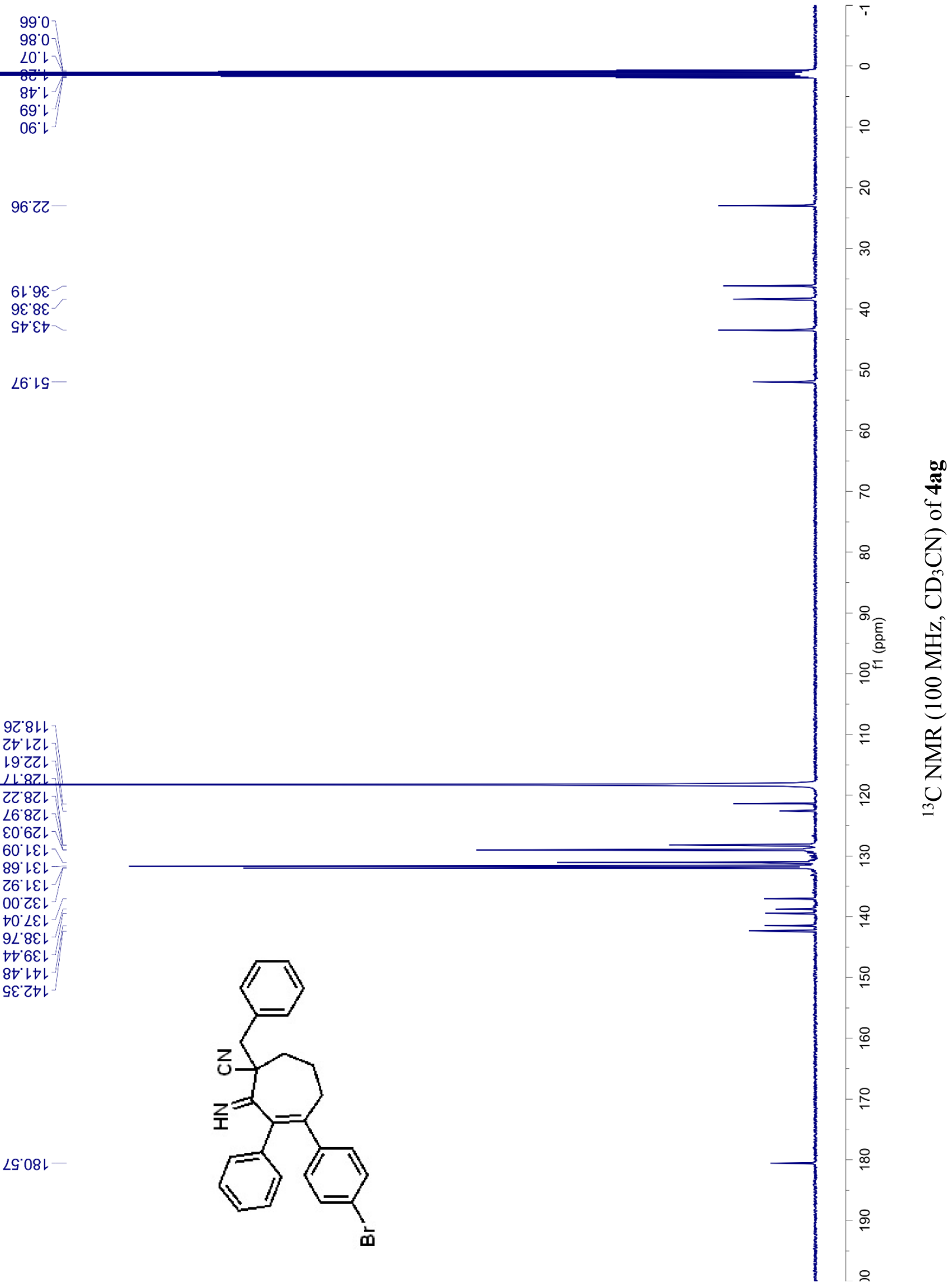


$\angle 69^{\circ}$ '

$9 L L L$

次 ᄂ

8ZLL

$6 \varepsilon L ' L$

$\angle \nabla L$

G98.

पट8'

$\varepsilon \varepsilon 乙 " 乙$

StZ $Z$

EG乙乙

$5 \mathrm{Z} \cdot \mathrm{Z}$

$\varepsilon \angle Z^{\prime} 乙$

†८ 乙

乙6乙'乙

レレ゙て

タレオ゙

8Et 乙

ยงจ"乙

GLV'乙

เ87"

$\angle \mathcal{E} 0^{\circ} \varepsilon$

$\angle 90^{\circ} \varepsilon$

$\angle 90^{\circ} \varepsilon$

ᄂ $60^{\circ} \varepsilon$

$960^{\circ} \varepsilon$

$66 \varepsilon^{\circ} \varepsilon$

เ89" $\varepsilon$

$109^{\circ} \varepsilon$

$\neg 29^{\circ} \varepsilon$

$8 \nabla 9^{\circ} \varepsilon$

$899^{\circ} \varepsilon$

$6 \downarrow L ' 9$

6SL' 9

ZLL'9-

608'9-

LZ8.9

$9 \succ 8.9$

6L6" 9

\&86.9-

966.9

$000^{\circ} \mathrm{L}$

$09 L^{\circ} \mathrm{L}$

$29 t^{\circ} L$

86t L

$\angle I G^{\circ} \angle$

$\nabla G 8^{\circ} \mathrm{L}$

$698^{\circ} \mathrm{L}$

$798^{\circ} \mathrm{L}$

GL8 $L$

$088^{\circ} \mathrm{L}$

$8 \varepsilon L^{\circ} 6$

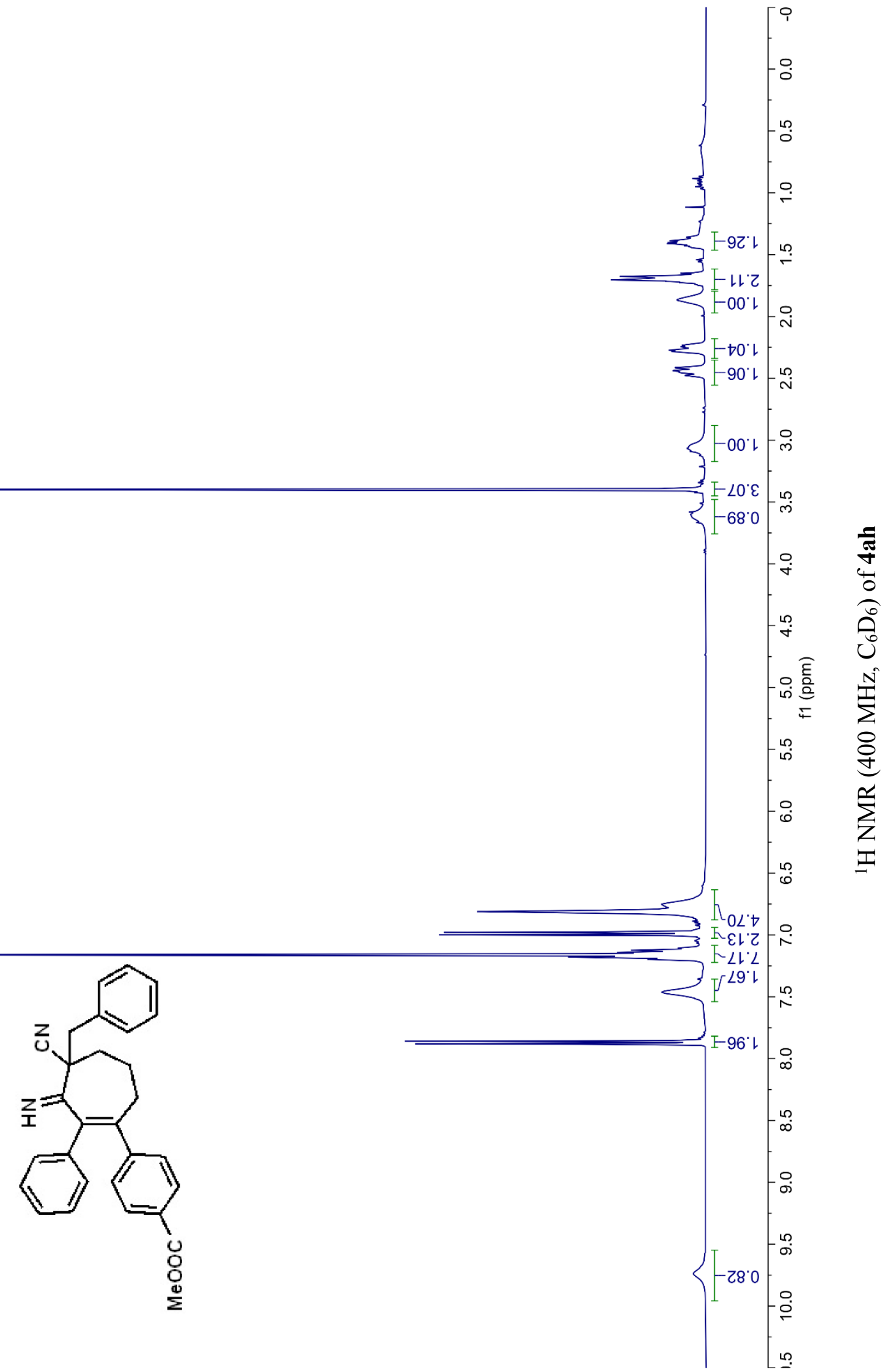


68`てー

$\nabla L S \varepsilon^{-}$

$\checkmark 6.8 \varepsilon-$

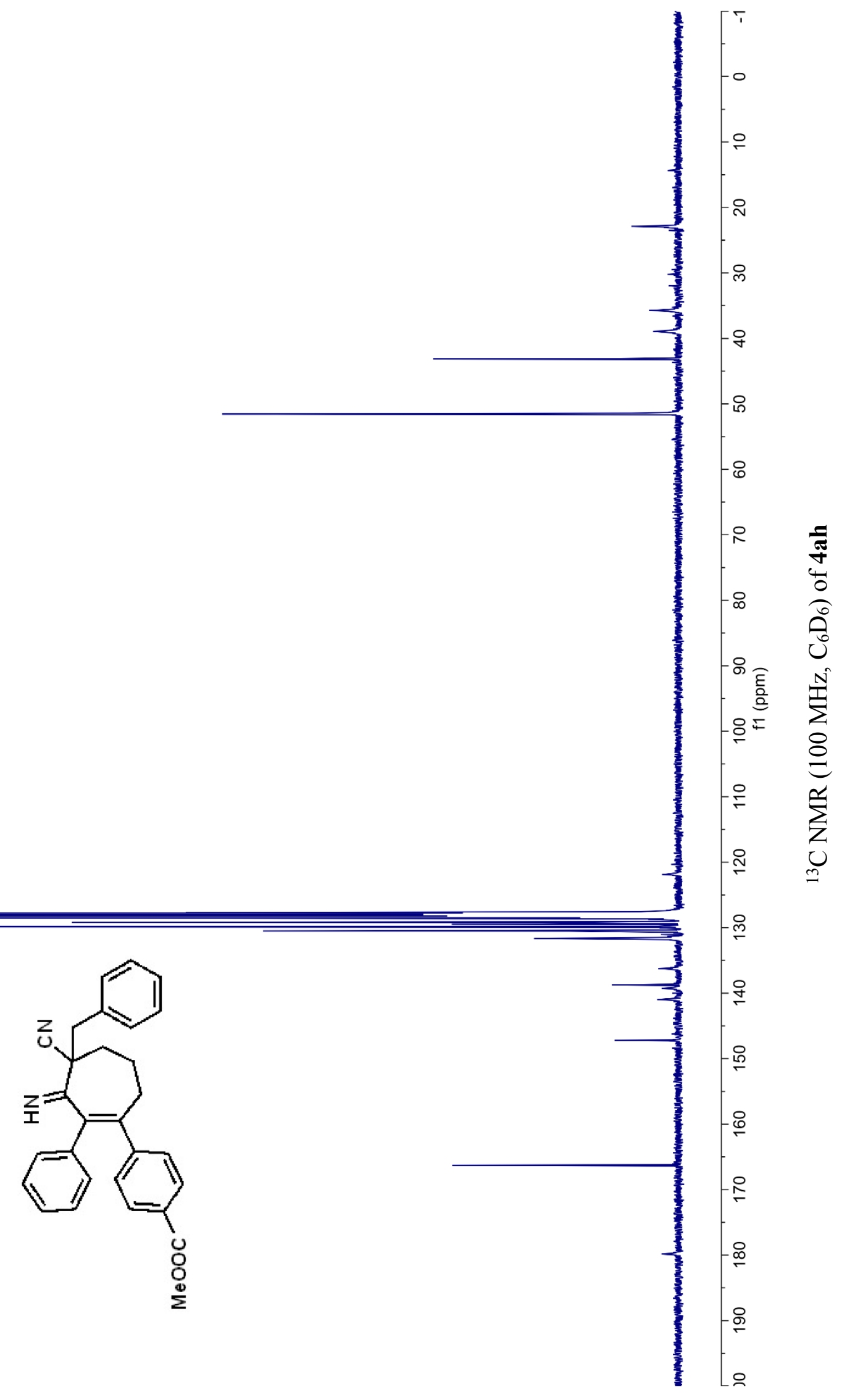

98'レレ

$19 . \angle Z L$

$0 \angle \angle Z L$

$Z 8^{\circ} \angle Z L$

$0 \varepsilon^{\circ} 8 Z L$

tt'8ZL

\&G'8ZL

ᄂ乙6ZL

Lt6ZL

c8.6ZL

S9. LEL

¿Z' $9 \varepsilon 1-$

$\varepsilon L^{\prime} 8 \varepsilon L-$

$\rightarrow Z 6 \varepsilon l$

$\angle 60 t 1$

6เレナレー

8Z.991-

$786 L$ 
$\varepsilon 8 \varepsilon^{\circ} \varepsilon$

†८० 9

8Z8' 9

†८8'9

เ 8. 9

St8. 9

878.9

$120 \mathrm{~L}$

$920^{\circ} \mathrm{L}$

IEO $L$

$\angle E O^{\circ} L$

$\varepsilon \nabla 0^{\circ} L$

$\angle \forall O^{\circ} \angle$

$670^{\circ} \mathrm{L}$

$\triangle G 0^{\circ} \mathrm{L}$

$890^{\circ} \mathrm{L}$

$190^{\circ} \mathrm{L}$

$990^{\circ} \mathrm{L}$

$0 \angle 0^{\circ} \mathrm{L}$

$\checkmark \angle 0^{\circ} \mathrm{L}$

$8 \angle 0^{\circ} \mathrm{L}$

$\angle 80^{\circ} \mathrm{L}$

GOL'L

OEL L

GEL'L

Z†L'L

$\angle \forall L^{\circ} \angle$

SGLL

$09 L^{\circ} \mathrm{L}$

$89 L^{\circ} \mathrm{L}$

LLL

09Z' $L$

$\angle L C ' L$
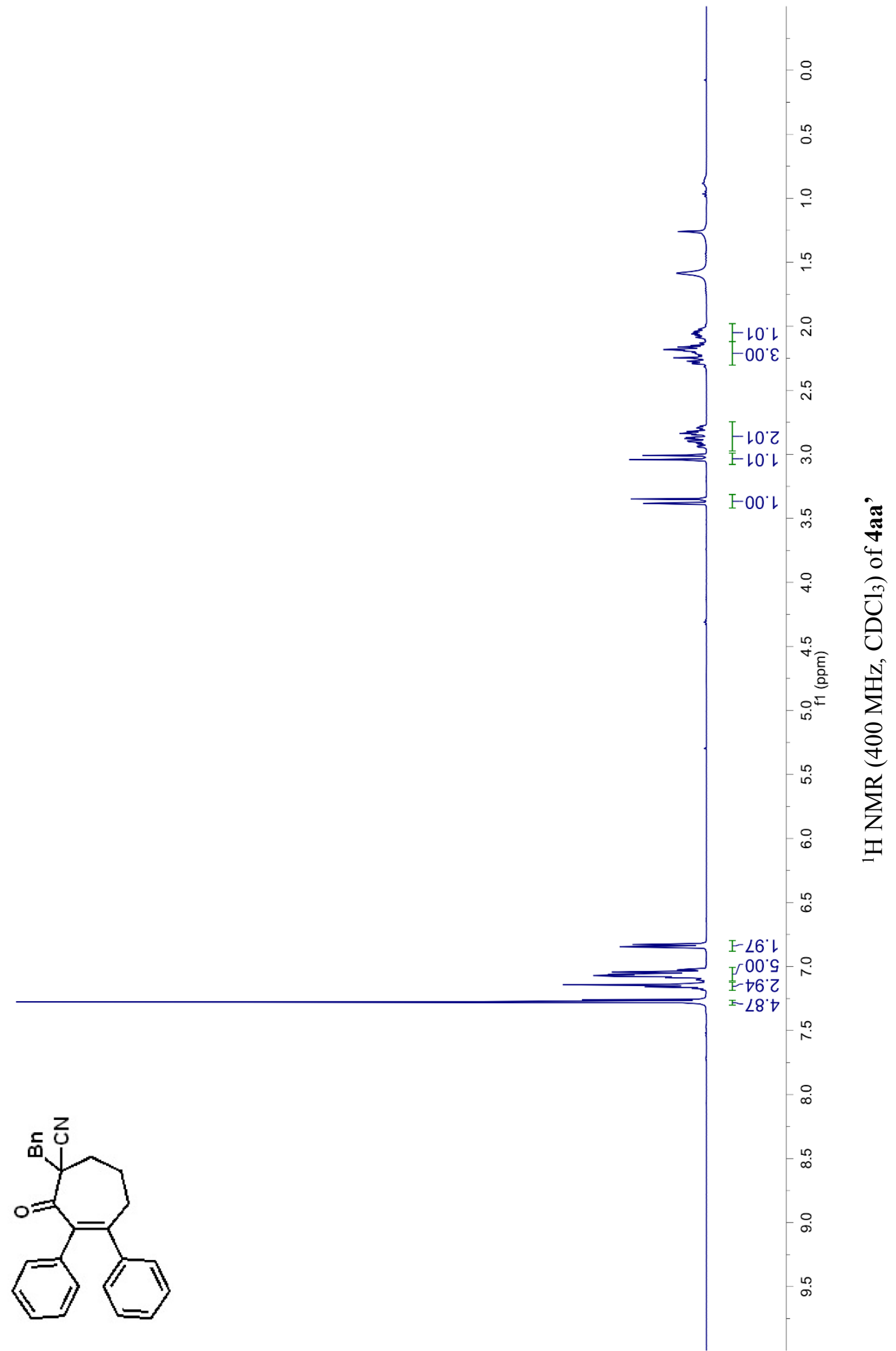
เฤ๕乙-

$99^{\circ} \mathrm{-}-$
乙9. $9 \varepsilon^{-}$

88 .t

OS.S -
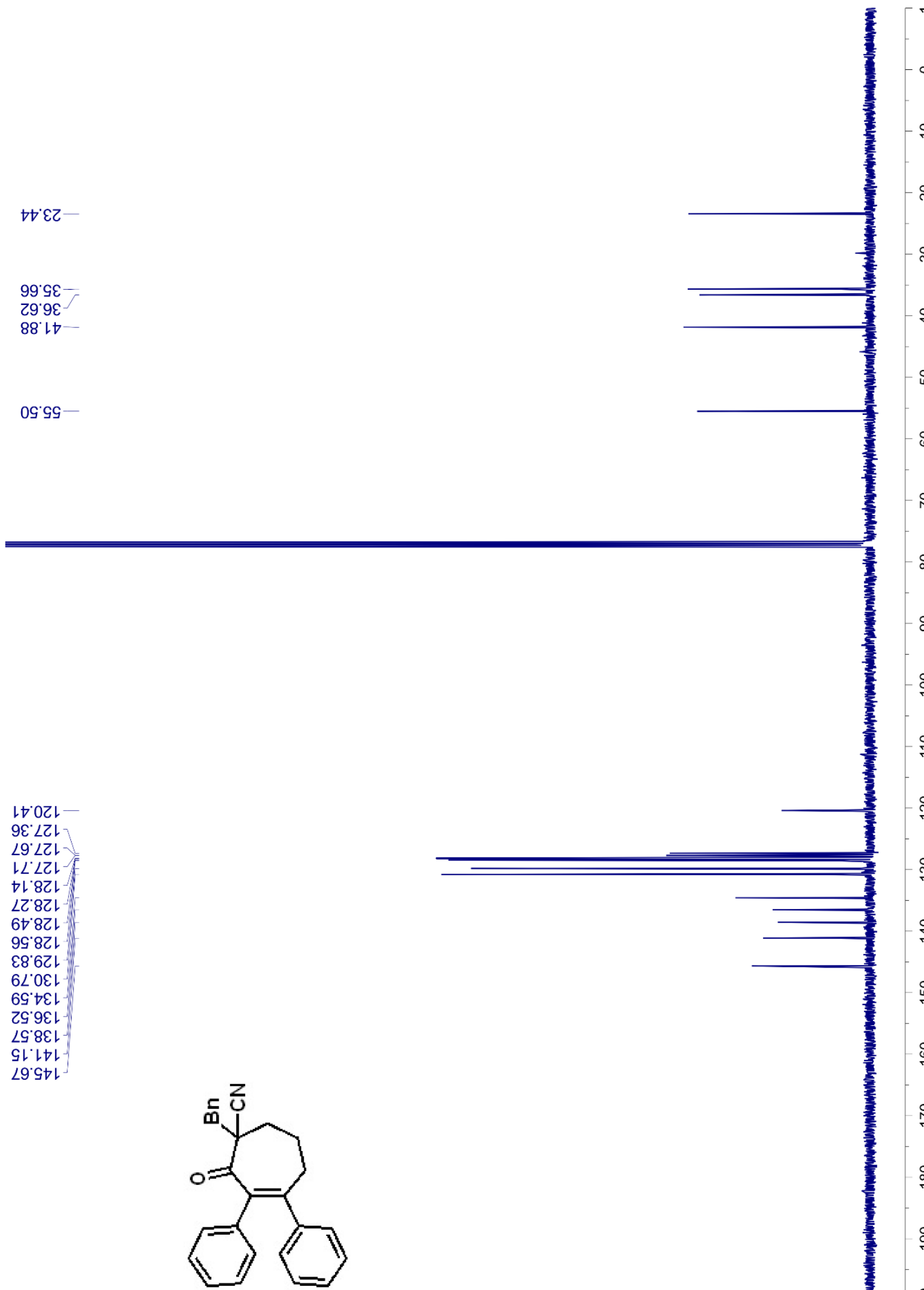

LC LOZ

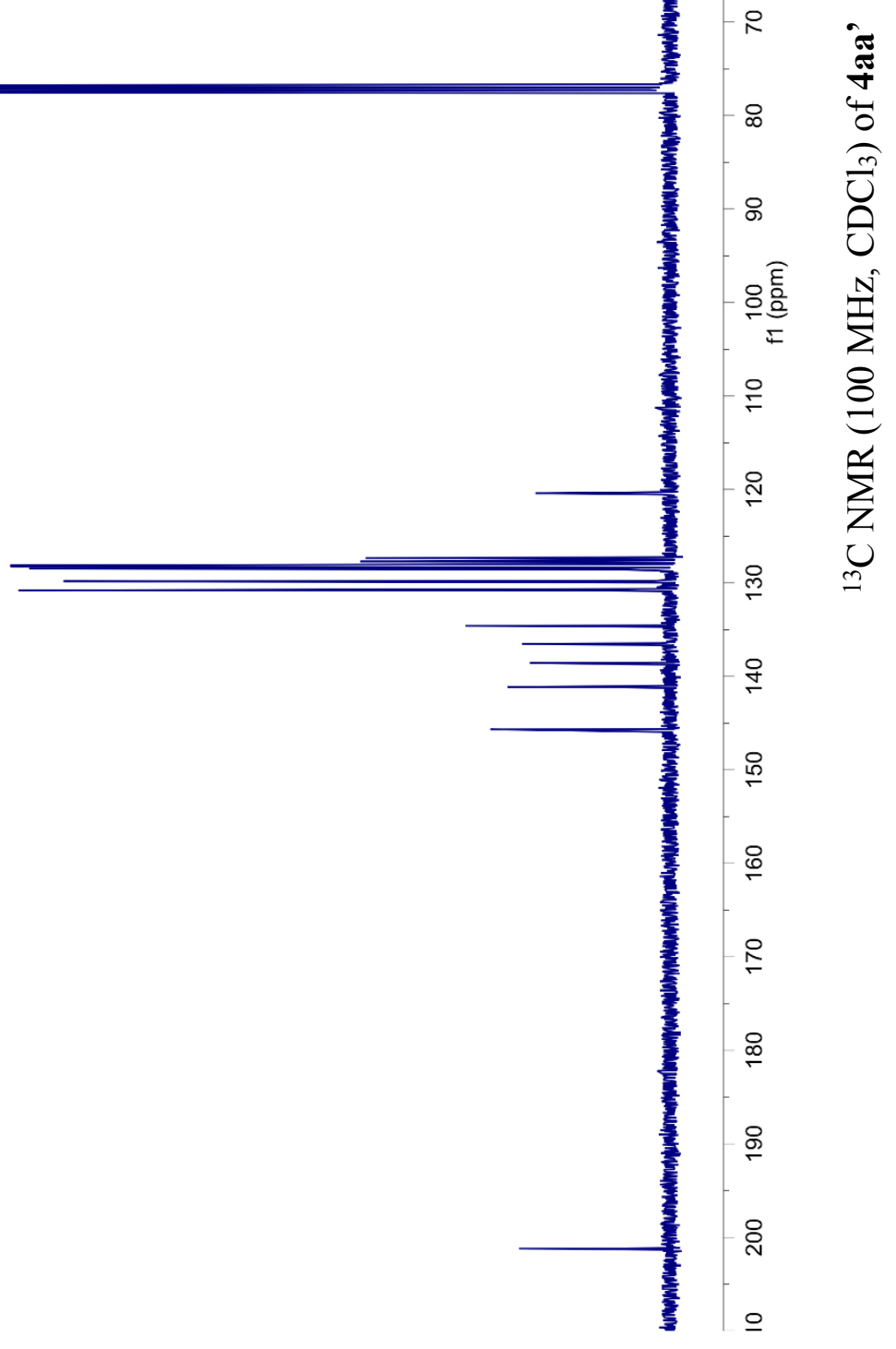




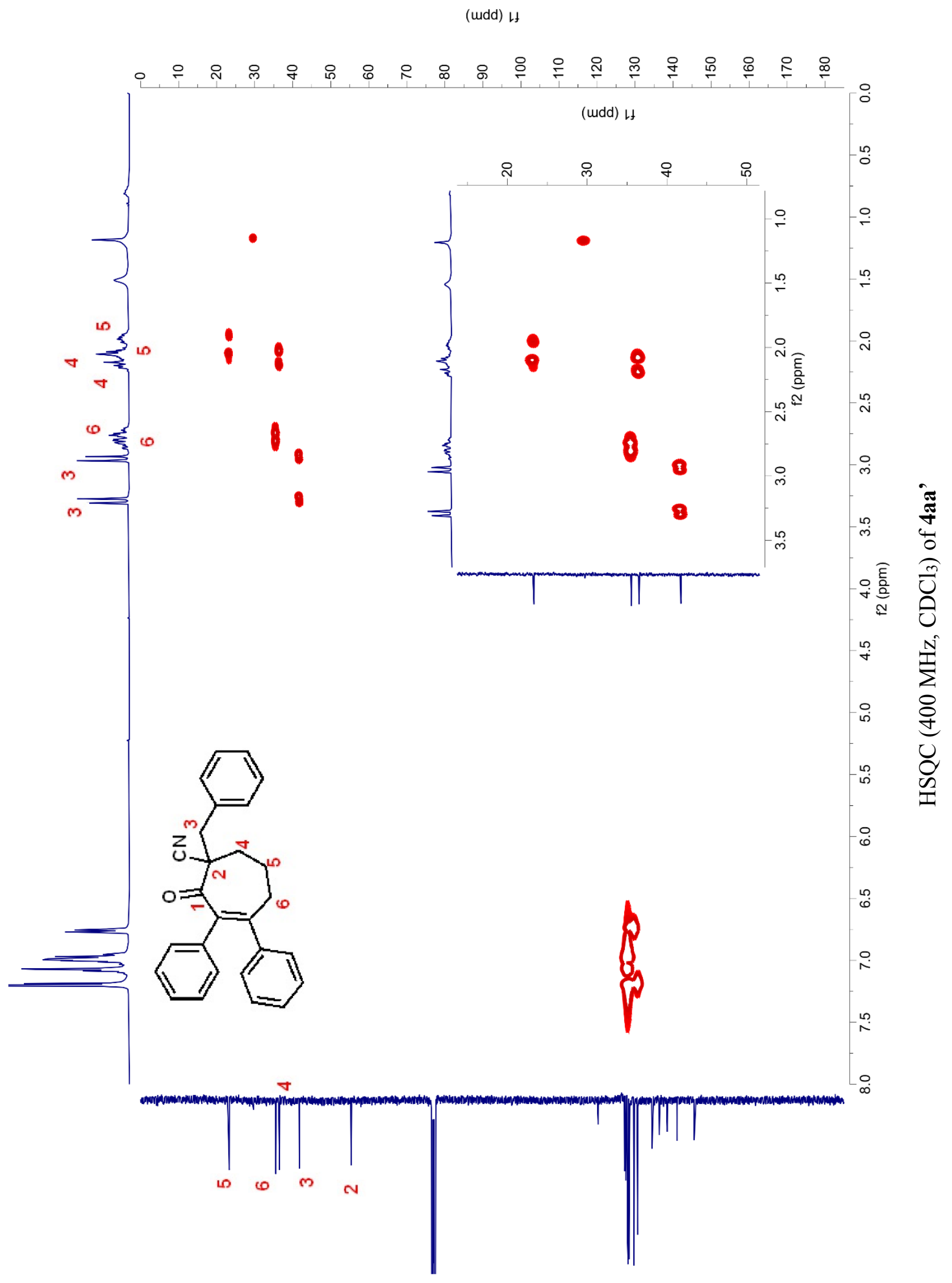


(udd) if

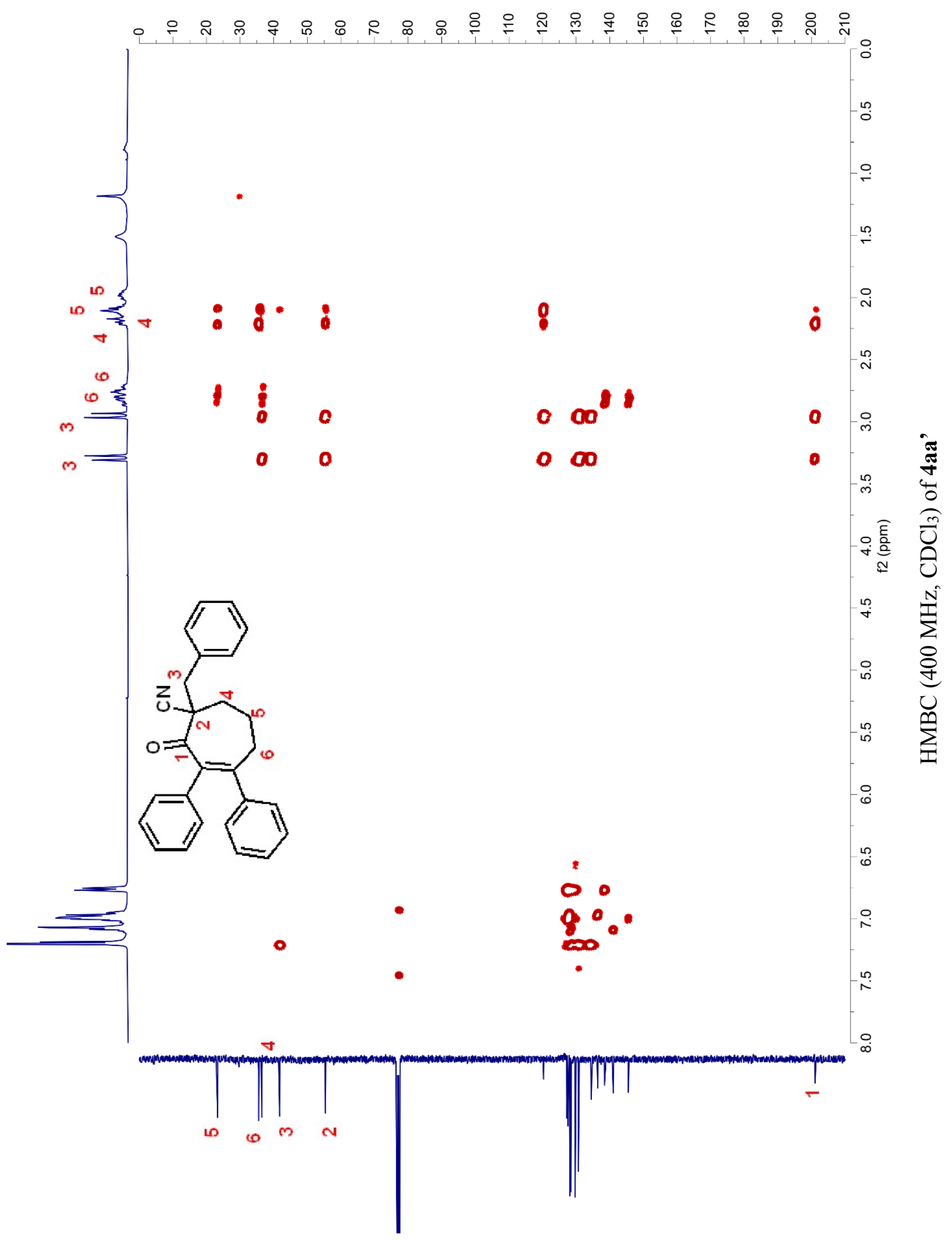



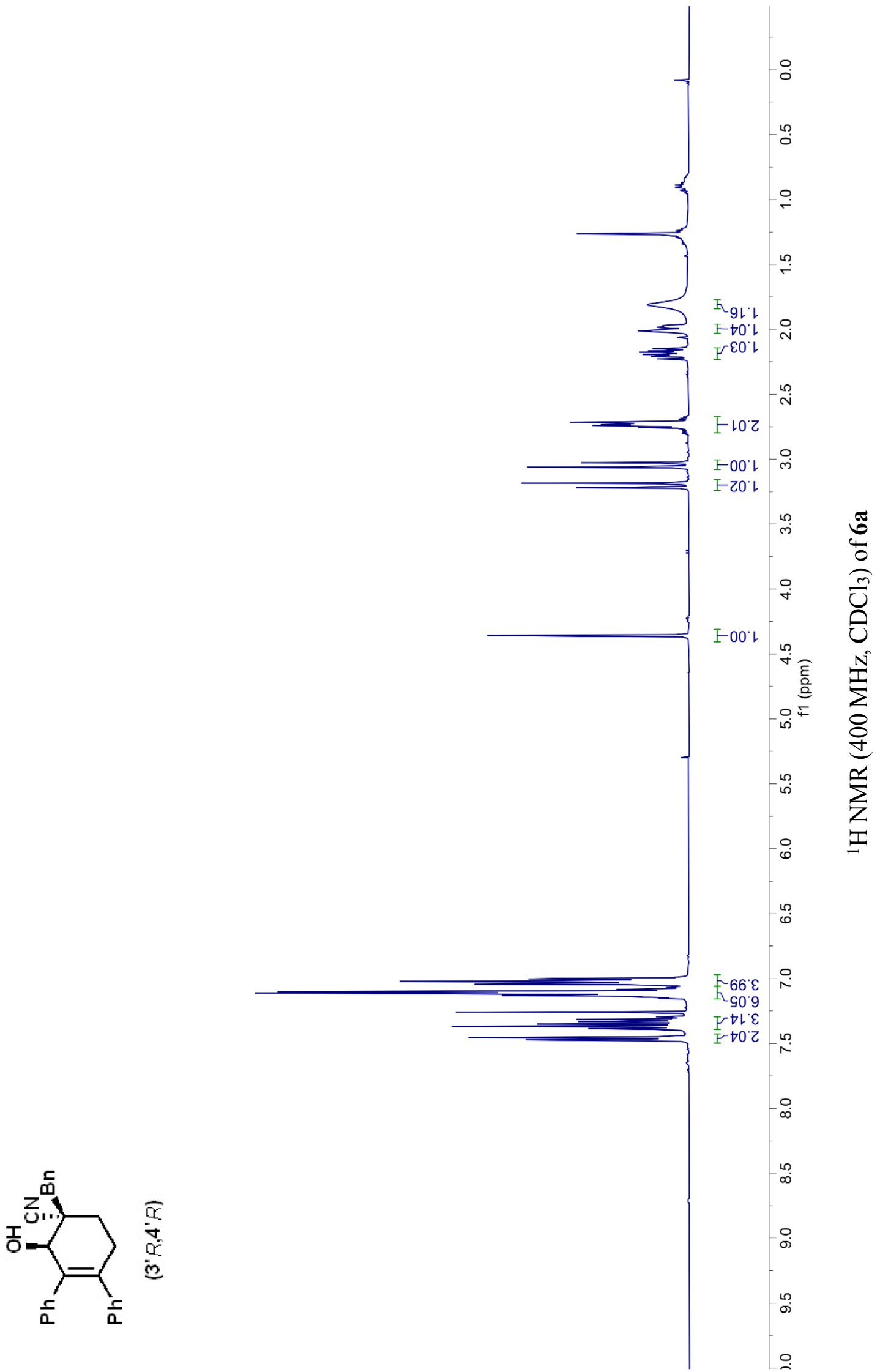

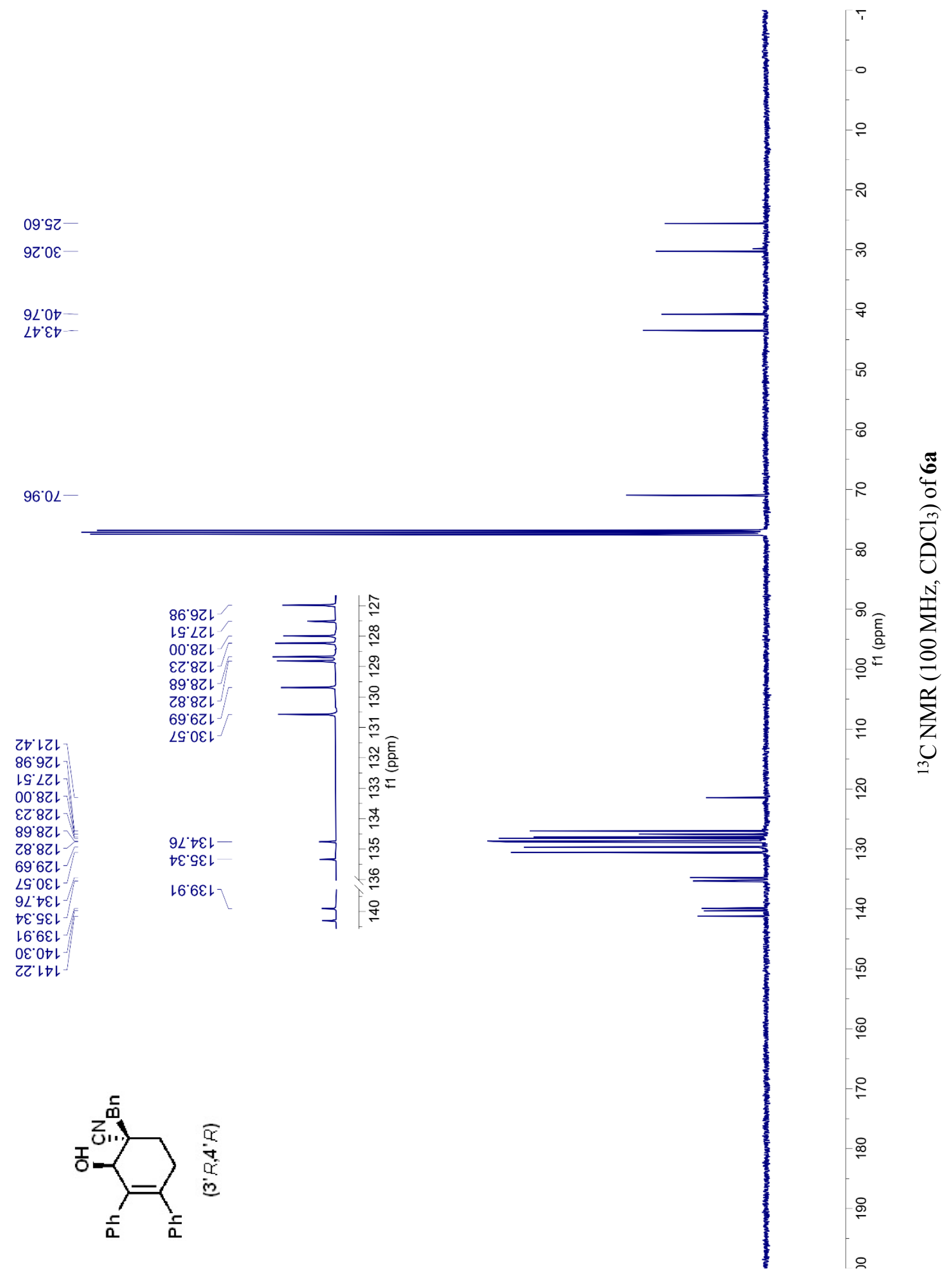

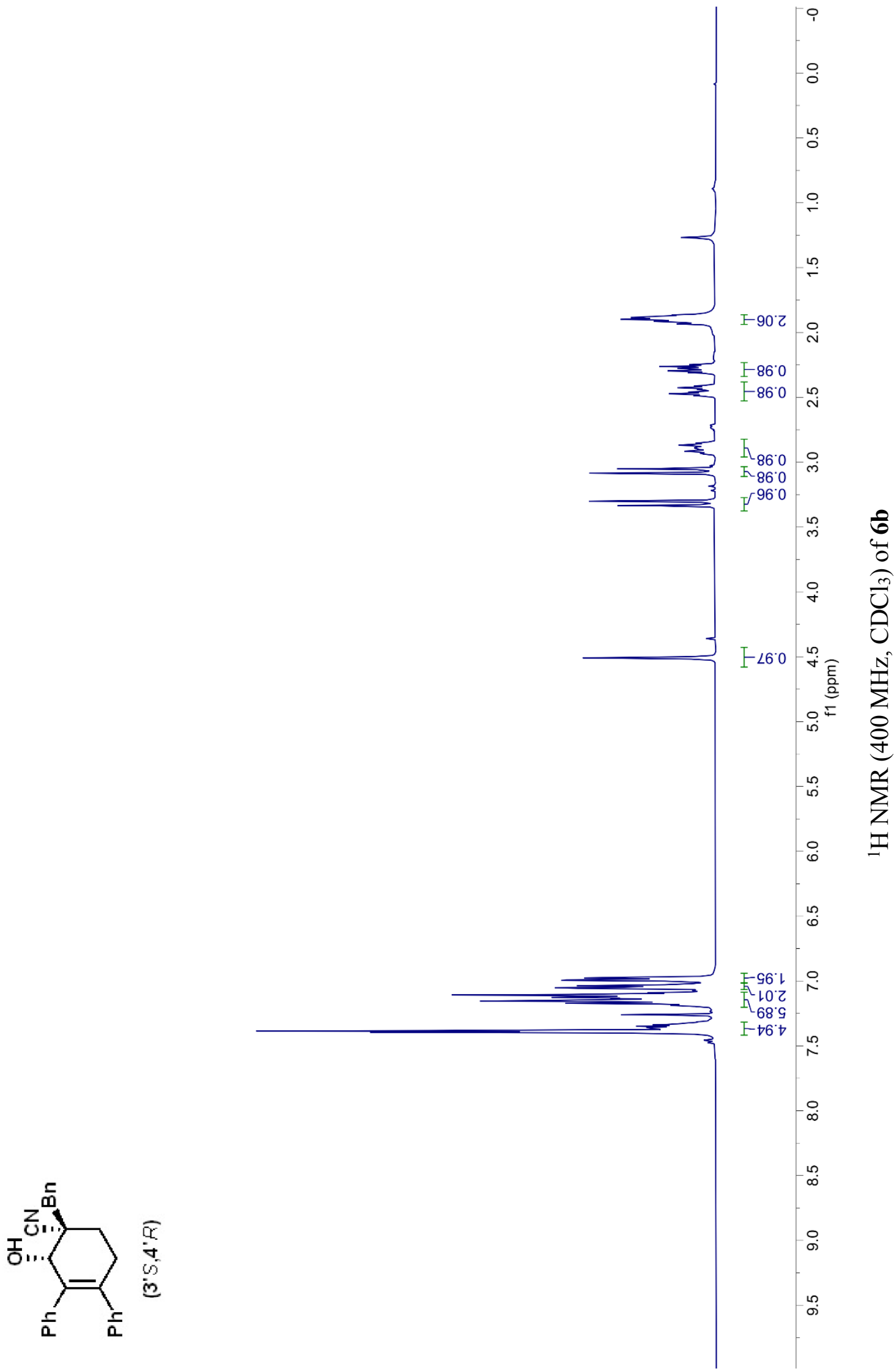
ง6.9乙

จレ'6乙

LLOD

$\nabla 6^{\circ} \nabla \nabla$

LLZL-

89レてレ

88.9ZL

七Z LZL

$99^{\circ} \angle Z L$

0 .8Z

与E'8乙レ

87.8ZL-

$69^{\circ} 8 \mathrm{ZL}$

66.6乙レ

$0 L^{\circ} 0 \varepsilon^{\circ}$

乙† $\downarrow \varepsilon\llcorner$

เ6 $6^{\circ} \nabla \varepsilon$

$\varepsilon 6^{\circ} \angle \varepsilon L$

乙レ $8 \varepsilon\llcorner$.

9ヤ・เレ

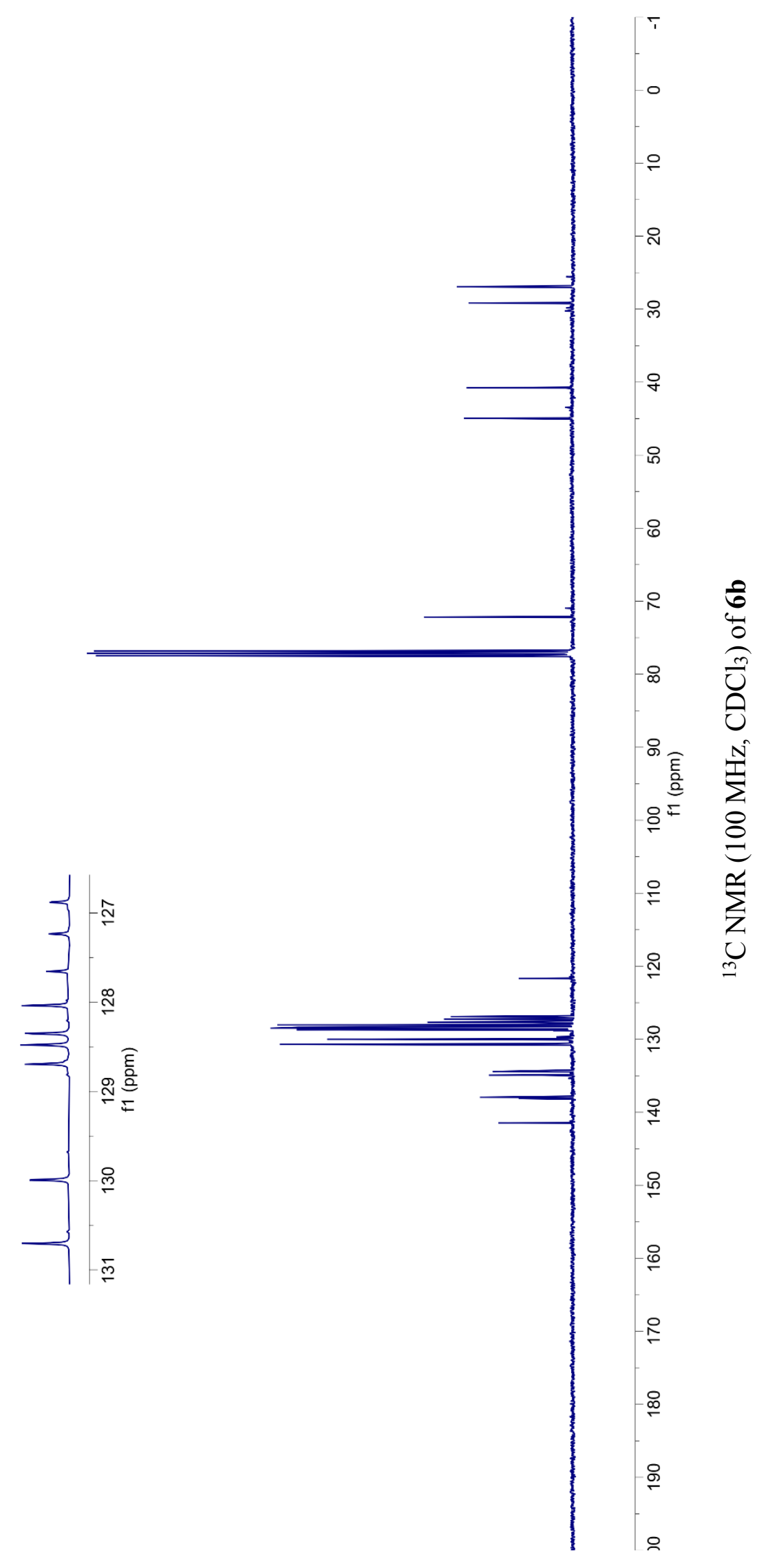




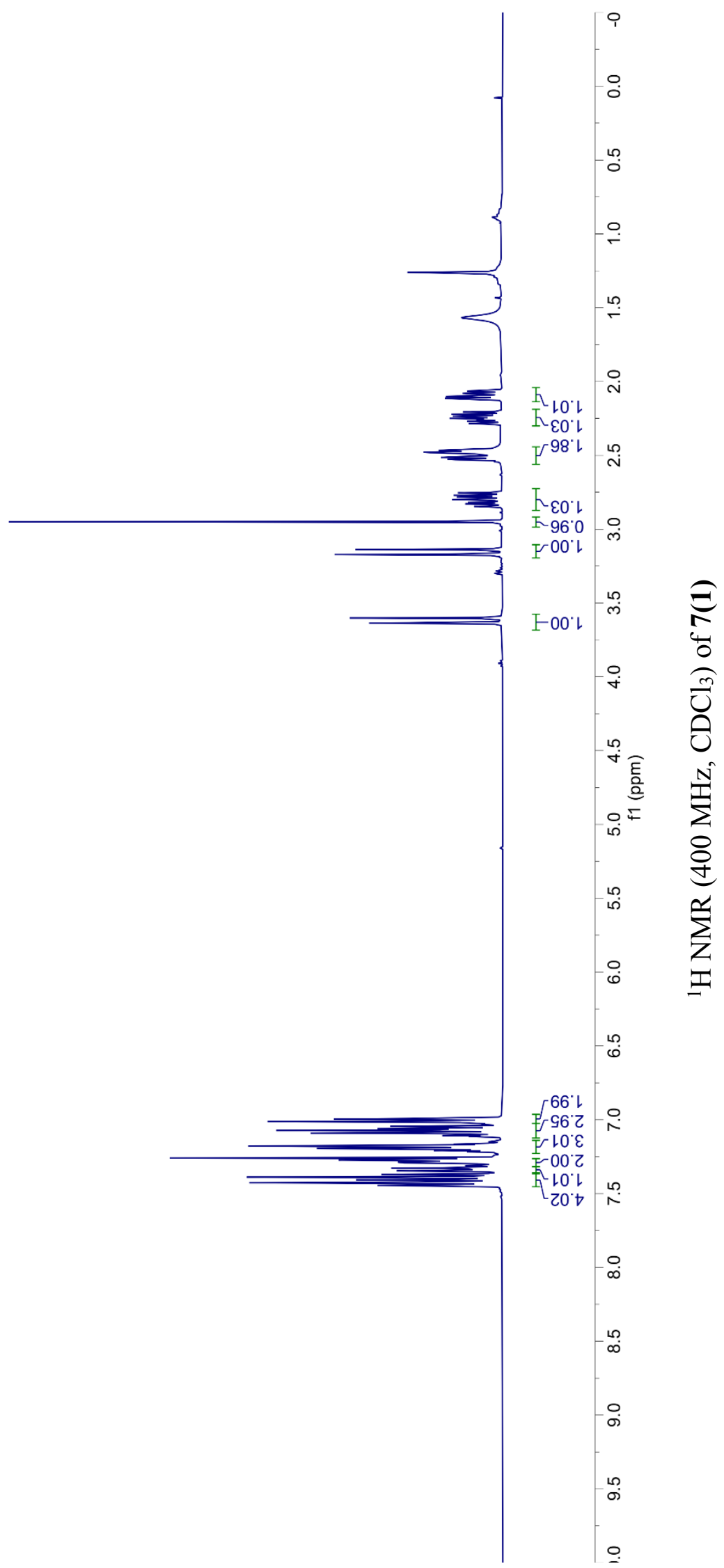


$00^{\circ} \angle Z^{--}$

t9.62-

$1968-$

18.87
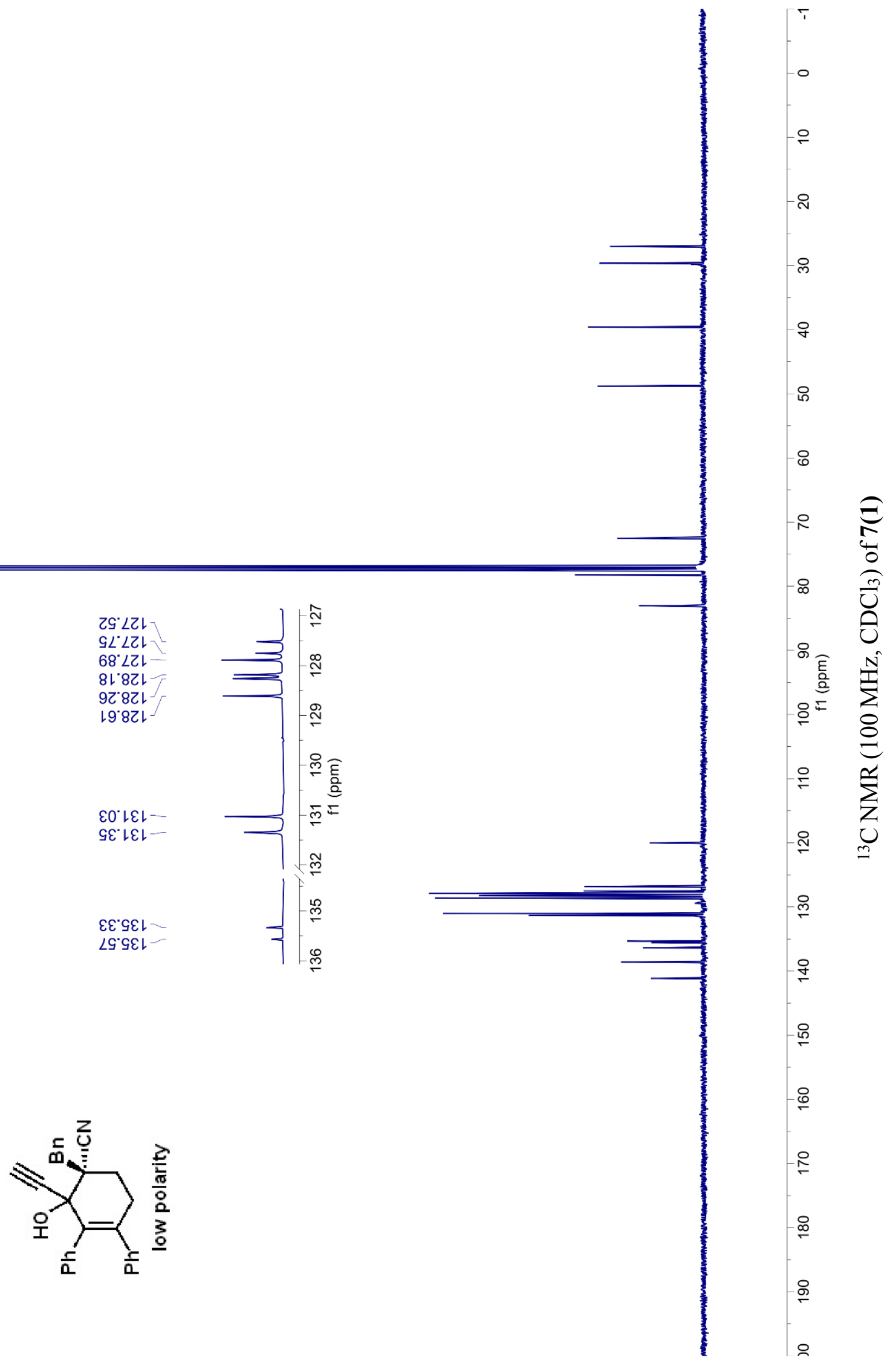

$99^{\circ} Z L$
$t Z^{\prime} 8 L$

c००

$\checkmark 8.9 Z 1$

$Z G^{\circ} \angle Z L$

GL' $\angle Z L-$

$68 . \angle Z L$

$818 \mathrm{LL}$

9Z'8ZL

$\varepsilon 0^{\circ} L E L$

$\varsigma \varepsilon^{\prime}\llcorner\varepsilon \downarrow$

$\varepsilon \varepsilon^{\prime} \varsigma \varepsilon \downarrow$

$\angle G$ 'SEL

$8 \varepsilon^{\prime} 9 \varepsilon \downarrow$

$89^{\circ} 8 \varepsilon L$

$Z G^{\circ} \angle Z L$
$G L^{\circ} \angle Z L$
$68^{\circ} \angle Z L$
$8 L^{\circ} 8 Z L$
$9 Z^{\circ} 8 Z L$
$19^{\circ} 8 Z L$

$\angle \mathcal{G}^{\circ} \mathrm{G}$ L

GE'LEL-

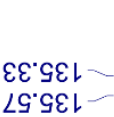

Glut 
060"乙

GOL'Z

0レレ

9てレ乙

$\nabla$ 드

LGL乙

เ9เ'乙

ELL

$\angle L G^{\circ} Z$

069'乙

ह69'乙

S09'乙

0ะ9.乙

099.

999'乙

$0 \angle 9^{\circ} \mathrm{Z}$

$989^{\circ} \mathrm{Z}$

GEL'乙

$\varepsilon \angle 0^{\circ} \varepsilon$

$80 L^{\circ} \varepsilon$

$8 \nabla \mathcal{G}^{\circ} \mathcal{E}$

$89^{\circ} \varepsilon$

乙86. 9

$986^{\circ} 9$

$066^{\circ} 9$

$966^{\circ} 9$

$200^{\circ} \mathrm{L}$

$900^{\circ} \mathrm{L}$

$\checkmark 90^{\circ} \mathrm{L}$

$\angle 90^{\circ} \mathrm{L}$

$1 \angle O^{\circ} \mathrm{L}$

$9 \angle 0^{\circ} \mathrm{L}$

$880^{\circ} \mathrm{L}$

$\angle 80^{\circ} \mathrm{L}$

$960^{\circ} \mathrm{L}$

101.2

$\mathrm{LOL} 2$

8LL'L

EटL

Zヤレ

$87 L^{\circ} L$

$ד G L^{\circ} L$

$19 L^{\circ} \mathrm{L}$

$99 L^{\circ} L$

$\nabla \angle L^{\circ} L$

$\angle L L^{\circ} L$

98L'L

$06 L^{\circ} \mathrm{L}$

26L'L

$66 \mathrm{~L}^{\circ} \mathrm{L}$

†OZ" 2

ZLZ"L

SLZ L

$8 \mathrm{LZ} L$

$\triangle Z Z{ }^{\circ}$

†ध 'L

$8 \nabla \varepsilon^{\circ} L$

乙GE'L

9GE'L

$\varepsilon L \varepsilon^{\circ} L$

$\angle L E^{\circ} L$

$\angle 8 \varepsilon^{\circ} \angle$

Z6E'L

$\rightarrow 0 \nabla^{\circ} L$

$60 \nabla^{\circ} L$

ELt

$6 \varepsilon \nabla^{\circ} L$

乙ヤヤ L

$9 \forall \nabla^{\circ} L$

ESt"L

89t"L

$\varepsilon 9 \nabla^{\circ} \mathrm{L}$

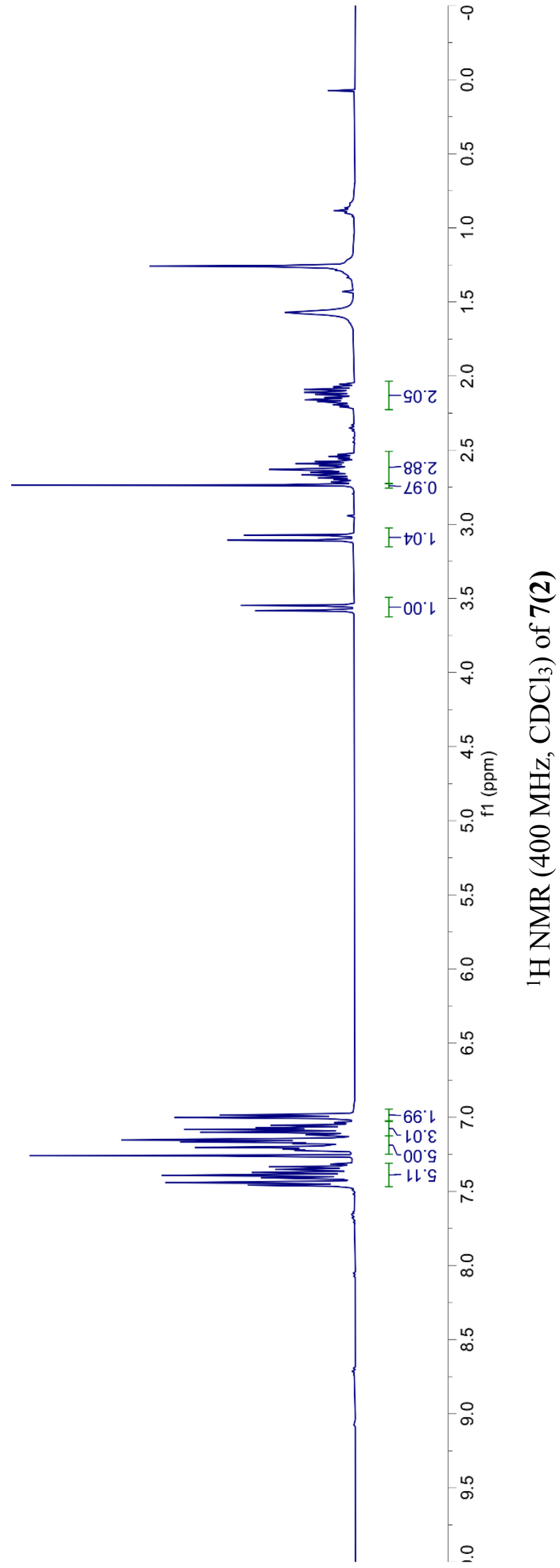


98

乙レ*6Z-

$\nabla \mathcal{G}^{*} \angle \mathcal{E}$

५ะ.8†-
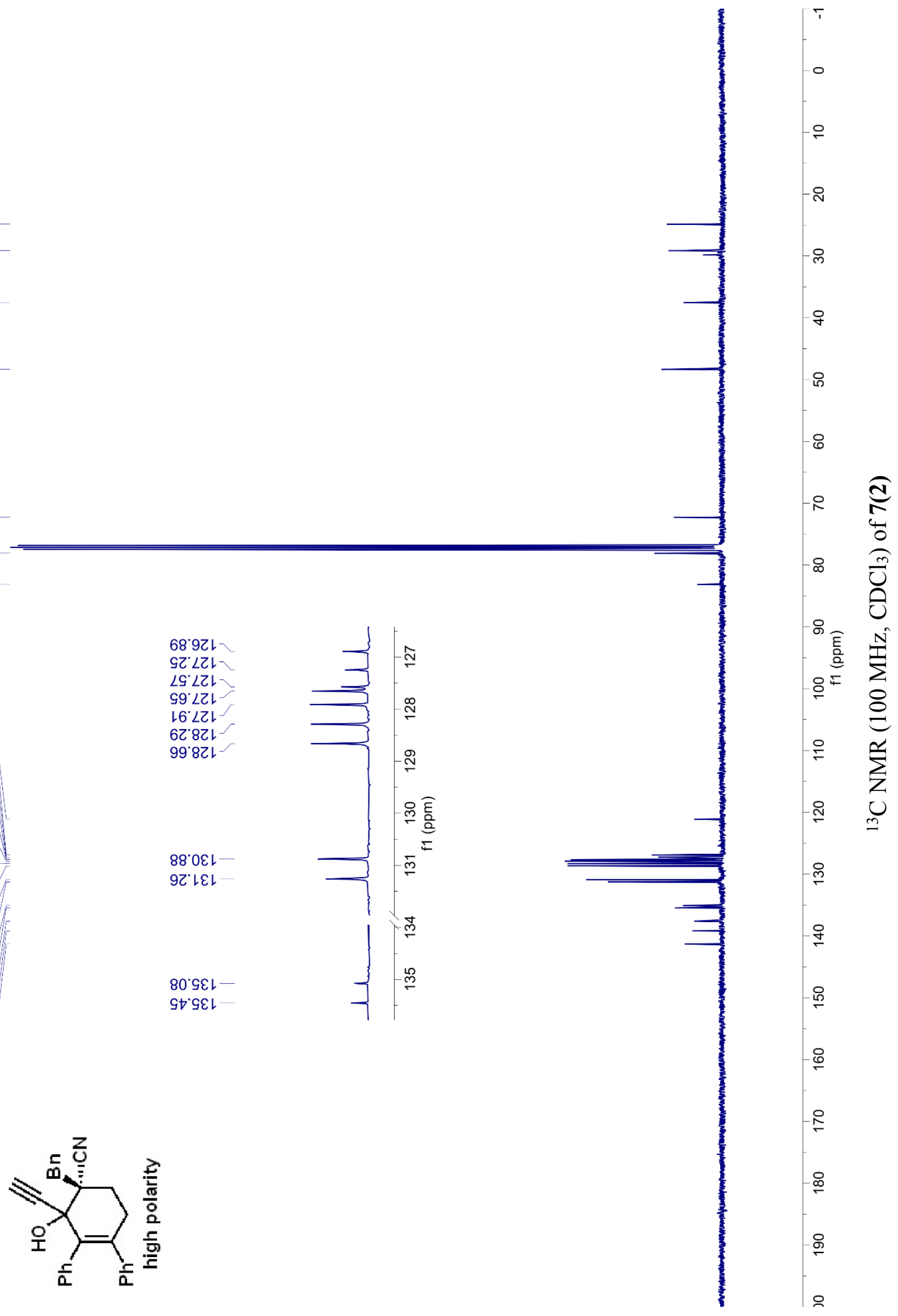

ZL.LZL

68.9ZL

GZ LZL

$\angle G \angle Z L$

$9 . \angle Z L$
$L 6 . \angle Z L$

$6 Z 8 Z 1$

99.8ZL

88.0ع

$9 Z^{\prime}\llcorner\varepsilon \downarrow$

80. $\varsigma \varepsilon$

Gt $\subseteq \varepsilon L$

$6 L^{\circ} 6 \varepsilon L$

$0 \varepsilon^{\prime}\llcorner เ$ 

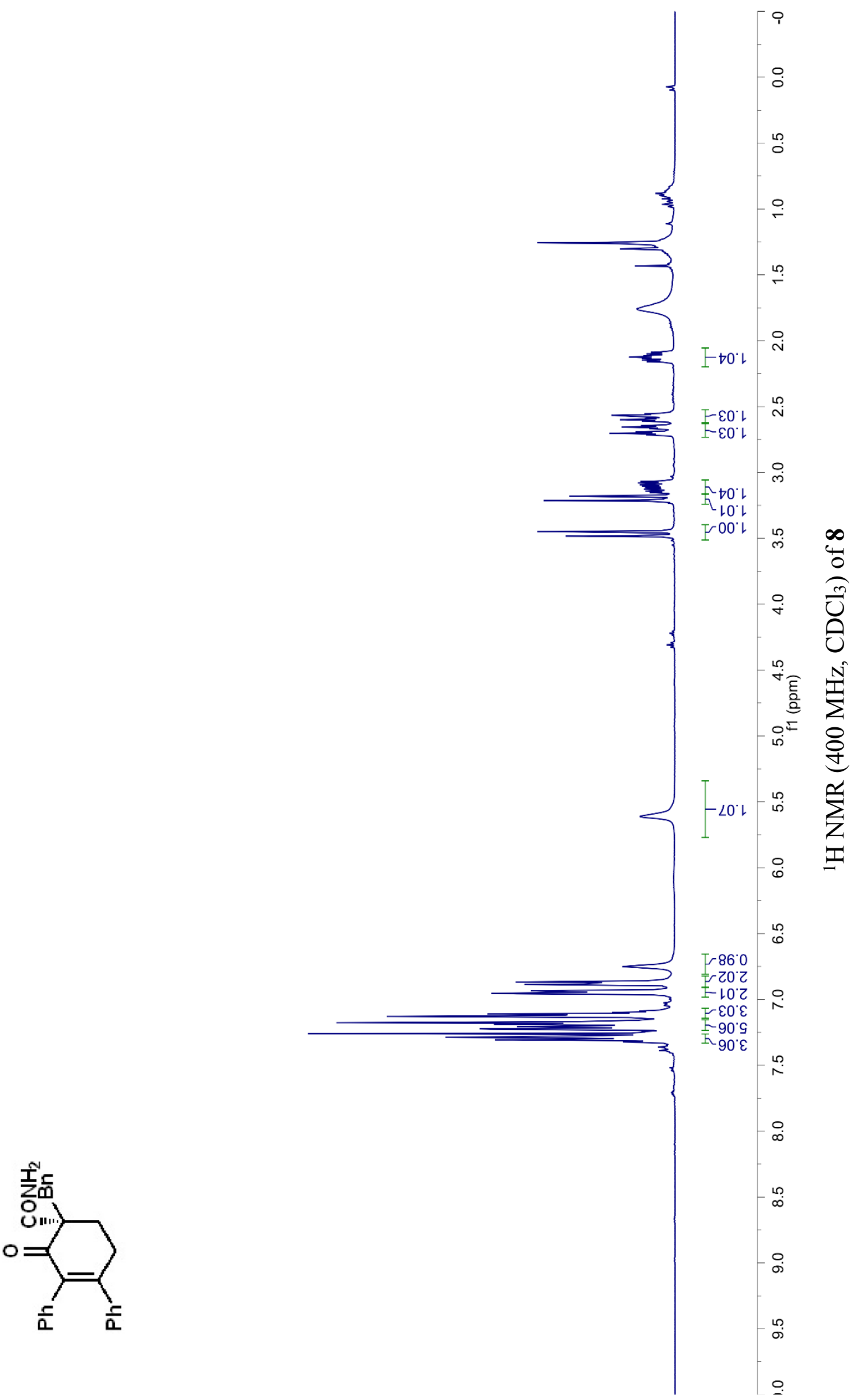


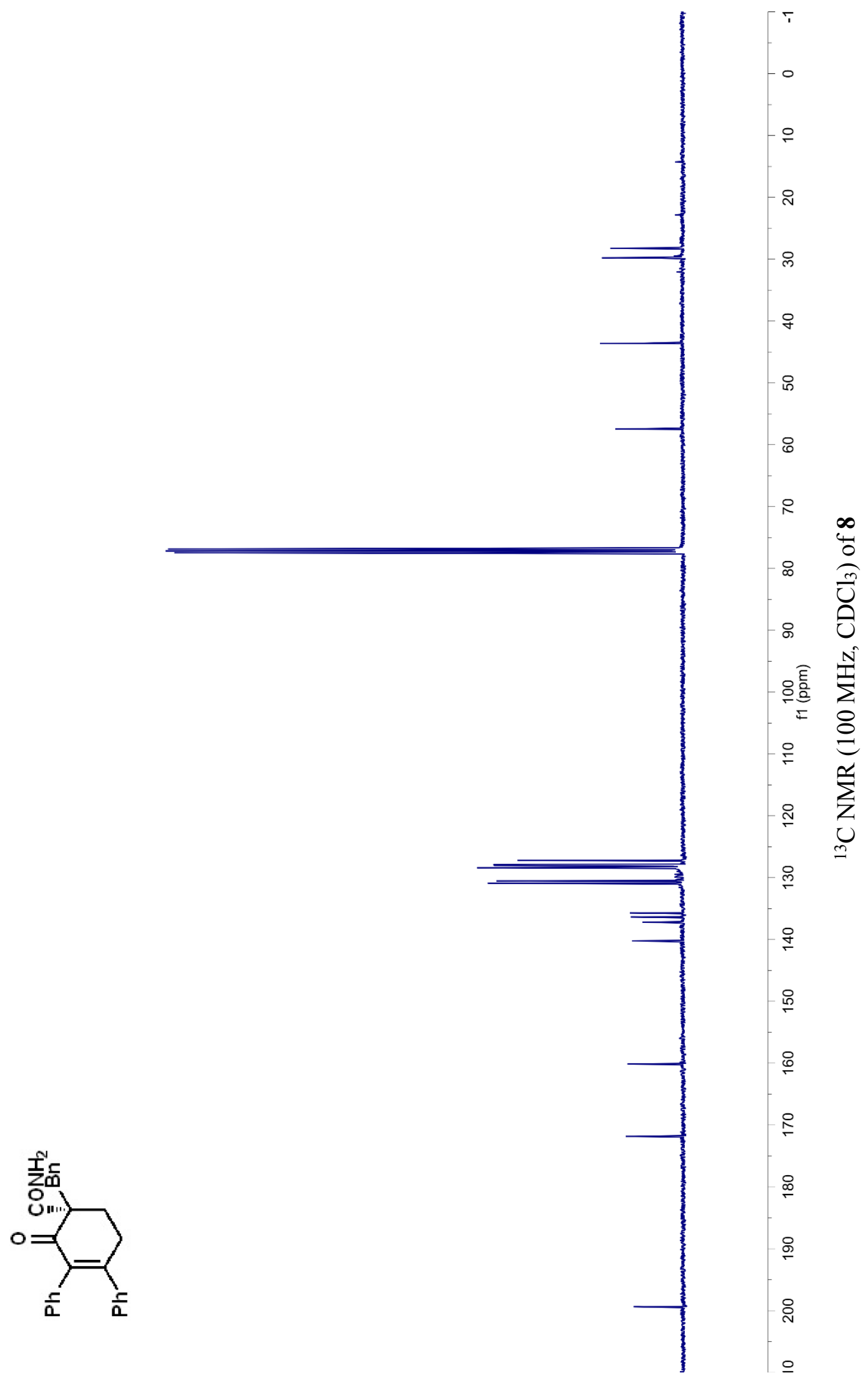

6L $\angle Z L$

$\angle 8^{\circ} \angle Z L$

$66^{\circ}$ LZL

$70.8 \mathrm{ZL}$

เE'8ZL

0† 8Zレ

$\varepsilon G^{\circ} 0 \varepsilon \mathrm{L}$

$68^{\circ} 0 \mathrm{EL}$

$0 \angle \mathrm{C} G \mathrm{~L}$

$\angle \varepsilon^{\prime} 9 \varepsilon L$

$0 乙 \angle \varepsilon L$

OZ'0七レ

$\varepsilon L^{\circ} 09 l-$

E8 LLL-

LE.66เ-

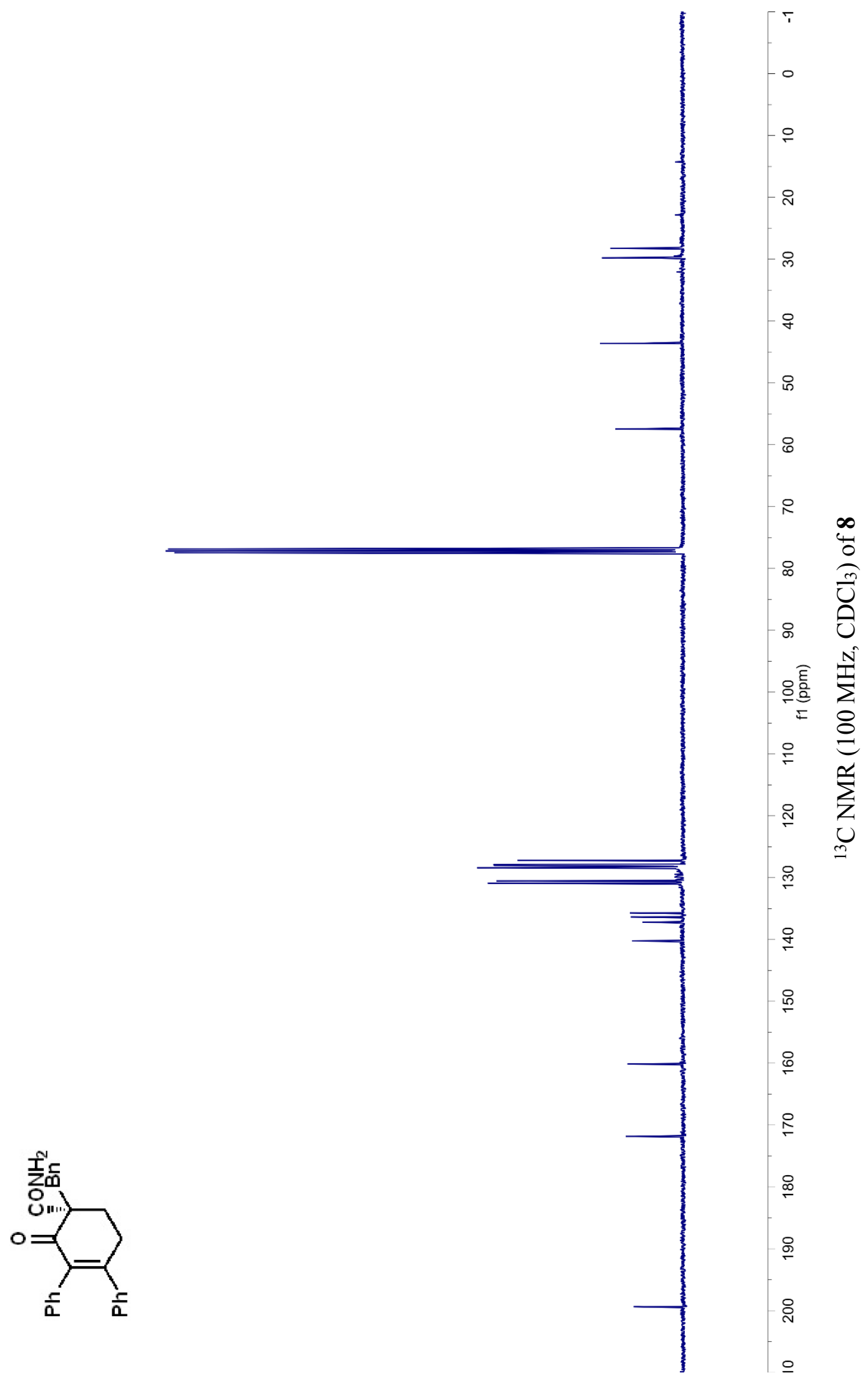



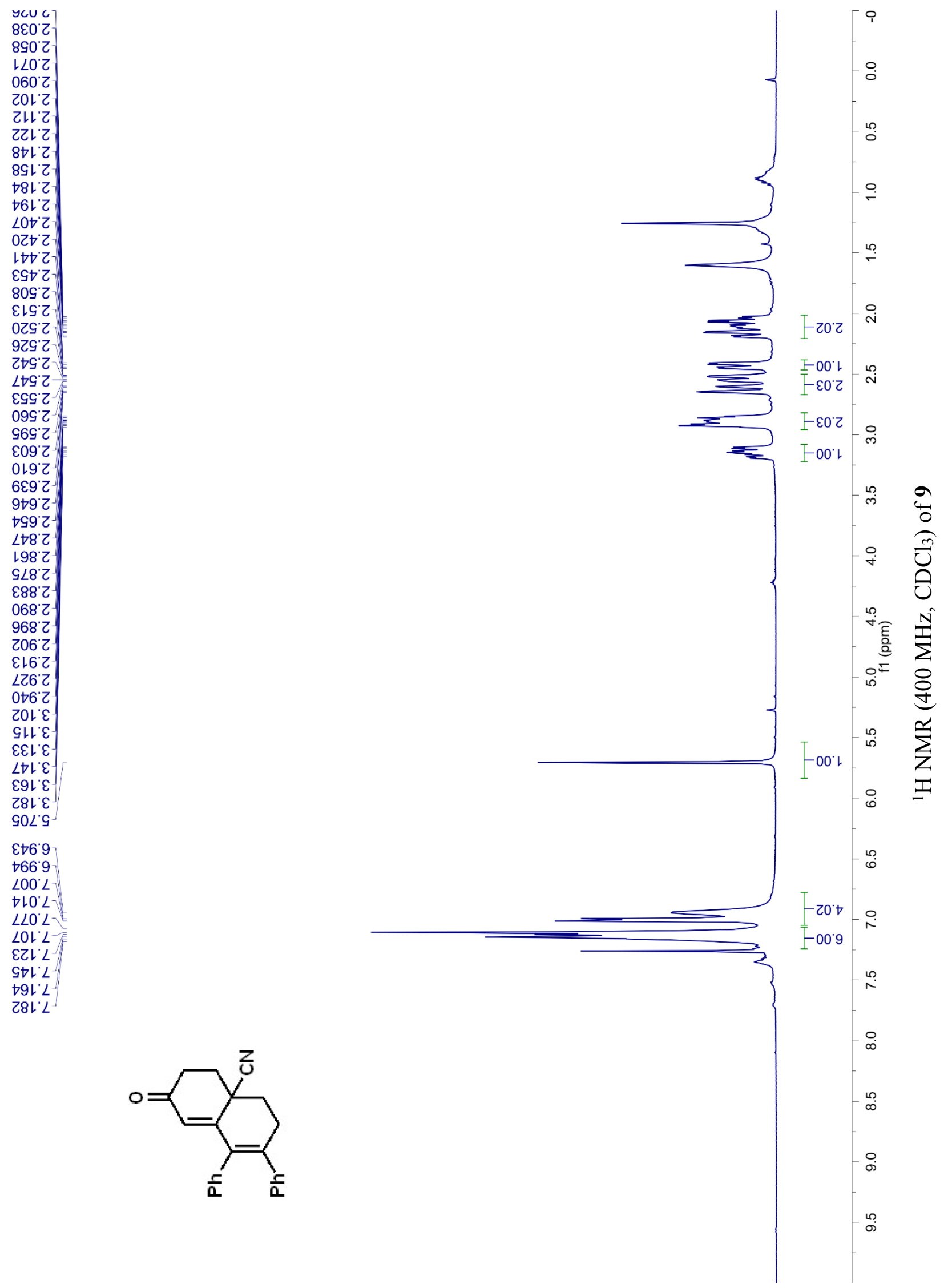


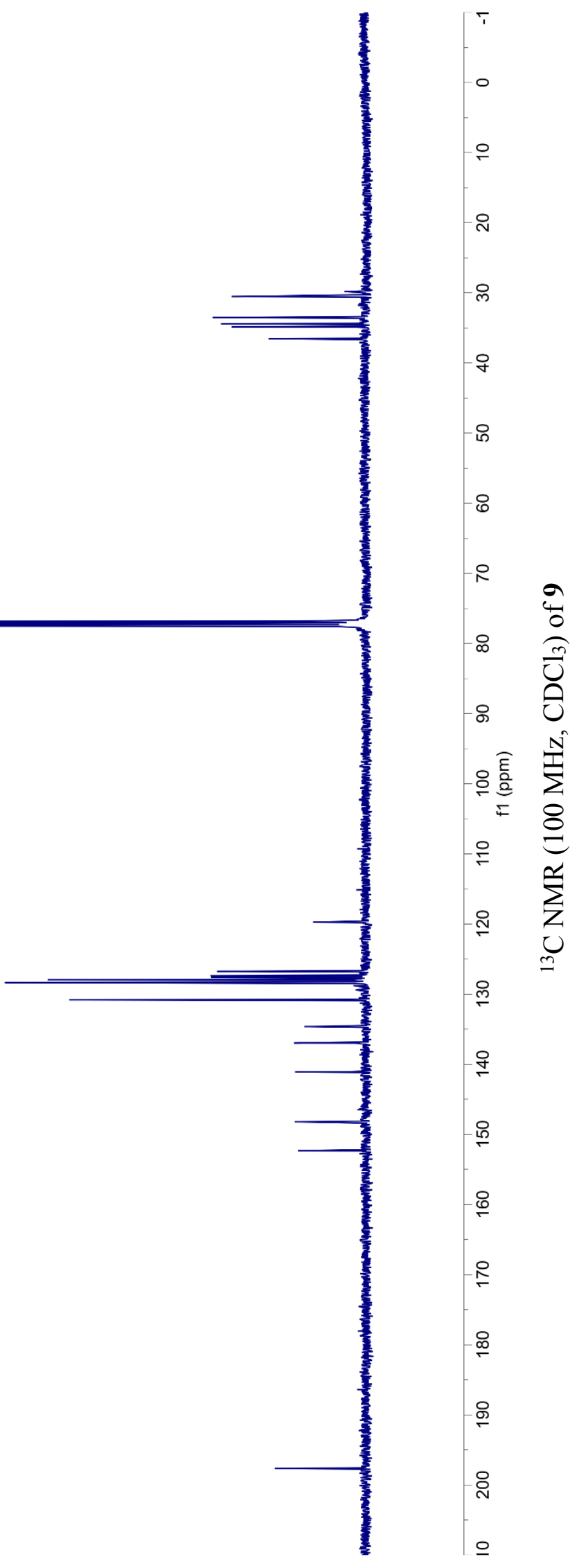

$\varepsilon 9^{\circ} \nabla \varepsilon L$

$86^{\circ} 9 \varepsilon \downarrow$

01ㄴt

して8†

$\downarrow \varepsilon^{\prime} Z S \downarrow$

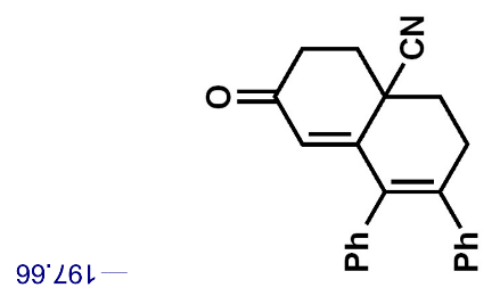

